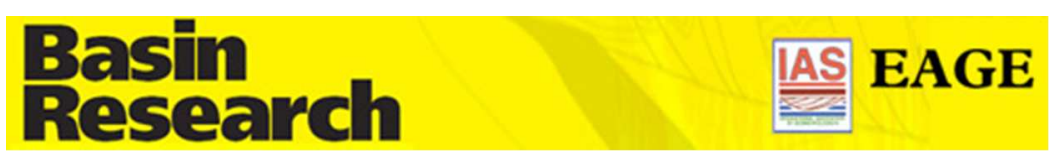

\title{
The zircon story of the Nile: time-structure maps of source rocks and discontinuous propagation of detrital signals
}

\begin{tabular}{|r|l|}
\hline Journal: & Basin Research \\
\hline Manuscript ID & BRE-134-2017.R1 \\
\hline Danuscript Type: & Original Article \\
\hline Complete List of Authors: & $\begin{array}{l}\text { Garzanti, Eduardo; University of Milano-Bicocca, Laboratory for provenance } \\
\text { studies, Department of Earth and Environmental Sciences } \\
\text { Vermeesch, Pieter; University College London, Geochronology Centre, } \\
\text { Department of Earth Sciences } \\
\text { Rittner, Martin; University College London, Geochronology Centre, } \\
\text { Department of Earth Sciences } \\
\text { Simmons, Matthew; University College London, Geochronology Centre, } \\
\text { Department of Earth Sciences }\end{array}$ \\
\hline Keywords: & $\begin{array}{l}\text { Nile River, Selection bias in detrital-zircon geochronology, Sm-Nd model } \\
\text { ages, Time structure of source terranes, Segmented sediment-routing } \\
\text { systems, Sediment budgets }\end{array}$ \\
\hline & \\
\hline
\end{tabular}

SCHOLARONE ${ }^{\text {m }}$

Manuscripts 


\title{
The zircon story of the Nile: time-structure maps of source rocks and discontinuous propagation of detrital signals
}

\author{
Eduardo Garzanti ${ }^{1 *}$, Pieter Vermeesch ${ }^{2}$, Martin Rittner ${ }^{2}$, Matthew Simmons ${ }^{2}$ \\ ${ }^{1}$ Laboratory for Provenance Studies, Department of Earth and Environmental Sciences, University \\ of Milano-Bicocca, 20126 Milano, Italy \\ ${ }^{2}$ London Geochronology Centre, Department of Earth Sciences, University College London, \\ London, WC1E 6BT, UK \\ * Corresponding author. Tel.: +39-02-64482088; fax: +39-02-64482073. \\ E-mail addresses: eduardo.garzanti@unimib.it (E. Garzanti), p.vermeesch@ucl.ac.uk \\ (P.Vermeesch), m.rittner@ucl.ac.uk (M.Rittner), mrsimmons77@icloud.com (M.Simmons) \\ ${ }^{1}$ Tel.: +39-02-64482088; fax: +39-02-64482073 \\ ${ }^{2}$ Tel.: +44-020-76792418; fax: +44-020-76792867
}

Keywords: Nile River; Selection bias in detrital-zircon geochronology; Sm-Nd model ages; Time structure of source terranes; Segmented sediment-routing systems; Sediment budgets. 


\section{ABSTRACT}

2 A new dimension has recently been added to provenance analysis by the rapid development of

3 detrital-geochronology techniques. The application of any dating method to sediments allows the

4 definition of a unique age pattern of parent rocks, a "time structure" that represents an essential

5 complement to the information on their lithological structure obtained by traditional petrographic

6

7 and mineralogical methods. This detrital-geochronology study illustrates the distribution of U-Pb zircon ages in all parts of the Nile catchment from its equatorial headwaters to the Delta, and surveys how the provenance signal is formed, transmitted, and modified along this huge sedimentrouting system. Age-spectra obtained by targeting specific parts of zircon grains after cathodoluminesce imaging and by our Automated Phase Mapping + LAICPMS "blind-dating strategy" were compared. The former approach emphasizes specific magmatic or metamorphic events in source areas, whereas the latter aims at minimizing selection bias and focuses on consistency among samples. Grain-size and hydraulic-sorting controls were also checked, but found to have only a minor effect on zircon-age distributions. The trimodal age spectrum of Kagera zircons sourced from the rift highlands of Burundi and Rwanda, characterized by prominent late Neoarchean (Aruan) and mid-Mesoproterozoic (Kibaran) peaks with a wider mid-Paleoproterozoic (Ubendian) cluster, is lost in the Lake Victoria sediment sink. The sharp unimodal Aruan peak displayed by zircon grains in both Victoria and Albert Nile is supplemented and finally superseded by Neoproterozoic grains across the vast marshlands of South Sudan, where detritus produced in equatorial regions is eventually stored. All Nile tributaries in Sudan and Egypt carry zircon grains yielding predominantly Neoproterozoic ages, with a major peak around $0.6 \mathrm{Ga}$ associated with clusters around 0.8 and $1.0 \mathrm{Ga}$. A few Oligocene zircons represent the key diagnostic feature of Ethiopian provenance, the Ariadne's golden thread that allowed retracing the paleo-Nile to its Ethiopian sources back in the Oligocene. The Nile presents a text-book case of discontinuous transmission of provenance signals along a segmented ultra-long drainage system. Zircon-age fingerprints as well as all other detrital signatures are lost repeatedly in large Ugandan lakes, reconstituted or replaced, lost again in the vast marshlands of South Sudan, and finally homogenized downstream from Ethiopia and Sudan to the Mediterranean Sea. 


\section{INTRODUCTION}

The formidable development of detrital geochronology in the last decade fostered real progress in provenance analysis, allowing the determination of the age of source terranes by a wide spectrum of techniques, the most frequently used being U-Pb dating in zircon, rutile and apatite, ${ }^{40} \mathrm{Ar} /{ }^{39} \mathrm{Ar}$ dating in white mica, and fission track dating of zircon and apatite (von Eynatten \& Dunkl, 2012). As a function of the targeted mineral and isotopic system, each technique reproduces a different age distribution across source areas, providing information on their complex thermal evolution through successive episodes of crustal growth (Vermeesch et al., 2009). In parallel, model mantle extraction ages (i.e., the inferred time of separation of continental crust from the mantle based on a geochemical model of the evolving crust-mantle system) can be derived by the analysis of isotopic parent-daughter tracers such as Sm-Nd or Lu-Hf (e.g., DePaolo \& Wasserburg, 1976; Goldstein et al., 1984; Vervoort et al., 1999). A variety of geochronological or model-age maps of source areas can thus be produced by the determination of isotopic ratios in bedrocks (e.g., Bennett \& DePaolo, 1987; DePaolo et al., 1991; Champion, 2013) or in sediments (Goldstein et al., 1997; Padoan et al., 2011). In provenance analysis, each of such diverse "time structures" (i.e., age patterns) of parent rocks obtained by applying a geochronological or isotopic method to daughter sediments represents a valuable complement to the description of their lithological structure as obtained by traditional petrographic and mineralogical approaches (e.g., Folk, 1968; Dickinson, 1970; Mange \& Morton, 2007).

Detrital geochronology thus provides a fundamental additional dimension to the classical interpretation of detrital modes, which are per se unable to determine whether igneous or metamorphic rocks exposed in source terranes are young or old, and hence whether those terranes are active orogenic belts or ancient orogenic roots exposed along rift shoulders or at the core of a craton (Garzanti, 2016). Inferring plate-tectonic setting of ancient sedimentary successions without the help of detrital geochronology can be very misleading (e.g., von Eynatten et al., 1996). 
In the case of modern sands, the lithological structure and the diverse time structures of source terranes are generally known through geological mapping and bedrock geochronology, respectively. Bedrock data, however, are seldom distributed evenly throughout an entire orogen or continental block. Detrital geochronology may thus help us to achieve a complete coverage of entire regions, to identify sources overlooked by bedrock geochronology, and to assess the relative importance of crustal-growth episodes provided that mineral fertilities and erosion rates in different sources are taken into due account (Moecher \& Samson, 2006; Vezzoli et al., 2016).

In the huge Nile River catchment, parts of which are scarcely accessible because of political and logistic reasons, detrital studies provide the only viable efficient approach to comprehensively investigate source terranes. To this end, bulk Sm-Nd isotope geochemistry on size classes ranging from cohesive mud to medium sand (Padoan et al., 2011) allowed us to produce the first timestructure map of source rocks across the entire catchment in terms of Sm-Nd model ages (fig. 10 in Garzanti et al., 2015). More recently, a range of methods have been deployed to characterize age signatures in sediments of the trunk river and its major branches, including zircon U-Pb geochronology integrated by $\mathrm{Hf}$ isotope compositions and Lu-Hf model ages, Ar-Ar data on white mica and plagioclase, $\mathrm{U}-\mathrm{Pb}$ data on rutile, and $\mathrm{Sr}, \mathrm{Nd}$ and $\mathrm{Hf}$ isotope analysis on mud samples (Fielding et al., 2017). The present study focuses on detrital-zircon geochronology to produce the $\mathrm{U}-\mathrm{Pb}$ zircon time-structure map of the entire Nile catchment, from its equatorial headwaters of Rwanda and Burundi to the Delta in the Mediterranean Sea, and investigates how provenance signals are formed, transmitted, lost, reconstituted, replaced, or modified along the successive tracts of this ultra-long segmented sediment-routing system.

\section{THE NILE}

The Nile River drains an area of $3 \cdot 10^{6} \mathrm{~km}^{2}$ and flows for $\sim 6700 \mathrm{~km}$ northward from $3^{\circ} 55^{\prime} \mathrm{S}$ to $31^{\circ} 30^{\prime}$ N (Fig. 1A; Woodward et al., 2015). Climatic conditions across this wide latitudinal range, spanning both the Equator and the Tropic of Cancer, range from warm humid in the south to hot hyperarid in the 
82

north. The Kagera-White Nile, the southern equatorial branch, is sourced from rift highlands of Burundi, Rwanda, Uganda and western Kenya, where 1-2 m of rainfall are distributed in two rainy seasons in autumn and spring. After receiving the outflow of Lakes Victoria, Kyoga and Albert, half of White Nile waters are lost in the Sudd marshes of South Sudan, which acts as a real dam (Sudd means "dam" in Arabic) for sediments derived from the south. The vast South Sudan marshlands represent the surface expression of buried late Mesozoic rift basins hosting up to 9-10 km-thick Lower Cretaceous to Quaternary sedimentary successions (McHargue et al., 1992; Mohamed et al., 2001; Dou et al., 2007). At the northern edge of the marshes, annual water discharge is restored to $\sim 30 \mathrm{~km}^{3}$ by the Sobat River, and the White Nile eventually flows slowly in its broad channel towards Khartoum, across an area that was occupied by a vast seasonal paleolake in the late Pleistocene (Lombardini, 1865; Williams et al., 2015). The two other major branches, the Blue Nile and Atbara Rivers, drain Ethiopian rift highlands, where elevations are mainly between 2000 and 3000 m a.s.l. with several peaks above $4000 \mathrm{~m}$. Climate is determined by the seasonal migration of the intertropical convergence zone from south to north and back, so that total annual precipitation progressively decreases northward from $>2000 \mathrm{~mm}$ to $\leq 1000 \mathrm{~mm}$ together with the length of the summer rain and runoff season (Fig. 1B). Fluvial regimes vary consequently from perennial in the subequatorial south (e.g., Baro River, the main branch of the Sobat), to seasonal for the Blue Nile, to markedly seasonal for the Dinder, Rahad, and Tekeze-Atbara rivers that virtually dry out from December to May. In August, the Blue Nile sees a third of its $48 \mathrm{~km}^{3}$ annual water discharge, and the Atbara nearly half of its $11 \mathrm{~km}^{3}$ discharge (Sutcliffe \& Parks, 1999). The main Nile receives no significant tributary water north of Atbara town, and hardly any rainfall $(<50 \mathrm{~mm} / \mathrm{a})$ across the Sahara. Its regime thus reflects evenly distributed runoff from equatorial Africa, with the superposed pulse of summer monsoonal floods from Ethiopian highlands. About $85 \%$ of the Blue Nile flow is concentrated from July to October, whereas high levels in the White Nile persist from late September to January. The White Nile provides the main Nile with $83 \%$ of its low-season flow, but only $10 \%$ of its peak flow (Fig. 1C). 
107 The ultra-long course of the Nile across almost 36 degrees of latitude represents a unique feature on the Earth's surface, strictly linked to the tectonic development of the East African divergent plate margin. Since the early Oligocene, dynamic uplift associated with magmatic upwelling in the south has created a prominent northwestward tilt in the northeastern African lithosphere of Sudan and Egypt, favouring the initial establishment of a paleo-Nile drainage (Adamson et al., 1993; Pik et al., 2003; Faccenna et al., 2018; Fielding et al., 2018). The linear course of the river, broadly following the geological boundary between the Pan-African Orogen and the Saharan Metacraton (Abdelsalam et al., 2003; Johnson et al., 2011), is confined by the Red Sea rift shoulder to the east (Fig. 1D). Nile waters are thus forced to flow northward for $>2000 \mathrm{~km}$ across the Sahara Desert, and only where the rift deviates towards the northeast along the Levant transform boundary can river waters eventually meet the sea.

\section{METHODS AND RESULTS}

Complete information on the petrographic and mineralogical composition of the 34 samples selected for this study are given in Garzanti et al. (2015), which also includes petrographic and heavymineral data on several samples collected and studied by Fielding et al. (2017). Detrital zircons were identified on heavy-mineral separates by Automated Phase Mapping (APM) with a QEMSCAN ${ }^{\circledR}$ WellSite $^{\mathrm{TM}}$ instrument (Vermeesch et al., 2017). One of the advantages of the APM approach is that all zircons are picked, including murky grains easily discarded by visual inspection but invariably confirmed to be zircon by LAICPMS analysis. This workflow allowed us to calculate also the area of each dated zircon grain from the number of pixels registered as zircon by QEMSCAN ${ }^{\circledR}$. Such grain-size information is however biased by the effect of variable polish depth during sample preparation: smaller grains below the level of polish are missed and larger grains may be preferentially polished away (Simmons, 2016 pp. 38-39).

$\mathrm{U}-\mathrm{Pb}$ zircon ages were determined at the London Geochronology Centre using an Agilent 7700x LAICPMS (laser ablation-inductively coupled plasma-mass spectrometry) system, employing a 
134 NewWave NWR193 Excimer Laser operated at $10 \mathrm{~Hz}$ with a $20 \mu \mathrm{m}$ spot size and $\sim 2.5 \mathrm{~J} / \mathrm{cm}^{2}$ 135 fluence. No cathodo-luminesce (CL) imaging was done, and the laser spot was always placed 136 "blindly" in the middle of zircon grains in order to treat all samples equally and avoid bias in 137 intersample comparison. Data reduction was performed using GLITTER 4.4.2 software (Griffin et

138 al., 2008). We used ${ }^{206} \mathrm{~Pb} /{ }^{238} \mathrm{U}$ and ${ }^{207} \mathrm{~Pb} /{ }^{206} \mathrm{~Pb}$ ages for zircons younger and older than $1100 \mathrm{Ma}$, respectively. No common $\mathrm{Pb}$ correction was applied (for further methodological information see supplementary material in Rittner et al., 2016). Grains with $>+5 /-15 \%$ age discordance were discarded, and 2413 concordant ages were obtained overall. The main age clusters observed in Nile sediments are referred to as the Neoproterozoic Pan African (Johnson et al., 2011), midMesoproterozoic Kibaran (Tack et al., 2010), mid-Paleoproterozoic Ubendian, and late Neoarchean Aruan (Link et al., 2010) thermal events (Fig. 2). The full geochronological dataset is provided in Appendix B.

\section{The White Nile}

The Kagera River, draining the Karagwe-Ankole Belt in Burundi and Rwanda (Fernandez-Alonso et al., 2012), carries zircon grains with mainly Kibaran (peak at $1355 \mathrm{Ma}$ ), Ubendian (polymodal cluster around $2 \mathrm{Ga}$ ) and Aruan ages (peak at $2613 \mathrm{Ma}$ ) to Lake Victoria. The youngest ages are Pan-African and the oldest Mesoarchean or even Paleoarchean (Table 1).

Both Victoria and Albert Nile sands are largely derived from high-grade Archean gneisses of the Congo Craton in Uganda (Link et al., 2010), as reflected by dominantly Aruan zircon ages (peak at $2583 \mathrm{Ma}$ ). The youngest ages are Pan-African and the oldest Mesoarchean. Paleoproterozoic ages occur in Victoria Nile sand but are minor in Albert Nile sand. These very same two samples were analysed also by Fielding et al. (2017) after collecting cathodo-luminescence images. Interlaboratory data comparison indicates similarly dominant Aruan ages, although Pan-African zircons are more common for the Victoria Nile than for the Albert Nile sample in this case (Table 1). Both datasets show a wide age gap between 1 and $2 \mathrm{Ga}$. 
In South Sudan to the north, Aruan zircon ages are still common in Bahr el Jebel sand at Juba (peak at 2557), but a major polymodal Pan-African cluster appears (peaks around $650 \mathrm{Ma}$ and $970 \mathrm{Ma}$, with a minor cluster around $840 \mathrm{Ma}$ ). In the Bor sample collected $160 \mathrm{~km}$ downstream, the Aruan peak is much smaller, and the Pan-African cluster predominant. Similar zircon-age spectra, with a dominant polymodal Pan-African cluster (major peak around $670 \mathrm{Ma}$ ) and an Aruan peak at 2583 Ma characterize sand of the Lol tributary, draining high-grade gneisses south of the political border with the Congo. Zircon grains carried by the Sobat tributary draining Neoproterozoic granitoid gneisses in southern Ethiopia yield dominant Pan-African ages, with a few Paleozoic and Aruan ages. Finally, zircon grains in White Nile sand between the Sudd marshes and the Gezira alluvial fan built by the Blue Nile (Williams \& Adamson, 1982) display a dominant, broad and asymmetrical Pan-African cluster (peak around $620 \mathrm{Ma}$ ), with one Cretaceous, one Carboniferous, and few Aruan ages (Table 1). The single Oligocene age obtained by Fielding et al. (2017) in the White Nile sample collected farther north reveals mixing with Blue Nile sediments of the Gezira fan.

\section{The Blue Nile and Atbara-Tekeze}

Detrital zircons carried by all Ethiopian branches of the Nile, draining Neoproterozoic basement and the overlying Mesozoic strata capped by Oligocene continental flood basalts (Merla et al., 1979; Gani et al., 2009) are invariably dominated by a trimodal Neoproterozoic cluster. Blue Nile sand displays a major peak around $820 \mathrm{Ma}$, a subordinate one around $620 \mathrm{Ma}$, and scattered ages around $1000 \mathrm{Ma}$, Atbara sand a major peak around $620 \mathrm{Ma}$, a subordinate one at $800 \mathrm{Ma}$, and a minor one around $940 \mathrm{Ma}$. Oligocene ages (24 - $32 \mathrm{Ma}$ ) represent 2\% of total U-Pb zircon ages in most samples (Fig. 3); the youngest zircon was found in Tekeze sand (11 Ma). Mesozoic and Paleozoic ages are also represented, together with a few Paleoproterozoic to Neoarchean ages. Blue Nile sand yielded a few Cretaceous and Jurassic zircons, Tekeze and Atbara sands more Paleoproterozoic and Archean zircons. From Blue Nile, Tekeze and Atbara samples collected in 
188

189

190

191

192

193

194

195

196

197

198

199

200

201

202

203

204

205

206

207

208

209

210

211

212

213

214

nearby sites, Fielding et al. (2017) obtained similar results, with somewhat richer Oligocene populations (Table 1).

\section{The main Nile from Sudan to the Delta}

The Nile River hardly receives any tributary sediment north of Atbara town and across the Sahara Desert. Zircon grains in Wadi Milk, draining the Saharan Metacraton and its Phanerozoic siliciclastic covers (Abdelsalam et al., 2003), yielded a dominant polymodal Pan-African cluster (main peak at $\sim 600 \mathrm{Ma}$ with shoulder around $670 \mathrm{Ma}$, subordinate peak around $770 \mathrm{Ma}$ with shoulder around $870 \mathrm{Ma}$ and tail to $1020 \mathrm{Ma}$ ) together with a few Cretaceous, Paleozoic, midPaleoproterozoic, and late Neoarchean ages.

Main Nile sand collected in Nubia before the closure of the Merowe Dam is dominated by the broad polymodal cluster of Neoproterozoic zircons typical of Ethiopian branches (major peak around 770 Ma, subordinate one around $610 \mathrm{Ma}$ ), and yielded only one Oligocene age, a few Early Triassic and early Paleozoic ages, and minor mid-Paleoproterozoic to late Neoarchean ages. A slightly higher percentage of Oligocene zircons is reported by Fielding et al. (2017).

Detrital zircons from Wadi Qena, draining Neoproterozoic basement and cover rocks of the Red Sea Hills, also yielded a dominant asymmetric polymodal Pan-African cluster with prominent peak at $\sim 610 \mathrm{Ma}$; the youngest age is Silurian whereas the oldest is late Neoarchean. Zircon ages obtained by Fielding et al. (2017) on sand of Wadi Hammamat, also draining the Red Sea Hills in southeastern Egypt, display a very similar distribution, dominated by an asymmetric polymodal Pan-African cluster with prominent peak at $\sim 610 \mathrm{Ma}$ and a few Oligocene and Cretaceous ages suggesting eolian contamination with both Nile and Saharan sand; mid-Paleoproterozoic and late Neoarchean ages also occur. Dune sand from the Western Desert of Egypt to the north, analysed by Fielding et al. (2018), yielded the same broad asymmetrical zircon-age cluster with peak at $\sim 610$ Ma. The youngest age is earliest Devonian. A mid-Paleoproterozoic cluster centered around 1950 Ma and a smaller late Neoarchean one also occur. 
215 Main Nile sands collected near Cairo before closure of the Aswan High Dam and in the modern

216 Delta are dominated by the polymodal and asymmetrical Pan-African cluster. The main peak is 217 invariably around $600 \mathrm{Ma}$, the subordinate one around $800 \mathrm{Ma}$, and minor clusters occur around 2181000 Ma. Oligocene ages represent 1\% of total zircons. Mesozoic to Ordovician ages are few. A 219 mid-Paleoproterozoic cluster and a few late Neoarchean ages are represented. The sample analysed in the lower Nile by Fielding et al. (2017) did not yield any zircon age younger than the Late 221 Triassic.

222

223

224

225

226

227

228

229

\section{POTENTIAL CONTROLS ON ZIRCON-AGE SPECTRA}

Zircon, an ultradense mineral (density $\sim 4.65 \mathrm{~g} / \mathrm{cm}^{3}$ ), is expected to be systematically concentrated in the fine tail of the size distribution of any sorted sediment deposited by a tractive current (Rubey, 1933; Garzanti et al., 2008). Different source rocks may shed populations of zircon grains characterized by different age and different size, as found for instance in Amazon River sediments (Lawrence et al., 2011). Measuring the size of dated zircon grains, looking for size-age relationships, and checking for hydrodynamic fractionation may thus provide fundamental clues for provenance diagnoses based on detrital-zircon geochronological data.

\section{Grain-size control}

The mineralogical composition of Nile sediments depends strongly on grain size. As readily visible in the field, white fluvial bars of litho-feldspatho-quartzose sand supplied mainly from crystalline basement contrasts sharply with the dark-coloured silty sand of levee deposits, dominantly derived from volcanic rocks (Garzanti et al., 2006, 2015). The intrasample and intersample mineralogical variability related to mixing of these two different detrital populations in different proportions is most evident in Blue Nile sand. We thus tested whether grain-size control was reflected in the distribution of zircon ages as well, and specifically whether volcanic-derived zircon grains yielding young Oligocene ages were preferentially smaller than Neoproterozoic grains derived from 
basement rocks. The relationship between grain size and U-Pb ages of 264 zircon grains from the 32-355 size window of Blue Nile sand sample 2964 collected just upstream of the Ethiopia/Sudan boundary, however, failed to reveal a clear grain-size dependence of zircon ages (Fig. 4).

\section{Hydraulic-sorting control}

All zircon grains need not display the same physical behaviour. Very old and U-rich zircons have undergone greater radiation damage and thus contain far more defects in their crystal lattice than young and U-poor zircons. The durability as well as the density of these metamictic grains may thus be reduced, and they could be preferentially leached chemically, destroyed mechanically, or removed selectively (Garzanti et al., 2009). In order to test this latter possibility, we compared the age spectra of detrital zircons in Nile Delta beach and beach-placer sands affected by hydrodynamic processes to very different degrees, confident that all zircons including dark murky grains were properly identified by the adopted APM + LAICPMS analytical method in all of the samples. However, despite the extreme density sorting undergone by the Rosetta beach placer, where ultradense grains have been concentrated to the highest degree, its zircon-age spectrum is not notably different from that of "normal" Delta beach samples, and even includes a greater percentage of old zircon grains. Thus, no major effect of hydraulic sorting on zircon-age distribution was detected (Fig. 3).

\section{BLIND VS. TARGETED DATING STRATEGIES}

Some of our samples were previously analysed by Fielding et al. (2017) using a strategy notably different from ours. These authors: a) separated zircon grains by hand-picking prior to U-Pb analysis, taking care to reduce selection bias by choosing all grains identified by the operator as zircon in one field of view; b) subjected all the selected grains to cathodo-luminescence imaging; c) used this data to identify which grains were simple and which were complex; d) in the former case they used transmitted-light images to target laser spots to avoid domain boundaries, cracks, and 
271 inclusions, whereas in the latter case they targeted laser spots to sample distinct domains within the

272

273

274

crystals, with the main aim to obtain meaningful ages and identify specific sediment sources. Instead, we spotted all grains objectively identified by Automated Phase Mapping as zircon in the heavy-mineral separate and next always placed the laser spot "blindly" in the middle of zircon grains. At the cost of losing detailed provenance information, this time-saving protocol represents the easiest way to ensure consistency among samples, which is a fundamental prerequisite in provenance studies where different age distributions are compared with each other like fingerprints. A possible effect of this simpler strategy could be a shift of the U-Pb age spectra towards older ages, because of the preferential sampling of old inherited zircon cores. Aiming for the grain interior also runs the risk of missing the potentially very informative young magmatic overgrowths that are found in the rims of multi-cycle zircon crystals. The risk of introducing spurious mixed ages (e.g., where spots penetrated different intragrain boundaries and growth zones or intersected common- $\mathrm{Pb}$-bearing inclusions) is instead prevented, because such averaging produces discordant $\mathrm{U}-\mathrm{Pb}$ compositions that are removed from the dataset (in the present case we have discarded grains with $>+5 /-15 \%$ age discordance, as explained above).

Direct comparison of our results with those of Fielding et al. (2017) provides us with a unique opportunity to evaluate the different outcomes of our "blind-dating" versus their "targeted-dating" approach, and to discuss advantages and disadvantages involved in either strategy. If the young end of the age spectrum is not considered, then the zircon $\mathrm{U}-\mathrm{Pb}$ age spectra of most of the eight couples of replicate samples from all major branches of the Nile catchment analysed by us with the APM+LAICPMS approach and by Fielding et al. (2017) after hand-picking and CL-imaging are barely distinguishable (Fig. 5). The lack of any marked selection bias is coherent with an internal consistency check of the Fielding et al. (2017) data, which revealed the preponderance of simple zircon grains of igneous origin in the trunk river and its main Ethiopian tributaries, where only a few grains include older, $\sim 1$ Ga cores (I. Millar, written comm. 2018). As a consequence, differences between the detrital-zircon $\mathrm{U}-\mathrm{Pb}$ age distributions of grain interiors and edges are minor 
297 for most samples (Fig. 6). In favorable cases such as this, the simpler and quicker "blind" analytical

298 protocol does not diminish our power to detect the presence of specific sediment sources.

299

300

301

302

303

304

305

306

307

308

309

310

311

312

313

314

315

316

317

The "blind-dating" and "targeted-dating" approaches, however, do produce a different outcome if a number of zircon grains display complex structure (Fig. 7). This is best shown by Victoria Nile and Albert Nile sands, where the very same samples WN17 $=3692$ and WN16 $=3690-$ collected jointly and only labeled differently by Y. Najman and E. Garzanti in 2007 - were analysed following different protocols. In the Albert Nile sample WN16 $=3690$, the interior and edge of zircon grains yielded broadly similar age distributions. In the Victoria Nile sample, instead, Fielding et al. (2017) obtained a higher number of Neoproterozoic ages. Specifically, cathodo-luminescence images highlight two populations of zircon cores, aged as $\sim 2.5 \mathrm{Ga}$ and 800-900 Ma, and zircon rims aged as $\sim 600$ Ma occurring on both (whereas 800-900 Ma zircons do not occur as rims on Archaean cores; I. Millar, written comm., 2018), a level of detail obviously lost by the "blind" approach. Growth of Neoproterozoic zircon rims reflects a tectono-metamorphic event documented by kyanite-garnet schists exposed in the Victoria Nile catchment and yielding U-Th-Pb monazite ages of $\sim 0.63 \mathrm{Ga}$ (Appel et al., 2005; Schenk et al., 2007). Such a Pan-African rejuvenation was extensive throughout the Saharan Metacraton and affected NW Uganda as well, but only marginally the gneissic rocks drained by the Albert Nile (fig. 1 in Abdelsalam et al., 2003).

Another major difference between the two datasets stems from the fact that Fielding et al. (2017) first discovered and next expressly targeted young Cenozoic grains to trace provenance from Ethiopian volcanic highlands (Fielding et al., 2018). This effort led them to strongly emphasize the percentage of zircon grains with ages $<40 \mathrm{Ma}$, representing as much as $14.3 \%$ and $7.5 \%$ of detrital zircons in their dataset for the Blue Nile and Atbara samples, but only $2.4 \%$ for both rivers in our replicate samples (Fig. 5). Conversely, the "blind dating" approach allowed us to obtain an unbiased estimate of the age distribution in the interior of zircon grains.

\section{TIME STRUCTURES OF SOURCE ROCKS}


The age information extracted from the isotopic signature of a detrital component and condensed in one number may correspond to a single major magmatic or metamorphic event, but it may also reflect a superposition of successive events or mixing from different crystal domains in the same grain or from different sources in the case of bulk-sediment analyses. Different isotopic systems generally yield very different age numbers, and the comparison of the time-structure maps thus produced may lead us to reconstruct the polyphase geological evolution of a vast region.

In the case of the Nile catchment, the time-structure maps produced by U-Pb zircon-age and $\mathrm{Sm}-\mathrm{Nd}$ fingerprinting display similar patterns overall, but they characterize the major geological domains with notably different age labels (Fig. 8). Sm-Nd $t_{\mathrm{DM}}$ model ages up to 1 Ga older than the age of source rocks characterize sediments sourced from the Karagwe-Ankole belt and Virunga volcanoes in Burundi and Rwanda. This reflects repeated reworking and re-melting of early Paleoproterozoic crust through the late Paleoproterozoic and Mesoproterozoic (Tack et al., 2010), and contamination with Paleoproterozoic crust underlying the volcanoes (Rogers et al., 1998). Instead, Sm-Nd $t_{\mathrm{DM}}$ model ages are only $\sim 0.2 \mathrm{Ga}$ older than $\mathrm{U}-\mathrm{Pb}$ zircon ages for Ethiopian flood basalts underlain by Neoproterozoic crust. Another major difference between the two maps in Fig. 8 reflects the inability of $\mathrm{U}-\mathrm{Pb}$ zircon age-spectra to differentiate clearly between ultimate provenance from the Saharan Metacraton plus cover strata versus Pan-African terranes exposed all along the Red Sea rift shoulder, both sources being characterized by a dominant multimodal cluster of Neoproterozoic ages.

\section{ZIRCON AGES AND SEDIMENT BUDGETS}

Zircon, widespread in recycled sands and ancient sandstones because of its durability, is the most commonly targeted mineral in detrital geochronology. U-Pb ages of detrital zircons, determined routinely at a reasonable cost, faithfully reflect the crystallization ages of exposed magmatic and metamorphic rocks. As any other technique, however, detrital-zircon geochronology has its own pitfalls. Source rocks that contain little zircon or none at all (e.g., mafic and ultramafic rocks, 
351 limestone, chert) remain in the shadow, whereas felsic igneous and metaigneous rocks rich in zircon 352 crystals are obviously overrepresented ("fertility" bias; Moecher \& Samson, 2006; Dickinson, 2008;

353

354

355

356

357

358

359

360

361

362

363

364

365

366

367

368

369

370

371

372

373

374

375

376

377

Malusà et al., 2013, 2016). Durability, which is on the one hand the principal reason why zircon is so precious in provenance research, may on the other hand represent a disadvantage, because grains may be recycled over and over from one sedimentary cycle to the next, with age-spectra remaining unchanged through time and space and therefore ceasing to represent useful provenance tracers (Thomas et al., 2004; Dickinson et al., 2009; Garzanti et al., 2013a).

\section{Hints for the calculation of a zircon budget}

In all three major Nile branches (White Nile, Blue Nile, and Atbara), as well as in wadi sands from Nubia and the Red Sea Hills and in eolian sands of the Western Desert, U-Pb age spectra of detrital zircons are all alike, and invariably dominated by a multimodal Neoproterozoic cluster (Fig. 2). Such a similarity makes the calculation of a zircon provenance budget based on the comparison among age spectra hardly feasible. The problem can be tackled in reverse, by first estimating zircon concentration in the main contributing sources. Zircon concentration can be calculated from integrated petrographic, heavy-mineral, and geochemical data, under the assumption that zircon contains on average $465,000 \mathrm{ppm} \mathrm{Zr}$ and contributes between $60 \%$ and $80 \%$ of total $\mathrm{Zr}$ to sand derived largely from metamorphic basement (Garzanti et al., 2010). In sand derived from continental flood basalts, volcanic rock fragments are the major contributor of $\mathrm{Zr}$, and zircon grains released from the associated subordinate felsic products may contribute only $20 \%$ of total $\mathrm{Zr}$, as calculated for Atbara sand (Padoan et al., 2011). Under these assumptions, based on the integrated petrographic, heavy-mineral, and geochemical dataset in Garzanti et al. (2015), zircon concentration is estimated to be $0.02-0.05 \%$ in White Nile sand, and thus notably higher than in Blue Nile (0.01 - 0.02\%) and Atbara sands ( 0.01\%).

Although petrographic and mineralogical data have long documented that the White Nile, Nubian widyan (plural of wadi = "dry valley"), and the Red Sea Hills in Egypt supply very little sediment to 
378 the main Nile (<5\%; Shukri, 1950 p. 521; Garzanti et al., 2006), the low zircon concentration in

379 Blue Nile and Atbara sands suggests that sources other than Ethiopian highlands may contribute

380

381

382

383

384

385

386

387

388

389

390

391

392

393

394

395

396

397

398

399

400

401

402

403 significantly to the zircon budget. In particular, the slightly higher amount of Paleoproterozoic to Neoarchean ages observed in the Egyptian lower tract of the Nile and in the Delta points to additional zircon supply from the Red Sea Hills and/or Saharan Metacraton with its widespread cover strata of "Nubian" sandstones (Klitzsch \& Squyres, 1990). Although dominant in the Albert Nile, these old grains are not derived from Uganda, because the Aruan trace is lost in the White Nile downstream of the Sudd. Among Ethiopian sources, only the Tekeze contributes a few zircons with Aruan ages, which remain very scarce in the main Nile across Sudan.

\section{PROPAGATION OF DETRITAL SIGNALS IN A SEGMENTED RIVER SYSTEM}

The transmission across large river basins of detrital signals triggered by tectonic, climatic or eustatic processes (Blum \& Törnqvist, 2000; Castelltort \& Van Den Driessche, 2003), the effect of temporary or permanent internal sediment storage (Wittman et al., 2016), and time lags between erosion and deposition and consequent potential mismatches between terrestrial and marine records (Nie et al., 2015) are widely debated issues in tectonic sedimentology (Leeder, 2011; Hinderer, 2012). Each big river on Earth is a sediment-routing system with its own peculiarities. Most have internal sediment sinks of various origin, but perhaps none as many and varied as the Nile, where they range from major Ugandan lakes (Victoria, Kyoga, George, Albert) variously related to rift tectonics and geomorphology, to the vast subsident Sudd and Machar marshlands of South Sudan, and to the artificial reservoirs created in Sudan and Egypt by man in the last hundred years (e.g., Roseires, Sennar, Khashm el Girba, Merowe, LakeNubia / Nasser). The Nile is unique in being controlled from the headwaters to the Delta by rift-related tectonic features (Adamson et al., 1993), active since Cretaceous times (McHargue et al., 1992) and cutting across old cratonic basement extensively remobilized during the Neoproterozoic orogeny (Abdelsalam et al., 2002). From the 
404 study of the Nile River we learn that sediment transport in a long drainage system may be complex 405 and very discontinuous. As a consequence, the transmission of provenance signals can be repeatedly blocked, regenerated or lost, get mixed or overwhelmed repeatedly along the course of the river, thus introducing major uncertainties in the calculation of sediment budgets.

\section{Discontinuous propagation of the zircon-age provenance signal}

The $\mathrm{U}-\mathrm{Pb}$ age signature of zircon grains changes drastically and repeatedly along the White Nile in Uganda and South Sudan, whereas it remains remarkably homogeneous from downstream of the Sudd marshes to the Delta (Fig. 3). In Uganda, the upstream signal is lost in correspondence with any big lake, representing an effective barrier to sediment transport. And each time it is replaced by a different or even by a similar signal, depending on the age of zircons contained in the rocks and sedimentary deposits eroded downstream of the lake. The trimodal Kibaran + Ubendian + Aruan zircon-age spectrum characterizing Kagera sediments and inherited from source rocks exposed in rift highlands of Burundi and Rwanda cannot overstep Lake Victoria. The sharp unimodal Aruan peak, characterizing zircon grains both in the Victoria Nile downstream of Lake Kyoga and in the Albert Nile downstream of Lake Albert, combines with Neoproterozoic grains increasing progressively in frequency across the lowlands of South Sudan. The Aruan signal does not pass the Sudd marshes, and White Nile zircons in Sudan display the same Neoproterozoic polymodal cluster as wadi and eolian sand derived from the Saharan Metacraton and its sedimentary covers in Nubia (Wadi Milk, Western Desert dunes) or from the Red Sea Hills (Wadi Qena, Wadi Hammamat). This Neoproterozoic signal is similar to that of Blue Nile, Atbara and main Nile sediments all the way to the Delta except for its lack of Oligocene zircon ages (Fig. 2). The Saharan Metacraton extensively remobilized during the Neoproterozoic - and its "Nubian" siliciclastic covers thus supply first-cycle and polycyclic detritus characterized by almost the same zircon-age signal as that of the Neoproterozoic Pan-African belt exposed all along the Red Sea rift shoulder from Ethiopia to Egypt. The rather homogeneous age-distribution shown by Nile zircons from Khartoum to the Delta 
431 retains virtually no memory of provenance signals (i.e., Kibaran, Ubendian, and especially Aruan 432 ages) emitted in the vast White Nile basin upstream of the Sudd (Fig. 2).

433

434

435

436

437

438

439

440

441

442

443

444

445

446

447

448

449

450

451

452

453

454

455

456

457

\section{Discontinuous propagation of other provenance signals}

All provenance signals share the same fate as that of the zircon-age signal. As far as sand petrography and heavy minerals are concerned, the quartzose sand of the Kagera River containing staurolite and kyanite is stored in Lake Victoria. In the upper Victoria Nile downstream of Lake Victoria, feldspar-rich sand with epidote-dominated heavy-mineral suites is regenerated locally, only to be stored next in Lake Kyoga. In the lower Victoria Nile downstream of Lake Kyoga, quartzose sand with zircon-rich suites is contributed locally by the Kafu tributary, but it is soon overwhelmed by feldspatho-quartzose detritus with amphibole-dominated suites that is successively stored in Lake Albert. In the Albert Nile downstream of Lake Albert, quartzose sand with epidote, kyanite and amphibole reworked locally is soon overwhelmed by feldspatho-quartzose detritus with amphibole-dominated suites transported by the Bahr el Jebel and finally stored in the Sudd marshes. The White Nile downstream of the Sudd carries only a small amount of pure quartzose sand with epidote and amphibole recycled locally, which is overwhelmed at Khartoum by the sediment mass flushed from Ethiopia by the Blue Nile. The composition of Nile sediments from Khartoum to the Delta is strongly size-dependent, ranging from quartzo-feldspatho-lithic volcaniclastic for silty sand carried in suspension to litho-feldspatho-quartzose for coarser sand bedload.

$\mathrm{Sr}$ and $\mathrm{Nd}$ isotopes provide perhaps the clearest evidence of such a discontinuous transmission of provenance signals along the segmented White Nile sediment-routing system (fig. 17 in Garzanti et al., 2015). The isotopic signatures of Kagera sediments (high ${ }^{87} \mathrm{Sr} /{ }^{86} \mathrm{Sr}$ ratio, negative $\varepsilon \mathrm{Nd}_{(0)}$, and Paleoproterozoic Sm-Nd model age) are all lost in Lake Victoria. Victoria Nile and Albert Nile sediments are characterized by lower ${ }^{87} \mathrm{Sr} /{ }^{86} \mathrm{Sr}$ ratio, much more strongly negative $\varepsilon \mathrm{Nd}_{(0)}$, and Mesoarchean to Paleoarchean Sm-Nd model ages. This fingerprint gets blurred progressively across

South Sudan, where the ${ }^{87} \mathrm{Sr} /{ }^{86} \mathrm{Sr}$ ratio decreases further, $\varepsilon \mathrm{Nd}_{(0)}$ becomes much less strongly 
458 negative, and Sm-Nd model ages are first Neoarchean, next Paleoproterozoic, and finally 459 Mesoproterozoic (Fig. 9). The original isotopic signatures of Sobat River sediments sourced from 460 Pan-African terranes in southern Ethiopia (low ${ }^{87} \mathrm{Sr} /{ }^{86} \mathrm{Sr}$ ratio, only slightly negative $\varepsilon \mathrm{Nd}_{(0)}$, and 461 Neoproterozoic Sm-Nd model age) change as well across the marshes, but in the opposite direction, 462 ending up with the same ${ }^{87} \mathrm{Sr} /{ }^{86} \mathrm{Sr}$ ratio, moderately negative $\varepsilon \mathrm{Nd}_{(0)}$, and Mesoproterozoic $\mathrm{Sm}-\mathrm{Nd}$

463

464

465

466

467

468

469

470

471

472

473

474

475

476

477

478

479

480

481

482

483 model age as White Nile sediments. This indicates that even detritus derived from Ethiopia via the Sobat River is dumped in the marshes and replaced by local reworking within South Sudan lowlands.

The climatic imprint carried by clay minerals is also lost across the Sudd marshes (Fig. 9), where kaolinite-dominated muds transported from hot and humid Uganda are deposited and replaced by smectite-dominated muds reworked locally and carried by the White Nile downstream. This indicates that not even the finest sediment fraction can pass the marshlands. Smectite represents the dominant clay mineral from Ethiopia and Sudan to the Mediterranean sea floors (El-Attar \& Jackson, 1973; Stanley \& Wingerath, 1996).

The transmission of provenance signals is thus disrupted very effectively by long-lived natural sediment sinks (Fig. 9). Instead, only minor changes in sediment signatures are observed downstream of artificial reservoirs, even where the sediment flux is reduced drastically or stopped altogether. Sand samples collected before and after closure of the Aswan High Dam, which has effectively forced the displacement of the ultimate Nile sediment sink a thousand km inland from the Mediterranean Sea to the upstream end of Lake Nubia / Nasser, do not show marked systematic changes in petrographic, heavy-mineral, or isotopic signatures. This is ascribed to prompt regeneration of the original compositional fingerprints by reworking of channel and overbank sediments deposited downstream at earlier times. The effect of the Aswan High Dam on sediment composition, as observed after half a century, is limited to somewhat increased mixing proportions of Nile sediments with wind-blown quartz, as most evident in coarser sand fractions (Garzanti et al., 2015), and to a marked local increase in wind-blown kaolinite in the clay fraction (Stanley \& 
484

485

486

487

488

489

490

491

492

493

494

495

496

497

498

499

500

501

502

503

504

505

506

507

508

509

Wingerath, 1996). These only minor compositional effects point to virtually negligible tributary sediment supply in Egypt, owing to arid climate in the narrow and mildly elevated Red Sea Hills, and reflect the main direction of prevailing winds, which blow roughly parallel to the Nile Valley rather than across it (Hereher, 2014).

At times in the past, however, the transmission of detrital signals could have been disrupted periodically also in the final tract of the lower Nile. During arid stages, water discharge decreased sharply and the trunk river may have been subdivided into a series of disconnected tracts. During the extremely dry conditions of Marine Isotope Stage 2, for instance, invasion of eolian dunes from the Western Desert is believed to have formed a series of lakes where late Paleolithic human populations of hunter-fisher-gatherers could find ideal conditions for subsistence (Vermeersch \& Van Meer, 2015). Long rivers draining across polyhistory cratonic basements hosting recent or old rejuvenated rift sags (Adamson et al., 1993; Salama, 1997), especially if flowing across desert areas and therefore depending on water reservoirs located in humid faraway source regions, are thus likely to be characterized by sediment-routing systems as segmented as those of heavily dammed rivers in the Anthropocene (e.g., Indus River; Garzanti et al., 2005). This represents a major element of uncertainty to be taken into account in paleogeographic and paleoclimatic reconstructions based on the study of stratigraphic successions accumulated in the terminal sediment sink (e.g., Hinderer, 2012; Nie et al., 2015).

\section{SUMMARY}

This geochronological study provides a complete coverage of the ultra-long Nile River catchment, from the equatorial sources in humid central Africa, across the hyperarid Sahara, and as far as the Mediterranean Sea (Fig. 1). It monitors where, how, and why a provenance signal is generated, transmitted, lost, recreated, or replaced along such a huge segmented sediment-routing system. It also discusses the advantages and disadvantages of "blind" versus "targeted" approaches to zircon- 
510 dating in provenance studies aimed at defining ages of source rocks or calculating sediment 511 budgets.

512 Zircon grains in all Nile branches from Ethiopia and Sudan to the Delta yield a dominant polymodal 513 cluster of Neoproterozoic $\mathrm{U}-\mathrm{Pb}$ ages, reflecting accretion of the Arabian-Nubian Shield and 514 rejuvenation of the Saharan Metacraton during the major polyphase "Pan-African" Orogeny (Fig. 515 2). Young zircons with Oligocene ages, occurring in Blue Nile and Tekeze-Atbara sands and traced 516 as far as the Delta, provide a unique fingerprint of provenance from the Ethiopian volcanic plateau 517 (Fielding et al., 2017, 2018). The very different signals emitted from equatorial rift highlands, 518 including the trimodal Mesoproterozoic (Kibaran), Paleoproterozoic (Ubendian) and Neoarchean 519 (Aruan) peaks characterizing Kagera sand, and the unimodal Aruan peak with subordinate 520 Neoproterozoic ages characterizing Victoria Nile and Albert Nile sands, do not propagate beyond provenance signals, independently of grain size (Fig. 9). Sand petrography, heavy-mineral assemblages, $\mathrm{Rb}-\mathrm{Sr}$ and $\mathrm{Sm}-\mathrm{Nd}$ isotopic ratios, and clay minerals indicate that all sediment fractions are dumped repeatedly and trapped efficiently in major Ugandan lakes first, in South Sudan marshlands next, and finally in artificial reservoirs built in Sudan and Egypt in the last century by man.

An exceptional opportunity to investigate the effect of selection bias on detrital-zircon $\mathrm{U}-\mathrm{Pb}$ age spectra was offered by previous analyses of eight couples of replicate samples from all major branches of the Nile catchment following a different protocol. Fielding et al. (2017) used cathodoluminescence images to guide the placement of the laser spot, whereas we consistently analysed "blindly" the interior of randomly selected zircon grains. In most cases, hardly any difference is observed in the age-spectra obtained on the same sample set when CL-imaging was used and when it was not (Fig. 5), which is explained by the fact that many zircons in Nile sands are simple igneous grains without complex zoning. Where complex zircon grains make up a considerable proportion of the sample under study, as in the Ugandan branches of the White Nile, our "blind- 
536 dating" strategy provided consistent results but failed to identify all details of the multi-step 537 geological evolution of source rocks during Neoproterozoic orogenic events highlighted instead by the "targeted-dating" approach (Fig. 7). Another major difference is seen for the minor but provenance-diagnostic population of Oligocene zircons, which were specifically targeted by Fielding et al. (2017) to trace provenance from Ethiopian volcanic highlands and are consequently over-represented in their Blue Nile and Atbara spectra (Fig. 5).

Diverse detrital-geochronology techniques allow the definition of multiple "time structures" of source rocks (Fig. 8), and thus provide a fundamental additional dimension to the traditional interpretation of detrital modes, which cannot determine by themselves whether igneous or metamorphic rocks exposed in source terranes are young or old, and hence whether those terranes are active orogenic belts or ancient cratonic blocks.

\section{ACKNOWLEDGMENTS}

Samples were collected in the field with Lilly Bau, Leo, Cecilia, Ada Ali Abdel Megid, Marta Padoan and Yani Najman, obtained with the help of Mohammed Khaled, Maurizio Levi and Beatrice Spadaccini, or retrieved from the Geological Museum of the Fouad I University at Cairo thanks to Ahmed El Kammar and Ibtsam Arafa. The article benefited greatly from the extremely useful constructive comments provided by Reviewers Ian Millar, Yani Najman, and Scott Samson. Ian Millar very kindly provided cathodo-luminescence images and many pieces of detailed information on the zircon grains analysed by Fielding et al. (2017).

\section{SUPPORTING INFORMATION}

Supplementary data associated with this article can be found in the online version, at http://doi. Appendix A includes information on sampling sites (Table A1), and the bulk-sand petrography (Table A2), heavy-mineral (Table A3), and geochemical (Table A4) 
1

2

3

4

5

6

7

8

9

10

11

12

13

14

15

16

17

18

19

20

21

22

23

24

25

26

27

28

29

30

31

32

33

34

35

36

37

38

39

40

41

42

43

44

45

46

47

48

49

50

51

52

53

54

55

56

57

58

59

60

562 datasets. Appendix B provides the complete U-Pb zircon geochronological dataset. The Google-

563 Earth $^{\mathrm{TM}}$ map of sampling sites Nilezircon. $\mathrm{kmz}$ is also provided. 
564

565

566

567

568

569

570

571

572

573

574

575

576

577

578

579

580

581

582

583

584

585

586

587

588

589

\section{FIGURE CAPTIONS}

Figure 1. The Nile River catchment. A) Location of samples selected for U-Pb detrital-zircon geochronological analysis (yellow circles = samples collected between 2002 and 2009 before closure of the Merowe Dam; orange circles = samples collected by the Egyptian Irrigation Department before construction of the Aswan High Dam; full information on sampling sites provided in Appendix Table A1 and Google-Earth ${ }^{\mathrm{TM}}$ map Nilezircon.kmz); $\mathrm{R}=$ Rwanda; $\mathrm{B}=$ Burundi; C.A.R. = Central Africa Republic. B) Rainfall map. Nile cataracts and major dams are shown, together with location of modern sands (white circles) analysed for U-Pb detrital zircon geochronology by Fielding et al. (2017). C) Water discharge (Sutcliffe \& Parks, 1999) and sediment fluxes (Garzanti et al., 2006). D) Geological map (redrawn from Asga-Unesco, 1963 and other sources cited in text).

Figure 2. U-Pb age spectra of detrital zircons (age vs. frequencies plotted as Kernel Density Estimates using the provenance package of Vermeesch et al., 2016). Sediments carried by the Kagera River are dumped in Lake Victoria, where the Kibaran and Ubendian signals are lost. The Aruan signal is restored in the Victoria Nile, and once again in the Albert Nile downstream of Lake Albert, but it is then progressively lost across the Sudd marshes of South Sudan, where White Nile sand becomes dominated by the Pan-African signal. Polymodal Pan-African ages are dominant not only in Blue Nile and Atbara sediments derived from Ethiopia, but also in sediments of widyan draining the Saharan Metacraton and its siliciclastic covers in Nubia and the Red Sea Hills in Egypt. Zircons of Oligocene age are diagnostic of provenance from Ethiopian volcanic highlands.

Figure 3. Discontinuous downstream propagation of the zircon-age provenance signal. Note the stark contrast between age-spectra upstream and downstream of Lake Victoria in Uganda, and between upstream and downstream of the Sudd marshes in South Sudan. Spectra become 
590 homogeneously dominated by Neoproterozoic ages from Khartoum to the Delta. The young 591 population of Oligocene grains derives from the Ethiopian volcanic plateau drained by the Atbara

592

593

594 and Blue Nile. In the Delta, similar spectra for the Rosetta magnetite placer and other beaches indicate minor effect of selective-entrainment processes on zircon-age distributions.

Figure 4. Grain-size information obtained during QEMSCAN $^{\circledR}$ analysis (Simmons, 2016). Upper panel: U-Pb age versus circular equivalent diameter of zircon grains from Blue Nile sample 2964 (age color-code as in Fig. 3). Evidence for grain-size control is lacking, although it may be masked by bias introduced by variable polish depth during sample preparation. Lower panel: Size distribution of zircon grains (blue) versus that of the total heavy-mineral population (black).

Figure 5. Comparison of U-Pb zircon-age distributions obtained with different analytical protocols from eight couples of replicate sand samples. Above in magenta: CL-guided "targeted-dating strategy" (data from Fielding et al., 2017); below in blue: "blind-dating strategy" (this study). The two methods yielded different results in the young end of the spectrum (yellow arrows) because grains of Oligocene age, specifically targeted as tracers of Ethiopian volcanic provenance by Fielding et al. (2017), are consequently over-represented in their dataset. WN17 $=3692$ and WN16 $=3690$ are the very same two samples collected in the Victoria and Albert Nile by Y. Najman and E. Garzanti in 2007. Each of the other six couples was collected in the same or in nearby localities by different operators in different years.

Figure 6. Overall consistency of $\mathrm{U}-\mathrm{Pb}$ ages obtained targeting the grain interior (above in magenta) or the grain edges (below in orange) of detrital zircons. Most studied grains were in fact simple, with no xenocrystic cores or late overgrowths (all data after Fielding et al., 2017). A notable exception - illustrated further in Fig. 7 - is the higher frequency of Neoproterozoic ages yielded by zircon rims in the Victoria Nile sample. 
616

617

618

619

620

621

622

623

624

625

626

627

628

629

630

631

632

633

634

635

636

637

638

639

640

641

Figure 7. Notable differences in U-Pb zircon-age distributions are obtained with "blind" and "targeted" approaches for samples including numerous grains with complex age structure. This is the case of the White Nile in Uganda (data from subsamples WN17 and WN16 after Fielding et al., 2017), where a higher frequency of Neoproterozoic (Npz) ages was obtained by the CL-guided strategy for the Victoria Nile sample, especially from zircon rims. This reflects the Pan-African rejuvenation event recorded extensively throughout the Saharan Metacraton as far as NW Uganda but only marginally by gneissic rocks in the Albert Nile catchment.

Figure 8. Different time-structure maps of source rocks in the Nile catchment based on detrital zircon geochronology (left) and Sm-Nd fingerprinting (right; Padoan et al., 2011; Garzanti et al., 2013b). Dominant Neoproterozoic U-Pb zircon ages characterize both Pan-African basement exposed along the Red Sea rift shoulder and drained by all Ethiopian rivers (Sobat, Blue Nile, Atbara and their tributaries) and the Saharan Metacraton with its siliciclastic covers (Abdelsalam et al., 2003). Neogene Virunga volcanoes and Oligocene Ethiopian lavas yield detritus with late Mesoproterozoic and Permo-Triassic Sm-Nd model age, respectively, revealing interaction of rising magmas with Paleoproterozoic and Neoproterozoic basement rocks, respectively. Sample locations are shown; details of map contours are drawn according to the geological map (Fig. 1).

Figure 9. Repeated decoupling of provenance signals along the White Nile sediment-routing system. Detrital fingerprints of Kagera sand are lost in Lake Victoria. Sand derived from the Archean Congo-Tanzania craton is carried by the Victoria Nile to Lake Albert, and by the Albert Nile/Bahr el Jebel to the Sudd marshes. Sediments exiting the Sudd marshes have lost all provenance signals from headwater sources. Repeated trapping in natural sediment sinks explains the very minor sediment contribution of the White Nile to the main Nile downstream of Khartoum. Petrographic, heavy-mineral and isotopic data after Garzanti et al. (2015); clay-mineral data after 
642 Buursink (1971), El-Attar \& Jackson (1973), De Vivo et al. (1981), and Nyakairu \& Koeberl 643 (2001).

644

645 Table 1. Distribution of U-Pb zircon ages along the Nile sediment-routing system. ${ }^{\circ}$ Samples 646 collected before closure of the Aswan High Dam; * data after Fielding et al. (2017). 


\section{REFERENCES}

ABDELSAlAM, M.G., LIÉGEOIS, J.P. \& STERN, R.J. (2002) The Saharan Metacraton. Review. Journal of African Earth Sciences, 34, 119-136.

ABDELSALAM, M.G., ABDEL-RAHMAN, E.S.M., EL-FAKI, E.F.M., AL-HUR, B., EL-BASHIER, F.R.M., STERN, R.J. \& THURMOND, A.K. (2003) Neoproterozoic deformation in the northeastern part of the Saharan Metacraton, northern Sudan. Precambrian Research, 123, 203-221.

ADAMSON, D., MCEVEDY, R. \& WILLIAMS, M.A.J. (1993) Tectonic inheritance in the Nile basin and adjacent areas. Israel Journal of Earth Sciences, 41, 75-85.

APPEL, P., SCHENK, V. \& SCHUMANN, A. (2005) P-T path and metamorphic ages of pelitic schists at Murchison Falls, NW Uganda: evidence for a Pan-African tectonometamorphic event in the Congo Craton. European Journal of Mineralogy, 17, 655-664.

ASGA-UNESCO (1963) Geological map of Africa, sheet N.3, scale 1:5,000,000. Association of African Geological Surveys, United Nations Educational, Scientific and Cultural Organization, Paris.

BENNETT, V.C. \& DEPAOLO, D.J. (1987) Proterozoic crustal history of the western United States as determined by neodymium isotopic mapping. Geological Society of America Bulletin, 99, 674-685.

BLUM, M.D. \& TÖRNQVIST, T.E. (2000) Fluvial responses to climate and sea-level change: a review and look forward. Sedimentology, 47, 2-48.

BUURSINK, J. (1971) Soils of central Sudan. Grafisch Bedrijf Schotanus \& Jens, Utrecht, The Netherlands, $248 \mathrm{p}$.

CASTELLTORT, S. \& VAN DEN DRIESSCHE, J. (2003) How plausible are high-frequency sediment supply-driven cycles in the stratigraphic record? Sedimentary Geology, 157, 3-13.

CHAMPION, D.C. (2013) Neodymium depleted mantle model age map of Australia: explanatory notes and user guide. Geoscience Australia, Canberra, doi.org/10.11636/Record.2013.044.

DEPAOLO, D.J. \& WASSERBURG, G.J. (1976) Nd isotopic variations and petrogenetic models. Geophysical Research Letters, 3, 249-252.

DEPAOLO, D.J., LINN, A.M. \& SCHUBERT, G. (1991) The continental crustal age distribution: methods of determining mantle separation ages from $\mathrm{Sm}-\mathrm{Nd}$ isotopic data and application to the southwestern United States. Journal of Geophysical Research: Solid Earth, 96(B2), 2071-2088. 
DE VIVO, B., GiUnta, G., LiMA, A., RAMAGlia, V.M., ORSI, G., PERrONE, V. \& ZUPPETTA, A. (1981) An application of moving average analysis to a regional geochemical reconnaissance on residual soils of southern Sudan. Rendiconti della Società Italiana di Mineralogia e Petrologia, 37, 387-406.

DICKINSON, W.R. (1970) Interpreting detrital modes of graywacke and arkose. Journal of Sedimentary Petrology, 40, 695-707.

DICKINSON, W.R. (2008) Impact of differential zircon fertility of granitoid basement rocks in North America on age populations of detrital zircons and implications for granite petrogenesis. Earth and Planetary Science Letters, 275, 80-92.

DICKINSON, W.R., LAWTON, T.F. \& GEHRELS, G.E. (2009) Recycling detrital zircons: A case study from the Cretaceous Bisbee Group of southern Arizona. Geology, 37, 503-506.

DOU, L., XIAO, K., CHENG, D., SHI, B. \& LI, Z. (2007) Petroleum geology of the Melut Basin and the Great Palogue Field, Sudan. Marine and Petroleum Geology, 24, 129-144.

EL-ATTAR, H.A. \& JACKSON, M.L. (1973) Montmorillonitic soils developed in Nile river sediments. Soil Sciences, 116, 191-201.

FACCENNA, C., GLIŠOVIĆ, P., FORTE, A., BECKER, T.W., GARZANTI, E. \& SEMBRONI, A. (2018) Long-lived river systems controlled by a mantle conveyor belt: the Nile. Nature Geoscience, in review.

FERNANDEZ-ALONSO, M., CUTTEN, H., DE WAELE, B., TACK, L., TAHON, A., BAUDET, D. \& BARRITT, S.D. (2012) The Mesoproterozoic Karagwe-Ankole Belt (former NE Kibaran belt): the result of prolonged extensional intracratonic basin development punctuated by two short-lived far-field compressional events. Precambrian Research, 216-219, 63-86.

FIELDING, L., NAJMAN, Y., MILlAR, I., BUTTERWORTH, P., ANDÒ, S., PADOAN, M., BARFOD, D. \& KNELLER, B. (2017) A detrital record of the Nile River and its catchment. Journal of the Geological Society London, 174, 301-317.

FIELDING, L., NAJMAN, Y., MILlAR, I., BUTTERWORTH, P., GARZANTI, E., VEZZOLI, G., BARFOD, D. \& KNELLER, B. (2018) The initiation and evolution of the River Nile. Earth and Planetary Science Letters, 489, 166-178.

FOLK, R.L. (1968) Petrology of Sedimentary Rocks. Hemphill Publishing Co., Austin, US, 182 p.

GANI, N.D., ABDELSALAM, M.G., GERA, S. \& GANI, M.R. (2009) Stratigraphic and structural evolution of the Blue Nile Basin, Northwestern Ethiopian Plateau. Geological Journal, 44, 30-56. 
GARZANTI, E. (2016) From static to dynamic provenance analysis - Sedimentary petrology upgraded. Sedimentary Geology, 336, 3-13.

GARZANTI, E., VEZZOLI, G., ANDÒ, S., PAPARELlA, P. \& CLIFT, P.D. (2005) Petrology of Indus River sands : a key to interpret erosion history of the Western Himalayan Syntaxis. Earth and Planetary Science Letters, 229, 287-302.

GARZANTI, E., ANDÒ, S., VEZZOLI, G., ALI ABDEL MEGID, A. \& EL KAMMAR, A. (2006) Petrology of Nile River sands (Ethiopia and Sudan): sediment budgets and erosion patterns. Earth and Planetary Science Letters, 252, 327-341.

GARZANTI, E., ANDÒ, S. \& VEZZOLI, G. (2008). Settling-equivalence of detrital minerals and grain-size dependence of sediment composition. Earth and Planetary Science Letters, 273, 138151.

GARZANTI, E., ANDÒ, S. \& VEZZOLI, G. (2009) Grain-size dependence of sediment composition and environmental bias in provenance studies. Earth and Planetary Science Letters, 277, 422-432.

GARZANTI, E., ANDÒ, S., FRANCE-LANORD, C., VEZZOLI, G., GALY, V. \& NAJMAN, Y. (2010) Mineralogical and chemical variability of fluvial sediments. 1. Bedload sand (GangaBrahmaputra, Bangladesh). Earth and Planetary Science Letters, 299, 368-381.

GARZANTI, E., VERMEESCH, P., ANDÒ, S., VEZZOLI, G., VAlAGUSSA, M., ALLEN, K., KHADI, K.A. \& AL-JUBOURY, I.A. (2013a) Provenance and recycling of Arabian desert sand. Earth-Science Reviews, 120, 1-19.

GARZANTI, E., PADOAN, M., SETTI, M., PERUTA, L., NAJMAN, Y. \& VILLA, I.M. (2013b) Weathering geochemistry and Sr-Nd fingerprints of equatorial upper Nile and Congo muds. Geochemistry, Geophysics, Geosystems, 14, 292-316.

GARZANTI, E., ANDÒ, S., PADOAN, M., VEZZOLI, G. \& EL KAMMAR, A. (2015) The modern Nile sediment system: Processes and products. Quaternary Science Reviews, 130, 9-56.

GOLDSTEIN, S.L., O'NIONS, R.K. \& HAMILTON, P.J. (1984) A Sm-Nd isotopic study of atmospheric dusts and particulates from major river systems. Earth and Planetary Science Letters, 70, 221-236.

GOLDSTEIN, S.L., ARNDT, N.T. \& STALLARD, R.F. (1997) The history of a continent from U-Pb ages of zircons from Orinoco River sand and $\mathrm{Sm}-\mathrm{Nd}$ isotopes in Orinoco basin river sediments. Chemical Geology, 139, 271-286. 
GRIFFIN, W.L., POWELL, W.J., PEARSON, N.J. \& O'REILLY, S.Y. (2008) GLITTER: data reduction software for laser ablation ICP-MS, in Sylvester, P., ed., Laser ablation-ICP-MS in the earth sciences: current practices and outstanding issues. Mineralogical Association of Canada, Short Course, 40, p. 204-207.

HEREHER, M.E. (2014) Assessment of sand drift potential along the Nile Valley and Delta using climatic and satellite data. Applied Geography, 55, 39-47.

HINDERER, M. (2012) From gullies to mountain belts: a review of sediment budgets at various scales. Sedimentary Geology, 280, 21-59.

JOHNSON, P.R., ANDRESEN, A., COLlinS, A.S., FOWLER, A.R., FRITZ, H., GHEBREAB, W., KUSKY, T. \& STERN, R.J. (2011) Late Cryogenian-Ediacaran history of the Arabian-Nubian Shield: a review of depositional, plutonic, structural, and tectonic events in the closing stages of the northern East African Orogen. Journal of African Earth Sciences, 61, 167-232.

KLITZSCH, E.H. \& SQUYRES, C.H. (1990) Paleozoic and Mesozoic geological history of north eastern Africa based upon new interpretation of Nubian strata. American Association of Petroleum Geologists Bulletin, 74, 1203-1211.

LAWRENCE, R.L., COX, R.,MAPES, R.W. \& COLEMAN, D.S. (2011) Hydrodynamic fractionation of zircon age populations. Geological Society of America Bulletin, 123, 295-305.

LEEDER, M.R. (2011) Tectonic sedimentology: sediment systems deciphering global to local tectonics. Sedimentology, 58, 2-56.

LINK, K., KOEHN, D., BARTH, M.G., TIBERINDWA, J.V., BARIFAIJO, E., AANYU, K. \& FOLEY, S.F. (2010) Continuous cratonic crust between the Congo and Tanzania blocks in western Uganda. International Journal of Earth Sciences, 99, 1559-1573.

LOMBARDINI, E. (1865) Essai sur l' hydrologie du Nil. Bernardini, Paris/Milan, 72 p.

MALUSÀ, M.G., CARTER, A., LIMONCELLI, M., VILLA, I.M. \& GARZANTI, E. (2013) Bias in detrital zircon geochronology and thermochronometry. Chemical Geology, 359, 90-107.

MALUSÀ, M.G., RESENTINI, A. \& GARZANTI, E. (2016) Hydraulic sorting and mineral fertility bias in detrital geochronology. Gondwana Research, 31, 1-19.

MANGE, M.A. \& MORTON, A.C. (2007) Geochemistry of heavy minerals. In: Heavy Minerals in Use (Ed. by Mange, M.A. \& Wright, D.T.), Developments in Sedimentology, 58, 345-391. Elsevier, Amsterdam. 
MCHARGUE, T.R., HEIDRICK, T.L. \& LIVINGSTON, J.E. (1992) Tectonostratigraphic development of the interior Sudan rifts, Central Africa. Tectonophysics, 213, 187-202.

MERLA, G., ABBATE, E., AZZAROLI, A., BRUNI, P., CANUTI, P., FAZZUOLI, M., SAGRI, M. \& TACCONI, P. (1979) A geological map of Ethiopia and Somalia, 1:2,000,000 and comment. University of Florence, Italy.

MOECHER, D.P. \& SAMSON, S.D. (2006) Differential zircon fertility of source terranes and natural bias in the detrital zircon record: implications for sedimentary provenance analysis. Earth and Planetary Science Letters, 247, 252-266.

MOHAMED, A.Y., ASHCROFT, W.A. \& WHITEMAN, A.J. (2001) Structural development and crustal stretching in the Muglad Basin, southern Sudan. Journal of African Earth Sciences, 32, 179191.

NIE, J., STEVEnS, T., RITTNER, M., STOCKLI, D., GARZANTI, E., LIMONTA, M., BIRD, A., ANDÒ, S., VERMEESCH, P., SAYLOR, J., LU, H., BREECKER, D., HU, X., LIU, S., RESENTINI, A., VEZZOLI, G., PENG, W., CARTER, A., JI, S. \& PAN, B. (2015) Loess Plateau storage of Northeastern Tibetan Plateau-derived Yellow River sediment. Nature Communications, 6, DOI: $10.1038 /$ ncomms9511.

NYAKAIRU, G.W. \& KOEBERL, C. (2001) Mineralogical and chemical composition and distribution of rare earth elements in clay-rich sediments from central Uganda. Geochemical Journal, 35, 13-28.

PADOAN, M., GARZANTI, E., HARLAVAN, Y. \& VILLA, I.M. (2011) Tracing Nile sediment sources by $\mathrm{Sr}$ and $\mathrm{Nd}$ isotope signatures (Uganda, Ethiopia, Sudan). Geochimica et Cosmochimica Acta, 75, 3627-3644.

PIK, R., MARTY, B., CARIGNAN, J. \& LAVÉ, J. (2003) Stability of the Upper Nile drainage network (Ethiopia) deduced from (U/Th)/He thermochronometry: implications for uplift and erosion of the Afar plume dome. Earth and Planetary Science Letters, 215, 73-88.

RITTNER, M., VERMEESCH, P., CARTER, A., BIRD, A., STEVENS, T., GARZANTI, E., ANDÒ, S., VEZZOLI, G., DUTT, R., XU, Z. \& LU, H. (2016) The provenance of Taklamakan desert sand. Earth and Planetary Science Letters, 437, 127-137.

ROGERS, N.W., JAMES, D., KELLEY, S.P. \& DE MULDER, M. (1998) The generation of potassic lavas from the eastern Virunga Province, Rwanda. Journal of Petrology, 39, 1223-1247. 
798

799

800

801

802

803

804

805

806

807

808

809

810

811

812

813

814

815

816

817

818

819

820

821

822

823

824

825

826

827

828

RUBEY, W.W. (1933) The size-distribution of heavy minerals within a water-laid sandstone. Journal of Sedimentary Petrology, 3, 3-29.

SALAMA, R.B., 1997. Rift basins of the Sudan. Sedimentary basins of the world, 3, 105-149.

SCHENK, V., APPEL, P., JONS, N., LOOSE, D., SCHUMANN, A. \& WEGNER, H. (2007) Metamorphic reworking of the Congo craton in Uganda. Geochimica et Cosmochimica Acta, 71, A887.

SHUKRI, N.M. (1950) The mineralogy of some Nile sediments. Quarterly Journal of the Geological Society, 106, 466-467.

SIMMONS, M. (2016) Detrital Geochronology of the Nile. MSci Independent Project GEOLM905, University College London, $81 \mathrm{p}$.

STANLEY, J.D. \& WINGERATH, J.G. (1996) Nile sediment dispersal altered by the Aswan High Dam: the kaolinite trace. Marine Geology, 133, 1-9.

SUTCLIFFE, J.V. \& PARKS, Y.P. (1999) The hydrology of the Nile. International Association of Hydrological Sciences, Special Publication, 5, 179 p.

TACK, L., WINGATE, M.T.D., DE WAELE, B., MEERTE, J., BELOUSOVA, E., GRIFFIN, B., TAHON, A. \& FERNANDEZ-ALONSO, M. (2010) The 1375 Ma "Kibaran event" in Central Africa: prominent emplacement of bimodal magmatism under extensional regime. Precambrian Research, 180, 63-84.

THOMAS, W.A., BECKER, T.P., SAMSON, S.D. \& HAMILTON, M.A. (2004) Detrital zircon evidence of a recycled orogenic foreland provenance for Alleghanian clastic-wedge sandstones. The Journal of Geology, 112, 23-37.

VERMEERSCH, P.M. \& VAN NEER, W. (2015) Nile behaviour and late Palaeolithic humans in upper Egypt during the Late Pleistocene. Quaternary Science Reviews, 130, 155-167.

VERMEESCH, P., AVIGAD, D. \& MCWILliAMS, M.O. (2009) 500 m.y. of thermal history elucidated by multi-method detrital thermochronology of North Gondwana Cambrian sandstone (Eilat area, Israel). Geological Society of America Bulletin, 121, 1204-1216.

VERMEESCH, P., RESENTINI, A. \& GARZANTI, E. (2016) An R package for statistical provenance analysis. Sedimentary Geology, 336, 14-25.

VERMEESCH, P., RITTNER, M., PETROU, E., OMMA, J., MATTINSON, C. \& GARZANTI, E. (2017) High throughput petrochronology and sedimentary provenance analysis by automated phase mapping and LAICPMS. Geochemistry, Geophysics, Geosystems, doi: 10.1002/2017GC007109. 
VERVOORT, J.D., PATCHETT, P.J., BLICHERT-TOFT, J. \& ALBARÈDE, F. (1999) Relationships between Lu-Hf and Sm-Nd isotopic systems in the global sedimentary system. Earth and Planetary Science Letters, 168, 79-99.

VEZZOLI, G., GARZANTI, E., LIMONTA, M., ANDÓ, S. \& YANG, S. ( 2016) Erosion patterns in the Changjiang (Yangtze River) catchment revealed by bulk-sample versus single-mineral provenance budgets. Geomorphology, 261, 177-192.

VON EYNATTEN, H. \& DUNKL, I. (2012) Assessing the sediment factory: the role of single grain analysis. Earth-Science Reviews, 115, 97-120.

VON EYNATTEN, H., GAUPP, R. \& WIJBRANS, J.R. (1996) ${ }^{40} \mathrm{Ar} /{ }^{39} \mathrm{Ar}$ laser-probe dating of detrital white micas from Cretaceous sedimentary rocks of the Eastern Alps: evidence for Variscan high-pressure metamorphism and implications for Alpine orogeny. Geology, 24, 691-694.

WILLIAMS, M.A.J. \& ADAMSON, D. (1982) A land between two Niles. Balkema, Rotterdam, 246 p.

WILLIAMS, M.A.J., DULLER, G.A.T., WILLIAMS, F.M., WOODWARD, J.C., MACKLIN, M.G., EL TOM, O.A.M., MUNRO, R.N., EL HAJAZ, Y. \& BARROWS, T.T. (2015) Causal links between Nile floods and eastern Mediterranean sapropel formation during the past $250 \mathrm{kyr}$ confirmed by OSL and radiocarbon dating of Blue and White Nile sediments. Quaternary Science Reviews, 130, 89-108.

WITTMAN, H., MALUSÀ, M.G., RESENTINI, A., GARZANTI, E. \& NIEDERMANN, S. (2016) The cosmogenic record of mountain erosion transmitted across a foreland basin: source-to-sink analysis of in situ ${ }^{10} \mathrm{Be},{ }^{26} \mathrm{Al}$ and ${ }^{21} \mathrm{Ne}$ in sediment of the Po river catchment. Earth and Planetary Science Letters, 452, 258-271.

WOODWARD, J.C., WILliAMS, M.A.J., GARZANTI, E., MACKLIN, M.G. \& MARRINER, N. (2015) From source to sink: exploring the Quaternary history of the Nile. Quaternary Science Reviews, 130, 230 p. 
2

3

4

5

6

7

8

9

10

11

12

13

14

15

16

17

18

19

20

21

22

23

24

25

26

27

28

29

30

31

32

33

34

35

36

37

38

39

40

41

42

43

44

45

46

47

48

49

50

51

52

53

54

55

56

57

58

59

60

$\mathrm{U}-\mathrm{Pb}$ age spectra of detrital zircons are monitored throughout the ultra-long Nile drainage basin

All provenance signals are disrupted repeatedly along this huge segmented sediment-routing system

"CL-guided" versus 'blind' strategies to zircon-dating are confronted, and selection bias discussed

Different time-structure maps of source areas are defined by detrital-zircon and Sm-Nd isotope data

Zircon-age distributions do not reveal any significant grain-size or hydraulic-sorting control 

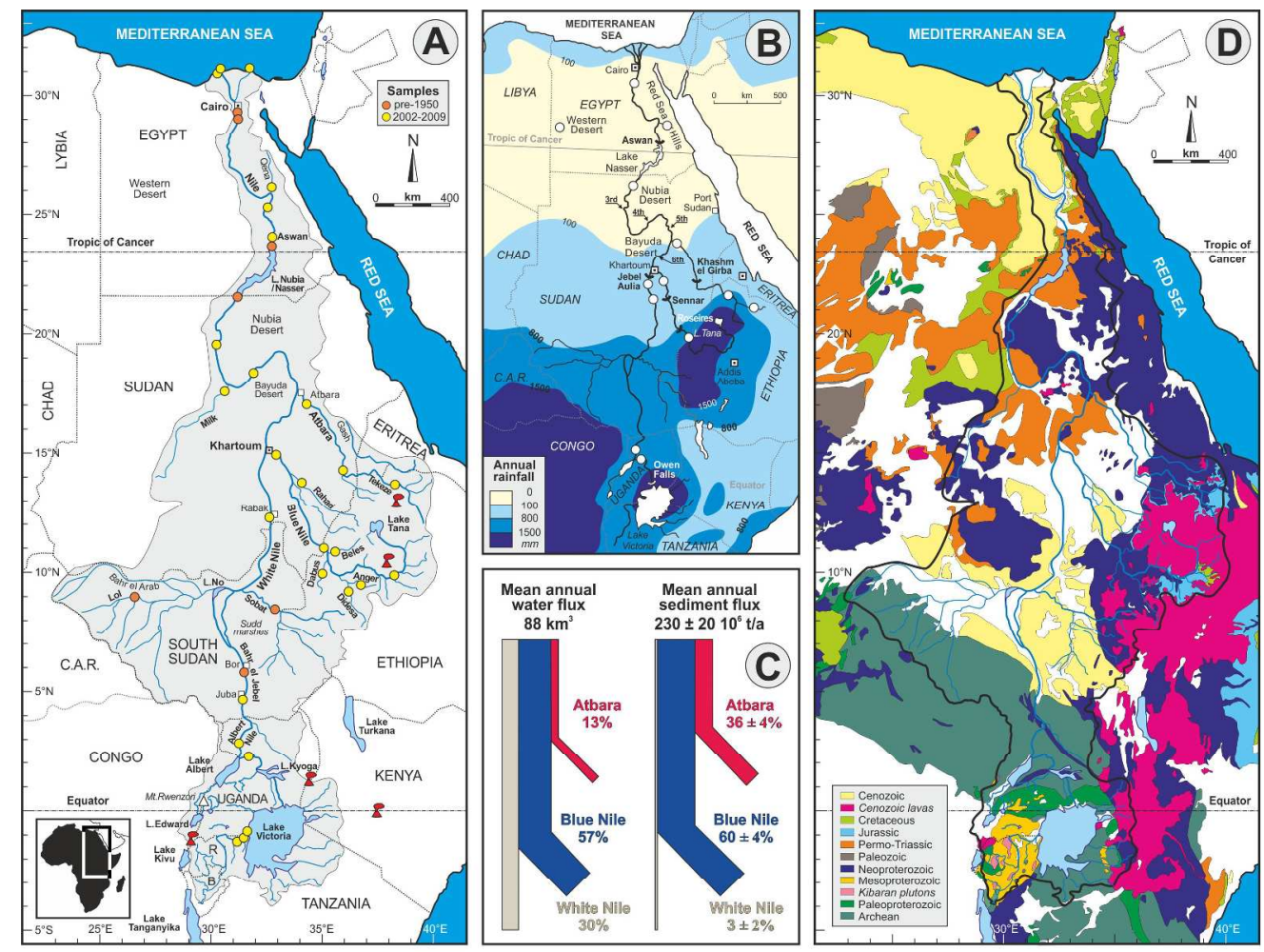

Figure 1 Nile zircon

Figure 1. The Nile River catchment. A) Location of samples selected for $\mathrm{U}-\mathrm{Pb}$ detrital-zircon geochronological analysis (yellow circles = samples collected between 2002 and 2009 before closure of the Merowe Dam; orange circles = samples collected by the Egyptian Irrigation Department before construction of the Aswan High Dam; full information on sampling sites provided in Appendix Table A1 and Google-EarthTM map Nilezircon.kmz); R = Rwanda; $B=$ Burundi; C.A.R. = Central Africa Republic. B) Rainfall map. Nile cataracts and major dams are shown, together with location of modern sands (white circles) analysed for U-Pb detrital zircon geochronology by Fielding et al. (2017). C) Water discharge (Sutcliffe \& Parks, 1999) and sediment fluxes (Garzanti et al., 2006). D) Geological map (redrawn from Asga-Unesco, 1963 and other sources cited in text).

$278 \times 223 \mathrm{~mm}(300 \times 300 \mathrm{DPI})$ 


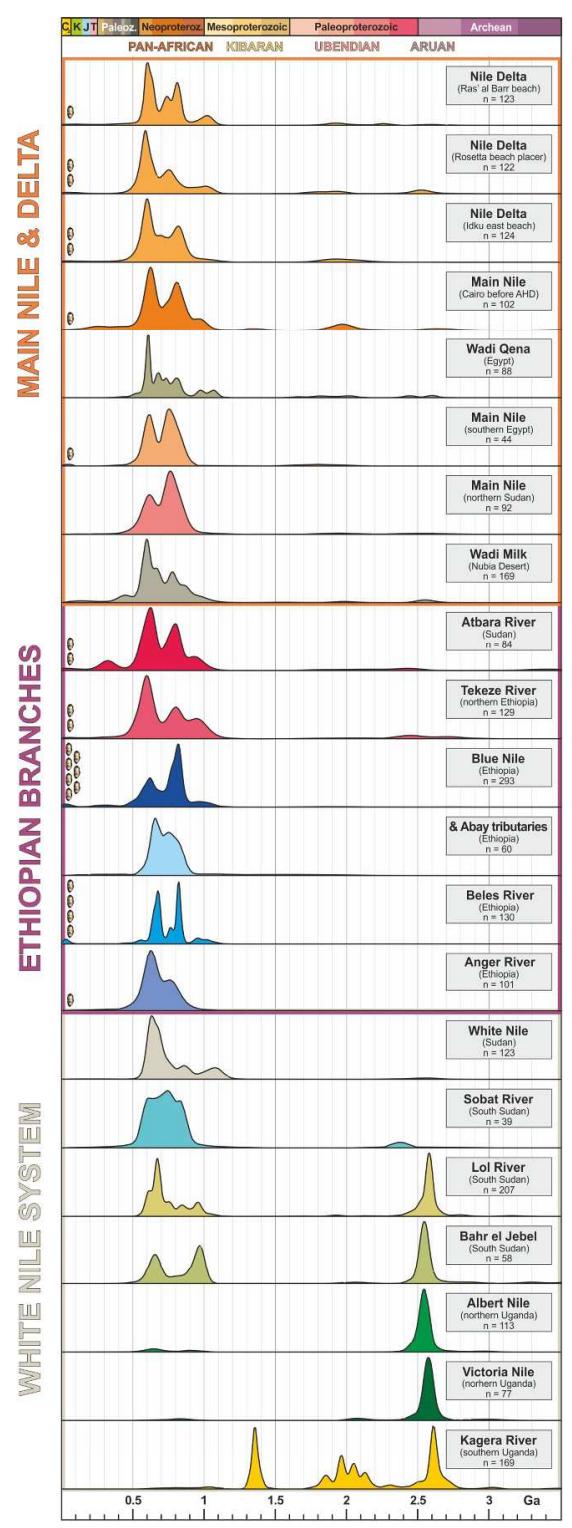

Figure 2 Nile zircon

Figure 2. U-Pb age spectra of detrital zircons (age vs. frequencies plotted as Kernel Density Estimates using the provenance package of Vermeesch et al., 2016). Sediments carried by the Kagera River are dumped in Lake Victoria, where the Kibaran and Ubendian signals are lost. The Aruan signal is restored in the Victoria Nile, and once again in the Albert Nile downstream of Lake Albert, but it is then progressively lost across the Sudd marshes of South Sudan, where White Nile sand becomes dominated by the Pan-African signal. Polymodal Pan-African ages are dominant not only in Blue Nile and Atbara sediments derived from Ethiopia, but also in sediments of widyan draining the Saharan Metacraton and its siliciclastic covers in Nubia and the Red Sea Hills in Egypt. Zircons of Oligocene age are diagnostic of provenance from Ethiopian volcanic highlands. 


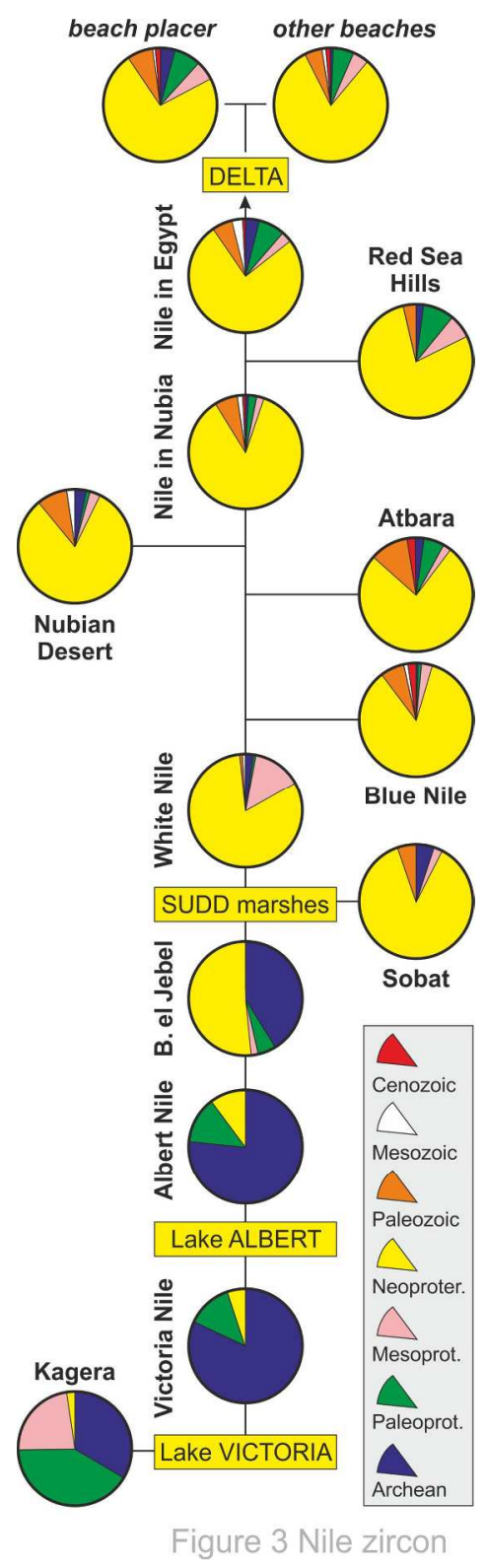

Figure 3. Discontinuous downstream propagation of the zircon-age provenance signal. Note the stark contrast between age-spectra upstream and downstream of Lake Victoria in Uganda, and between upstream and downstream of the Sudd marshes in South Sudan. Spectra become homogeneously dominated by Neoproterozoic ages from Khartoum to the Delta. The young population of Oligocene grains derives from the Ethiopian volcanic plateau drained by the Atbara and Blue Nile. In the Delta, similar spectra for the Rosetta magnetite placer and other beaches indicate minor effect of selective-entrainment processes on zircon-age distributions.

$$
80 \times 270 \mathrm{~mm}(300 \times 300 \mathrm{DPI})
$$



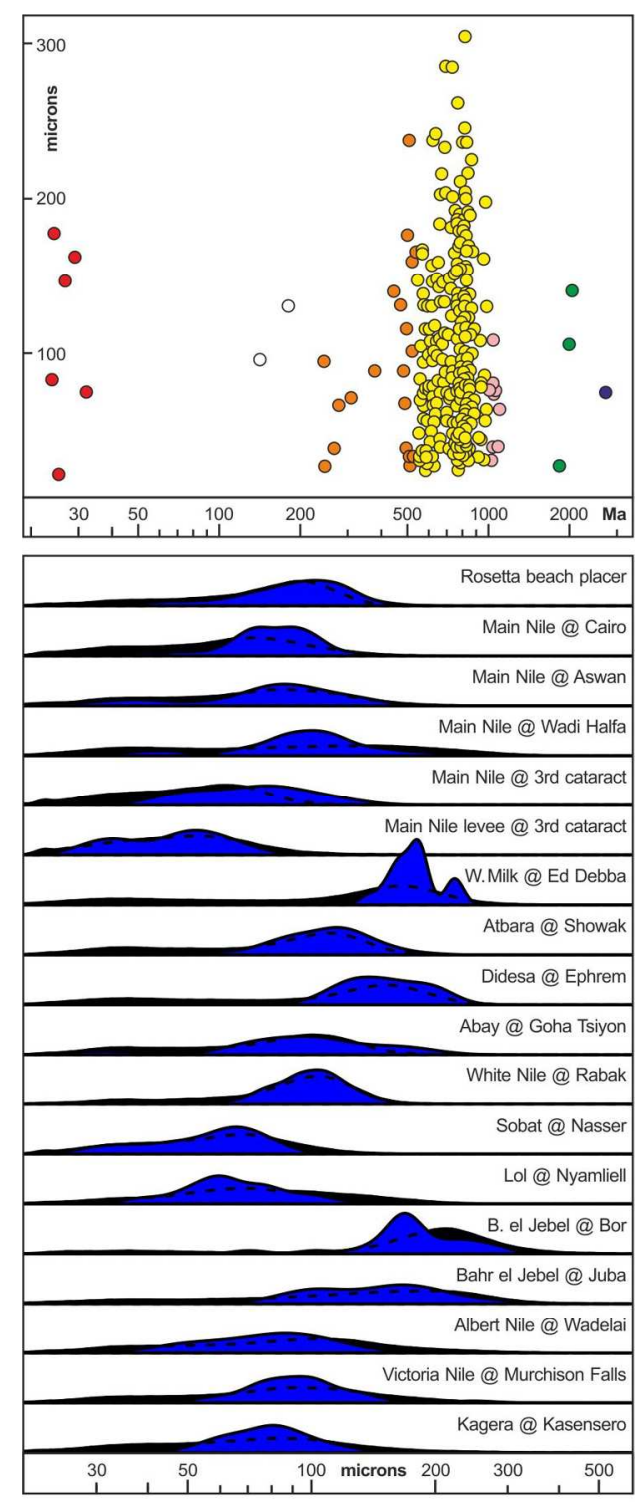

Figure 4 Nile zircon

Figure 4. Grain-size information obtained during QEMSCAN $®$ analysis (Simmons, 2016). Upper panel: U-Pb age versus circular equivalent diameter of zircon grains from Blue Nile sample 2964 (age color-code as in Fig. 3). Evidence for grain-size control is lacking, although it may be masked by bias introduced by variable polish depth during sample preparation. Lower panel: Size distribution of zircon grains (blue) versus that of the total heavy-mineral population (black).

$$
76 \times 194 \mathrm{~mm}(300 \times 300 \text { DPI })
$$




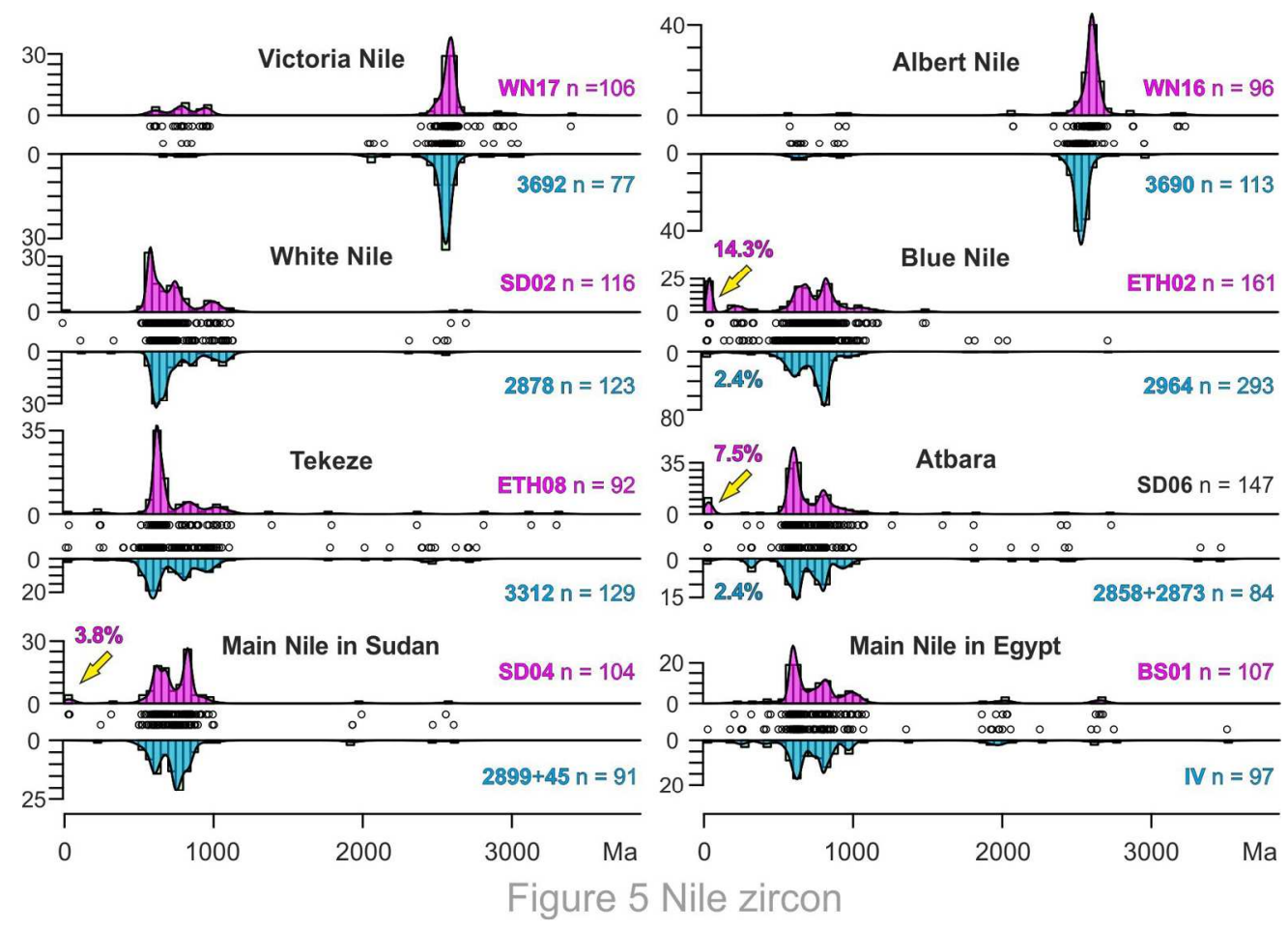

Figure 5. Comparison of U-Pb zircon-age distributions obtained with different analytical protocols from eight couples of replicate sand samples. Above in magenta: CL-guided "targeted-dating strategy" (data from

Fielding et al., 2017); below in blue: "blind-dating strategy" (this study). The two methods yielded different results in the young end of the spectrum (yellow arrows) because grains of Oligocene age, specifically targeted as tracers of Ethiopian volcanic provenance by Fielding et al. (2017), are consequently overrepresented in their dataset. WN17 $=3692$ and WN16 $=3690$ are the very same two samples collected in the Victoria and Albert Nile by Y. Najman and E. Garzanti in 2007. Each of the other six couples was collected in the same or in nearby localities by different operators in different years.

$159 \times 114 \mathrm{~mm}(300 \times 300 \mathrm{DPI})$ 

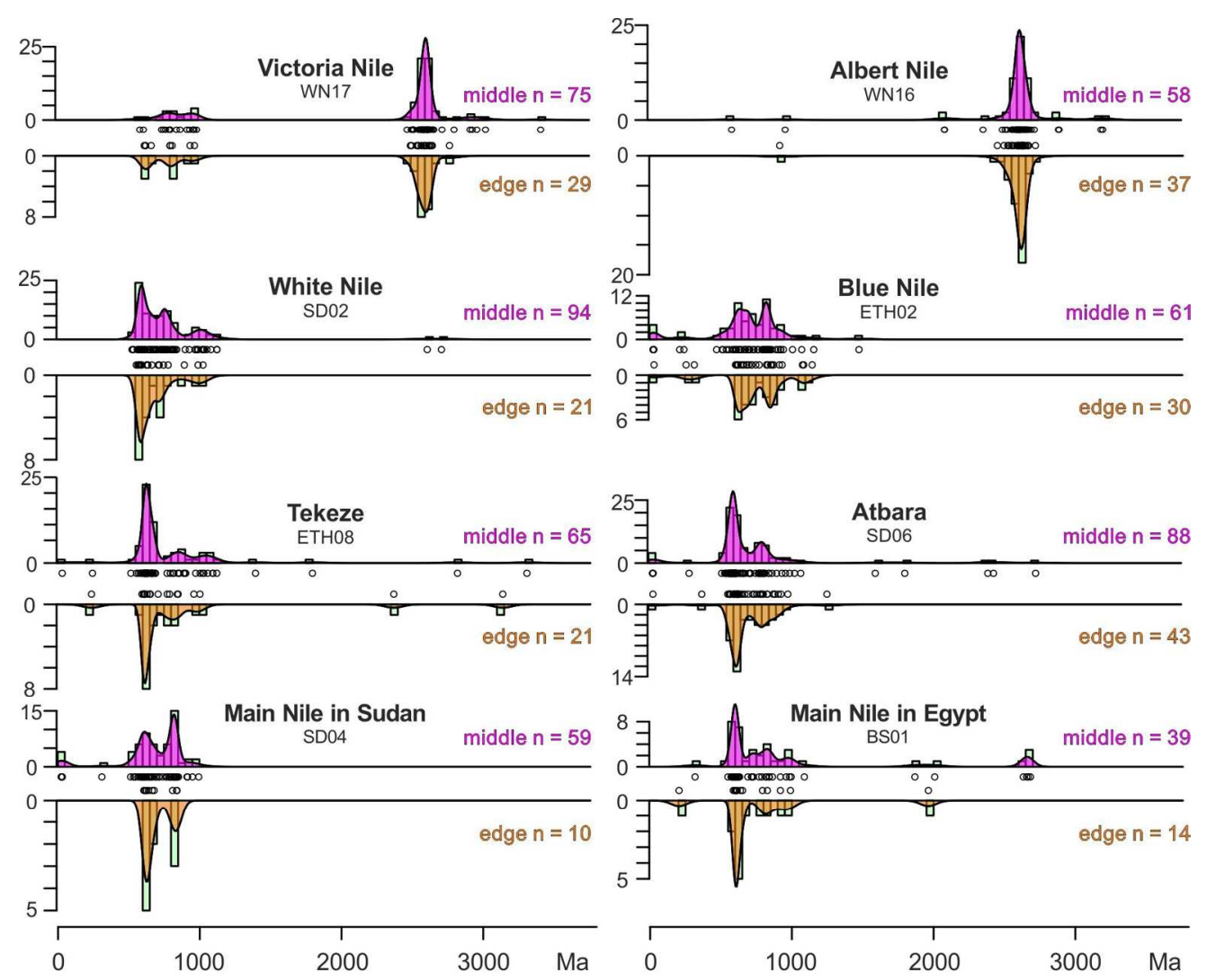

Figure 6 Nile zircon

Figure 6. Overall consistency of $\mathrm{U}-\mathrm{Pb}$ ages obtained targeting the grain interior (above in magenta) or the grain edges (below in orange) of detrital zircons. Most studied grains were in fact simple, with no xenocrystic cores or late overgrowths (all data after Fielding et al., 2017). A notable exception - illustrated further in Fig. 7 - is the higher frequency of Neoproterozoic ages yielded by zircon rims in the Victoria Nile sample.

$160 \times 139 \mathrm{~mm}(300 \times 300 \mathrm{DPI})$ 
Figure 7. Notable differences in U-Pb zircon-age distributions are obtained with "blind" and "targeted" approaches for samples including numerous grains with complex age structure. This is the case of the White Nile in Uganda (data from subsamples WN17 and WN16 after Fielding et al., 2017), where a higher frequency of Neoproterozoic (Npz) ages was obtained by the CL-guided strategy for the Victoria Nile sample, especially from zircon rims. This reflects the Pan-African rejuvenation event recorded extensively throughout the Saharan Metacraton as far as NW Uganda but only marginally by gneissic rocks in the Albert Nile catchment.

$88 \times 76 \mathrm{~mm}(300 \times 300$ DPI $)$ 

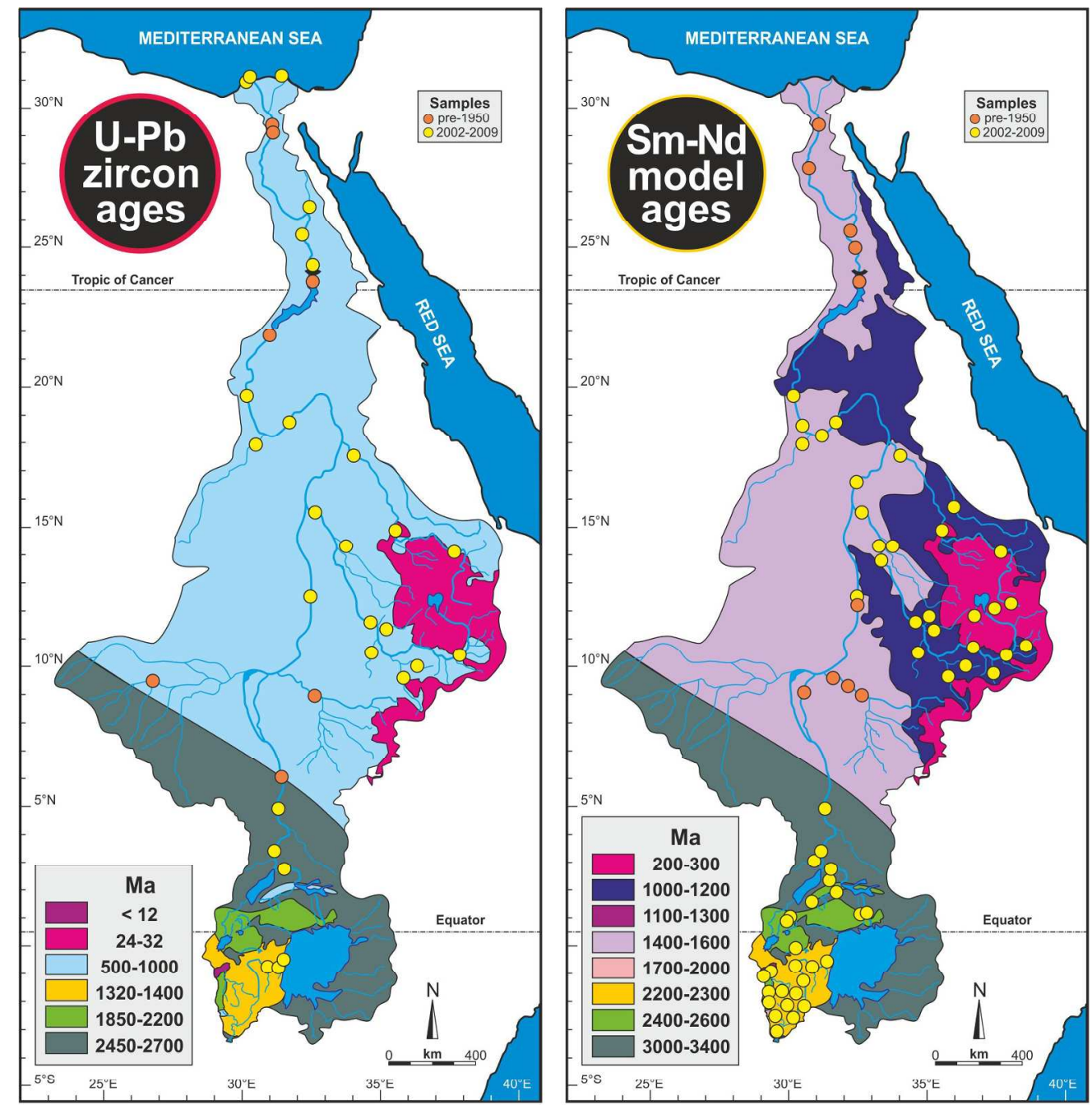

\section{Figure 8 Nile zircon}

Figure 8. Different time-structure maps of source rocks in the Nile catchment based on detrital zircon geochronology (left) and Sm-Nd fingerprinting (right; Padoan et al., 2011; Garzanti et al., 2013b). Dominant Neoproterozoic U-Pb zircon ages characterize both Pan-African basement exposed along the Red Sea rift shoulder and drained by all Ethiopian rivers (Sobat, Blue Nile, Atbara and their tributaries) and the Saharan Metacraton with its siliciclastic covers (Abdelsalam et al., 2003). Neogene Virunga volcanoes and Oligocene Ethiopian lavas yield detritus with late Mesoproterozoic and Permo-Triassic Sm-Nd model age, respectively, revealing interaction of rising magmas with Paleoproterozoic and Neoproterozoic basement rocks, respectively. Sample locations are shown; details of map contours are drawn according to the geological map (Fig. 1). 


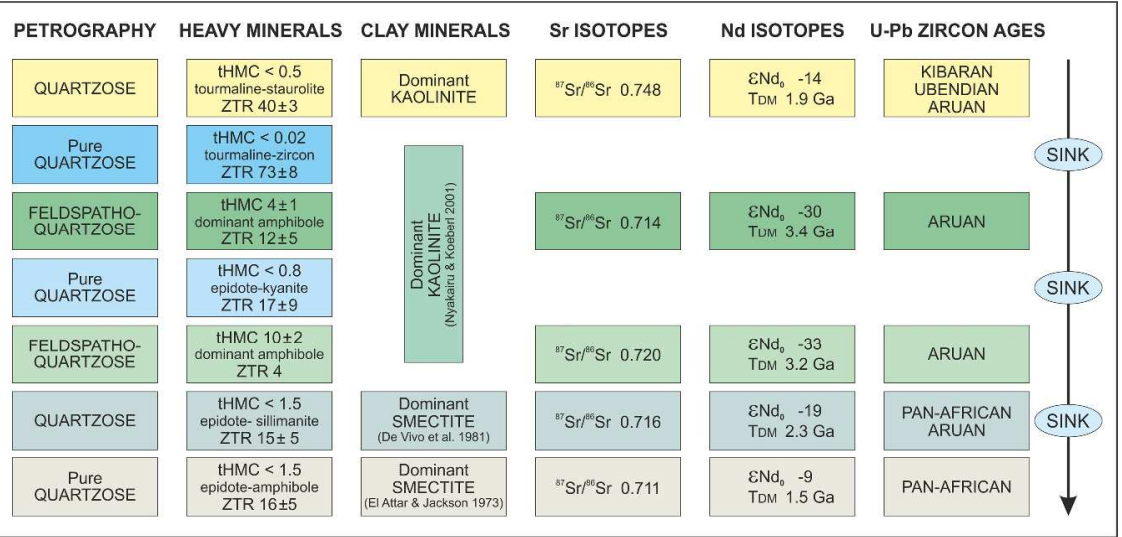

Figure 9 Nile zircon

Figure 9. Repeated decoupling of provenance signals along the White Nile sediment-routing system. Detrital fingerprints of Kagera sand are lost in Lake Victoria. Sand derived from the Archean Congo-Tanzania craton is carried by the Victoria Nile to Lake Albert, and by the Albert Nile/Bahr el Jebel to the Sudd marshes. Sediments exiting the Sudd marshes have lost all provenance signals from headwater sources. Repeated trapping in natural sediment sinks explains the very minor sediment contribution of the White Nile to the main Nile downstream of Khartoum. Petrographic, heavy-mineral and isotopic data after Garzanti et al. (2015); clay-mineral data after Buursink (1971), El-Attar \& Jackson (1973), De Vivo et al. (1981), and Nyakairu \& Koeberl (2001).

$447 \times 197 \mathrm{~mm}(300 \times 300$ DPI $)$ 
6 Kagera River

7 Victoria Nile

8 Victoria Nile*

9 Albert Nile

$10_{\text {Bahr el Jebel }}^{\text {Albert Nile* }^{*}}$

11 Lol River ${ }^{\circ}$

12 Sobat River

$13^{\text {White Nile }}$

$13^{\text {White Nile }}$

$14_{\text {Blue Nile }}^{\text {White Nile }}$

15 Blue Nile* $^{*}$

16 Anger River

17 Beles River
$\&$ Abay tributarie

$18_{\text {Tekeze River }}^{\& \text { Abay tribut }}$

19 Tekeze River $^{*}$

20Atbara River

21 Atbara River

$22^{\text {Wadi Milk }}$

23 Nile in Nubia

24wadi Qena

25Western Desert*

$26^{\text {Nile at Cairo }}{ }^{\circ}$

Nile at Cairo

27 Nile Delta

\begin{tabular}{|c|c|c|c|c|c|c|c|c|c|c|c|c|c|c|c|c|c|c|c|c|c|c|c|c|c|c|}
\hline \multirow{3}{*}{$\begin{array}{c}\mathbf{n}^{\circ} \text { ages } \\
\text { Total } \\
3644\end{array}$} & \multicolumn{2}{|c|}{ CENOZOIC } & \multicolumn{2}{|c|}{ MESOZOIC } & \multicolumn{2}{|c|}{ PALEOZOIC } & \multicolumn{2}{|c|}{\begin{tabular}{|l} 
NEOPROTEROZ. \\
\end{tabular}} & \multicolumn{4}{|c|}{ MESOPROTEROZOIC } & \multicolumn{8}{|c|}{ PALEOPROTEROZOIC } & \multicolumn{5}{|c|}{ ARCHEAN } & \\
\hline & $\mathrm{Ma}$ & $\%$ time & $\mathrm{Ma}$ & $\%$ time & $\mathrm{Ma}$ & $\%$ time & $\mathrm{Ma}$ & $\%$ time & Ste & & Ectasian/C & mnian & Stath & & Oros & & Rhya & & Side & & Neoarc & hean & Mesoz & rchean & Eo/Paleoarchean & \\
\hline & $0-66$ & $2 \%$ & $66-252$ & $5 \%$ & 252-541 & $8 \%$ & 541-1000 & $12 \%$ & $1000-1200$ & $5 \%$ & $1200-1600$ & $11 \%$ & 1600-1800 & $5 \%$ & 1800-2050 & $7 \%$ & $2050-2300$ & $7 \%$ & $2300-2500$ & $5 \%$ & $2500-2800$ & $8 \%$ & $2800-3200$ & $11 \%$ & $3200-3800 \quad 16 \%$ & \\
\hline
\end{tabular}

\begin{tabular}{|c|c|c|c|c|c|c|c|c|c|c|c|c|c|c|c|c|c|c|c|c|c|c|c|c|c|c|c|}
\hline 169 & & & & & & & $663-966$ & $2 \%$ & 1034-1055 & $1 \%$ & $1316-1444$ & $22 \%$ & $1761-1786$ & $1 \%$ & 1819-2048 & $24 \%$ & 2056-2292 & $12 \%$ & $2304-2470$ & $4 \%$ & $2509-2784$ & $32 \%$ & 3014-3037 & $1 \%$ & 3489 & $0.6 \%$ & $100.0 \%$ \\
\hline 77 & & & & & & & $675-867$ & $5 \%$ & & & & & & & & & 2053-2162 & $5 \%$ & 2384-2488 & $8 \%$ & $2504-2680$ & $77 \%$ & 2832-3060 & $5 \%$ & & & $100.0 \%$ \\
\hline 106 & & & & & & & 588-989 & $24 \%$ & & & & & & & & & & & 2409-2498 & $3 \%$ & $2721-2774$ & $68 \%$ & $2806-3028$ & $5 \%$ & 3417 & $0.9 \%$ & $100.0 \%$ \\
\hline 113 & & & & & & & 590-953 & $11 \%$ & & & & & & & & & & & 2388-2499 & $13 \%$ & 2502-2767 & $74 \%$ & 2969-2970 & $2 \%$ & & & $100.0 \%$ \\
\hline 97 & & & & & & & 587-965 & $3 \%$ & & & & & & & & & 2088-2090 & $2 \%$ & $2361-2497$ & $4 \%$ & $2517-2723$ & $86 \%$ & 2893-3193 & $4 \%$ & 3206 & $1 \%$ & $100.0 \%$ \\
\hline 58 & & & & & & & 551-991 & $52 \%$ & 1008 & $2 \%$ & & & & & & & 2065 & $2 \%$ & 2463-2487 & $3 \%$ & $2521-2685$ & $34 \%$ & 2868 & $2 \%$ & $3297-3788$ & $5 \%$ & $100.0 \%$ \\
\hline 207 & & & & & & & 558-992 & $55 \%$ & 1013-1060 & $2 \%$ & & & 1679 & $0.5 \%$ & 1926-1929 & $1 \%$ & 2119 & $0.5 \%$ & 2354-2494 & $4 \%$ & $2500-2794$ & $36 \%$ & 2833-3191 & $1 \%$ & & & $100.0 \%$ \\
\hline 39 & & & & & $337-501$ & $5 \%$ & 553-924 & $87 \%$ & 1059 & $3 \%$ & & & & & & & & & & & $2520-2538$ & $5 \%$ & & & & & $100.0 \%$ \\
\hline 123 & & & 119 & $1 \%$ & 341 & $0.8 \%$ & $558-988$ & $81 \%$ & $1003-1144$ & $14 \%$ & & & & & & & & & 2328 & $0.8 \%$ & 2514-2587 & $2 \%$ & & & & & $100.0 \%$ \\
\hline 116 & 0.4 & $0.9 \%$ & & & 527-537 & $3 \%$ & 557-993 & $87 \%$ & $1021-1126$ & $8 \%$ & & & & & & & & & & & $2610-2710$ & $2 \%$ & & & & & $100.0 \%$ \\
\hline 293 & $24-32$ & $2 \%$ & $142-247$ & $1 \%$ & $267-522$ & $7 \%$ & 542-984 & $84 \%$ & $1000-1096$ & $3 \%$ & & & 1786 & $0.3 \%$ & $1828-2046$ & $1 \%$ & & & & & 2722 & $0.3 \%$ & & & & & $100.0 \%$ \\
\hline 245 & 24-32 & $9 \%$ & $139-247$ & $4 \%$ & $258-529$ & $4 \%$ & 542-965 & $77 \%$ & $1009-1159$ & $4 \%$ & $1460-1469$ & $0.8 \%$ & & & 1964 & $0.4 \%$ & & & & & & & & & & & $100.0 \%$ \\
\hline 101 & 31 & $1 \%$ & & & $258-531$ & $9 \%$ & 552-991 & $85 \%$ & 1021 & $1 \%$ & & & 1779 & $1 \%$ & 1916 & $1 \%$ & & & & & 2992-3041 & $2 \%$ & & & & & $100.0 \%$ \\
\hline 130 & 29-31 & $3 \%$ & & & $472-527$ & $2 \%$ & 551-997 & $92 \%$ & 1011-1072 & $4 \%$ & & & & & & & & & & & & & & & & & $100.0 \%$ \\
\hline 60 & & & & & $258-343$ & $3 \%$ & 565-995 & $92 \%$ & & & 1374 & $2 \%$ & 1601 & $2 \%$ & 1922 & $2 \%$ & & & & & & & & & & & $100.0 \%$ \\
\hline 129 & $11-28$ & $2 \%$ & 239 & $1 \%$ & $265-539$ & $11 \%$ & 546-994 & $72 \%$ & $1005-1110$ & $5 \%$ & & & 1789 & $0.8 \%$ & 2021 & $0.8 \%$ & 2189 & $0.8 \%$ & 2404-2495 & $4 \%$ & 2634-2772 & $3 \%$ & & & & & $100.0 \%$ \\
\hline 92 & 32 & $1 \%$ & $239-517$ & $3 \%$ & & & $556-974$ & $80 \%$ & $1004-1123$ & $9 \%$ & 1396 & $1 \%$ & 1798 & $1 \%$ & & & & & 2372 & $1 \%$ & & & 2821-3141 & $2 \%$ & 3311 & $1 \%$ & $100.0 \%$ \\
\hline 84 & 29-31 & $2 \%$ & & & $255-533$ & $11 \%$ & 544-961 & $76 \%$ & $1005-1006$ & $2 \%$ & & & & & 1818 & $1 \%$ & 2069-2232 & $2 \%$ & $2431-2458$ & $2 \%$ & & & & & $3343-3481$ & $2 \%$ & $100.0 \%$ \\
\hline 147 & $27-34$ & $7 \%$ & & & $287-538$ & $3 \%$ & 542-997 & $84 \%$ & $1031-1075$ & $2 \%$ & 1260 & $0.7 \%$ & 1601 & $0.7 \%$ & 1809 & $0.7 \%$ & & & $2395-2435$ & $1 \%$ & 2732 & $0.7 \%$ & & & & & $100.0 \%$ \\
\hline 136 & & & $103-137$ & $2 \%$ & 263-533 & $9 \%$ & 549-982 & $82 \%$ & $1020-1070$ & $2 \%$ & 1556 & $0.7 \%$ & & & 1983-1984 & $1 \%$ & & & & & 2539-2583 & $3 \%$ & & & & & $100.0 \%$ \\
\hline 135 & 24 & $0.7 \%$ & $246-251$ & $1 \%$ & $447-534$ & $7 \%$ & 555-997 & $86 \%$ & $1008-1163$ & $2 \%$ & & & & & $1938-1940$ & $1 \%$ & 2478 & $0.7 \%$ & 2619 & $0.7 \%$ & & & & & & & $100.0 \%$ \\
\hline 104 & 25-32 & $4 \%$ & & & $312-535$ & $3 \%$ & 545-995 & $91 \%$ & & & & & & & 1993.085 & $1 \%$ & & & & & 2558.857 & $1 \%$ & & & & & $100.0 \%$ \\
\hline 88 & & & & & $435-524$ & $3 \%$ & 543-982 & $78 \%$ & $1029-1090$ & $7 \%$ & & & $1662-1787$ & $2 \%$ & $1836-2043$ & $5 \%$ & & & $2429-2461$ & $2 \%$ & 2591-2612 & $2 \%$ & & & & & $100.0 \%$ \\
\hline 217 & & & & & $417-522$ & $2 \%$ & $550-998$ & $68 \%$ & $1000-1131$ & $14 \%$ & 1441 & $0.5 \%$ & $1686-1791$ & $1 \%$ & 1804-2045 & $10 \%$ & 2051-2200 & $2 \%$ & & & $2560-2687$ & $2.8 \%$ & & & & & $100.0 \%$ \\
\hline 102 & 25 & $1 \%$ & $175-252$ & $3 \%$ & 259-524 & $6 \%$ & 551-999 & $75 \%$ & 1071 & $1 \%$ & 1337-1359 & $2 \%$ & & & $1870-2007$ & $6 \%$ & 2062-2258 & $2 \%$ & & & $2603-2756$ & $3 \%$ & & & 3517 & $1 \%$ & $100.0 \%$ \\
\hline 107 & & & 204 & $0.9 \%$ & $317-521$ & $4 \%$ & 549-998 & $81 \%$ & $1028-1088$ & $6 \%$ & & & & & $1868-2040$ & $5 \%$ & & & & & 2633-2683 & $3.8 \%$ & & & & & $100.0 \%$ \\
\hline 369 & $27-32$ & $1 \%$ & $109-240$ & $1.1 \%$ & $280-536$ & $6 \%$ & $544-975$ & $79 \%$ & 1003-1069 & $5 \%$ & & & $1691-1783$ & $0.5 \%$ & $1803-2042$ & $4 \%$ & 2087-2260 & $1 \%$ & $2364-2496$ & $0.5 \%$ & $2511-2663$ & $2 \%$ & & & & & $100.0 \%$ \\
\hline
\end{tabular}


APPENDIX A - APPENDIX TABLES CAPTIONS

"The zircon story of the Nile: time-structure maps of source rocks and discontinuous propagation of detrital signals"

by Eduardo Garzanti, Pieter Vermeesch, Martin Rittner, and Matthew Simmons

Table A1. Sample location. Sampling sites and provenance of the studied sediment samples (see also the Google Earth file Nilezircon. $\mathrm{kmz}$ ). Provenance classification after Garzanti \& Andò (2007a) and Garzanti et al. (2007).

Table A2. Sand petrography. GSZ = grain size. $Q=$ quartz $(\mathrm{Qp}=$ polycrystalline $) ; \mathrm{F}=$ feldspars $(\mathrm{KF}=\mathrm{K}$-feldspar, including $\mathrm{Mi}=$ microcline; $\mathrm{P}=$ plagioclase $) ; \mathrm{L}=$ aphanitic lithic grains $(\mathrm{Lv}=$ volcanic; Lvf $=$ felsic volcanic and subvolcanic; Lvm = mafic volcanic and subvolcanic; Ls $=$ sedimentary; $\mathrm{Lc}=$ carbonate $; \mathrm{Lp}=$ shale/siltstone; $\mathrm{Lch}=$ chert $\mathrm{Lm}=$ metamorphic; $\mathrm{Lms}=$ low-rank metasedimentary; Lmv = low-rank metavolcanic; Lmf $=$ medium-rank and high-rank felsic metamorphic; Lmb = medium-rank and high-rank metabasite; Lu = ultramafic); $\mathrm{ms}=$ muscovite; $b t$ = biotite; $\mathrm{HM}=$ heavy minerals; n.d. = not determined. The Metamorphic Indices MI and MI*, expressing the average rank of rock fragments in each sample, vary respectively from 0 (in detritus shed by exclusively sedimentary and volcanic cover rocks) or from 100 (in very-low-rank detritus shed by exclusively very low-grade metamorphic rocks) to 500 (in very-high-rank detritus shed by exclusively high-grade basement rocks; Garzanti \& Vezzoli, 2003). Values in bold are weighted averages of multiple-window analyses.

Table A3. Heavy minerals. The ZTR index (sum of zircon, tourmaline and rutile over total transparent heavy minerals; Hubert, 1962) evaluates the "mineralogical stability" of the detrital assemblage (Garzanti, 2017). The HCI (Hornblende Colour Index) and MMI (Metasedimentary Minerals Index) vary from 0 in detritus from greenschist-facies to lowermost amphibolite-facies rocks yielding exclusively blue/green amphibole and chloritoid, to 100 in detritus from granulitefacies rocks yielding exclusively brown hornblende and sillimanite, and are used to estimate the average metamorphic grade of metaigneous and metasedimentary source rocks, respectively (Andò et al., 2014). $\mathrm{H} \mathrm{wt} \%=$ weight percent of the heavy fraction $\left(>2.90 \mathrm{~g} / \mathrm{cm}^{3}\right.$; Garzanti \& Andò, 2007b); 
$\mathrm{HM}=$ heavy minerals; n.d. = not determined. Values in bold are weighted averages of multiplewindow analyses.

Table A4. Sand geochemistry. Chemical weathering indices are after Nesbitt \& Young (1982; CIA), Garzanti et al. (2014a, 2014b; CIX), Harnois (1988; CIW), Fedo et al. (1995; PIA), Parker (1970, WIP); $\alpha^{\mathrm{Al}}$ values calculated with reference to UCC (Gaillardet et al., 1999) were normalized to immobile Al to avoid bias caused by hydraulic concentration of heavy minerals hosting Ti, REE, and Th (Garzanti et al., 2013a, 2013b). La $\mathrm{La}_{\mathrm{N}} / \mathrm{Yb}_{\mathrm{N}}, \mathrm{La}_{\mathrm{N}} / \mathrm{Sm}_{\mathrm{N}}, \mathrm{Gd}_{\mathrm{N}} / \mathrm{Ho}_{\mathrm{N}}$ and $\mathrm{Ho}_{\mathrm{N}} / \mathrm{Yb}_{\mathrm{N}}$ ratios are normalized to CI carbonaceous chondrites (McDonough \& Sun, 1995). The Eu anomaly Eu/Eu* is the measured chondrite-normalized $\mathrm{Eu}$ value over the value that $\mathrm{Eu}$ would have in a linear extrapolation between chondrite-normalized values of $\mathrm{Sm}$ and $\mathrm{Gd}$. The $\mathrm{Ce}$ anomaly $\mathrm{Ce} / \mathrm{Ce}$ *, indicative of redox state, is the measured PAAS-normalized Ce value over the value that Ce would have in a linear extrapolation between PAAS-normalized values of La and Pr. MREE is the average of $\mathrm{Eu}, \mathrm{Gd}, \mathrm{Tb}$ and Dy normalized to PAAS, MREE* the average of LREE (La, Ce, Pr, Nd) and HREE (Er, Tm, Yb, Lu) values (Haley et al., 2004). LOI = loss on ignition; D.L. = detection limit; n.d. = not determined. Elements analysed at ACME Laboratories by aqua regia digestion (a.r.d.) may be underestimated because of only partial leaching of refractory minerals (for further information on adopted procedures, geostandards used and precision for various elements of group 4A-4B see http://acmelab.com.). Values in bold are weighted averages of multiple-window analyses.

\section{CITED REFERENCES}

Andò, S., Morton, A., Garzanti, E., 2014. Metamorphic grade of source rocks revealed by chemical fingerprints of detrital amphibole and garnet. In: Scott, R., Smyth. H., Morton, A., Richardson, N. (Eds.), Sediment provenance studies in hydrocarbon exploration and production. Geological Society London Special Publication 386, 351-371.

Fedo, C.M., Nesbitt, H.W., Young G.M., 1995. Unraveling the effects of potassium metasomatism in sedimentary rocks and paleosols, with implications for paleoweathering conditions and provenance. Geology 23, 921-924.

Gaillardet, J., Dupré, B., Allègre, C.J., 1999. Geochemistry of large river suspended sediments: silicate weathering or recycling tracer? Geochimica et Cosmochimica Acta 63, 4037-4051.

Garzanti E., 2017. The maturity myth in sedimentology and provenance analysis. Journal of Sedimentary Research, 87, 353-365 
Garzanti, E., Andò, S., 2007a. Plate tectonics and heavy-mineral suites of modern sands. In: Mange, M.A., Wright, D.T. (Eds.), Heavy Minerals in Use. Amsterdam, Elsevier, Developments in Sedimentology Series 58, pp. 741-763.

Garzanti, E., Andò, S., 2007b. Heavy-mineral concentration in modern sands: implications for provenance interpretation. In: Mange, M.A., Wright, D.T. (Eds.), Heavy Minerals in Use. Elsevier, Amsterdam, Developments in Sedimentology Series 58, pp. 517-545.

Garzanti, E., Vezzoli, G., 2003. A classification of metamorphic grains in sands based on their composition and grade. Journal of Sedimentary Research 73, 830-837.

Garzanti, E., Doglioni, C., Vezzoli, G., Andò, S., 2007. Orogenic belts and orogenic sediment provenances. The Journal of Geology 115, 315-334.

Garzanti, E., Padoan, M., Andò, S., Resentini, A., Vezzoli, G., Lustrino, M., 2013a. Weathering and relative durability of detrital minerals in equatorial climate: sand petrology and geochemistry in the East African Rift. The Journal of Geology 121, 547-580.

Garzanti, E., Padoan, M., Setti, M., Peruta, L., Najman, Y., Villa, I.M., 2013b. Weathering geochemistry and Sr-Nd fingerprints of equatorial upper Nile and Congo muds. Geochemistry Geophysics Geosystems 14, 292-316.

Garzanti, E., Vermeesch, P., Padoan, M., Resentini, A., Vezzoli, G., Andò, S., 2014a. Provenance of passive-margin sand (southern Africa). The Journal of Geology 122, 17-42.

Garzanti, E., Padoan, M., Setti, M., López-Galindo, A., Villa, I.M., 2014b. Provenance versus weathering control on the composition of tropical river mud (southern Africa). Chemical Geology 366, 61-74.

Haley, B.A., Klinkhammer, G.P., McManus, J., 2004. Rare earth elements in pore waters of marine sediments. Geochimica et Cosmochimica Acta 68, 1265-1279.

Harnois, L., 1988. The CIW index: a new chemical index of weathering. Sedimentary Geology 55, 319-322.

Hubert, J.F., 1962. A zircon-tourmaline-rutile maturity index and the interdependence of the composition of heavy minerals assemblages with the gross composition and texture of sandstones. Journal of Sedimentary Petrology 32, 440-450.

McDonough, W.F., Sun, S.S., 1995. The composition of the Earth. Chemical Geology 120, 223-253.

Nesbitt, H.W., Young, G.M., 1982. Early Proterozoic climates and plate motions inferred from major element chemistry of lutites. Nature 299, 715-717.

Parker, A., 1970. An index of weathering for silicate rocks. Geological Magazine 107, 501-504. 
Table A1. Sample location

1

2

3

4

Sample $\quad$ River / Lake Sit

WHITE NILESYSTEM

3641Ls Kage

3642S Lake Victoria

3692S /WN17 Victoria Nile

3690S/WN16 Albert Nile

$4052 S$

Bahr El Jebel

13

Bahr el Jebel

Lol

Sobat

24

2878L,S White Nile

BLUE NILE SYSTEM

3 2947L,S Abay

$142975 s \quad$ Anger

$12976 \mathrm{~L}, \mathrm{~S} \quad$ Dides

3295L,S Dabus

6 2970L,S Beles

17 2964L,S Blue Nile

18 2876s Rahad

19

20

22

23

24

\begin{tabular}{|c|c|c|c|c|c|c|}
\hline Latitude & Longitude & Altitude & $\begin{array}{l}\text { Collected/ } \\
\text { provided by }\end{array}$ & Country & Year & Main source rocks \\
\hline S 0 0 ${ }^{\circ} 14^{\prime} 57^{\prime \prime}$ & E $31^{\circ} 25^{\prime} 07^{\prime \prime}$ & $1150 \mathrm{~m}$ & E.Garzanti & Tanzania & 2007 & Karagwe-Ankole Belt \\
\hline S $00^{\circ} 56^{\prime} 23^{\prime \prime}$ & E $31^{\circ} 45^{\prime} 49^{\prime \prime}$ & $1135 \mathrm{~m}$ & E.Garzanti & Uganda & 2007 & Karagwe-Ankole Belt \\
\hline S $00^{\circ} 55^{\prime} 00^{\prime \prime}$ & E $31^{\circ} 45^{\prime} 44^{\prime \prime}$ & $1135 \mathrm{~m}$ & E.Garzanti & Uganda & 2007 & Kagera delta \\
\hline N $02^{\circ} 16^{\prime} 31^{\prime \prime}$ & E $31^{\circ} 40^{\prime} 36^{\prime \prime}$ & $637 \mathrm{~m}$ & E.Garzanti & Uganda & 2007 & Uganda basements \\
\hline N $02^{\circ} 43^{\prime} 25^{\prime \prime}$ & E $31^{\circ} 26^{\prime} 36^{\prime \prime}$ & $620 \mathrm{~m}$ & E.Garzanti & Uganda & 2007 & Northwestern Uganda basement \\
\hline N $04^{\circ} 49^{\prime} 30^{\prime \prime}$ & E $31^{\circ} 37^{\prime}$ & $453 \mathrm{~m}$ & M.Khaled & South Sudan & 2009 & Uganda / South Sudan basements \\
\hline N $06^{\circ} 12^{\prime} 14^{\prime \prime}$ & E $31^{\circ} 33^{\prime} 09^{\prime \prime}$ & $426 \mathrm{~m}$ & Fouad I University & South Sudan & before 1950 & Uganda / South Sudan basements \\
\hline N $09^{\circ} 09^{\prime} 12^{\prime \prime}$ & E $26^{\circ} 58^{\prime} 57^{\prime \prime}$ & $440 \mathrm{~m}$ & Fouad I University & South Sudan & before 1950 & South Sudan basement \\
\hline N $08^{\circ} 36^{\prime} 45^{\prime \prime}$ & E $33^{\circ} 04^{\prime} 20^{\prime \prime}$ & $404 \mathrm{~m}$ & Fouad I University & South Sudan & before 1950 & Ethiopian basement/S.Sudan lowlands \\
\hline N $13^{\circ} 10^{\prime} 26^{\prime \prime}$ & E $32^{\circ} 41^{\prime} 58^{\prime \prime}$ & $385 \mathrm{~m}$ & E.Garzanti & Sudan & 2003 & Sudanese lowlands recycled \\
\hline N $10^{\circ} 04^{\prime} 22^{\prime \prime}$ & E $38^{\circ} 11^{\prime} 23^{\prime \prime}$ & $1039 \mathrm{~m}$ & E.Garzanti & Ethiopia & 2004 & Mostly Ethiopian Traps \\
\hline N 09²6'01" & E $36^{\circ} 32^{\prime} 29^{\prime \prime}$ & $1290 \mathrm{~m}$ & E.Garzanti & Ethiopia & 2004 & Mainly Ethiopian basement \\
\hline N $09^{\circ} 01^{\prime} 51^{\prime \prime}$ & E $36^{\circ} 09^{\prime} 19^{\prime \prime}$ & $1130 \mathrm{~m}$ & E.Garzanti & Ethiopia & 2004 & Mainly Ethiopian basement \\
\hline N $09^{\circ} 45^{\prime} 52^{\prime \prime}$ & E $34^{\circ} 48^{\prime} 34^{\prime \prime}$ & $1339 \mathrm{~m}$ & A.A.Abdel Megid & Ethiopia & 2005 & Mainly Ethiopian basement \\
\hline N 1107'56" & E $35^{\circ} 28^{\prime} 12^{\prime \prime}$ & $605 \mathrm{~m}$ & E.Garzanti & Ethiopia & 2004 & Ethiopian Traps + basement \\
\hline N $11^{\circ} 07^{\prime} 11^{\prime \prime}$ & E $35^{\circ} 11^{\prime} 48^{\prime \prime}$ & $519 \mathrm{~m}$ & E.Garzanti & Ethiopia & 2004 & Ethiopian Traps + basement \\
\hline N $14^{\circ} 06^{\prime} 08^{\prime \prime}$ & E $33^{\circ} 55^{\prime} 38^{\prime \prime}$ & $415 \mathrm{~m}$ & E.Garzanti & Sudan & 2003 & Ethiopian Traps + basement \\
\hline N 153'ㄱ' & E $32^{\circ} 34^{\prime} 42^{\prime \prime}$ & $379 \mathrm{~m}$ & E.Garzanti & Sudan & 2003 & Ethiopian Traps + basement \\
\hline N $13^{\circ} 44^{\prime} 10^{\prime \prime}$ & E $38^{\circ} 11^{\prime} 35^{\prime \prime}$ & $853 \mathrm{~m}$ & Fouad I University & Ethiopia & 2005 & Mostly Ethiopian Traps \\
\hline N $14^{\circ} 23^{\prime} 56^{\prime \prime}$ & E $35^{\circ} 52^{\prime} 52^{\prime \prime}$ & $476 \mathrm{~m}$ & E.Garzanti & Sudan & 2003 & Mostly Ethiopian Traps \\
\hline N $17^{\circ} 37^{\prime} 11^{\prime \prime}$ & E $34^{\circ} 08^{\prime} 36^{\prime \prime}$ & $356 \mathrm{~m}$ & E.Garzanti & Sudan & 2003 & Mostly Ethiopian Traps \\
\hline N $18^{\circ} 32^{\prime} 38^{\prime \prime}$ & E $31^{\circ} 51^{\prime} 22^{\prime \prime}$ & $247 \mathrm{~m}$ & E.Garzanti & Sudan & 2003 & Ethiopian Traps + Continental Block \\
\hline N $19^{\circ} 22^{\prime} 27^{\prime \prime}$ & E $33^{\circ} 22^{\prime} 52^{\prime \prime}$ & $313 \mathrm{~m}$ & Y.Najman/L.Fiedling & Sudan & 2013 & Ethiopian Traps + Continental Block \\
\hline N 1801'38" & E $30^{\circ} 56^{\prime} 44^{\prime \prime}$ & $245 \mathrm{~m}$ & E.Garzanti & Sudan & 2003 & Mainly Nubian Sandstone \\
\hline N $19^{\circ} 40^{\prime}$ & E $30^{\circ} 24^{\prime}$ & $226 \mathrm{~m}$ & M.Levi & Sudan & 2003 & Ethiopian Traps + Continental Block \\
\hline N $21^{\circ} 20^{\prime} 47^{\prime \prime}$ & E $30^{\circ} 55^{\prime} 14^{\prime \prime}$ & $183 \mathrm{~m}$ & Y.Najman/L.Fiedling & Sudan & 2013 & Ethiopian Traps + Continental Block \\
\hline N 21ํ⒋'34" & E $31^{\circ} 14^{\prime} 04^{\prime \prime}$ & $180 \mathrm{~m}$ & Fouad I University & Sudan & before 1950 & Ethiopian Traps + Continental Block \\
\hline N $24^{\circ} 04^{\prime}$ & $E 32^{\circ} 52^{\prime}$ & $90 \mathrm{~m}$ & Fouad I University & Egypt & before 1950 & Ethiopian Traps + Continental Block \\
\hline N 24운 & $E 32^{\circ} 52^{\prime}$ & $90 \mathrm{~m}$ & A.A.Abdel Megid & Egypt & 2003 & Ethiopian Traps + Continental Block \\
\hline N $25^{\circ} 32^{\prime}$ & $E 32^{\circ} 38^{\prime}$ & $68 \mathrm{~m}$ & A.A.Abdel Megid & Egypt & 2003 & Ethiopian Traps + Continental Block \\
\hline N $27^{\circ} 40^{\prime} 11^{\prime \prime}$ & E $32^{\circ} 34^{\prime} 34^{\prime \prime}$ & $477 \mathrm{~m}$ & Y.Najman/L.Fiedling & Egypt & 2013 & Nubian Sandstone + Eocene limestone \\
\hline N 2659'53" & E $32^{\circ} 44^{\prime} 56^{\prime \prime}$ & $274 \mathrm{~m}$ & Y.Najman/L.Fiedling & Egypt & 2013 & Nubian Sandstone + Eocene limestone \\
\hline N 2615'11" & E $32^{\circ} 45^{\prime} 19^{\prime \prime}$ & $95 \mathrm{~m}$ & E.Garzanti & Egypt & 2002 & Mesozoic covers $+\mathrm{RSH}$ basement \\
\hline N 2956'37" & E $31^{\circ} 16^{\prime} 09^{\prime \prime}$ & $15 \mathrm{~m}$ & Fouad I University & Egypt & before 1950 & Ethiopian Traps + Continental Block \\
\hline N 2957'18" & E $31^{\circ} 14^{\prime} 50^{\prime \prime}$ & $15 \mathrm{~m}$ & Fouad I University & Egypt & before 1950 & Ethiopian Traps + Continental Block \\
\hline N 31ํ2'16" & E $30^{\circ} 20^{\prime} 27^{\prime \prime}$ & $0 \mathrm{~m}$ & A.A.Abdel Megid & Egypt & 2005 & Ethiopian Traps + Continental Block \\
\hline N 31ํ26'38" & E $30^{\circ} 21^{\prime} 29^{\prime \prime}$ & $0 \mathrm{~m}$ & E.Garzanti & Egypt & 2007 & Ethiopian Traps + Continental Block \\
\hline N 31ํ2'ㄹ" & E $31^{\circ} 46^{\prime} 46$ & $0 \mathrm{~m}$ & A.A.Abdel Megid & Egypt & 2005 & Ethiopian Traps + Continental Block \\
\hline N $25^{\circ} 10^{\prime} 39^{\prime \prime}$ & E $27^{\circ} 24^{\prime} 43^{\prime \prime}$ & $455 \mathrm{~m}$ & Y.Najman/L.Fiedling & Egypt & 2013 & Nubian Sandstone \\
\hline N 2614'39" & E $27^{\circ} 35^{\prime} 10^{\prime \prime}$ & $232 \mathrm{~m}$ & Y.Najman/L.Fiedling & Egypt & 2013 & Nubian Sandstone \\
\hline N 26⒌ $59^{\prime} 05^{\prime \prime}$ & E $25^{\circ} 14^{\prime} 05^{\prime \prime}$ & $190 \mathrm{~m}$ & Y.Najman/L.Fiedling & Egypt & 2013 & Nubian Sandstone \\
\hline
\end{tabular}

Latitude Longitude Altitude Collected/ Country Year Main source rocks provenance

Continental Block Continental Block Continental Block DCB metamorphic DCB metamorphic DCB metamorphic DCB metamorphic DCB metamorphic DCB metamorphic Recycled clastic

Anorogenic Volcanic DCB metamorphic DCB metamorphic DCB metamorphic Anorogenic mixed Anorogenic mixed Anorogenic mixed Anorogenic mixed

Anorogenic Volcanic Anorogenic Volcanic Anorogenic Volcanic

Anorogenic mixed Anorogenic mixed Undissected C. Block Anorogenic mixed Anorogenic mixed Anorogenic mixed Anorogenic mixed Anorogenic mixed Anorogenic mixed Undissected C. Block Undissected C. Block Transitional C. Block Anorogenic mixed

Anorogenic mixed

Anorogenic mixed Anorogenic mixed Anorogenic mixed

Undissected C. Block Undissected C. Block Undissected C. Block

Site
Kyaka
Kasensero
Kasensero
Murchison Fall
Wadelai
Juba
Bor
Nyamlell
Nasser
Rabak
Blue Nile

Goha Tsiyon Tsige Maryam Ephrem Bambesi Enat Beles Bambudi Hufeira Khartoum

Togo Ber Showak Abu Ammar

Karima SD05 Ed Debba

3th cataract SD04

Wadi Halfa

Aswan

Aswan

Luxor 2401B

$4727 S$ 1989S $51 \quad$ Nile NILE DELTA 3290S beach 3709P beach placer 3289S beach WESTERN DESERT 4729E eolian dune 4730E eolian dune 4728E eolian dune
RSH3A

RSH5A

Qena

Cairo Tora

Cairo Ma'adi

Idku east

Rosetta

Ras El Barr

WD19C

WD20C

WD03C 


\section{S Kagera Kyak}

$\begin{array}{ll}3643 \mathrm{~S} & \text { Kagera } \\ 3641 \mathrm{~L} & \text { Kagera }\end{array}$

$\begin{array}{ll}3641 \mathrm{~S} & \text { Kagera } \\ 3642 \mathrm{~S} & \text { Lake Victoria }\end{array}$

$3692 S$ /WN17 Victoria Nile

3690S/WN16 Albert Nile

3690S/WN16 Albert Nile

4052S Bahr El Jebel

$\begin{array}{ll}24 & \text { Sobat } \\ 2878 \mathrm{~L} & \text { White Nile }\end{array}$

Kyaka
Kyaka

G.Vezzoli

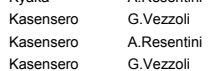

$\begin{array}{ll}\text { Kasensero } & \text { G.Vezzol } \\ \text { Murchison Falls } & \text { GVezzol }\end{array}$

$\begin{array}{ll}\text { Murchison Falls } & \text { G.Vezzoli } \\ \text { Murchison Falls } & \text { A.Resentini }\end{array}$

Wadelai G.Vezzoli

$\begin{array}{ll}\text { Wadelai } & \text { A.Resentin } \\ \text { Juba } & \text { G.Vezzoli }\end{array}$

Juba G.Vezzo

$2878 S$ White Nile
BLUE NILE SYSTEM

$\begin{array}{ll}2947 L & \text { Abay } \\ 2947 S & \text { Abay }\end{array}$

$\begin{array}{ll}\text { Nasser } & \text { S.Andò } \\ \text { Rabak } & \text { A.A.Megid }\end{array}$

29755 Anger

$\begin{array}{lll}2976 \mathrm{~L} & \text { Didesa } \\ 2976 \mathrm{~S} & \text { Didesa }\end{array}$

$\begin{array}{lll}3295 \mathrm{~L} & \text { Dabus } \\ 3295 \mathrm{~S} & \text { Dabus }\end{array}$

$14 \quad 2905020$

$\begin{array}{ll}2970 \mathrm{~L} & \text { Beles } \\ 2970 \mathrm{~S} & \text { Beles }\end{array}$

$5 \quad 2904$

2964L Blue Nile

$162964 \mathrm{~S} \quad$ Blue Nile

12 Blue Nile

82 Blue Nile

$\begin{array}{lll}2964 \mathrm{~S} & \text { Blue Nile } \\ 192964 \mathrm{~S} & \text { Blue Nile }\end{array}$

$28765 \quad$ Rahad

$\begin{array}{lll}2843 \mathrm{~L} & \text { Blue Nile } \\ 1243 \mathrm{~S} & \text { Blue Nile }\end{array}$

$\begin{array}{lll}21 & 2843 S & \text { Blue Nile } \\ 2 & 28435 & \text { Blue Nile }\end{array}$

22 Blue Nile 2843S Blue Nile
ATBARA SYSTEM

Khartoum

A.A.Megid
A.A.Megid

A.A.Megid
A.A.Megid

A.A.Megid
A.A.Megid

A.A.Megid
A.A.Megid

A.A.Megid
A.A.Megid

$\begin{array}{ll}{ }_{33125} & \text { Tekeze } \\ \text { Tekezeze }\end{array}$

Togo Ber A.A.Megid

$\begin{array}{ll}\text { Togo Ber } & \text { A.A.Megid } \\ \text { Showak } & \text { A.A.Megid }\end{array}$

$\begin{array}{ll}2873 \mathrm{~L} & \text { Atbara } \\ 2873 \mathrm{~S} & \text { Atbara }\end{array}$

2873S Atbara

$\begin{array}{lll}28 & 28735 & \text { Atbara } \\ 29 & 28735 & \text { Atbara } \\ 28582 & \text { Athara }\end{array}$

Showak
Showak

A.A.Megid

$\begin{array}{ll}\text { Showak } & \text { A.A.Megid } \\ \text { Showak } & \text { A.A.Megid } \\ \text { Showak } & \text { A.A.Megid }\end{array}$

$\begin{array}{ll}\text { Abu Ammar } & \text { A.A.A.Megid } \\ \text { Abu Ammar } & \text { A.A.Megid }\end{array}$

Abbara

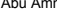

$\begin{array}{lll}4735 L & \text { Nile } \\ 28475 & \text { Milk } \\ 2 & \end{array}$

SD05
Ed Debba

G.Vezzoli
A.A.Megid

2852L Nile

Karima

Karima

A.A.Megid

3

Karma
Karima

A.A.Megid

Karima
Karima

A.A.Megid

Karima AAMegic

$\begin{array}{ll}\text { 3rd cataract } & \text { A.A.Megid } \\ \text { 3rd cataract } & \text { A.A.Megid }\end{array}$

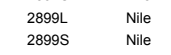

3rd cataract

A.A.Megid
A.A.Megid

$\begin{array}{ll}\text { 3rd cataract } & \text { A.A.Megid } \\ \text { 3rd cataract } & \text { A.A.Megid }\end{array}$

$\begin{array}{lll}28995 & \text { Nile } \\ 28995 & \text { Nile }\end{array}$

3rd cataract

AA.Megid
A.A.Megid
A.

AAMegid

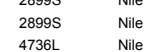

3rd cataract

G.Vezzoli
G.Vezzoli

A.A.Megid

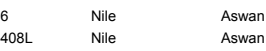

$41 \quad 240818$

Aswan
Luxor

G.Vezzoli

47265
47275

Unm omeney

RSH5A

$44 \quad 31$

2005 S- Nile

Qena

G.Vezzoli
A.A.Megid

A.A.Megid
G.Vezzoli
G

G.Vezzoli

G.Verzoli
G.Verzoli
G.

G.Vezzoli
G.Verzoli
G.

G.Vezzoli

G.Vezzoli
G.Vezzoli

G.Vezzoli
G.Vezzoli

G.Vezzzoli

G.Vezzzoli

G.Vezzoli

Delta beach placer Rose

Delta beach placer Rosett

Della beach placer Roselta
Deta beach placer Rosetta
63-250

250-1000

250-1000

250-1000

250-1000

250-1000

$63-250$
$250-1000$

63-250 n.

378 bulk

$\begin{array}{lllllllllllllllllllll}31 \% & 92.2 & 2.1 & 2.1 & 0.7 & 0.0 & 0.0 & 0.3 & 0.0 & 0.7 & 0.0 & 0.7 & 0.0 & 0.0 & 0.3 & 0.0 & 1.0 & 100.0 & 233 & 175 & 8\end{array}$

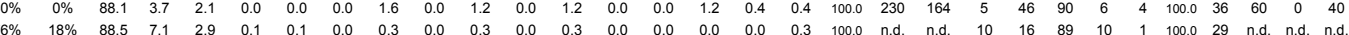

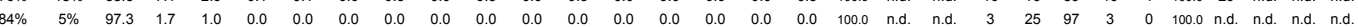

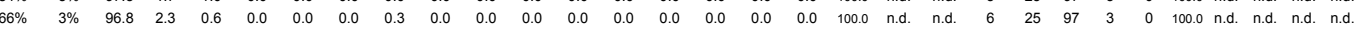

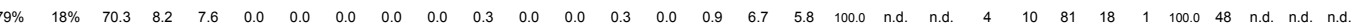
$\begin{array}{llllllllllllllllllllllllllllllll} & 1 \% & 1 \% & 61.3 & 11.9 & 14.5 & 0.0 & 0.0 & 0.0 & 0.0 & 0.0 & 0.4 & 0.0 & 2.2 & 0.0 & 0.0 & 1.5 & 1.5 & 6.7 & 100.0 & 347 & 347 & 8 & 8 & 68 & 29 & 3 & 100.0 & 55 & \text { n.d. n.d. n.d. }\end{array}$ $\begin{array}{lllllllllllllllllllllllllllllll} & 39 \% & 52.9 & 14.1 & 13.2 & 0.0 & 0.0 & 0.0 & 0.0 & 0.0 & 0.3 & 0.3 & 0.6 & 0.3 & 0.0 & 0.5 & 2.3 & 15.6 & 100.0 & 363 & 363 & 10 & 14 & 65 & 33 & 2 & 100.0 & 48 & \text { n.d. } & \text { n.d. } & \text { n.d. }\end{array}$

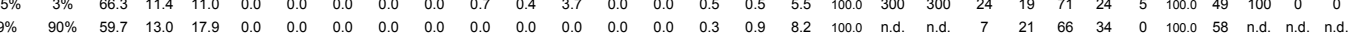
$\begin{array}{llllllllllllllllllllllllllllllllllll}18 \% & 64.7 & 12.8 & 19.8 & 0.0 & 0.3 & 0.0 & 0.0 & 0.0 & 0.0 & 0.0 & 0.0 & 0.0 & 0.0 & 0.0 & 0.0 & 2.3 & 100.0 & 413 & 330 & 26 & 11 & 66 & 33 & 0 & 100.0 & 61 & \text { n.d. } & \text { n.d. } & \text { n.d. }\end{array}$ $\begin{array}{cccccccccccccccccccccccccccccc}37.0 & 10.9 & 50.0 & 0.0 & 0.0 & 0.0 & 0.0 & 0.0 & 0.0 & 0.0 & 0.0 & 0.0 & 0.0 & 0.0 & 1.1 & 1.1 & 100.0 & \text { n.d. } & \text { n.d. } & 0 & 0 & 38 & 62 & 0 & 100.0 & 82 & \text { n.d. } & \text { n.d. } & \text { n.d. } \\ \quad 98.1 & 1.1 & 0.4 & 0.0 & 0.0 & 0.0 & 0.0 & 0.0 & 0.0 & 0.0 & 0.0 & 0.0 & 0.0 & 0.0 & 0.0 & 0.4 & 100.0 & \text { n.d. } & \text { n.d. } & 3 & \text { n.d. } & 98 & 2 & 0 & 100.0 & \text { n.d. } & \text { n.d. } & \text { n.d. } & \text { n.d. }\end{array}$

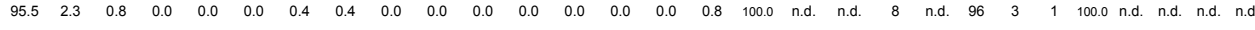
274 bulk bulk $\begin{array}{lllllllllllllllllllllllllllll}19.9 & 0.0 & 6.6 & 2.6 & 55.9 & 5.3 & 0.0 & 0.0 & 0.0 & 0.0 & 0.0 & 0.0 & 0.0 & 0.0 & 0.0 & 9.6 & 100.0 & \text { n.d. } & 0 & 13 & 0 & 22 & 7 & 71 & 100.0 & 100 & 0 & 92 & 8\end{array}$ $\begin{array}{lllllllllllllllllllllllllllllll}60.0 & 15.2 & 8.7 & 2.0 & 4.1 & 0.0 & 0.0 & 0.0 & 0.0 & 0.9 & 2.0 & 0.7 & 0.0 & 0.0 & 1.3 & 5.2 & 100.0 & 375 & 237 & 16 & 36 & 64 & 26 & 10 & 100.0 & 36 & 36 & 64 & 0\end{array}$ $\begin{array}{llllllllllllllllllllllllllllll}71.6 & 4.3 & 8.1 & 0.0 & 0.0 & 0.0 & 0.0 & 0.0 & 0.0 & 0.0 & 0.0 & 0.0 & 0.0 & 0.0 & 3.3 & 12.8 & 100.0 & \text { n.d. } & \text { n.d. } & 3 & 36 & 85 & 15 & 0 & 100.0 & 65 & \text { n.d. } & \text { n.d. } & \text { n.d. } \\ 56.8 & 9.7 & 18.7 & 0.9 & 2.6 & 0.0 & 0.0 & 0.0 & 0.0 & 0.0 & 0.0 & 0.0 & 0.0 & 0.6 & 0.2 & 10.5 & 100.0 & 500 & 265 & 29 & 26 & 64 & 32 & 4 & 100.0 & 66 & \text { n.d. } & \text { n.d. } & \text { n.d. }\end{array}$ $\begin{array}{lllllllllllllllllllllllllllll}56.4 & 2.0 & 11.9 & 0.0 & 3.0 & 0.0 & 0.0 & 0.0 & 0.0 & 4.0 & 0.0 & 0.0 & 0.0 & 0.0 & 5.9 & 16.8 & 100.0 & 257 & 180 & 6 & 15 & 73 & 18 & 9 & 100.0 & 86 & 57 & 43 & 0\end{array}$

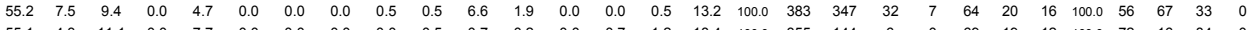

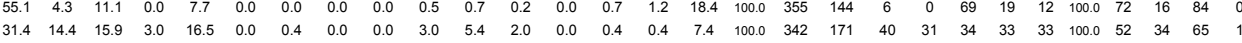

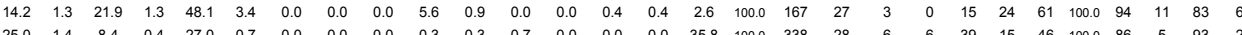

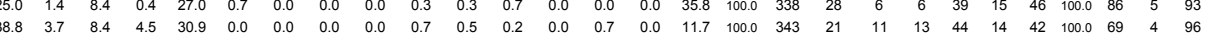

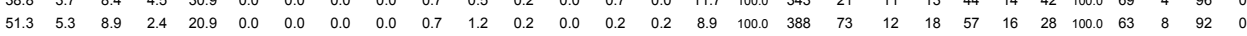
$\begin{array}{lllllllllllllllllllllllllllll}58.7 & 8.9 & 16.6 & 2.1 & 5.3 & 0.0 & 0.0 & 0.0 & 0.0 & 0.4 & 1.8 & 1.1 & 0.0 & 0.0 & 0.0 & 5.2 & 100.0 & 394 & 187 & 28 & 25 & 62 & 27 & 11 & 100.0 & 65 & 31 & 69 & 0\end{array}$

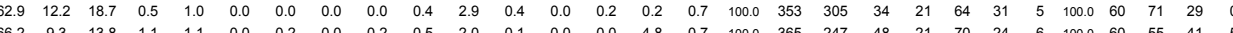
$\begin{array}{lllllllllllllllllllllllllllllllll}\mathbf{7 1 0 - 1 0 0 0} & 6.7 \% & & 66.2 & 9.3 & 13.8 & 1.1 & 1.1 & 0.0 & 0.2 & 0.0 & 0.2 & 0.5 & 2.0 & 0.1 & 0.0 & 0.0 & 4.8 & 0.7 & 100.0 & 365 & 247 & 48 & 21 & 70 & 24 & 6 & 100.0 & 60 & 55 & 41 & 5 \\ \mathbf{1 2 5 - 1 0 0 0} & \mathbf{9 7 . 5 \%} & \mathbf{1 . 4} \% & \mathbf{5 3 . 6} & \mathbf{7 . 5} & \mathbf{1 3 . 1} & \mathbf{2 . 1} & \mathbf{1 3 . 3} & \mathbf{0 . 0} & \mathbf{0 . 0} & \mathbf{0 . 0} & \mathbf{0 . 0} & \mathbf{0 . 5} & \mathbf{1 . 6} & \mathbf{0 . 5} & \mathbf{0 . 0} & \mathbf{0 . 2} & \mathbf{0 . 4} & \mathbf{7 . 2} & \mathbf{1 0 0 . 0} & \mathbf{3 5 9} & \mathbf{1 3 0} & \mathbf{2 0} & \mathbf{1 9} & \mathbf{5 8} & \mathbf{2 2} & \mathbf{2 0} & \mathbf{1 0 0 . 0} & \mathbf{6 4} & \mathbf{1 4} & \mathbf{8 5} & \mathbf{0}\end{array}$

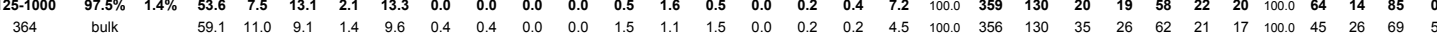
$\begin{array}{ll}364 & \text { bulk } \\ 235 & \text { bulk }\end{array}$ $\begin{array}{cc}72 & \text { bulk } \\ 125-180 & 3.0 \%\end{array}$ $180-250 \quad 16.9 \%$ $250-355 \quad 41.3 \%$ $\begin{array}{lllllllllllllllllllllllllllllllll}24.6 & 1.0 & 11.8 & 7.5 & 40.3 & 0.3 & 0.0 & 0.0 & 0.3 & 1.7 & 0.2 & 0.8 & 0.0 & 0.0 & 1.0 & 10.4 & 100.0 & 250 & 23 & 4 & 3 & 28 & 14 & 58 & 100.0 & 92 & 6 & 93 & 1\end{array}$ $\begin{array}{lllllllllllllllllllllllllllllllll}21.7 & 2.8 & 3.4 & 0.0 & 8.4 & 0.0 & 0.0 & 0.0 & 0.0 & 0.6 & 0.2 & 1.1 & 0.0 & 0.0 & 0.3 & 61.5 & 100.0 & 315 & 89 & 9 & 23 & 57 & 16 & 27 & 100.0 & 55 & 18 & 82 & 0 \\ 50.6 & 8.4 & 10.6 & 0.6 & 13.1 & 0.0 & 0.0 & 0.0 & 0.0 & 0.0 & 1.1 & 0.8 & 0.0 & 0.0 & 0.0 & 14.8 & 100.0 & 342 & 98 & 11 & 22 & 59 & 22 & 18 & 100.0 & 56 & 12 & 88 & 0\end{array}$ $\begin{array}{lllllllllllllllllllllllllllllllllll}64.5 & 6.8 & 17.2 & 0.8 & 2.9 & 0.0 & 0.0 & 0.0 & 0.0 & 0.7 & 1.2 & 1.5 & 0.0 & 0.2 & 0.5 & 3.7 & 100.0 & 376 & 247 & 12 & 18 & 67 & 25 & 7 & 100.0 & 72 & 48 & 52 & 0\end{array}$ $\begin{array}{ccccccccccccccccccccccccccccc}71.6 & 9.4 & 13.3 & 0.3 & 1.2 & 0.0 & 0.0 & 0.0 & 0.0 & 1.4 & 1.1 & 0.4 & 0.0 & 0.0 & 0.0 & 1.4 & 100.0 & 327 & 225 & 23 & 18 & 73 & 23 & 4 & 100.0 & 59 & 67 & 33 & 0 \\ 72.5 & 12.2 & 7.3 & 0.7 & 1.4 & 0.0 & 0.3 & 0.0 & 0.0 & 0.0 & 0.7 & 0.0 & 0.0 & 0.0 & 4.5 & 0.3 & 100.0 & \text { n.d. } & 118 & 27 & 36 & 76 & 21 & 3 & 100.0 & 38 & \text { n.d. } & \text { n.d. } & \text { n.d. } \\ \end{array}$

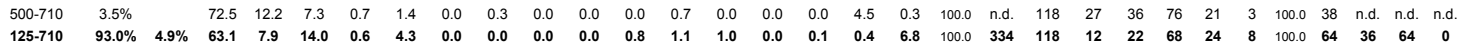
$\begin{array}{cccccccccccccccccccccccccccccccc}125-710 & 93.0 \% & 4.9 \% & 63.1 & 7.9 & 14.0 & 0.6 & 4.3 & 0.0 & 0.0 & 0.0 & 0.0 & 0.8 & 1.1 & 1.0 & 0.0 & 0.1 & 0.4 & 6.8 & 100.0 & 334 & 118 & 12 & 22 & 68 & 24 & 8 & 100.0 & 64 & 36 & 64 & 0 \\ 277 & \text { bulk } & & 65.1 & 12.3 & 15.1 & 1.1 & 1.1 & 0.4 & 0.0 & 0.0 & 0.0 & 1.8 & 0.0 & 0.0 & 0.0 & 0.0 & 1.1 & 2.1 & 100.0 & 291 & 229 & 20 & 35 & 67 & 28 & 4 & 100.0 & 55 & 42 & 50 & 8\end{array}$

$\begin{array}{ccc}83 & \text { bulk } & \\ 120 & \text { bukk } & \\ 90 & \text { bulk } & \\ 125-180 & 34.8 \% & \\ 180-250 & 36.3 \% & \\ 250-355 & 7.5 \% & \\ \mathbf{1 2 5 - 3 5 5} & \mathbf{7 8 . 7 \%} & \mathbf{3 . 9 \%} \\ 157 & \text { buk } & \\ 77 & \text { bulk } & \\ & & \end{array}$

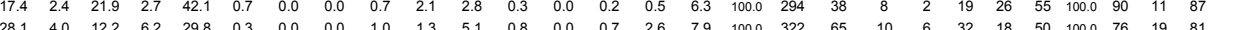

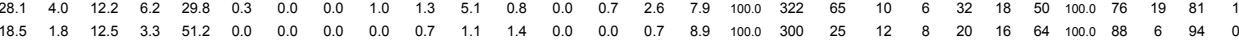

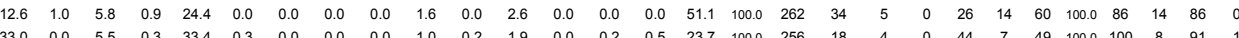
$\begin{array}{cccccccccccccccccccccccccccccc}33.0 & 0.0 & 5.5 & 0.3 & 33.4 & 0.3 & 0.0 & 0.0 & 0.0 & 1.0 & 0.2 & 1.9 & 0.0 & 0.2 & 0.5 & 23.7 & 100.0 & 256 & 18 & 4 & 0 & 44 & 7 & 49 & 100.0 & 100 & 8 & 91 & 1 \\ 47.8 & 2.4 & 7.8 & 3.3 & 25.9 & 0.0 & 0.0 & 0.0 & 1.4 & 0.3 & 1.0 & 2.7 & 0.0 & 0.0 & 0.0 & 7.5 & 100.0 & 295 & 57 & 9 & 5 & 52 & 11 & 37 & 100.0 & 77 & 16 & 84 & 0 & \\ \end{array}$

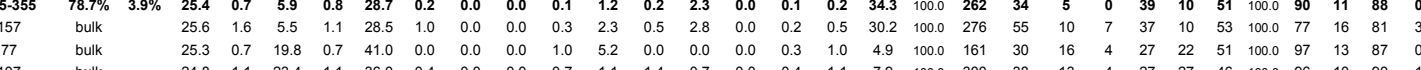

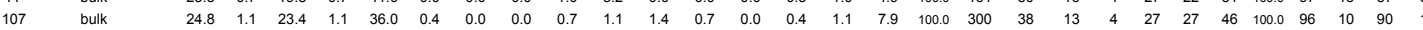

\begin{tabular}{|c|c|c|}
\hline & & \\
\hline 318 & bulk & \\
\hline 96 & bulk & \\
\hline $125-180$ & $3.2 \%$ & \\
\hline $180-250$ & $21.1 \%$ & \\
\hline $250-355$ & $68.0 \%$ & \\
\hline $355-500$ & $4.3 \%$ & \\
\hline $125-500$ & $96.7 \%$ & $0.6 \%$ \\
\hline 264 & bulk & \\
\hline 75 & bulk & \\
\hline $125-180$ & $5.8 \%$ & \\
\hline $180-250$ & $19.2 \%$ & \\
\hline $250-355$ & $58.4 \%$ & \\
\hline $355-500$ & $7.8 \%$ & \\
\hline $80-500$ & $91.2 \%$ & $3.7 \%$ \\
\hline 242 & bulk & \\
\hline 80 & bulk & \\
\hline 120 & bulk & \\
\hline 70 & bulk & \\
\hline 340 & bulk & \\
\hline 220 & bulk & \\
\hline 260 & bulk & \\
\hline 450 & bulk & \\
\hline 125 & bulk & \\
\hline $80-106$ & $2.1 \%$ & \\
\hline $06-125$ & $2.7 \%$ & \\
\hline $25-150$ & $8.3 \%$ & \\
\hline $50-180$ & $22.2 \%$ & \\
\hline $80-212$ & $38.4 \%$ & \\
\hline $12-250$ & $18.5 \%$ & \\
\hline $50-300$ & $4.8 \%$ & \\
\hline $00-355$ & $1.3 \%$ & \\
\hline 30-355 & $98.3 \%$ & $1.1 \%$ \\
\hline 80-212 & $5.7 \%$ & \\
\hline $2-250$ & $1.8 \%$ & \\
\hline & $0.6 \%$ & \\
\hline & $0.1 \%$ & \\
\hline
\end{tabular}
$\begin{array}{llllllllllllllllllllllllllllll}25.3 & 2.1 & 16.8 & 0.0 & 18.9 & 1.1 & 0.5 & 0.0 & 0.3 & 0.0 & 0.8 & 0.3 & 0.0 & 0.0 & 1.6 & 32.3 & 100.0 & 314 & 27 & 8 & 3 & 38 & 29 & 33 & 100.0 & 89 & 6 & 87 & 7\end{array}$ $\begin{array}{lllllllllllllllllllllllllll}0.4 & 1.5 & 0.0 & 0.0 & 0.0 & 0.0 & 0.0 & 0.0 & 0.0 & 0.0 & 0.0 & 0.0 & 0.0 & 0.0 & 0.0 & 1000 & \text { n.d. } & \text { n.d. } & 13 & 38 & 95 & 5 & 0 & 100.0 & 31 & \text { n.d. n.d. } & \text { n.d. }\end{array}$

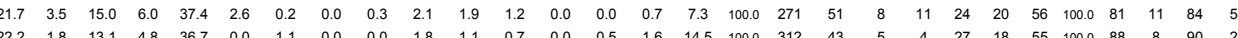
$\begin{array}{ccccccccccccccccccccccccccccc}42.2 & 1.8 & 13.1 & 4.8 & 36.7 & 0.0 & 1.1 & 0.0 & 0.0 & 1.8 & 1.1 & 0.7 & 0.0 & 0.5 & 1.6 & 14.5 & 100.0 & 312 & 43 & 5 & 4 & 27 & 18 & 55 & 100.0 & 88 & 8 & 90 & 2 \\ 4.2 & 9.4 & 1.3 & 19.5 & 0.0 & 0.0 & 0.0 & 0.0 & 2.0 & 0.5 & 2.8 & 0.0 & 0.0 & 0.0 & 18.2 & 100.0 & 281 & 63 & 12 & 19 & 51 & 17 & 32 & 100.0 & 69 & 20 & 80 & 0\end{array}$ $\begin{array}{llllllllllllllllllllllllllllllllllll}6.5 & 5.3 & 11.6 & 1.7 & 16.2 & 0.0 & 0.0 & 0.0 & 0.0 & 1.0 & 1.2 & 0.5 & 0.0 & 0.0 & 0.0 & 2.0 & 100.0 & 314 & 62 & 14 & 5 & 62 & 17 & 21 & 100.0 & 69 & 13 & 87 & 0\end{array}$ $\begin{array}{ccccccccccccccccccccccccccccc}69.2 & 9.8 & 9.8 & 2.5 & 5.7 & 0.0 & 0.0 & 0.3 & 0.0 & 0.3 & 0.3 & 0.0 & 0.0 & 0.0 & 1.0 & 1.0 & 100.0 & 300 & 56 & 16 & 33 & 71 & 20 & 9 & 100.0 & 50 & 7 & 89 & 4\end{array}$

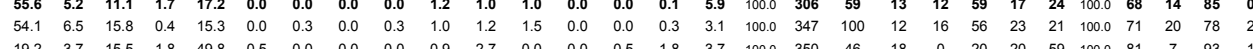
$\begin{array}{lllllllllllllllllllllllllllllll}19.2 & 3.7 & 15.5 & 1.8 & 49.8 & 0.5 & 0.0 & 0.0 & 0.0 & 0.9 & 2.7 & 0.0 & 0.0 & 0.5 & 1.8 & 3.7 & 100.0 & 350 & 46 & 18 & 0 & 20 & 20 & 59 & 100.0 & 81 & 7 & 93 & 1 \\ 40.0 & 3.7 & 16.9 & 3.0 & 19.0 & 0.0 & 0.0 & 0.0 & 0.3 & 1.0 & 3.2 & 0.5 & 0.0 & 0.0 & 0.3 & 11.9 & 100.0 & 320 & 76 & 8 & 7 & 46 & 24 & 31 & 100.0 & 82 & 19 & 81 & 0\end{array}$ $\begin{array}{lllllllllllllllllllllllllllllllllll}4.0 & 16.9 & 3.0 & 19.0 & 0.0 & 0.0 & 0.0 & 0.3 & 1.0 & 3.2 & 0.5 & 0.0 & 0.0 & 0.3 & 11.9 & 100.0 & 320 & 76 & 8 & 7 & 46 & 24 & 31 & 100.0 & 82 & 19 & 81 & 0\end{array}$

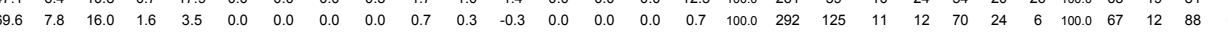
$\begin{array}{lllllllllllllllllllllllllllllllllll}75.5 & 10.1 & 7.7 & 3.1 & 1.8 & 0.0 & 0.3 & 0.0 & 0.3 & 0.0 & 0.5 & 0.2 & 0.0 & 0.0 & 0.3 & 0.0 & 100.0 & 357 & 114 & 22 & 24 & 76 & 18 & 6 & 100.0 & 43 & 17 & 78 & 6\end{array}$ $\begin{array}{llllllllllllllllllllllllllllll}63.5 & 7.5 & 14.3 & 1.6 & 7.4 & 0.0 & 0.0 & 0.0 & 0.1 & 0.9 & 0.7 & 0.1 & 0.0 & 0.0 & 0.1 & 3.8 & 100.0 & 306 & 95 & 11 & 18 & 66 & 23 & 11 & 100.0 & 66 & 16 & 83 & 0 \\ 61.3 & 5.7 & 12.4 & 0.8 & 11.2 & 0.0 & 0.0 & 0.0 & 0.0 & 0.7 & 0.0 & 1.4 & 0.0 & 0.0 & 0.0 & 6.4 & 100.0 & 344 & 76 & 17 & 17 & 66 & 19 & 15 & 100.0 & 69 & 15 & 85 & 0\end{array}$ $\begin{array}{rllllllllllllllllllllllllllll}34.0 & 5.4 & 26.1 & 2.1 & 15.5 & 1.1 & 0.8 & 0.0 & 1.1 & 0.8 & 0.5 & 0.3 & 0.0 & 0.0 & 1.9 & 10.3 & 100.0 & 230 & 29 & 10 & 5 & 39 & 36 & 25 & 100.0 & 83 & 12 & 79 & 9 \\ 47.9 & 6.6 & 123 & 0.4 & 14.9 & 0.6 & 0.3 & 0.0 & 0.3 & 0.9 & 1.9 & 0.1 & 0.3 & 0.0 & 12 & 123 & 1000 & 287 & 75 & 12 & 11 & 55 & 22 & 23 & 1000 & 65 & 17 & 78 & 5\end{array}$

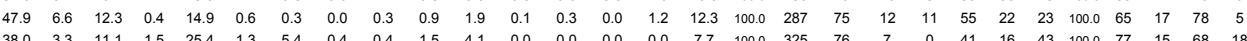
$\begin{array}{cccccccccccccccccccccccccccccc}38.0 & 3.3 & 11.1 & 1.5 & 25.4 & 1.3 & 5.4 & 0.4 & 0.4 & 1.5 & 4.1 & 0.0 & 0.0 & 0.0 & 0.0 & 7.7 & 100.0 & 325 & 76 & 7 & 0 & 41 & 16 & 43 & 100.0 & 77 & 15 & 68 & 18 \\ 62.6 & 7.5 & 13.2 & 2.2 & 8.8 & 0.2 & 0.2 & 0.4 & 0.4 & 0.7 & 0.7 & 0.0 & 0.0 & 0.0 & 0.0 & 3.2 & 100.0 & 250 & 48 & 10 & 15 & 65 & 21 & 14 & 100.0 & 64 & 13 & 82 & 5\end{array}$ $\begin{array}{llllllllllllllllllllllllllllll}61.0 & 2.5 & 1.8 & 0.6 & 0.0 & 27.9 & 3.7 & 0.0 & 0.0 & 0.0 & 0.0 & 0.0 & 0.0 & 0.0 & 0.3 & 2.1 & 100.0 & \text { n.d. } & \text { n.d. } & 4 & 0 & 63 & 4 & 33 & 100.0 & 43 & 0 & 2 & 98\end{array}$ $\begin{array}{lllllllllllllllllllllllllllllllll}8.5 & 1.8 & 2.9 & 1.4 & 0.4 & 13.2 & 1.2 & 0.0 & 0.0 & 0.3 & 0.0 & 0.0 & 0.0 & 0.0 & 0.0 & 0.3 & 100.0 & \text { n.d. } & 0 & 10 & 7 & 79 & 5 & 17 & 100.0 & 63 & 2 & 11 & 88\end{array}$ $\begin{array}{llllllllllllllllllllllllllllllllllll}50.6 & 10.6 & 13.3 & 1.1 & 1.6 & 13.1 & 1.3 & 7.2 & 0.0 & 0.8 & 0.0 & 0.0 & 0.0 & 0.0 & 0.0 & 0.4 & 100.0 & 286 & 114 & 16 & 0 & 51 & 24 & 25 & 100.0 & 56 & 3 & 11 & 86\end{array}$ $\begin{array}{ccccccccccccccccccccccccccccc}47.0 & 7.5 & 9.0 & 0.7 & 18.7 & 1.2 & 0.3 & 0.0 & 0.3 & 0.6 & 0.6 & 0.3 & 0.0 & 0.3 & 1.4 & 12.2 & 100.0 & 333 & 46 & 10 & 12 & 55 & 19 & 26 & 100.0 & 54 & 8 & 86 & 6 \\ 16.3 & 0.9 & 3.3 & 0.0 & 5.4 & 0.9 & 0.0 & 0.0 & 0.0 & 0.0 & 0.2 & 1.1 & 0.0 & 0.0 & 0.0 & 72.0 & 100.0 & \text { n.d. } & 62 & 4 & 0 & 58 & 15 & 27 & 100.0 & 79 & 16 & 72 & 12\end{array}$ $\begin{array}{llllllllllllllllllllllllllllllllllll}33.2 & 3.3 & 6.9 & 0.0 & 9.1 & 0.0 & 0.0 & 0.0 & 0.0 & 0.3 & 0.3 & 0.9 & 0.0 & 0.0 & 0.0 & 45.9 & 100.0 & 375 & 79 & 8 & 7 & 61 & 19 & 20 & 100.0 & 68 & 14 & 86 & 0\end{array}$ $\begin{array}{llllllllllllllllllllllllllllll}47.4 & 2.7 & 9.1 & 0.6 & 8.7 & 0.3 & 0.0 & 0.0 & 0.0 & 0.6 & 1.2 & 1.5 & 0.0 & 0.3 & 0.0 & 27.5 & 100.0 & 300 & 84 & 6 & 3 & 66 & 16 & 18 & 100.0 & 77 & 26 & 72 & 2 \\ 672 & 4.1 & 5.8 & 0.6 & 6.4 & 0.3 & 0.0 & 0.0 & 0.3 & 0.3 & 0.0 & 0.3 & 0.0 & 0.0 & 0.0 & 14.8 & 100.0 & 220 & 39 & 9 & 9 & 79 & 12 & 10 & 1000 & 59 & 11 & 86 & 4\end{array}$ $\begin{array}{llllllllllllllllllllllllllllllll}67.2 & 4.1 & 5.8 & 0.6 & 6.4 & 0.3 & 0.0 & 0.0 & 0.3 & 0.3 & 0.0 & 0.3 & 0.0 & 0.0 & 0.0 & 14.8 & 100.0 & 220 & 39 & 9 & 9 & 79 & 12 & 10 & 100.0 & 59 & 11 & 86 & 4 \\ 80.1 & 4.9 & 5.8 & 0.3 & 4.0 & 0.0 & 0.0 & 0.0 & 0.6 & 0.0 & 0.6 & 0.0 & 0.0 & 0.3 & 0.0 & 3.4 & 100.0 & 214 & 71 & 7 & 6 & 83 & 11 & 6 & 100.0 & 54 & 22 & 78 & 0\end{array}$

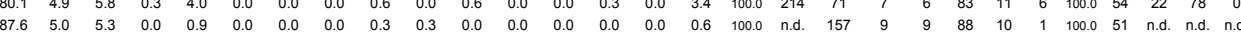

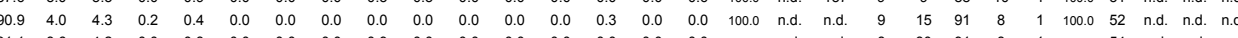
$\begin{array}{llllllllllllllllllllllllllllllllll}1.1 & 3.6 & 4.2 & 0.0 & 0.3 & 0.0 & 0.0 & 0.0 & 0.9 & 0.0 & 0.0 & 0.0 & 0.0 & 0.0 & 0.0 & 0.0 & 100.0 & \text { n.d. } & \text { n.d. } & 8 & 30 & 91 & 8 & 1 & 100.0 & 54 & \text { n.d. } & \text { n.d. } & \text { n.d. }\end{array}$

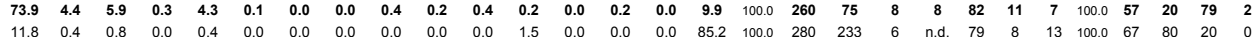

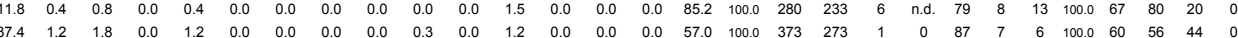

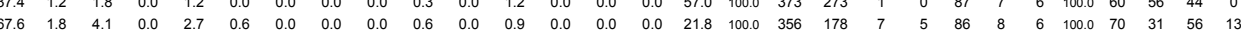
$\begin{array}{cccccccccccccccccccccccccccccccc}300-355 & 0.1 \% & & 67.8 & 1.8 & 3.0 & 0.0 & 1.2 & 0.0 & 0.0 & 0.0 & 0.0 & 0.3 & 0.6 & 0.6 & 0.0 & 0.0 & 0.0 & 24.8 & 100.0 & 279 & 217 & 10 & 29 & 90 & 6 & 4 & 100.0 & 63 & \text { n.d. } & \text { n.d. } & \text { n.d. } \\ \mathbf{1 8 0 - 3 5 5} & \mathbf{8 . 1 \%} & \mathbf{0 . 0} \% & \mathbf{2 1 . 9} & \mathbf{0 . 7} & \mathbf{1 . 2} & \mathbf{0 . 0} & \mathbf{0 . 7} & \mathbf{0 . 0} & \mathbf{0 . 0} & \mathbf{0 . 0} & \mathbf{0 . 0} & \mathbf{0 . 1} & \mathbf{0 . 0} & \mathbf{1 . 4} & \mathbf{0 . 0} & \mathbf{0 . 0} & \mathbf{0 . 0} & \mathbf{7 3 . 9} & \mathbf{1 0 0 . 0} & \mathbf{3 1 8} & \mathbf{2 2 5} & \mathbf{7} & \mathbf{5} & \mathbf{8 4} & \mathbf{7} & \mathbf{9} & \mathbf{1 0 0 . 0} & \mathbf{6 5} & \mathbf{6 6} & \mathbf{3 2} & \mathbf{2}\end{array}$

$\begin{array}{ll}140 & >95 \\ 150 & >95\end{array}$ 


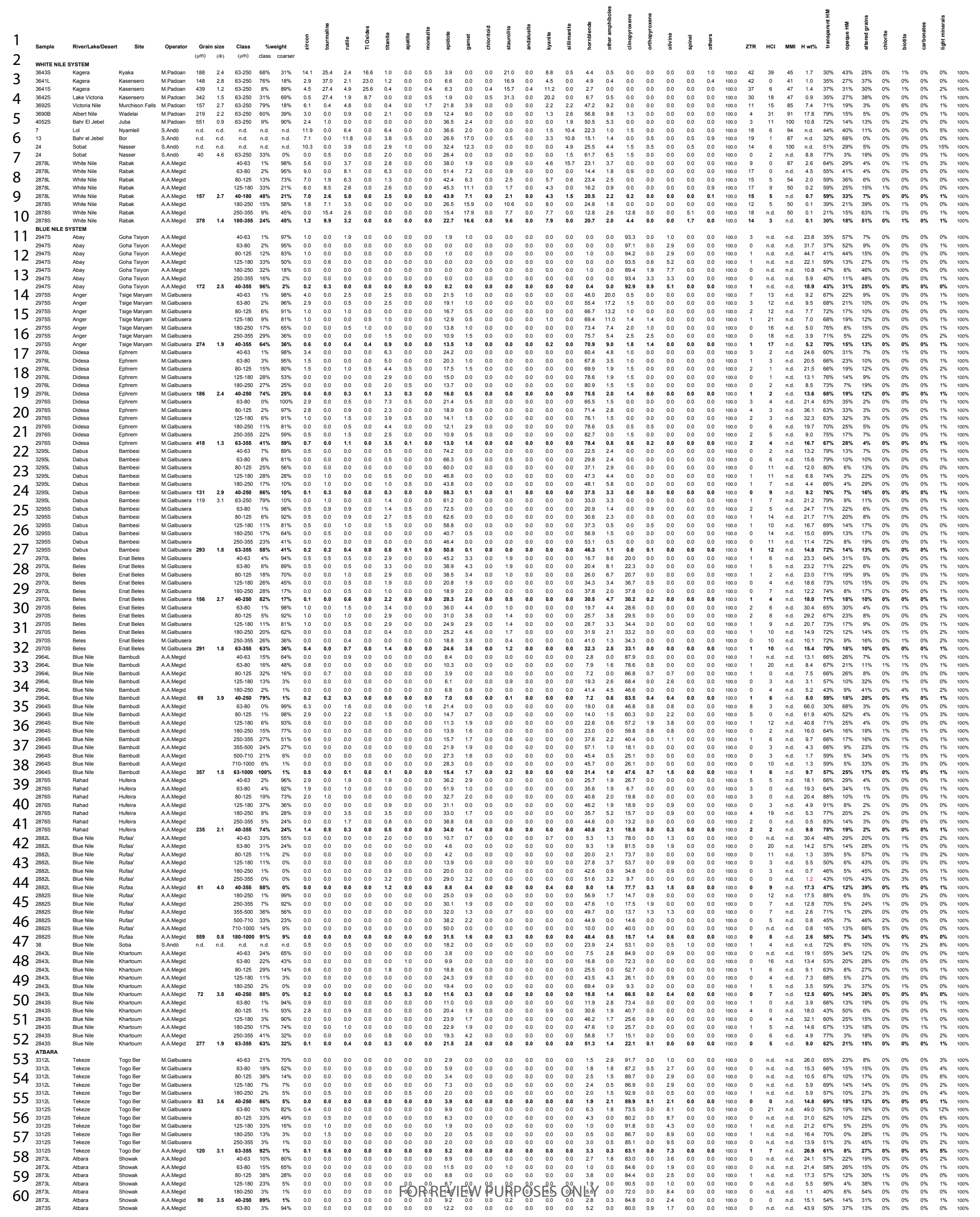




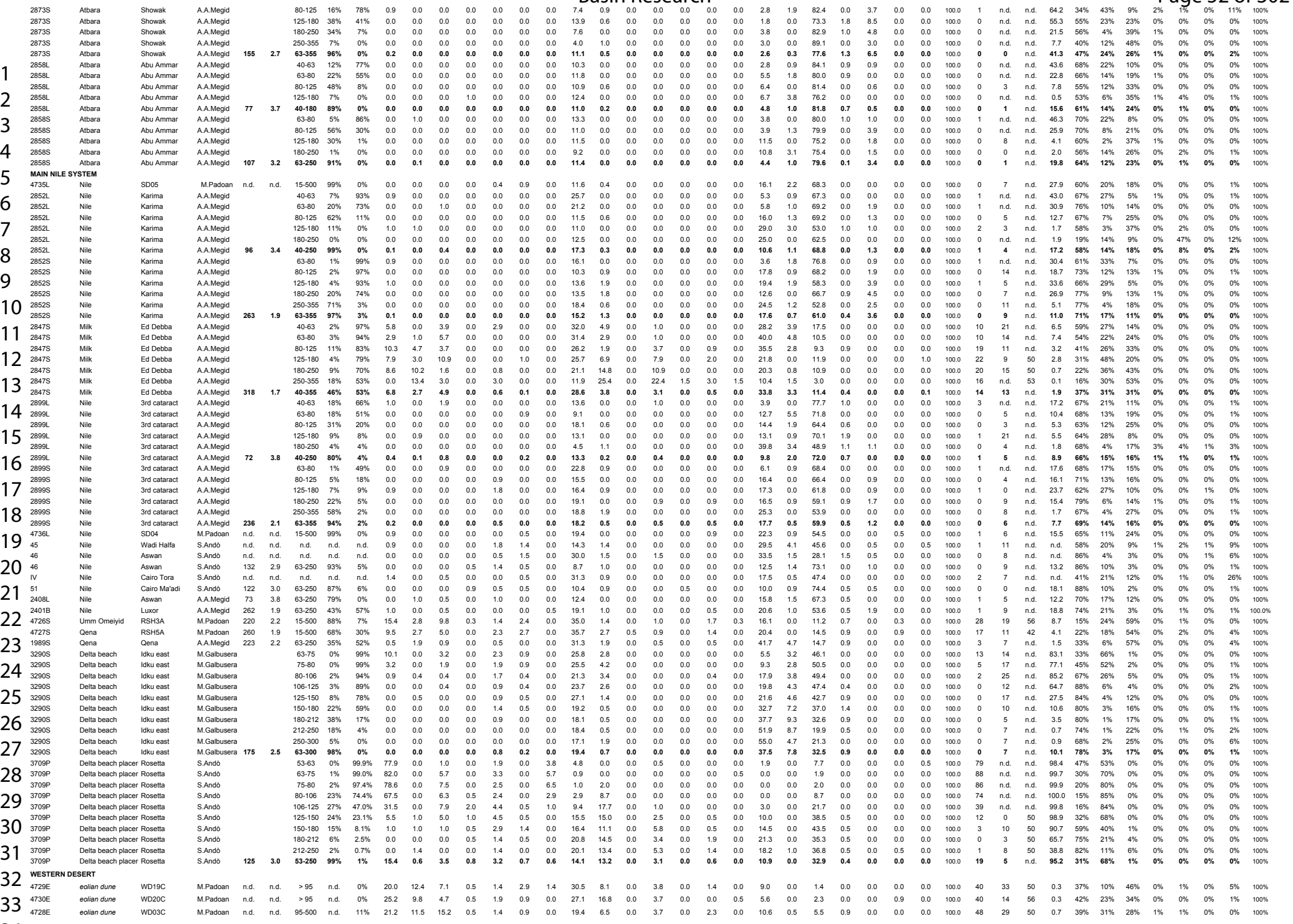


Sample 3643S Kagera @ Kyaka 149 grain analysed 117 concordant ages

\begin{tabular}{|c|c|c|c|c|c|c|}
\hline \multirow[b]{2}{*}{ grain } & \multicolumn{3}{|c|}{ concentrations } & \multicolumn{3}{|l|}{ isotopic ratios } \\
\hline & U [ppm] & $\mathrm{Pb}[\mathrm{ppm}]$ & $\mathrm{Th} / \mathrm{U}$ & Pb207/Pb206 & $2 \sigma 76$ & Pb207/U235 \\
\hline S3643_G001 & 574.1 & 221.4 & 0.3289 & 0.12449 & 0.00294 & 6.18009 \\
\hline S3643_G002 & 73.7 & 35.2 & 1.3574 & 0.11893 & 0.00336 & 5.78375 \\
\hline S3643_G003 & 54.4 & 33.9 & 0.7592 & 0.18013 & 0.0048 & 12.59804 \\
\hline S3643_G004 & 119.7 & 44.9 & 0.5101 & 0.11296 & 0.003 & 5.24326 \\
\hline S3643_G005 & 869.5 & 366.8 & 0.8318 & 0.37666 & 0.0087 & 20.12351 \\
\hline S3643_G006 & 1217.8 & 278.6 & 0.6722 & 0.13395 & 0.00314 & 3.93597 \\
\hline S3643 G007 & 624.8 & 252.3 & 2.6122 & 0.20481 & 0.00484 & 7.53907 \\
\hline S3643_G008 & 70.6 & 29.3 & 0.4298 & 0.13244 & 0.00374 & 6.89661 \\
\hline S3643_G009 & 60.1 & 28.2 & 1.5647 & 0.11224 & 0.00342 & 5.12747 \\
\hline S3643_G010 & 916.1 & 263.4 & 0.2189 & 0.17427 & 0.00408 & 7.01695 \\
\hline S3643_G011 & 251 & 59.1 & 0.1594 & 0.08699 & 0.00228 & 2.85174 \\
\hline S3643_G012 & 142.7 & 59.3 & 0.8049 & 0.11506 & 0.003 & 5.4999 \\
\hline S3643_G013 & 63.3 & 37.5 & 0.7119 & 0.17453 & 0.00462 & 11.82816 \\
\hline S3643_G014 & 466.9 & 109.1 & 0.1547 & 0.08671 & 0.00214 & 2.83333 \\
\hline S3643_G015 & 214.9 & 156.7 & 1.3271 & 0.18229 & 0.00438 & 13.18747 \\
\hline S3643_G016 & 247.8 & 132.9 & 0.5079 & 0.16542 & 0.00398 & 10.69436 \\
\hline S3643_G017 & 1013.3 & 414.3 & 0.431 & 0.1269 & 0.00298 & 6.57451 \\
\hline S3643_G018 & 211.8 & 95.4 & 0.9274 & 0.12014 & 0.00302 & 6.03699 \\
\hline S3643_G019 & 180.9 & 46.4 & 0.5589 & 0.08701 & 0.0024 & 2.78474 \\
\hline S3643_G020 & 680.8 & 305.5 & 1.9617 & 0.21582 & 0.0051 & 9.86672 \\
\hline S3643_G021 & 119.7 & 50.5 & 0.527 & 0.12636 & 0.00332 & 6.51169 \\
\hline S3643_G022 & 219.1 & 60.5 & 0.7271 & 0.08756 & 0.00234 & 2.88222 \\
\hline S3643_G023 & 189.3 & 83.5 & 0.6327 & 0.12606 & 0.00318 & 6.62399 \\
\hline S3643_G024 & 807.3 & 172 & 0.4081 & 0.091 & 0.00222 & 2.56474 \\
\hline S3643_G025 & 898.8 & 347 & 0.6268 & 0.12455 & 0.00296 & 5.85505 \\
\hline S3643_G026 & 86.3 & 55.5 & 0.8161 & 0.18284 & 0.00468 & 12.92299 \\
\hline S3643_G027 & 120.3 & 74 & 0.6065 & 0.18424 & 0.00462 & 13.11971 \\
\hline S3643_G028 & 107.2 & 42.6 & 0.9722 & 0.11116 & 0.00306 & 4.91535 \\
\hline S3643_G029 & 231.6 & 93.9 & 0.8263 & 0.11409 & 0.00288 & 5.3117 \\
\hline S3643_G030 & 820.9 & 123.6 & 0.0137 & 0.07071 & 0.00178 & 1.57523 \\
\hline S3643_G031 & & & & 0.86874 & 0.03074 & \\
\hline S3643_G032 & 508.2 & 258 & 0.1393 & 0.17407 & 0.00414 & 11.69351 \\
\hline S3643_G033 & 201.3 & 116 & 0.7329 & 0.1758 & 0.0043 & 11.66708 \\
\hline S3643_G034 & 578.8 & 203.8 & 0.0984 & 0.12002 & 0.0029 & 5.83595 \\
\hline S3643_G035 & 96.2 & 25.6 & 0.6445 & 0.0867 & 0.00268 & 2.81213 \\
\hline S3643_G036 & 282.4 & 178.2 & 0.8178 & 0.17555 & 0.00424 & 12.26249 \\
\hline S3643_G037 & 190.8 & 46 & 0.2724 & 0.08692 & 0.0024 & 2.82496 \\
\hline S3643_G038 & 109.3 & 28.9 & 0.6204 & 0.08628 & 0.00264 & 2.79966 \\
\hline S3643_G039 & 159 & 92.8 & 0.7766 & 0.17179 & 0.0043 & 11.33851 \\
\hline S3643_G040 & 142.2 & 65.1 & 0.4185 & 0.13375 & 0.00346 & 7.60302 \\
\hline S3643_G041 & 484.7 & 209 & 1.5079 & 0.12133 & 0.00296 & 6.07073 \\
\hline S3643_G042 & 553.7 & 230.5 & 0.6579 & 0.11984 & 0.0029 & 5.98576 \\
\hline S3643_G043 & 527.1 & 174.6 & 1.7985 & 0.16471 & 0.00402 & 6.44035 \\
\hline S3643_G044 & 120.3 & 28.8 & 0.3272 & 0.08559 & 0.00256 & 2.72865 \\
\hline S3643_G045 & 209.7 & 53.2 & 0.5942 & 0.11755 & 0.00312 & 3.73049 \\
\hline S3643_G046 & 486.3 & 120 & 0.7578 & 0.0959 & 0.00244 & 2.8466 \\
\hline S3643_G047 & 233.2 & 171.7 & 0.7944 & 0.22779 & 0.00554 & 18.46875 \\
\hline S3643_G048 & 230.1 & 57.8 & 0.4999 & 0.08603 & 0.00232 & 2.73935 \\
\hline S3643_G049 & 503 & 183.1 & 0.9645 & 0.13434 & 0.0033 & 5.7911 \\
\hline S3643_G050 & 359.2 & 155.6 & 0.7171 & 0.12181 & 0.00302 & 6.1832 \\
\hline
\end{tabular}




\begin{tabular}{|c|c|c|c|c|c|c|}
\hline S3643_G051 & 740.4 & 383.9 & 0.4734 & 0.17449 & 0.0042 & 11.36914 \\
\hline S3643_G052 & 2087.3 & 176.3 & 2.6116 & 0.26143 & 0.00646 & 2.11148 \\
\hline S3643_G053 & 182 & 45.4 & 0.3861 & 0.08548 & 0.0024 & 2.78238 \\
\hline S3643_G054 & 286 & 166.2 & 2.5047 & 0.18356 & 0.00456 & 9.4752 \\
\hline S3643_G055 & 356.6 & 85.7 & 0.1016 & 0.08589 & 0.00226 & 2.91623 \\
\hline S3643_G056 & 748.8 & 287.8 & 2.0974 & 0.17604 & 0.0043 & 6.72487 \\
\hline S3643 G057 & 238.4 & 76.5 & 0.5479 & 0.1359 & 0.00348 & 5.6505 \\
\hline S3643_G058 & 3861.9 & 254.3 & 0.6436 & 0.43191 & 0.01044 & 3.79607 \\
\hline S3643_G059 & 226.9 & 122.2 & 0.5682 & 0.17811 & 0.00442 & 11.85297 \\
\hline S3643_G060 & 92 & 37.1 & 0.8289 & 0.11648 & 0.00332 & 5.39283 \\
\hline S3643_G061 & 165.8 & 96.5 & 0.5246 & 0.17533 & 0.00442 & 12.10224 \\
\hline S3643_G062 & 370.2 & 350.6 & 4.2067 & 0.17055 & 0.00422 & 9.34045 \\
\hline S3643_G063 & 160.5 & 67.2 & 0.4863 & 0.12592 & 0.0033 & 6.52879 \\
\hline S3643_G064 & 313.2 & 196.1 & 0.7346 & 0.17842 & 0.0044 & 12.50978 \\
\hline S3643_G065 & 304.8 & 174.4 & 0.6297 & 0.1774 & 0.00438 & 11.9508 \\
\hline S3643_G066 & 435.6 & 199.1 & 1.2051 & 0.12352 & 0.00308 & 6.0067 \\
\hline S3643_G067 & 132.3 & 77.2 & 0.3465 & 0.22461 & 0.00572 & 15.78747 \\
\hline S3643_G068 & 254.6 & 104.2 & 0.2823 & 0.12746 & 0.00326 & 6.77811 \\
\hline S3643_G069 & 261.4 & 59.8 & 0.2472 & 0.08503 & 0.00232 & 2.67744 \\
\hline S3643_G070 & 175.7 & 49.3 & 0.8032 & 0.08674 & 0.00246 & 2.8555 \\
\hline S3643_G071 & 167.8 & 71.4 & 0.5891 & 0.12438 & 0.00328 & 6.37571 \\
\hline S3643_G072 & 174.6 & 107.6 & 0.5704 & 0.17835 & 0.00452 & 12.74104 \\
\hline S3643_G073 & 40.3 & & 0.2682 & 0.30368 & 0.00812 & 30.3433 \\
\hline S3643_G074 & 329.4 & 144.4 & 0.5941 & 0.13143 & 0.00332 & 6.95477 \\
\hline S3643_G075 & 1270.6 & 285.4 & 0.4968 & 0.08823 & 0.0022 & 2.59525 \\
\hline S3643_G076 & 323.1 & 124 & 1.5125 & 0.16652 & 0.00422 & 7.57829 \\
\hline S3643_G077 & 78.4 & 37.1 & 0.0481 & 0.16139 & 0.0044 & 10.34461 \\
\hline S3643_G078 & 280.8 & 53.5 & 0.4907 & 0.08752 & 0.00244 & 2.14515 \\
\hline S3643_G079 & 148 & 48.6 & 0.233 & 0.10922 & 0.003 & 4.81315 \\
\hline S3643_G080 & 55.4 & 23.6 & 0.8192 & 0.11963 & 0.0037 & 5.83281 \\
\hline S3643_G081 & 339.3 & 145.7 & 0.8706 & 0.12183 & 0.0031 & 6.00129 \\
\hline S3643_G082 & 181.4 & 49.5 & 0.8478 & 0.08529 & 0.00244 & 2.6952 \\
\hline S3643_G083 & 135.9 & 56.4 & 0.994 & 0.13383 & 0.00364 & 6.24232 \\
\hline S3643_G084 & 1343.3 & 212.3 & 1.0973 & 0.12161 & 0.00308 & 2.12574 \\
\hline S3643_G085 & 154.8 & 69.5 & 0.7609 & 0.13001 & 0.00348 & 6.76092 \\
\hline S3643_G086 & 96.7 & 59.7 & 0.5742 & 0.17571 & 0.00466 & 12.50655 \\
\hline S3643_G087 & 731.5 & 359.7 & 0.9675 & 0.17325 & 0.0043 & 10.02822 \\
\hline S3643_G088 & 145.9 & 39 & 0.645 & 0.08574 & 0.00254 & 2.79729 \\
\hline S3643_G089 & 106.7 & 48.1 & 1.2787 & 0.11431 & 0.00324 & 5.35572 \\
\hline S3643_G090 & 806.8 & 191.4 & 0.2174 & 0.08808 & 0.00224 & 2.8698 \\
\hline S3643_G091 & 744.6 & 342.7 & 1.5683 & 0.13285 & 0.00334 & 6.65642 \\
\hline S3643_G092 & 709 & 235.7 & 0.9714 & 0.13741 & 0.00346 & 5.70217 \\
\hline S3643_G093 & 236.3 & 55.6 & 0.2081 & 0.08671 & 0.0024 & 2.80563 \\
\hline S3643_G094 & 490.5 & 116.4 & 0.2562 & 0.08651 & 0.00226 & 2.8392 \\
\hline S3643_G095 & 178.3 & 64.5 & 1.0304 & 0.12401 & 0.00336 & 5.06579 \\
\hline S3643_G096 & 122.4 & 60.5 & 0.935 & 0.13246 & 0.00362 & 7.25725 \\
\hline S3643_G097 & 186.7 & 109.1 & 0.4236 & 0.17735 & 0.00458 & 12.52629 \\
\hline S3643_G098 & 84.2 & 55.4 & 0.7216 & 0.18784 & 0.00506 & 13.81706 \\
\hline S3643_G099 & 823.5 & 391.8 & 0.9301 & 0.15137 & 0.0038 & 8.6559 \\
\hline S3643_G100 & 115.6 & 46.1 & 0.6817 & 0.11276 & 0.00318 & 5.33618 \\
\hline S3643_G101 & 94.1 & 54.2 & 0.5521 & 0.16989 & 0.00462 & 11.54835 \\
\hline S3643_G102 & 108.2 & 65.2 & 0.639 & 0.17287 & 0.00464 & 11.97059 \\
\hline S3643_G103 & 680.8 & 259.3 & 0.7733 & 0.17873 & 0.00452 & 8.63438 \\
\hline S3643_G104 & 369.1 & 104.5 & 0.815 & 0.08668 & 0.00234 & 2.86024 \\
\hline S3643_G105 & 275.6 & 149.6 & 1.4768 & 0.17756 & 0.00458 & 10.403 \\
\hline
\end{tabular}




\begin{tabular}{|c|c|c|c|c|c|c|}
\hline S3643_G106 & 644.7 & 217.6 & 0.3873 & 0.15087 & 0.00384 & 6.72855 \\
\hline S3643_G107 & 352.4 & 150.2 & 1.0206 & 0.1222 & 0.00318 & 5.87499 \\
\hline S3643_G108 & 233.2 & 106.1 & 1.0244 & 0.12002 & 0.0032 & 5.95722 \\
\hline S3643_G109 & 974.6 & 348.4 & 2.5946 & 0.18238 & 0.00464 & 6.00636 \\
\hline S3643_G110 & 362.9 & 184.3 & 1.5813 & 0.17657 & 0.00456 & 9.82176 \\
\hline S3643_G111 & 448.6 & 212 & 0.7449 & 0.21677 & 0.00556 & 12.21024 \\
\hline S3643_G112 & 409.9 & 254.2 & 0.4132 & 0.18917 & 0.00484 & 14.02347 \\
\hline S3643_G113 & 373.3 & 230.8 & 0.8626 & 0.17655 & 0.00454 & 12.10019 \\
\hline S3643_G114 & 206 & 91.8 & 0.9464 & 0.12003 & 0.00324 & 5.95677 \\
\hline S3643_G115 & 97.3 & 44.8 & 1.3151 & 0.11327 & 0.00332 & 5.27694 \\
\hline S3643_G116 & 469.5 & 208.4 & 1.9088 & 0.12525 & 0.00328 & 5.31573 \\
\hline S3643_G117 & 107.7 & 37.7 & 0.5236 & 0.1077 & 0.00316 & 4.76342 \\
\hline S3643_G118 & 180.4 & 45.4 & 0.4519 & 0.08723 & 0.00254 & 2.82957 \\
\hline S3643_G119 & 104.6 & 54.2 & 1.4088 & 0.17568 & 0.00486 & 10.06014 \\
\hline S3643_G120 & 165.2 & 47.9 & 0.8259 & 0.0877 & 0.00258 & 2.95881 \\
\hline S3643_G121 & 147.4 & 37 & 0.4129 & 0.08687 & 0.0026 & 2.83212 \\
\hline S3643_G122 & 628.5 & 388.7 & 1.2542 & 0.24766 & 0.00634 & 16.01942 \\
\hline S3643_G123 & 83.1 & 37.3 & 1.065 & 0.13726 & 0.004 & 6.98497 \\
\hline S3643_G124 & 595 & 164.7 & 0.772 & 0.21042 & 0.00548 & 6.82536 \\
\hline S3643_G125 & 142.2 & 83.4 & 2.2965 & 0.12115 & 0.00338 & 6.00811 \\
\hline S3643_G126 & 100.9 & 61.8 & 1.1243 & 0.17568 & 0.00488 & 12.15026 \\
\hline S3643_G127 & 296.5 & 185.8 & 0.4889 & 0.18917 & 0.00494 & 14.06847 \\
\hline S3643_G128 & 168.4 & 96.5 & 0.4097 & 0.17844 & 0.00476 & 12.46171 \\
\hline S3643_G129 & 487.3 & 165.8 & 1.1553 & 0.13594 & 0.00358 & 5.42793 \\
\hline S3643_G130 & 376.5 & 239.8 & 0.7391 & 0.17575 & 0.00458 & 12.52269 \\
\hline S3643_G131 & 58 & 17.8 & 1.1859 & 0.08524 & 0.00312 & 2.80497 \\
\hline S3643_G132 & 104.6 & 29.5 & 0.9713 & 0.11906 & 0.00366 & 3.76807 \\
\hline S3643_G133 & 69 & 28.6 & 0.3214 & 0.13189 & 0.00394 & 7.04089 \\
\hline S3643_G134 & 184.6 & 117.2 & 0.7016 & 0.19491 & 0.00518 & 14.46793 \\
\hline S3643_G135 & 53.3 & 22.9 & 0.599 & 0.12777 & 0.00402 & 6.61629 \\
\hline S3643_G136 & 133.9 & 55.7 & 1.2182 & 0.1276 & 0.00362 & 6.0842 \\
\hline S3643_G137 & 241 & 136.6 & 0.3728 & 0.17405 & 0.00462 & 12.11169 \\
\hline S3643_G138 & 112.9 & 33.2 & 0.9269 & 0.10303 & 0.00322 & 3.40736 \\
\hline S3643_G139 & 322.6 & 140.4 & 0.74 & 0.1215 & 0.00326 & 6.15723 \\
\hline S3643_G140 & 433.5 & 264.5 & 1.0761 & 0.1807 & 0.00474 & 12.24147 \\
\hline S3643_G141 & 244.2 & 110.5 & 1.0584 & 0.12114 & 0.0033 & 6.1553 \\
\hline S3643_G142 & 199.2 & 48.8 & 0.3448 & 0.08871 & 0.0026 & 2.89105 \\
\hline S3643_G143 & 321.6 & 132.4 & 0.8177 & 0.11355 & 0.00308 & 5.38501 \\
\hline S3643_G144 & 252.5 & 139.8 & 1.0411 & 0.18684 & 0.005 & 12.25908 \\
\hline S3643_G145 & 181.4 & 45.5 & 0.3057 & 0.08812 & 0.00262 & 2.95365 \\
\hline S3643_G146 & 74.8 & 30.2 & 0.7639 & 0.11604 & 0.00362 & 5.45856 \\
\hline S3643_G147 & 377.5 & 154.4 & 0.4817 & 0.12788 & 0.00344 & 6.6412 \\
\hline S3643_G148 & 247.3 & 164.6 & 1.2059 & 0.17056 & 0.00458 & 11.59954 \\
\hline S3643_G149 & 229.5 & 59.5 & 0.4758 & 0.08646 & 0.00252 & 2.8574 \\
\hline
\end{tabular}




\section{5\% concordant}

\begin{tabular}{|c|c|c|c|c|c|c|}
\hline $2 \sigma 75$ & Pb206/U238 & 2068 & $\begin{array}{c}\text { ages } \\
\text { age } 206 / 238\end{array}$ & $2 \sigma$ age 68 & age $207 / 235$ & $2 \sigma$ age 75 \\
\hline 0.1636 & 0.36017 & 0.00882 & 1983 & 41.8 & 2001.6 & 29.8 \\
\hline 0.17614 & 0.35284 & 0.00928 & 1948.1 & 44.2 & 1944 & 33.4 \\
\hline 0.36996 & 0.50745 & 0.01348 & 2645.7 & 57.6 & 2650.1 & 35.4 \\
\hline 0.15248 & 0.33677 & 0.00862 & 1871.1 & 41.6 & 1859.7 & 31.4 \\
\hline 0.52534 & 0.38764 & 0.00946 & 2111.9 & 44 & 3097.3 & 32.6 \\
\hline 0.10392 & 0.21319 & 0.0052 & 1245.8 & 27.6 & 1621.1 & 27.4 \\
\hline 0.19962 & 0.26707 & 0.00656 & 1525.9 & 33.4 & 2177.6 & 30.6 \\
\hline 0.21032 & 0.37781 & 0.01002 & 2066 & 46.8 & 2098.2 & 34.4 \\
\hline 0.16608 & 0.33145 & 0.00896 & 1845.4 & 43.4 & 1840.7 & 34.6 \\
\hline 0.18498 & 0.29215 & 0.00714 & 1652.3 & 35.6 & 2113.6 & 30 \\
\hline 0.0819 & 0.23785 & 0.00598 & 1375.5 & 31.2 & 1369.3 & 27.4 \\
\hline 0.15742 & 0.34683 & 0.0088 & 1919.4 & 42.2 & 1900.6 & 31.2 \\
\hline 0.34546 & 0.49171 & 0.01294 & 2578.1 & 56 & 2590.9 & 35 \\
\hline 0.07792 & 0.23707 & 0.00586 & 1371.4 & 30.6 & 1364.4 & 26.2 \\
\hline 0.35614 & 0.52488 & 0.01306 & 2719.8 & 55.2 & 2693.2 & 32.6 \\
\hline 0.2888 & 0.46907 & 0.01164 & 2479.5 & 51 & 2496.9 & 32 \\
\hline 0.17386 & 0.3759 & 0.00918 & 2057.1 & 43 & 2055.9 & 29.8 \\
\hline 0.16792 & 0.3646 & 0.00914 & 2003.9 & 43.2 & 1981.2 & 31 \\
\hline 0.0832 & 0.23221 & 0.00592 & 1346.1 & 31 & 1351.5 & 28 \\
\hline 0.26196 & 0.3317 & 0.00814 & 1846.6 & 39.4 & 2422.4 & 31.4 \\
\hline 0.18772 & 0.37391 & 0.00956 & 2047.8 & 44.8 & 2047.5 & 32.2 \\
\hline 0.08374 & 0.23882 & 0.00602 & 1380.6 & 31.4 & 1377.3 & 27.6 \\
\hline 0.18554 & 0.38124 & 0.0096 & 2082.1 & 44.8 & 2062.5 & 31.4 \\
\hline 0.06978 & 0.20448 & 0.00504 & 1199.3 & 27 & 1290.6 & 25.4 \\
\hline 0.15604 & 0.34107 & 0.00836 & 1891.8 & 40.2 & 1954.6 & 29.6 \\
\hline 0.36804 & 0.51283 & 0.01324 & 2668.7 & 56.4 & 2674.1 & 34.2 \\
\hline 0.36634 & 0.51668 & 0.01314 & 2685.1 & 55.8 & 2688.3 & 33.6 \\
\hline 0.14714 & 0.32083 & 0.0083 & 1793.8 & 40.6 & 1804.9 & 31.8 \\
\hline 0.1487 & 0.33779 & 0.00846 & 1876 & 40.8 & 1870.7 & 30.4 \\
\hline 0.04372 & 0.16162 & 0.00398 & 965.8 & 22 & 960.5 & 21.8 \\
\hline 0.31328 & 0.4874 & 0.012 & 2559.4 & 52 & 2580.2 & 32 \\
\hline 0.32 & 0.48151 & 0.01206 & 2533.8 & 52.4 & 2578.1 & 32.8 \\
\hline 0.15768 & 0.35279 & 0.00868 & 1947.9 & 41.4 & 1951.8 & 29.8 \\
\hline 0.09208 & 0.23535 & 0.00622 & 1362.5 & 32.4 & 1358.8 & 30.4 \\
\hline 0.3328 & 0.50682 & 0.01258 & 2643 & 53.8 & 2624.7 & 32.6 \\
\hline 0.08438 & 0.23581 & 0.006 & 1364.9 & 31.2 & 1362.2 & 28.2 \\
\hline 0.09064 & 0.23543 & 0.00618 & 1362.9 & 32.2 & 1355.4 & 30.2 \\
\hline 0.31654 & 0.47889 & 0.01212 & 2522.4 & 52.8 & 2551.4 & 33.2 \\
\hline 0.21686 & 0.41244 & 0.01048 & 2226.1 & 47.8 & 2185.2 & 32.6 \\
\hline 0.1654 & 0.36302 & 0.00898 & 1996.5 & 42.4 & 1986.1 & 30.2 \\
\hline 0.16252 & 0.36239 & 0.00894 & 1993.5 & 42.4 & 1973.8 & 30 \\
\hline 0.17534 & 0.2837 & 0.00702 & 1610 & 35.2 & 2037.8 & 30.6 \\
\hline 0.08682 & 0.2313 & 0.00604 & 1341.3 & 31.6 & 1336.3 & 29.6 \\
\hline 0.10822 & 0.23026 & 0.00584 & 1335.9 & 30.6 & 1577.9 & 29.4 \\
\hline 0.07992 & 0.21537 & 0.00536 & 1257.4 & 28.4 & 1367.9 & 26.8 \\
\hline 0.50478 & 0.58827 & 0.01468 & 2982.4 & 59.6 & 3014.5 & 33.6 \\
\hline 0.0807 & 0.23104 & 0.00584 & 1339.9 & 30.6 & 1339.2 & 27.4 \\
\hline 0.15854 & 0.31277 & 0.00774 & 1754.3 & 38 & 1945.1 & 30.2 \\
\hline 0.17082 & 0.36829 & 0.00916 & 2021.3 & 43.2 & 2002.1 & 30.6 \\
\hline
\end{tabular}




\begin{tabular}{|c|c|c|c|c|c|c|}
\hline 0.3075 & 0.47274 & 0.01164 & 2495.6 & 51 & 2553.9 & 32.2 \\
\hline 0.05778 & 0.0586 & 0.00146 & 367.1 & 8.8 & 1152.6 & 24.2 \\
\hline 0.08446 & 0.23617 & 0.00604 & 1366.8 & 31.4 & 1350.8 & 28.4 \\
\hline 0.26204 & 0.37451 & 0.00936 & 2050.6 & 43.8 & 2385.1 & 32.4 \\
\hline 0.08404 & 0.24636 & 0.00618 & 1419.7 & 32 & 1386.1 & 27.4 \\
\hline 0.18342 & 0.27717 & 0.00686 & 1577.1 & 34.6 & 2075.9 & 30.8 \\
\hline 0.1599 & 0.30167 & 0.0076 & 1699.6 & 37.6 & 1923.8 & 31 \\
\hline 0.10262 & 0.06377 & 0.00158 & 398.5 & 9.6 & 1591.9 & 27.8 \\
\hline 0.3292 & 0.48284 & 0.0121 & 2539.6 & 52.6 & 2592.9 & 33 \\
\hline 0.1664 & 0.33592 & 0.00882 & 1867 & 42.6 & 1883.7 & 33.2 \\
\hline 0.3404 & 0.50081 & 0.01266 & 2617.3 & 54.4 & 2612.4 & 33.4 \\
\hline 0.25812 & 0.39736 & 0.0099 & 2156.9 & 45.6 & 2372 & 32.2 \\
\hline 0.18902 & 0.37618 & 0.00958 & 2058.4 & 44.8 & 2049.8 & 32.2 \\
\hline 0.34584 & 0.50873 & 0.01268 & 2651.2 & 54.2 & 2643.5 & 33 \\
\hline 0.33088 & 0.48877 & 0.0122 & 2565.4 & 52.8 & 2600.6 & 32.8 \\
\hline 0.16724 & 0.35284 & 0.00878 & 1948.1 & 41.8 & 1976.8 & 30.6 \\
\hline 0.44726 & 0.50997 & 0.01302 & 2656.5 & 55.6 & 2864 & 34.4 \\
\hline 0.19194 & 0.38583 & 0.0097 & 2103.5 & 45.2 & 2082.9 & 31.8 \\
\hline 0.07972 & 0.22845 & 0.0058 & 1326.4 & 30.4 & 1322.2 & 27.6 \\
\hline 0.08754 & 0.23885 & 0.00614 & 1380.7 & 32 & 1370.3 & 28.8 \\
\hline 0.18488 & 0.37192 & 0.00946 & 2038.4 & 44.4 & 2028.9 & 32.2 \\
\hline 0.35986 & 0.51832 & 0.0131 & 2692.1 & 55.6 & 2660.7 & 33.6 \\
\hline 0.90948 & 0.72496 & 0.01976 & 3514.6 & 73.8 & 3498 & 37.6 \\
\hline 0.1954 & 0.38394 & 0.0096 & 2094.7 & 44.8 & 2105.7 & 31.6 \\
\hline 0.07208 & 0.21342 & 0.00528 & 1247 & 28 & 1299.3 & 25.8 \\
\hline 0.2131 & 0.3302 & 0.00828 & 1839.4 & 40.2 & 2182.3 & 32 \\
\hline 0.3096 & 0.46505 & 0.0122 & 2461.8 & 53.6 & 2466.1 & 35 \\
\hline 0.0648 & 0.17784 & 0.00454 & 1055.2 & 24.8 & 1163.5 & 26.2 \\
\hline 0.14454 & 0.31973 & 0.00824 & 1788.4 & 40.2 & 1787.2 & 31.6 \\
\hline 0.19288 & 0.35376 & 0.00968 & 1952.5 & 46 & 1951.3 & 35.8 \\
\hline 0.1701 & 0.35739 & 0.00896 & 1969.8 & 42.6 & 1976 & 31 \\
\hline 0.08318 & 0.22927 & 0.0059 & 1330.7 & 31 & 1327.1 & 28.6 \\
\hline 0.18574 & 0.33843 & 0.00874 & 1879.1 & 42 & 2010.4 & 32.8 \\
\hline 0.0596 & 0.12683 & 0.00316 & 769.8 & 18 & 1157.2 & 24.6 \\
\hline 0.19864 & 0.3773 & 0.00966 & 2063.7 & 45.2 & 2080.6 & 32.8 \\
\hline 0.36746 & 0.51644 & 0.0134 & 2684.1 & 57 & 2643.2 & 34.8 \\
\hline 0.27858 & 0.41998 & 0.01042 & 2260.4 & 47.4 & 2437.4 & 32.4 \\
\hline 0.08874 & 0.23672 & 0.00616 & 1369.6 & 32.2 & 1354.8 & 29.4 \\
\hline 0.16488 & 0.33993 & 0.00888 & 1886.3 & 42.8 & 1877.8 & 33 \\
\hline 0.08118 & 0.2364 & 0.00588 & 1368 & 30.6 & 1374 & 26.8 \\
\hline 0.1863 & 0.36353 & 0.00904 & 1998.9 & 42.8 & 2066.9 & 31.2 \\
\hline 0.1601 & 0.30109 & 0.0075 & 1696.7 & 37.2 & 1931.7 & 30.6 \\
\hline 0.08482 & 0.23476 & 0.00598 & 1359.4 & 31.2 & 1357 & 28.2 \\
\hline 0.08202 & 0.23812 & 0.00596 & 1376.9 & 31 & 1366 & 27.2 \\
\hline 0.15052 & 0.29638 & 0.0076 & 1673.3 & 37.8 & 1830.4 & 31.6 \\
\hline 0.21748 & 0.3975 & 0.01028 & 2157.5 & 47.4 & 2143.6 & 33.6 \\
\hline 0.35942 & 0.51245 & 0.013 & 2667.1 & 55.4 & 2644.7 & 34 \\
\hline 0.41154 & 0.5337 & 0.01398 & 2757 & 58.8 & 2737.2 & 35.6 \\
\hline 0.24262 & 0.4149 & 0.01032 & 2237.3 & 47 & 2302.5 & 32.2 \\
\hline 0.16344 & 0.34334 & 0.00892 & 1902.7 & 42.8 & 1874.7 & 32.8 \\
\hline 0.34558 & 0.49318 & 0.01288 & 2584.4 & 55.6 & 2568.5 & 35.2 \\
\hline 0.35476 & 0.5024 & 0.01302 & 2624.1 & 55.8 & 2602.1 & 35 \\
\hline 0.24374 & 0.3505 & 0.00874 & 1937 & 41.8 & 2300.2 & 32.4 \\
\hline 0.08444 & 0.23942 & 0.00604 & 1383.7 & 31.4 & 1371.5 & 27.8 \\
\hline 0.29842 & 0.42508 & 0.01074 & 2283.5 & 48.6 & 2471.3 & 33.4 \\
\hline
\end{tabular}




\begin{tabular}{|c|c|c|c|c|c|c|}
\hline 0.19104 & 0.32357 & 0.00808 & 1807.1 & 39.4 & 2076.4 & 31.6 \\
\hline 0.16974 & 0.34882 & 0.00878 & 1929 & 42 & 1957.5 & 31.4 \\
\hline 0.17508 & 0.36011 & 0.00916 & 1982.7 & 43.4 & 1969.6 & 32 \\
\hline 0.1703 & 0.23895 & 0.00596 & 1381.2 & 31 & 1976.8 & 31 \\
\hline 0.28156 & 0.40358 & 0.01016 & 2185.5 & 46.6 & 2418.2 & 33.2 \\
\hline 0.34828 & 0.40869 & 0.01026 & 2208.9 & 47 & 2620.7 & 33.6 \\
\hline 0.39954 & 0.53784 & 0.01348 & 2774.4 & 56.6 & 2751.3 & 34 \\
\hline 0.34618 & 0.49726 & 0.0125 & 2602 & 53.8 & 2612.2 & 33.8 \\
\hline 0.17704 & 0.36005 & 0.0092 & 1982.4 & 43.6 & 1969.6 & 32.4 \\
\hline 0.16686 & 0.33802 & 0.00892 & 1877.1 & 43 & 1865.1 & 33.6 \\
\hline 0.15392 & 0.30792 & 0.00776 & 1730.5 & 38.2 & 1871.4 & 31 \\
\hline 0.1507 & 0.3209 & 0.00844 & 1794.1 & 41.2 & 1778.5 & 33 \\
\hline 0.08906 & 0.23535 & 0.0061 & 1362.5 & 31.8 & 1363.4 & 29.2 \\
\hline 0.3046 & 0.41547 & 0.01088 & 2239.9 & 49.6 & 2440.3 & 35.2 \\
\hline 0.094 & 0.24479 & 0.00636 & 1411.6 & 33 & 1397.1 & 29.8 \\
\hline 0.09122 & 0.23653 & 0.00618 & 1368.6 & 32.2 & 1364.1 & 29.8 \\
\hline 0.45746 & 0.4693 & 0.01176 & 2480.5 & 51.6 & 2878 & 34.2 \\
\hline 0.2203 & 0.36922 & 0.00982 & 2025.7 & 46.2 & 2109.5 & 35 \\
\hline 0.1968 & 0.23534 & 0.00592 & 1362.4 & 30.8 & 2089 & 32 \\
\hline 0.18346 & 0.35981 & 0.00932 & 1981.3 & 44.2 & 1977 & 33.2 \\
\hline 0.37154 & 0.50179 & 0.01322 & 2621.5 & 56.8 & 2616.1 & 36 \\
\hline 0.408 & 0.53956 & 0.01364 & 2781.6 & 57.2 & 2754.3 & 34.4 \\
\hline 0.36788 & 0.50668 & 0.01298 & 2642.4 & 55.6 & 2639.8 & 34.8 \\
\hline 0.15838 & 0.28969 & 0.0073 & 1640 & 36.4 & 1889.3 & 31.2 \\
\hline 0.36274 & 0.51696 & 0.01302 & 2686.3 & 55.4 & 2644.4 & 34 \\
\hline 0.10664 & 0.23876 & 0.00672 & 1380.2 & 35 & 1356.9 & 34.6 \\
\hline 0.12326 & 0.22962 & 0.00614 & 1332.5 & 32.2 & 1586 & 32.6 \\
\hline 0.22756 & 0.38731 & 0.01042 & 2110.3 & 48.4 & 2116.6 & 35.8 \\
\hline 0.42594 & 0.53855 & 0.01376 & 2777.4 & 57.6 & 2780.9 & 35 \\
\hline 0.22284 & 0.37569 & 0.01036 & 2056.1 & 48.6 & 2061.5 & 37 \\
\hline 0.18822 & 0.34594 & 0.00902 & 1915.2 & 43.2 & 1988 & 33.6 \\
\hline 0.35628 & 0.50487 & 0.01284 & 2634.7 & 55 & 2613.1 & 34.4 \\
\hline 0.11318 & 0.23995 & 0.00642 & 1386.4 & 33.4 & 1506.1 & 32.2 \\
\hline 0.18254 & 0.36768 & 0.00934 & 2018.5 & 44 & 1998.4 & 32.2 \\
\hline 0.3571 & 0.4915 & 0.0124 & 2577.2 & 53.6 & 2623.1 & 34.2 \\
\hline 0.18484 & 0.36866 & 0.00942 & 2023.1 & 44.4 & 1998.1 & 32.6 \\
\hline 0.09166 & 0.23644 & 0.00614 & 1368.2 & 32 & 1379.6 & 29.6 \\
\hline 0.16074 & 0.34408 & 0.00874 & 1906.3 & 42 & 1882.5 & 31.8 \\
\hline 0.36248 & 0.47603 & 0.01214 & 2510 & 53 & 2624.4 & 34.8 \\
\hline 0.09446 & 0.24317 & 0.00634 & 1403.2 & 32.8 & 1395.8 & 30 \\
\hline 0.18206 & 0.34128 & 0.00924 & 1892.8 & 44.4 & 1894.1 & 35.4 \\
\hline 0.19688 & 0.37678 & 0.00956 & 2061.2 & 44.8 & 2064.8 & 32.6 \\
\hline 0.34418 & 0.49342 & 0.01258 & 2585.5 & 54.4 & 2572.6 & 34.6 \\
\hline 0.09004 & 0.23978 & 0.0062 & 1385.6 & 32.2 & 1370.8 & 29.4 \\
\hline
\end{tabular}




\begin{tabular}{|c|c|c|c|c|c|}
\hline age $207 / 206$ & $2 \sigma$ age 76 & $\begin{array}{l}\text { discordance } \\
\Delta 68-75[\%]\end{array}$ & $\Delta 68-76[\%]$ & $\begin{array}{c}\text { preferred age } \\
\text { age }\end{array}$ & $2 \sigma$ age \\
\hline 2021.6 & 41.8 & -0.9 & -1.9 & 2021.6 & 41.8 \\
\hline 1940.2 & 50.6 & 0.2 & 0.4 & 1940.2 & 50.6 \\
\hline 2654.1 & 44.2 & -0.2 & -0.3 & 2654.1 & 44.2 \\
\hline 1847.6 & 48 & 0.6 & 1.3 & 1847.6 & 48 \\
\hline 3818.4 & 35 & -31.8 & -44.7 & & \\
\hline 2150.4 & 41 & -23.2 & -42.1 & & \\
\hline 2864.9 & 38.4 & -29.9 & -46.7 & & \\
\hline 2130.6 & 49.4 & -1.5 & -3 & 2130.6 & 49.4 \\
\hline 1836 & 55.2 & 0.3 & 0.5 & 1836 & 55.2 \\
\hline 2599.1 & 39 & -21.8 & -36.4 & & \\
\hline 1360.3 & 50.6 & 0.5 & 1.1 & 1360.3 & 50.6 \\
\hline 1880.8 & 47 & 1 & 2.1 & 1880.8 & 47 \\
\hline 2601.6 & 44.2 & -0.5 & -0.9 & 2601.6 & 44.2 \\
\hline 1354.1 & 47.6 & 0.5 & 1.3 & 1354.1 & 47.6 \\
\hline 2673.8 & 39.8 & 1 & 1.7 & 2673.8 & 39.8 \\
\hline 2511.8 & 40.4 & -0.7 & -1.3 & 2511.8 & 40.4 \\
\hline 2055.5 & 41.4 & 0.1 & 0.1 & 2055.5 & 41.4 \\
\hline 1958.3 & 44.8 & 1.1 & 2.3 & 1958.3 & 44.8 \\
\hline 1360.7 & 53.2 & -0.4 & -1.1 & 1360.7 & 53.2 \\
\hline 2949.8 & 38.2 & -23.8 & -37.4 & & \\
\hline 2047.9 & 46.4 & 0 & 0 & 2047.9 & 46.4 \\
\hline 1372.8 & 51.4 & 0.2 & 0.6 & 1372.8 & 51.4 \\
\hline 2043.7 & 44.6 & 0.9 & 1.9 & 2043.7 & 44.6 \\
\hline 1446.6 & 46.4 & -7.1 & -17.1 & & \\
\hline 2022.4 & 42.2 & -3.2 & -6.5 & 2022.4 & 42.2 \\
\hline 2678.8 & 42.4 & -0.2 & -0.4 & 2678.8 & 42.4 \\
\hline 2691.4 & 41.4 & -0.1 & -0.2 & 2691.4 & 41.4 \\
\hline 1818.5 & 50 & -0.6 & -1.4 & 1818.5 & 50 \\
\hline 1865.6 & 45.6 & 0.3 & 0.6 & 1865.6 & 45.6 \\
\hline 949 & 51.6 & 0.6 & 1.8 & 965.8 & 22 \\
\hline 2597.2 & 39.6 & -0.8 & -1.5 & 2597.2 & 39.6 \\
\hline 2613.6 & 40.8 & -1.7 & -3.1 & 2613.6 & 40.8 \\
\hline 1956.5 & 43.2 & -0.2 & -0.4 & 1956.5 & 43.2 \\
\hline 1353.8 & 59.6 & 0.3 & 0.6 & 1353.8 & 59.6 \\
\hline 2611.3 & 40.2 & 0.7 & 1.2 & 2611.3 & 40.2 \\
\hline 1358.7 & 53.2 & 0.2 & 0.5 & 1358.7 & 53.2 \\
\hline 1344.5 & 59 & 0.5 & 1.4 & 1344.5 & 59 \\
\hline 2575.2 & 41.8 & -1.1 & -2 & 2575.2 & 41.8 \\
\hline 2147.8 & 45.2 & 1.9 & 3.6 & 2147.8 & 45.2 \\
\hline 1975.9 & 43.4 & 0.5 & 1 & 1975.9 & 43.4 \\
\hline 1953.8 & 43.2 & 1 & 2 & 1953.8 & 43.2 \\
\hline 2504.6 & 41 & -21 & -35.7 & & \\
\hline 1328.9 & 57.8 & 0.4 & 0.9 & 1328.9 & 57.8 \\
\hline 1919.3 & 47.6 & -15.3 & -30.4 & & \\
\hline 1545.8 & 47.8 & -8.1 & -18.7 & & \\
\hline 3036.6 & 39 & -1.1 & -1.8 & 3036.6 & 39 \\
\hline 1338.9 & 52.2 & 0.1 & 0.1 & 1338.9 & 52.2 \\
\hline 2155.5 & 42.8 & -9.8 & -18.6 & & \\
\hline 1982.9 & 44.2 & 1 & 1.9 & 1982.9 & 44.2 \\
\hline
\end{tabular}




\begin{tabular}{|c|c|c|c|c|c|}
\hline 2601.2 & 40.2 & -2.3 & -4.1 & 2601.2 & 40.2 \\
\hline 3255.3 & 39 & -68.1 & -88.7 & & \\
\hline 1326.4 & 54.4 & 1.2 & 3 & 1326.4 & 54.4 \\
\hline 2685.3 & 41 & -14 & -23.6 & & \\
\hline 1335.7 & 50.8 & 2.4 & 6.3 & & \\
\hline 2615.9 & 40.6 & -24 & -39.7 & & \\
\hline 2175.6 & 44.6 & -11.7 & -21.9 & & \\
\hline 4024.1 & 36 & -75 & -90.1 & & \\
\hline 2635.4 & 41.2 & -2.1 & -3.6 & 2635.4 & 41.2 \\
\hline 1902.9 & 51.2 & -0.9 & -1.9 & 1902.9 & 51.2 \\
\hline 2609.2 & 42 & 0.2 & 0.3 & 2609.2 & 42 \\
\hline 2563 & 41.4 & -9.1 & -15.8 & & \\
\hline 2041.8 & 46.4 & 0.4 & 0.8 & 2041.8 & 46.4 \\
\hline 2638.2 & 41 & 0.3 & 0.5 & 2638.2 & 41 \\
\hline 2628.7 & 41 & -1.4 & -2.4 & 2628.7 & 41 \\
\hline 2007.7 & 44.2 & -1.5 & -3 & 2007.7 & 44.2 \\
\hline 3014 & 40.8 & -7.2 & -11.9 & 3014 & 40.8 \\
\hline 2063.2 & 45.2 & 1 & 1.9 & 2063.2 & 45.2 \\
\hline 1316.2 & 53 & 0.3 & 0.8 & 1316.2 & 53 \\
\hline 1354.7 & 54.6 & 0.8 & 1.9 & 1354.7 & 54.6 \\
\hline 2020 & 46.8 & 0.5 & 0.9 & 2020 & 46.8 \\
\hline 2637.6 & 42 & 1.2 & 2.1 & 2637.6 & 42 \\
\hline 3489.1 & 41.4 & 0.5 & 0.7 & 3489.1 & 41.4 \\
\hline 2117.2 & 44.2 & -0.5 & -1.1 & 2117.2 & 44.2 \\
\hline 1387.5 & 47.8 & -4 & -10.1 & 1387.5 & 47.8 \\
\hline 2523 & 42.6 & -15.7 & -27.1 & & \\
\hline 2470.3 & 46 & -0.2 & -0.3 & 2470.3 & 46 \\
\hline 1372 & 53.6 & -9.3 & -23.1 & 1055.2 & 24.8 \\
\hline 1786.4 & 50 & 0.1 & 0.1 & 1786.4 & 50 \\
\hline 1950.7 & 55.2 & 0.1 & 0.1 & 1950.7 & 55.2 \\
\hline 1983.2 & 45.2 & -0.3 & -0.7 & 1983.2 & 45.2 \\
\hline 1322.1 & 55.4 & 0.3 & 0.6 & 1322.1 & 55.4 \\
\hline 2148.8 & 47.6 & -6.5 & -12.6 & 2148.8 & 47.6 \\
\hline 1980 & 45 & -33.5 & -61.1 & & \\
\hline 2098.1 & 47 & -0.8 & -1.6 & 2098.1 & 47 \\
\hline 2612.8 & 44.2 & 1.5 & 2.7 & 2612.8 & 44.2 \\
\hline 2589.3 & 41.4 & -7.3 & -12.7 & 2589.3 & 41.4 \\
\hline 1332.3 & 57.4 & 1.1 & 2.8 & 1332.3 & 57.4 \\
\hline 1869 & 51.2 & 0.5 & 0.9 & 1869 & 51.2 \\
\hline 1384.2 & 48.8 & -0.4 & -1.2 & 1384.2 & 48.8 \\
\hline 2136 & 44 & -3.3 & -6.4 & 2136 & 44 \\
\hline 2194.8 & 43.8 & -12.2 & -22.7 & & \\
\hline 1354.1 & 53.4 & 0.2 & 0.4 & 1354.1 & 53.4 \\
\hline 1349.6 & 50.4 & 0.8 & 2 & 1349.6 & 50.4 \\
\hline 2014.7 & 48 & -8.6 & -16.9 & & \\
\hline 2130.8 & 47.8 & 0.7 & 1.3 & 2130.8 & 47.8 \\
\hline 2628.2 & 43 & 0.8 & 1.5 & 2628.2 & 43 \\
\hline 2723.3 & 44.4 & 0.7 & 1.2 & 2723.3 & 44.4 \\
\hline 2361.5 & 42.8 & -2.8 & -5.3 & 2361.5 & 42.8 \\
\hline 1844.4 & 51 & 1.5 & 3.2 & 1844.4 & 51 \\
\hline 2556.6 & 45.6 & 0.6 & 1.1 & 2556.6 & 45.6 \\
\hline 2585.6 & 44.8 & 0.8 & 1.5 & 2585.6 & 44.8 \\
\hline 2641.1 & 42 & -15.8 & -26.7 & & \\
\hline 1353.4 & 52 & 0.9 & 2.2 & 1353.4 & 52 \\
\hline 2630.2 & 42.8 & -7.6 & -13.2 & 2630.2 & 42.8 \\
\hline
\end{tabular}




\begin{tabular}{|c|c|c|c|c|c|}
\hline 2355.8 & 43.4 & -13 & -23.3 & & \\
\hline 1988.6 & 46.2 & -1.5 & -3 & 1988.6 & 46.2 \\
\hline 1956.5 & 47.6 & 0.7 & 1.3 & 1956.5 & 47.6 \\
\hline 2674.6 & 42.2 & -30.1 & -48.4 & & \\
\hline 2620.9 & 43 & -9.6 & -16.6 & & \\
\hline 2956.9 & 41.4 & -15.7 & -25.3 & & \\
\hline 2735 & 42.2 & 0.8 & 1.4 & 2735 & 42.2 \\
\hline 2620.7 & 42.8 & -0.4 & -0.7 & 2620.7 & 42.8 \\
\hline 1956.7 & 48.2 & 0.7 & 1.3 & 1956.7 & 48.2 \\
\hline 1852.5 & 53 & 0.6 & 1.3 & 1852.5 & 53 \\
\hline 2032.3 & 46.4 & -7.5 & -14.9 & 2032.3 & 46.4 \\
\hline 1760.9 & 53.6 & 0.9 & 1.9 & 1760.9 & 53.6 \\
\hline 1365.6 & 56 & -0.1 & -0.2 & 1365.6 & 56 \\
\hline 2612.5 & 46 & -8.2 & -14.3 & 2612.5 & 46 \\
\hline 1375.9 & 56.6 & 1 & 2.6 & 1375.9 & 56.6 \\
\hline 1357.6 & 57.6 & 0.3 & 0.8 & 1357.6 & 57.6 \\
\hline 3169.9 & 40.6 & -13.8 & -21.7 & & \\
\hline 2192.9 & 50.6 & -4 & -7.6 & 2192.9 & 50.6 \\
\hline 2908.8 & 42.2 & -34.8 & -53.2 & & \\
\hline 1973.2 & 49.8 & 0.2 & 0.4 & 1973.2 & 49.8 \\
\hline 2612.5 & 46.2 & 0.2 & 0.3 & 2612.5 & 46.2 \\
\hline 2735 & 43 & 1 & 1.7 & 2735 & 43 \\
\hline 2638.4 & 44.4 & 0.1 & 0.2 & 2638.4 & 44.4 \\
\hline 2176.1 & 45.8 & -13.2 & -24.6 & & \\
\hline 2613.2 & 43.4 & 1.6 & 2.8 & 2613.2 & 43.4 \\
\hline 1321 & 71 & 1.7 & 4.5 & 1321 & 71 \\
\hline 1942.2 & 55 & -16 & -31.4 & & \\
\hline 2123.3 & 52.4 & -0.3 & -0.6 & 2123.3 & 52.4 \\
\hline 2784 & 43.6 & -0.1 & -0.2 & 2784 & 43.6 \\
\hline 2067.5 & 55.4 & -0.3 & -0.6 & 2067.5 & 55.4 \\
\hline 2065.2 & 50 & -3.7 & -7.3 & 2065.2 & 50 \\
\hline 2597 & 44.2 & 0.8 & 1.5 & 2597 & 44.2 \\
\hline 1679.4 & 57.8 & -7.9 & -17.4 & & \\
\hline 1978.4 & 47.8 & 1 & 2 & 1978.4 & 47.8 \\
\hline 2659.3 & 43.4 & -1.8 & -3.1 & 2659.3 & 43.4 \\
\hline 1973.1 & 48.6 & 1.2 & 2.5 & 1973.1 & 48.6 \\
\hline 1397.9 & 56.2 & -0.8 & -2.1 & 1397.9 & 56.2 \\
\hline 1857 & 49 & 1.3 & 2.7 & 1857 & 49 \\
\hline 2714.5 & 44.2 & -4.4 & -7.5 & 2714.5 & 44.2 \\
\hline 1385.1 & 57 & 0.5 & 1.3 & 1385.1 & 57 \\
\hline 1896.1 & 56 & -0.1 & -0.2 & 1896.1 & 56 \\
\hline 2069 & 47.4 & -0.2 & -0.4 & 2069 & 47.4 \\
\hline 2563.1 & 45 & 0.5 & 0.9 & 2563.1 & 45 \\
\hline 1348.5 & 56.2 & 1.1 & 2.7 & 1348.5 & 56.2 \\
\hline
\end{tabular}


Sample 3641L Kagera @ Kasensero 58 grain analysed 38 concordant ages

\begin{tabular}{|c|c|c|c|c|c|c|}
\hline \multirow[b]{2}{*}{ grain } & \multicolumn{3}{|c|}{ concentrations } & \multicolumn{3}{|l|}{ isotopic ratios } \\
\hline & U [ppm] & $\mathrm{Pb}$ [ppm] & Th/U & Pb207/Pb206 & $2 \sigma 76$ & Pb207/U235 \\
\hline X3641B G001 & 122.9 & 19.2 & 0.4172 & 0.06545 & 0.00258 & 1.16201 \\
\hline X3641B_G002 & 98.2 & 45.3 & 0.6238 & 0.13097 & 0.00372 & 7.19681 \\
\hline X3641B_G003 & 396.2 & 176.9 & 0.5981 & 0.12621 & 0.00316 & 6.75814 \\
\hline X3641B_G004 & 87.1 & 55 & 0.774 & 0.18187 & 0.00506 & 12.87784 \\
\hline X3641B_G005 & 135.5 & 82.4 & 0.5751 & 0.17786 & 0.00452 & 12.57766 \\
\hline X3641B G006 & 234.2 & 140.4 & 0.6698 & 0.17547 & 0.00452 & 12.11741 \\
\hline X3641B G007 & 522.8 & 248.6 & 0.796 & 0.17791 & 0.00442 & 10.1965 \\
\hline X3641B_G008 & 208.1 & 47.8 & 0.148 & 0.08678 & 0.00248 & 2.79078 \\
\hline X3641B_G009 & 1598.3 & 581.4 & 2.8972 & 0.17757 & 0.0043 & 5.55537 \\
\hline X3641B_G010 & 202.5 & 55.6 & 0.467 & 0.08912 & 0.00274 & 3.12311 \\
\hline X3641B_G011 & 4170.6 & 204.4 & 0.6957 & 0.16991 & 0.00434 & 1.0851 \\
\hline X3641B_G012 & 259.8 & 77.9 & 1.018 & 0.08783 & 0.0025 & 2.95339 \\
\hline X3641B G013 & 358 & 42.6 & 0.6047 & 0.0599 & 0.00202 & 0.89369 \\
\hline X3641B_G014 & 211.8 & 94.1 & 0.1797 & 0.14641 & 0.00378 & 8.61914 \\
\hline X3641B_G015 & 424.6 & 197.3 & 0.8657 & 0.12608 & 0.0032 & 6.63312 \\
\hline X3641B_G016 & 184.8 & 84 & 1.0174 & 0.1205 & 0.00326 & 6.02866 \\
\hline X3641B_G017 & 50.7 & 14.3 & 0.8263 & 0.0888 & 0.00386 & 2.94088 \\
\hline X3641B_G018 & 547.5 & 318 & 0.6953 & 0.17378 & 0.00424 & 11.85819 \\
\hline X3641B G019 & 142.9 & 45.4 & 1.1609 & 0.08681 & 0.00276 & 2.97069 \\
\hline X3641B_G020 & 950.7 & 247.2 & 1.5872 & 0.17515 & 0.00432 & 5.42605 \\
\hline X3641B_G021 & 99.6 & 29 & 0.8975 & 0.0851 & 0.00292 & 2.84697 \\
\hline X3641B_G022 & 148.1 & 39.8 & 0.5114 & 0.08687 & 0.00272 & 2.9534 \\
\hline X3641B_G023 & 81 & 24.7 & 1.061 & 0.08523 & 0.0031 & 2.88253 \\
\hline X3641B_G024 & 1013.1 & 387.6 & 3.7178 & 0.19022 & 0.00486 & 5.10858 \\
\hline X3641B_G025 & & & & 0.76734 & 0.01938 & \\
\hline X3641B_G026 & 206.7 & 93 & 0.8356 & 0.12758 & 0.00338 & 6.60543 \\
\hline X3641B_G027 & 101 & 29.5 & 0.8033 & 0.08755 & 0.00298 & 3.00169 \\
\hline X3641B_G028 & 213.2 & 107.4 & 0.9971 & 0.18136 & 0.0047 & 10.4898 \\
\hline X3641B_G029 & 328.7 & 194.5 & 0.6324 & 0.17374 & 0.00446 & 11.9496 \\
\hline X3641B_G030 & 150.8 & 69.4 & 0.9485 & 0.12041 & 0.00332 & 6.14126 \\
\hline X3641B G031 & 164.3 & 41.6 & 0.6194 & 0.08655 & 0.00266 & 2.77143 \\
\hline X3641B_G032 & 222.1 & 131.6 & 0.4846 & 0.18447 & 0.00484 & 13.1075 \\
\hline X3641B_G033 & 288.2 & 163.7 & 0.3571 & 0.17276 & 0.00452 & 12.08018 \\
\hline X3641B_G034 & 195.1 & 54.4 & 0.8078 & 0.08724 & 0.00256 & 2.87653 \\
\hline X3641B_G035 & 217.9 & 152.9 & 0.9177 & 0.1785 & 0.00472 & 13.25036 \\
\hline X3641B_G036 & 1148.6 & & 1.8138 & 0.15346 & 0.00394 & 3.8916 \\
\hline X3641B_G037 & 186.2 & 59.3 & 0.5542 & 0.05595 & 0.00216 & 2.20054 \\
\hline X3641B_G038 & 148.1 & 82.3 & 0.8183 & 0.15456 & 0.00416 & 9.65869 \\
\hline X3641B_G039 & 122 & 55.3 & 0.7763 & 0.12834 & 0.00362 & 6.72756 \\
\hline X3641B_G040 & 210.9 & 77.9 & 0.1585 & 0.12136 & 0.00332 & 6.06518 \\
\hline X3641B_G041 & & & & 0.76549 & 4.07956 & -54.93647 \\
\hline X3641B_G042 & 608 & 349.8 & 1.7181 & 0.16057 & 0.0041 & 8.92574 \\
\hline X3641B_G043 & 98.7 & 59.1 & 0.5616 & 0.17547 & 0.00506 & 12.31024 \\
\hline X3641B_G044 & 1026.6 & 376.8 & 1.5048 & 0.17682 & 0.00468 & 7.64201 \\
\hline X3641B_G045 & 484.2 & 226.4 & 0.9653 & 0.16579 & 0.00428 & 9.03234 \\
\hline X3641B_G046 & 573.6 & 118.8 & 0.2592 & 0.0889 & 0.0024 & 2.5675 \\
\hline X3641B_G047 & 74 & 41.2 & 0.5904 & 0.1653 & 0.00474 & 10.832 \\
\hline X3641B_G048 & 280.7 & 124.4 & 0.6541 & 0.13252 & 0.00354 & 7.1307 \\
\hline X3641B_G049 & 670.4 & 332 & 0.9722 & 0.16518 & 0.00432 & 9.49005 \\
\hline X3641B G050 & 415.8 & 236 & 0.4722 & 0.17584 & 0.00458 & 12.10025 \\
\hline
\end{tabular}




$\begin{array}{lcccccc}\text { X3641B_G051 } & 717.4 & 170.8 & 0.1559 & 0.08557 & 0.00228 & 2.84972 \\ \text { X3641B_G052 } & 3114.2 & 217 & 1.5637 & 0.2178 & 0.0057 & 1.64625 \\ \text { X3641B_G053 } & 3485.3 & 299 & 3.0691 & 0.17211 & 0.00512 & 1.25458 \\ \text { X3641B_G054 } & 190 & 33.7 & 0.2654 & 0.08655 & 0.00278 & 2.07589 \\ \text { X3641B_G055 } & & & & -0.1536 & 0.87812 & \\ \text { X3641B_G056 } & 313.8 & 202.8 & 0.9766 & 0.17671 & 0.00478 & 12.3199 \\ \text { X3641B_G057 } & 1936.3 & 347.1 & 3.527 & 0.17426 & 0.00462 & 2.36166 \\ \text { X3641B_G058 } & 859.4 & 269.6 & 2.1666 & 0.16627 & 0.00448 & 5.19294\end{array}$




\section{$65.5 \%$ concordant}

\begin{tabular}{|c|c|c|c|c|c|c|}
\hline $2 \sigma 75$ & Pb206/U238 & $2 \sigma 68$ & $\begin{array}{c}\text { ages } \\
\text { age } 206 / 238\end{array}$ & $2 \sigma$ age 68 & age $207 / 235$ & $2 \sigma$ age 75 \\
\hline 0.04548 & 0.1288 & 0.00338 & 781 & 19.4 & 782.9 & 25.8 \\
\hline 0.213 & 0.39868 & 0.01 & 2163 & 46 & 2136.1 & 33.8 \\
\hline 0.17892 & 0.38847 & 0.00908 & 2115.7 & 42.2 & 2080.3 & 30.2 \\
\hline 0.37802 & 0.51372 & 0.0132 & 2672.5 & 56.2 & 2670.8 & 35.6 \\
\hline 0.33914 & 0.51306 & 0.0123 & 2669.7 & 52.4 & 2648.6 & 32.8 \\
\hline 0.3304 & 0.50103 & 0.0121 & 2618.2 & 52 & 2613.5 & 33.2 \\
\hline 0.26834 & 0.41581 & 0.00976 & 2241.4 & 44.4 & 2452.8 & 31.6 \\
\hline 0.08246 & 0.23333 & 0.00564 & 1351.9 & 29.4 & 1353.1 & 28 \\
\hline 0.14294 & 0.22698 & 0.00522 & 1318.7 & 27.4 & 1909.2 & 28.8 \\
\hline 0.09848 & 0.25425 & 0.00634 & 1460.4 & 32.6 & 1438.4 & 30.4 \\
\hline 0.02888 & 0.04633 & 0.00108 & 292 & 6.6 & 746.1 & 18.2 \\
\hline 0.0869 & 0.24397 & 0.0059 & 1407.3 & 30.6 & 1395.7 & 28.4 \\
\hline 0.03052 & 0.10824 & 0.00266 & 662.5 & 15.4 & 648.3 & 20 \\
\hline 0.2352 & 0.4271 & 0.0102 & 2292.6 & 46 & 2298.6 & 32 \\
\hline 0.17816 & 0.38168 & 0.00898 & 2084.1 & 41.8 & 2063.8 & 30.6 \\
\hline 0.17042 & 0.36298 & 0.00878 & 1996.3 & 41.6 & 1980 & 31.6 \\
\hline 0.12688 & 0.24028 & 0.00708 & 1388.2 & 36.8 & 1392.5 & 39.8 \\
\hline 0.30742 & 0.49506 & 0.01144 & 2592.5 & 49.4 & 2593.3 & 31.4 \\
\hline 0.09678 & 0.24828 & 0.00628 & 1429.6 & 32.4 & 1400.2 & 30.8 \\
\hline 0.14158 & 0.22476 & 0.0052 & 1307 & 27.4 & 1889 & 29 \\
\hline 0.09908 & 0.24273 & 0.00632 & 1400.9 & 32.8 & 1368 & 32.4 \\
\hline 0.09432 & 0.24667 & 0.00618 & 1421.3 & 32 & 1395.7 & 30.4 \\
\hline 0.10574 & 0.24536 & 0.00656 & 1414.5 & 34 & 1377.4 & 34 \\
\hline 0.13688 & 0.19484 & 0.00458 & 1147.5 & 24.8 & 1837.5 & 29.4 \\
\hline 131.99006 & 24.17686 & 1.21936 & & & & \\
\hline 0.184 & 0.37564 & 0.009 & 2055.9 & 42.2 & 2060.1 & 31.6 \\
\hline 0.10374 & 0.24873 & 0.00646 & 1431.9 & 33.4 & 1408 & 32.6 \\
\hline 0.2866 & 0.41962 & 0.01004 & 2258.8 & 45.6 & 2479 & 32.6 \\
\hline 0.3249 & 0.49898 & 0.01188 & 2609.4 & 51 & 2600.5 & 32.8 \\
\hline 0.1768 & 0.37004 & 0.009 & 2029.6 & 42.4 & 1996.1 & 32.2 \\
\hline 0.08724 & 0.23232 & 0.00576 & 1346.6 & 30.2 & 1347.9 & 29.4 \\
\hline 0.3632 & 0.51549 & 0.01246 & 2680 & 53 & 2687.4 & 33.6 \\
\hline 0.33368 & 0.50731 & 0.0122 & 2645.1 & 52.2 & 2610.6 & 33.4 \\
\hline 0.08726 & 0.23922 & 0.00584 & 1382.6 & 30.4 & 1375.8 & 28.8 \\
\hline 0.37048 & 0.53856 & 0.01306 & 2777.4 & 54.8 & 2697.7 & 33.8 \\
\hline 0.105 & 0.18398 & 0.0043 & 1088.7 & 23.4 & 1611.9 & 28 \\
\hline 0.08676 & 0.28537 & 0.007 & 1618.4 & 35.2 & 1181.2 & 32 \\
\hline 0.27328 & 0.45336 & 0.011 & 2410.2 & 48.8 & 2402.8 & 33.4 \\
\hline 0.19816 & 0.3803 & 0.00938 & 2077.7 & 43.8 & 2076.2 & 33.2 \\
\hline 0.17362 & 0.36257 & 0.00874 & 1994.3 & 41.4 & 1985.3 & 31.8 \\
\hline 231.44258 & -0.52066 & 1.9931 & & & & \\
\hline 0.2406 & 0.40329 & 0.0094 & 2184.2 & 43.2 & 2330.4 & 31.6 \\
\hline 0.37244 & 0.50897 & 0.01308 & 2652.2 & 55.8 & 2628.4 & 36.2 \\
\hline 0.21162 & 0.31355 & 0.00746 & 1758.2 & 36.6 & 2189.8 & 32 \\
\hline 0.246 & 0.39526 & 0.00928 & 2147.2 & 42.8 & 2341.3 & 32 \\
\hline 0.0723 & 0.20952 & 0.00494 & 1226.3 & 26.4 & 1291.4 & 26.2 \\
\hline 0.32492 & 0.47542 & 0.01202 & 2507.3 & 52.6 & 2508.8 & 35.6 \\
\hline 0.19998 & 0.39039 & 0.00928 & 2124.6 & 43 & 2127.9 & 31.8 \\
\hline 0.26146 & 0.41683 & 0.00984 & 2246.1 & 44.8 & 2386.6 & 32.4 \\
\hline 0.33136 & 0.49926 & 0.01172 & 2610.6 & 50.4 & 2612.2 & 32.8 \\
\hline
\end{tabular}




$\begin{array}{lcccccc}0.07972 & 0.2416 & 0.00566 & 1395 & 29.4 & 1368.7 & 26.6 \\ 0.04494 & 0.05484 & 0.00128 & 344.2 & 7.8 & 988.1 & 22.2 \\ 0.03756 & 0.05289 & 0.0013 & 332.2 & 8 & 825.5 & 21.8 \\ 0.06764 & 0.174 & 0.00434 & 1034.1 & 23.8 & 1140.9 & 27.8 \\ & & & & & & \\ 0.35044 & 0.50579 & 0.0122 & 2638.6 & 52.2 & 2629.1 & 34 \\ 0.06532 & 0.09832 & 0.0023 & 604.6 & 13.4 & 1231.1 & 25.2 \\ 0.14602 & 0.22659 & 0.00536 & 1316.6 & 28.2 & 1851.5 & 30.6\end{array}$




\begin{tabular}{|c|c|c|c|c|c|}
\hline age $207 / 206$ & $2 \sigma$ age 76 & $\begin{array}{l}\text { discordance } \\
\Delta 68-75[\%]\end{array}$ & $\Delta 68-76[\%]$ & $\begin{array}{c}\text { preferred age } \\
\text { age }\end{array}$ & $2 \sigma$ age \\
\hline 788.8 & 82.8 & -0.2 & -1 & 781 & 19.4 \\
\hline 2111 & 49.8 & 1.3 & 2.5 & 2111 & 49.8 \\
\hline 2045.8 & 44.2 & 1.7 & 3.4 & 2045.8 & 44.2 \\
\hline 2670 & 46 & 0.1 & 0.1 & 2670 & 46 \\
\hline 2633 & 42.2 & 0.8 & 1.4 & 2633 & 42.2 \\
\hline 2610.5 & 42.8 & 0.2 & 0.3 & 2610.5 & 42.8 \\
\hline 2633.5 & 41.2 & -8.6 & -14.9 & 2633.5 & 41.2 \\
\hline 1355.6 & 55.2 & -0.1 & -0.3 & 1355.6 & 55.2 \\
\hline 2630.3 & 40.2 & -30.9 & -49.9 & & \\
\hline 1406.7 & 58.8 & 1.5 & 3.8 & 1406.7 & 58.8 \\
\hline 2556.8 & 42.8 & -60.9 & -88.6 & & \\
\hline 1378.8 & 54.8 & 0.8 & 2.1 & 1378.8 & 54.8 \\
\hline 600 & 73 & 2.2 & 10.4 & 662.5 & 15.4 \\
\hline 2304.4 & 44.4 & -0.3 & -0.5 & 2304.4 & 44.4 \\
\hline 2044 & 44.8 & 1 & 2 & 2044 & 44.8 \\
\hline 1963.6 & 48.2 & 0.8 & 1.7 & 1963.6 & 48.2 \\
\hline 1399.8 & 83.4 & -0.3 & -0.8 & 1399.8 & 83.4 \\
\hline 2594.4 & 40.6 & 0 & -0.1 & 2594.4 & 40.6 \\
\hline 1356.3 & 61.4 & 2.1 & 5.4 & & \\
\hline 2607.5 & 41 & -30.8 & -49.9 & & \\
\hline 1317.8 & 66.6 & 2.4 & 6.3 & & \\
\hline 1357.6 & 60.4 & 1.8 & 4.7 & 1357.6 & 60.4 \\
\hline 1320.8 & 70.4 & 2.7 & 7.1 & & \\
\hline 2744.1 & 42 & -37.5 & -58.2 & & \\
\hline 2064.9 & 46.8 & -0.2 & -0.4 & 2064.9 & 46.8 \\
\hline 1372.6 & 65.4 & 1.7 & 4.3 & 1372.6 & 65.4 \\
\hline 2665.3 & 43 & -8.9 & -15.3 & & \\
\hline 2594 & 42.8 & 0.3 & 0.6 & 2594 & 42.8 \\
\hline 1962.3 & 49.2 & 1.7 & 3.4 & 1962.3 & 49.2 \\
\hline 1350.5 & 59.4 & -0.1 & -0.3 & 1350.5 & 59.4 \\
\hline 2693.5 & 43.4 & -0.3 & -0.5 & 2693.5 & 43.4 \\
\hline 2584.6 & 43.6 & 1.3 & 2.3 & 2584.6 & 43.6 \\
\hline 1365.8 & 56.6 & 0.5 & 1.2 & 1365.8 & 56.6 \\
\hline 2639 & 44 & 3 & 5.2 & & \\
\hline 2384.8 & 43.8 & -32.5 & -54.3 & & \\
\hline 450.4 & 85.8 & 37 & 259.3 & & \\
\hline 2397 & 45.8 & 0.3 & 0.5 & 2397 & 45.8 \\
\hline 2075.4 & 49.6 & 0.1 & 0.1 & 2075.4 & 49.6 \\
\hline 1976.3 & 48.8 & 0.5 & 0.9 & 1976.3 & 48.8 \\
\hline 2461.7 & 43.2 & -6.3 & -11.3 & 2461.7 & 43.2 \\
\hline 2610.5 & 48 & 0.9 & 1.6 & 2610.5 & 48 \\
\hline 2623.3 & 44 & -19.7 & -33 & & \\
\hline 2515.6 & 43.4 & -8.3 & -14.6 & 2515.6 & 43.4 \\
\hline 1402 & 51.8 & -5 & -12.5 & 1402 & 51.8 \\
\hline 2510.6 & 48.2 & -0.1 & -0.1 & 2510.6 & 48.2 \\
\hline 2131.6 & 46.8 & -0.2 & -0.3 & 2131.6 & 46.8 \\
\hline 2509.4 & 44 & -5.9 & -10.5 & 2509.4 & 44 \\
\hline 2614 & 43.4 & -0.1 & -0.1 & 2614 & 43.4 \\
\hline
\end{tabular}




$\begin{array}{cccccc}1328.5 & 51.6 & 1.9 & 5 & & \\ 2964.5 & 42.2 & -65.2 & -88.4 & & \\ 2578.3 & 49.6 & -59.8 & -87.1 & & \\ 1350.5 & 62 & -9.4 & -23.4 & 1034.1 & 23.8 \\ & & & & & \\ 2622.2 & 45 & 0.4 & 0.6 & 2622.2 & 45 \\ 2599 & 44.2 & -50.9 & -76.7 & & \\ 2520.4 & 45.2 & -28.9 & -47.8 & & \end{array}$


Sample $3642 S$ Lake Victoria beach @ Kasensero 28 grain analysed 14 conc

\begin{tabular}{cccc|ccc} 
& \multicolumn{1}{c}{ concentrations } & & & isotopic ratios & & \\
grain & $\mathbf{U}$ [ppm] & Pb [ppm] & Th/U & Pb207/Pb206 & 20 76 & Pb207/U235 \\
\hline S3642_G001 & 889.9 & 209.4 & 0.2273 & 0.08688 & 0.00204 & 2.80354 \\
S3642_G002 & 163.1 & 73 & 0.5791 & 0.13677 & 0.00338 & 7.4268 \\
S3642_G003 & 60.7 & 31 & 10.1679 & 0.0854 & 0.00362 & 1.59573 \\
S3642_G004 & 885.7 & 315.7 & 1.7628 & 0.13918 & 0.00324 & 5.9332 \\
S3642_G005 & 391.6 & 208.5 & 0.2573 & 0.17397 & 0.00406 & 11.7821 \\
S3642_G006 & 95.7 & 43.8 & 1.0644 & 0.11867 & 0.00322 & 5.88721 \\
S3642_G007 & 333.6 & 167.4 & 0.9498 & 0.1702 & 0.00402 & 9.6367 \\
S3642_G008 & 2788.5 & 150.1 & 2.1868 & 0.13108 & 0.00332 & 0.71723 \\
S3642_G009 & 356.1 & 161.3 & 0.3735 & 0.14641 & 0.00346 & 8.41081 \\
S3642_G010 & 1067.2 & 21.3 & 5.8152 & 0.78262 & 0.02128 & 3.2998 \\
S3642_G011 & & & & 0.48465 & 3.9126 & \\
X3642n_G001 & 592.1 & 80.3 & 1.6552 & 0.15535 & 0.00408 & 2.38741 \\
X3642n_G002 & 599.3 & 131.7 & 0.386 & 0.09088 & 0.00236 & 2.69993 \\
X3642n_G003 & 429.9 & 111.2 & 0.9035 & 0.118 & 0.00306 & 3.81989 \\
X3642n_G004 & 143.1 & 62.9 & 0.3598 & 0.13535 & 0.00352 & 7.55175 \\
X3642n_G005 & 113.4 & 63.8 & 0.9208 & 0.17949 & 0.00482 & 11.71832 \\
X3642n_G006 & 289.6 & 96.4 & 1.7208 & 0.13907 & 0.00358 & 5.12738 \\
X3642n_G007 & & & & 0.77066 & 0.02272 & \\
X3642n_G008 & 95.7 & 30.5 & 0.5361 & 0.14855 & 0.00464 & 6.07985 \\
X3642n_G009 & 32.1 & 14.1 & 6.4362 & 0.08241 & 0.00386 & 1.58343 \\
X3642n_G010 & 73.2 & 33.9 & 1.2129 & 0.11878 & 0.0035 & 5.78193 \\
X3642n_G011 & 158 & 93.7 & 0.6171 & 0.17621 & 0.00456 & 12.14129 \\
X3642n_G012 & 4843.8 & 276.8 & 1.2251 & 0.388 & 0.00988 & 2.75403 \\
X3642n_G013 & 795.1 & 5.3 & 5.0438 & 0.76453 & 0.0207 & 2.50694 \\
X3642n_G014 & 230.2 & 106.5 & 0.4006 & 0.14532 & 0.0037 & 8.45231 \\
X3642n_G015 & 274.3 & 93.9 & 0.9682 & 0.12353 & 0.0034 & 4.70089 \\
X3642n_G016 & 1112.5 & 148.2 & 1.9378 & 0.13263 & 0.0034 & 1.84964 \\
X3642n_G017 & 248.9 & 155.4 & 1.1148 & 0.1735 & 0.00458 & 11.47396
\end{tabular}




\section{ordant ages $\quad \mathbf{5 0 . 0} \%$ concordant}

\begin{tabular}{|c|c|c|c|c|c|c|}
\hline $2 \sigma 75$ & Pb206/U238 & $2 \sigma 68$ & $\begin{array}{c}\text { ages } \\
\text { age } 206 / 238\end{array}$ & $2 \sigma$ age 68 & age $207 / 235$ & $2 \sigma$ age 75 \\
\hline 0.0741 & 0.23412 & 0.0057 & 1356.1 & 29.8 & 1356.5 & 25.4 \\
\hline 0.20406 & 0.39397 & 0.00988 & 2141.2 & 45.6 & 2164.2 & 31.6 \\
\hline 0.06744 & 0.13557 & 0.00396 & 819.6 & 22.4 & 968.5 & 32.2 \\
\hline 0.15524 & 0.30929 & 0.00754 & 1737.2 & 37.2 & 1966.1 & 29.2 \\
\hline 0.30974 & 0.49139 & 0.01206 & 2576.7 & 52.2 & 2587.2 & 31.6 \\
\hline 0.17364 & 0.35995 & 0.00932 & 1981.9 & 44.2 & 1959.4 & 32.6 \\
\hline 0.2553 & 0.41081 & 0.01012 & 2218.6 & 46.2 & 2400.7 & 31.4 \\
\hline 0.01982 & 0.0397 & 0.00098 & 251 & 6 & 549 & 15 \\
\hline 0.22344 & 0.41681 & 0.01026 & 2246 & 46.6 & 2276.3 & 31 \\
\hline 0.09046 & 0.03059 & 0.00084 & 194.2 & 5.2 & 1481 & 30.2 \\
\hline 0.06654 & 0.11151 & 0.0027 & 681.5 & 15.6 & 1238.8 & 25.6 \\
\hline 0.07518 & 0.21557 & 0.00516 & 1258.4 & 27.4 & 1328.4 & 26.2 \\
\hline 0.10596 & 0.23489 & 0.00564 & 1360.1 & 29.4 & 1596.9 & 28.4 \\
\hline 0.21028 & 0.40486 & 0.0098 & 2191.4 & 45 & 2179.2 & 31.8 \\
\hline 0.33634 & 0.47372 & 0.01184 & 2499.9 & 51.8 & 2582.2 & 34.4 \\
\hline 0.14114 & 0.26752 & 0.00642 & 1528.2 & 32.6 & 1840.7 & 30 \\
\hline 1608.64014 & 82.82088 & 15.12962 & & & & \\
\hline 0.19636 & 0.29698 & 0.00792 & 1676.3 & 39.4 & 1987.4 & 35.8 \\
\hline 0.07304 & 0.13942 & 0.00416 & 841.4 & 23.6 & 963.7 & 34.6 \\
\hline 0.17878 & 0.35322 & 0.00904 & 1950 & 43 & 1943.7 & 33.8 \\
\hline 0.33736 & 0.49997 & 0.01216 & 2613.7 & 52.2 & 2615.4 & 33.4 \\
\hline 0.0745 & 0.0515 & 0.00124 & 323.7 & 7.6 & 1343.2 & 26.2 \\
\hline 0.069 & 0.02379 & 0.0006 & 151.6 & 3.8 & 1274 & 26.8 \\
\hline 0.2319 & 0.42204 & 0.01012 & 2269.7 & 45.8 & 2280.8 & 31.8 \\
\hline 0.1369 & 0.27613 & 0.0068 & 1571.8 & 34.4 & 1767.4 & 31 \\
\hline 0.05058 & 0.10119 & 0.0024 & 621.4 & 14 & 1063.3 & 23 \\
\hline 0.3247 & 0.47986 & 0.01182 & 2526.7 & 51.4 & 2562.5 & 33.8 \\
\hline
\end{tabular}




\begin{tabular}{|c|c|c|c|c|c|}
\hline age $207 / 206$ & $2 \sigma$ age 76 & $\begin{array}{l}\text { discordance } \\
\Delta 68-75[\%]\end{array}$ & $\Delta 68-76[\%]$ & $\begin{array}{c}\text { preferred age } \\
\text { age }\end{array}$ & $2 \sigma$ age \\
\hline 1357.8 & 45.2 & 0 & -0.1 & 1357.8 & 45.2 \\
\hline 2186.7 & 43 & -1.1 & -2.1 & 2186.7 & 43 \\
\hline 1324.6 & 82 & -15.4 & -38.1 & & \\
\hline 2217 & 40.4 & -11.6 & -21.6 & & \\
\hline 2596.2 & 39 & -0.4 & -0.8 & 2596.2 & 39 \\
\hline 1936.3 & 48.6 & 1.2 & 2.4 & 1936.3 & 48.6 \\
\hline 2559.6 & 39.6 & -7.6 & -13.3 & 2559.6 & 39.6 \\
\hline 2112.5 & 44.4 & -54.3 & -88.1 & & \\
\hline 2304.4 & 40.6 & -1.3 & -2.5 & 2304.4 & 40.6 \\
\hline 4890.4 & 38.8 & -86.9 & -96 & & \\
\hline 2405.7 & 44.6 & -45 & -71.7 & & \\
\hline 1444.1 & 49.4 & -5.3 & -12.9 & 1444.1 & 49.4 \\
\hline 1926.2 & 46.4 & -14.8 & -29.4 & & \\
\hline 2168.5 & 45.4 & 0.6 & 1.1 & 2168.5 & 45.4 \\
\hline 2648.2 & 44.6 & -3.2 & -5.6 & 2648.2 & 44.6 \\
\hline 2215.7 & 44.6 & -17 & -31 & & \\
\hline 2329.3 & 53.6 & -15.7 & -28 & & \\
\hline 1255.3 & 91.6 & -12.7 & -33 & 841.4 & 23.6 \\
\hline 1938 & 52.8 & 0.3 & 0.6 & 1938 & 52.8 \\
\hline 2617.5 & 43 & -0.1 & -0.1 & 2617.5 & 43 \\
\hline 3863.2 & 38.4 & -75.9 & -91.6 & & \\
\hline 4857.1 & 38.6 & -88.1 & -96.9 & & \\
\hline 2291.6 & 43.8 & -0.5 & -1 & 2291.6 & 43.8 \\
\hline 2007.8 & 48.8 & -11.1 & -21.7 & & \\
\hline 2133.1 & 44.8 & -41.6 & -70.9 & & \\
\hline 2591.7 & 44 & -1.4 & -2.5 & 2591.7 & 44 \\
\hline
\end{tabular}


Sample 3692SVictoria Nile @ downstream Murchison Falls 104 grain analyse

\begin{tabular}{|c|c|c|c|c|c|c|}
\hline \multirow[b]{2}{*}{ grain } & \multicolumn{3}{|l|}{ concentrations } & \multicolumn{3}{|l|}{ isotopic ratios } \\
\hline & U [ppm] & $\mathrm{Pb}[p p m]$ & $T h / U$ & Pb207/Pb206 & $2 \sigma 76$ & Pb207/U235 \\
\hline X3692_G001 & & & & 0.88286 & 0.06682 & 337.52625 \\
\hline X3692_G002 & 406.4 & 325.3 & 2.5116 & 0.1782 & 0.0043 & 11.68989 \\
\hline X3692_G003 & 1887.7 & 3.7 & 8.9467 & 0.28648 & 0.00706 & 2.14376 \\
\hline X3692_G004 & 700.3 & 387.3 & 0.7483 & 0.17 & 0.00406 & 10.8631 \\
\hline X3692_G005 & 505.6 & 283.8 & 0.5058 & 0.17418 & 0.00418 & 11.73236 \\
\hline X3692_G006 & 299.5 & 165.6 & 0.5404 & 0.16811 & 0.00412 & 11.09019 \\
\hline X3692_G007 & 571.4 & 223.1 & 0.5534 & 0.1751 & 0.00424 & 9.07035 \\
\hline X3692 G008 & 1586 & 452.9 & 0.5987 & 0.18481 & 0.00442 & 6.94309 \\
\hline X3692_G009 & 252.1 & 145.2 & 0.6936 & 0.17355 & 0.00428 & 11.54016 \\
\hline X3692_G010 & 451 & 244.4 & 0.6329 & 0.17307 & 0.0042 & 11.08563 \\
\hline X3692_G011 & 333.5 & 126.5 & 0.2192 & 0.13477 & 0.00336 & 6.85359 \\
\hline X3692_G012 & 52.4 & 8.2 & 0.5336 & 0.07092 & 0.00348 & 1.40661 \\
\hline X3692_G013 & 124.6 & 68.9 & 0.4931 & 0.16769 & 0.00432 & 11.16758 \\
\hline X3692_G014 & 473.7 & 214.8 & 0.1781 & 0.17405 & 0.00424 & 10.62145 \\
\hline X3692_G015 & 601.1 & 243 & 0.0977 & 0.19552 & 0.00474 & 11.16034 \\
\hline X3692_G016 & 319.3 & 170.6 & 0.4924 & 0.17144 & 0.00422 & 11.13721 \\
\hline X3692_G017 & 471.6 & 227.9 & 0.1937 & 0.16732 & 0.00408 & 10.61849 \\
\hline X3692_G018 & 1429.6 & 550.6 & 0.3217 & 0.15858 & 0.00382 & 8.36362 \\
\hline X3692_G019 & 165.7 & 72.2 & 0.8398 & 0.12671 & 0.00334 & 6.32017 \\
\hline X3692_G020 & 127.4 & 72 & 0.9599 & 0.16966 & 0.0044 & 10.61415 \\
\hline X3692_G021 & 191.2 & 96.1 & 0.6516 & 0.16018 & 0.00408 & 9.56216 \\
\hline X3692_G022 & 132.4 & 80 & 0.6875 & 0.17059 & 0.00438 & 11.73944 \\
\hline X3692_G023 & 3803 & 968.2 & 0.1375 & 0.14289 & 0.00344 & 5.22846 \\
\hline X3692_G024 & 97 & 54.9 & 0.9931 & 0.17288 & 0.00462 & 10.76897 \\
\hline X3692_G025 & 188.3 & 93.5 & 0.7634 & 0.16558 & 0.00424 & 9.61625 \\
\hline X3692_G026 & 570.7 & 185.4 & 1.8841 & 0.16072 & 0.004 & 6.24366 \\
\hline X3692_G027 & 175.6 & 91.2 & 0.479 & 0.17427 & 0.00444 & 11.13049 \\
\hline X3692_G028 & 662.7 & 323.2 & 0.0378 & 0.16973 & 0.00414 & 11.21013 \\
\hline X3692_G029 & 344.8 & 169.5 & 0.5541 & 0.17544 & 0.00436 & 10.62081 \\
\hline X3692_G030 & 576.4 & 217.3 & 0.1251 & 0.15335 & 0.0038 & 8.01109 \\
\hline X3692_G031 & & & & -0.48241 & 0.586 & 153.37337 \\
\hline X3692_G032 & 270.5 & 185.7 & 1.8127 & 0.1707 & 0.0043 & 10.9109 \\
\hline X3692_G033 & 886.5 & 179.9 & 0.9272 & 0.16702 & 0.00424 & 4.301 \\
\hline X3692_G034 & 960.1 & 114.8 & 0.5767 & 0.08978 & 0.00236 & 1.38199 \\
\hline X3692_G035 & 157.9 & 99.1 & 0.7663 & 0.17054 & 0.0044 & 11.89911 \\
\hline X3692_G036 & 118.2 & 60.7 & 0.5366 & 0.16245 & 0.00432 & 10.08445 \\
\hline X3692_G037 & 264.1 & 155.3 & 0.8703 & 0.17684 & 0.00446 & 11.62756 \\
\hline X3692_G038 & 2034.2 & 885.8 & 0.2316 & 0.17067 & 0.00416 & 9.90609 \\
\hline X3692_G039 & 219.5 & 124.3 & 0.6156 & 0.17558 & 0.00446 & 11.69613 \\
\hline X3692_G040 & 206.8 & 126.4 & 0.9226 & 0.1752 & 0.00448 & 11.73666 \\
\hline X3692_G041 & & & & 0.88066 & 0.02802 & 90.23416 \\
\hline X3692_G042 & 464.5 & 273.5 & 2.0019 & 0.18386 & 0.0046 & 10.36045 \\
\hline X3692_G043 & 393.7 & 225.9 & 0.8053 & 0.17272 & 0.00432 & 11.2598 \\
\hline X3692_G044 & 741.3 & 380.5 & 0.2637 & 0.17792 & 0.0044 & 11.76166 \\
\hline X3692_G045 & 170.6 & 75.4 & 0.8381 & 0.12769 & 0.00344 & 6.43356 \\
\hline X3692_G046 & 126 & 68.3 & 0.6106 & 0.17042 & 0.00452 & 10.96967 \\
\hline X3692_G047 & 219.5 & 33.9 & 0.6515 & 0.06461 & 0.00208 & 1.23268 \\
\hline X3692_G048 & 336.3 & 42.1 & 0.0866 & 0.06495 & 0.00196 & 1.18034 \\
\hline X3692_G049 & 397.9 & 145.9 & 0.8934 & 0.17453 & 0.00446 & 7.90508 \\
\hline X3692_G050 & 2485.3 & 97.3 & 0.1032 & 0.16079 & 0.00438 & 0.86001 \\
\hline
\end{tabular}


X3692_G051

X3692_G052

X3692_G053

X3692_G054

X3692_G055

X3692_G056

X3692_G057

X3692_G058

X3692_G059

X3692_G060

X3692_G061

X3692_G062

X3692_G063

X3692_G064

X3692_G065

X3692_G066

X3692_G067

X3692_G068

X3692_G069

X3692_G070

X3692_G071

X3692_G072

X3692_G073

X3692_G074

X3692_G075

X3692_G076

X3692_G077

X3692_G078

X3692_G079

X3692_G080

X3692_G081

X3692_G082

X3692_G083

X3692_G084

X3692_G085

X3692_G086

X3692_G087

X3692_G088

X3692_G089

X3692_G090

X3692_G091

X3692_G092

X3692_G093

X3692_G094

X3692_G095

X3692_G096

X3692_G097

X3692_G098

X3692_G099

X3692_G100

X3692_G101

X3692_G102

X3692_G103

X3692_G104
402.9

298.8

136.7

206

1527.3

446.8

556.5

945.3

490.7

279

262

474.4

923.3

383.1

1290.1

235.1

353.3

351.2

385.2

410

257.7

262.7

451.7

233.7

572.1

404.3

517.6

1137.8

701

169.9

257

283.2

631.6

645.7

7773.7

1165.5

848.3

186.9

410

346.9

235.1

225.9

332.8

460.2

381.6

261.3

1268.8

190.5

60.9

152.2

\begin{tabular}{cc}
180.8 & 0.2388 \\
184.9 & 0.9933 \\
81.2 & 0.7701 \\
106.9 & 0.6699 \\
770.9 & 0.8546 \\
245.3 & 0.4922 \\
67.2 & 0.5147 \\
298.4 & 1.5299 \\
217.3 & 0.1817 \\
147.1 & 0.8922 \\
133.9 & 0.2872 \\
229.3 & 0.6298 \\
374.2 & 0.1804 \\
192.4 & 0.3294 \\
226 & 4.6279 \\
126.2 & 0.5125 \\
196.6 & 0.7278 \\
160.1 & 0.1427 \\
197.5 & 0.5923 \\
224.6 & 0.9895 \\
146.7 & 1.135 \\
135 & 0.3671 \\
244 & 0.4924 \\
137.5 & 0.2996 \\
360 & 0.841 \\
211.6 & 0.4311 \\
132.5 & 3.629 \\
223.4 & 3.9005 \\
309.5 & 0.1159 \\
114.3 & 1.1879 \\
143.4 & 1.1626 \\
144.6 & 0.6144 \\
222.8 & 1.3517 \\
& \\
\hline 265 & 0.6828 \\
\hline
\end{tabular}

265

$-130.5$

112.5

508.1

108.9

252.3

128.4

145.2

125.9

228.2

216.7

176

134.2

565.8

96.5

22.6

98.5

0.6828
$0.85808 \quad 0.04284$

$0.17558 \quad 0.00446$

$0.17338 \quad 0.00444$

$0.1733 \quad 0.00462$

$0.16194 \quad 0.00426$

$0.1674 \quad 0.00418$

$0.17145 \quad 0.00436$

$0.07692 \quad 0.0022$

$0.18075 \quad 0.00458$

$0.17183 \quad 0.00438$

$0.17624 \quad 0.00458$

$0.16844 \quad 0.0044$

$0.16892 \quad 0.00432$

$0.16308 \quad 0.00412$

$0.17603 \quad 0.00452$

$0.1278 \quad 0.00358$

$0.18297 \quad 0.00478$

$0.17262 \quad 0.00446$

0.17028

0.17203

0.174

0.16843

0.18088

0.17108

0.17816

0.20069

0.17256

0.12921

0.1034

0.1706

0.20891

0.17372

0.16992

0.18194

0.84929

0.17486

0.95561

0.48287

0.17526

0.174

0.23116

0.16305

0.91856

0.1743

0.17101

0.22487

0.1679

0.1646

0.79085

0.16925

0.16777

0.17212

0.12961

0.18086
0.0044

0.00444

0.0045

0.00446

0.00476

0.00444

0.00484

0.00524

0.00456

0.00374

0.00294

0.00444

0.00564

0.00466

0.00452

0.00498

0.03934

0.00458

0.03136

0.01398

0.00456

0.00474

0.00608

0.00442

0.17156

0.00476

0.00484

0.00606

0.00452

0.00442

0.07966

0.00462

0.00442

0.00476

0.0043

0.005
10.46708

11.57794

11.63702

9.88933

10.50967

11.38799

1.17005

6.67336

10.37596

10.75878

10.96056

9.97374

9.15003

11.324

1.13264

12.01682

11.10968

10.46024

10.63904

10.6767

10.25057

11.75534

11.19647

12.89172

14.2413

11.18286

2.36433

1.34044

10.22578

14.8672

10.54593

10.39378

7.59834

486.63724

8.77624

6.45552

12.18136

12.19969

11.47802

16.45126

7.70898

285.53705

11.50922

10.53601

18.45086

9.21456

10.31916

10.23118

10.14773

10.86254

5.76106

12.42507 


\section{7 concordant ages $74.0 \%$ concordant}

\begin{tabular}{|c|c|c|c|c|c|c|}
\hline $2 \sigma 75$ & $\mathrm{~Pb} 206 / \mathrm{U} 238$ & $2 \sigma 68$ & $\begin{array}{c}\text { ages } \\
\text { age } 206 / 238\end{array}$ & $2 \sigma$ age 68 & age $207 / 235$ & $2 \sigma$ age 75 \\
\hline 54.95414 & 2.77386 & 0.46596 & & & & \\
\hline 0.27914 & 0.47595 & 0.00996 & 2509.6 & 43.6 & 2579.9 & 29.8 \\
\hline 0.05134 & 0.05429 & 0.00114 & 340.8 & 7 & 1163 & 22.4 \\
\hline 0.257 & 0.46362 & 0.00962 & 2455.5 & 42.4 & 2511.5 & 29.4 \\
\hline 0.27898 & 0.48871 & 0.01018 & 2565.1 & 44 & 2583.3 & 29.8 \\
\hline 0.26866 & 0.47865 & 0.0101 & 2521.4 & 44 & 2530.7 & 30.2 \\
\hline 0.21642 & 0.37585 & 0.00782 & 2056.9 & 36.6 & 2345.1 & 29.2 \\
\hline 0.16376 & 0.27258 & 0.00562 & 1553.9 & 28.4 & 2104.2 & 28 \\
\hline 0.28164 & 0.48245 & 0.01022 & 2537.9 & 44.4 & 2567.8 & 30.4 \\
\hline 0.26566 & 0.46474 & 0.0097 & 2460.5 & 42.6 & 2530.4 & 29.8 \\
\hline 0.16896 & 0.36897 & 0.0078 & 2024.6 & 36.8 & 2092.7 & 29 \\
\hline 0.06626 & 0.14391 & 0.00396 & 866.7 & 22.4 & 891.7 & 33.4 \\
\hline 0.28624 & 0.4832 & 0.01062 & 2541.2 & 46.2 & 2537.2 & 31.6 \\
\hline 0.25544 & 0.44278 & 0.00926 & 2363.1 & 41.4 & 2490.6 & 29.8 \\
\hline 0.26702 & 0.41414 & 0.00862 & 2233.8 & 39.2 & 2536.6 & 29.8 \\
\hline 0.27124 & 0.47133 & 0.00994 & 2489.4 & 43.6 & 2534.7 & 30.2 \\
\hline 0.2564 & 0.46045 & 0.00964 & 2441.5 & 42.6 & 2490.3 & 29.8 \\
\hline 0.19862 & 0.38265 & 0.0079 & 2088.7 & 36.8 & 2271.2 & 28.8 \\
\hline 0.16428 & 0.36189 & 0.00788 & 1991.1 & 37.2 & 2021.3 & 30 \\
\hline 0.27274 & 0.45391 & 0.00996 & 2412.6 & 44.2 & 2489.9 & 31.6 \\
\hline 0.2406 & 0.43313 & 0.00932 & 2319.8 & 42 & 2393.5 & 30.6 \\
\hline 0.29952 & 0.4993 & 0.0109 & 2610.8 & 46.8 & 2583.8 & 31.6 \\
\hline 0.12424 & 0.26548 & 0.00546 & 1517.8 & 27.8 & 1857.3 & 27 \\
\hline 0.28632 & 0.45195 & 0.0102 & 2403.9 & 45.2 & 2503.4 & 32.6 \\
\hline 0.24368 & 0.42136 & 0.00912 & 2266.7 & 41.4 & 2398.7 & 30.8 \\
\hline 0.15318 & 0.28185 & 0.00592 & 1600.7 & 29.8 & 2010.6 & 28.6 \\
\hline 0.28142 & 0.46339 & 0.01002 & 2454.5 & 44.2 & 2534.1 & 31.2 \\
\hline 0.27098 & 0.4792 & 0.00998 & 2523.8 & 43.4 & 2540.8 & 30 \\
\hline 0.26146 & 0.43922 & 0.00928 & 2347.1 & 41.6 & 2490.5 & 30.2 \\
\hline 0.19562 & 0.37903 & 0.00794 & 2071.8 & 37.2 & 2232.3 & 29.2 \\
\hline 532.70624 & -2.30674 & 7.67178 & & & & \\
\hline 0.27214 & 0.46375 & 0.00988 & 2456.1 & 43.6 & 2515.6 & 30.6 \\
\hline 0.1071 & 0.18684 & 0.00396 & 1104.2 & 21.6 & 1693.6 & 27.2 \\
\hline 0.03558 & 0.11169 & 0.00236 & 682.6 & 13.6 & 881.3 & 19.8 \\
\hline 0.30574 & 0.50624 & 0.01104 & 2640.6 & 47.2 & 2596.5 & 31.6 \\
\hline 0.26666 & 0.45039 & 0.01002 & 2397 & 44.6 & 2442.5 & 32 \\
\hline 0.2903 & 0.47705 & 0.01016 & 2514.4 & 44.4 & 2574.9 & 30.8 \\
\hline 0.2389 & 0.42111 & 0.0087 & 2265.5 & 39.4 & 2426.1 & 29.4 \\
\hline 0.2949 & 0.4833 & 0.01036 & 2541.6 & 45 & 2580.4 & 31 \\
\hline 0.2975 & 0.48604 & 0.01046 & 2553.5 & 45.4 & 2583.6 & 31.2 \\
\hline 3.17458 & 0.7434 & 0.0261 & & & & \\
\hline 0.25574 & 0.40885 & 0.0086 & 2209.7 & 39.4 & 2467.5 & 30.2 \\
\hline 0.27898 & 0.47298 & 0.00996 & 2496.6 & 43.6 & 2544.9 & 30.4 \\
\hline 0.28784 & 0.47963 & 0.01 & 2525.7 & 43.6 & 2585.6 & 30.2 \\
\hline 0.17124 & 0.36555 & 0.00802 & 2008.4 & 37.8 & 2036.9 & 30.6 \\
\hline 0.28836 & 0.46703 & 0.01032 & 2470.5 & 45.4 & 2520.6 & 32.2 \\
\hline 0.03882 & 0.13843 & 0.0031 & 835.8 & 17.6 & 815.6 & 22 \\
\hline 0.03484 & 0.13185 & 0.00288 & 798.4 & 16.4 & 791.5 & 20.4 \\
\hline 0.19918 & 0.32862 & 0.00698 & 1831.7 & 33.8 & 2220.3 & 30 \\
\hline 0.02262 & 0.03881 & 0.00084 & 245.5 & 5.2 & 630.1 & 16.4 \\
\hline
\end{tabular}




\begin{tabular}{|c|c|c|c|c|c|c|}
\hline 1145.08448 & 28.42135 & 9.70214 & & & & \\
\hline 0.26242 & 0.43252 & 0.00914 & 2317.1 & 41.2 & 2477 & 30.6 \\
\hline 0.2937 & 0.48449 & 0.01034 & 2546.8 & 45 & 2570.9 & 31.2 \\
\hline 0.30884 & 0.4872 & 0.0108 & 2558.6 & 46.8 & 2575.7 & 32.4 \\
\hline 0.25796 & 0.44307 & 0.00964 & 2364.4 & 43 & 2424.5 & 31.4 \\
\hline 0.25954 & 0.45551 & 0.00948 & 2419.7 & 42 & 2480.8 & 30.2 \\
\hline 0.28604 & 0.48192 & 0.01018 & 2535.6 & 44.2 & 2555.4 & 30.8 \\
\hline 0.0327 & 0.11036 & 0.00238 & 674.8 & 13.8 & 786.7 & 19.6 \\
\hline 0.16678 & 0.26788 & 0.00562 & 1530 & 28.6 & 2069.1 & 29 \\
\hline 0.26108 & 0.43812 & 0.00924 & 2342.2 & 41.4 & 2468.9 & 30.6 \\
\hline 0.2762 & 0.44291 & 0.0095 & 2363.7 & 42.4 & 2502.5 & 31.4 \\
\hline 0.28404 & 0.4721 & 0.0102 & 2492.8 & 44.6 & 2519.8 & 31.6 \\
\hline 0.25222 & 0.42839 & 0.00906 & 2298.5 & 40.8 & 2432.4 & 30.6 \\
\hline 0.22896 & 0.40708 & 0.00852 & 2201.6 & 39 & 2353.1 & 30 \\
\hline 0.28798 & 0.46675 & 0.00992 & 2469.3 & 43.6 & 2550.2 & 31 \\
\hline 0.03074 & 0.0643 & 0.0014 & 401.7 & 8.4 & 769 & 19.2 \\
\hline 0.31086 & 0.4765 & 0.01026 & 2512 & 44.8 & 2605.7 & 31.8 \\
\hline 0.28398 & 0.46695 & 0.00994 & 2470.2 & 43.6 & 2532.4 & 31.2 \\
\hline 0.26782 & 0.44569 & 0.00948 & 2376.1 & 42.2 & 2476.4 & 31 \\
\hline 0.27218 & 0.44869 & 0.00954 & 2389.4 & 42.4 & 2492.1 & 31 \\
\hline 0.27274 & 0.44518 & 0.00944 & 2373.8 & 42.2 & 2495.4 & 31 \\
\hline 0.26896 & 0.44155 & 0.00958 & 2357.6 & 42.8 & 2457.7 & 31.6 \\
\hline 0.30642 & 0.47152 & 0.01018 & 2490.2 & 44.6 & 2585.1 & 31.8 \\
\hline 0.28764 & 0.47482 & 0.0101 & 2504.7 & 44.2 & 2539.6 & 31.2 \\
\hline 0.34852 & 0.525 & 0.01172 & 2720.4 & 49.6 & 2671.8 & 33.2 \\
\hline 0.36918 & 0.51485 & 0.01108 & 2677.3 & 47.2 & 2765.9 & 32.2 \\
\hline 0.29224 & 0.47018 & 0.01014 & 2484.4 & 44.4 & 2538.5 & 31.8 \\
\hline 0.06652 & 0.13276 & 0.00294 & 803.6 & 16.8 & 1231.9 & 26 \\
\hline 0.03722 & 0.09406 & 0.00204 & 579.5 & 12 & 863.4 & 20.8 \\
\hline 0.26338 & 0.43487 & 0.00924 & 2327.6 & 41.6 & 2455.4 & 31 \\
\hline 0.39838 & 0.51631 & 0.01144 & 2683.5 & 48.6 & 2806.8 & 33.2 \\
\hline 0.28048 & 0.44044 & 0.00962 & 2352.6 & 43 & 2484 & 32 \\
\hline 0.2733 & 0.4438 & 0.00958 & 2367.6 & 42.8 & 2470.5 & 31.8 \\
\hline 0.20444 & 0.30299 & 0.00666 & 1706.1 & 33 & 2184.7 & 31.6 \\
\hline 52.01994 & 4.15714 & 0.45004 & & & & \\
\hline 0.22666 & 0.36415 & 0.00772 & 2001.8 & 36.4 & 2315 & 30.8 \\
\hline 0.18324 & 0.04901 & 0.00136 & 308.4 & 8.4 & 2039.9 & 37.8 \\
\hline 0.33804 & 0.18302 & 0.00438 & 1083.5 & 23.8 & 2618.5 & 35.2 \\
\hline 0.31366 & 0.50502 & 0.01066 & 2635.3 & 45.6 & 2619.9 & 31.4 \\
\hline 0.3102 & 0.47859 & 0.01054 & 2521.1 & 46 & 2562.8 & 32.8 \\
\hline 0.429 & 0.51632 & 0.01106 & 2683.6 & 47 & 2903.4 & 32.4 \\
\hline 0.2064 & 0.34302 & 0.00746 & 1901.2 & 35.8 & 2197.6 & 31.2 \\
\hline 107.49616 & 2.25528 & 0.88524 & & & & \\
\hline 0.31202 & 0.47906 & 0.01056 & 2523.2 & 46 & 2565.3 & 32.8 \\
\hline 0.29612 & 0.44698 & 0.01016 & 2381.8 & 45.2 & 2483.1 & 33.6 \\
\hline 0.49502 & 0.5953 & 0.01304 & 3010.9 & 52.6 & 3013.5 & 33.4 \\
\hline 0.24526 & 0.39818 & 0.0086 & 2160.7 & 39.6 & 2359.6 & 31.6 \\
\hline 0.27492 & 0.45483 & 0.00982 & 2416.7 & 43.6 & 2463.8 & 31.8 \\
\hline 410.7407 & 9.99401 & 3.79616 & & & & \\
\hline 0.277 & 0.43858 & 0.0096 & 2344.3 & 43 & 2455.9 & 32.4 \\
\hline 0.26384 & 0.43882 & 0.00926 & 2345.4 & 41.4 & 2448.3 & 31.2 \\
\hline 0.29768 & 0.45788 & 0.01012 & 2430.2 & 44.8 & 2511.4 & 33 \\
\hline 0.18784 & 0.32248 & 0.00798 & 1801.8 & 38.8 & 1940.6 & 35.8 \\
\hline 0.34168 & 0.49842 & 0.01108 & 2607 & 47.6 & 2637.1 & 33.4 \\
\hline
\end{tabular}




\begin{tabular}{|c|c|c|c|c|c|}
\hline age $207 / 206$ & $2 \sigma$ age 76 & $\begin{array}{l}\text { discordance } \\
\Delta 68-75[\%]\end{array}$ & $\Delta 68-76[\%]$ & $\begin{array}{c}\text { preferred age } \\
\text { age }\end{array}$ & $2 \sigma$ age \\
\hline 2636.2 & 40 & -2.7 & -4.8 & 2636.2 & 40 \\
\hline 3398.6 & 38.4 & -70.7 & -90 & & \\
\hline 2557.6 & 40 & -2.2 & -4 & 2557.6 & 40 \\
\hline 2598.2 & 40 & -0.7 & -1.3 & 2598.2 & 40 \\
\hline 2538.9 & 41 & -0.4 & -0.7 & 2538.9 & 41 \\
\hline 2607 & 40.4 & -12.3 & -21.1 & & \\
\hline 2696.5 & 39.6 & -26.2 & -42.4 & & \\
\hline 2592.2 & 41.2 & -1.2 & -2.1 & 2592.2 & 41.2 \\
\hline 2587.6 & 40.4 & -2.8 & -4.9 & 2587.6 & 40.4 \\
\hline 2161 & 43.4 & -3.3 & -6.3 & 2161 & 43.4 \\
\hline 955.1 & 100.4 & -2.8 & -9.3 & 866.7 & 22.4 \\
\hline 2534.7 & 43.2 & 0.2 & 0.3 & 2534.7 & 43.2 \\
\hline 2597 & 40.6 & -5.1 & -9 & 2597 & 40.6 \\
\hline 2789.2 & 39.6 & -11.9 & -19.9 & & \\
\hline 2571.8 & 41.2 & -1.8 & -3.2 & 2571.8 & 41.2 \\
\hline 2531 & 41 & -2 & -3.5 & 2531 & 41 \\
\hline 2440.6 & 40.8 & -8 & -14.4 & 2440.6 & 40.8 \\
\hline 2052.8 & 46.6 & -1.5 & -3 & 2052.8 & 46.6 \\
\hline 2554.3 & 43.4 & -3.1 & -5.5 & 2554.3 & 43.4 \\
\hline 2457.6 & 43 & -3.1 & -5.6 & 2457.6 & 43 \\
\hline 2563.4 & 43 & 1 & 1.8 & 2563.4 & 43 \\
\hline 2262.5 & 41.6 & -18.3 & -32.9 & & \\
\hline 2585.7 & 44.6 & -4 & -7 & 2585.7 & 44.6 \\
\hline 2513.5 & 43 & -5.5 & -9.8 & 2513.5 & 43 \\
\hline 2463.2 & 42 & -20.4 & -35 & & \\
\hline 2599.1 & 42.4 & -3.1 & -5.6 & 2599.1 & 42.4 \\
\hline 2555 & 40.8 & -0.7 & -1.2 & 2555 & 40.8 \\
\hline 2610.2 & 41.4 & -5.8 & -10.1 & 2610.2 & 41.4 \\
\hline 2383.6 & 42.2 & -7.2 & -13.1 & 2383.6 & 42.2 \\
\hline 2564.5 & 42.2 & -2.4 & -4.2 & 2564.5 & 42.2 \\
\hline 2528 & 42.6 & -34.8 & -56.3 & & \\
\hline 1420.9 & 50.2 & -22.6 & -52 & & \\
\hline 2562.9 & 43.2 & 1.7 & 3 & 2562.9 & 43.2 \\
\hline 2481.3 & 44.8 & -1.9 & -3.4 & 2481.3 & 44.8 \\
\hline 2623.5 & 42 & -2.3 & -4.2 & 2623.5 & 42 \\
\hline 2564.2 & 40.8 & -6.6 & -11.6 & 2564.2 & 40.8 \\
\hline 2611.6 & 42.2 & -1.5 & -2.7 & 2611.6 & 42.2 \\
\hline 2607.9 & 42.6 & -1.2 & -2.1 & 2607.9 & 42.6 \\
\hline 2688 & 41.4 & -10.5 & -17.8 & & \\
\hline 2584.2 & 41.8 & -1.9 & -3.4 & 2584.2 & 41.8 \\
\hline 2633.6 & 41 & -2.3 & -4.1 & 2633.6 & 41 \\
\hline 2066.4 & 47.4 & -1.4 & -2.8 & 2066.4 & 47.4 \\
\hline 2561.8 & 44.4 & -2 & -3.6 & 2561.8 & 44.4 \\
\hline 761.7 & 67.8 & 2.5 & 9.7 & 835.8 & 17.6 \\
\hline 772.7 & 63.6 & 0.9 & 3.3 & 798.4 & 16.4 \\
\hline 2601.6 & 42.6 & -17.5 & -29.6 & & \\
\hline 2464 & 46 & -61 & -90 & & \\
\hline
\end{tabular}




\begin{tabular}{|c|c|c|c|c|c|}
\hline 2611.6 & 42.2 & -6.5 & -11.3 & 2611.6 & 42.2 \\
\hline 2590.5 & 42.8 & -0.9 & -1.7 & 2590.5 & 42.8 \\
\hline 2589.8 & 44.4 & -0.7 & -1.2 & 2589.8 & 44.4 \\
\hline 2476 & 44.4 & -2.5 & -4.5 & 2476 & 44.4 \\
\hline 2531.8 & 41.8 & -2.5 & -4.4 & 2531.8 & 41.8 \\
\hline 2571.8 & 42.6 & -0.8 & -1.4 & 2571.8 & 42.6 \\
\hline 1119.1 & 57 & -14.2 & -39.7 & 674.8 & 13.8 \\
\hline 2659.8 & 42 & -26.1 & -42.5 & & \\
\hline 2575.5 & 42.6 & -5.1 & -9.1 & 2575.5 & 42.6 \\
\hline 2617.8 & 43.2 & -5.5 & -9.7 & 2617.8 & 43.2 \\
\hline 2542.2 & 43.8 & -1.1 & -1.9 & 2542.2 & 43.8 \\
\hline 2547 & 42.8 & -5.5 & -9.8 & 2547 & 42.8 \\
\hline 2487.8 & 42.6 & -6.4 & -11.5 & 2487.8 & 42.6 \\
\hline 2615.8 & 42.8 & -3.2 & -5.6 & 2615.8 & 42.8 \\
\hline 2067.9 & 49.4 & -47.8 & -80.6 & & \\
\hline 2680 & 43.2 & -3.6 & -6.3 & 2680 & 43.2 \\
\hline 2583.2 & 43.2 & -2.5 & -4.4 & 2583.2 & 43.2 \\
\hline 2560.4 & 43.2 & -4.1 & -7.2 & 2560.4 & 43.2 \\
\hline 2577.5 & 43.2 & -4.1 & -7.3 & 2577.5 & 43.2 \\
\hline 2596.5 & 43.2 & -4.9 & -8.6 & 2596.5 & 43.2 \\
\hline 2542.1 & 44.4 & -4.1 & -7.3 & 2542.1 & 44.4 \\
\hline 2661 & 43.6 & -3.7 & -6.4 & 2661 & 43.6 \\
\hline 2568.2 & 43.4 & -1.4 & -2.5 & 2568.2 & 43.4 \\
\hline 2635.8 & 45.2 & 1.8 & 3.2 & 2635.8 & 45.2 \\
\hline 2831.8 & 42.6 & -3.2 & -5.5 & 2831.8 & 42.6 \\
\hline 2582.6 & 44.2 & -2.1 & -3.8 & 2582.6 & 44.2 \\
\hline 2087.2 & 51 & -34.8 & -61.5 & & \\
\hline 1686 & 52.4 & -32.9 & -65.6 & & \\
\hline 2563.5 & 43.6 & -5.2 & -9.2 & 2563.5 & 43.6 \\
\hline 2897.1 & 43.8 & -4.4 & -7.4 & 2897.1 & 43.8 \\
\hline 2593.8 & 44.8 & -5.3 & -9.3 & 2593.8 & 44.8 \\
\hline 2556.9 & 44.6 & -4.2 & -7.4 & 2556.9 & 44.6 \\
\hline 2670.6 & 45.4 & -21.9 & -36.1 & & \\
\hline 2604.7 & 43.6 & -13.5 & -23.1 & & \\
\hline 5173.5 & 46.2 & -84.9 & -94 & & \\
\hline 4189.7 & 42.8 & -58.6 & -74.1 & & \\
\hline 2608.5 & 43.4 & 0.6 & 1 & 2608.5 & 43.4 \\
\hline 2596.5 & 45.4 & -1.6 & -2.9 & 2596.5 & 45.4 \\
\hline 3060.1 & 42 & -7.6 & -12.3 & 3060.1 & 42 \\
\hline 2487.5 & 45.6 & -13.5 & -23.6 & & \\
\hline 2599.4 & 45.6 & -1.6 & -2.9 & 2599.4 & 45.6 \\
\hline 2567.6 & 47.4 & -4.1 & -7.2 & 2567.6 & 47.4 \\
\hline 3015.9 & 43.2 & -0.1 & -0.2 & 3015.9 & 43.2 \\
\hline 2536.8 & 45.2 & -8.4 & -14.8 & 2536.8 & 45.2 \\
\hline 2503.5 & 45.2 & -1.9 & -3.5 & 2503.5 & 45.2 \\
\hline 2550.2 & 45.8 & -4.5 & -8.1 & 2550.2 & 45.8 \\
\hline 2535.5 & 44.2 & -4.2 & -7.5 & 2535.5 & 44.2 \\
\hline 2578.4 & 46.2 & -3.2 & -5.7 & 2578.4 & 46.2 \\
\hline 2092.7 & 58.4 & -7.1 & -13.9 & 2092.7 & 58.4 \\
\hline 2660.8 & 45.8 & -1.1 & -2 & 2660.8 & 45. \\
\hline
\end{tabular}


Sample 3690B Albert Nile @ Wadelai 150 grain analysed 113 concordant a

\begin{tabular}{|c|c|c|c|c|c|c|}
\hline \multirow[b]{2}{*}{ grain } & \multicolumn{3}{|c|}{ concentrations } & \multicolumn{3}{|l|}{ isotopic ratios } \\
\hline & U [ppm] & $\mathrm{Pb}$ [ppm] & $T h / U$ & Pb207/Pb206 & $2 \sigma 76$ & Pb207/U235 \\
\hline X3690F_G001 & 329.3 & 163.1 & 0.6519 & 0.1654 & 0.00396 & 9.77639 \\
\hline X3690F_G002 & & & & 0.90308 & 0.05218 & 941.45526 \\
\hline X3690F_G003 & 73.5 & 32.7 & 0.5438 & 0.16802 & 0.00464 & 9.23257 \\
\hline X3690F_G004 & 297.2 & 171.4 & 0.2896 & 0.1769 & 0.00422 & 12.59087 \\
\hline X3690F_G005 & 907.5 & 283.1 & 0.118 & 0.1568 & 0.00376 & 6.96666 \\
\hline X3690F_G006 & 764.7 & 228.4 & 0.3295 & 0.14927 & 0.00358 & 6.01664 \\
\hline X3690F_G007 & 3393.6 & 298.8 & 0.0028 & 0.05802 & 0.0014 & 0.7663 \\
\hline X3690F_G008 & & & & 0.08144 & 1.18054 & 24.27058 \\
\hline X3690F_G009 & 517.1 & 199.9 & 0.0909 & 0.16509 & 0.00396 & 8.95127 \\
\hline X3690F_G010 & 643 & 389.7 & 0.7379 & 0.1725 & 0.00416 & 11.77868 \\
\hline X3690F- G011 & 246.2 & 128 & 0.9155 & 0.16449 & 0.00404 & 9.37308 \\
\hline X3690F_G012 & 1053.6 & 522 & 0.7037 & 0.17162 & 0.00404 & 10.44863 \\
\hline X3690F_G013 & 668.7 & 348.3 & 0.2463 & 0.17206 & 0.00408 & 11.46764 \\
\hline X3690F_G014 & 48.2 & 33.2 & 1.0793 & 0.17079 & 0.00474 & 12.05511 \\
\hline X3690F_G015 & 234.2 & 125.9 & 0.6887 & 0.17006 & 0.00412 & 10.67729 \\
\hline X3690F_G016 & 529.5 & 249.1 & 0.1086 & 0.16897 & 0.00402 & 10.69648 \\
\hline X3690F_G017 & 560.8 & 226.2 & 0.7535 & 0.16301 & 0.00388 & 8.07454 \\
\hline X3690F_G018 & & & & 0.06047 & 1.04164 & 6.11681 \\
\hline X3690F_G019 & 1022.8 & 440.8 & 0.1046 & 0.16756 & 0.00394 & 9.98442 \\
\hline X3690F_G020 & 1566.1 & 288.9 & 0.106 & 0.13179 & 0.00314 & 3.52661 \\
\hline X3690F_G021 & 20.7 & 11.1 & 0.1832 & 0.16641 & 0.00562 & 11.40369 \\
\hline X3690F_G022 & & & & 0.72655 & 0.68734 & \\
\hline X3690F_G023 & 433.6 & 39.3 & 0.0132 & 0.06081 & 0.00178 & 0.82308 \\
\hline X3690F_G024 & 406 & 281.1 & 0.7392 & 0.21854 & 0.0052 & 17.08258 \\
\hline X3690F_G025 & 293.9 & 126.9 & 0.5703 & 0.16337 & 0.00404 & 8.75516 \\
\hline X3690F_G026 & & & & -0.20343 & 2.09734 & \\
\hline X3690F_G027 & 147 & 77.3 & 0.4627 & 0.16914 & 0.0043 & 10.91474 \\
\hline X3690F_G028 & 243.9 & 138 & 1.2289 & 0.18553 & 0.00456 & 11.24362 \\
\hline X3690F_G029 & & & & 0.68084 & 0.02552 & 34.14083 \\
\hline X3690F_G030 & 43.6 & 24.7 & 0.5217 & 0.17161 & 0.00516 & 11.52783 \\
\hline X3690F_G031 & 0.9 & 0.1 & 0.1412 & 0.30378 & 0.08498 & 5.1933 \\
\hline X3690F_G032 & & & & 0.616 & 0.29742 & 110.25622 \\
\hline X3690F_G033 & 199.8 & 108.1 & 0.6516 & 0.1701 & 0.00418 & 10.80805 \\
\hline X3690F_G034 & 158.5 & 75.8 & 0.336 & 0.17296 & 0.0043 & 10.56262 \\
\hline X3690F_G035 & 190.1 & 122.3 & 0.581 & 0.17444 & 0.00436 & 12.7307 \\
\hline X3690F_G036 & & & & 0.83676 & 0.03698 & 115.20857 \\
\hline X3690F G037 & 613.6 & 94.9 & 0.8617 & 0.06586 & 0.00174 & 1.17749 \\
\hline X3690F_G038 & 81.8 & 44.2 & 0.2641 & 0.16901 & 0.00452 & 11.51715 \\
\hline X3690F_G039 & 27.1 & 16 & 2.7126 & 0.15094 & 0.0063 & 7.24448 \\
\hline X3690F_G040 & 401.4 & 202.4 & 0.3369 & 0.16822 & 0.00418 & 10.76477 \\
\hline X3690F_G041 & 796.4 & 397.3 & 0.9934 & 0.17511 & 0.00424 & 10.26584 \\
\hline X3690F_G042 & 198.9 & 103.5 & 0.6065 & 0.16962 & 0.0043 & 10.57749 \\
\hline X3690F_G043 & 405.5 & 211.4 & 0.574 & 0.16784 & 0.00414 & 10.50633 \\
\hline X3690F_G044 & 175 & 74.3 & 1.3278 & 0.15346 & 0.00412 & 6.90157 \\
\hline X3690F_G045 & 650.3 & 360 & 0.719 & 0.1723 & 0.00418 & 11.13128 \\
\hline X3690F_G046 & 644.8 & 325.8 & 0.2166 & 0.16927 & 0.0041 & 11.08611 \\
\hline X3690F_G047 & 1104.6 & 473.1 & 0.1657 & 0.16388 & 0.00406 & 9.5282 \\
\hline X3690F_G048 & & & & 0.50992 & 0.59478 & \\
\hline X3690F_G049 & 416.6 & 239.4 & 0.4427 & 0.17207 & 0.00422 & 11.85949 \\
\hline X3690F_G050 & 634.3 & 295.2 & 0.7202 & 0.17175 & 0.0042 & 9.68344 \\
\hline
\end{tabular}




\begin{tabular}{|c|c|c|c|c|c|c|}
\hline X3690F_G051 & 390.4 & 232 & 0.6818 & 0.17346 & 0.00434 & 11.80342 \\
\hline X3690F_G052 & 193.4 & 98.6 & 0.6377 & 0.16866 & 0.00428 & 10.29629 \\
\hline X3690F_G053 & 1035.2 & 433.6 & 0.4965 & 0.16721 & 0.00406 & 9.35614 \\
\hline X3690F_G054 & 384 & 174.2 & 0.2021 & 0.17244 & 0.00434 & 10.53307 \\
\hline X3690F_G055 & 296.2 & 183.1 & 0.8753 & 0.17674 & 0.00442 & 12.06794 \\
\hline X3690F_G056 & 173.6 & 111.1 & 0.9968 & 0.17264 & 0.00436 & 11.84738 \\
\hline X3690F_G057 & 146.5 & 80.8 & 0.7585 & 0.16906 & 0.00432 & 10.77331 \\
\hline X3690F_G058 & 45.5 & 5.2 & 0.3823 & 0.06846 & 0.0037 & 1.01112 \\
\hline X3690F_G059 & 112.5 & 16.9 & 0.2114 & 0.07789 & 0.0026 & 1.62415 \\
\hline X3690F_G060 & 146.5 & 20.5 & 0.0614 & 0.07271 & 0.0023 & 1.47718 \\
\hline X3690F_G061 & 76.7 & 45.3 & 1.2839 & 0.16615 & 0.0045 & 10.10964 \\
\hline X3690F_G062 & 120.3 & 61.1 & 0.6758 & 0.1601 & 0.00424 & 9.62046 \\
\hline X3690F_G063 & & & & 0.95817 & 0.06772 & \\
\hline X3690F_G064 & 733.9 & 312.7 & 0.3059 & 0.16758 & 0.00426 & 9.55491 \\
\hline X3690F_G065 & 186.9 & 86.4 & 1.0661 & 0.16765 & 0.00436 & 8.87168 \\
\hline X3690F_G066 & 57.9 & 39.9 & 1.0097 & 0.17021 & 0.00512 & 12.27652 \\
\hline X3690F_G067 & 67.1 & 45.1 & 0.8484 & 0.16815 & 0.00468 & 12.13651 \\
\hline X3690F_G068 & 103.8 & 31 & 0.673 & 0.11944 & 0.00402 & 4.23656 \\
\hline X3690F G069 & 296.2 & 172 & 0.4575 & 0.21846 & 0.0055 & 15.8957 \\
\hline X3690F_G070 & 220 & 132.6 & 0.8661 & 0.16679 & 0.00432 & 11.11731 \\
\hline X3690F_G071 & 260.9 & 146.3 & 0.8244 & 0.16923 & 0.00438 & 10.78661 \\
\hline X3690F_G072 & 768.8 & 453.6 & 1.1142 & 0.18373 & 0.00456 & 11.95167 \\
\hline X3690F_G073 & 140.5 & 74.1 & 0.8002 & 0.17121 & 0.00454 & 10.44214 \\
\hline \multicolumn{7}{|l|}{ X3690F G074 } \\
\hline X3690F_G075 & 253.1 & 119.1 & 0.1797 & 0.1657 & 0.00428 & 10.32546 \\
\hline X3690F_G076 & 352.3 & 152.7 & 0.3188 & 0.16411 & 0.00458 & 9.27133 \\
\hline X3690F_G077 & 243.4 & 79.9 & 0.0011 & 0.14413 & 0.0039 & 6.8463 \\
\hline X3690F_G078 & 8.7 & & 2.5298 & 0.69282 & 0.11326 & 9.86505 \\
\hline X3690F_G079 & 124 & 66.9 & 0.5123 & 0.16929 & 0.00472 & 11.06805 \\
\hline X3690F_G080 & 291.2 & 128.8 & 0.3195 & 0.16333 & 0.00422 & 9.40702 \\
\hline X3690F_G081 & 262.7 & 119.6 & 0.3049 & 0.16192 & 0.00426 & 9.5687 \\
\hline X3690F_G082 & 632.9 & 329.7 & 0.1659 & 0.16524 & 0.00418 & 11.14912 \\
\hline X3690F_G083 & 185.5 & 93.5 & 0.263 & 0.16481 & 0.0043 & 10.6713 \\
\hline X3690F_G084 & 305.4 & 137.6 & 0.3638 & 0.16975 & 0.0044 & 10.01158 \\
\hline X3690F_G085 & & & & 0.97557 & 0.03546 & \\
\hline X3690F_G086 & 69.4 & 37.9 & 0.7658 & 0.16928 & 0.00512 & 10.70534 \\
\hline X3690F_G087 & 275.6 & 141 & 0.787 & 0.17256 & 0.00448 & 10.31722 \\
\hline X3690F_G088 & 261.3 & 53.1 & 0.1079 & 0.11629 & 0.00328 & 3.4086 \\
\hline X3690F_G089 & 977.8 & 420.1 & 0.0253 & 0.15978 & 0.00404 & 9.53313 \\
\hline X3690F_G090 & 13.8 & 8.1 & 0.5616 & 0.17202 & 0.00678 & 11.80129 \\
\hline X3690F_G091 & 462.5 & 174.2 & 0.1762 & 0.15372 & 0.00398 & 7.90303 \\
\hline X3690F_G092 & 6 & 3.1 & 0.1874 & 0.17724 & 0.0087 & 11.88231 \\
\hline X3690F_G093 & 158 & 87.1 & 0.8848 & 0.17144 & 0.0046 & 10.70519 \\
\hline X3690F_G094 & 469.4 & 231.1 & 0.5601 & 0.16298 & 0.0042 & 9.75954 \\
\hline X3690F_G095 & 1189.5 & 140.2 & 0.8034 & 0.0709 & 0.00192 & 1.06212 \\
\hline X3690F_G096 & 1017.3 & 433.3 & 0.6024 & 0.17017 & 0.00438 & 9.04196 \\
\hline X3690F_G097 & 468 & 224 & 0.4115 & 0.16692 & 0.00434 & 10.10948 \\
\hline X3690F_G098 & 134.1 & 73.2 & 1.1863 & 0.15923 & 0.00436 & 9.23384 \\
\hline X3690F_G099 & 218.2 & 104.9 & 0.4388 & 0.16655 & 0.00444 & 10.06478 \\
\hline X3690F_G100 & 64.8 & 32.6 & 0.5957 & 0.15805 & 0.00464 & 9.53438 \\
\hline X3690F_G101 & 57.4 & 23.7 & 0.3144 & 0.15946 & 0.00478 & 8.65057 \\
\hline X3690F_G102 & 351.8 & 165.4 & 0.8393 & 0.16507 & 0.00436 & 9.87783 \\
\hline X3690F_G103 & 232.4 & 145.6 & 0.6181 & 0.17954 & 0.00478 & 12.95515 \\
\hline X3690F_G104 & 392.7 & 200 & 1.0292 & 0.17324 & 0.0046 & 10.11684 \\
\hline X3690F_G105 & 320.6 & 185.8 & 0.8413 & 0.16979 & 0.00452 & 11.11952 \\
\hline
\end{tabular}




\begin{tabular}{|c|c|c|c|c|c|c|}
\hline X3690F_G106 & 287.5 & 161.6 & 1.1077 & 0.16089 & 0.00434 & 9.88271 \\
\hline X3690F_G107 & 95.1 & 61.5 & 1.0905 & 0.16858 & 0.00498 & 11.51352 \\
\hline X3690F_G108 & 71.6 & 47 & 0.899 & 0.17456 & 0.00518 & 12.37843 \\
\hline X3690F_G109 & 744 & 73.5 & 0.0917 & 0.06643 & 0.00198 & 0.95842 \\
\hline X3690F_G110 & 695.8 & 72.1 & 0.0137 & 0.0595 & 0.0018 & 0.91988 \\
\hline X3690F_G111 & 424.8 & 662 & 5.1404 & 0.16712 & 0.00472 & 7.56596 \\
\hline X3690F_G112 & & & & -18.06665 & 782.08074 & \\
\hline X3690F G113 & 305 & 170.7 & 0.4225 & 0.19291 & 0.00518 & 13.37921 \\
\hline X3690F_G114 & 446.9 & 223.6 & 0.4489 & 0.16535 & 0.00446 & 10.2918 \\
\hline X3690F_G115 & 335.7 & 162.5 & 0.7658 & 0.16546 & 0.0045 & 9.62866 \\
\hline X3690F_G116 & 10.1 & 5.3 & 0.27 & 0.1705 & 0.00858 & 11.35037 \\
\hline X3690F_G117 & 226 & 120.8 & 0.324 & 0.16778 & 0.00464 & 11.22025 \\
\hline X3690F_G118 & 298.5 & 162.7 & 0.434 & 0.16893 & 0.00458 & 11.27816 \\
\hline X3690F_G119 & 2075.9 & 919.6 & 0.1222 & 0.16922 & 0.0045 & 10.21856 \\
\hline X3690F_G120 & 343.1 & 179.5 & 0.7385 & 0.16743 & 0.00456 & 10.30565 \\
\hline X3690F_G121 & 1036.1 & 288.1 & 0.5704 & 0.15628 & 0.00422 & 5.92759 \\
\hline X3690F_G122 & 659.5 & 323.4 & 0.3968 & 0.17596 & 0.00476 & 11.01242 \\
\hline X3690F_G123 & 74.4 & 38.2 & 0.2808 & 0.16784 & 0.005 & 11.00051 \\
\hline X3690F_G124 & 8.7 & 0.8 & 0.0613 & 0.06605 & 0.01072 & 0.94058 \\
\hline X3690F_G125 & 792.3 & 303.9 & 0.137 & 0.16303 & 0.00442 & 8.67826 \\
\hline X3690F_G126 & 248.9 & 135.7 & 0.7955 & 0.16769 & 0.00466 & 10.54518 \\
\hline X3690F_G127 & 1740.7 & 264.1 & 0.1994 & 0.21461 & 0.00596 & 4.87356 \\
\hline X3690F_G128 & 461.6 & 222.2 & 0.4238 & 0.1678 & 0.00462 & 10.18399 \\
\hline X3690F_G129 & 197 & 132.5 & 1.5497 & 0.16621 & 0.00472 & 10.97666 \\
\hline X3690F_G130 & 432.2 & 233.7 & 0.8499 & 0.17001 & 0.0047 & 10.60459 \\
\hline X3690F_G131 & 639.8 & 354.3 & 0.7794 & 0.16051 & 0.00442 & 10.20945 \\
\hline X3690F_G132 & 389.9 & 214.3 & 0.3915 & 0.16993 & 0.00476 & 11.49321 \\
\hline X3690F_G133 & 49.1 & & 2.0975 & 0.13572 & 0.00504 & 5.60676 \\
\hline X3690F_G134 & 1610.2 & 240.5 & 0.0345 & 0.06963 & 0.00198 & 1.52842 \\
\hline X3690F_G135 & 113.4 & 52.4 & 0.522 & 0.1672 & 0.00534 & 9.66059 \\
\hline X3690F_G136 & 387.2 & 264.1 & 1.431 & 0.17179 & 0.0048 & 11.73243 \\
\hline X3690F_G137 & 124.9 & 78.7 & 0.951 & 0.17216 & 0.00516 & 11.82594 \\
\hline X3690F_G138 & 408.8 & 133.5 & 0.7821 & 0.15862 & 0.00454 & 6.50117 \\
\hline X3690F_G139 & 278.8 & 177.8 & 0.8274 & 0.17338 & 0.0049 & 12.22433 \\
\hline X3690F_G140 & 1145.4 & 436 & 0.3175 & 0.17135 & 0.00478 & 8.88452 \\
\hline X3690F_G141 & 653.1 & 281.9 & 0.3358 & 0.17261 & 0.00484 & 9.83314 \\
\hline X3690F_G142 & 66.1 & 43.2 & 1.1696 & 0.16744 & 0.00514 & 11.39245 \\
\hline X3690F_G143 & 67.1 & 47.5 & 2.2332 & 0.16596 & 0.00516 & 10.12685 \\
\hline X3690F_G144 & 450.6 & 261.7 & 0.7975 & 0.16159 & 0.00456 & 10.61481 \\
\hline X3690F_G145 & 154.3 & 27.9 & 0.9907 & 0.07168 & 0.00268 & 1.50098 \\
\hline X3690F_G146 & 231.9 & 128.6 & 0.4692 & 0.17178 & 0.00496 & 11.59523 \\
\hline X3690F_G147 & 314.6 & 155.5 & 0.0623 & 0.16779 & 0.0048 & 11.1317 \\
\hline X3690F_G148 & 314.6 & 150.9 & 0.7557 & 0.16606 & 0.00478 & 9.44588 \\
\hline X3690F_G149 & 446 & 124.3 & 0.2949 & 0.15005 & 0.00434 & 5.73109 \\
\hline X3690F G150 & 179.6 & 96.3 & 0.508 & 0.17003 & 0.00496 & 11.10114 \\
\hline
\end{tabular}




\section{ges $\quad 75.3 \%$ concordant}

\begin{tabular}{|c|c|c|c|c|c|c|}
\hline $2 \sigma 75$ & $\mathrm{~Pb} 206 / \mathrm{U} 238$ & $2 \sigma 68$ & $\begin{array}{c}\text { ages } \\
\text { age } 206 / 238\end{array}$ & $2 \sigma$ age 68 & age $207 / 235$ & $2 \sigma$ age 75 \\
\hline 0.2434 & 0.42887 & 0.00966 & 2300.6 & 43.6 & 2413.9 & 30.2 \\
\hline 177.0274 & 7.56415 & 1.4417 & & & & \\
\hline 0.2618 & 0.39871 & 0.00986 & 2163.1 & 45.4 & 2361.4 & 34 \\
\hline 0.31404 & 0.51643 & 0.01166 & 2684 & 49.6 & 2649.5 & 30.8 \\
\hline 0.1734 & 0.32239 & 0.00724 & 1801.4 & 35.2 & 2107.2 & 29.2 \\
\hline 0.15028 & 0.29246 & 0.00658 & 1653.8 & 32.8 & 1978.3 & 28.6 \\
\hline 0.01912 & 0.09582 & 0.00212 & 589.9 & 12.4 & 577.6 & 14.4 \\
\hline 354.49484 & 2.16238 & 4.73778 & & & & \\
\hline 0.22344 & 0.39341 & 0.00886 & 2138.6 & 41 & 2333 & 30 \\
\hline 0.29584 & 0.49544 & 0.01124 & 2594.2 & 48.4 & 2587 & 31 \\
\hline 0.2396 & 0.41346 & 0.00948 & 2230.7 & 43.2 & 2375.2 & 30.8 \\
\hline 0.25632 & 0.44174 & 0.00984 & 2358.4 & 44 & 2475.4 & 30 \\
\hline 0.2832 & 0.4836 & 0.01082 & 2542.9 & 47 & 2562 & 30.4 \\
\hline 0.34762 & 0.51214 & 0.01292 & 2665.8 & 55 & 2608.7 & 35.2 \\
\hline 0.26946 & 0.45556 & 0.01036 & 2419.9 & 45.8 & 2495.5 & 30.8 \\
\hline 0.26496 & 0.45932 & 0.0103 & 2436.6 & 45.4 & 2497.1 & 30.4 \\
\hline 0.1999 & 0.35941 & 0.00804 & 1979.4 & 38.2 & 2239.4 & 29.6 \\
\hline 104.9258 & 0.7339 & 1.42552 & & & & \\
\hline 0.2452 & 0.43235 & 0.00962 & 2316.3 & 43.2 & 2433.3 & 29.8 \\
\hline 0.08746 & 0.19416 & 0.00434 & 1143.9 & 23.4 & 1533.2 & 25.8 \\
\hline 0.3974 & $\begin{array}{c}0.49723 \\
-12.71118\end{array}$ & $\begin{array}{c}0.01456 \\
71.70052\end{array}$ & 2601.9 & 62.6 & 2556.7 & 41.8 \\
\hline 0.02434 & 0.0982 & 0.00228 & 603.9 & 13.4 & 609.8 & 17.2 \\
\hline 0.42524 & 0.56717 & 0.01278 & 2896.2 & 52.6 & 2939.5 & 31.4 \\
\hline 0.22434 & 0.38886 & 0.0089 & 2117.5 & 41.4 & 2312.8 & 30.8 \\
\hline 0.2883 & 0.46822 & 0.01098 & 2475.8 & 48.2 & 2515.9 & 32.2 \\
\hline 0.28806 & 0.43973 & 0.0101 & 2349.4 & 45.2 & 2543.5 & 31.4 \\
\hline 1.2601 & 0.36384 & 0.01394 & & & & \\
\hline 0.35904 & 0.48739 & 0.01302 & 2559.4 & 56.4 & 2566.8 & 37.6 \\
\hline 1.22524 & 0.12404 & 0.02316 & 753.8 & 132.8 & 1851.5 & 286.4 \\
\hline 66.74922 & 1.2987 & 0.67126 & & & & \\
\hline 0.27662 & 0.46103 & 0.01054 & 2444.1 & 46.6 & 2506.8 & 31.2 \\
\hline 0.27348 & 0.44312 & 0.01022 & 2364.6 & 45.6 & 2485.4 & 31.4 \\
\hline 0.33096 & 0.52954 & 0.01226 & 2739.5 & 51.6 & 2659.9 & 32 \\
\hline 6.53098 & 0.99901 & 0.0598 & & & & \\
\hline 0.03188 & 0.12973 & 0.00294 & 786.3 & 16.8 & 790.1 & 19.2 \\
\hline 0.32024 & 0.49446 & 0.01204 & 2589.9 & 52 & 2566 & 33.8 \\
\hline 0.30128 & 0.34824 & 0.01134 & 1926.2 & 54.2 & 2142 & 47.2 \\
\hline 0.27834 & 0.46431 & 0.01068 & 2458.6 & 47 & 2503 & 31.4 \\
\hline 0.25832 & 0.42537 & 0.00958 & 2284.8 & 43.4 & 2459 & 30.6 \\
\hline 0.2787 & 0.45246 & 0.01054 & 2406.2 & 46.8 & 2486.7 & 32 \\
\hline 0.26982 & 0.45419 & 0.01038 & 2413.9 & 46 & 2480.5 & 31.2 \\
\hline 0.18992 & 0.32633 & 0.00778 & 1820.6 & 37.8 & 2098.9 & 31.8 \\
\hline 0.28066 & 0.46874 & 0.01056 & 2478 & 46.4 & 2534.2 & 30.8 \\
\hline 0.2797 & 0.4752 & 0.0107 & 2506.3 & 46.8 & 2530.4 & 30.8 \\
\hline 0.24528 & 0.42186 & 0.00962 & 2268.9 & 43.6 & 2390.3 & 31 \\
\hline 0.30302 & 0.50008 & 0.01136 & 2614.1 & 48.8 & 2593.4 & 31.4 \\
\hline 0.246 & 0.40909 & 0.00924 & 2210.8 & 42.2 & 2405.1 & 30.6 \\
\hline
\end{tabular}




\begin{tabular}{|c|c|c|c|c|c|c|}
\hline 0.30746 & 0.49373 & 0.01136 & 2586.8 & 49 & 2588.9 & 31.8 \\
\hline 0.27068 & 0.44295 & 0.01024 & 2363.8 & 45.8 & 2461.8 & 31.8 \\
\hline 0.2362 & 0.40599 & 0.0091 & 2196.6 & 41.8 & 2373.5 & 30.4 \\
\hline 0.27546 & 0.44321 & 0.0102 & 2365 & 45.6 & 2482.8 & 31.6 \\
\hline 0.31374 & 0.49543 & 0.01136 & 2594.1 & 49 & 2609.7 & 31.8 \\
\hline 0.31144 & 0.49793 & 0.01152 & 2604.9 & 49.6 & 2592.4 & 32 \\
\hline 0.28622 & 0.46236 & 0.01076 & 2450 & 47.4 & 2503.8 & 32.2 \\
\hline 0.05282 & 0.10716 & 0.00318 & 656.2 & 18.6 & 709.4 & 31.4 \\
\hline 0.054 & 0.1513 & 0.00372 & 908.2 & 20.8 & 979.6 & 26 \\
\hline 0.04702 & 0.14741 & 0.00356 & 886.4 & 20 & 921.1 & 24.2 \\
\hline 0.28238 & 0.44149 & 0.01064 & 2357.3 & 47.6 & 2444.9 & 33.4 \\
\hline \multirow[t]{2}{*}{0.26352} & 0.436 & 0.01034 & 2332.7 & 46.4 & 2399.1 & 32.6 \\
\hline & 77.08825 & 85.53718 & & & & \\
\hline 0.25148 & 0.41369 & 0.00952 & 2231.8 & 43.4 & 2392.8 & 31.6 \\
\hline 0.23868 & 0.38395 & 0.00896 & 2094.7 & 41.8 & 2324.9 & 31.8 \\
\hline 0.38306 & 0.52333 & 0.01386 & 2713.3 & 58.6 & 2625.8 & 37.6 \\
\hline 0.3502 & 0.52369 & 0.01298 & 2714.8 & 55 & 2615 & 35 \\
\hline 0.14234 & 0.25736 & 0.00676 & 1476.3 & 34.6 & 1681.1 & 35 \\
\hline 0.41618 & 0.52795 & 0.01214 & 2732.8 & 51.2 & 2870.5 & 32.6 \\
\hline 0.29822 & 0.48363 & 0.01126 & 2543.1 & 49 & 2533 & 32.4 \\
\hline 0.28904 & 0.46248 & 0.01076 & 2450.5 & 47.4 & 2504.9 & 32.4 \\
\hline 0.30748 & 0.47198 & 0.01062 & 2492.2 & 46.6 & 2600.6 & 31.4 \\
\hline 0.2867 & 0.44254 & 0.01048 & 2362 & 46.8 & 2474.8 & 33 \\
\hline 0.2756 & 0.45215 & 0.01044 & 2404.8 & 46.4 & 2464.4 & 32 \\
\hline 0.26556 & 0.4099 & 0.00998 & 2214.5 & 45.6 & 2365.2 & 34 \\
\hline 0.18994 & 0.34465 & 0.00812 & 1909 & 39 & 2091.7 & 31.8 \\
\hline 1.16312 & 0.10331 & 0.01474 & 633.8 & 86.2 & 2422.2 & 200 \\
\hline 0.31918 & 0.47438 & 0.01164 & 2502.7 & 50.8 & 2528.9 & 34.6 \\
\hline 0.25126 & 0.4179 & 0.00962 & 2250.9 & 43.8 & 2378.5 & 31.8 \\
\hline 0.2597 & 0.42878 & 0.00998 & 2300.2 & 45 & 2394.2 & 32.2 \\
\hline 0.29256 & 0.48956 & 0.01112 & 2568.8 & 48.2 & 2535.7 & 31.6 \\
\hline 0.2883 & 0.46981 & 0.0109 & 2482.7 & 47.8 & 2494.9 & 32.4 \\
\hline 0.26802 & 0.42794 & 0.00986 & 2296.4 & 44.6 & 2435.9 & 32 \\
\hline 106.99716 & 7.93795 & 0.8005 & & & & \\
\hline 0.333 & 0.45884 & 0.01194 & 2434.4 & 52.8 & 2497.9 & 37 \\
\hline 0.27706 & 0.4338 & 0.01 & 2322.8 & 45 & 2463.6 & 32.2 \\
\hline 0.09766 & 0.21268 & 0.00502 & 1243.1 & 26.6 & 1506.4 & 28.8 \\
\hline 0.24998 & 0.43292 & 0.00976 & 2318.9 & 44 & 2390.7 & 31.2 \\
\hline 0.47682 & 0.49776 & 0.01632 & 2604.2 & 70.2 & 2588.8 & 48 \\
\hline 0.2113 & 0.37302 & 0.00852 & 2043.6 & 40 & 2220 & 31.2 \\
\hline 0.5961 & 0.48642 & 0.01954 & 2555.2 & 84.8 & 2595.2 & 59.4 \\
\hline 0.29662 & 0.45307 & 0.01066 & 2408.9 & 47.2 & 2497.9 & 33.2 \\
\hline 0.26006 & 0.43448 & 0.00988 & 2325.9 & 44.4 & 2412.3 & 31.6 \\
\hline 0.0293 & 0.1087 & 0.00248 & 665.2 & 14.4 & 734.9 & 18.6 \\
\hline 0.2407 & 0.38553 & 0.00876 & 2102.1 & 40.8 & 2342.3 & 31.4 \\
\hline 0.27142 & 0.43945 & 0.01004 & 2348.2 & 45 & 2444.8 & 32 \\
\hline 0.25966 & 0.42076 & 0.00996 & 2263.9 & 45.2 & 2361.5 & 33.2 \\
\hline 0.27632 & 0.43847 & 0.01018 & 2343.8 & 45.6 & 2440.7 & 32.6 \\
\hline 0.28648 & 0.43768 & 0.0109 & 2340.2 & 48.8 & 2390.9 & 35.4 \\
\hline 0.26484 & 0.39361 & 0.00992 & 2139.5 & 45.8 & 2301.9 & 35.6 \\
\hline 0.26926 & 0.43418 & 0.00998 & 2324.5 & 44.8 & 2423.4 & 32.2 \\
\hline 0.35642 & 0.52355 & 0.01214 & 2714.2 & 51.4 & 2676.4 & 33.2 \\
\hline 0.27622 & 0.4237 & 0.00974 & 2277.3 & 44.2 & 2445.5 & 32.4 \\
\hline 0.30546 & 0.47517 & 0.01098 & 2506.2 & 48 & 2533.2 & 32.8 \\
\hline
\end{tabular}




\begin{tabular}{|c|c|c|c|c|c|c|}
\hline 0.27484 & 0.44568 & 0.01038 & 2376 & 46.2 & 2423.9 & 32.8 \\
\hline 0.35024 & 0.49552 & 0.01248 & 2594.5 & 53.8 & 2565.7 & 36.2 \\
\hline 0.3784 & 0.51451 & 0.01304 & 2675.9 & 55.6 & 2633.5 & 36.6 \\
\hline 0.02882 & 0.10468 & 0.00244 & 641.8 & 14.2 & 682.5 & 18.8 \\
\hline 0.0281 & 0.11217 & 0.00262 & 685.3 & 15.2 & 662.3 & 18.6 \\
\hline 0.2183 & 0.32848 & 0.00784 & 1831 & 38 & 2180.8 & 33.2 \\
\hline 716.87194 & 0.09561 & 4.1446 & & & & \\
\hline 0.37004 & 0.50322 & 0.01162 & 2627.6 & 49.8 & 2706.8 & 33.4 \\
\hline 0.28514 & 0.4516 & 0.01042 & 2402.4 & 46.2 & 2461.4 & 32.8 \\
\hline 0.26948 & 0.42224 & 0.0098 & 2270.6 & 44.4 & 2399.9 & 32.8 \\
\hline 0.5814 & 0.48301 & 0.01938 & 2540.4 & 84.2 & 2552.4 & 60 \\
\hline 0.31894 & 0.48521 & 0.0114 & 2549.9 & 49.4 & 2541.6 & 33.8 \\
\hline 0.31446 & 0.48439 & 0.0112 & 2546.4 & 48.6 & 2546.4 & 33.2 \\
\hline 0.27974 & 0.43815 & 0.00996 & 2342.4 & 44.6 & 2454.8 & 32.4 \\
\hline 0.28888 & 0.44661 & 0.01034 & 2380.2 & 46 & 2462.6 & 33 \\
\hline 0.16442 & 0.2752 & 0.0063 & 1567.1 & 31.8 & 1965.3 & 30.8 \\
\hline 0.30574 & 0.45408 & 0.01042 & 2413.4 & 46.2 & 2524.2 & 33 \\
\hline 0.33578 & 0.47554 & 0.01184 & 2507.8 & 51.8 & 2523.2 & 36.2 \\
\hline 0.14642 & 0.10332 & 0.0065 & 633.8 & 38 & 673.2 & 85.6 \\
\hline 0.24172 & 0.38622 & 0.00884 & 2105.3 & 41.2 & 2304.8 & 32.4 \\
\hline 0.301 & 0.45626 & 0.01066 & 2423 & 47.2 & 2483.9 & 33.6 \\
\hline 0.1379 & 0.16476 & 0.00382 & 983.2 & 21.2 & 1797.7 & 30.4 \\
\hline 0.28784 & 0.44036 & 0.01018 & 2352.3 & 45.6 & 2451.6 & 33.2 \\
\hline 0.31906 & 0.47915 & 0.01134 & 2523.6 & 49.4 & 2521.2 & 34.4 \\
\hline 0.30066 & 0.45258 & 0.01048 & 2406.7 & 46.6 & 2489.1 & 33.4 \\
\hline 0.2888 & 0.4615 & 0.01064 & 2446.2 & 47 & 2453.9 & 33.2 \\
\hline 0.32984 & 0.49073 & 0.01146 & 2573.8 & 49.6 & 2564 & 34 \\
\hline 0.20714 & 0.29972 & 0.00838 & 1689.9 & 41.6 & 1917.1 & 40 \\
\hline 0.04428 & 0.15927 & 0.00366 & 952.7 & 20.4 & 941.9 & 22.4 \\
\hline 0.3144 & 0.41922 & 0.0109 & 2256.9 & 49.6 & 2403 & 37.8 \\
\hline 0.33664 & 0.49552 & 0.01152 & 2594.5 & 49.6 & 2583.3 & 34 \\
\hline 0.36256 & 0.4984 & 0.01232 & 2606.9 & 53 & 2590.7 & 36.4 \\
\hline 0.1894 & 0.29736 & 0.00696 & 1678.2 & 34.6 & 2046.1 & 32.6 \\
\hline 0.35394 & 0.51154 & 0.01196 & 2663.2 & 51 & 2621.8 & 34.4 \\
\hline 0.25332 & 0.3762 & 0.00866 & 2058.5 & 40.6 & 2326.2 & 33 \\
\hline 0.28228 & 0.41333 & 0.00956 & 2230.1 & 43.6 & 2419.3 & 33.6 \\
\hline 0.35726 & 0.49367 & 0.01236 & 2586.5 & 53.4 & 2555.8 & 37 \\
\hline 0.321 & 0.44273 & 0.01116 & 2362.9 & 49.8 & 2446.4 & 37 \\
\hline 0.30712 & 0.4766 & 0.01106 & 2512.4 & 48.2 & 2490 & 34 \\
\hline 0.05558 & 0.15193 & 0.00386 & 911.8 & 21.6 & 930.8 & 27.6 \\
\hline 0.34228 & 0.48975 & 0.01156 & 2569.6 & 50 & 2572.3 & 34.8 \\
\hline 0.3254 & 0.48136 & 0.01124 & 2533.2 & 49 & 2534.2 & 34.4 \\
\hline 0.2774 & 0.41272 & 0.00966 & 2227.3 & 44 & 2382.3 & 34 \\
\hline 0.16876 & 0.27712 & 0.00646 & 1576.8 & 32.6 & 1936.1 & 32.2 \\
\hline 0.3313 & 0.4737 & 0.01124 & 2499.8 & 49.2 & 2531.7 & 35 \\
\hline
\end{tabular}




\begin{tabular}{|c|c|c|c|c|c|}
\hline age $207 / 206$ & $2 \sigma$ age 76 & $\begin{array}{l}\text { discordance } \\
\Delta 68-75[\%]\end{array}$ & $\Delta 68-76[\%]$ & $\begin{array}{c}\text { preferred age } \\
\text { age }\end{array}$ & $2 \sigma$ age \\
\hline 2511.6 & 40.2 & -4.7 & -8.4 & 2511.6 & 40.2 \\
\hline 2538 & 46.4 & -8.4 & -14.8 & 2538 & 46.4 \\
\hline 2624 & 39.6 & 1.3 & 2.3 & 2624 & 39.6 \\
\hline 2421.4 & 40.6 & -14.5 & -25.6 & & \\
\hline 2337.6 & 41 & -16.4 & -29.3 & & \\
\hline 530.5 & 52.8 & 2.1 & 11.2 & 589.9 & 12.4 \\
\hline 2508.5 & 40.4 & -8.3 & -14.7 & 2508.5 & 40.4 \\
\hline 2582 & 40.2 & 0.3 & 0.5 & 2582 & 40.2 \\
\hline 2502.3 & 41.4 & -6.1 & -10.9 & 2502.3 & 41.4 \\
\hline 2573.5 & 39.4 & -4.7 & -8.4 & 2573.5 & 39.4 \\
\hline 2577.8 & 39.6 & -0.7 & -1.4 & 2577.8 & 39.6 \\
\hline 2565.4 & 46.4 & 2.2 & 3.9 & 2565.4 & 46.4 \\
\hline 2558.2 & 40.6 & -3 & -5.4 & 2558.2 & 40.6 \\
\hline 2547.5 & 39.8 & -2.4 & -4.4 & 2547.5 & 39.8 \\
\hline 2487.1 & 40.2 & -11.6 & -20.4 & & \\
\hline 2533.4 & 39.4 & -4.8 & -8.6 & 2533.4 & 39.4 \\
\hline 2122 & 41.8 & -25.4 & -46.1 & & \\
\hline 2521.9 & 56.8 & 1.8 & 3.2 & 2521.9 & 56.8 \\
\hline 632.5 & 63 & -1 & -4.5 & 603.9 & 13.4 \\
\hline 2970 & 38.4 & -1.5 & -2.5 & 2970 & 38.4 \\
\hline 2490.8 & 41.6 & -8.4 & -15 & 2490.8 & 41.6 \\
\hline 2549.2 & 42.6 & -1.6 & -2.9 & 2549.2 & 42.6 \\
\hline 2702.9 & 40.6 & -7.6 & -13.1 & 2702.9 & 40.6 \\
\hline 2573.4 & 50.2 & -0.3 & -0.5 & 2573.4 & 50.2 \\
\hline 3489.6 & 432.8 & -59.3 & -78.4 & & \\
\hline 2558.6 & 41.2 & -2.5 & -4.5 & 2558.6 & 41.2 \\
\hline 2586.5 & 41.4 & -4.9 & -8.6 & 2586.5 & 41.4 \\
\hline 2600.7 & 41.6 & 3 & 5.3 & & \\
\hline 801.9 & 55.4 & -0.5 & -1.9 & 786.3 & 16.8 \\
\hline 2547.9 & 44.8 & 0.9 & 1.7 & 2547.9 & 44.8 \\
\hline 2356.6 & 71.2 & -10.1 & -18.3 & & \\
\hline 2540 & 41.6 & -1.8 & -3.2 & 2540 & 41.6 \\
\hline 2607.1 & 40.4 & -7.1 & -12.4 & 2607.1 & 40.4 \\
\hline 2553.9 & 42.4 & -3.2 & -5.8 & 2553.9 & 42.4 \\
\hline 2536.2 & 41.4 & -2.7 & -4.8 & 2536.2 & 41.4 \\
\hline 2384.8 & 45.8 & -13.3 & -23.7 & & \\
\hline 2580.1 & 40.6 & -2.2 & -4 & 2580.1 & 40.6 \\
\hline 2550.4 & 40.6 & -1 & -1.7 & 2550.4 & 40.6 \\
\hline 2496.1 & 41.8 & -5.1 & -9.1 & 2496.1 & 41.8 \\
\hline 2577.9 & 41 & 0.8 & 1.4 & 2577.9 & 41 \\
\hline 2574.8 & 40.8 & -8.1 & -14.1 & 2574.8 & 40.8 \\
\hline
\end{tabular}




\begin{tabular}{|c|c|c|c|c|c|}
\hline 2591.3 & 41.8 & -0.1 & -0.2 & 2591.3 & 41.8 \\
\hline 2544.4 & 42.6 & -4 & -7.1 & 2544.4 & 42.6 \\
\hline 2529.9 & 40.8 & -7.5 & -13.2 & 2529.9 & 40.8 \\
\hline 2581.5 & 42 & -4.7 & -8.4 & 2581.5 & 42 \\
\hline 2622.5 & 41.6 & -0.6 & -1.1 & 2622.5 & 41.6 \\
\hline 2583.4 & 42.2 & 0.5 & 0.8 & 2583.4 & 42.2 \\
\hline 2548.4 & 42.8 & -2.1 & -3.9 & 2548.4 & 42.8 \\
\hline 882.5 & 111.8 & -7.5 & -25.6 & 656.2 & 18.6 \\
\hline 1144.1 & 66.4 & -7.3 & -20.6 & 908.2 & 20.8 \\
\hline 1005.9 & 64.2 & -3.8 & -11.9 & 886.4 & 20 \\
\hline 2519.2 & 45.6 & -3.6 & -6.4 & 2519.2 & 45.6 \\
\hline 2456.7 & 44.8 & -2.8 & -5 & 2456.7 & 44.8 \\
\hline 2533.6 & 42.6 & -6.7 & -11.9 & 2533.6 & 42.6 \\
\hline 2534.3 & 43.6 & -9.9 & -17.3 & & \\
\hline 2559.7 & 50.4 & 3.3 & 6 & & \\
\hline 2539.3 & 46.6 & 3.8 & 6.9 & & \\
\hline 1947.9 & 60.2 & -12.2 & -24.2 & & \\
\hline 2969.4 & 40.6 & -4.8 & -8 & 2969.4 & 40.6 \\
\hline 2525.7 & 43.4 & 0.4 & 0.7 & 2525.7 & 43.4 \\
\hline 2550 & 43.4 & -2.2 & -3.9 & 2550 & 43.4 \\
\hline 2686.8 & 41 & -4.2 & -7.2 & 2686.8 & 41 \\
\hline 2569.5 & 44.4 & -4.6 & -8.1 & 2569.5 & 44.4 \\
\hline 2514.7 & 43.4 & -2.4 & -4.4 & 2514.7 & 43.4 \\
\hline 2498.5 & 47 & -6.4 & -11.4 & 2498.5 & 47 \\
\hline 2277.4 & 46.6 & -8.7 & -16.2 & & \\
\hline 4715.9 & 235 & -73.8 & -86.6 & & \\
\hline 2550.6 & 46.6 & -1 & -1.9 & 2550.6 & 46.6 \\
\hline 2490.4 & 43.6 & -5.4 & -9.6 & 2490.4 & 43.6 \\
\hline 2475.8 & 44.4 & -3.9 & -7.1 & 2475.8 & 44.4 \\
\hline 2510 & 42.6 & 1.3 & 2.3 & 2510 & 42.6 \\
\hline 2505.6 & 43.8 & -0.5 & -0.9 & 2505.6 & 43.8 \\
\hline 2555.2 & 43.4 & -5.7 & -10.1 & 2555.2 & 43.4 \\
\hline 2550.5 & 50.6 & -2.5 & -4.6 & 2550.5 & 50.6 \\
\hline 2582.6 & 43.4 & -5.7 & -10.1 & 2582.6 & 43.4 \\
\hline 1900 & 50.6 & -17.5 & -34.6 & & \\
\hline 2453.3 & 42.8 & -3 & -5.5 & 2453.3 & 42.8 \\
\hline 2577.4 & 65.8 & 0.6 & 1 & 2577.4 & 65.8 \\
\hline 2387.7 & 44 & -7.9 & -14.4 & 2387.7 & 44 \\
\hline 2627.2 & 81.6 & -1.5 & -2.7 & 2627.2 & 81.6 \\
\hline 2571.8 & 44.8 & -3.6 & -6.3 & 2571.8 & 44.8 \\
\hline 2486.8 & 43.4 & -3.6 & -6.5 & 2486.8 & 43.4 \\
\hline 954.5 & 55.4 & -9.5 & -30.3 & 665.2 & 14.4 \\
\hline 2559.3 & 43 & -10.3 & -17.9 & & \\
\hline 2527 & 43.6 & -4 & -7.1 & 2527 & 43.6 \\
\hline 2447.5 & 46.4 & -4.1 & -7.5 & 2447.5 & 46.4 \\
\hline 2523.3 & 44.8 & -4 & -7.1 & 2523.3 & 44.8 \\
\hline 2434.9 & 49.8 & -2.1 & -3.9 & 2434.9 & 49.8 \\
\hline 2449.9 & 50.8 & -7.1 & -12.7 & 2449.9 & 50.8 \\
\hline 2508.3 & 44.4 & -4.1 & -7.3 & 2508.3 & 44.4 \\
\hline 2648.6 & 44.2 & 1.4 & 2.5 & 2648.6 & 44.2 \\
\hline 2589.2 & 44.4 & -6.9 & -12 & 2589.2 & 44.4 \\
\hline 2555.6 & 44.6 & -1.1 & -1.9 & 2555.6 & 44.6 \\
\hline
\end{tabular}




\begin{tabular}{|c|c|c|c|c|c|}
\hline 2465 & 45.6 & -2 & -3.6 & 2465 & 45.6 \\
\hline 2543.6 & 49.6 & 1.1 & 2 & 2543.6 & 49.6 \\
\hline 2601.9 & 49.4 & 1.6 & 2.8 & 2601.9 & 49.4 \\
\hline 820 & 62.2 & -6 & -21.7 & 641.8 & 14.2 \\
\hline 585.4 & 65.6 & 3.5 & 17.1 & 685.3 & 15.2 \\
\hline 2529 & 47.4 & -16 & -27.6 & & \\
\hline 2767.1 & 44 & -2.9 & -5 & 2767.1 & 44 \\
\hline 2511.1 & 45.4 & -2.4 & -4.3 & 2511.1 & 45.4 \\
\hline 2512.2 & 45.8 & -5.4 & -9.6 & 2512.2 & 45.8 \\
\hline 2562.6 & 84.2 & -0.5 & -0.9 & 2562.6 & 84.2 \\
\hline 2535.6 & 46.4 & 0.3 & 0.6 & 2535.6 & 46.4 \\
\hline 2547.1 & 45.4 & 0 & 0 & 2547.1 & 45.4 \\
\hline 2549.9 & 44.6 & -4.6 & -8.1 & 2549.9 & 44.6 \\
\hline 2532.1 & 45.6 & -3.3 & -6 & 2532.1 & 45.6 \\
\hline 2415.8 & 45.8 & -20.3 & -35.1 & & \\
\hline 2615.2 & 45 & -4.4 & -7.7 & 2615.2 & 45 \\
\hline 2536.2 & 50 & -0.6 & -1.1 & 2536.2 & 50 \\
\hline 808 & 339.6 & -5.8 & -21.6 & 633.8 & 38 \\
\hline 2487.3 & 45.6 & -8.7 & -15.4 & & \\
\hline 2534.7 & 46.6 & -2.5 & -4.4 & 2534.7 & 46.6 \\
\hline 2940.7 & 44.8 & -45.3 & -66.6 & & \\
\hline 2535.8 & 46.2 & -4.1 & -7.2 & 2535.8 & 46.2 \\
\hline 2519.8 & 47.8 & 0.1 & 0.1 & 2519.8 & 47.8 \\
\hline 2557.7 & 46.2 & -3.3 & -5.9 & 2557.7 & 46.2 \\
\hline 2461 & 46.6 & -0.3 & -0.6 & 2461 & 46.6 \\
\hline 2557 & 46.8 & 0.4 & 0.7 & 2557 & 46.8 \\
\hline 2173.3 & 64.6 & -11.9 & -22.2 & & \\
\hline 917.5 & 58.4 & 1.2 & 3.8 & 952.7 & 20.4 \\
\hline 2529.8 & 53.6 & -6.1 & -10.8 & 2529.8 & 53.6 \\
\hline 2575.2 & 46.6 & 0.4 & 0.8 & 2575.2 & 46.6 \\
\hline 2578.8 & 50 & 0.6 & 1.1 & 2578.8 & 50 \\
\hline 2441 & 48.4 & -18 & -31.2 & & \\
\hline 2590.5 & 47.2 & 1.6 & 2.8 & 2590.5 & 47. \\
\hline 2570.9 & 46.6 & -11.5 & -19.9 & & \\
\hline 2583.1 & 46.8 & -7.8 & -13.7 & 2583.1 & 46.8 \\
\hline 2532.2 & 51.6 & 1.2 & 2.1 & 2532.2 & 51.6 \\
\hline 2517.3 & 52.2 & -3.4 & -6.1 & 2517.3 & 52. \\
\hline 2472.4 & 47.6 & 0.9 & 1.6 & 2472.4 & 47.6 \\
\hline 976.9 & 76.2 & -2 & -6.7 & 911.8 & 21.6 \\
\hline 2575.1 & 48.2 & -0.1 & -0.2 & 2575.1 & 48.2 \\
\hline 2535.7 & 48 & 0 & -0.1 & 2535.7 & 48 \\
\hline 2518.3 & 48.4 & -6.5 & -11.6 & 2518.3 & 48 \\
\hline 2346.5 & 49.4 & -18.6 & -32.8 & & \\
\hline 2557.9 & 48.8 & -1.3 & -2.3 & 2557.9 & 48. \\
\hline
\end{tabular}


Sample 4052S Bahr El Jebel @ Juba 52 grain analysed 40 concordant ages

\begin{tabular}{|c|c|c|c|c|c|c|}
\hline \multirow[b]{2}{*}{ grain } & \multicolumn{3}{|c|}{ concentrations } & \multicolumn{3}{|l|}{ isotopic ratios } \\
\hline & U [ppm] & $\mathrm{Pb}$ [ppm] & $\mathrm{Th} / \mathrm{U}$ & Pb207/Pb206 & $2 \sigma 76$ & $\mathrm{~Pb} 207 / \mathrm{U} 235$ \\
\hline X4052S_G001 & 68.6 & 12.1 & 1.0249 & 0.06876 & 0.00306 & 1.37469 \\
\hline X4052S_G002 & & & & 0.58916 & 0.06566 & 18.31006 \\
\hline X4052S_G003 & 1039.6 & 219.5 & 0.077 & 0.14836 & 0.00352 & 4.5556 \\
\hline X4052S_G004 & 693.7 & 154.6 & 1.6391 & 0.072 & 0.0019 & 1.60651 \\
\hline X4052S_G005 & 527.8 & 298.6 & 0.2434 & 0.18353 & 0.00436 & 13.09815 \\
\hline X4052S_G006 & 204.6 & 160.7 & 2.2227 & 0.16725 & 0.00416 & 11.07086 \\
\hline X4052S_G007 & 72.6 & 12.1 & 0.9626 & 0.06839 & 0.00328 & 1.31825 \\
\hline X4052S G008 & 74 & 15.2 & 0.6794 & 0.13367 & 0.00502 & 3.22436 \\
\hline X4052S_G009 & 92.6 & 16.9 & 0.6582 & 0.06818 & 0.00308 & 1.53597 \\
\hline X4052S_G010 & 3.3 & 0.7 & 0.3557 & 0.26404 & 0.05876 & 7.33716 \\
\hline X4052S_G011 & 80 & 14.1 & 0.7824 & 0.07216 & 0.0031 & 1.50923 \\
\hline X4052S_G012 & 283.2 & 200 & 0.1092 & 0.26838 & 0.0064 & 24.15015 \\
\hline X4052S_G013 & 84 & 13.7 & 0.4103 & 0.16526 & 0.00562 & 3.68886 \\
\hline X4052S_G014 & 372.5 & 188.3 & 0.6898 & 0.163 & 0.00402 & 9.75351 \\
\hline X4052S_G015 & 108.6 & 56.3 & 1.0254 & 0.16069 & 0.00442 & 9.23518 \\
\hline X4052S_G016 & 195.9 & 17.5 & 0.3004 & 0.05887 & 0.00242 & 0.72412 \\
\hline X4052S_G017 & 282.6 & 152.2 & 0.6927 & 0.17126 & 0.00424 & 10.88511 \\
\hline X4052S_G018 & 300.5 & 252.1 & 0.7378 & 0.32179 & 0.0077 & 31.62114 \\
\hline X4052S_G019 & 17.3 & 7.5 & 0.7306 & 0.12761 & 0.0066 & 6.53543 \\
\hline X4052S_G020 & 2 & 0.2 & 0.0468 & 0.62611 & 0.05456 & 28.01887 \\
\hline X4052S G021 & 161.3 & 110.9 & 1.5472 & 0.1717 & 0.0044 & 11.55434 \\
\hline X4052S_G022 & 110.6 & 19.7 & 0.5596 & 0.06928 & 0.00268 & 1.55692 \\
\hline X4052S_G023 & 137.9 & 76.7 & 0.8222 & 0.17153 & 0.00452 & 10.90618 \\
\hline X4052S_G024 & 96.6 & 18.7 & 0.7735 & 0.07266 & 0.00284 & 1.69488 \\
\hline X4052S_G025 & 90.6 & 17.4 & 0.7993 & 0.07319 & 0.00284 & 1.67687 \\
\hline X4052S_G026 & 62 & 11.8 & 0.7926 & 0.07171 & 0.0033 & 1.63519 \\
\hline X4052S_G027 & 4496.9 & 663.4 & 0.0401 & 0.06903 & 0.0017 & 1.49412 \\
\hline X4052S_G028 & 245.9 & 103 & 0.4367 & 0.16592 & 0.00428 & 8.99493 \\
\hline X4052S_G029 & 106.6 & 89.5 & 0.7474 & 0.36913 & 0.0093 & 38.30989 \\
\hline X4052S_G030 & 858.3 & 97.1 & 0.4957 & 0.06974 & 0.00196 & 1.06667 \\
\hline X4052S_G031 & 973.6 & 373.1 & 0.5685 & 0.16801 & 0.0042 & 8.07637 \\
\hline X4052S_G032 & 356.5 & 49.1 & 1.5331 & 0.0612 & 0.0021 & 0.85597 \\
\hline X4052S_G033 & 398.5 & 42.2 & 0.1886 & 0.05238 & 0.01068 & 0.7736 \\
\hline X4052S_G034 & 465.2 & 46.2 & 0.3404 & 0.05786 & 0.00192 & 0.77917 \\
\hline X4052S_G035 & 59.3 & 9.6 & 0.88 & 0.07198 & 0.0043 & 1.33892 \\
\hline X4052S_G036 & 62 & 7.9 & 0.7992 & 0.05663 & 0.00376 & 0.86721 \\
\hline X4052S_G037 & 367.9 & 200.1 & 0.9087 & 0.17328 & 0.00444 & 10.67279 \\
\hline X4052S_G038 & 245.2 & & 0.5817 & 0.19846 & 0.00508 & 16.58076 \\
\hline X4052S_G039 & 176.6 & 102.4 & 0.6171 & 0.16996 & 0.00452 & 11.49067 \\
\hline X4052S_G040 & 64.6 & 12.7 & 1.0105 & 0.07231 & 0.00352 & 1.60772 \\
\hline X4052S_G041 & 157.9 & 82.7 & 0.7914 & 0.16669 & 0.00472 & 10.11443 \\
\hline X4052S_G042 & 142.6 & 81.1 & 0.6679 & 0.16983 & 0.0046 & 11.26836 \\
\hline X4052S_G043 & 64 & 13.7 & 0.4035 & 0.09248 & 0.00368 & 2.61571 \\
\hline X4052S_G044 & 591.8 & 286.3 & 0.2757 & 0.1766 & 0.0045 & 11.1287 \\
\hline X4052S_G045 & 915.6 & 93.2 & 0.1889 & 0.07271 & 0.00214 & 1.03212 \\
\hline X4052S G046 & 202.6 & 32.7 & 0.1536 & 0.07109 & 0.00248 & 1.62677 \\
\hline X4052S_G047 & 353.2 & 143.5 & 0.144 & 0.17201 & 0.00458 & 9.63666 \\
\hline X4052S_G048 & 77.3 & 44 & 0.6513 & 0.16991 & 0.00508 & 11.30002 \\
\hline X4052S_G049 & 722.4 & 376.3 & 0.5553 & 0.16674 & 0.00432 & 10.5357 \\
\hline X4052S_G050 & 233.2 & 43 & 0.67 & 0.07126 & 0.00238 & 1.61591 \\
\hline
\end{tabular}




$\begin{array}{llllllc}\text { X4052S_G051 } & 852.3 & 355.4 & 0.3333 & 0.16633 & 0.00436 & 9.19043 \\ \text { X4052S_G052 } & 618.4 & 287.8 & 0.2104 & 0.16758 & 0.00442 & 10.32215\end{array}$




\begin{tabular}{|c|c|c|c|c|c|c|}
\hline $2 \sigma 75$ & Pb206/U238 & $2 \sigma 68$ & $\begin{array}{c}\text { ages } \\
\text { age } 206 / 238\end{array}$ & $2 \sigma$ age 68 & age $207 / 235$ & $2 \sigma$ age 75 \\
\hline 0.05976 & 0.14506 & 0.00392 & 873.2 & 22 & 878.2 & 30.6 \\
\hline 1.71016 & 0.22549 & 0.023 & & & & \\
\hline 0.11054 & 0.22278 & 0.00488 & 1296.5 & 25.8 & 1741.2 & 26.8 \\
\hline 0.04272 & 0.16188 & 0.00362 & 967.2 & 20 & 972.7 & 21.6 \\
\hline 0.31922 & 0.5178 & 0.01144 & 2689.8 & 48.6 & 2686.7 & 30.6 \\
\hline 0.28292 & 0.48027 & 0.01096 & 2528.4 & 47.8 & 2529.1 & 31.4 \\
\hline 0.06156 & 0.13985 & 0.00392 & 843.8 & 22.2 & 853.7 & 32 \\
\hline 0.11692 & 0.17501 & 0.00484 & 1039.7 & 26.6 & 1463 & 36.2 \\
\hline 0.06756 & 0.16344 & 0.00444 & 975.9 & 24.6 & 944.9 & 32.4 \\
\hline 1.47738 & 0.20162 & 0.0276 & 1184 & 148 & 2153.3 & 233.4 \\
\hline 0.06322 & 0.15175 & 0.00406 & 910.8 & 22.8 & 934.1 & 31 \\
\hline 0.59444 & 0.65287 & 0.0146 & 3239.4 & 57 & 3274.5 & 31.8 \\
\hline 0.12104 & 0.16195 & 0.00432 & 967.6 & 24 & 1569 & 34.6 \\
\hline 0.24572 & 0.43414 & 0.00976 & 2324.4 & 43.8 & 2411.8 & 30.8 \\
\hline 0.25812 & 0.41699 & 0.01008 & 2246.8 & 45.8 & 2361.6 & 33.6 \\
\hline 0.02896 & 0.08924 & 0.00224 & 551 & 13.2 & 553.1 & 20.6 \\
\hline 0.27548 & 0.46114 & 0.0104 & 2444.6 & 45.8 & 2513.4 & 31.2 \\
\hline 0.78056 & 0.71296 & 0.01594 & 3469.6 & 60 & 3538.6 & 32.2 \\
\hline 0.3356 & 0.37157 & 0.0137 & 2036.8 & 64.4 & 2050.7 & 56 \\
\hline 2.2374 & 0.32469 & 0.02834 & 1812.6 & 138 & 3419.8 & 121 \\
\hline 0.30334 & 0.48825 & 0.0113 & 2563.1 & 49 & 2569 & 32.2 \\
\hline 0.059 & 0.16305 & 0.00416 & 973.7 & 23 & 953.2 & 28.6 \\
\hline 0.29344 & 0.46131 & 0.01086 & 2445.3 & 48 & 2515.2 & 32.8 \\
\hline 0.0648 & 0.16924 & 0.00438 & 1007.9 & 24.2 & 1006.6 & 30 \\
\hline 0.06366 & 0.16623 & 0.00428 & 991.3 & 23.6 & 999.8 & 29.6 \\
\hline 0.0733 & 0.16545 & 0.0046 & 987 & 25.4 & 983.9 & 33.8 \\
\hline 0.03736 & 0.15704 & 0.00342 & 940.3 & 19 & 928 & 20 \\
\hline 0.23638 & 0.39334 & 0.00902 & 2138.3 & 41.8 & 2337.5 & 31.6 \\
\hline 1.00358 & 0.753 & 0.01778 & 3618.6 & 65.4 & 3728 & 34.2 \\
\hline 0.03002 & 0.11098 & 0.0025 & 678.4 & 14.6 & 737.1 & 18.8 \\
\hline 0.20524 & 0.34878 & 0.00776 & 1928.8 & 37 & 2239.6 & 30.2 \\
\hline 0.02908 & 0.10147 & 0.00242 & 623 & 14.2 & 627.9 & 19.6 \\
\hline 0.15238 & 0.10717 & 0.0077 & 656.3 & 44.8 & 581.8 & 95.8 \\
\hline 0.02562 & 0.09771 & 0.00228 & 601 & 13.4 & 585 & 18 \\
\hline 0.07696 & 0.13496 & 0.00428 & 816.1 & 24.4 & 862.8 & 39.4 \\
\hline 0.05564 & 0.11111 & 0.00348 & 679.2 & 20.2 & 634.1 & 34.6 \\
\hline 0.27898 & 0.44687 & 0.01012 & 2381.3 & 45 & 2495.1 & 31.8 \\
\hline 0.4343 & 0.60616 & 0.01382 & 3054.6 & 55.4 & 2910.9 & 32.8 \\
\hline 0.31232 & 0.49052 & 0.01144 & 2572.9 & 49.4 & 2563.8 & 33 \\
\hline 0.07608 & 0.16132 & 0.00462 & 964.1 & 25.6 & 973.2 & 35.4 \\
\hline 0.29126 & 0.44024 & 0.0107 & 2351.7 & 47.8 & 2445.3 & 34.4 \\
\hline 0.31122 & 0.4814 & 0.01132 & 2533.4 & 49.2 & 2545.6 & 33.4 \\
\hline 0.10188 & 0.20521 & 0.00554 & 1203.2 & 29.6 & 1305.1 & 35.4 \\
\hline 0.2887 & 0.4572 & 0.0102 & 2427.2 & 45.2 & 2534 & 31.6 \\
\hline 0.03038 & 0.10299 & 0.00236 & 631.9 & 13.8 & 720 & 19.2 \\
\hline 0.05608 & 0.16603 & 0.00406 & 990.2 & 22.4 & 980.6 & 26.8 \\
\hline 0.26004 & 0.40648 & 0.0093 & 2198.8 & 42.6 & 2400.7 & 32.2 \\
\hline 0.34394 & 0.48254 & 0.01222 & 2538.3 & 53.2 & 2548.2 & 36.6 \\
\hline 0.27734 & 0.45844 & 0.01024 & 2432.7 & 45.2 & 2483.1 & 31.8 \\
\hline 0.05346 & 0.16452 & 0.00394 & 981.8 & 21.8 & 976.4 & 25.8 \\
\hline
\end{tabular}




$\begin{array}{llllllc}0.24404 & 0.40089 & 0.00896 & 2173.1 & 41.2 & 2357.2 & 31.6 \\ 0.27612 & 0.44689 & 0.01004 & 2381.4 & 44.8 & 2464.1 & 32\end{array}$




\begin{tabular}{cc|cc|cc|}
\hline & & discordance & preferred age & \\
age $207 / 206$ & 20 age 76 & $\Delta$ 68-75 [\%] & $\Delta$ 68-76 [\%] & age & 20 age \\
\hline 891.5 & 92 & -0.6 & -2.1 & 873.2 & 22
\end{tabular}

\begin{tabular}{|c|c|c|c|c|c|}
\hline 2327.1 & 40.6 & -25.5 & -44.3 & & \\
\hline 985.9 & 53.8 & -0.6 & -1.9 & 967.2 & 20 \\
\hline 2685 & 39.2 & 0.1 & 0.2 & 2685 & 39.2 \\
\hline 2530.3 & 41.8 & 0 & -0.1 & 2530.3 & 41.8 \\
\hline 880.4 & 99.2 & -1.2 & -4.2 & 843.8 & 22.2 \\
\hline 2146.7 & 65.6 & -28.9 & -51.6 & & \\
\hline 874 & 93.6 & 3.3 & 11.7 & 975.9 & 24.6 \\
\hline 3271 & 350 & -45 & -63.8 & & \\
\hline 990.5 & 87.4 & -2.5 & -8 & 910.8 & 22.8 \\
\hline 3296.6 & 37.4 & -1.1 & -1.7 & 3296.6 & 37.4 \\
\hline 2510.2 & 57.2 & -38.3 & -61.5 & & \\
\hline 2487 & 41.6 & -3.6 & -6.5 & 2487 & 41.6 \\
\hline 2462.9 & 46.4 & -4.9 & -8.8 & 2462.9 & 46.4 \\
\hline 562.3 & 89.6 & -0.4 & -2 & 551 & 13.2 \\
\hline 2570 & 41.4 & -2.7 & -4.9 & 2570 & 41.4 \\
\hline 3578.4 & 36.8 & -1.9 & -3 & 3578.4 & 36.8 \\
\hline 2065.3 & 91.2 & -0.7 & -1.4 & 2065.3 & 91.2 \\
\hline 4569.8 & 126.2 & -47 & -60.3 & & \\
\hline 2574.3 & 42.8 & -0.2 & -0.4 & 2574.3 & 42.8 \\
\hline 907.1 & 79.6 & 2.1 & 7.3 & 973.7 & 23 \\
\hline 2572.6 & 44 & -2.8 & -4.9 & 2572.6 & 44 \\
\hline 1004.5 & 79.4 & 0.1 & 0.3 & 1007.9 & 24.2 \\
\hline 1019.2 & 78.6 & -0.8 & -2.7 & 991.3 & 23.6 \\
\hline 977.7 & 93.8 & 0.3 & 0.9 & 987 & 25.4 \\
\hline 899.6 & 50.8 & 1.3 & 4.5 & 940.3 & 19 \\
\hline 2516.9 & 43.4 & -8.5 & -15 & & \\
\hline 3787.9 & 38.2 & -2.9 & -4.5 & 3787.9 & 38.2 \\
\hline 920.7 & 57.8 & -8 & -26.3 & 678.4 & 14.6 \\
\hline 2537.9 & 42 & -13.9 & -24 & & \\
\hline 646.3 & 73.8 & -0.8 & -3.6 & 623 & 14.2 \\
\hline 302 & 464.8 & 12.8 & 117.3 & & \\
\hline 524.5 & 72.8 & 2.7 & 14.6 & 601 & 13.4 \\
\hline 985.4 & 121.6 & -5.4 & -17.2 & 816.1 & 24.4 \\
\hline 477.2 & 146.8 & 7.1 & 42.3 & & \\
\hline 2589.6 & 42.8 & -4.6 & -8 & 2589.6 & 42.8 \\
\hline 2813.6 & 41.8 & 4.9 & 8.6 & & \\
\hline 2557.2 & 44.6 & 0.4 & 0.6 & 2557.2 & 44.6 \\
\hline 994.7 & 99 & -0.9 & -3.1 & 964.1 & 25.6 \\
\hline 2524.7 & 47.6 & -3.8 & -6.9 & 2524.7 & 47.6 \\
\hline 2556 & 45.4 & -0.5 & -0.9 & 2556 & 45.4 \\
\hline 1477.3 & 75.4 & -7.8 & -18.5 & & \\
\hline 2621.2 & 42.4 & -4.2 & -7.4 & 2621.2 & 42.4 \\
\hline 1005.9 & 59.8 & -12.2 & -37.2 & 631.9 & 13.8 \\
\hline 960 & 71.2 & 1 & 3.1 & 990.2 & 22.4 \\
\hline 2577.3 & 44.4 & -8.4 & -14.7 & 2577.3 & 44.4 \\
\hline 2556.8 & 50 & -0.4 & -0.7 & 2556.8 & 50 \\
\hline 2525.2 & 43.6 & -2 & -3.7 & 2525.2 & 43.6 \\
\hline 964.9 & 68.2 & 0.6 & 1.8 & 981.8 & 21.8 \\
\hline
\end{tabular}


2521

2533.6
44

44.2
$-7.8$

$-3.4$
$-13.8$

$-6$
2521

2533.6
44

44.2
10

11

12

13

14

15

16

17

18

19

20

21

22

23

24

25

26

27

28

29

30

31

32

33

34

35

36

37

38

39

40

41

42

43

44

45

46

47

48

49

50

51

52

53

54

55

56

57

58

59

60 
Sample $13 \quad$ Bahr el Jebel @ Bor 22 grain analysed 18 concordant ages 81

\begin{tabular}{cccc|ccc} 
& concentrations & & & isotopic ratios & & \\
grain & $\begin{array}{c}\mathbf{U}[\mathbf{p p m}] \\
\text { Xb [ppm] }\end{array}$ & $\mathrm{Th} \mathbf{U}$ & $\mathbf{P b 2 0 7 / P b 2 0 6}$ & $\mathbf{2 \sigma} \mathbf{7 6}$ & $\mathbf{P b 2 0 7 / U 2 3 5}$ \\
\hline X13_G001 & 98.1 & 62.5 & 0.6506 & 0.20518 & 0.00556 & 15.19762 \\
X13_G002 & 99.2 & 11.1 & 0.392 & 0.06013 & 0.00296 & 0.89368 \\
X13_G003 & 363.4 & 209 & 0.753 & 0.16872 & 0.00428 & 11.11128 \\
X13_G004 & 117.3 & 19.6 & 0.5478 & 0.07109 & 0.00314 & 1.50656 \\
X13_G005 & & & & -0.20432 & 3.23534 & 3.91885 \\
X13_G006 & 115.1 & 12.1 & 0.1311 & 0.06235 & 0.00334 & 0.93549 \\
X13_G007 & 213.2 & 28.5 & 0.506 & 0.06297 & 0.00232 & 1.07969 \\
X13_G008 & 266.4 & 146.9 & 0.6282 & 0.16977 & 0.00448 & 11.0656 \\
X13_G009 & 227.5 & 38 & 0.442 & 0.06942 & 0.00244 & 1.51598 \\
X13_G010 & 119.5 & 13.8 & 0.5374 & 0.05877 & 0.00286 & 0.8668 \\
X13_G011 & 177.1 & 18.5 & 0.2205 & 0.05754 & 0.00258 & 0.84253 \\
X13_G012 & 15.9 & 5 & 0.3869 & 0.13864 & 0.00786 & 5.76878 \\
X13_G013 & 38.9 & 5 & 0.7682 & 0.06006 & 0.0044 & 0.92527 \\
X13_G014 & 411.7 & 54.6 & 0.4179 & 0.06301 & 0.00204 & 1.0995 \\
X13_G015 & 214.9 & 128.7 & 0.8528 & 0.16734 & 0.0048 & 11.12812 \\
X13_G016 & & & & -14.69411 & 486.05422 & 283.38635 \\
X13_G017 & 169.9 & 18.2 & 0.1252 & 0.05892 & 0.0026 & 0.90866 \\
X13_G018 & 1491 & 692.8 & 0.1006 & 0.16633 & 0.00476 & 10.43826 \\
X13_G019 & 200.6 & 36.8 & 0.8992 & 0.06843 & 0.00264 & 1.45307 \\
X13_G020 & 180.3 & 90.9 & 1.8862 & 0.15092 & 0.00488 & 7.11264 \\
X13_G021 & 323.4 & 32.1 & 0.2161 & 0.05944 & 0.00236 & 0.82656 \\
X13_G022 & 154 & 92 & 0.6832 & 0.16907 & 0.00544 & 11.55639 \\
& & & & &
\end{tabular}




\section{$.8 \%$ concordant}

\begin{tabular}{ccc|cccc}
\hline $\mathbf{2 \sigma} \mathbf{7 5}$ & Pb206/U238 & $\mathbf{2 \sigma} \mathbf{6 8}$ & $\begin{array}{c}\text { ages } \\
\text { age } \mathbf{2 0 6 / 2 3 8}\end{array}$ & $\mathbf{2 \sigma}$ age $\mathbf{6 8}$ & age $\mathbf{2 0 7 / 2 3 5}$ & $\mathbf{2 \sigma}$ age $\mathbf{7 5}$ \\
\hline 0.41942 & 0.53742 & 0.01292 & 2772.6 & 54.2 & 2827.7 & 34.6 \\
0.04264 & 0.10783 & 0.00294 & 660.1 & 17.2 & 648.3 & 27 \\
0.28568 & 0.47783 & 0.01066 & 2517.8 & 46.4 & 2532.5 & 31.4 \\
0.06458 & 0.15375 & 0.00414 & 922 & 23.2 & 933 & 31.6 \\
60.54912 & -0.13915 & 0.55662 & & & & \\
0.04832 & 0.10886 & 0.0031 & 666.1 & 18 & 670.5 & 29.8 \\
0.03892 & 0.1244 & 0.00302 & 755.8 & 17.4 & 743.5 & 23.2 \\
0.29566 & 0.4729 & 0.01068 & 2496.3 & 46.8 & 2528.7 & 32.4 \\
0.05254 & 0.15844 & 0.00384 & 948.1 & 21.4 & 936.9 & 26.2 \\
0.04102 & 0.10701 & 0.0029 & 655.4 & 16.8 & 633.8 & 26.2 \\
0.03676 & 0.10624 & 0.00276 & 650.9 & 16 & 620.5 & 24 \\
0.31888 & 0.30189 & 0.01168 & 1700.7 & 57.8 & 1941.7 & 59.4 \\
0.0653 & 0.11178 & 0.00376 & 683.1 & 21.8 & 665.1 & 39.4 \\
0.03514 & 0.1266 & 0.00294 & 768.4 & 16.8 & 753.1 & 21.2 \\
0.32106 & 0.48247 & 0.01116 & 2538 & 48.6 & 2533.9 & 34.4 \\
417.20666 & -0.13992 & 4.63212 & & & & \\
0.03908 & 0.11188 & 0.0029 & 683.7 & 16.8 & 656.3 & 24.8 \\
0.2996 & 0.45529 & 0.01024 & 2418.7 & 45.4 & 2474.5 & 33.8 \\
0.05494 & 0.15406 & 0.00386 & 923.7 & 21.6 & 911.1 & 27.6 \\
0.22848 & 0.34191 & 0.00834 & 1895.9 & 40 & 2125.6 & 36 \\
0.03222 & 0.10089 & 0.0025 & 619.6 & 14.6 & 611.7 & 21.6 \\
0.37304 & 0.49588 & 0.01212 & 2596.1 & 52.2 & 2569.2 & 37.8
\end{tabular}




\begin{tabular}{cc|cc|cc}
\hline & & discordance & & preferred age & \\
age $\mathbf{2 0 7 / 2 0 6}$ & $\mathbf{2 \sigma}$ age $\mathbf{7 6}$ & $\mathbf{\Delta} \mathbf{6 8 - 7 5}[\%]$ & $\boldsymbol{\Delta} \mathbf{6 8 - 7 6}[\%]$ & age & $\mathbf{2 \sigma}$ age \\
\hline 2867.9 & 44 & -1.9 & -3.3 & 2867.9 & 44 \\
608.3 & 106.4 & 1.8 & 8.5 & 660.1 & 17.2 \\
2545 & 42.6 & -0.6 & -1.1 & 2545 & 42.6 \\
960 & 90.2 & -1.2 & -4 & 922 & 23.2 \\
& & & & & \\
686.1 & 114.4 & -0.7 & -2.9 & 666.1 & 18 \\
707.2 & 78.4 & 1.7 & 6.9 & 755.8 & 17.4 \\
2555.4 & 44.2 & -1.3 & -2.3 & 2555.4 & 44.2 \\
911.2 & 72.4 & 1.2 & 4 & 948.1 & 21.4 \\
558.6 & 106 & 3.4 & 17.3 & 655.4 & 16.8 \\
512.3 & 98.6 & 4.9 & 27.1 & 650.9 & 16 \\
2210.3 & 98.4 & -12.4 & -23.1 & & \\
605.7 & 158.4 & 2.7 & 12.8 & 683.1 & 21.8 \\
708.6 & 68.8 & 2 & 8.4 & 768.4 & 16.8 \\
2531.2 & 48.2 & 0.2 & 0.3 & 2531.2 & 48.2 \\
564.1 & 96.2 & 4.2 & 21.2 & 683.7 & 16.8 \\
2521 & 48 & -2.3 & -4.1 & 2521 & 48 \\
881.6 & 79.8 & 1.4 & 4.8 & 923.7 & 21.6 \\
2356.4 & 55.2 & -10.8 & -19.5 & & \\
583.2 & 86.2 & 1.3 & 6.2 & 619.6 & 14.6 \\
2548.5 & 54 & 1 & 1.9 & 2548.5 & 54
\end{tabular}


$\begin{array}{llllll}\text { Sample } 7 & \text { Lol @ Nyamlell } \quad 295 \text { grain analysed } 207 \text { concordant ages } \quad 70.2\end{array}$

\begin{tabular}{|c|c|c|c|c|c|c|}
\hline \multirow[b]{2}{*}{ grain } & \multicolumn{3}{|c|}{ concentrations } & \multicolumn{3}{|l|}{ isotopic ratios } \\
\hline & U [ppm] & $\mathrm{Pb}$ [ppm] & Th/U & Pb207/Pb206 & $2 \sigma 76$ & Pb207/U235 \\
\hline X7_G001 & 149.7 & 116 & 1.7126 & 0.20089 & 0.00444 & 14.83058 \\
\hline X7_G0-G02 & 653.6 & 192.2 & 0.8217 & 0.15866 & 0.00346 & 5.61013 \\
\hline X7_G003 & 434.9 & 143.7 & 0.7639 & 0.1642 & 0.0036 & 6.7053 \\
\hline X7_G004 & 84 & 44.4 & 0.4825 & 0.17389 & 0.00406 & 11.2557 \\
\hline X7_G005 & 57.4 & 31.7 & 1.1044 & 0.17619 & 0.0043 & 10.52598 \\
\hline X7_G006 & 514.9 & 50.3 & 0.2409 & 0.05932 & 0.00148 & 0.80549 \\
\hline X7_G0-G007 & 759.2 & 33.5 & 0.8744 & 0.06284 & 0.00172 & 0.32302 \\
\hline X7_G008 & 120.3 & 23.8 & 0.7628 & 0.07512 & 0.00214 & 1.78212 \\
\hline X7 ${ }^{-} \mathrm{G} 009$ & 197.9 & 125.3 & 1.059 & 0.17524 & 0.00388 & 11.80587 \\
\hline X7_G0_G10 & 143.3 & 66.8 & 0.4777 & 0.17271 & 0.0039 & 10.10445 \\
\hline X7_G011 & 190.6 & 31 & 0.5197 & 0.06978 & 0.0019 & 1.45086 \\
\hline X7_G012 & 282.9 & 36.9 & 0.3254 & 0.06631 & 0.00172 & 1.16196 \\
\hline X7_G013 & 74.9 & 14.9 & 1.2531 & 0.07237 & 0.00242 & 1.52509 \\
\hline X7_G014 & 662.3 & 59.9 & 0.0292 & 0.06195 & 0.00148 & 0.83322 \\
\hline X7_G015 & 340.3 & 39.2 & 0.4423 & 0.0627 & 0.00166 & 0.94527 \\
\hline X7_G016 & 244.8 & 29.7 & 0.3251 & 0.06295 & 0.0017 & 1.03197 \\
\hline X7_G017 & 400 & 44.7 & 0.3559 & 0.06149 & 0.00158 & 0.92078 \\
\hline X7_G018 & 198.4 & 150.5 & 0.6983 & 0.24286 & 0.00528 & 20.61989 \\
\hline X7_G019 & 363.7 & 188.6 & 0.4693 & 0.17416 & 0.00378 & 11.13243 \\
\hline X7_G020 & 418.4 & 45.7 & 0.1894 & 0.06375 & 0.00158 & 0.98144 \\
\hline $\mathrm{X} 7 \_\mathrm{G} 021$ & 341.7 & 177.1 & 1.2534 & 0.17004 & 0.0037 & 10.41065 \\
\hline $\mathrm{X} 7^{-} \mathrm{G} 022$ & 513.9 & 69.1 & 0.5796 & 0.06413 & 0.00156 & 1.08895 \\
\hline X7_G023 & 159.8 & 109.4 & 1.8922 & 0.17248 & 0.00388 & 10.658 \\
\hline X7_G024 & 815.2 & 104.4 & 0.3055 & 0.06784 & 0.00156 & 1.17091 \\
\hline X7_G025 & 73.5 & 13 & 0.6031 & 0.07364 & 0.00248 & 1.6173 \\
\hline X7_G026 & 70.3 & 0.4 & 0.681 & 0.09078 & 0.01736 & 0.06832 \\
\hline X7_G027 & 536 & 317.3 & 2.9515 & 0.16716 & 0.00366 & 8.02169 \\
\hline X7_G028 & 1770.1 & 546.1 & 0.0952 & 0.18474 & 0.00396 & 8.04218 \\
\hline X7_G029 & 154.3 & 92.6 & 0.7916 & 0.17857 & 0.004 & 12.10458 \\
\hline X7_G030 & 81.8 & 10 & 0.5685 & 0.06595 & 0.00254 & 1.01424 \\
\hline X7_G031 & 215.9 & 24.6 & 0.3413 & 0.06195 & 0.0018 & 0.95098 \\
\hline X7_G032 & 445.5 & 233.7 & 1.0473 & 0.17611 & 0.00382 & 10.39842 \\
\hline X7_G033 & 60.2 & 6.2 & 0.6394 & 0.06174 & 0.00292 & 0.78885 \\
\hline X7_G034 & 77.6 & 8.2 & 0.5754 & 0.05661 & 0.00242 & 0.75472 \\
\hline X7_G035 & 131.4 & 67 & 0.5342 & 0.17021 & 0.00386 & 10.56879 \\
\hline X7_G036 & 138.7 & 78.7 & 0.9657 & 0.17071 & 0.00388 & 10.69012 \\
\hline X7_G037 & & & & 0.78318 & 0.02462 & 52.28482 \\
\hline X7_G038 & 693.5 & 365.4 & 0.6926 & 0.17369 & 0.00374 & 10.79994 \\
\hline X7_G039 & 270.5 & 122 & 0.778 & 0.16429 & 0.00364 & 9.02631 \\
\hline X7_G040 & 53.7 & 6.6 & 0.6145 & 0.06149 & 0.00288 & 0.93488 \\
\hline X7_G041 & 27.1 & 3.2 & 0.6197 & 0.06135 & 0.00422 & 0.91409 \\
\hline X7_G042 & 145.1 & 20.2 & 0.6416 & 0.07158 & 0.00214 & 1.23102 \\
\hline X7_G043 & 363.7 & 148.5 & 0.3393 & 0.16722 & 0.00368 & 9.00673 \\
\hline X7_G044 & 383 & 40.3 & 0.2527 & 0.06007 & 0.00158 & 0.8771 \\
\hline X7_G045 & 203.5 & 28.3 & 1.2847 & 0.06478 & 0.00192 & 0.91865 \\
\hline X7_G046 & 450.1 & 209.8 & 0.3999 & 0.17725 & 0.00384 & 10.6853 \\
\hline X7_G047 & 425.3 & 252.2 & 0.978 & 0.17092 & 0.00372 & 11.07587 \\
\hline X7_G048 & 147 & 17.1 & 0.48 & 0.06173 & 0.00198 & 0.93133 \\
\hline X7_G049 & 934.2 & 412.6 & 0.5087 & 0.17124 & 0.00368 & 9.72252 \\
\hline X7_G050 & 270.1 & 131.5 & 0.3666 & 0.17039 & 0.00374 & 10.55239 \\
\hline
\end{tabular}




\begin{tabular}{|c|c|c|c|c|c|c|}
\hline X7_G051 & 54.7 & 8.2 & 0.8226 & 0.06567 & 0.00282 & 1.15497 \\
\hline X7_G052 & 306.8 & 38.8 & 0.8292 & 0.0612 & 0.00166 & 0.91056 \\
\hline X7_G053 & 82.2 & 45.3 & 0.9124 & 0.17771 & 0.0042 & 11.0523 \\
\hline X7_G054 & 264.1 & 112.2 & 0.3685 & 0.17776 & 0.00394 & 9.98567 \\
\hline X7_G055 & 263.2 & 39.7 & 0.5323 & 0.06686 & 0.0018 & 1.26474 \\
\hline X7_G056 & 193.4 & 22.6 & 0.4202 & 0.06573 & 0.00194 & 1.01907 \\
\hline X7_G057 & 216.3 & 28.3 & 0.8717 & 0.0604 & 0.00178 & 0.92217 \\
\hline X7_G058 & 1589.6 & 149.6 & 0.1594 & 0.14635 & 0.00324 & 1.82064 \\
\hline X7_G059 & 217.7 & 21.1 & 0.2561 & 0.06098 & 0.00184 & 0.82065 \\
\hline X7_G060 & 1114.2 & 238.5 & 0.4597 & 0.12977 & 0.00282 & 3.63585 \\
\hline X7_G061 & 500.2 & 257.5 & 0.5322 & 0.17325 & 0.00378 & 10.90497 \\
\hline X7_G062 & 128.1 & 19.8 & 0.4607 & 0.06936 & 0.00208 & 1.38967 \\
\hline X7_G063 & 928.2 & 387.8 & 0.3683 & 0.20583 & 0.00444 & 11.81014 \\
\hline X7_G064 & 169 & 75.4 & 0.6425 & 0.16927 & 0.00384 & 9.19727 \\
\hline X7_G065 & 103.8 & 49.2 & 0.8767 & 0.17337 & 0.00406 & 9.38113 \\
\hline X7_G066 & 56.5 & 9 & 0.6119 & 0.07316 & 0.00284 & 1.44571 \\
\hline X7_G067 & 64.3 & 7.7 & 1.1419 & 0.06218 & 0.00288 & 0.80923 \\
\hline X7_G068 & 74.4 & 9.6 & 0.4782 & 0.06517 & 0.00252 & 1.08387 \\
\hline X7_G069 & 196.6 & 23.2 & 0.4904 & 0.06304 & 0.00188 & 0.96239 \\
\hline X7_G070 & 302.7 & 29.7 & 0.2498 & 0.06051 & 0.00168 & 0.82344 \\
\hline X7_G071 & 277.9 & 199.9 & 0.1748 & 0.25098 & 0.00546 & 22.12582 \\
\hline X7_G072 & 513.9 & 184.4 & 0.7403 & 0.16239 & 0.00358 & 7.10723 \\
\hline X7_G073 & 345.8 & 169.4 & 0.3569 & 0.17073 & 0.00376 & 10.64455 \\
\hline X7_GöG4 & 172.2 & 93.1 & 0.6377 & 0.17324 & 0.0039 & 11.08506 \\
\hline X7_G075 & 487.8 & 261.2 & 0.7433 & 0.17409 & 0.0038 & 11.04255 \\
\hline X7_G076 & 662.7 & 96.4 & 0.6663 & 0.06894 & 0.00162 & 1.25436 \\
\hline X7_G077 & 589.3 & 97.7 & 0.274 & 0.09913 & 0.00226 & 2.27616 \\
\hline X7_G078 & 247.1 & 33.3 & 0.125 & 0.06641 & 0.00178 & 1.28107 \\
\hline X7_G079 & 153.4 & 18.3 & 0.7428 & 0.06233 & 0.00204 & 0.89221 \\
\hline X7_G080 & 835 & 464.5 & 0.8196 & 0.24681 & 0.00532 & 16.74857 \\
\hline X7_G081 & 145.1 & 74.8 & 0.5374 & 0.1708 & 0.0039 & 10.70922 \\
\hline X7_G082 & 73.5 & 12.2 & 0.6417 & 0.075 & 0.00264 & 1.52394 \\
\hline X7_G083 & 23 & 2.4 & 0.4061 & 0.05659 & 0.00454 & 0.78023 \\
\hline X7_G084 & 274.6 & 155.9 & 1.3585 & 0.17265 & 0.00384 & 10.21463 \\
\hline X7_G085 & & & & -3.02419 & 12.77544 & 319.47839 \\
\hline X7_G086 & 127.7 & 30.4 & 0.7342 & 0.11791 & 0.00304 & 3.60419 \\
\hline X7_G087 & 1581.8 & 274.1 & 0.8267 & 0.15215 & 0.00334 & 3.36474 \\
\hline X7_G0_G8 & 176.8 & 73.9 & 0.4752 & 0.16039 & 0.00366 & 8.49567 \\
\hline X7_G089 & 474 & 184.5 & 0.2964 & 0.15074 & 0.00332 & 7.71928 \\
\hline X7_G090 & 141 & 16.8 & 0.3002 & 0.06368 & 0.00204 & 1.03454 \\
\hline X7_G091 & 162.1 & 93 & 0.7618 & 0.17484 & 0.00396 & 11.47642 \\
\hline X7_G092 & 135 & 49 & 0.4932 & 0.16556 & 0.0039 & 7.65792 \\
\hline X7_G093 & 74.4 & 33.4 & 0.5659 & 0.17256 & 0.00426 & 9.49809 \\
\hline X7_G094 & 213.1 & 101.3 & 0.8681 & 0.16276 & 0.00368 & 8.92614 \\
\hline X7_G095 & 175.4 & 94.8 & 1.0666 & 0.16584 & 0.00378 & 9.78676 \\
\hline X7_G096 & 667.8 & 72.1 & 0.2592 & 0.06324 & 0.00154 & 0.94353 \\
\hline X7_G097 & 110.7 & 13.6 & 0.3376 & 0.08138 & 0.00266 & 1.31966 \\
\hline X7_G098 & 215.9 & 39.7 & 0.5105 & 0.07298 & 0.00192 & 1.71236 \\
\hline X7_G099 & & & & 3.46795 & 15.13388 & 322.16617 \\
\hline X7_G100 & 273.3 & 26.4 & 0.1305 & 0.06048 & 0.00174 & 0.83916 \\
\hline X7_G101 & 173.1 & 20 & 0.4134 & 0.0619 & 0.00194 & 0.94347 \\
\hline X7_G102 & 112.1 & 46.6 & 0.7139 & 0.17366 & 0.00414 & 8.64961 \\
\hline X7_G103 & 78.5 & 49.1 & 1.3903 & 0.17047 & 0.00412 & 10.76961 \\
\hline X7_G104 & 201.6 & 27.7 & 0.4317 & 0.06698 & 0.0019 & 1.20365 \\
\hline X7_G105 & 3591.1 & 877 & 0.1092 & 0.16242 & 0.00352 & 5.75666 \\
\hline
\end{tabular}




\begin{tabular}{|c|c|c|c|c|c|c|}
\hline X7_G106 & 225.5 & 26.6 & 0.4136 & 0.06212 & 0.00182 & 0.96788 \\
\hline X7_G107 & 181 & 72.7 & 0.2449 & 0.19563 & 0.00444 & 10.85787 \\
\hline X7_G108 & 386.3 & 42.4 & 0.4365 & 0.07758 & 0.002 & 1.12532 \\
\hline X7_G109 & 130.4 & 16 & 0.4932 & 0.07413 & 0.00236 & 1.16216 \\
\hline X7_G110 & 250.3 & 134.4 & 1.0503 & 0.17422 & 0.00392 & 10.74296 \\
\hline X7_G111 & 575.9 & 70.3 & 0.6141 & 0.06261 & 0.00156 & 0.95362 \\
\hline X7_G112 & 276.5 & 134.6 & 0.629 & 0.17186 & 0.00386 & 10.1107 \\
\hline X7_G113 & 126.8 & 73.2 & 0.6336 & 0.1747 & 0.00404 & 11.78505 \\
\hline X7_G114 & 823.9 & 94.7 & 0.4974 & 0.07317 & 0.00176 & 1.08205 \\
\hline X7 G115 & 254.4 & 129.2 & 0.5977 & 0.17144 & 0.00384 & 10.50725 \\
\hline X7_G116 & 34.9 & 25.2 & 1.7771 & 0.17249 & 0.00462 & 11.5195 \\
\hline X7_G117 & 565.8 & 113.9 & 0.233 & 0.14901 & 0.00336 & 4.10789 \\
\hline X7_G118 & 165.3 & 91 & 1.5532 & 0.16735 & 0.00386 & 9.24343 \\
\hline X7_G119 & 67.5 & 7.5 & 0.3639 & 0.06166 & 0.0027 & 0.91397 \\
\hline X7_G120 & 127.2 & 22.2 & 0.4831 & 0.07004 & 0.0021 & 1.57197 \\
\hline X7_G121 & 660.4 & 276 & 0.1104 & 0.17908 & 0.00394 & 10.41416 \\
\hline X7_G122 & 108.4 & 14 & 0.6774 & 0.06174 & 0.0022 & 0.98198 \\
\hline X7_G123 & 158.5 & 86 & 0.769 & 0.17255 & 0.00396 & 10.80832 \\
\hline X7_G124 & 276.9 & 36.4 & 1.4189 & 0.05976 & 0.00174 & 0.80321 \\
\hline X7_G125 & 260.4 & 148.1 & 0.8241 & 0.17784 & 0.004 & 11.53297 \\
\hline X7_G126 & 575 & 55 & 0.0994 & 0.0605 & 0.00154 & 0.84201 \\
\hline X7 G127 & 21.6 & 9.5 & 2.1946 & 0.15696 & 0.0056 & 6.27577 \\
\hline X7_G128 & 389 & 138.4 & 0.754 & 0.18431 & 0.00416 & 7.97672 \\
\hline X7_G129 & 231.9 & 35.4 & 0.4527 & 0.15401 & 0.00384 & 2.87498 \\
\hline X7_G130 & 223.2 & 48.2 & 1.4331 & 0.07215 & 0.00198 & 1.57705 \\
\hline X7_G131 & 178.2 & 99.1 & 0.4969 & 0.17387 & 0.00398 & 11.65317 \\
\hline X7_G132 & 147.9 & 15.6 & 0.2825 & 0.06047 & 0.00212 & 0.8774 \\
\hline X7_G133 & 42.7 & 4.9 & 0.4282 & 0.06498 & 0.00332 & 0.97745 \\
\hline X7_G134 & 620.5 & 63.8 & 0.1146 & 0.06223 & 0.00156 & 0.92417 \\
\hline X7_G135 & 10.6 & 3.2 & 0.2492 & 0.1464 & 0.00656 & 6.05922 \\
\hline X7_G136 & 213.1 & 37.6 & 0.6099 & 0.07212 & 0.00196 & 1.58265 \\
\hline X7_G137 & & & & 0.97411 & 0.0321 & \\
\hline X7_G138 & 530 & 261.5 & 0.5319 & 0.17449 & 0.00388 & 10.69594 \\
\hline X7 G139 & 530 & 228.6 & 0.4442 & 0.16624 & 0.0037 & 9.14035 \\
\hline X7_G140 & 967.2 & 129.2 & 1.4445 & 0.0888 & 0.0021 & 1.18909 \\
\hline X7_G141 & 85 & 9.6 & 0.4479 & 0.06391 & 0.00256 & 0.94869 \\
\hline X7_G142 & 189.2 & 23 & 0.5164 & 0.06424 & 0.002 & 0.9992 \\
\hline X7_G143 & 80.8 & 10.3 & 0.5895 & 0.06226 & 0.00252 & 0.99531 \\
\hline X7_G144 & 75.8 & 15.5 & 1.1747 & 0.06916 & 0.00248 & 1.52349 \\
\hline X7_G145 & 252.1 & 170.1 & 1.8928 & 0.17443 & 0.00396 & 10.89108 \\
\hline X7_G146 & 1573 & 444.6 & 0.4368 & 0.16326 & 0.00362 & 6.10929 \\
\hline X7_G147 & 272.8 & 155.5 & 1.0073 & 0.17473 & 0.00396 & 11.06745 \\
\hline X7_G148 & 685.2 & 126.4 & 0.9142 & 0.07212 & 0.00172 & 1.54003 \\
\hline X7_G149 & 446.9 & 166.4 & 1.0154 & 0.16252 & 0.00368 & 6.9253 \\
\hline X7_G150 & 1442.6 & 225.9 & 1.7708 & 0.18896 & 0.00424 & 3.29606 \\
\hline X7 G001 & 313.3 & 36.1 & 0.3386 & 0.06002 & 0.0018 & 0.93075 \\
\hline X7_G002 & 227.4 & 31.7 & 0.241 & 0.08722 & 0.00258 & 1.64426 \\
\hline X7_G003 & 204.4 & 121.6 & 1.2799 & 0.16908 & 0.00422 & 10.409 \\
\hline X7_G004 & 124.6 & 15.4 & 0.5888 & 0.06296 & 0.0025 & 0.97819 \\
\hline X7_G005 & 672.3 & 78.4 & 0.3039 & 0.08369 & 0.0022 & 1.32976 \\
\hline X7_G006 & 57.5 & 9 & 0.3865 & 0.10064 & 0.00416 & 1.92922 \\
\hline X7_G007 & & & & 0.83037 & 0.0279 & \\
\hline X7_G008 & 198.7 & 24.2 & 0.1623 & 0.06474 & 0.00216 & 1.1213 \\
\hline X7_G009 & 127.2 & 14.7 & 0.3213 & 0.05786 & 0.00252 & 0.88311 \\
\hline X7_G010 & 283.2 & 33.5 & 0.4764 & 0.06524 & 0.0023 & 1.00122 \\
\hline
\end{tabular}




\begin{tabular}{|c|c|c|c|c|c|c|}
\hline X7 G011 & 304.6 & 92.8 & 0.054 & 0.15375 & 0.00406 & 6.74101 \\
\hline X7 G012 & 285.4 & 49.6 & 0.5108 & 0.07126 & 0.0021 & 1.58925 \\
\hline X7_G013 & 1379.1 & 119 & 0.1481 & 0.11328 & 0.00328 & 1.33231 \\
\hline X7_G014 & 295.4 & 38.2 & 0.384 & 0.12834 & 0.00358 & 2.0756 \\
\hline X7_G015 & 203.9 & & 1.8866 & 0.0609 & 0.00208 & 0.90554 \\
\hline X7_G016 & 401.7 & 49.5 & 0.7973 & 0.04695 & 0.00164 & 0.68557 \\
\hline X7_G017 & 557.3 & 259.5 & 0.6428 & 0.17026 & 0.00418 & 9.74217 \\
\hline X7_G018 & 154.2 & 18.1 & 0.1597 & 0.06104 & 0.0022 & 1.01596 \\
\hline X7_G019 & 241 & 155.6 & 1.4149 & 0.17195 & 0.00428 & 11.13504 \\
\hline X7_-G020 & 247.5 & 36.6 & 0.4776 & 0.06606 & 0.002 & 1.26216 \\
\hline X7_G021 & & & & -0.1785 & 0.43448 & -46.3695 \\
\hline X7_-G022 & 1128.5 & 419.8 & 0.2023 & 0.1536 & 0.00372 & 7.83813 \\
\hline X7_G023 & & & & 0.26956 & 0.95424 & -66.01025 \\
\hline X7_G024 & & & & 0.80831 & 0.02058 & \\
\hline X7_G025 & & & & 0.82223 & 0.02008 & \\
\hline X7_G026 & 177.3 & 97.1 & 1.0206 & 0.17279 & 0.00442 & 10.43762 \\
\hline X7_G027 & 111.5 & 13.4 & 0.4679 & 0.0623 & 0.0026 & 0.96946 \\
\hline X7_G028 & 104.1 & 49.7 & 1.2813 & 0.11798 & 0.00334 & 5.8404 \\
\hline X7_G029 & 268.8 & 127.7 & 0.4808 & 0.16795 & 0.00424 & 9.96411 \\
\hline X7_G030 & 27 & 3.3 & 0.4639 & 0.06608 & 0.00482 & 1.05337 \\
\hline X7_-G031 & 861.9 & 412 & 0.0721 & 0.16563 & 0.00406 & 10.69979 \\
\hline X7_G032 & 478.9 & 110.8 & 0.0352 & 0.13185 & 0.00334 & 4.48794 \\
\hline X7_G033 & 109.4 & 16.8 & 0.6282 & 0.06601 & 0.00248 & 1.25746 \\
\hline X7_G034 & 356.4 & 52 & 0.908 & 0.06166 & 0.00204 & 1.04062 \\
\hline X7_G035 & 75.4 & 0.1 & 1.4598 & 0.42374 & 0.1795 & 0.05007 \\
\hline X7_G036 & & & & 0.16395 & 3.71722 & -4.20145 \\
\hline X7_G037 & 442.3 & 45.2 & 0.6437 & 0.07701 & 0.00234 & 1.01173 \\
\hline X7_G038 & 78 & 8.5 & 0.1992 & 0.0567 & 0.0032 & 0.86485 \\
\hline X7_G039 & 124.6 & 12.4 & 0.1223 & 0.06392 & 0.00264 & 0.91246 \\
\hline X7_G040 & 78.9 & 49.8 & 1.2162 & 0.1026 & 0.00412 & 6.38568 \\
\hline X7_G041 & 578.6 & 73.3 & 0.9108 & 0.09944 & 0.00274 & 1.6159 \\
\hline X7_G042 & 169.9 & 91.8 & 0.775 & 0.16621 & 0.00438 & 10.37772 \\
\hline X7_G043 & 260.1 & 32.5 & 0.9113 & 0.06084 & 0.00214 & 0.86599 \\
\hline X7_G044 & 224 & 143.6 & 0.7532 & 0.17234 & 0.00444 & 12.31272 \\
\hline X7_G045 & 617.9 & 122.3 & 0.6388 & 0.07026 & 0.00192 & 1.71787 \\
\hline X7_G046 & 309.4 & 167.5 & 0.4498 & 0.16827 & 0.0043 & 11.13327 \\
\hline X7_G047 & 122.9 & 15.7 & 0.8565 & 0.05828 & 0.00248 & 0.80351 \\
\hline X7_G048 & 288.9 & 175.4 & 1.2435 & 0.16639 & 0.00428 & 10.68436 \\
\hline X7_G049 & 546.4 & 57.5 & 0.2961 & 0.06121 & 0.00178 & 0.8784 \\
\hline X7_G050 & & & & -0.83379 & 2.6122 & \\
\hline X7_G051 & 114.2 & 19.1 & 0.9809 & 0.06987 & 0.00262 & 1.32798 \\
\hline X7_G052 & 186.5 & 17.7 & 0.4124 & 0.05711 & 0.00222 & 0.71202 \\
\hline X7_G053 & & & & -2.36281 & 15.75376 & 197.71669 \\
\hline X7_G054 & 224 & 27.3 & 1.0035 & 0.0588 & 0.0021 & 0.81156 \\
\hline X7_G055 & 327.7 & 26.6 & 0.6027 & 0.08278 & 0.00268 & 0.85534 \\
\hline X7_G056 & 64.9 & 7.8 & 0.9131 & 0.0833 & 0.0041 & 1.13901 \\
\hline X7_G057 & 208.7 & 127.9 & 0.909 & 0.19608 & 0.00516 & 13.1844 \\
\hline X7_G058 & 359.5 & 168.3 & 0.6859 & 0.17123 & 0.00448 & 9.71367 \\
\hline X7_G059 & 312.4 & 155 & 0.4061 & 0.16843 & 0.00442 & 10.51014 \\
\hline X7_G060 & 36.2 & 7.7 & 1.3325 & 0.06739 & 0.00386 & 1.5075 \\
\hline X7_G061 & 811.3 & 365.2 & 0.0762 & 0.15864 & 0.0041 & 9.74022 \\
\hline X7_G062 & 76.7 & 46.7 & 1.0567 & 0.17266 & 0.00504 & 11.33358 \\
\hline X7_G063 & 64.9 & 43.5 & 1.5547 & 0.15965 & 0.00482 & 10.35446 \\
\hline X7_G064 & 467.5 & 55.1 & 0.6791 & 0.06 & 0.00198 & 0.87163 \\
\hline X7_G065 & 156.4 & 30.4 & 0.8406 & 0.06872 & 0.00258 & 1.57639 \\
\hline
\end{tabular}




\begin{tabular}{|c|c|c|c|c|c|c|}
\hline X7_G066 & 587.8 & 166.1 & 0.4369 & 0.14135 & 0.00376 & 5.15638 \\
\hline X7_G067 & 315 & 44.2 & 0.6319 & 0.06493 & 0.00206 & 1.13189 \\
\hline X7_G068 & 135.1 & 15.1 & 0.3493 & 0.06283 & 0.00256 & 0.94573 \\
\hline X7_G069 & 55.8 & 7.9 & 0.9504 & 0.06117 & 0.00368 & 0.99921 \\
\hline X7_G070 & 208.3 & 118.5 & 0.589 & 0.17135 & 0.00466 & 11.50367 \\
\hline X7_G071 & 110.2 & 18.8 & 0.9504 & 0.06565 & 0.00268 & 1.28027 \\
\hline X7_G072 & 441 & 61.5 & 0.62 & 0.06371 & 0.00194 & 1.11041 \\
\hline X7_G073 & 386.5 & 213.1 & 0.6499 & 0.16807 & 0.00448 & 10.87795 \\
\hline X7_G074 & 157.3 & 27.8 & 0.5043 & 0.12165 & 0.00372 & 2.82799 \\
\hline X7_G075 & 660.1 & 355.9 & 1.7397 & 0.19544 & 0.00516 & 12.41644 \\
\hline X7_G076 & & & & -0.44886 & 1.24842 & 29.53474 \\
\hline X7_G077 & 607.8 & 278.4 & 0.2377 & 0.16286 & 0.00434 & 9.82078 \\
\hline X7_G078 & 208.3 & 116.5 & 0.7935 & 0.16689 & 0.00458 & 10.67773 \\
\hline X7_G079 & 370.4 & 136.3 & 0.2089 & 0.11816 & 0.00324 & 5.81632 \\
\hline X7_G080 & & & & 0.82377 & 0.02262 & 933.68616 \\
\hline X7_G081 & 167.3 & 40.3 & 0.9936 & 0.1645 & 0.00492 & 4.27326 \\
\hline X7_G082 & 833.1 & 103.7 & 0.7219 & 0.06025 & 0.00178 & 0.91558 \\
\hline X7_G083 & & & & -0.00449 & 0.15588 & -0.04885 \\
\hline X7_G084 & 197.4 & 84.1 & 0.4748 & 0.15904 & 0.00452 & 8.57847 \\
\hline X7_G085 & 227.4 & 145.3 & 1.0037 & 0.17117 & 0.0048 & 11.76639 \\
\hline X7_G086 & 575.6 & 252.8 & 0.7842 & 0.17685 & 0.0048 & 9.37706 \\
\hline X7_G087 & 1125 & 101.5 & 0.0819 & 0.06401 & 0.00186 & 0.8444 \\
\hline X7_G088 & 294.1 & 125.8 & 1.1136 & 0.13157 & 0.00372 & 6.30991 \\
\hline X7_G089 & 326.4 & 190.2 & 1.2821 & 0.17282 & 0.00478 & 10.56238 \\
\hline X7_G090 & & & & 0.88547 & 0.03446 & 136.54172 \\
\hline X7_G091 & 213.1 & 30.3 & 0.3123 & 0.06443 & 0.00224 & 1.24585 \\
\hline X7_G092 & 566.4 & 228.7 & 1.1589 & 0.17101 & 0.00472 & 8.09241 \\
\hline X7_G093 & 446.2 & 221.6 & 0.4059 & 0.16989 & 0.00468 & 10.93351 \\
\hline X7_G094 & 77.1 & 45.7 & 0.5792 & 0.17343 & 0.00516 & 12.02997 \\
\hline X7_G095 & 550.8 & 73.3 & 0.7921 & 0.06236 & 0.00192 & 0.99207 \\
\hline X7_G096 & & & & 0.89251 & 0.02432 & \\
\hline X7_G097 & & & & 1.25383 & 8.11676 & 81.83141 \\
\hline X7_G098 & 407 & 280.4 & 0.7998 & 0.18164 & 0.00506 & 13.47494 \\
\hline X7_G099 & 114.2 & 35.1 & 0.9711 & 0.10298 & 0.00344 & 3.52941 \\
\hline X7_G100 & 184.7 & 35.1 & 0.8596 & 0.07236 & 0.00258 & 1.61004 \\
\hline X7_G101 & 878.4 & 108.8 & 0.276 & 0.06912 & 0.00206 & 1.16832 \\
\hline X7_G102 & 193.9 & 101.8 & 0.5473 & 0.17352 & 0.00506 & 11.08496 \\
\hline X7_G103 & 12.2 & 1.4 & 0.3661 & 0.06175 & 0.0104 & 0.90577 \\
\hline X7_G104 & 397.4 & 48.2 & 0.6056 & 0.06114 & 0.0022 & 0.93266 \\
\hline X7_G105 & 160.8 & 41.3 & 0.5759 & 0.11026 & 0.00364 & 3.589 \\
\hline X7_G106 & 290.2 & 158.7 & 0.8533 & 0.17493 & 0.0051 & 10.93842 \\
\hline X7_G107 & 117.6 & 22.7 & 1.1407 & 0.07308 & 0.00318 & 1.54009 \\
\hline X7_G108 & 460.6 & 282 & 0.5934 & 0.18166 & 0.0052 & 12.95529 \\
\hline X7_G109 & 155.1 & 94.3 & 0.6106 & 0.17484 & 0.00526 & 12.27859 \\
\hline X7_G110 & & & & 801.42871 & & \\
\hline X7_G111 & 874.9 & 137.5 & 1.3557 & 0.09209 & 0.00276 & 1.60035 \\
\hline X7_G112 & 227.9 & 149.9 & 1.2087 & 0.17155 & 0.005 & 11.62283 \\
\hline X7_G113 & 110.2 & 37.1 & 0.423 & 0.18103 & 0.00566 & 8.03521 \\
\hline X7_G114 & 735.1 & 73.3 & 0.0419 & 0.06094 & 0.0019 & 0.8992 \\
\hline X7_G115 & 768.2 & 355.5 & 1.4011 & 0.17817 & 0.00512 & 9.1665 \\
\hline X7_G116 & 217.9 & 63.9 & 1.0962 & 0.10835 & 0.00338 & 3.66615 \\
\hline X7_G117 & & & & -0.00017 & 0.96656 & \\
\hline X7_G118 & & & & 2.09234 & 12.34322 & -39.07341 \\
\hline X7_G119 & & & & 0.83233 & 0.03732 & 970.76117 \\
\hline X7_G120 & 93.2 & 17.3 & 0.3749 & 0.07495 & 0.00294 & 1.84673 \\
\hline
\end{tabular}




$\begin{array}{lcc}\text { X7_G121 } & 100.7 & 15.6 \\ \text { X7_G122 } & & \\ \text { X7_G123 } & 472.8 & 79.7 \\ \text { X7_G124 } & 495.9 & 200 \\ \text { X7_G125 } & 180.8 & 19.5 \\ \text { X7_G126 } & 544.7 & 221.6 \\ \text { X7_G127 } & 227.4 & 21 \\ \text { X7_G128 } & 404.8 & 219.4 \\ \text { X7_G129 } & 105.4 & 16.5 \\ \text { X7_G130 } & 284.5 & 150 \\ \text { X7_G131 } & 438.3 & 79.3 \\ \text { X7_G132 } & 73.2 & 14.7 \\ \text { X7_G133 } & 64.1 & 11.9 \\ \text { X7_G134 } & 324.2 & 34.5 \\ \text { X7_G135 } & 149.9 & 24.9 \\ \text { X7_G136 } & 1140.7 & 267.9 \\ \text { X7_G137 } & 262.7 & 30.7 \\ \text { X7_G138 } & 34 & 3.9 \\ \text { X7_G139 } & 249.7 & 141 \\ \text { X7_G140 } & 792.2 & 126.3 \\ \text { X7_G141 } & 194.3 & 20.3 \\ \text { X7_G142 } & 527.2 & 56.6 \\ \text { X7_G143 } & 24 & 14 \\ \text { X7_G144 } & 391.7 & 44.7 \\ \text { X7_G145 } & 379.1 & 222.6\end{array}$

0.5031

0.669
0.2689
0.5709
0.7699
0.0399
0.5971
0.478
0.6405
1.3998
1.1707
0.8226
0.1869
0.5324
0.5448
0.3384
0.7693
0.5159
1.3085
0.5702
0.2271
0.6194
0.3599
0.5062

0.07135
0.53392
0.06929
0.16366
0.07076
0.18011
0.06014
0.17077
0.19145
0.18187
0.08272
0.06911
0.07214
0.06024
0.10071
0.1464
0.06115
0.05869
0.17249
0.13678
0.05816
0.06089
0.17098
0.06186
0.1748

0.0029
0.64868
0.0022
0.00482
0.0028
0.00534
0.00236
0.00512
0.0071
0.00554
0.00274
0.00322
0.00346
0.00218
0.0039
0.00444
0.00254
0.00528
0.00532
0.00428
0.00246
0.00206
0.00652
0.00218
0.0054

1.42173

10.67298

1.43284

8.82593

0.95572

9.18994

0.8237

11.07165

3.56125

11.38308

1.56556

1.51902

1.59218

0.90766

2.17408

4.45817

0.96575

0.82478

11.68236

2.72305

0.77455

0.91605

11.64391

0.95009

12.19067 


\section{$\%$ concordant}

\begin{tabular}{|c|c|c|c|c|c|c|}
\hline $2 \sigma 75$ & Pb206/U238 & $2 \sigma 68$ & $\begin{array}{c}\text { ages } \\
\text { age } 206 / 238\end{array}$ & $2 \sigma$ age 68 & age $207 / 235$ & $2 \sigma$ age 75 \\
\hline 0.36496 & 0.53556 & 0.0127 & 2764.8 & 53.4 & 2804.4 & 30.8 \\
\hline 0.1359 & 0.25652 & 0.006 & 1472 & 30.8 & 1917.7 & 27.6 \\
\hline 0.16342 & 0.29626 & 0.00694 & 1672.7 & 34.6 & 2073.3 & 28.4 \\
\hline 0.28882 & 0.46957 & 0.01142 & 2481.7 & 50 & 2544.5 & 31.4 \\
\hline 0.27956 & 0.4334 & 0.01078 & 2321 & 48.4 & 2482.2 & 32.4 \\
\hline 0.02158 & 0.09851 & 0.00234 & 605.7 & 13.8 & 599.9 & 15.6 \\
\hline 0.0093 & 0.03729 & 0.0009 & 236 & 5.6 & 284.2 & 9 \\
\hline 0.05342 & 0.17211 & 0.00426 & 1023.7 & 23.4 & 1039 & 24.6 \\
\hline 0.29094 & 0.48875 & 0.01158 & 2565.3 & 50.2 & 2589.1 & 30.4 \\
\hline 0.25194 & 0.42443 & 0.01012 & 2280.6 & 45.8 & 2444.4 & 30.4 \\
\hline 0.04164 & 0.15084 & 0.00366 & 905.7 & 20.6 & 910.2 & 22 \\
\hline 0.0322 & 0.12712 & 0.00304 & 771.4 & 17.4 & 782.9 & 19.2 \\
\hline 0.0523 & 0.15289 & 0.00394 & 917.1 & 22 & 940.5 & 25.8 \\
\hline 0.02174 & 0.09757 & 0.0023 & 600.2 & 13.6 & 615.4 & 15.4 \\
\hline 0.02652 & 0.10937 & 0.00262 & 669.1 & 15.2 & 675.6 & 17.6 \\
\hline 0.02952 & 0.11893 & 0.00286 & 724.4 & 16.4 & 719.9 & 18.6 \\
\hline 0.0252 & 0.10863 & 0.00258 & 664.8 & 15 & 662.8 & 17 \\
\hline 0.50206 & 0.61596 & 0.01454 & 3093.8 & 58 & 3120.9 & 31 \\
\hline 0.27036 & 0.46373 & 0.01088 & 2456 & 48 & 2534.3 & 29.8 \\
\hline 0.0263 & 0.11169 & 0.00266 & 682.6 & 15.4 & 694.3 & 17.2 \\
\hline 0.25324 & 0.44417 & 0.01042 & 2369.3 & 46.6 & 2472 & 29.6 \\
\hline 0.02878 & 0.12319 & 0.00292 & 748.9 & 16.8 & 748 & 18 \\
\hline 0.26532 & 0.4483 & 0.01068 & 2387.7 & 47.6 & 2493.8 & 30.4 \\
\hline 0.02952 & 0.12522 & 0.00294 & 760.5 & 16.8 & 787.1 & 18 \\
\hline 0.05564 & 0.15933 & 0.00412 & 953.1 & 23 & 976.9 & 26.6 \\
\hline 0.01246 & 0.00546 & 0.00034 & 35.1 & 2.2 & 67.1 & 13 \\
\hline 0.19538 & 0.34815 & 0.00818 & 1925.8 & 39.2 & 2233.5 & 29 \\
\hline 0.19262 & 0.31582 & 0.00734 & 1769.3 & 36 & 2235.8 & 28.6 \\
\hline 0.30082 & 0.49177 & 0.01172 & 2578.3 & 50.6 & 2612.5 & 30.6 \\
\hline 0.03938 & 0.11157 & 0.00296 & 681.9 & 17.2 & 711 & 24 \\
\hline 0.02882 & 0.11137 & 0.00272 & 680.7 & 15.8 & 678.6 & 18.8 \\
\hline 0.2518 & 0.42836 & 0.01004 & 2298.3 & 45.4 & 2470.9 & 29.6 \\
\hline 0.03692 & 0.09269 & 0.0026 & 571.4 & 15.4 & 590.5 & 24.6 \\
\hline 0.03224 & 0.09671 & 0.0026 & 595.1 & 15.2 & 571 & 22 \\
\hline 0.2659 & 0.45046 & 0.0108 & 2397.3 & 48 & 2486 & 30.6 \\
\hline 0.26956 & 0.45429 & 0.01092 & 2414.3 & 48.4 & 2496.6 & 30.8 \\
\hline 1.74854 & 0.48433 & 0.01716 & & & & \\
\hline 0.26058 & 0.45109 & 0.01054 & 2400.1 & 46.8 & 2506.1 & 29.6 \\
\hline 0.22248 & 0.39859 & 0.00942 & 2162.5 & 43.4 & 2340.7 & 29.6 \\
\hline 0.04336 & 0.1103 & 0.0031 & 674.5 & 18 & 670.2 & 26.8 \\
\hline 0.06122 & 0.1081 & 0.00358 & 661.7 & 20.8 & 659.2 & 37 \\
\hline 0.03826 & 0.12476 & 0.0031 & 757.9 & 17.8 & 814.8 & 21.8 \\
\hline 0.22052 & 0.39075 & 0.0092 & 2126.3 & 42.6 & 2338.7 & 29.4 \\
\hline 0.02458 & 0.10593 & 0.00254 & 649.1 & 14.8 & 639.4 & 16.8 \\
\hline 0.02844 & 0.10288 & 0.00254 & 631.3 & 14.8 & 661.6 & 18.8 \\
\hline 0.25942 & 0.43734 & 0.01026 & 2338.7 & 46 & 2496.1 & 29.6 \\
\hline 0.26912 & 0.47013 & 0.01104 & 2484.1 & 48.4 & 2529.5 & 29.8 \\
\hline 0.03076 & 0.10945 & 0.00274 & 669.6 & 16 & 668.3 & 20 \\
\hline 0.2345 & 0.41191 & 0.00962 & 2223.6 & 44 & 2408.8 & 29.2 \\
\hline 0.25906 & 0.44931 & 0.0106 & 2392.2 & 47.2 & 2484.5 & 29.8 \\
\hline
\end{tabular}




\begin{tabular}{|c|c|c|c|c|c|c|}
\hline 0.0494 & 0.12758 & 0.00352 & 774 & 20.2 & 779.6 & 27.8 \\
\hline 0.02612 & 0.10793 & 0.0026 & 660.7 & 15.2 & 657.4 & 17.6 \\
\hline 0.28782 & 0.4512 & 0.01108 & 2400.6 & 49.2 & 2527.5 & 31.8 \\
\hline 0.24694 & 0.40755 & 0.00966 & 2203.7 & 44.2 & 2433.5 & 30 \\
\hline 0.03612 & 0.13724 & 0.00332 & 829 & 18.8 & 830 & 20.6 \\
\hline 0.03142 & 0.11248 & 0.00278 & 687.1 & 16.2 & 713.4 & 19.8 \\
\hline 0.02844 & 0.11077 & 0.00272 & 677.2 & 15.8 & 663.5 & 18.6 \\
\hline 0.04466 & 0.09025 & 0.00212 & 557 & 12.6 & 1052.9 & 21.2 \\
\hline 0.02572 & 0.09764 & 0.00242 & 600.6 & 14.2 & 608.4 & 17.8 \\
\hline 0.08848 & 0.20326 & 0.00476 & 1192.8 & 25.6 & 1557.4 & 25.4 \\
\hline 0.2657 & 0.45664 & 0.01074 & 2424.7 & 47.6 & 2515.1 & 29.8 \\
\hline 0.04346 & 0.14536 & 0.00364 & 874.9 & 20.4 & 884.6 & 23 \\
\hline 0.28558 & 0.41628 & 0.00974 & 2243.6 & 44.4 & 2589.5 & 29.8 \\
\hline 0.23152 & 0.39418 & 0.00944 & 2142.2 & 43.6 & 2357.8 & 30.2 \\
\hline 0.24214 & 0.39256 & 0.00956 & 2134.7 & 44.2 & 2376 & 31 \\
\hline 0.05628 & 0.14335 & 0.00388 & 863.6 & 21.8 & 908.1 & 28.4 \\
\hline 0.03718 & 0.09442 & 0.00266 & 581.6 & 15.6 & 602 & 24.6 \\
\hline 0.04208 & 0.12066 & 0.00322 & 734.4 & 18.6 & 745.5 & 24.8 \\
\hline 0.0298 & 0.11076 & 0.00274 & 677.2 & 16 & 684.5 & 19.2 \\
\hline 0.02426 & 0.09873 & 0.0024 & 607 & 14 & 610 & 17 \\
\hline 0.54088 & 0.63957 & 0.01512 & 3187.3 & 59.4 & 3189.3 & 31.2 \\
\hline 0.17468 & 0.31752 & 0.00748 & 1777.6 & 36.6 & 2124.9 & 28.8 \\
\hline 0.26188 & 0.45231 & 0.01068 & 2405.5 & 47.4 & 2492.6 & 30 \\
\hline 0.27802 & 0.4642 & 0.01112 & 2458.1 & 49 & 2530.3 & 30.6 \\
\hline 0.26978 & 0.46018 & 0.01082 & 2440.4 & 47.8 & 2526.7 & 29.8 \\
\hline 0.03242 & 0.13201 & 0.00312 & 799.3 & 17.8 & 825.4 & 18.8 \\
\hline 0.05744 & 0.16659 & 0.00394 & 993.3 & 21.8 & 1204.9 & 23.2 \\
\hline 0.03668 & 0.13995 & 0.0034 & 844.4 & 19.2 & 837.3 & 20.6 \\
\hline 0.03004 & 0.10384 & 0.00262 & 636.9 & 15.4 & 647.6 & 19.8 \\
\hline 0.40596 & 0.49233 & 0.01154 & 2580.7 & 49.8 & 2920.6 & 30.6 \\
\hline 0.27156 & 0.45488 & 0.01096 & 2416.9 & 48.6 & 2498.2 & 30.8 \\
\hline 0.05468 & 0.14742 & 0.0039 & 886.5 & 22 & 940.1 & 27 \\
\hline 0.06108 & 0.10003 & 0.00346 & 614.6 & 20.2 & 585.6 & 38.8 \\
\hline 0.25308 & 0.42922 & 0.01018 & 2302.2 & 46 & 2454.4 & 30 \\
\hline 719.7044 & -0.76642 & 3.49302 & & & & \\
\hline 0.0998 & 0.22177 & 0.00548 & 1291.2 & 29 & 1550.5 & 28.4 \\
\hline 0.0824 & 0.16044 & 0.00376 & 959.2 & 20.8 & 1496.2 & 25.2 \\
\hline 0.2153 & 0.38428 & 0.00922 & 2096.2 & 43 & 2285.5 & 30 \\
\hline 0.19008 & 0.37151 & 0.00876 & 2036.5 & 41.2 & 2198.8 & 29 \\
\hline 0.0343 & 0.11786 & 0.00298 & 718.2 & 17.2 & 721.2 & 21 \\
\hline 0.28992 & 0.4762 & 0.01144 & 2510.7 & 50 & 2562.7 & 30.8 \\
\hline 0.198 & 0.33558 & 0.00816 & 1865.4 & 39.4 & 2191.7 & 30.4 \\
\hline 0.25562 & 0.39934 & 0.00998 & 2166 & 46 & 2387.4 & 32.2 \\
\hline 0.2244 & 0.39789 & 0.00952 & 2159.3 & 44 & 2330.5 & 30 \\
\hline 0.24766 & 0.42813 & 0.01028 & 2297.3 & 46.4 & 2414.9 & 30.6 \\
\hline 0.02496 & 0.10825 & 0.00258 & 662.6 & 15 & 674.7 & 16.8 \\
\hline 0.04412 & 0.11764 & 0.00304 & 717 & 17.6 & 854.4 & 24 \\
\hline 0.04818 & 0.17023 & 0.00414 & 1013.4 & 22.8 & 1013.2 & 23 \\
\hline 462.39788 & 0.67398 & 2.98772 & & & & \\
\hline 0.0254 & 0.10066 & 0.00246 & 618.3 & 14.4 & 618.7 & 17.4 \\
\hline 0.03068 & 0.11059 & 0.00278 & 676.2 & 16.2 & 674.7 & 19.8 \\
\hline 0.22606 & 0.36136 & 0.00886 & 1988.6 & 42 & 2301.8 & 31.2 \\
\hline 0.28678 & 0.45835 & 0.01138 & 2432.3 & 50.4 & 2503.4 & 32.2 \\
\hline 0.03598 & 0.13038 & 0.0032 & 790 & 18.2 & 802.3 & 20.8 \\
\hline 0.14006 & 0.25714 & 0.00602 & 1475.2 & 30.8 & 1939.9 & 27.6 \\
\hline
\end{tabular}




\begin{tabular}{|c|c|c|c|c|c|c|}
\hline 0.02964 & 0.11303 & 0.00278 & 690.3 & 16.2 & 687.4 & 19.2 \\
\hline 0.27404 & 0.40267 & 0.00968 & 2181.3 & 44.4 & 2511 & 30.8 \\
\hline 0.03114 & 0.10523 & 0.00254 & 645 & 14.8 & 765.5 & 19 \\
\hline 0.03814 & 0.11374 & 0.0029 & 694.4 & 16.8 & 783 & 22.2 \\
\hline 0.26922 & 0.44737 & 0.01068 & 2383.5 & 47.6 & 2501.1 & 30.4 \\
\hline 0.02564 & 0.1105 & 0.00264 & 675.7 & 15.4 & 680 & 17.2 \\
\hline 0.25288 & 0.42683 & 0.01018 & 2291.4 & 46 & 2444.9 & 30.2 \\
\hline 0.30216 & 0.48941 & 0.01186 & 2568.1 & 51.4 & 2587.5 & 31.4 \\
\hline 0.02836 & 0.10728 & 0.00256 & 656.9 & 15 & 744.6 & 17.8 \\
\hline 0.26318 & 0.44464 & 0.0106 & 2371.4 & 47.4 & 2480.6 & 30.4 \\
\hline 0.33462 & 0.48453 & 0.0128 & 2547 & 55.6 & 2566.2 & 35.2 \\
\hline 0.10296 & 0.2 & 0.00476 & 1175.3 & 25.6 & 1655.9 & 26.8 \\
\hline 0.23636 & 0.40074 & 0.00968 & 2172.4 & 44.6 & 2362.4 & 30.6 \\
\hline 0.03988 & 0.10754 & 0.00298 & 658.4 & 17.4 & 659.2 & 25.2 \\
\hline 0.0492 & 0.16282 & 0.0041 & 972.4 & 22.8 & 959.2 & 24.4 \\
\hline 0.2568 & 0.42191 & 0.00996 & 2269.1 & 45.2 & 2472.3 & 29.8 \\
\hline 0.03554 & 0.11539 & 0.00298 & 704 & 17.2 & 694.6 & 22.2 \\
\hline 0.27546 & 0.45444 & 0.01096 & 2415 & 48.6 & 2506.8 & 31 \\
\hline 0.02456 & 0.09752 & 0.0024 & 599.9 & 14 & 598.6 & 17.2 \\
\hline 0.28946 & 0.47049 & 0.01124 & 2485.7 & 49.2 & 2567.3 & 30.6 \\
\hline 0.0231 & 0.10097 & 0.00242 & 620.1 & 14.2 & 620.3 & 16.2 \\
\hline 0.22756 & 0.29008 & 0.0087 & 1641.9 & 43.4 & 2015.1 & 40.8 \\
\hline 0.20036 & 0.314 & 0.0075 & 1760.4 & 36.8 & 2228.4 & 29.6 \\
\hline 0.07714 & 0.13543 & 0.00332 & 818.8 & 18.8 & 1375.4 & 26.4 \\
\hline 0.04586 & 0.15858 & 0.0039 & 948.9 & 21.6 & 961.2 & 23 \\
\hline 0.2966 & 0.48624 & 0.01172 & 2554.4 & 50.8 & 2576.9 & 31 \\
\hline 0.03136 & 0.10527 & 0.00272 & 645.2 & 15.8 & 639.6 & 20.6 \\
\hline 0.04908 & 0.10913 & 0.00322 & 667.7 & 18.8 & 692.3 & 29.6 \\
\hline 0.02502 & 0.10774 & 0.00258 & 659.6 & 15 & 664.6 & 17 \\
\hline 0.27068 & 0.30028 & 0.01032 & 1692.7 & 51.2 & 1984.4 & 49.2 \\
\hline \multirow[t]{2}{*}{0.04568} & 0.15921 & 0.0039 & 952.4 & 21.6 & 963.4 & 22.8 \\
\hline & 218.64545 & 98.02118 & & & & \\
\hline 0.2661 & 0.44472 & 0.01054 & 2371.7 & 47 & 2497.1 & 30.2 \\
\hline 0.22754 & 0.39892 & 0.00946 & 2164.1 & 43.6 & 2352.2 & 29.8 \\
\hline 0.03088 & 0.09715 & 0.00232 & 597.7 & 13.6 & 795.5 & 18.6 \\
\hline 0.03822 & 0.10769 & 0.0029 & 659.3 & 16.8 & 677.4 & 23.8 \\
\hline 0.0322 & 0.11285 & 0.00284 & 689.3 & 16.4 & 703.4 & 20.4 \\
\hline 0.04056 & 0.11599 & 0.00314 & 707.4 & 18.2 & 701.4 & 24.6 \\
\hline 0.0559 & 0.15983 & 0.00424 & 955.8 & 23.6 & 939.9 & 27.4 \\
\hline 0.27608 & 0.453 & 0.01086 & 2408.6 & 48.2 & 2513.9 & 30.8 \\
\hline 0.15148 & 0.27149 & 0.00642 & 1548.4 & 32.6 & 1991.6 & 28.2 \\
\hline 0.28022 & 0.45955 & 0.01102 & 2437.6 & 48.6 & 2528.8 & 30.8 \\
\hline 0.04028 & 0.15493 & 0.0037 & 928.5 & 20.6 & 946.5 & 20.8 \\
\hline 0.17482 & 0.30916 & 0.00738 & 1736.6 & 36.4 & 2101.9 & 29.2 \\
\hline 0.08242 & 0.12655 & 0.003 & 768.2 & 17.2 & 1480.1 & 25.4 \\
\hline 0.02718 & 0.11251 & 0.00246 & 687.3 & 14.2 & 668 & 18.2 \\
\hline 0.04752 & 0.13676 & 0.00306 & 826.3 & 17.4 & 987.3 & 23.4 \\
\hline 0.25836 & 0.44662 & 0.00966 & 2380.2 & 43 & 2471.9 & 30.6 \\
\hline 0.03762 & 0.11271 & 0.00276 & 688.5 & 16 & 692.7 & 23.4 \\
\hline 0.03422 & 0.11528 & 0.00246 & 703.3 & 14.2 & 858.8 & 19.6 \\
\hline 0.07628 & 0.13907 & 0.00374 & 839.4 & 21.2 & 1091.3 & 33 \\
\hline 884.23328 & 37.88874 & 7.73188 & & & & \\
\hline 0.0365 & 0.12565 & 0.00288 & 763 & 16.4 & 763.6 & 21.8 \\
\hline 0.03714 & 0.11073 & 0.00276 & 677 & 16 & 642.7 & 23.8 \\
\hline 0.03424 & 0.11134 & 0.0026 & 680.5 & 15 & 704.4 & 21.4 \\
\hline
\end{tabular}




\begin{tabular}{|c|c|c|c|c|c|c|}
\hline 0.17602 & 0.31808 & 0.00706 & 1780.3 & 34.6 & 2078 & 30.6 \\
\hline 0.04594 & 0.1618 & 0.00358 & 966.8 & 19.8 & 966 & 23 \\
\hline 0.03732 & 0.08532 & 0.0019 & 527.8 & 11.2 & 859.9 & 21.2 \\
\hline 0.05616 & 0.11733 & 0.0026 & 715.2 & 15 & 1140.8 & 24.4 \\
\hline 0.03008 & 0.10788 & 0.00246 & 660.4 & 14.4 & 654.7 & 19.8 \\
\hline 0.0235 & 0.10594 & 0.00232 & 649.1 & 13.6 & 530.1 & 17 \\
\hline 0.23802 & 0.41511 & 0.00882 & 2238.2 & 40.2 & 2410.7 & 29.8 \\
\hline 0.03568 & 0.12075 & 0.00282 & 734.9 & 16.2 & 711.9 & 22 \\
\hline 0.27592 & 0.46982 & 0.01008 & 2482.8 & 44.2 & 2534.5 & 30.6 \\
\hline 0.03732 & 0.13862 & 0.00308 & 836.9 & 17.4 & 828.9 & 21.2 \\
\hline 136.4517 & 1.88467 & 4.32478 & & & & \\
\hline 0.1887 & 0.37021 & 0.00774 & 2030.4 & 36.4 & 2212.6 & 28.8 \\
\hline 225.86502 & -1.77661 & 3.31936 & & & & \\
\hline 103.49984 & 17.93616 & 0.90882 & & & & \\
\hline 60.59224 & 16.4647 & 0.49974 & & & & \\
\hline 0.26526 & 0.43825 & 0.00956 & 2342.8 & 42.8 & 2474.4 & 31.2 \\
\hline 0.03898 & 0.1129 & 0.0028 & 689.6 & 16.2 & 688.2 & 24.2 \\
\hline 0.1642 & 0.35914 & 0.00822 & 1978.1 & 39 & 1952.4 & 31.6 \\
\hline 0.25 & 0.43042 & 0.00926 & 2307.6 & 41.8 & 2431.5 & 30.6 \\
\hline 0.07358 & 0.11565 & 0.00394 & 705.5 & 22.8 & 730.6 & 42 \\
\hline 0.26052 & 0.46869 & 0.00984 & 2477.8 & 43.2 & 2497.4 & 30 \\
\hline 0.11258 & 0.24694 & 0.00526 & 1422.7 & 27.2 & 1728.7 & 27.4 \\
\hline 0.04584 & 0.13821 & 0.00332 & 834.5 & 18.8 & 826.8 & 25.2 \\
\hline 0.0334 & 0.12244 & 0.00278 & 744.6 & 16 & 724.2 & 20.8 \\
\hline 0.01608 & 0.00086 & 0.00024 & 5.5 & 1.6 & 49.6 & 24.6 \\
\hline 91.19034 & -0.18592 & 1.25776 & & & & \\
\hline 0.02982 & 0.09532 & 0.00212 & 586.9 & 12.4 & 709.7 & 19.2 \\
\hline 0.0472 & 0.11067 & 0.00312 & 676.6 & 18.2 & 632.8 & 29.8 \\
\hline 0.03632 & 0.10356 & 0.00256 & 635.2 & 15 & 658.4 & 23.4 \\
\hline 0.25694 & 0.45153 & 0.01142 & 2402.1 & 50.8 & 2030.3 & 41.6 \\
\hline 0.04368 & 0.11789 & 0.00256 & 718.4 & 14.8 & 976.4 & 22 \\
\hline 0.27238 & 0.45298 & 0.01002 & 2408.5 & 44.4 & 2469.1 & 31.8 \\
\hline 0.0297 & 0.10327 & 0.00238 & 633.5 & 14 & 633.4 & 19.8 \\
\hline 0.31568 & 0.51833 & 0.01126 & 2692.1 & 47.8 & 2628.5 & 31.6 \\
\hline 0.04614 & 0.17738 & 0.00382 & 1052.6 & 21 & 1015.2 & 22.4 \\
\hline 0.28324 & 0.48002 & 0.01032 & 2527.4 & 45 & 2534.3 & 31.2 \\
\hline 0.03306 & 0.10002 & 0.00246 & 614.5 & 14.4 & 598.8 & 22.2 \\
\hline 0.27266 & 0.46586 & 0.01002 & 2465.4 & 44 & 2496.1 & 31.2 \\
\hline 0.02506 & 0.10412 & 0.00226 & 638.5 & 13.2 & 640.1 & 17.2 \\
\hline 0.04816 & 0.1379 & 0.00332 & 832.8 & 18.8 & 858 & 25.8 \\
\hline 0.02672 & 0.09045 & 0.00214 & 558.2 & 12.6 & 545.9 & 19.2 \\
\hline 361.5033 & -0.6071 & 4.07758 & & & & \\
\hline 0.02816 & 0.10013 & 0.00232 & 615.2 & 13.6 & 603.3 & 19.4 \\
\hline 0.02682 & 0.07497 & 0.0017 & 466 & 10.2 & 627.6 & 18.6 \\
\hline 0.05346 & 0.09921 & 0.00278 & 609.8 & 16.4 & 772 & 30.6 \\
\hline 0.34502 & 0.48782 & 0.01066 & 2561.2 & 46.2 & 2692.9 & 32.2 \\
\hline 0.25134 & 0.41157 & 0.00886 & 2222.1 & 40.4 & 2408 & 31.2 \\
\hline 0.27358 & 0.45272 & 0.00978 & 2407.3 & 43.4 & 2480.8 & 31.6 \\
\hline 0.08338 & 0.1623 & 0.00488 & 969.5 & 27 & 933.4 & 39.4 \\
\hline 0.24926 & 0.44546 & 0.00948 & 2375 & 42.2 & 2410.5 & 30.8 \\
\hline 0.33086 & 0.47623 & 0.01138 & 2510.8 & 49.6 & 2551 & 35.2 \\
\hline 0.3126 & 0.47054 & 0.0115 & 2485.9 & 50.4 & 2467 & 36 \\
\hline 0.02816 & 0.10539 & 0.00238 & 645.9 & 13.8 & 636.5 & 19 \\
\hline 0.05754 & 0.16642 & 0.00404 & 992.4 & 22.4 & 960.9 & 27.8 \\
\hline
\end{tabular}




\begin{tabular}{|c|c|c|c|c|c|c|}
\hline 0.13522 & 0.26467 & 0.00568 & 1513.7 & 29 & 1845.4 & 29 \\
\hline 0.03512 & 0.12648 & 0.00284 & 767.8 & 16.2 & 768.7 & 21 \\
\hline 0.0373 & 0.1092 & 0.00268 & 668.1 & 15.6 & 675.9 & 23.4 \\
\hline 0.05788 & 0.11852 & 0.00354 & 722 & 20.4 & 703.4 & 34 \\
\hline 0.31006 & 0.48707 & 0.01074 & 2558 & 46.6 & 2564.9 & 32.8 \\
\hline 0.05058 & 0.14149 & 0.00352 & 853.1 & 19.8 & 837 & 27.2 \\
\hline 0.03312 & 0.12646 & 0.0028 & 767.6 & 16 & 758.4 & 20.2 \\
\hline 0.287 & 0.46956 & 0.01012 & 2481.6 & 44.4 & 2512.8 & 31.8 \\
\hline 0.08422 & 0.16866 & 0.00386 & 1004.7 & 21.2 & 1363 & 28.6 \\
\hline 0.3247 & 0.46093 & 0.00986 & 2443.7 & 43.6 & 2636.4 & 32 \\
\hline 76.2161 & -0.47738 & 0.89392 & & & & \\
\hline 0.25876 & 0.43752 & 0.00938 & 2339.5 & 42 & 2418.1 & 31.6 \\
\hline 0.29074 & 0.46421 & 0.01024 & 2458.1 & 45 & 2495.5 & 32.6 \\
\hline 0.15776 & 0.35713 & 0.00776 & 1968.6 & 36.8 & 1948.8 & 30.4 \\
\hline 36.13388 & 8.22324 & 0.29486 & & & & \\
\hline 0.12456 & 0.18847 & 0.00434 & 1113.1 & 23.6 & 1688.2 & 31 \\
\hline 0.02664 & 0.11025 & 0.0024 & 674.2 & 14 & 660 & 17.8 \\
\hline 1.69364 & 0.07885 & 0.0811 & & & & \\
\hline 0.2407 & 0.39134 & 0.00878 & 2129 & 40.6 & 2294.3 & 33 \\
\hline 0.32754 & 0.49874 & 0.01116 & 2608.4 & 48 & 2586 & 33.6 \\
\hline 0.25204 & 0.38469 & 0.0083 & 2098.2 & 38.6 & 2375.6 & 31.8 \\
\hline 0.02414 & 0.0957 & 0.00208 & 589.2 & 12.2 & 621.6 & 16.8 \\
\hline 0.17588 & 0.34795 & 0.00768 & 1924.8 & 36.8 & 2019.8 & 31.4 \\
\hline 0.28924 & 0.44341 & 0.0097 & 2365.9 & 43.4 & 2485.4 & 32.8 \\
\hline 6.7263 & 1.11878 & 0.05548 & & & & \\
\hline 0.0421 & 0.14028 & 0.00324 & 846.2 & 18.4 & 821.5 & 23.6 \\
\hline 0.22038 & 0.34333 & 0.00744 & 1902.7 & 35.8 & 2241.4 & 31.8 \\
\hline 0.29784 & 0.46692 & 0.01012 & 2470 & 44.4 & 2517.5 & 32.6 \\
\hline 0.35656 & 0.50326 & 0.01182 & 2627.8 & 50.6 & 2606.7 & 35.6 \\
\hline \multirow[t]{2}{*}{0.02992} & 0.11543 & 0.00256 & 704.2 & 14.8 & 699.8 & 19.2 \\
\hline & & 238.97634 & & & & \\
\hline 234.80584 & 0.47351 & 2.90638 & & & & \\
\hline 0.37218 & 0.53822 & 0.01176 & 2776 & 49.2 & 2713.5 & 33.4 \\
\hline 0.11512 & 0.24866 & 0.00594 & 1431.6 & 30.6 & 1533.8 & 32.4 \\
\hline 0.0561 & 0.16144 & 0.00384 & 964.8 & 21.4 & 974.1 & 26.8 \\
\hline 0.03414 & 0.12264 & 0.00268 & 745.7 & 15.4 & 785.9 & 20.2 \\
\hline 0.32012 & 0.46347 & 0.01048 & 2454.9 & 46.2 & 2530.3 & 34.4 \\
\hline 0.1478 & 0.10642 & 0.00614 & 651.9 & 35.8 & 654.8 & 86 \\
\hline 0.0327 & 0.11068 & 0.00258 & 676.7 & 15 & 669 & 21 \\
\hline 0.11576 & 0.23615 & 0.0056 & 1366.7 & 29.2 & 1547.1 & 32.2 \\
\hline 0.31522 & 0.45367 & 0.01018 & 2411.5 & 45.2 & 2517.9 & 34.2 \\
\hline 0.06472 & 0.15289 & 0.00398 & 917.1 & 22.2 & 946.5 & 31.2 \\
\hline 0.36624 & 0.51743 & 0.01136 & 2688.3 & 48.2 & 2676.4 & 34 \\
\hline \multirow[t]{2}{*}{0.3675} & 0.50951 & 0.01184 & 2654.5 & 50.6 & 2625.9 & 35.6 \\
\hline & -0.05533 & 0.69806 & & & & \\
\hline 0.047 & 0.12608 & 0.00278 & 765.5 & 16 & 970.3 & 23.2 \\
\hline 0.33546 & 0.49157 & 0.01096 & 2577.5 & 47.4 & 2574.5 & 34.4 \\
\hline 0.24566 & 0.32203 & 0.00764 & 1799.6 & 37.2 & 2235 & 35.4 \\
\hline 0.0276 & 0.10706 & 0.00238 & 655.7 & 13.8 & 651.3 & 18.4 \\
\hline 0.26012 & 0.37327 & 0.00814 & 2044.8 & 38.2 & 2354.8 & 33 \\
\hline 0.11248 & 0.2455 & 0.00558 & 1415.2 & 28.8 & 1564 & 30.8 \\
\hline 65.3931 & -0.13549 & 0.81294 & & & & \\
\hline 128.9943 & 8.46201 & 1.12632 & & & & \\
\hline 0.07062 & 0.17877 & 0.00446 & 1060.3 & 24.4 & 1062.3 & 30.6 \\
\hline
\end{tabular}




$\begin{array}{ccccccc}0.05594 & 0.14457 & 0.00362 & 870.5 & 20.4 & 898.1 & 28.4 \\ 10.26214 & 0.14503 & 0.12102 & & & & \\ 0.04458 & 0.15004 & 0.00336 & 901.2 & 18.8 & 902.7 & 23.2 \\ 0.2568 & 0.39127 & 0.00862 & 2128.7 & 40 & 2320.2 & 33.6 \\ 0.03646 & 0.098 & 0.00238 & 602.7 & 14 & 681.1 & 23 \\ 0.26854 & 0.3702 & 0.00816 & 2030.3 & 38.4 & 2357.1 & 33.8 \\ 0.03128 & 0.09936 & 0.00238 & 610.7 & 14 & 610.1 & 21 \\ 0.32714 & 0.47038 & 0.01048 & 2485.2 & 46 & 2529.2 & 34.8 \\ 0.12564 & 0.13496 & 0.00354 & 816.1 & 20.2 & 1540.9 & 36 \\ 0.34206 & 0.45409 & 0.01026 & 2413.4 & 45.4 & 2555 & 35.4 \\ 0.0507 & 0.13732 & 0.00314 & 829.5 & 17.8 & 956.7 & 25 \\ 0.06838 & 0.15947 & 0.00426 & 953.8 & 23.6 & 938.1 & 32.8 \\ 0.07392 & 0.16013 & 0.0044 & 957.5 & 24.4 & 967.2 & 34.4 \\ 0.03214 & 0.10931 & 0.00254 & 668.7 & 14.8 & 655.8 & 20.8 \\ 0.08136 & 0.15662 & 0.00396 & 938 & 22 & 1172.8 & 32.2 \\ 0.13296 & 0.22095 & 0.00488 & 1286.9 & 25.8 & 1723.2 & 31.2 \\ 0.03896 & 0.11458 & 0.00282 & 699.3 & 16.4 & 686.3 & 24 \\ 0.07142 & 0.10196 & 0.00378 & 625.9 & 22.2 & 610.7 & 44.6 \\ 0.35562 & 0.49139 & 0.0111 & 2576.7 & 48 & 2579.3 & 35.8 \\ 0.08332 & 0.14444 & 0.00324 & 869.7 & 18.2 & 1334.8 & 28.6 \\ 0.03166 & 0.09662 & 0.00238 & 594.6 & 14 & 582.4 & 21.6 \\ 0.03042 & 0.10915 & 0.00248 & 667.8 & 14.4 & 660.3 & 19.8 \\ 0.44476 & 0.49409 & 0.01416 & 2588.3 & 61 & 2576.2 & 44.6 \\ 0.03266 & 0.11144 & 0.00256 & 681.1 & 14.8 & 678.1 & 20.8 \\ 0.3712 & 0.50599 & 0.01132 & 2639.5 & 48.4 & 2619.2 & 35.8\end{array}$




\begin{tabular}{|c|c|c|c|c|c|}
\hline age $207 / 206$ & $2 \sigma$ age 76 & $\begin{array}{l}\text { discordance } \\
\Delta 68-75[\%]\end{array}$ & $\Delta 68-76[\%]$ & $\begin{array}{c}\text { preferred age } \\
\text { age }\end{array}$ & $2 \sigma$ age \\
\hline 2833.4 & 36 & -1.4 & -2.4 & 2833.4 & 36 \\
\hline 2441.4 & 37 & -23.2 & -39.7 & & \\
\hline 2499.4 & 37 & -19.3 & -33.1 & & \\
\hline 2595.4 & 39 & -2.5 & -4.4 & 2595.4 & 39 \\
\hline 2617.3 & 40.6 & -6.5 & -11.3 & 2617.3 & 40.6 \\
\hline 578.9 & 54.2 & 1 & 4.6 & 605.7 & 13.8 \\
\hline 702.8 & 58.2 & -17 & -66.4 & & \\
\hline 1071.7 & 57.2 & -1.5 & -4.5 & 1023.7 & 23.4 \\
\hline 2608.3 & 36.8 & -0.9 & -1.7 & 2608.3 & 36.8 \\
\hline 2584.1 & 37.6 & -6.7 & -11.7 & 2584.1 & 37.6 \\
\hline 921.9 & 56 & -0.5 & -1.8 & 905.7 & 20.6 \\
\hline 816.2 & 54.2 & -1.5 & -5.5 & 771.4 & 17.4 \\
\hline 996.4 & 68 & -2.5 & -8 & 917.1 & 22 \\
\hline 672.4 & 51.2 & -2.5 & -10.7 & 600.2 & 13.6 \\
\hline 698.1 & 56.4 & -1 & -4.2 & 669.1 & 15.2 \\
\hline 706.5 & 57.4 & 0.6 & 2.5 & 724.4 & 16.4 \\
\hline 656.4 & 55.2 & 0.3 & 1.3 & 664.8 & 15 \\
\hline 3138.8 & 34.6 & -0.9 & -1.4 & 3138.8 & 34.6 \\
\hline 2598 & 36.2 & -3.1 & -5.5 & 2598 & 36.2 \\
\hline 733.3 & 52.4 & -1.7 & -6.9 & 682.6 & 15.4 \\
\hline 2558 & 36.4 & -4.2 & -7.4 & 2558 & 36.4 \\
\hline 745.9 & 51.4 & 0.1 & 0.4 & 748.9 & 16.8 \\
\hline 2581.9 & 37.6 & -4.3 & -7.5 & 2581.9 & 37.6 \\
\hline 863.7 & 47.6 & -3.4 & -11.9 & 760.5 & 16.8 \\
\hline 1031.6 & 68 & -2.4 & -7.6 & 953.1 & 23 \\
\hline 1442 & 364.4 & -47.7 & -97.6 & & \\
\hline 2529.4 & 36.8 & -13.8 & -23.9 & & \\
\hline 2695.9 & 35.4 & -20.9 & -34.4 & & \\
\hline 2639.6 & 37.2 & -1.3 & -2.3 & 2639.6 & 37.2 \\
\hline 804.8 & 80.6 & -4.1 & -15.3 & 681.9 & 17.2 \\
\hline 672.4 & 62.2 & 0.3 & 1.2 & 680.7 & 15.8 \\
\hline 2616.6 & 36 & -7 & -12.2 & 2616.6 & 36 \\
\hline 665.1 & 101.2 & -3.2 & -14.1 & 571.4 & 15.4 \\
\hline 476.4 & 94.6 & 4.2 & 24.9 & 595.1 & 15.2 \\
\hline 2559.7 & 38 & -3.6 & -6.3 & 2559.7 & 38 \\
\hline 2564.6 & 38 & -3.3 & -5.9 & 2564.6 & 38 \\
\hline 2593.5 & 36 & -4.2 & -7.5 & 2593.5 & 36 \\
\hline 2500.3 & 37.2 & -7.6 & -13.5 & 2500.3 & 37.2 \\
\hline 656.4 & 100.4 & 0.6 & 2.8 & 674.5 & 18 \\
\hline 651.5 & 147.6 & 0.4 & 1.6 & 661.7 & 20.8 \\
\hline 974 & 61 & -7 & -22.2 & 757.9 & 17.8 \\
\hline 2530 & 37 & -9.1 & -16 & & \\
\hline 606.1 & 56.8 & 1.5 & 7.1 & 649.1 & 14.8 \\
\hline 767.2 & 62.4 & -4.6 & -17.7 & 631.3 & 14.8 \\
\hline 2627.3 & 36 & -6.3 & -11 & 2627.3 & 36 \\
\hline 2566.7 & 36.4 & -1.8 & -3.2 & 2566.7 & 36.4 \\
\hline 664.8 & 68.8 & 0.2 & 0.7 & 669.6 & 16 \\
\hline 2569.8 & 36 & -7.7 & -13.5 & 2569.8 & 36 \\
\hline 2561.5 & 36.8 & -3.7 & -6.6 & 2561.5 & 36.8 \\
\hline
\end{tabular}




\begin{tabular}{|c|c|c|c|c|c|}
\hline 795.9 & 90 & -0.7 & -2.7 & 774 & 20.2 \\
\hline 646.3 & 58.2 & 0.5 & 2.2 & 660.7 & 15.2 \\
\hline 2631.6 & 39.2 & -5 & -8.8 & 2631.6 & 39.2 \\
\hline 2632.1 & 36.8 & -9.4 & -16.3 & & \\
\hline 833.4 & 56.2 & -0.1 & -0.5 & 829 & 18.8 \\
\hline 797.8 & 61.8 & -3.7 & -13.9 & 687.1 & 16.2 \\
\hline 617.9 & 63.6 & 2.1 & 9.6 & 677.2 & 15.8 \\
\hline 2303.7 & 38 & -47.1 & -75.8 & & \\
\hline 638.5 & 65 & -1.3 & -5.9 & 600.6 & 14.2 \\
\hline 2094.9 & 38.2 & -23.4 & -43.1 & & \\
\hline 2589.3 & 36.4 & -3.6 & -6.4 & 2589.3 & 36.4 \\
\hline 909.5 & 61.8 & -1.1 & -3.8 & 874.9 & 20.4 \\
\hline 2873 & 35 & -13.4 & -21.9 & & \\
\hline 2550.4 & 38 & -9.1 & -16 & & \\
\hline 2590.4 & 39 & -10.2 & -17.6 & & \\
\hline 1018.4 & 78.6 & -4.9 & -15.2 & 863.6 & 21.8 \\
\hline 680.3 & 99 & -3.4 & -14.5 & 581.6 & 15.6 \\
\hline 779.8 & 81.2 & -1.5 & -5.8 & 734.4 & 18.6 \\
\hline 709.6 & 63.4 & -1.1 & -4.6 & 677.2 & 16 \\
\hline 621.9 & 59.8 & -0.5 & -2.4 & 607 & 14 \\
\hline 3191 & 34.4 & -0.1 & -0.1 & 3191 & 34.4 \\
\hline 2480.7 & 37.2 & -16.3 & -28.3 & & \\
\hline 2564.8 & 36.8 & -3.5 & -6.2 & 2564.8 & 36.8 \\
\hline 2589.2 & 37.6 & -2.9 & -5.1 & 2589.2 & 37.6 \\
\hline 2597.4 & 36.4 & -3.4 & -6 & 2597.4 & 36.4 \\
\hline 896.9 & 48.4 & -3.2 & -10.9 & 799.3 & 17.8 \\
\hline 1607.8 & 42.4 & -17.6 & -38.2 & & \\
\hline 819.3 & 56 & 0.8 & 3.1 & 844.4 & 19.2 \\
\hline 685.5 & 69.8 & -1.6 & -7.1 & 636.9 & 15.4 \\
\hline 3164.4 & 34.2 & -11.6 & -18.4 & & \\
\hline 2565.5 & 38.2 & -3.3 & -5.8 & 2565.5 & 38.2 \\
\hline 1068.5 & 70.8 & -5.7 & -17 & 886.5 & 22 \\
\hline 475.6 & 177.4 & 4.9 & 29.2 & 614.6 & 20.2 \\
\hline 2583.5 & 37.2 & -6.2 & -10.9 & 2583.5 & 37.2 \\
\hline 1924.8 & 46.2 & -16.7 & -32.9 & & \\
\hline 2370.2 & 37.4 & -35.9 & -59.5 & & \\
\hline 2459.8 & 38.6 & -8.3 & -14.8 & 2459.8 & 38.6 \\
\hline 2354.3 & 37.6 & -7.4 & -13.5 & 2354.3 & 37.6 \\
\hline 731 & 67.8 & -0.4 & -1.7 & 718.2 & 17.2 \\
\hline 2604.5 & 37.8 & -2 & -3.6 & 2604.5 & 37.8 \\
\hline 2513.3 & 39.6 & -14.9 & -25.8 & & \\
\hline 2582.6 & 41.2 & -9.3 & -16.1 & & \\
\hline 2484.5 & 38.2 & -7.3 & -13.1 & 2484.5 & 38.2 \\
\hline 2516.1 & 38.4 & -4.9 & -8.7 & 2516.1 & 38.4 \\
\hline 716.3 & 51.8 & -1.8 & -7.5 & 662.6 & 15 \\
\hline 1230.6 & 64.2 & -16.1 & -41.7 & & \\
\hline 1013.4 & 53.4 & 0 & 0 & 1013.4 & 22.8 \\
\hline 620.8 & 62 & -0.1 & -0.4 & 618.3 & 14.4 \\
\hline 670.7 & 67 & 0.2 & 0.8 & 676.2 & 16.2 \\
\hline 2593.2 & 39.8 & -13.6 & -23.3 & & \\
\hline 2562.3 & 40.4 & -2.8 & -5.1 & 2562.3 & 40.4 \\
\hline 837.2 & 59 & -1.5 & -5.6 & 790 & 18.2 \\
\hline 2481 & 36.6 & -24 & -40.5 & & \\
\hline
\end{tabular}




\begin{tabular}{|c|c|c|c|c|c|}
\hline 678.2 & 62.6 & 0.4 & 1.8 & 690.3 & 16.2 \\
\hline 2790.1 & 37.2 & -13.1 & -21.8 & & \\
\hline 1136.1 & 51.2 & -15.7 & -43.2 & & \\
\hline 1045 & 64.2 & -11.3 & -33.5 & 694.4 & 16.8 \\
\hline 2598.6 & 37.6 & -4.7 & -8.3 & 2598.6 & 37.6 \\
\hline 695 & 53.2 & -0.6 & -2.8 & 675.7 & 15.4 \\
\hline 2575.8 & 37.6 & -6.3 & -11 & 2575.8 & 37.6 \\
\hline 2603.2 & 38.6 & -0.7 & -1.3 & 2603.2 & 38.6 \\
\hline 1018.7 & 48.8 & -11.8 & -35.5 & 656.9 & 15 \\
\hline 2571.8 & 37.4 & -4.4 & -7.8 & 2571.8 & 37.4 \\
\hline 2582 & 44.8 & -0.7 & -1.4 & 2582 & 44.8 \\
\hline 2334.6 & 38.6 & -29 & -49.7 & & \\
\hline 2531.3 & 38.8 & -8 & -14.2 & 2531.3 & 38.8 \\
\hline 662.3 & 93.8 & -0.1 & -0.6 & 658.4 & 17.4 \\
\hline 929.5 & 61.6 & 1.4 & 4.6 & 972.4 & 22.8 \\
\hline 2644.4 & 36.6 & -8.2 & -14.2 & 2644.4 & 36.6 \\
\hline 665.1 & 76.4 & 1.3 & 5.8 & 704 & 17.2 \\
\hline 2582.5 & 38.4 & -3.7 & -6.5 & 2582.5 & 38.4 \\
\hline 594.9 & 63 & 0.2 & 0.8 & 599.9 & 14 \\
\hline 2632.8 & 37.4 & -3.2 & -5.6 & 2632.8 & 37.4 \\
\hline 621.5 & 55 & 0 & -0.2 & 620.1 & 14.2 \\
\hline 2423.2 & 60.6 & -18.5 & -32.2 & & \\
\hline 2692 & 37.2 & -21 & -34.6 & & \\
\hline 2390.9 & 42.4 & -40.5 & -65.8 & & \\
\hline 990.2 & 55.8 & -1.3 & -4.2 & 948.9 & 21.6 \\
\hline 2595.2 & 38.2 & -0.9 & -1.6 & 2595.2 & 38.2 \\
\hline 620.4 & 75.6 & 0.9 & 4 & 645.2 & 15.8 \\
\hline 773.7 & 107.6 & -3.6 & -13.7 & 667.7 & 18.8 \\
\hline 682 & 53.6 & -0.7 & -3.3 & 659.6 & 15 \\
\hline 2304.3 & 77 & -14.7 & -26.5 & & \\
\hline 989.3 & 55.2 & -1.1 & -3.7 & 952.4 & 21.6 \\
\hline 2601.2 & 37 & -5 & -8.8 & 2601.2 & 37 \\
\hline 2520.1 & 37.4 & -8 & -14.1 & 2520.1 & 37.4 \\
\hline 1399.8 & 45.4 & -24.9 & -57.3 & & \\
\hline 738.7 & 84.8 & -2.7 & -10.7 & 659.3 & 16.8 \\
\hline 749.5 & 65.8 & -2 & -8 & 689.3 & 16.4 \\
\hline 683.1 & 86.4 & 0.9 & 3.6 & 707.4 & 18.2 \\
\hline 903.5 & 74 & 1.7 & 5.8 & 955.8 & 23.6 \\
\hline 2600.6 & 37.8 & -4.2 & -7.4 & 2600.6 & 37.8 \\
\hline 2489.7 & 37.4 & -22.3 & -37.8 & & \\
\hline 2603.5 & 37.8 & -3.6 & -6.4 & 2603.5 & 37.8 \\
\hline 989.3 & 48.6 & -1.9 & -6.1 & 928.5 & 20.6 \\
\hline 2482 & 38.2 & -17.4 & -30 & & \\
\hline 2733.1 & 37 & -48.1 & -71.9 & & \\
\hline 604.3 & 64.8 & 2.9 & 13.7 & 687.3 & 14.2 \\
\hline 1365.4 & 57 & -16.3 & -39.5 & & \\
\hline 2548.6 & 41.8 & -3.7 & -6.6 & 2548.6 & 41.8 \\
\hline 706.9 & 84.4 & -0.6 & -2.6 & 688.5 & 16 \\
\hline 1285.4 & 51.2 & -18.1 & -45.3 & & \\
\hline 1635.9 & 76.8 & -23.1 & -48.7 & & \\
\hline 765.9 & 70.2 & -0.1 & -0.4 & 763 & 16.4 \\
\hline 524.5 & 95.6 & 5.3 & 29.1 & & \\
\hline 782.1 & 74 & -3.4 & -13 & 680.5 & 15 \\
\hline
\end{tabular}




\begin{tabular}{|c|c|c|c|c|c|}
\hline 2388.1 & 45 & -14.3 & -25.4 & & \\
\hline 964.9 & 60.2 & 0.1 & 0.2 & 966.8 & 19.8 \\
\hline 1852.7 & 52.4 & -38.6 & -71.5 & & \\
\hline 2075.4 & 49.2 & -37.3 & -65.5 & & \\
\hline 635.7 & 73.6 & 0.9 & 3.9 & 660.4 & 14.4 \\
\hline 46.7 & 83.4 & 22.4 & 1290.5 & & \\
\hline 2560.2 & 41 & -7.2 & -12.6 & 2560.2 & 41 \\
\hline 640.6 & 77.6 & 3.2 & 14.7 & 734.9 & 16.2 \\
\hline 2576.7 & 41.6 & -2 & -3.6 & 2576.7 & 41.6 \\
\hline 808.3 & 63.4 & 1 & 3.5 & 836.9 & 17.4 \\
\hline 2386.4 & 41.2 & -8.2 & -14.9 & 2386.4 & 41.2 \\
\hline 2584.9 & 42.8 & -5.3 & -9.4 & 2584.9 & 42.8 \\
\hline 684.4 & 89 & 0.2 & 0.8 & 689.6 & 16.2 \\
\hline 1925.9 & 50.8 & 1.3 & 2.7 & 1925.9 & 50.8 \\
\hline 2537.3 & 42.4 & -5.1 & -9.1 & 2537.3 & 42.4 \\
\hline 808.9 & 152.6 & -3.4 & -12.8 & 705.5 & 22.8 \\
\hline 2514 & 41.2 & -0.8 & -1.4 & 2514 & 41.2 \\
\hline 2122.7 & 44.4 & -17.7 & -33 & & \\
\hline 806.7 & 78.6 & 0.9 & 3.5 & 834.5 & 18.8 \\
\hline 662.3 & 70.8 & 2.8 & 12.4 & 744.6 & 16 \\
\hline 3995.5 & 633.6 & -88.8 & -99.9 & & \\
\hline 1121.4 & 60.6 & -17.3 & -47.7 & & \\
\hline 479.9 & 124.8 & 6.9 & 41 & & \\
\hline 739 & 87.4 & -3.5 & -14 & 635.2 & 15 \\
\hline 1671.7 & 74.2 & 18.3 & 43.7 & & \\
\hline 1613.6 & 51.4 & -26.4 & -55.5 & & \\
\hline 2519.8 & 44.2 & -2.5 & -4.4 & 2519.8 & 44.2 \\
\hline 633.6 & 75.8 & 0 & 0 & 633.5 & 14 \\
\hline 2580.5 & 43 & 2.4 & 4.3 & 2580.5 & 43 \\
\hline 936 & 56 & 3.7 & 12.5 & 1052.6 & 21 \\
\hline 2540.5 & 42.8 & -0.3 & -0.5 & 2540.5 & 42.8 \\
\hline 540.3 & 93 & 2.6 & 13.7 & 614.5 & 14.4 \\
\hline 2521.7 & 43.2 & -1.2 & -2.2 & 2521.7 & 43.2 \\
\hline 646.6 & 62.4 & -0.3 & -1.3 & 638.5 & 13.2 \\
\hline 924.5 & 77 & -2.9 & -9.9 & 832.8 & 18.8 \\
\hline 495.8 & 85.6 & 2.2 & 12.6 & 558.2 & 12.6 \\
\hline 559.7 & 77.8 & 2 & 9.9 & 615.2 & 13.6 \\
\hline 1264 & 63.2 & -25.7 & -63.1 & & \\
\hline 1276.3 & 96 & -21 & -52.2 & & \\
\hline 2793.8 & 43 & -4.9 & -8.3 & 2793.8 & 43 \\
\hline 2569.7 & 43.8 & -7.7 & -13.5 & 2569.7 & 43.8 \\
\hline 2542.1 & 44 & -3 & -5.3 & 2542.1 & 44 \\
\hline 849.9 & 119 & 3.9 & 14.1 & 969.5 & 27 \\
\hline 2441.2 & 43.8 & -1.5 & -2.7 & 2441.2 & 43.8 \\
\hline 2583.6 & 48.8 & -1.6 & -2.8 & 2583.6 & 48.8 \\
\hline 2452 & 51 & 0.8 & 1.4 & 2452 & 51 \\
\hline 603.6 & 71.4 & 1.5 & 7 & 645.9 & 13.8 \\
\hline 890.3 & 77.6 & 3.3 & 11.5 & 992.4 & 22.4 \\
\hline
\end{tabular}




\begin{tabular}{|c|c|c|c|c|c|}
\hline 2243.8 & 46 & -18 & -32.5 & & \\
\hline 772.1 & 66.8 & -0.1 & -0.6 & 767.8 & 16.2 \\
\hline 702.5 & 86.8 & -1.2 & -4.9 & 668.1 & 15.6 \\
\hline 645.2 & 129.2 & 2.6 & 11.9 & 722 & 20.4 \\
\hline 2570.9 & 45.4 & -0.3 & -0.5 & 2570.9 & 45.4 \\
\hline 795.2 & 85.6 & 1.9 & 7.3 & 853.1 & 19.8 \\
\hline 732 & 64.6 & 1.2 & 4.9 & 767.6 & 16 \\
\hline 2538.5 & 44.6 & -1.2 & -2.2 & 2538.5 & 44.6 \\
\hline 1980.6 & 54.4 & -26.3 & -49.3 & & \\
\hline 2788.5 & 43.2 & -7.3 & -12.4 & 2788.5 & 43.2 \\
\hline 2485.6 & 45 & -3.2 & -5.9 & 2485.6 & 45 \\
\hline 2526.7 & 46 & -1.5 & -2.7 & 2526.7 & 46 \\
\hline 1928.6 & 49.2 & 1 & 2.1 & 1928.6 & 49.2 \\
\hline 2502.4 & 50.4 & -34.1 & -55.5 & & \\
\hline 612.6 & 63.8 & 2.1 & 10.1 & 674.2 & 14 \\
\hline 2445.5 & 48.2 & -7.2 & -12.9 & 2445.5 & 48.2 \\
\hline 2569.1 & 46.8 & 0.9 & 1.5 & 2569.1 & 46.8 \\
\hline 2623.5 & 45.2 & -11.7 & -20 & & \\
\hline 742 & 61.4 & -5.2 & -20.6 & 589.2 & 12.2 \\
\hline 2119 & 49.6 & -4.7 & -9.2 & 2119 & 49.6 \\
\hline 2585.1 & 46.2 & -4.8 & -8.5 & 2585.1 & 46.2 \\
\hline 755.8 & 73.4 & 3 & 12 & 846.2 & 18.4 \\
\hline 2567.6 & 46.2 & -15.1 & -25.9 & & \\
\hline 2556.6 & 46.2 & -1.9 & -3.4 & 2556.6 & 46.2 \\
\hline 2591 & 49.6 & 0.8 & 1.4 & 2591 & 49 \\
\hline 686.5 & 65.8 & 0.6 & 2.6 & 704.2 & 14.8 \\
\hline 2667.9 & 46.2 & 2.3 & 4.1 & 2667.9 & 46.2 \\
\hline 1678.5 & 61.8 & -6.7 & -14.7 & 1678.5 & 61.8 \\
\hline 996.1 & 72.4 & -1 & -3.1 & 964.8 & 21.4 \\
\hline 902.3 & 61.4 & -5.1 & -17.4 & 745.7 & 15.4 \\
\hline 2591.9 & 48.6 & -3 & -5.3 & 2591.9 & 48.6 \\
\hline 665.5 & 360.6 & -0.4 & -2 & 651.9 & 35.8 \\
\hline 644.2 & 77.4 & 1.1 & 5.1 & 676.7 & 15 \\
\hline 1803.7 & 60 & -11.7 & -24.2 & & \\
\hline 2605.4 & 48.6 & -4.2 & -7.4 & 2605.4 & 48 \\
\hline 1016.2 & 88.2 & -3.1 & -9.7 & 917.1 & 22.2 \\
\hline 2668.1 & 47.4 & 0.4 & 0.8 & 2668.1 & 47.4 \\
\hline 2604.5 & 50.2 & 1.1 & 1.9 & 2604.5 & 50.2 \\
\hline 1469.2 & 56.8 & -21.1 & -47.9 & & \\
\hline 2572.8 & 48.8 & 0.1 & 0.2 & 2572.8 & 48.8 \\
\hline 2662.3 & 51.8 & -19.5 & -32.4 & & \\
\hline 637.1 & 67 & 0.7 & 2.9 & 655.7 & 13.8 \\
\hline 2635.9 & 47.8 & -13.2 & -22.4 & & \\
\hline 1771.9 & 57 & -9.5 & -20.1 & & \\
\hline 1067.2 & 78.8 & -0.2 & -0.6 & 1060.3 & 24.4 \\
\hline
\end{tabular}




$\begin{array}{cccccc}967.5 & 83 & -3.1 & -10 & 870.5 & 20.4 \\ 907.4 & 65.4 & -0.2 & -0.7 & 901.2 & 18.8 \\ 2493.8 & 49.6 & -8.3 & -14.6 & 2493.8 & 49.6 \\ 950.5 & 81 & -11.5 & -36.6 & 602.7 & 14 \\ 2653.9 & 49.2 & -13.9 & -23.5 & & \\ 608.6 & 84.8 & 0.1 & 0.3 & 610.7 & 14 \\ 2565.2 & 50.2 & -1.7 & -3.1 & 2565.2 & 50.2 \\ 2754.7 & 61 & -47 & -70.4 & & \\ 2670 & 50.4 & -5.5 & -9.6 & 2670 & 50.4 \\ 1262.6 & 64.8 & -13.3 & -34.3 & 829.5 & 17.8 \\ 902 & 96 & 1.7 & 5.7 & 953.8 & 23.6 \\ 989.9 & 97.6 & -1 & -3.3 & 957.5 & 24.4 \\ 612.2 & 78.2 & 2 & 9.2 & 668.7 & 14.8 \\ 1637.2 & 72 & -20 & -42.7 & & \\ 2304.3 & 52 & -25.3 & -44.2 & & \\ 644.5 & 89.2 & 1.9 & 8.5 & 699.3 & 16.4 \\ 555.6 & 196.2 & 2.5 & 12.6 & 625.9 & 22.2 \\ 2582 & 51.4 & -0.1 & -0.2 & 2582 & 51.4 \\ 2186.8 & 54.4 & -34.8 & -60.2 & & \\ 535.8 & 92.6 & 2.1 & 11 & 594.6 & 14 \\ 635.4 & 72.8 & 1.1 & 5.1 & 667.8 & 14.4 \\ 2567.3 & 63.8 & 0.5 & 0.8 & 2567.3 & 63.8 \\ 669.3 & 75.4 & 0.4 & 1.8 & 681.1 & 14.8 \\ 2604.1 & 51.4 & 0.8 & 1.4 & 2604.1 & 51.4\end{array}$


Sample 24 Sobat @ Nasser 52 grain analysed 39 concordant ages $\quad 75.0 \%$

\begin{tabular}{|c|c|c|c|c|c|c|}
\hline \multirow[b]{2}{*}{ grain } & \multicolumn{3}{|c|}{ concentrations } & \multicolumn{3}{|l|}{ isotopic ratios } \\
\hline & U [ppm] & $\mathrm{Pb}$ [ppm] & $\mathrm{Th} / \mathrm{U}$ & Pb207/Pb206 & $2 \sigma 76$ & Pb207/U235 \\
\hline X24_G001 & 629.5 & 32 & 0.1014 & 0.05302 & 0.0019 & 0.39256 \\
\hline X24_G002 & & & & 0.65344 & 6.06476 & 28.29853 \\
\hline X24 G003 & 142.8 & 18 & 0.5237 & 0.0638 & 0.00282 & 1.04274 \\
\hline X24_G004 & 416.7 & 38.3 & 0.1682 & 0.06094 & 0.00204 & 0.80873 \\
\hline X24_G005 & 458.1 & 63.5 & 0.5703 & 0.06295 & 0.00196 & 1.10179 \\
\hline X24_G006 & 1752 & 95.3 & 1.1919 & 0.10059 & 0.00292 & 0.64784 \\
\hline X24_G007 & 1121.1 & 124.4 & 0.849 & 0.05646 & 0.00168 & 0.73584 \\
\hline X24G008 & 867.9 & 91.7 & 0.8186 & 0.06323 & 0.00194 & 0.79444 \\
\hline X24_G009 & 155.6 & 15.7 & 0.0767 & 0.05837 & 0.00254 & 0.86845 \\
\hline X24_G010 & 182.7 & 20.2 & 0.0618 & 0.07399 & 0.00288 & 1.18459 \\
\hline X24_G011 & 263 & 38.9 & 1.0559 & 0.06281 & 0.00226 & 1.06483 \\
\hline X24_G012 & 120.7 & 12 & 0.0915 & 0.05737 & 0.00298 & 0.83044 \\
\hline X24_G013 & 285.7 & 33.6 & 0.299 & 0.06171 & 0.0023 & 0.98244 \\
\hline X24_G014 & 1449.6 & 122.8 & 0.5496 & 0.1044 & 0.00296 & 1.10974 \\
\hline X24_G015 & 299.5 & 29.6 & 0.3044 & 0.05481 & 0.00224 & 0.73818 \\
\hline X24_G016 & 15.8 & 8.5 & 0.5739 & 0.16801 & 0.00852 & 10.79651 \\
\hline X24_G017 & 311.8 & 39.9 & 0.6416 & 0.06693 & 0.00246 & 1.06808 \\
\hline X24_G018 & 240.9 & 27.3 & 0.7461 & 0.05476 & 0.00228 & 0.7522 \\
\hline X24_G019 & 1037.8 & 134.5 & 0.3693 & 0.06438 & 0.0019 & 1.11646 \\
\hline X24_G020 & 140.4 & 17.6 & 0.3769 & 0.06538 & 0.00284 & 1.09351 \\
\hline X24_G021 & 1218.6 & 98.6 & 0.2377 & 0.06965 & 0.00208 & 0.77533 \\
\hline X24_G022 & 391.1 & 68.3 & 1.1162 & 0.06707 & 0.00214 & 1.29448 \\
\hline X24_G023 & 938.3 & 104.2 & 0.5614 & 0.05676 & 0.00178 & 0.80094 \\
\hline X24_G024 & 237.9 & 120.2 & 0.3809 & 0.16623 & 0.00462 & 10.5476 \\
\hline X24_G025 & 212.8 & 25.5 & 0.8927 & 0.0576 & 0.00238 & 0.80174 \\
\hline X24_G026 & 1012.7 & 94.2 & 0.1882 & 0.05592 & 0.00174 & 0.73601 \\
\hline X24_G027 & 114.8 & 17.8 & 0.7769 & 0.06174 & 0.00312 & 1.14656 \\
\hline X24_G028 & 198 & 21.2 & 0.7678 & 0.06089 & 0.00268 & 0.78487 \\
\hline X24_G029 & 589.1 & 101.9 & 1.0718 & 0.06435 & 0.002 & 1.24341 \\
\hline X24_G030 & 741.8 & 92.4 & 0.7394 & 0.06786 & 0.00214 & 1.05172 \\
\hline X24_G031 & 767.9 & 80.4 & 0.3208 & 0.05817 & 0.00188 & 0.85612 \\
\hline X24_G032 & 171.9 & 25.7 & 0.544 & 0.06334 & 0.00246 & 1.20275 \\
\hline X24_G033 & 362 & 38.1 & 0.1475 & 0.05987 & 0.00216 & 0.89725 \\
\hline X24_G034 & 2032.8 & 173.1 & 0.0787 & 0.06338 & 0.0019 & 0.782 \\
\hline X24_G035 & 813.7 & 131.5 & 0.703 & 0.0632 & 0.00188 & 1.24757 \\
\hline X24_G036 & 159.6 & 27 & 0.689 & 0.06241 & 0.00252 & 1.28474 \\
\hline X24_G037 & 192.1 & 29.3 & 0.4191 & 0.06456 & 0.0024 & 1.29016 \\
\hline X24_G038 & 1245.7 & 230.2 & 1.8547 & 0.06511 & 0.00206 & 1.12393 \\
\hline X24_G039 & 1443.2 & 206.2 & 2.6793 & 0.08682 & 0.0027 & 1.06632 \\
\hline X24_G040 & 241.4 & 37.6 & 0.2799 & 0.06685 & 0.00254 & 1.41871 \\
\hline X24_G041 & 631.5 & 82 & 0.3264 & 0.06069 & 0.002 & 1.06502 \\
\hline X24_G042 & 158.1 & 16.5 & 0.2415 & 0.06125 & 0.00308 & 0.88171 \\
\hline X24_G043 & 384.7 & 58.2 & 0.6556 & 0.06249 & 0.00214 & 1.16724 \\
\hline X24_G044 & 2300.7 & 212.7 & 0.2244 & 0.06151 & 0.00188 & 0.797 \\
\hline X24_G045 & 556.1 & 76.6 & 0.6581 & 0.06633 & 0.00218 & 1.16364 \\
\hline X24_G046 & 187.2 & 28.1 & 0.7672 & 0.06098 & 0.00254 & 1.23318 \\
\hline X24_G047 & & & & 0.82604 & 0.11426 & 259.33405 \\
\hline X24_G048 & 271.4 & 38.7 & 0.5575 & 0.05938 & 0.00226 & 1.07121 \\
\hline X24_G049 & 96.5 & 18.1 & 0.3743 & 0.0819 & 0.0036 & 2.01301 \\
\hline X24_G050 & 321.1 & 46.7 & 0.4698 & 0.06336 & 0.00224 & 1.20159 \\
\hline
\end{tabular}




$\begin{array}{lccccccc}1 & & & & & & \\ 2 & \text { X24_G051 } & 407.3 & 42.2 & 0.5232 & 0.11439 & 0.00372 & 1.52573 \\ 3 & \text { X24_G052 } & 367 & 55.2 & 0.4064 & 0.06914 & 0.00234 & 1.36853\end{array}$




\section{concordant}

\begin{tabular}{|c|c|c|c|c|c|c|}
\hline $2 \sigma 75$ & Pb206/U238 & 2068 & $\begin{array}{c}\text { ages } \\
\text { age } 206 / 238\end{array}$ & $2 \sigma$ age 68 & age $207 / 235$ & $2 \sigma$ age 75 \\
\hline 0.0138 & 0.05373 & 0.00126 & 337.4 & 7.8 & 336.2 & 12.2 \\
\hline 180.49908 & 0.31423 & 2.23982 & & & & \\
\hline 0.04496 & 0.11858 & 0.00312 & 722.4 & 18 & 725.3 & 26.6 \\
\hline 0.02672 & 0.09629 & 0.00226 & 592.6 & 13.2 & 601.7 & 18.6 \\
\hline 0.03412 & 0.12699 & 0.00292 & 770.7 & 16.8 & 754.2 & 20.6 \\
\hline 0.01866 & 0.04673 & 0.00106 & 294.4 & 6.6 & 507.1 & 14.8 \\
\hline 0.0219 & 0.09457 & 0.00214 & 582.5 & 12.6 & 560 & 16 \\
\hline 0.0243 & 0.09117 & 0.00208 & 562.5 & 12.2 & 593.7 & 17.2 \\
\hline 0.03682 & 0.10795 & 0.00278 & 660.8 & 16.2 & 634.7 & 23.8 \\
\hline 0.04512 & 0.11618 & 0.00294 & 708.5 & 17 & 793.4 & 25.6 \\
\hline 0.03788 & 0.12302 & 0.00298 & 747.9 & 17.2 & 736.2 & 22.8 \\
\hline 0.04178 & 0.10504 & 0.00292 & 643.9 & 17 & 613.9 & 27.2 \\
\hline 0.0361 & 0.11552 & 0.00282 & 704.7 & 16.2 & 694.9 & 22.4 \\
\hline 0.03144 & 0.07713 & 0.00174 & 479 & 10.4 & 758 & 19.4 \\
\hline 0.02966 & 0.09772 & 0.00244 & 601 & 14.4 & 561.3 & 20.6 \\
\hline 0.55468 & 0.46629 & 0.01874 & 2467.3 & 82.4 & 2505.8 & 60.2 \\
\hline 0.03846 & 0.1158 & 0.00284 & 706.3 & 16.4 & 737.8 & 23.2 \\
\hline 0.0306 & 0.09968 & 0.0025 & 612.5 & 14.6 & 569.5 & 21.2 \\
\hline 0.033 & 0.12585 & 0.00286 & 764.1 & 16.4 & 761.3 & 20 \\
\hline 0.04642 & 0.12137 & 0.0032 & 738.4 & 18.4 & 750.2 & 27 \\
\hline 0.02306 & 0.08078 & 0.00184 & 500.8 & 11 & 582.8 & 16.6 \\
\hline 0.04126 & 0.14006 & 0.00328 & 845 & 18.6 & 843.3 & 22.6 \\
\hline 0.02516 & 0.10241 & 0.00236 & 628.5 & 13.8 & 597.4 & 17.6 \\
\hline 0.29706 & 0.46047 & 0.01064 & 2441.6 & 47 & 2484.1 & 33.6 \\
\hline 0.03246 & 0.10102 & 0.00256 & 620.4 & 15 & 597.8 & 22 \\
\hline 0.02284 & 0.09551 & 0.0022 & 588 & 13 & 560.1 & 16.6 \\
\hline 0.0565 & 0.13477 & 0.0038 & 815 & 21.6 & 775.6 & 31.4 \\
\hline 0.03358 & 0.09355 & 0.00244 & 576.5 & 14.4 & 588.3 & 22.8 \\
\hline 0.03868 & 0.14024 & 0.00326 & 846 & 18.4 & 820.4 & 21.8 \\
\hline 0.03304 & 0.11248 & 0.00262 & 687.1 & 15.2 & 729.7 & 20.4 \\
\hline 0.02758 & 0.10681 & 0.0025 & 654.2 & 14.6 & 628 & 18.6 \\
\hline 0.04602 & 0.13782 & 0.00346 & 832.3 & 19.6 & 801.9 & 25.6 \\
\hline 0.03206 & 0.10877 & 0.00264 & 665.6 & 15.4 & 650.3 & 20.8 \\
\hline 0.02352 & 0.08956 & 0.00206 & 552.9 & 12.2 & 586.6 & 16.8 \\
\hline 0.03736 & 0.14327 & 0.00328 & 863.1 & 18.4 & 822.3 & 21.2 \\
\hline 0.05116 & 0.14942 & 0.00382 & 897.7 & 21.4 & 839 & 27.2 \\
\hline 0.04748 & 0.14505 & 0.0036 & 873.2 & 20.2 & 841.4 & 25.6 \\
\hline 0.03566 & 0.12531 & 0.00294 & 761.1 & 16.8 & 764.9 & 21.2 \\
\hline 0.0331 & 0.08915 & 0.00208 & 550.5 & 12.4 & 736.9 & 20.4 \\
\hline 0.05312 & 0.15406 & 0.00388 & 923.7 & 21.6 & 896.8 & 27.2 \\
\hline 0.03504 & 0.1274 & 0.00302 & 773 & 17.2 & 736.3 & 21.2 \\
\hline 0.04306 & 0.1045 & 0.00292 & 640.7 & 17 & 641.9 & 27.4 \\
\hline 0.03976 & 0.13559 & 0.00326 & 819.7 & 18.6 & 785.4 & 22.8 \\
\hline 0.02436 & 0.09406 & 0.00218 & 579.5 & 12.8 & 595.1 & 17.2 \\
\hline 0.0383 & 0.12736 & 0.00304 & 772.8 & 17.4 & 783.7 & 22.2 \\
\hline 0.05038 & 0.14682 & 0.0038 & 883.1 & 21.4 & 815.8 & 27.6 \\
\hline 70.037 & 2.27931 & 0.64052 & & & & \\
\hline 0.04044 & 0.13098 & 0.00326 & 793.5 & 18.6 & 739.3 & 23.8 \\
\hline 0.08646 & 0.17845 & 0.00496 & 1058.5 & 27.2 & 1119.9 & 35.2 \\
\hline 0.04222 & 0.1377 & 0.00336 & 831.6 & 19 & 801.3 & 23.8 \\
\hline
\end{tabular}




$\begin{array}{llllllll}1 & 0.04932 & 0.09684 & 0.00234 & 595.9 & 13.8 & 940.8 & 24.8 \\ 2 & 0.04616 & 0.14371 & 0.00346 & 865.6 & 19.6 & 875.5 & 24.4 \\ 3 & & & \end{array}$




\begin{tabular}{cc|cc|cc|}
\hline & & discordance & preferred age & \\
age 207/206 & 20 age 76 & $\Delta$ 68-75 [\%] & $\Delta$ 68-76 [\%] & age & 2 $\sigma$ age \\
\hline 329.7 & 81.2 & 0.3 & 2.3 & 337.4 & 7.8
\end{tabular}

\begin{tabular}{|c|c|c|c|c|c|}
\hline 735 & 93.6 & -0.4 & -1.7 & 722.4 & 18 \\
\hline 637.1 & 72 & -1.5 & -7 & 592.6 & 13.2 \\
\hline 706.5 & 66.2 & 2.2 & 9.1 & 770.7 & 16.8 \\
\hline 1635 & 54 & -41.9 & -82 & & \\
\hline 470.5 & 65.8 & 4 & 23.8 & 582.5 & 12.6 \\
\hline 716 & 65.2 & -5.3 & -21.4 & 562.5 & 12.2 \\
\hline 543.7 & 95.2 & 4.1 & 21.5 & 660.8 & 16.2 \\
\hline 1041.2 & 78.6 & -10.7 & -31.9 & 708.5 & 17 \\
\hline 701.8 & 76.6 & 1.6 & 6.6 & 747.9 & 17.2 \\
\hline 505.8 & 114.2 & 4.9 & 27.3 & 643.9 & 17 \\
\hline 664.1 & 79.8 & 1.4 & 6.1 & 704.7 & 16.2 \\
\hline 1703.8 & 52.2 & -36.8 & -71.9 & & \\
\hline 404.5 & 91.4 & 7.1 & 48.6 & & \\
\hline 2537.9 & 85 & -1.5 & -2.8 & 2537.9 & 85 \\
\hline 835.6 & 76.6 & -4.3 & -15.5 & 706.3 & 16.4 \\
\hline 402.4 & 93.2 & 7.6 & 52.2 & & \\
\hline 754.1 & 62.2 & 0.4 & 1.3 & 764.1 & 16.4 \\
\hline 786.6 & 91.2 & -1.6 & -6.1 & 738.4 & 18.4 \\
\hline 918.1 & 61.4 & -14.1 & -45.5 & 500.8 & 11 \\
\hline 839.9 & 66.4 & 0.2 & 0.6 & 845 & 18.6 \\
\hline 482.2 & 69.2 & 5.2 & 30.3 & & \\
\hline 2520 & 46.6 & -1.7 & -3.1 & 2520 & 46.6 \\
\hline 514.6 & 90.8 & 3.8 & 20.6 & 620.4 & 15 \\
\hline 449.2 & 69.2 & 5 & 30.9 & 588 & 13 \\
\hline 665.1 & 108.2 & 5.1 & 22.5 & & \\
\hline 635.4 & 94.8 & -2 & -9.3 & 576.5 & 14.4 \\
\hline 753.2 & 65.6 & 3.1 & 12.3 & 846 & 18.4 \\
\hline 864.3 & 65.4 & -5.8 & -20.5 & 687.1 & 15.2 \\
\hline 536.2 & 70.8 & 4.2 & 22 & 654.2 & 14.6 \\
\hline 719.7 & 82.4 & 3.8 & 15.7 & 832.3 & 19.6 \\
\hline 598.9 & 78.2 & 2.4 & 11.1 & 665.6 & 15.4 \\
\hline 721 & 63.6 & -5.7 & -23.3 & 552.9 & 12.2 \\
\hline 715 & 63.2 & 5 & 20.7 & 863.1 & 18.4 \\
\hline 688.2 & 86.2 & 7 & 30.4 & & \\
\hline 760 & 78.4 & 3.8 & 14.9 & 873.2 & 20.2 \\
\hline 777.9 & 66.6 & -0.5 & -2.2 & 761.1 & 16.8 \\
\hline 1356.5 & 60 & -25.3 & -59.4 & & \\
\hline 833.1 & 79.2 & 3 & 10.9 & 923.7 & 21.6 \\
\hline 628.3 & 71 & 5 & 23 & 773 & 17.2 \\
\hline 648 & 108 & -0.2 & -1.1 & 640.7 & 17 \\
\hline 690.9 & 73 & 4.4 & 18.6 & 819.7 & 18.6 \\
\hline 657.1 & 65.6 & -2.6 & -11.8 & 579.5 & 12.8 \\
\hline 816.8 & 68.6 & -1.4 & -5.4 & 772.8 & 17.4 \\
\hline 638.5 & 89.6 & 8.3 & 38.3 & & \\
\hline 581.1 & 82.6 & 7.3 & 36.6 & & \\
\hline 1243.1 & 86.2 & -5.5 & -14.9 & 1058.5 & 27.2 \\
\hline 720.3 & 75 & 3.8 & 15.5 & 831.6 & 19 \\
\hline
\end{tabular}




$\begin{array}{lcccccc}1 & 1870.3 & 58.6 & -36.7 & -68.1 & & \\ 2 & 902.9 & 69.8 & -1.1 & -4.1 & 865.6 & 19.6 \\ 3 & & & & & & \end{array}$


Sample 2878S White Nile @ Rabak 150 grain analysed 123 concordant ages

\begin{tabular}{|c|c|c|c|c|c|c|}
\hline \multirow[b]{2}{*}{ grain } & \multicolumn{3}{|c|}{ concentrations } & \multicolumn{3}{|l|}{ isotopic ratios } \\
\hline & U [ppm] & $\mathrm{Pb}$ [ppm] & Th/U & Pb207/Pb206 & $2 \sigma 76$ & Pb207/U235 \\
\hline X2878S_G001 & 113.4 & 18 & 0.5163 & 0.07024 & 0.00272 & 1.42799 \\
\hline X2878S_G002 & 185.2 & 22.1 & 0.9463 & 0.08513 & 0.00314 & 1.14183 \\
\hline X2878S_G003 & 86.9 & 11 & 0.7613 & 0.06001 & 0.00312 & 0.91014 \\
\hline X2878S_G004 & 20.2 & 2.2 & 0.567 & 0.06694 & 0.00746 & 0.93147 \\
\hline X2878S_G005 & 80.6 & 16.2 & 0.4477 & 0.07769 & 0.00302 & 2.01204 \\
\hline X2878S_G006 & 492.7 & 64.6 & 0.4902 & 0.07818 & 0.00196 & 1.32361 \\
\hline X2878S_G007 & 160.8 & 23.2 & 0.3492 & 0.06818 & 0.0023 & 1.31928 \\
\hline X2878S_G008 & & & & 32.52293 & & \\
\hline X2878S G009 & 101.5 & 11.4 & 0.6448 & 0.06193 & 0.0027 & 0.86597 \\
\hline X2878S G010 & 181.2 & 20.6 & 0.7388 & 0.05883 & 0.00232 & 0.80538 \\
\hline X2878S_G011 & 623.2 & 77.2 & 0.2526 & 0.06605 & 0.00168 & 1.12818 \\
\hline X2878S_G012 & 914.4 & 63.3 & 0.0874 & 0.08442 & 0.00224 & 0.83773 \\
\hline X2878S_G013 & 431.9 & 8.9 & 0.6148 & 0.04805 & 0.00302 & 0.12324 \\
\hline X2878S_G014 & 98.4 & 19.6 & 0.7952 & 0.07284 & 0.00266 & 1.71745 \\
\hline X2878S_G015 & 153.9 & 23.1 & 0.4548 & 0.06745 & 0.0023 & 1.31471 \\
\hline X2878S_G016 & 319.8 & 33.8 & 0.3465 & 0.06037 & 0.0019 & 0.85708 \\
\hline X2878S_G017 & 101.2 & 13.4 & 0.5948 & 0.06307 & 0.0029 & 1.04671 \\
\hline X2878S_G018 & 82.7 & 11.2 & 0.526 & 0.06125 & 0.00306 & 1.05645 \\
\hline X2878S_G019 & 83.2 & 14.9 & 0.507 & 0.0705 & 0.00282 & 1.6111 \\
\hline X2878S_G020 & 608.3 & 74.3 & 0.14 & 0.06588 & 0.00158 & 1.14921 \\
\hline X2878S_G021 & 83.9 & 8 & 0.3333 & 0.17576 & 0.00616 & 2.35055 \\
\hline X2878S_G022 & 350 & 55 & 0.6601 & 0.0669 & 0.00176 & 1.2911 \\
\hline X2878S_G023 & 29.3 & 5.9 & 0.554 & 0.07113 & 0.00398 & 1.80758 \\
\hline X2878S_G024 & 177.4 & 26.4 & 0.4048 & 0.07404 & 0.00244 & 1.43909 \\
\hline X2878S_G025 & 54.4 & 12 & 0.811 & 0.07422 & 0.00332 & 1.92974 \\
\hline X2878S_G026 & 76.8 & 17.3 & 0.9421 & 0.07517 & 0.0029 & 1.92786 \\
\hline X2878S_G027 & 231.4 & 35.5 & 0.7283 & 0.06426 & 0.002 & 1.19049 \\
\hline X2878S_G028 & 77.4 & 34.3 & 0.3971 & 0.16562 & 0.00424 & 9.42031 \\
\hline X2878S_G029 & 157.5 & 16.5 & 0.448 & 0.06081 & 0.00238 & 0.83563 \\
\hline X2878S_G030 & 655.9 & 77.5 & 0.1538 & 0.08252 & 0.002 & 1.35188 \\
\hline X2878S_G031 & 321.1 & 34.1 & 0.3385 & 0.06 & 0.00182 & 0.86008 \\
\hline X2878S_G032 & 145.8 & 17.4 & 0.9154 & 0.05731 & 0.0023 & 0.78738 \\
\hline X2878S_G033 & 158.2 & 23.2 & 0.4753 & 0.06571 & 0.0023 & 1.24621 \\
\hline X2878S_G034 & 251.2 & 29.4 & 0.3861 & 0.05908 & 0.00186 & 0.92187 \\
\hline X2878S_G035 & & & & 0.50644 & 0.92864 & \\
\hline X2878S_G036 & 155.5 & 20.7 & 0.4103 & 0.06502 & 0.00248 & 1.14046 \\
\hline X2878S_G037 & 130.4 & 13.5 & 0.3051 & 0.06099 & 0.00246 & 0.86108 \\
\hline X2878S_G038 & 386.8 & 37.3 & 0.1267 & 0.05779 & 0.0017 & 0.80426 \\
\hline X2878S_G039 & 74.7 & 13.2 & 0.4033 & 0.07262 & 0.00294 & 1.69139 \\
\hline X2878S_G040 & 106.4 & 11.9 & 0.3024 & 0.06143 & 0.0026 & 0.93858 \\
\hline X2878S_G041 & 302.9 & 164.2 & 0.7643 & 0.17304 & 0.00376 & 10.86369 \\
\hline X2878S_G042 & 443.5 & 54.4 & 1.0003 & 0.05901 & 0.00162 & 0.81358 \\
\hline X2878S_G043 & 135.2 & 14.6 & 0.4163 & 0.05902 & 0.00228 & 0.84012 \\
\hline X2878S_G044 & 121.9 & 2.9 & -0.2604 & 2.13708 & 0.15144 & 7.24328 \\
\hline X2878S_G045 & 1109.4 & 108.3 & 0.0256 & 0.06287 & 0.00152 & 0.91399 \\
\hline X2878S_G046 & 67.9 & 14.3 & 0.5449 & 0.07421 & 0.00302 & 1.97496 \\
\hline X2878S_G047 & 238.9 & 41.1 & 0.874 & 0.06644 & 0.00198 & 1.33113 \\
\hline X2878S_G048 & 226.9 & 26.5 & 0.759 & 0.05965 & 0.00204 & 0.8385 \\
\hline X2878S_G049 & 79.9 & 8.5 & 0.4866 & 0.05739 & 0.00308 & 0.79289 \\
\hline X2878S_G050 & 74.8 & 8.2 & 0.5084 & 0.06112 & 0.0032 & 0.86482 \\
\hline
\end{tabular}




\begin{tabular}{|c|c|c|c|c|c|c|}
\hline X2878S_G051 & 263.3 & 32.1 & 0.2006 & 0.06296 & 0.0019 & 1.07748 \\
\hline X2878S_G052 & 488.7 & 82.9 & 0.262 & 0.07364 & 0.00176 & 1.71097 \\
\hline X2878S_G053 & 263 & 34.6 & 0.7843 & 0.06246 & 0.00188 & 0.97698 \\
\hline X2878S_G054 & 172.7 & 17.8 & 0.2762 & 0.05948 & 0.00218 & 0.84605 \\
\hline X2878S_G055 & 2271.1 & 166.7 & 0.9069 & 0.09761 & 0.00242 & 0.91855 \\
\hline X2878S_G056 & 259.8 & 26.7 & 0.2593 & 0.06461 & 0.00206 & 0.92134 \\
\hline X2878S_G057 & 173 & 17.8 & 0.5517 & 0.0573 & 0.00258 & 0.74846 \\
\hline X2878S_G058 & 402.2 & 51 & 0.222 & 0.06327 & 0.00174 & 1.11348 \\
\hline X2878S_G059 & 552 & 100 & 2.4974 & 0.06062 & 0.00158 & 0.90083 \\
\hline X2878S_G060 & 111.9 & 13.1 & 0.9169 & 0.05724 & 0.00272 & 0.76873 \\
\hline X2878S_G061 & 210.6 & 26.3 & 0.2491 & 0.06329 & 0.00206 & 1.09139 \\
\hline X2878S_G062 & 205.4 & 24.9 & 0.2321 & 0.0627 & 0.00204 & 1.05517 \\
\hline X2878S G063 & 186.8 & 29.3 & 0.4832 & 0.06993 & 0.00222 & 1.39449 \\
\hline X2878S_G064 & 121.1 & 25.7 & 1.3368 & 0.07158 & 0.00254 & 1.58571 \\
\hline X2878S_G065 & 471.1 & 50.7 & 0.9954 & 0.09634 & 0.00274 & 1.30468 \\
\hline X2878S_G066 & 52.7 & 6 & 0.9197 & 0.05791 & 0.004 & 0.76623 \\
\hline X2878S_G067 & 40.4 & 8.5 & 0.5984 & 0.07659 & 0.00388 & 1.9944 \\
\hline X2878S_G068 & 323.4 & 44.7 & 0.4297 & 0.0726 & 0.00228 & 1.25706 \\
\hline X2878S_G069 & 59.9 & 7.6 & 0.6048 & 0.05949 & 0.00344 & 0.93888 \\
\hline X2878S_G070 & 1108.3 & 119.7 & 1.3608 & 0.17279 & 0.00392 & 2.14816 \\
\hline X2878S_G071 & 46.5 & 6.9 & 0.6293 & 0.04444 & 0.00316 & 0.83798 \\
\hline X2878S_G072 & 285 & 31.9 & 0.1543 & 0.07068 & 0.00214 & 1.10994 \\
\hline X2878S_G073 & 199.3 & 20.8 & 0.3152 & 0.05837 & 0.00204 & 0.8272 \\
\hline X2878S_G074 & 1338.9 & 140.8 & 0.1801 & 0.12864 & 0.00304 & 1.79863 \\
\hline X2878S_G075 & 518.7 & 53.7 & 0.1786 & 0.0611 & 0.00162 & 0.89632 \\
\hline X2878S_G076 & 77.4 & 16.1 & 0.7584 & 0.07066 & 0.00276 & 1.75291 \\
\hline X2878S_G077 & & & & 0.82565 & 0.02662 & \\
\hline X2878S_G078 & 110 & -2800.9 & 0.3192 & -0.04428 & 0.00416 & -0.83483 \\
\hline X2878S_G079 & 1689.9 & 637 & 0.0225 & 0.14847 & 0.00308 & 7.92402 \\
\hline X2878S_G080 & 94.9 & 18.5 & 0.6969 & 0.07339 & 0.00274 & 1.7426 \\
\hline X2878S_G081 & 73.5 & 7.7 & 0.3899 & 0.06457 & 0.00336 & 0.9575 \\
\hline X2878S_G082 & 231.7 & 20.7 & 0.0177 & 0.05933 & 0.00204 & 0.79047 \\
\hline X2878S_G083 & 607.9 & 77.7 & 0.4793 & 0.08058 & 0.00232 & 1.32316 \\
\hline X2878S_G084 & 258.4 & 25.4 & 0.0932 & 0.06018 & 0.00198 & 0.86231 \\
\hline X2878S_G085 & 104.2 & 12.1 & 0.6395 & 0.05963 & 0.00282 & 0.8607 \\
\hline X2878S_G086 & 38.5 & 7.4 & 0.4456 & 0.07443 & 0.00374 & 1.84571 \\
\hline X2878S_G087 & 243.9 & 28.1 & 0.1522 & 0.06318 & 0.00204 & 1.03736 \\
\hline X2878S_G088 & 912.8 & 113.2 & 0.4508 & 0.07181 & 0.00172 & 1.15078 \\
\hline X2878S_G089 & 82.2 & 20.6 & 1.0648 & 0.07685 & 0.00278 & 2.12453 \\
\hline X2878S_G090 & & & & 0.55281 & 1.16062 & 121.59718 \\
\hline X2878S_G091 & 104.7 & 17.5 & 0.6705 & 0.06779 & 0.00292 & 1.38264 \\
\hline X2878S_G092 & 538.7 & 59.5 & 0.6688 & 0.05964 & 0.00162 & 0.81372 \\
\hline X2878S G093 & 95.1 & 10.8 & 0.4945 & 0.06011 & 0.0028 & 0.88297 \\
\hline X2878S_G094 & 81 & 8.9 & 0.231 & 0.06437 & 0.00318 & 0.98279 \\
\hline X2878S_G095 & 337.9 & 42.1 & 0.7422 & 0.06078 & 0.0018 & 0.91288 \\
\hline X2878S_G096 & 159.8 & 15.1 & 0.2042 & 0.05699 & 0.00238 & 0.75825 \\
\hline X2878S_G097 & 176.7 & 23.1 & 0.8392 & 0.06214 & 0.00234 & 0.95855 \\
\hline X2878S_G098 & 204.7 & 32.4 & 0.7025 & 0.06941 & 0.00214 & 1.33725 \\
\hline X2878S_G099 & 423.6 & 44 & 0.5941 & 0.05861 & 0.00174 & 0.76467 \\
\hline X2878S_G100 & 894.1 & 45.8 & 0.0936 & 0.05317 & 0.00148 & 0.39889 \\
\hline X2878S_G101 & 51.2 & 11.8 & 1.0493 & 0.07787 & 0.00364 & 2.01761 \\
\hline X2878S_G102 & 194.4 & 26.4 & 0.6644 & 0.06216 & 0.00204 & 1.03782 \\
\hline X2878S_G103 & 91.5 & 11.4 & 0.4664 & 0.06236 & 0.0027 & 1.01439 \\
\hline X2878S_G104 & 191.6 & 24 & 0.741 & 0.06318 & 0.00218 & 0.95413 \\
\hline X2878S_G105 & 3187.6 & 112.9 & 0.2651 & 0.18077 & 0.00416 & 0.82763 \\
\hline
\end{tabular}




\begin{tabular}{|c|c|c|c|c|c|c|}
\hline X2878S_G106 & 231.5 & 21.2 & 0.3138 & 0.0596 & 0.00204 & 0.74341 \\
\hline X2878S_G107 & 101.8 & 13.2 & 0.8221 & 0.06181 & 0.00292 & 0.95305 \\
\hline X2878S_G108 & 236.5 & 62.2 & 1.7188 & 0.07253 & 0.00198 & 1.82639 \\
\hline X2878S_G109 & 1049 & 159.8 & 3.0484 & 0.10005 & 0.00232 & 1.26873 \\
\hline X2878S_G110 & 154.6 & 20.3 & 0.8123 & 0.06067 & 0.00222 & 0.94039 \\
\hline X2878S_G111 & 40.6 & 4.8 & 0.8422 & 0.05888 & 0.00424 & 0.8154 \\
\hline X2878S G112 & 85.3 & 9.6 & 0.6637 & 0.0613 & 0.00348 & 0.85058 \\
\hline X2878S_G113 & 261.5 & 28 & 0.5539 & 0.05984 & 0.00194 & 0.81253 \\
\hline X2878S_G114 & 122.4 & 14.2 & 0.3036 & 0.06092 & 0.0026 & 0.96349 \\
\hline X2878S_G115 & 108.5 & 19.4 & 0.4481 & 0.07271 & 0.00248 & 1.69019 \\
\hline X2878S_G116 & 123.1 & 13.7 & 0.7152 & 0.05638 & 0.00264 & 0.76977 \\
\hline X2878S_G117 & & & & 0.06277 & 0.00786 & 0.95049 \\
\hline X2878S_G118 & 55.5 & 11.8 & 0.8312 & 0.09111 & 0.00366 & 2.25453 \\
\hline X2878S_G119 & 263.7 & 38.4 & 0.2616 & 0.06889 & 0.00194 & 1.38099 \\
\hline X2878S_G120 & 344.2 & 90 & 2.3469 & 0.16663 & 0.00402 & 3.86674 \\
\hline X2878S_G121 & 143.8 & 16.4 & 0.3753 & 0.06036 & 0.00226 & 0.92153 \\
\hline X2878S_G122 & 87.6 & 10.9 & 0.5953 & 0.06207 & 0.00294 & 0.9736 \\
\hline X2878S_G123 & 695 & 68.7 & 0.2068 & 0.05908 & 0.00154 & 0.82191 \\
\hline X2878S_G124 & 252.8 & 38.4 & 0.3232 & 0.06671 & 0.0019 & 1.36832 \\
\hline X2878S_G125 & 94.2 & 10.8 & 0.5734 & 0.06137 & 0.00278 & 0.89057 \\
\hline X2878S_G126 & 191.7 & 21.8 & 0.3269 & 0.0616 & 0.00214 & 0.95166 \\
\hline X2878S_G127 & 173.1 & 26.9 & 0.5299 & 0.065 & 0.00214 & 1.28619 \\
\hline X2878S_G128 & 66 & 8.1 & 0.5819 & 0.06048 & 0.00356 & 0.93673 \\
\hline X2878S_G129 & & & & -0.95313 & 1.71592 & \\
\hline X2878S_G130 & 90.1 & 57 & 0.9814 & 0.17089 & 0.0041 & 11.62015 \\
\hline X2878S_G131 & 151.4 & 18.9 & 0.6511 & 0.06216 & 0.00232 & 0.96358 \\
\hline X2878S_G132 & & & & -0.14935 & 0.65782 & 214.00204 \\
\hline X2878S_G133 & 61 & 13.1 & 0.647 & 0.07663 & 0.00322 & 2.01807 \\
\hline X2878S_G134 & 1673.4 & 174 & 0.0921 & 0.06126 & 0.00138 & 0.92764 \\
\hline X2878S_G135 & 214.5 & 23.5 & 0.2609 & 0.0606 & 0.00208 & 0.91617 \\
\hline X2878S_G136 & 390.8 & 41.6 & 0.3475 & 0.06034 & 0.00172 & 0.86732 \\
\hline X2878S_G137 & 59.6 & 12.2 & 0.6702 & 0.07254 & 0.00314 & 1.81792 \\
\hline X2878S_G138 & 294.5 & 37.4 & 1.0697 & 0.0575 & 0.00184 & 0.80977 \\
\hline X2878S_G139 & 330.1 & 36.5 & 0.3825 & 0.06015 & 0.00178 & 0.88655 \\
\hline X2878S_G140 & 406.7 & 46.4 & 0.3163 & 0.06011 & 0.00168 & 0.9292 \\
\hline X2878S_G141 & 577.1 & 119.6 & 0.5443 & 0.07613 & 0.00176 & 1.99201 \\
\hline X2878S_G142 & 374.9 & 43.9 & 0.2618 & 0.06687 & 0.00186 & 1.07567 \\
\hline X2878S_G143 & 327.1 & 36.6 & 0.3756 & 0.05951 & 0.00178 & 0.89011 \\
\hline X2878S_G144 & 327.6 & 32.7 & 0.3882 & 0.0627 & 0.0019 & 0.83144 \\
\hline X2878S_G145 & 558.4 & 77.9 & 0.9048 & 0.06172 & 0.00158 & 0.99352 \\
\hline X2878S_G146 & 2044.9 & 198.4 & 0.3634 & 0.10954 & 0.00238 & 1.39268 \\
\hline X2878S_G147 & 199.3 & 21.4 & 0.314 & 0.06009 & 0.0021 & 0.87547 \\
\hline X2878S_G148 & 20.2 & 6.5 & 235.5725 & 0.47216 & 0.04498 & 1.69132 \\
\hline X2878S_G149 & 48.1 & 5.6 & 0.5676 & 0.06175 & 0.00378 & 0.91596 \\
\hline X2878S_G150 & 54 & 10.4 & 1.0238 & 0.07287 & 0.00388 & 1.58099 \\
\hline
\end{tabular}




\section{$82.0 \%$ concordant}

\begin{tabular}{|c|c|c|c|c|c|c|}
\hline $2 \sigma 75$ & Pb206/U238 & $2 \sigma 68$ & $\begin{array}{c}\text { ages } \\
\text { age } 206 / 238\end{array}$ & $2 \sigma$ age 68 & age $207 / 235$ & $2 \sigma$ age 75 \\
\hline 0.05484 & 0.14737 & 0.00386 & 886.2 & 21.6 & 900.7 & 28 \\
\hline 0.04156 & 0.09722 & 0.00254 & 598.1 & 15 & 773.4 & 24.4 \\
\hline 0.04616 & 0.10992 & 0.00314 & 672.3 & 18.2 & 657.1 & 28.6 \\
\hline 0.09984 & 0.10086 & 0.00462 & 619.4 & 27 & 668.4 & 59 \\
\hline 0.07798 & 0.18771 & 0.00506 & 1109 & 27.4 & 1119.6 & 32 \\
\hline 0.0347 & 0.12272 & 0.00282 & 746.2 & 16.2 & 856.1 & 19.6 \\
\hline 0.0449 & $\begin{array}{l}0.14025 \\
-0.1101\end{array}$ & $\begin{array}{c}0.0035 \\
16.02776\end{array}$ & 846.1 & 19.8 & 854.2 & 24.2 \\
\hline 0.0372 & 0.10135 & 0.00272 & 622.3 & 16 & 633.4 & 24.2 \\
\hline 0.03162 & 0.09923 & 0.00254 & 609.9 & 14.8 & 599.9 & 21.2 \\
\hline 0.02996 & 0.12381 & 0.00284 & 752.5 & 16.2 & 766.9 & 18.4 \\
\hline 0.02296 & 0.07193 & 0.00168 & 447.8 & 10.2 & 617.9 & 16.4 \\
\hline 0.00752 & 0.01859 & 0.00054 & 118.7 & 3.4 & 118 & 7.8 \\
\hline 0.06258 & 0.17089 & 0.00444 & 1017 & 24.4 & 1015.1 & 28.8 \\
\hline 0.0452 & 0.14128 & 0.00352 & 851.9 & 19.8 & 852.2 & 24.4 \\
\hline 0.0274 & 0.1029 & 0.00248 & 631.4 & 14.4 & 628.5 & 18.6 \\
\hline 0.0473 & 0.1203 & 0.0033 & 732.3 & 19 & 727.3 & 27.8 \\
\hline 0.05184 & 0.12502 & 0.00356 & 759.4 & 20.4 & 732.1 & 30 \\
\hline 0.06404 & 0.16565 & 0.00444 & 988.1 & 24.6 & 974.5 & 30.2 \\
\hline 0.02924 & 0.12643 & 0.00288 & 767.5 & 16.4 & 776.9 & 18 \\
\hline 0.07882 & 0.09694 & 0.0027 & 596.5 & 15.8 & 1227.7 & 31.8 \\
\hline 0.0354 & 0.13988 & 0.00324 & 844 & 18.4 & 841.8 & 20 \\
\hline 0.09918 & 0.1842 & 0.00584 & 1089.9 & 31.8 & 1048.2 & 42 \\
\hline 0.04774 & 0.14088 & 0.0035 & 849.6 & 19.8 & 905.3 & 24.8 \\
\hline 0.08522 & 0.18846 & 0.00538 & 1113 & 29.2 & 1091.4 & 35.4 \\
\hline 0.07428 & 0.18589 & 0.00498 & 1099.1 & 27 & 1090.8 & 31.4 \\
\hline 0.03764 & 0.13429 & 0.00324 & 812.3 & 18.4 & 796.2 & 21.8 \\
\hline 0.25558 & 0.41227 & 0.01028 & 2225.3 & 47 & 2379.8 & 32.8 \\
\hline 0.03236 & 0.0996 & 0.00256 & 612.1 & 15 & 616.7 & 21.6 \\
\hline 0.0345 & 0.11875 & 0.00272 & 723.4 & 15.6 & 868.4 & 19.4 \\
\hline 0.02646 & 0.1039 & 0.00246 & 637.2 & 14.4 & 630.2 & 18 \\
\hline 0.03134 & 0.09958 & 0.00256 & 611.9 & 15 & 589.7 & 21.4 \\
\hline 0.04368 & 0.13747 & 0.00344 & 830.3 & 19.4 & 821.7 & 24.2 \\
\hline 0.02952 & 0.1131 & 0.00272 & 690.7 & 15.8 & 663.3 & 19.2 \\
\hline 0.04328 & 0.12713 & 0.00328 & 771.5 & 18.8 & 772.7 & 25 \\
\hline 0.0343 & 0.10234 & 0.00266 & 628.1 & 15.6 & 630.7 & 22.6 \\
\hline 0.02418 & 0.10087 & 0.00238 & 619.5 & 14 & 599.2 & 17 \\
\hline 0.06782 & 0.16882 & 0.00458 & 1005.6 & 25.2 & 1005.3 & 31 \\
\hline 0.03916 & 0.11074 & 0.00292 & 677 & 17 & 672.1 & 24.6 \\
\hline 0.2555 & 0.45505 & 0.01038 & 2417.7 & 46 & 2511.5 & 29.2 \\
\hline 0.02306 & 0.09993 & 0.00232 & 614 & 13.6 & 604.5 & 16.4 \\
\hline 0.03222 & 0.10318 & 0.00262 & 633 & 15.4 & 619.2 & 21.4 \\
\hline 0.19636 & 0.02457 & 0.00174 & 156.5 & 11 & 2141.8 & 89.4 \\
\hline 0.0234 & 0.10537 & 0.0024 & 645.8 & 14 & 659.2 & 16.2 \\
\hline 0.08016 & 0.1929 & 0.00528 & 1137.1 & 28.6 & 1107 & 33 \\
\hline 0.04056 & 0.14522 & 0.00348 & 874.1 & 19.6 & 859.4 & 22.2 \\
\hline 0.0289 & 0.10188 & 0.0025 & 625.4 & 14.6 & 618.3 & 19.4 \\
\hline 0.04162 & 0.10014 & 0.00288 & 615.2 & 16.8 & 592.8 & 27.4 \\
\hline 0.04414 & 0.10256 & 0.00296 & 629.4 & 17.4 & 632.8 & 28.2 \\
\hline
\end{tabular}




\begin{tabular}{|c|c|c|c|c|c|c|}
\hline 0.03308 & 0.12405 & 0.00296 & 753.8 & 17 & 742.4 & 20.2 \\
\hline 0.04338 & 0.16841 & 0.00384 & 1003.3 & 21.2 & 1012.6 & 21.2 \\
\hline 0.02996 & 0.11338 & 0.0027 & 692.3 & 15.6 & 692.1 & 19.2 \\
\hline 0.03092 & 0.1031 & 0.00258 & 632.6 & 15 & 622.5 & 20.6 \\
\hline 0.02372 & 0.06821 & 0.00158 & 425.4 & 9.6 & 661.6 & 16.4 \\
\hline 0.02974 & 0.10335 & 0.0025 & 634 & 14.6 & 663.1 & 19.4 \\
\hline 0.0331 & 0.09468 & 0.00254 & 583.2 & 15 & 567.3 & 22.8 \\
\hline 0.03162 & 0.12757 & 0.00298 & 774 & 17 & 759.8 & 19.2 \\
\hline 0.0244 & 0.10771 & 0.00248 & 659.4 & 14.4 & 652.2 & 16.8 \\
\hline 0.03582 & 0.09734 & 0.00266 & 598.8 & 15.6 & 579 & 24.2 \\
\hline 0.0358 & 0.12498 & 0.00304 & 759.2 & 17.4 & 749.2 & 21.6 \\
\hline 0.0346 & 0.12198 & 0.00298 & 741.9 & 17.2 & 731.4 & 21.2 \\
\hline 0.04488 & 0.14453 & 0.00354 & 870.2 & 20 & 886.6 & 23.8 \\
\hline 0.05648 & 0.16058 & 0.0041 & 960 & 22.8 & 964.6 & 27.2 \\
\hline 0.03758 & 0.09815 & 0.00236 & 603.6 & 13.8 & 847.8 & 21.4 \\
\hline 0.05116 & 0.09591 & 0.00314 & 590.4 & 18.4 & 577.6 & 33.6 \\
\hline 0.09918 & 0.18873 & 0.0058 & 1114.5 & 31.4 & 1113.6 & 40 \\
\hline 0.0399 & 0.12551 & 0.00306 & 762.2 & 17.6 & 826.6 & 22.4 \\
\hline 0.05304 & 0.1144 & 0.00346 & 698.3 & 20 & 672.3 & 32 \\
\hline 0.05148 & 0.09011 & 0.00206 & 556.2 & 12.2 & 1164.5 & 22.4 \\
\hline 0.05836 & 0.13667 & 0.00432 & 825.8 & 24.6 & 618 & 36 \\
\hline 0.03406 & 0.11383 & 0.00274 & 695 & 15.8 & 758.1 & 20.6 \\
\hline 0.02904 & 0.10273 & 0.00254 & 630.4 & 14.8 & 612.1 & 19.6 \\
\hline 0.04454 & 0.10134 & 0.00232 & 622.3 & 13.6 & 1045 & 21.4 \\
\hline 0.02458 & 0.10632 & 0.00246 & 651.3 & 14.4 & 649.8 & 16.8 \\
\hline 0.06828 & 0.1798 & 0.00478 & 1065.9 & 26.2 & 1028.2 & 30.6 \\
\hline 573.56958 & 29.73272 & 5.05226 & & & & \\
\hline 0.07814 & 0.13666 & 0.00362 & 825.7 & 20.6 & -1828.5 & -499.2 \\
\hline 0.1795 & 0.38686 & 0.0086 & 2108.2 & 40 & 2222.4 & 27.4 \\
\hline 0.06478 & 0.17211 & 0.0045 & 1023.7 & 24.8 & 1024.4 & 29.4 \\
\hline 0.04862 & 0.10748 & 0.00316 & 658.1 & 18.4 & 682 & 29.6 \\
\hline 0.02736 & 0.09658 & 0.00238 & 594.3 & 14 & 591.4 & 19 \\
\hline 0.03876 & 0.11902 & 0.00284 & 724.9 & 16.4 & 855.9 & 21.6 \\
\hline 0.02864 & 0.10385 & 0.00252 & 636.9 & 14.8 & 631.4 & 19.2 \\
\hline 0.04002 & 0.10462 & 0.00288 & 641.4 & 16.8 & 630.5 & 25.6 \\
\hline 0.09114 & 0.17975 & 0.00544 & 1065.6 & 29.8 & 1061.9 & 38.6 \\
\hline 0.03394 & 0.11902 & 0.0029 & 724.9 & 16.8 & 722.6 & 21 \\
\hline 0.0291 & 0.11615 & 0.00264 & 708.4 & 15.2 & 777.6 & 18 \\
\hline 0.07704 & 0.20037 & 0.00524 & 1177.3 & 28.2 & 1156.8 & 30.8 \\
\hline 263.38614 & 1.59434 & 3.34426 & & & & \\
\hline 0.05892 & 0.14784 & 0.00406 & 888.8 & 22.8 & 881.6 & 30 \\
\hline 0.02298 & 0.0989 & 0.0023 & 608 & 13.4 & 604.5 & 16.2 \\
\hline 0.0405 & 0.10647 & 0.00292 & 652.2 & 17 & 642.6 & 25.8 \\
\hline 0.04746 & 0.11067 & 0.00316 & 676.6 & 18.4 & 695 & 28.8 \\
\hline 0.02766 & 0.10886 & 0.00258 & 666.1 & 15 & 658.6 & 18.4 \\
\hline 0.03132 & 0.09643 & 0.0025 & 593.5 & 14.6 & 573 & 21.6 \\
\hline 0.0359 & 0.11181 & 0.00284 & 683.3 & 16.4 & 682.5 & 22.6 \\
\hline 0.04186 & 0.13964 & 0.00338 & 842.6 & 19.2 & 862 & 22.8 \\
\hline 0.0231 & 0.09457 & 0.00222 & 582.5 & 13 & 576.7 & 16.6 \\
\hline 0.0114 & 0.05438 & 0.00126 & 341.4 & 7.8 & 340.8 & 10.4 \\
\hline 0.09278 & 0.1878 & 0.00556 & 1109.4 & 30.2 & 1121.5 & 37.6 \\
\hline 0.03446 & 0.12101 & 0.00296 & 736.4 & 17 & 722.8 & 21.2 \\
\hline 0.0433 & 0.1179 & 0.00316 & 718.5 & 18.2 & 711.1 & 26 \\
\hline 0.033 & 0.10946 & 0.0027 & 669.6 & 15.6 & 680.3 & 21 \\
\hline 0.01994 & 0.03319 & 0.00076 & 210.5 & 4.8 & 612.3 & 15 \\
\hline
\end{tabular}




\begin{tabular}{|c|c|c|c|c|c|c|}
\hline 0.02564 & 0.09041 & 0.00222 & 558 & 13.2 & 564.4 & 18.2 \\
\hline 0.04422 & 0.11176 & 0.0031 & 683 & 18 & 679.7 & 27.2 \\
\hline 0.05146 & 0.18251 & 0.0043 & 1080.7 & 23.4 & 1055 & 23.6 \\
\hline 0.03106 & 0.09191 & 0.00208 & 566.8 & 12.2 & 831.8 & 18.4 \\
\hline 0.03442 & 0.11235 & 0.00282 & 686.4 & 16.4 & 673.1 & 21.8 \\
\hline 0.05688 & 0.10037 & 0.0034 & 616.6 & 20 & 605.5 & 36.2 \\
\hline 0.04684 & 0.10058 & 0.00304 & 617.8 & 17.8 & 625 & 30 \\
\hline 0.02652 & 0.09842 & 0.00238 & 605.1 & 14 & 603.9 & 18.4 \\
\hline 0.04074 & 0.11464 & 0.00304 & 699.6 & 17.6 & 685.1 & 25 \\
\hline 0.05794 & 0.16849 & 0.00426 & 1003.8 & 23.6 & 1004.8 & 27 \\
\hline 0.03542 & 0.09896 & 0.00268 & 608.3 & 15.8 & 579.6 & 23.8 \\
\hline 0.11672 & 0.10976 & 0.00436 & & & & \\
\hline 0.08956 & 0.17937 & 0.00502 & 1063.5 & 27.4 & 1198.2 & 34.4 \\
\hline 0.03996 & 0.14531 & 0.00344 & 874.6 & 19.4 & 880.9 & 21.6 \\
\hline 0.09744 & 0.1682 & 0.00396 & 1002.2 & 21.8 & 1606.8 & 27.2 \\
\hline 0.0345 & 0.11066 & 0.0028 & 676.6 & 16.2 & 663.2 & 22 \\
\hline 0.0451 & 0.1137 & 0.00316 & 694.2 & 18.2 & 690.3 & 27.6 \\
\hline 0.02224 & 0.10084 & 0.00232 & 619.3 & 13.6 & 609.1 & 16 \\
\hline 0.03996 & 0.14868 & 0.00352 & 893.6 & 19.8 & 875.4 & 21.8 \\
\hline 0.03956 & 0.10519 & 0.00286 & 644.8 & 16.6 & 646.7 & 25.2 \\
\hline 0.0332 & 0.11198 & 0.00278 & 684.2 & 16.2 & 679 & 21.2 \\
\hline 0.04288 & 0.14343 & 0.00354 & 864 & 20 & 839.6 & 23.4 \\
\hline 0.05354 & 0.11226 & 0.00346 & 685.9 & 20 & 671.2 & 32.6 \\
\hline 1514.6975 & 4.40396 & 9.23834 & & & & \\
\hline 0.29952 & 0.49286 & 0.01194 & 2583 & 51.6 & 2574.3 & 31.8 \\
\hline 0.03578 & 0.11236 & 0.00284 & 686.4 & 16.4 & 685.1 & 22.4 \\
\hline 897.52246 & -10.386 & 20.27402 & & & & \\
\hline 0.08422 & 0.19089 & 0.00532 & 1126.2 & 28.8 & 1121.6 & 34.2 \\
\hline 0.02228 & 0.10976 & 0.00246 & 671.4 & 14.2 & 666.4 & 15.6 \\
\hline 0.0316 & 0.10958 & 0.0027 & 670.3 & 15.6 & 660.3 & 20.6 \\
\hline 0.02548 & 0.10419 & 0.00244 & 638.9 & 14.2 & 634.1 & 17.4 \\
\hline 0.07774 & 0.18164 & 0.00508 & 1075.9 & 27.8 & 1051.9 & 33.8 \\
\hline 0.0262 & 0.10208 & 0.00246 & 626.6 & 14.4 & 602.3 & 18.2 \\
\hline 0.02684 & 0.10684 & 0.00252 & 654.4 & 14.6 & 644.5 & 18 \\
\hline 0.02672 & 0.11205 & 0.00262 & 684.6 & 15.2 & 667.2 & 17.8 \\
\hline 0.0491 & 0.18966 & 0.0043 & 1119.5 & 23.4 & 1112.8 & 21.8 \\
\hline 0.0308 & 0.11659 & 0.00274 & 710.9 & 15.8 & 741.5 & 19.2 \\
\hline 0.0271 & 0.10842 & 0.00256 & 663.6 & 14.8 & 646.4 & 18.2 \\
\hline 0.02568 & 0.09612 & 0.00228 & 591.6 & 13.4 & 614.4 & 17.8 \\
\hline 0.02652 & 0.11668 & 0.00268 & 711.4 & 15.4 & 700.5 & 17.4 \\
\hline 0.03248 & 0.09216 & 0.00206 & 568.3 & 12.2 & 885.8 & 18.4 \\
\hline 0.03076 & 0.1056 & 0.00262 & 647.1 & 15.2 & 638.5 & 20.4 \\
\hline 0.1173 & 0.02596 & 0.0019 & 165.2 & 12 & 1005.3 & 76.6 \\
\hline 0.05432 & 0.10752 & 0.00338 & 658.3 & 19.6 & 660.2 & 33.4 \\
\hline 0.08192 & 0.15726 & 0.0049 & 941.5 & 27.2 & 962.8 & 38.4 \\
\hline
\end{tabular}




\begin{tabular}{|c|c|c|c|c|c|}
\hline age $207 / 206$ & $2 \sigma$ age 76 & $\begin{array}{l}\text { discordance } \\
\Delta 68-75[\%]\end{array}$ & $\Delta 68-76[\%]$ & $\begin{array}{c}\text { preferred age } \\
\text { age }\end{array}$ & $2 \sigma$ age \\
\hline 935.4 & 79.4 & -1.6 & -5.3 & 886.2 & 21.6 \\
\hline 1318.5 & 71.4 & -22.7 & -54.6 & & \\
\hline 603.9 & 112.4 & 2.3 & 11.3 & 672.3 & 18.2 \\
\hline 835.9 & 232.2 & -7.3 & -25.9 & 619.4 & 27 \\
\hline 1139 & 77.4 & -0.9 & -2.6 & 1139 & 77.4 \\
\hline 1151.5 & 49.8 & -12.8 & -35.2 & 746.2 & 16.2 \\
\hline 874 & 69.8 & -1 & -3.2 & 846.1 & 19.8 \\
\hline 671.7 & 93.2 & -1.7 & -7.4 & 622.3 & 16 \\
\hline 560.8 & 86 & 1.7 & 8.8 & 609.9 & 14.8 \\
\hline 808 & 53.2 & -1.9 & -6.9 & 752.5 & 16.2 \\
\hline 1302.2 & 51.6 & -27.5 & -65.6 & & \\
\hline 101.7 & 148.6 & 0.6 & 16.7 & 118.7 & 3.4 \\
\hline 1009.5 & 74 & 0.2 & 0.7 & 1017 & 24.4 \\
\hline 851.7 & 70.8 & 0 & 0 & 851.9 & 19.8 \\
\hline 616.9 & 68 & 0.5 & 2.4 & 631.4 & 14.4 \\
\hline 710.6 & 97.8 & 0.7 & 3.1 & 732.3 & 19 \\
\hline 648 & 107.4 & 3.7 & 17.2 & 759.4 & 20.4 \\
\hline 943 & 82 & 1.4 & 4.8 & 988.1 & 24.6 \\
\hline 802.6 & 50.2 & -1.2 & -4.4 & 767.5 & 16.4 \\
\hline 2613.3 & 58.4 & -51.4 & -77.2 & & \\
\hline 834.7 & 54.8 & 0.3 & 1.1 & 844 & 18.4 \\
\hline 961.2 & 114.4 & 4 & 13.4 & 1089.9 & 31.8 \\
\hline 1042.6 & 66.4 & -6.2 & -18.5 & 849.6 & 19.8 \\
\hline 1047.5 & 90.2 & 2 & 6.3 & & \\
\hline 1073.1 & 77.4 & 0.8 & 2.4 & 1099.1 & 27 \\
\hline 750.2 & 65.8 & 2 & 8.3 & 812.3 & 18.4 \\
\hline 2513.9 & 43 & -6.5 & -11.5 & 2513.9 & 43 \\
\hline 632.5 & 84.2 & -0.8 & -3.2 & 612.1 & 15 \\
\hline 1257.9 & 47.4 & -16.7 & -42.5 & & \\
\hline 603.6 & 65.6 & 1.1 & 5.6 & 637.2 & 14.4 \\
\hline 503.5 & 88.4 & 3.8 & 21.5 & 611.9 & 15 \\
\hline 797.2 & 73.4 & 1.1 & 4.2 & 830.3 & 19.4 \\
\hline 570 & 68.6 & 4.1 & 21.2 & 690.7 & 15.8 \\
\hline 775 & 80.2 & -0.2 & -0.5 & 771.5 & 18.8 \\
\hline 638.9 & 86.8 & -0.4 & -1.7 & 628.1 & 15.6 \\
\hline 521.8 & 64.6 & 3.4 & 18.7 & 619.5 & 14 \\
\hline 1003.4 & 82.2 & 0 & 0.2 & 1005.6 & 25.2 \\
\hline 654.3 & 90.8 & 0.7 & 3.5 & 677 & 17 \\
\hline 2587.3 & 36.2 & -3.7 & -6.6 & 2587.3 & 36.2 \\
\hline 567.5 & 59.8 & 1.6 & 8.2 & 614 & 13.6 \\
\hline 567.8 & 84 & 2.2 & 11.5 & 633 & 15.4 \\
\hline 6284.3 & 96 & -92.7 & -97.5 & & \\
\hline 703.8 & 51.4 & -2 & -8.2 & 645.8 & 14 \\
\hline 1047.2 & 82 & 2.7 & 8.6 & & \\
\hline 820.3 & 62.2 & 1.7 & 6.6 & 874.1 & 19.6 \\
\hline 590.9 & 74.2 & 1.1 & 5.8 & 625.4 & 14.6 \\
\hline 506.6 & 118 & 3.8 & 21.5 & 615.2 & 16.8 \\
\hline 643.5 & 112.6 & -0.5 & -2.2 & 629.4 & 17.4 \\
\hline
\end{tabular}




\begin{tabular}{|c|c|c|c|c|c|}
\hline 706.9 & 64.2 & 1.5 & 6.6 & 753.8 & 17 \\
\hline 1031.6 & 48.4 & -0.9 & -2.7 & 1003.3 & 21.2 \\
\hline 689.9 & 64.2 & 0 & 0.4 & 692.3 & 15.6 \\
\hline 584.7 & 79.6 & 1.6 & 8.2 & 632.6 & 15 \\
\hline 1579 & 46.4 & -35.7 & -73.1 & & \\
\hline 761.7 & 67.2 & -4.4 & -16.8 & 634 & 14.6 \\
\hline 503.1 & 99 & 2.8 & 15.9 & 583.2 & 15 \\
\hline 717.3 & 58.4 & 1.9 & 7.9 & 774 & 17 \\
\hline 625.8 & 56.2 & 1.1 & 5.4 & 659.4 & 14.4 \\
\hline 500.8 & 104.6 & 3.4 & 19.6 & 598.8 & 15.6 \\
\hline 718 & 69.2 & 1.3 & 5.7 & 759.2 & 17.4 \\
\hline 698.1 & 69.4 & 1.4 & 6.3 & 741.9 & 17.2 \\
\hline 926.3 & 65.2 & -1.8 & -6.1 & 870.2 & 20 \\
\hline 974 & 72.4 & -0.5 & -1.4 & 960 & 22.8 \\
\hline 1554.4 & 53.4 & -28.8 & -61.2 & & \\
\hline 526.4 & 151.4 & 2.2 & 12.2 & 590.4 & 18.4 \\
\hline 1110.5 & 101.2 & 0.1 & 0.4 & 1110.5 & 101.2 \\
\hline 1002.8 & 63.8 & -7.8 & -24 & 762.2 & 17.6 \\
\hline 585.1 & 125.6 & 3.9 & 19.3 & 698.3 & 20 \\
\hline 2584.9 & 37.8 & -52.2 & -78.5 & & \\
\hline-86.3 & 174.2 & 33.6 & -1057.4 & & \\
\hline 948.2 & 62 & -8.3 & -26.7 & 695 & 15.8 \\
\hline 543.7 & 76.4 & 3 & 15.9 & 630.4 & 14.8 \\
\hline 2079.5 & 41.6 & -40.5 & -70.1 & & \\
\hline 642.8 & 57 & 0.2 & 1.3 & 651.3 & 14.4 \\
\hline \multirow[t]{2}{*}{947.6} & 80 & 3.7 & 12.5 & 1065.9 & 26.2 \\
\hline & & -145.2 & & & \\
\hline 2328.4 & 35.6 & -5.1 & -9.5 & 2328.4 & 35.6 \\
\hline 1024.7 & 75.6 & -0.1 & -0.1 & 1023.7 & 24.8 \\
\hline 760.4 & 109.8 & -3.5 & -13.4 & 658.1 & 18.4 \\
\hline 579.2 & 74.8 & 0.5 & 2.6 & 594.3 & 14 \\
\hline 1211.2 & 56.6 & -15.3 & -40.1 & & \\
\hline 610.1 & 71.2 & 0.9 & 4.4 & 636.9 & 14.8 \\
\hline 590.2 & 102.6 & 1.7 & 8.7 & 641.4 & 16.8 \\
\hline 1053.2 & 101.2 & 0.3 & 1.2 & 1065.6 & 29.8 \\
\hline 714.3 & 68.6 & 0.3 & 1.5 & 724.9 & 16.8 \\
\hline 980.6 & 48.8 & -8.9 & -27.8 & 708.4 & 15.2 \\
\hline 1117.3 & 72.2 & 1.8 & 5.4 & & \\
\hline 862.1 & 89.4 & 0.8 & 3.1 & 888.8 & 22.8 \\
\hline 590.5 & 59 & 0.6 & 2.9 & 608 & 13.4 \\
\hline 607.5 & 100.8 & 1.5 & 7.4 & 652.2 & 17 \\
\hline 753.8 & 104.2 & -2.6 & -10.2 & 676.6 & 18.4 \\
\hline 631.5 & 63.8 & 1.1 & 5.5 & 666.1 & 15 \\
\hline 491.1 & 92.2 & 3.6 & 20.8 & 593.5 & 14.6 \\
\hline 678.9 & 80.4 & 0.1 & 0.6 & 683.3 & 16.4 \\
\hline 910.9 & 63.4 & -2.3 & -7.5 & 842.6 & 19.2 \\
\hline 552.6 & 64.8 & 1 & 5.4 & 582.5 & 13 \\
\hline 336.1 & 63 & 0.2 & 1.6 & 341.4 & 7.8 \\
\hline 1143.6 & 93 & -1.1 & -3 & 1143.6 & 93 \\
\hline 679.6 & 70.2 & 1.9 & 8.4 & 736.4 & 17 \\
\hline 686.5 & 92.4 & 1 & 4.7 & 718.5 & 18.2 \\
\hline 714.3 & 73.4 & -1.6 & -6.3 & 669.6 & 15.6 \\
\hline 2659.9 & 38.2 & -65.6 & -92.1 & & \\
\hline
\end{tabular}




\begin{tabular}{|c|c|c|c|c|c|}
\hline 589.1 & 74.2 & -1.1 & -5.3 & 558 & 13.2 \\
\hline 667.5 & 101.2 & 0.5 & 2.3 & 683 & 18 \\
\hline 1000.8 & 55.4 & 2.4 & 8 & 1080.7 & 23.4 \\
\hline 1625 & 43.2 & -31.9 & -65.1 & & \\
\hline 627.6 & 78.8 & 2 & 9.4 & 686.4 & 16.4 \\
\hline 562.7 & 156.8 & 1.8 & 9.6 & 616.6 & 20 \\
\hline 649.8 & 121.8 & -1.1 & -4.9 & 617.8 & 17.8 \\
\hline 597.8 & 70.2 & 0.2 & 1.2 & 605.1 & 14 \\
\hline 636.4 & 91.8 & 2.1 & 9.9 & 699.6 & 17.6 \\
\hline 1005.9 & 69.2 & -0.1 & -0.2 & 1003.8 & 23.6 \\
\hline 467.4 & 103.6 & 4.9 & 30.2 & 608.3 & 15.8 \\
\hline 1448.9 & 76.4 & -11.2 & -26.6 & 1063.5 & 27.4 \\
\hline 895.4 & 58.2 & -0.7 & -2.3 & 874.6 & 19.4 \\
\hline 2524.1 & 40.6 & -37.6 & -60.3 & & \\
\hline 616.5 & 80.8 & 2 & 9.7 & 676.6 & 16.2 \\
\hline 676.5 & 101.2 & 0.6 & 2.6 & 694.2 & 18.2 \\
\hline 570 & 56.8 & 1.7 & 8.6 & 619.3 & 13.6 \\
\hline 828.7 & 59.4 & 2.1 & 7.8 & 893.6 & 19.8 \\
\hline 652.2 & 97.2 & -0.3 & -1.1 & 644.8 & 16.6 \\
\hline 660.3 & 74.4 & 0.8 & 3.6 & 684.2 & 16.2 \\
\hline 774.3 & 69.2 & 2.9 & 11.6 & 864 & 20 \\
\hline 620.8 & 127 & 2.2 & 10.5 & 685.9 & 20 \\
\hline 2566.4 & 40.2 & 0.3 & 0.6 & 2566.4 & 40.2 \\
\hline 679.6 & 79.8 & 0.2 & 1 & 686.4 & 16.4 \\
\hline 1111.6 & 84 & 0.4 & 1.3 & 1111.6 & 84 \\
\hline 648.4 & 48.4 & 0.7 & 3.5 & 671.4 & 14.2 \\
\hline 625.1 & 74 & 1.5 & 7.2 & 670.3 & 15.6 \\
\hline 615.8 & 61.6 & 0.8 & 3.8 & 638.9 & 14.2 \\
\hline 1001.1 & 87.8 & 2.3 & 7.5 & 1075.9 & 27.8 \\
\hline 510.8 & 70.4 & 4 & 22.7 & 626.6 & 14.4 \\
\hline 609 & 64 & 1.5 & 7.5 & 654.4 & 14.6 \\
\hline 607.5 & 60.4 & 2.6 & 12.7 & 684.6 & 15.2 \\
\hline 1098.5 & 46.2 & 0.6 & 1.9 & 1098.5 & 46.2 \\
\hline 833.7 & 58 & -4.1 & -14.7 & 710.9 & 15.8 \\
\hline 585.8 & 65 & 2.7 & 13.3 & 663.6 & 14.8 \\
\hline 698.1 & 64.6 & -3.7 & -15.2 & 591.6 & 13.4 \\
\hline 664.4 & 54.8 & 1.6 & 7.1 & 711.4 & 15.4 \\
\hline 1791.8 & 39.6 & -35.8 & -68.3 & & \\
\hline 606.8 & 75.6 & 1.3 & 6.6 & 647.1 & 15.2 \\
\hline 4156.6 & 141.2 & -83.6 & -96 & & \\
\hline 665.5 & 131 & -0.3 & -1.1 & 658.3 & 19.6 \\
\hline 1010.3 & 108 & -2.2 & -6.8 & 941.5 & 27.2 \\
\hline
\end{tabular}




\section{Sample 2947S Abay @ Goha Tsiyon 27 grain analysed 4 concordant ages}

\begin{tabular}{|c|c|c|c|c|c|c|}
\hline \multirow[b]{2}{*}{ grain } & \multicolumn{3}{|c|}{ concentrations } & \multicolumn{3}{|l|}{ isotopic ratios } \\
\hline & U [ppm] & $\mathrm{Pb}$ [ppm] & $\mathrm{Th} / \mathrm{U}$ & $\mathrm{Pb} 207 / \mathrm{Pb} 206$ & $2 \sigma 76$ & $\mathrm{~Pb} 207 / \mathrm{U} 235$ \\
\hline X2947S G001 & & & & 0.34273 & 1.82988 & 19.22721 \\
\hline X2947S_G002 & & & & 0.53893 & 0.36088 & 47.95984 \\
\hline X2947S_G003 & & & & 0.70656 & 0.11098 & 415.86508 \\
\hline X2947S_G004 & & & & 0.84341 & 0.04162 & 944.12683 \\
\hline X2947S_G005 & & & & 0.80089 & 0.04746 & \\
\hline X2947S_G006 & & & & 54.37289 & & 227.14677 \\
\hline X2947S G007 & & & & 0.81362 & 0.44976 & \\
\hline X2947S_G008 & & & & 0.82595 & 0.0722 & 277.06625 \\
\hline X2947S_G009 & & & & 0.82426 & 0.03724 & \\
\hline X2947S_G010 & & & & 0.89333 & 1.07674 & 486.72595 \\
\hline X2947S_G011 & & & & 0.82663 & 0.16908 & 143.5423 \\
\hline X2947S_G012 & & & & 3.02105 & 13.33338 & 247.33389 \\
\hline X2947S_G013 & & & & 0.80884 & 0.16122 & 179.79218 \\
\hline X2947S_G014 & & & & -0.89884 & 3.89154 & -73.59762 \\
\hline X2947S_G015 & & & & -6.19172 & 55.24828 & \\
\hline X2947S_G016 & & & & 9.89654 & 1.69788 & \\
\hline X2947S_G017 & & & & 0.75171 & 0.13072 & 82.12801 \\
\hline X2947S_G018 & & & & 0.56459 & 0.56 & 20.70885 \\
\hline X2947S G019 & & & & 0.72041 & 0.1733 & 628.38251 \\
\hline X2947S_G020 & & & & 0.84142 & 0.12068 & 40.47459 \\
\hline X2947S_G021 & 77.3 & 15.6 & 0.9728 & 0.07272 & 0.00372 & 1.66031 \\
\hline X2947S G022 & 241.9 & 97 & 0.9711 & 0.11773 & 0.00422 & 5.28007 \\
\hline X2947S_G023 & 1279.5 & 66.6 & 1.0518 & 0.12153 & 0.00436 & 0.67564 \\
\hline X2947S_G024 & 79.3 & 9.2 & 1.098 & 0.06039 & 0.0042 & 0.77127 \\
\hline X2947S_G025 & 362.5 & 15.7 & 0.4915 & 0.05195 & 0.00284 & 0.29254 \\
\hline X2947S_G026 & & & & -1.67749 & 6.99308 & -53.55665 \\
\hline X2947S_G027 & & & & -0.0638 & 0.80748 & -29.70832 \\
\hline
\end{tabular}




\section{$14.8 \%$ concordant}

\begin{tabular}{|c|c|c|c|c|c|c|}
\hline $2 \sigma 75$ & Pb206/U238 & $2 \sigma 68$ & $\begin{array}{c}\text { ages } \\
\text { age } 206 / 238\end{array}$ & $2 \sigma$ age 68 & age $207 / 235$ & $2 \sigma$ age 75 \\
\hline 92.6551 & 0.40702 & 0.9636 & & & & \\
\hline 33.21416 & 0.64565 & 0.25098 & & & & \\
\hline 148.7944 & 4.27023 & 1.55416 & & & & \\
\hline 130.08126 & 8.12156 & 1.12544 & & & & \\
\hline 664.2483 & 21.44522 & 6.03284 & & & & \\
\hline 159.22564 & 0.03031 & 2.64166 & & & & \\
\hline 1160.1997 & 10.80138 & 10.74022 & & & & \\
\hline 37.38434 & 2.43375 & 0.34732 & & & & \\
\hline 146.97506 & 9.29997 & 1.2956 & & & & \\
\hline 1643.13172 & 3.9529 & 13.6406 & & & & \\
\hline 40.2347 & 1.25983 & 0.37538 & & & & \\
\hline 302.0744 & 0.59397 & 2.6555 & & & & \\
\hline 47.25838 & 1.61268 & 0.4418 & & & & \\
\hline 205.35496 & 0.59404 & 2.13854 & & & & \\
\hline 972.48834 & 0.34766 & 3.27704 & & & & \\
\hline 224.21444 & 0.7376 & 0.20272 & & & & \\
\hline 15.85486 & 0.79264 & 0.17022 & & & & \\
\hline 17.58908 & 0.26611 & 0.15204 & & & & \\
\hline 395.98754 & 6.32823 & 3.94458 & & & & \\
\hline 5.51556 & 0.34898 & 0.05056 & & & & \\
\hline 0.0826 & 0.16563 & 0.00482 & 988 & 26.6 & 993.5 & 37.2 \\
\hline 0.18812 & 0.32537 & 0.00832 & 1815.9 & 40.4 & 1865.6 & 37.6 \\
\hline 0.02358 & 0.04033 & 0.001 & 254.9 & 6.2 & 524.1 & 17.8 \\
\hline 0.05176 & 0.09266 & 0.00302 & 571.2 & 17.8 & 580.5 & 34 \\
\hline 0.0155 & 0.04085 & 0.00112 & 258.1 & 7 & 260.6 & 14 \\
\hline 95.87198 & 0.23162 & 0.87948 & & & & \\
\hline 387.09698 & 3.37797 & 11.81528 & & & & \\
\hline
\end{tabular}




\begin{tabular}{|cc|cc|cc|}
\hline & & discordance & preferred age & \\
age 207/206 & $2 \sigma$ age 76 & $\Delta 68-75[\%]$ & $\Delta 68-76[\%]$ & age & 20 age \\
\hline
\end{tabular}

$\begin{array}{lccccc}1006.2 & 103.8 & -0.6 & -1.8 & 988 & 26.6 \\ 1922.1 & 64.2 & -2.7 & -5.5 & 1922.1 & 64.2 \\ 1978.8 & 63.8 & -51.4 & -87.1 & & \\ 617.6 & 150.2 & -1.6 & -7.5 & 571.2 & 17.8 \\ 283.2 & 125 & -0.9 & -8.9 & 258.1 & 7\end{array}$




\section{Sample 2975S Anger @ Tsige Maryam 116 grain analysed 101 concordant ag€}

\begin{tabular}{|c|c|c|c|c|c|c|}
\hline \multirow[b]{2}{*}{ grain } & \multicolumn{2}{|c|}{ concentrations } & \multicolumn{4}{|l|}{ isotopic ratios } \\
\hline & $\mathbf{U}[\mathrm{ppm}]$ & $T h / U$ & Pb207/Pb206 & $2 \sigma 76$ & Pb207/U235 & $2 \sigma 75$ \\
\hline 2975005 & 800.6 & 346.9 & 0.05939 & 0.00099 & 0.69095 & 0.01157 \\
\hline 2975012 & 1166.6 & 1579.9 & 0.06229 & 0.00086 & 0.88701 & 0.01267 \\
\hline 2975018 & 450.1 & 276.9 & 0.05985 & 0.0011 & 0.77883 & 0.01434 \\
\hline 2975020 & 554.9 & 428.6 & 0.05989 & 0.001 & 0.87054 & 0.01462 \\
\hline 2975_021 & 376.3 & 176.9 & 0.06855 & 0.0012 & 1.32989 & 0.02341 \\
\hline 2975_022 & 917.5 & 506.0 & 0.06153 & 0.00092 & 0.82845 & 0.01268 \\
\hline 2975023 & 183.8 & 219.9 & 0.09633 & 0.00193 & 1.61205 & 0.03153 \\
\hline 2975024 & 2395.0 & 1144.8 & 0.11615 & 0.0015 & 0.88916 & 0.01189 \\
\hline 2975025 & 216.5 & 167.0 & 0.06193 & 0.00146 & 0.86247 & 0.01994 \\
\hline 2975_029 & 97.9 & 8.0 & 0.06092 & 0.00216 & 0.85369 & 0.02923 \\
\hline 2975_031 & 542.9 & 309.0 & 0.06464 & 0.00104 & 1.10878 & 0.01799 \\
\hline 2975033 & 1515.4 & 1173.2 & 0.06223 & 0.00086 & 0.87017 & 0.01237 \\
\hline 2975035 & 2932.8 & 1634.2 & 0.06748 & 0.00082 & 1.14845 & 0.01475 \\
\hline 2975036 & 1340.1 & 88.9 & 0.06105 & 0.00087 & 0.80149 & 0.01176 \\
\hline 2975_040 & 1549.7 & 2134.4 & 0.06446 & 0.00091 & 0.79808 & 0.01159 \\
\hline 2975_042 & 1998.1 & 1375.8 & 0.08731 & 0.00122 & 1.08259 & 0.01549 \\
\hline 2975_045 & 36.1 & 9.0 & 0.06307 & 0.00342 & 1.17522 & 0.06167 \\
\hline 2975046 & 2360.6 & 2458.4 & 0.07706 & 0.00104 & 0.79365 & 0.01102 \\
\hline 2975047 & 4520.3 & 1568.6 & 0.1227 & 0.00148 & 1.14505 & 0.0145 \\
\hline 2975_052 & 386.6 & 331.5 & 0.05959 & 0.00123 & 0.85802 & 0.01753 \\
\hline 2975_055 & 963.8 & 1396.6 & 0.06476 & 0.001 & 0.75893 & 0.0119 \\
\hline 2975_056 & 1458.7 & 1070.5 & 0.06169 & 0.00085 & 0.88167 & 0.01253 \\
\hline 2975057 & 620.2 & 525.9 & 0.06643 & 0.00106 & 1.12773 & 0.01817 \\
\hline 2975058 & 218.2 & 168.4 & 0.06434 & 0.00143 & 1.17686 & 0.02583 \\
\hline 2975_062 & 1168.3 & 169.4 & 0.06302 & 0.00093 & 0.89816 & 0.01351 \\
\hline 2975_063 & 542.9 & 297.9 & 0.06139 & 0.00106 & 0.91325 & 0.01576 \\
\hline 2975_064 & 381.4 & 250.5 & 0.06113 & 0.0012 & 0.92396 & 0.01795 \\
\hline 2975_068 & 386.6 & 230.5 & 0.06235 & 0.00122 & 0.88454 & 0.01715 \\
\hline 2975074 & 175.2 & 80.1 & 0.06622 & 0.00161 & 1.164 & 0.02774 \\
\hline 2975078 & 391.7 & 349.1 & 0.06421 & 0.00126 & 0.92398 & 0.01796 \\
\hline 2975_080 & 261.1 & 373.2 & 0.06562 & 0.00156 & 0.80806 & 0.01879 \\
\hline 2975_083 & 634.0 & 392.4 & 0.05858 & 0.00139 & 0.33535 & 0.00776 \\
\hline 2975_084 & 496.5 & 309.2 & 0.06461 & 0.00112 & 1.12523 & 0.01955 \\
\hline 2975085 & 876.2 & 228.2 & 0.0643 & 0.00096 & 1.08725 & 0.01646 \\
\hline 2975087 & 1142.5 & 707.7 & 0.06041 & $9.00 \mathrm{E}-04$ & 0.83547 & 0.01267 \\
\hline 2975_088 & 139.2 & 94.0 & 0.05969 & 0.00178 & 0.83585 & 0.02415 \\
\hline 2975_089 & 24.1 & 0.2 & 0.06462 & 0.00479 & 0.98694 & 0.07056 \\
\hline 2975_091 & 491.4 & 446.2 & 0.06139 & 0.00113 & 0.82938 & 0.01523 \\
\hline 2975092 & 331.6 & 80.0 & 0.07007 & 0.0019 & 1.15365 & 0.03026 \\
\hline 2975093 & 1924.3 & 1242.5 & 0.06493 & 0.00116 & 0.73135 & 0.01297 \\
\hline 2975_098 & 345.3 & 162.9 & 0.06754 & 0.0013 & 1.14882 & 0.02184 \\
\hline 2975_100 & 218.2 & 55.4 & 0.06354 & 0.00152 & 1.02808 & 0.0241 \\
\hline 2975_101 & 441.5 & 150.5 & 0.06315 & 0.0012 & 0.92652 & 0.01752 \\
\hline $2975^{-} 102$ & 2168.2 & 412.1 & 0.06629 & 0.00089 & 1.05983 & 0.01461 \\
\hline 2975105 & 187.3 & 73.4 & 0.06837 & 0.00164 & 1.22468 & 0.02863 \\
\hline 2975_106 & 5848.4 & 1179.9 & 0.07415 & 0.001 & 0.48693 & 0.00675 \\
\hline 2975_107 & 154.6 & 161.8 & 0.06596 & 0.00191 & 0.94866 & 0.02666 \\
\hline 2975_109 & 5166.3 & 2818.6 & 0.05072 & 0.00072 & 0.28504 & 0.00415 \\
\hline $2975^{-} 114$ & 2235.2 & 840.4 & 0.07149 & 0.00093 & 1.56884 & 0.0211 \\
\hline 2975116 & 3376.0 & 561.3 & 0.07349 & 0.001 & 0.73004 & 0.01016 \\
\hline 2975_117 & 1912.2 & 2677.4 & 0.07371 & 0.0011 & 0.63585 & 0.00959 \\
\hline 2975_120 & 1432.9 & 654.3 & 0.06315 & 0.00092 & 0.9931 & 0.01469 \\
\hline 2975_121 & 1322.9 & 595.1 & 0.07064 & 0.00102 & 1.20957 & 0.01784 \\
\hline 2975_122 & 446.7 & 199.3 & 0.10877 & 0.00151 & 4.74785 & 0.06789 \\
\hline
\end{tabular}




\begin{tabular}{|c|c|c|c|c|c|c|}
\hline 2975_123 & 692.4 & 326.2 & 0.06115 & 0.00121 & 0.86898 & 0.01701 \\
\hline 2975_124 & 486.2 & 242.5 & 0.06634 & 0.00119 & 1.19783 & 0.0214 \\
\hline 2975_125 & 965.6 & 909.3 & 0.06776 & 0.00129 & 0.99614 & 0.01878 \\
\hline 2975_126 & 163.2 & 55.9 & 0.07028 & 0.00203 & 0.99768 & 0.02789 \\
\hline 2975_127 & 61.9 & 42.0 & 0.06643 & 0.00296 & 1.19891 & 0.05159 \\
\hline 2975_131 & 271.5 & 285.7 & 0.06004 & 0.00144 & 0.89293 & 0.0209 \\
\hline 2975_132 & 5970.3 & 5362.0 & 0.0942 & 0.00129 & 0.49352 & 0.00686 \\
\hline 2975_134 & 127.1 & 48.1 & 0.06391 & 0.00202 & 0.99822 & 0.0306 \\
\hline 2975_136 & 372.8 & 362.5 & 0.05933 & 0.00131 & 0.75735 & 0.0164 \\
\hline 2975_137 & 570.4 & 498.1 & 0.06233 & 0.00114 & 0.9361 & $0.016 s$ \\
\hline 2975_140 & 89.3 & 62.8 & 0.06 & 0.00267 & 0.7096 & 0.030 \\
\hline 2975_141 & 158.1 & 213.0 & 0.0599 & 0.00203 & 0.69973 & 0.022 \\
\hline 2975_142 & 795.5 & 587.3 & 0.06738 & 0.00108 & 1.20856 & 0.019 \\
\hline 2975_144 & 341.9 & 203.5 & 0.06817 & 0.00138 & 1.15664 & 0.023 \\
\hline 2975_146 & 304.1 & 185.1 & 0.05926 & 0.00139 & 0.91569 & 0.020 \\
\hline 2975_147 & 446.7 & 249.3 & 0.06743 & 0.00123 & 1.27167 & 0.023 \\
\hline 2975_148 & 381.4 & 75.4 & 0.06427 & 0.00129 & 1.14738 & 0.0226 \\
\hline 2975_149 & 1651.1 & 387.8 & 0.05893 & 0.00097 & 0.55288 & 0.009 \\
\hline 2975_152 & 515.4 & 255.5 & 0.04134 & 0.00505 & 0.02722 & 0.003 \\
\hline 2975153 & 403.8 & 130.5 & 0.07099 & 0.00138 & 1.07977 & 0.0207 \\
\hline 2975_157 & 379.7 & 444.7 & 0.06782 & 0.00145 & 0.92576 & 0.0193 \\
\hline 2975_158 & 845.3 & 243.3 & 0.06639 & 0.00105 & 1.21253 & 0.019 \\
\hline 2975_160 & 247.4 & 169.5 & 0.07147 & 0.00143 & 1.69118 & 0.033 \\
\hline 2975_161 & 190.7 & 78.2 & 0.07423 & 0.00164 & 1.70053 & $0.036 \varepsilon$ \\
\hline 2975_162 & 1503.3 & 358.6 & 0.06496 & 0.00095 & 1.12596 & 0.016 \\
\hline 2975_163 & 3766.0 & 5436.9 & 0.13009 & 0.00181 & 1.29649 & 0.018 \\
\hline 2975_165 & 1359.0 & 1252.9 & 0.07936 & 0.00117 & 1.30312 & 0.019 \\
\hline 2975_166 & 862.5 & 747.8 & 0.06377 & 0.00107 & 1.10548 & 0.018 \\
\hline 2975_168 & 1668.3 & 958.2 & 0.065 & 0.00105 & 0.85604 & 0.0139 \\
\hline 2975_169 & 49.8 & 14.4 & 0.0705 & 0.00332 & 1.2102 & $0.054 \varepsilon$ \\
\hline 2975_170 & 257.7 & 206.5 & 0.06626 & 0.00146 & 1.21081 & 0.02617 \\
\hline 2975_172 & 357.4 & 176.6 & 0.06451 & 0.00132 & 1.22057 & 0.024 \\
\hline 2975_173 & 3121.8 & 125.2 & 0.07072 & 0.00097 & 1.37432 & 0.019 \\
\hline 2975_174 & 323.0 & 161.4 & 0.06049 & 0.00145 & 0.84742 & 0.019 \\
\hline 2975_175 & 300.7 & 218.0 & 0.06134 & 0.00151 & 0.86337 & 0.020 \\
\hline 2975_176 & 577.3 & 496.6 & 0.06426 & 0.00125 & 0.90324 & 0.017 \\
\hline 2975_179 & 1527.4 & 689.2 & 0.05669 & 0.00097 & 0.57123 & 0.009 \\
\hline 2975_183 & 917.5 & 1682.6 & 0.22847 & 0.00303 & 16.46729 & $0.224 \varepsilon$ \\
\hline 2975_184 & 379.7 & 279.2 & 0.06246 & 0.0014 & 0.926 & 0.0202 \\
\hline 2975_185 & 419.2 & 159.1 & 0.06137 & 0.0013 & 0.92249 & 0.0191 \\
\hline 2975_187 & 1324.6 & 467.6 & 0.06614 & 0.00106 & 0.96109 & 0.015 \\
\hline 2975_188 & 1733.5 & 914.9 & 0.06214 & 0.00095 & 0.86221 & 0.0133 \\
\hline 2975_190 & 443.3 & 295.4 & 0.06509 & 0.00124 & 1.13784 & 0.021 \\
\hline 2975_191 & 1197.5 & 939.9 & 0.06203 & 0.001 & 0.88707 & 0.014 \\
\hline 2975_192 & 144.3 & 31.2 & 0.13941 & 0.00304 & 2.68258 & $0.055 \varepsilon$ \\
\hline 2975_193 & 2235.2 & 1056.3 & 0.06591 & 0.00102 & 0.62002 & 0.009 \\
\hline 2975_195 & 1848.7 & 922.6 & 0.06142 & 0.00094 & 0.82383 & 0.012 \\
\hline 2975_197 & 558.4 & 298.7 & 0.22157 & 0.00302 & 17.46339 & 0.2443 \\
\hline 2975_198 & 769.7 & 494.5 & 0.07179 & 0.00113 & 1.42628 & 0.0225 \\
\hline 2975_199 & 965.6 & 550.5 & 0.06401 & 0.00104 & 0.87982 & 0.0142 \\
\hline 2975_200 & 544.6 & 460.9 & 0.06336 & 0.00125 & 0.8865 & 0.017 \\
\hline 2975_201 & 130.6 & 88.3 & 0.07061 & 0.00174 & 1.38669 & 0.0333 \\
\hline 2975_202 & 2620.1 & 313.5 & 0.07484 & 0.00112 & 0.87984 & 0.013 \\
\hline 2975_204 & 518.9 & 818.5 & 0.06233 & 0.00115 & 0.877 & 0.016 \\
\hline 2975_206 & 551.5 & 187.3 & 0.07126 & 0.00117 & 1.44363 & 0.0237 \\
\hline 2975_207 & 417.5 & 293.0 & 0.06734 & 0.00123 & 1.1628 & 0.021 \\
\hline 2975_208 & 195.9 & 109.0 & 0.06047 & 0.00161 & 0.84459 & 0.0218 \\
\hline 2975_211 & 84.2 & 47.7 & 0.06194 & 0.00241 & 0.82844 & 0.0310 \\
\hline 2975_212 & 1341.8 & 1254.4 & 0.07893 & 0.00127 & 0.90229 & 0.014 \\
\hline
\end{tabular}




$\begin{array}{lcccccc}\text { 2975_213 } & 1682.0 & 1324.6 & 0.06722 & 0.00103 & 0.85745 & 0.0132 \\ \text { 2975_214 } & 1523.9 & 164.3 & 0.11733 & 0.00163 & 5.08745 & 0.07181 \\ \text { 2975_215 } & 563.5 & 343.5 & 0.05931 & 0.00113 & 0.81725 & 0.01531\end{array}$




\begin{tabular}{|c|c|c|c|c|c|c|}
\hline $\mathrm{Pb206/U238}$ & $2 \sigma 68$ & $\begin{array}{c}\text { ages } \\
\text { age } 206 / 238\end{array}$ & $2 \sigma$ age 68 & age $207 / 235$ & $2 \sigma$ age 75 & age $207 / 206$ \\
\hline 0.08443 & 0.00102 & 522.5 & 6.09 & 533.4 & 6.95 & 581.4 \\
\hline 0.10335 & 0.0012 & 634 & 7.03 & 644.8 & 6.82 & 684 \\
\hline 0.09444 & 0.00118 & 581.7 & 6.96 & 584.8 & 8.18 & 598.1 \\
\hline 0.10548 & 0.00128 & 646.5 & 7.48 & 635.9 & 7.94 & 599.7 \\
\hline 0.14079 & 0.00177 & 849.1 & 9.99 & 858.8 & 10.2 & 885.2 \\
\hline 0.09772 & 0.00116 & 601 & 6.79 & 612.8 & 7.04 & 657.7 \\
\hline 0.12145 & 0.00167 & 738.9 & 9.59 & 974.9 & 12.26 & 1554.1 \\
\hline 0.05555 & 0.00065 & 348.5 & 3.95 & 645.9 & 6.39 & 1897.8 \\
\hline 0.10106 & 0.00139 & 620.6 & 8.14 & 631.5 & 10.87 & 671.7 \\
\hline 0.1017 & 0.00171 & 624.3 & 9.98 & 626.7 & 16.01 & 636.4 \\
\hline 0.12448 & 0.00151 & 756.3 & 8.65 & 757.6 & 8.66 & 762.7 \\
\hline 0.10147 & 0.00118 & 623 & 6.89 & 635.7 & 6.72 & 682.1 \\
\hline 0.12351 & 0.0014 & 750.7 & 8.04 & 776.5 & 6.97 & 852.6 \\
\hline 0.09527 & 0.00111 & 586.6 & 6.55 & 597.7 & 6.63 & 641 \\
\hline 0.08985 & 0.00105 & 554.7 & 6.2 & 595.7 & 6.54 & 756.6 \\
\hline 0.08998 & 0.00106 & 555.4 & 6.27 & 744.9 & 7.55 & 1367.4 \\
\hline 0.13522 & 0.00309 & 817.6 & 17.53 & 789.1 & 28.79 & 710.6 \\
\hline 0.07474 & 0.00087 & 464.6 & 5.19 & 593.2 & 6.24 & 1122.7 \\
\hline 0.06772 & 0.00077 & 422.4 & 4.65 & 774.9 & 6.87 & 1995.9 \\
\hline 0.10449 & 0.00136 & 640.7 & 7.92 & 629 & 9.58 & 588.6 \\
\hline 0.08504 & 0.00101 & 526.1 & 6.02 & 573.4 & 6.87 & 766.6 \\
\hline 0.10371 & 0.0012 & 636.1 & 7.01 & 641.9 & 6.76 & 663.4 \\
\hline 0.12319 & 0.00149 & 748.9 & 8.54 & 766.7 & 8.67 & 820 \\
\hline 0.13272 & 0.00181 & 803.4 & 10.28 & 789.9 & 12.05 & 753 \\
\hline 0.10342 & 0.00122 & 634.4 & 7.1 & 650.7 & 7.23 & 709 \\
\hline 0.10795 & 0.00132 & 660.8 & 7.69 & 658.8 & 8.36 & 653 \\
\hline 0.10968 & 0.0014 & 670.9 & 8.14 & 664.5 & 9.47 & 643.9 \\
\hline 0.10295 & 0.00132 & 631.7 & 7.69 & 643.4 & 9.24 & 686.1 \\
\hline 0.12755 & 0.00181 & 773.9 & 10.36 & 783.8 & 13.02 & 813.3 \\
\hline 0.10442 & 0.00134 & 640.3 & 7.82 & 664.5 & 9.48 & 748.6 \\
\hline 0.08936 & 0.00124 & 551.7 & 7.32 & 601.4 & 10.55 & 794.3 \\
\hline 0.04154 & 0.00056 & 262.4 & 3.44 & 293.6 & 5.9 & 551.4 \\
\hline 0.12638 & 0.00156 & 767.2 & 8.92 & 765.5 & 9.34 & 761.6 \\
\hline 0.12271 & 0.00144 & 746.1 & 8.3 & 747.2 & 8.01 & 751.4 \\
\hline 0.10035 & 0.00118 & 616.5 & 6.89 & 616.6 & 7.01 & 618.3 \\
\hline 0.10161 & 0.00154 & 623.8 & 9.01 & 616.9 & 13.36 & 593 \\
\hline 0.11082 & 0.00306 & 677.5 & 17.74 & 697.2 & 36.06 & 762 \\
\hline 0.09804 & 0.00122 & 602.9 & 7.17 & 613.3 & 8.45 & 652.9 \\
\hline 0.11946 & 0.00182 & 727.5 & 10.46 & 779 & 14.27 & 930.5 \\
\hline 0.08174 & 0.00101 & 506.5 & 6.02 & 557.3 & 7.6 & 772 \\
\hline 0.12342 & 0.00158 & 750.2 & 9.08 & 776.7 & 10.32 & 854.5 \\
\hline 0.1174 & 0.00163 & 715.6 & 9.41 & 718 & 12.06 & 726.4 \\
\hline 0.10646 & 0.00135 & 652.2 & 7.84 & 665.8 & 9.24 & 713.3 \\
\hline 0.11601 & 0.00133 & 707.6 & 7.67 & 733.7 & 7.2 & 815.6 \\
\hline 0.12999 & 0.00184 & 787.8 & 10.47 & 811.9 & 13.07 & 879.7 \\
\hline 0.04765 & 0.00055 & 300.1 & 3.36 & 402.8 & 4.61 & 1045.6 \\
\hline 0.10437 & 0.00159 & 640 & 9.28 & 677.4 & 13.89 & 805 \\
\hline 0.04078 & 0.00047 & 257.7 & 2.89 & 254.6 & 3.28 & 228.2 \\
\hline 0.15923 & 0.00181 & 952.5 & 10.07 & 958 & 8.34 & 971.6 \\
\hline 0.07208 & 0.00083 & 448.7 & 4.97 & 556.6 & 5.96 & 1027.6 \\
\hline 0.06259 & 0.00073 & 391.4 & 4.45 & 499.7 & 5.95 & 1033.6 \\
\hline 0.11412 & 0.00133 & 696.6 & 7.67 & 700.3 & 7.48 & 713.1 \\
\hline 0.12424 & 0.00145 & 754.9 & 8.31 & 805 & 8.2 & 947.1 \\
\hline 0.31675 & 0.00377 & 1773.8 & 18.45 & 1775.7 & 11.99 & 1778.8 \\
\hline
\end{tabular}




\begin{tabular}{|c|c|c|c|c|c|c|}
\hline 0.10311 & 0.00131 & 632.6 & 7.68 & 635 & 9.24 & 644.6 \\
\hline 0.13103 & 0.00163 & 793.7 & 9.29 & 799.6 & 9.89 & 817 \\
\hline 0.10668 & 0.00136 & 653.4 & 7.9 & 701.8 & 9.55 & 861.2 \\
\hline 0.10301 & 0.00158 & 632 & 9.24 & 702.6 & 14.17 & 936.6 \\
\hline 0.13096 & 0.00257 & 793.3 & 14.67 & 800.1 & 23.82 & 819.9 \\
\hline 0.10792 & 0.00148 & 660.6 & 8.59 & 647.9 & 11.24 & 605 \\
\hline 0.03802 & 0.00044 & 240.5 & 2.71 & 407.3 & 4.67 & 1512.1 \\
\hline 0.11334 & 0.00181 & 692.1 & 10.45 & 702.9 & 15.55 & 738.7 \\
\hline 0.09263 & 0.00122 & 571.1 & 7.2 & 572.5 & 9.48 & 579.2 \\
\hline 0.10898 & 0.00135 & 666.8 & 7.84 & 670.8 & 8.89 & 685.4 \\
\hline 0.08582 & 0.00163 & 530.8 & 9.69 & 544.5 & 18.07 & 603.4 \\
\hline 0.08477 & 0.00137 & 524.5 & 8.13 & 538.6 & 13.7 & 599.9 \\
\hline 0.13016 & 0.00156 & 788.8 & 8.89 & 804.5 & 8.98 & 849.4 \\
\hline 0.12312 & 0.0016 & 748.5 & 9.19 & 780.4 & 10.87 & 873.6 \\
\hline 0.11212 & 0.00152 & 685.1 & 8.8 & 660.1 & 11.13 & 576.7 \\
\hline 0.13684 & 0.00171 & 826.8 & 9.72 & 833.1 & 10.34 & 851.1 \\
\hline 0.12953 & 0.00167 & 785.2 & 9.51 & 776 & 10.72 & 750.7 \\
\hline 0.06807 & 0.00081 & 424.5 & 4.87 & 446.9 & 5.94 & 564.7 \\
\hline 0.00478 & 0.00013 & 30.7 & 0.86 & 27.3 & 3.21 & 0.1 \\
\hline 0.11037 & 0.00142 & 674.9 & 8.22 & 743.5 & 10.11 & 957.1 \\
\hline 0.09905 & 0.00131 & 608.8 & 7.66 & 665.4 & 10.2 & 863.1 \\
\hline 0.13253 & 0.00157 & 802.3 & 8.96 & 806.4 & 8.87 & 818.6 \\
\hline 0.17169 & 0.00226 & 1021.4 & 12.43 & 1005.2 & 12.64 & 971 \\
\hline 0.16622 & 0.00229 & 991.3 & 12.66 & 1008.7 & 13.84 & 1047.8 \\
\hline 0.12576 & 0.00146 & 763.7 & 8.34 & 765.8 & 7.98 & 773.2 \\
\hline 0.07232 & 0.00084 & 450.1 & 5.04 & 844.2 & 8.05 & 2099.1 \\
\hline 0.11915 & 0.00139 & 725.7 & 8.01 & 847.1 & 8.54 & 1181.1 \\
\hline 0.12579 & 0.00151 & 763.8 & 8.67 & 756 & 8.94 & 734 \\
\hline 0.09556 & 0.00114 & 588.3 & 6.68 & 628 & 7.6 & 774.4 \\
\hline 0.12455 & 0.00261 & 756.7 & 14.98 & 805.3 & 25.21 & 943.1 \\
\hline 0.1326 & 0.00178 & 802.7 & 10.14 & 805.6 & 12.02 & 814.5 \\
\hline 0.1373 & 0.00178 & 829.4 & 10.07 & 810 & 11.23 & 758.3 \\
\hline 0.14102 & 0.0016 & 850.4 & 9.06 & 878 & 8.22 & 949.3 \\
\hline 0.10166 & 0.00138 & 624.1 & 8.09 & 623.2 & 10.86 & 621 \\
\hline 0.10214 & 0.00141 & 626.9 & 8.24 & 632 & 11.25 & 651 \\
\hline 0.10199 & 0.00129 & 626.1 & 7.54 & 653.5 & 9.26 & 750.3 \\
\hline 0.07312 & 0.00087 & 454.9 & 5.24 & 458.8 & 6.31 & 478.9 \\
\hline 0.523 & 0.00601 & 2711.9 & 25.45 & 2904.3 & 13.07 & 3041.4 \\
\hline 0.10758 & 0.00143 & 658.7 & 8.32 & 665.5 & 10.69 & 690 \\
\hline 0.10908 & 0.00141 & 667.4 & 8.22 & 663.7 & 10.11 & 652.2 \\
\hline 0.10545 & 0.00125 & 646.2 & 7.27 & 683.9 & 7.99 & 810.8 \\
\hline 0.10069 & 0.00117 & 618.4 & 6.86 & 631.3 & 7.25 & 678.9 \\
\hline 0.12686 & 0.00159 & 769.9 & 9.11 & 771.5 & 10.17 & 777.1 \\
\hline 0.10377 & 0.00122 & 636.5 & 7.14 & 644.8 & 7.68 & 675.1 \\
\hline 0.13963 & 0.00208 & 842.6 & 11.78 & 1323.7 & 15.4 & 2219.9 \\
\hline 0.06826 & $8.00 \mathrm{E}-04$ & 425.6 & 4.8 & 489.9 & 6.05 & 803.6 \\
\hline 0.09734 & 0.00113 & 598.8 & 6.63 & 610.2 & 7.03 & 653.9 \\
\hline 0.57193 & 0.00665 & 2915.7 & 27.29 & 2960.6 & 13.44 & 2992.1 \\
\hline 0.14416 & 0.0017 & 868.2 & 9.59 & 900 & 9.43 & 980 \\
\hline 0.09974 & 0.00118 & 612.9 & 6.91 & 640.9 & 7.7 & 742 \\
\hline 0.10153 & 0.00128 & 623.4 & 7.51 & 644.5 & 9.28 & 720.3 \\
\hline 0.1425 & 0.00204 & 858.8 & 11.51 & 883.3 & 14.18 & 946.3 \\
\hline 0.08531 & 0.00099 & 527.7 & 5.86 & 640.9 & 7.14 & 1064.2 \\
\hline 0.1021 & 0.00125 & 626.7 & 7.34 & 639.4 & 8.66 & 685.4 \\
\hline 0.14702 & 0.00176 & 884.2 & 9.89 & 907.2 & 9.86 & 964.7 \\
\hline 0.1253 & 0.00155 & 761 & 8.86 & 783.3 & 9.86 & 848.3 \\
\hline 0.10135 & 0.00145 & 622.3 & 8.49 & 621.7 & 12.02 & 620.4 \\
\hline 0.09706 & 0.00171 & 597.1 & 10.06 & 612.7 & 17.23 & 672 \\
\hline 0.08296 & 0.00098 & 513.7 & 5.85 & 653 & 7.72 & 1170.3 \\
\hline
\end{tabular}




$\begin{array}{lccccccc}1 & & & & & & \\ 2 & 0.09256 & 0.00107 & 570.7 & 6.34 & 628.7 & 7.22 & 844.6 \\ 3 & 0.31464 & 0.00359 & 1763.5 & 17.59 & 1834 & 11.98 & 1915.9 \\ 4 & 0.09999 & 0.00123 & 614.3 & 7.23 & 606.5 & 8.55 & 578.6\end{array}$




\begin{tabular}{|c|c|c|c|c|}
\hline $2 \sigma$ age 76 & $\begin{array}{l}\text { discordance } \\
\Delta 68-75[\%]\end{array}$ & $\Delta 68-76[\%]$ & $\begin{array}{c}\text { preferred age } \\
\text { age }\end{array}$ & $2 \sigma$ age \\
\hline 35.63 & 2.1 & 11.3 & 522.5 & 6.09 \\
\hline 29.23 & 1.7 & 7.9 & 634.0 & 7.03 \\
\hline 39.43 & 0.5 & 2.8 & 581.7 & 6.96 \\
\hline 35.61 & -1.6 & -7.2 & 646.5 & 7.48 \\
\hline 35.83 & 1.1 & 4.3 & 849.1 & 9.99 \\
\hline 31.81 & 2.0 & 9.4 & 601.0 & 6.79 \\
\hline 37.06 & 31.9 & 110.3 & & \\
\hline 23.1 & 85.3 & 444.6 & & \\
\hline 49.65 & 1.8 & 8.2 & 620.6 & 8.14 \\
\hline 74.36 & 0.4 & 1.9 & 624.3 & 9.98 \\
\hline 33.41 & 0.2 & 0.8 & 756.3 & 8.65 \\
\hline 29.12 & 2.0 & 9.5 & 623.0 & 6.89 \\
\hline 25.08 & 3.4 & 13.6 & 750.7 & 8.04 \\
\hline 30.41 & 1.9 & 9.3 & 586.6 & 6.55 \\
\hline 29.54 & 7.4 & 36.4 & 554.7 & 6.2 \\
\hline 26.67 & 34.1 & 146.2 & & \\
\hline 111.38 & -3.5 & -13.1 & 817.6 & 17.53 \\
\hline 26.6 & 27.7 & 141.6 & & \\
\hline 21.27 & 83.5 & 372.5 & & \\
\hline 44.18 & -1.8 & -8.1 & 640.7 & 7.92 \\
\hline 32.26 & 9.0 & 45.7 & & \\
\hline 29.23 & 0.9 & 4.3 & 636.1 & 7.01 \\
\hline 32.92 & 2.4 & 9.5 & 748.9 & 8.54 \\
\hline 46.34 & -1.7 & -6.3 & 803.4 & 10.28 \\
\hline 31.02 & 2.6 & 11.8 & 634.4 & 7.1 \\
\hline 36.48 & -0.3 & -1.2 & 660.8 & 7.69 \\
\hline 41.51 & -1.0 & -4.0 & 670.9 & 8.14 \\
\hline 41.21 & 1.9 & 8.6 & 631.7 & 7.69 \\
\hline 50.13 & 1.3 & 5.1 & 773.9 & 10.36 \\
\hline 40.95 & 3.8 & 16.9 & 640.3 & 7.82 \\
\hline 49.22 & 9.0 & 44.0 & 551.7 & 7.32 \\
\hline 51.02 & 11.9 & 110.1 & 262.4 & 3.44 \\
\hline 36.13 & -0.2 & -0.7 & 767.2 & 8.92 \\
\hline 31.07 & 0.1 & 0.7 & 746.1 & 8.3 \\
\hline 31.82 & 0.0 & 0.3 & 616.5 & 6.89 \\
\hline 62.7 & -1.1 & -4.9 & 623.8 & 9.01 \\
\hline 148.81 & 2.9 & 12.5 & 677.5 & 17.74 \\
\hline 39.13 & 1.7 & 8.3 & 602.9 & 7.17 \\
\hline 54.59 & 7.1 & 27.9 & 727.5 & 10.46 \\
\hline 37.08 & 10.0 & 52.4 & 506.5 & 6.02 \\
\hline 39.35 & 3.5 & 13.9 & 750.2 & 9.08 \\
\hline 49.97 & 0.3 & 1.5 & 715.6 & 9.41 \\
\hline 40.01 & 2.1 & 9.4 & 652.2 & 7.84 \\
\hline 27.67 & 3.7 & 15.3 & 707.6 & 7.67 \\
\hline 48.71 & 3.1 & 11.7 & 787.8 & 10.47 \\
\hline 27.03 & 34.2 & 248.4 & & \\
\hline 59.56 & 5.8 & 25.8 & 640.0 & 9.28 \\
\hline 32.56 & -1.2 & -11.4 & 257.7 & 2.89 \\
\hline 26.25 & 0.6 & 2.0 & 952.5 & 10.07 \\
\hline 26.89 & 24.0 & 129.0 & & \\
\hline 29.87 & 27.7 & 164.1 & & \\
\hline 30.54 & 0.5 & 2.4 & 696.6 & 7.67 \\
\hline 29.37 & 6.6 & 25.5 & 754.9 & 8.31 \\
\hline 25.15 & 0.1 & 0.3 & 1778.8 & 25.15 \\
\hline
\end{tabular}




\begin{tabular}{|c|c|c|c|c|}
\hline 42.02 & 0.4 & 1.9 & 632.6 & 7.68 \\
\hline 37.02 & 0.7 & 2.9 & 793.7 & 9.29 \\
\hline 39.09 & 7.4 & 31.8 & 653.4 & 7.9 \\
\hline 58.19 & 11.2 & 48.2 & 632.0 & 9.24 \\
\hline 90.28 & 0.9 & 3.4 & 793.3 & 14.67 \\
\hline 51.02 & -1.9 & -8.4 & 660.6 & 8.59 \\
\hline 25.54 & 69.4 & 528.7 & & \\
\hline 65.61 & 1.6 & 6.7 & 692.1 & 10.45 \\
\hline 47.27 & 0.2 & 1.4 & 571.1 & 7.2 \\
\hline 38.42 & 0.6 & 2.8 & 666.8 & 7.84 \\
\hline 93.59 & 2.6 & 13.7 & 530.8 & 9.69 \\
\hline 71.84 & 2.7 & 14.4 & 524.5 & 8.13 \\
\hline 33.07 & 2.0 & 7.7 & 788.8 & 8.89 \\
\hline 41.42 & 4.3 & 16.7 & 748.5 & 9.19 \\
\hline 50.07 & -3.6 & -15.8 & 685.1 & 8.8 \\
\hline 37.59 & 0.8 & 2.9 & 826.8 & 9.72 \\
\hline 41.71 & -1.2 & -4.4 & 785.2 & 9.51 \\
\hline 35.33 & 5.3 & 33.0 & 424.5 & 4.87 \\
\hline 17.96 & -11.1 & -99.7 & 30.7 & 0.86 \\
\hline 39.33 & 10.2 & 41.8 & 674.9 & 8.22 \\
\hline 43.68 & 9.3 & 41.8 & 608.8 & 7.66 \\
\hline 32.75 & 0.5 & 2.0 & 802.3 & 8.96 \\
\hline 40.37 & -1.6 & -4.9 & 1021.4 & 12.43 \\
\hline 43.84 & 1.8 & 5.7 & 991.3 & 12.66 \\
\hline 30.47 & 0.3 & 1.2 & 763.7 & 8.34 \\
\hline 24.19 & 87.6 & 366.4 & & \\
\hline 28.8 & 16.7 & 62.8 & & \\
\hline 35.14 & -1.0 & -3.9 & 763.8 & 8.67 \\
\hline 33.78 & 6.7 & 31.6 & 588.3 & 6.68 \\
\hline 93.69 & 6.4 & 24.6 & 756.7 & 14.98 \\
\hline 45.42 & 0.4 & 1.5 & 802.7 & 10.14 \\
\hline 42.48 & -2.3 & -8.6 & 829.4 & 10.07 \\
\hline 27.78 & 3.2 & 11.6 & 850.4 & 9.06 \\
\hline 50.73 & -0.1 & -0.5 & 624.1 & 8.09 \\
\hline 51.82 & 0.8 & 3.8 & 626.9 & 8.24 \\
\hline 40.69 & 4.4 & 19.8 & 626.1 & 7.54 \\
\hline 37.89 & 0.9 & 5.3 & 454.9 & 5.24 \\
\hline 21.11 & 7.1 & 12.2 & 3041.4 & 21.11 \\
\hline 47.03 & 1.0 & 4.8 & 658.7 & 8.32 \\
\hline 44.74 & -0.6 & -2.3 & 667.4 & 8.22 \\
\hline 33.2 & 5.8 & 25.5 & 646.2 & 7.27 \\
\hline 32.44 & 2.1 & 9.8 & 618.4 & 6.86 \\
\hline 39.56 & 0.2 & 0.9 & 769.9 & 9.11 \\
\hline 33.99 & 1.3 & 6.1 & 636.5 & 7.14 \\
\hline 37.29 & 57.1 & 163.5 & & \\
\hline 32.23 & 15.1 & 88.8 & & \\
\hline 32.36 & 1.9 & 9.2 & 598.8 & 6.63 \\
\hline 21.78 & 1.5 & 2.6 & 2992.1 & 21.78 \\
\hline 31.79 & 3.7 & 12.9 & 868.2 & 9.59 \\
\hline 33.97 & 4.6 & 21.1 & 612.9 & 6.91 \\
\hline 41.44 & 3.4 & 15.5 & 623.4 & 7.51 \\
\hline 49.78 & 2.9 & 10.2 & 858.8 & 11.51 \\
\hline 29.79 & 21.5 & 101.7 & 527.7 & 5.86 \\
\hline 38.95 & 2.0 & 9.4 & 626.7 & 7.34 \\
\hline 33.3 & 2.6 & 9.1 & 884.2 & 9.89 \\
\hline 37.54 & 2.9 & 11.5 & 761 & 8.86 \\
\hline 56.49 & -0.1 & -0.3 & 622.3 & 8.49 \\
\hline 81.11 & 2.6 & 12.5 & 597.1 & 10.06 \\
\hline 31.6 & 27.1 & 127.8 & & \\
\hline
\end{tabular}




$\begin{array}{lcccc}31.66 & 10.2 & 48.0 & 570.7 & 6.34 \\ 24.75 & 4.0 & 8.6 & 1915.9 & 24.75 \\ 40.76 & -1.3 & -5.8 & 614.3 & 7.23\end{array}$


Sample 2976S Didesa @ Ephrem 178 grain analysed 50 concordant ages

\begin{tabular}{|c|c|c|c|c|c|c|}
\hline \multirow[b]{2}{*}{ grain } & \multicolumn{3}{|c|}{ concentrations } & \multicolumn{3}{|l|}{ isotopic ratios } \\
\hline & U [ppm] & $\mathrm{Pb}$ [ppm] & Th/U & Pb207/Pb206 & $2 \sigma 76$ & Pb207/U235 \\
\hline X2976n_G001 & 16 & 1.6 & 0.291 & 0.05751 & 0.00536 & 0.75696 \\
\hline X2976n_G002 & 177 & 24.3 & 0.4332 & 0.06499 & 0.00192 & 1.1657 \\
\hline X2976n_G003 & & & & 0.75872 & 0.02336 & 78.06116 \\
\hline X2976n_G004 & 41.7 & 4.6 & 0.393 & 0.06858 & 0.00356 & 0.98405 \\
\hline X2976n_G005 & 134 & 14.9 & 0.5198 & 0.05958 & 0.00206 & 0.84921 \\
\hline X2976n G006 & 72.5 & 7.9 & 0.5384 & 0.05932 & 0.0026 & 0.81922 \\
\hline X2976n_G007 & 85.3 & 10.2 & 0.7598 & 0.05801 & 0.0024 & 0.83306 \\
\hline X2976n_G008 & & & & 0.60231 & 0.07074 & 1.86164 \\
\hline X2976n_G009 & 32.1 & 3.4 & 0.3975 & 0.0645 & 0.00392 & 0.90112 \\
\hline X2976n_G010 & & & & 0.10108 & 0.00382 & 1.33397 \\
\hline X2976n_G011 & & & & 0.09808 & 0.00362 & 1.31322 \\
\hline X2976n_G012 & 578.4 & 57.4 & 0.0335 & 0.06028 & 0.00146 & 0.88527 \\
\hline X2976n_G013 & & & & 0.30237 & 0.01524 & 6.63717 \\
\hline X2976n_G014 & & & & 0.20796 & 0.01322 & 3.98599 \\
\hline X2976n_G015 & & & & 0.26412 & 0.0108 & 4.14629 \\
\hline X2976n_G016 & & & & 0.26894 & 0.01074 & 4.42688 \\
\hline X2976n_G017 & & & & 0.31114 & 0.01816 & 6.43079 \\
\hline X2976n_G018 & 3693.6 & 489 & 1.2192 & 0.06404 & 0.00138 & 0.943 \\
\hline X2976n G019 & 143 & 17.7 & 0.3803 & 0.06218 & 0.00202 & 1.02308 \\
\hline X2976n_G020 & 111.6 & 15.6 & 0.2678 & 0.06653 & 0.0022 & 1.26179 \\
\hline X2976n_G021 & 190.5 & 24 & 0.4259 & 0.06684 & 0.00204 & 1.10287 \\
\hline X2976n_G022 & 153.3 & 18.8 & 0.4063 & 0.06314 & 0.00202 & 1.01957 \\
\hline X2976n_G023 & 173.8 & 9 & 0.5531 & 0.06682 & 0.00266 & 0.45606 \\
\hline X2976n_G024 & 343.1 & 52.7 & 0.6564 & 0.06465 & 0.00164 & 1.2201 \\
\hline X2976n_G025 & 417.5 & 68.9 & 1.0079 & 0.06384 & 0.0016 & 1.18302 \\
\hline X2976n_G026 & 130.8 & 17.9 & 1.0244 & 0.06106 & 0.00214 & 0.93359 \\
\hline X2976n_G027 & & & & 0.09972 & 0.00438 & 1.38319 \\
\hline X2976n_G028 & & & & 0.20489 & 0.00908 & 3.21837 \\
\hline X2976n_G029 & 1519.1 & 164.6 & 0.2426 & 0.06578 & 0.00148 & 1.01536 \\
\hline X2976n_G030 & & & & 0.75288 & 0.02562 & 69.02879 \\
\hline X2976n_G031 & 317.4 & 43.2 & 0.528 & 0.06455 & 0.00172 & 1.11949 \\
\hline X2976n_G032 & & & & 0.31322 & 0.01972 & 6.44893 \\
\hline X2976n_G033 & & & & 0.08303 & 0.00374 & 1.13108 \\
\hline X2976n_G034 & & & & 0.33735 & 0.0145 & 6.41324 \\
\hline X2976n_G035 & & & & 0.17326 & 0.00656 & 1.99344 \\
\hline X2976n_G036 & & & & 0.62268 & 0.08132 & 1.8858 \\
\hline X2976n_G037 & & & & 0.07246 & 0.00592 & 1.7086 \\
\hline X2976n_G038 & & & & 0.15207 & 0.00786 & 2.20416 \\
\hline X2976n_G039 & & & & 0.11929 & 0.0068 & 1.76919 \\
\hline X2976n_G040 & & & & 0.33255 & 0.01778 & 7.4822 \\
\hline X2976n_G041 & & & & 0.3464 & 0.0221 & 7.32055 \\
\hline X2976n_G042 & 139.8 & 16.4 & 0.1128 & 0.07536 & 0.00252 & 1.25998 \\
\hline X2976n_G043 & 281.5 & 30.5 & 0.3299 & 0.05914 & 0.0017 & 0.86235 \\
\hline X2976n_G044 & 410.4 & 40.1 & 0.0363 & 0.05899 & 0.00156 & 0.85327 \\
\hline X2976n_G045 & & & & 0.09422 & 0.00442 & 1.32263 \\
\hline X2976n_G046 & 5845.1 & 228.5 & 0.0203 & 0.06401 & 0.00142 & 0.3717 \\
\hline X2976n G047 & 479.7 & 59.6 & 0.0997 & 0.06735 & 0.00166 & 1.20604 \\
\hline X2976n_G048 & 2175.8 & 122.4 & 0.2776 & 0.21843 & 0.00482 & 1.57568 \\
\hline X2976n_G049 & & & & 0.3856 & 0.01774 & 9.1717 \\
\hline X2976n_G050 & 149.4 & 19.2 & 1.2409 & 0.05639 & 0.00198 & 0.76776 \\
\hline
\end{tabular}




\begin{tabular}{|c|c|c|c|c|c|c|}
\hline X2976n_G051 & 74.4 & 8.3 & 0.4576 & 0.05896 & 0.0026 & 0.85791 \\
\hline X2976n_G052 & & & & 0.74528 & 0.0964 & 113.0762 \\
\hline X2976n_G053 & & & & 0.55705 & 0.02868 & 17.80117 \\
\hline X2976n_G054 & & & & 0.08139 & 0.003 & 1.34296 \\
\hline X2976n_G055 & & & & 0.10594 & 0.00464 & 1.40369 \\
\hline X2976n_G056 & & & & 0.12489 & 0.00718 & 1.81185 \\
\hline X2976n_G057 & & & & 0.41273 & 0.02052 & 9.02442 \\
\hline X2976n_G058 & & & & 0.32407 & 0.00894 & 7.01736 \\
\hline X2976n_G059 & & & & 0.15644 & 0.0071 & 2.39191 \\
\hline X2976n_G060 & & & & 0.09005 & 0.00364 & 1.29126 \\
\hline X2976n_G061 & & & & 0.8088 & 0.0352 & 181.38142 \\
\hline X2976n_G062 & & & & 0.23047 & 0.00756 & 2.2598 \\
\hline X2976n_G063 & & & & 0.09978 & 0.00346 & 1.71107 \\
\hline X2976n_G064 & & & & 0.11032 & 0.00412 & 1.54001 \\
\hline X2976n_G065 & & & & 0.19465 & 0.00872 & 2.23864 \\
\hline X2976n_G066 & & & & 0.37073 & 0.01016 & 7.85058 \\
\hline X2976n_G067 & & & & 0.0909 & 0.00442 & 1.26852 \\
\hline X2976n_G068 & & & & 0.53392 & 0.15364 & 64.95598 \\
\hline X2976n_G069 & & & & 0.6233 & 0.03202 & 136.38998 \\
\hline X2976n_G070 & & & & 0.43603 & 0.01564 & 8.70551 \\
\hline X2976n_G071 & 62.8 & 8.1 & 0.3692 & 0.06216 & 0.00274 & 1.06977 \\
\hline X2976n_G072 & 61.6 & 7.8 & 0.3713 & 0.06235 & 0.00282 & 1.05819 \\
\hline X2976n_G073 & & & & 0.74367 & 0.03692 & 59.53342 \\
\hline X2976n_G074 & 3905.9 & 198.6 & 0.0383 & 0.06311 & 0.00144 & 0.47549 \\
\hline X2976n_G075 & & & & 0.16156 & 0.00928 & 2.53154 \\
\hline X2976n_G076 & & & & 0.34881 & 0.01714 & 7.1753 \\
\hline X2976n_G077 & & & & 0.28654 & 0.01366 & 5.1675 \\
\hline X2976n_G078 & & & & 0.36593 & 0.01516 & 7.42358 \\
\hline X2976n_G079 & & & & -0.18402 & 0.23474 & -32.243 \\
\hline X2976n_G080 & 207.1 & 29.5 & 0.345 & 0.06548 & 0.00192 & 1.24808 \\
\hline X2976n_G081 & & & & 0.09256 & 0.0062 & 1.503 \\
\hline X2976n_G082 & & & & 0.3872 & 0.02046 & 7.63708 \\
\hline X2976n_G083 & & & & 0.15963 & 0.00742 & 2.83084 \\
\hline X2976n_G084 & & & & 0.34665 & 0.01932 & 7.52496 \\
\hline X2976n_G085 & & & & 0.07562 & 0.00282 & 1.02833 \\
\hline X2976n_G086 & & & & 0.35445 & 0.01742 & 7.53545 \\
\hline X2976n_G087 & 67.3 & 8.1 & 0.7498 & 0.06257 & 0.00304 & 0.90324 \\
\hline X2976n_G088 & & & & 0.43272 & 0.02692 & 10.47614 \\
\hline X2976n_G089 & & & & 0.17981 & 0.00934 & 3.04973 \\
\hline X2976n_G090 & & & & 0.23424 & 0.0137 & 4.73792 \\
\hline X2976n_G091 & & & & 0.50388 & 0.02142 & 13.5848 \\
\hline X2976n_G092 & & & & 0.0927 & 0.0026 & 1.1878 \\
\hline X2976n_G093 & & & & 0.51943 & 0.01626 & 15.12985 \\
\hline X2976n_G094 & & & & 0.17765 & 0.00468 & 2.32572 \\
\hline X2976n_G095 & & & & 0.08334 & 0.01186 & 1.19285 \\
\hline X2976n_G096 & 1135 & 97.9 & 0.087 & 0.06695 & 0.0016 & 0.84514 \\
\hline X2976n_G097 & & & & 0.71187 & 1.32394 & \\
\hline X2976n_G098 & 49.4 & 5 & 0.1892 & 0.05997 & 0.0032 & 0.853 \\
\hline X2976n_G099 & & & & 0.2285 & 0.01388 & 3.64677 \\
\hline X2976n_G100 & & & & 0.09374 & 0.00404 & 1.53392 \\
\hline X2976n_G101 & 112.2 & 17.4 & 0.5655 & 0.06505 & 0.00234 & 1.26875 \\
\hline X2976n_G102 & & & & 0.17821 & 0.01194 & 2.75828 \\
\hline X2976n_G103 & & & & 0.20646 & 0.009 & 3.25944 \\
\hline X2976n_G104 & & & & 0.41746 & 0.02882 & 8.74378 \\
\hline X2976n_G105 & & & & 0.18563 & 0.01132 & 3.4923 \\
\hline
\end{tabular}


X2976n_G106

X2976n_G107

X2976n_G108

X2976n_G109

X2976n_G110

X2976n_G111

X2976n_G112

X2976n_G113

X2976n_G114

X2976n_G115

X2976n_G116

X2976n_G117

X2976n_G118

X2976n_G119

X2976n_G120

X2976n_G121

X2976n_G122

X2976n_G123

X2976n_G124

X2976n G125

X2976n G126

X2976n G127

X2976n_G128

X2976n_G129

X2976n_G130

X2976n_G131

X2976n_G132

X2976n_G133

X2976n_G134

X2976n_G135

X2976n_G136

X2976n_G137

X2976n_G138

X2976n_G139

X2976n_G140

X2976n_G141

X2976n_G142

X2976n_G143

X2976n_G144

X2976n_G145

X2976n_G146

X2976n_G147

X2976n_G148

X2976n_G149

X2976n_G150

X2976n_G151

X2976n_G152

X2976n_G153

X2976n_G154

X2976n_G155

X2976n_G156

X2976n_G157

X2976n_G158

X2976n_G159

X2976n_G160

\begin{tabular}{|c|c|c|}
\hline 0.49232 & 0.02784 & 12.1417 \\
\hline 0.27005 & 0.0134 & 4.97624 \\
\hline 0.12793 & 0.0047 & 1.76313 \\
\hline 0.51359 & 1.78876 & 166.51558 \\
\hline 0.2419 & 0.00908 & 4.08881 \\
\hline 0.06096 & 0.00166 & 0.96302 \\
\hline 0.26312 & 0.01616 & 4.55955 \\
\hline 0.08512 & 0.00358 & 1.25898 \\
\hline 0.3939 & 0.0227 & 9.58649 \\
\hline 0.82325 & 0.03846 & \\
\hline 0.14281 & 0.00852 & 2.14984 \\
\hline 3.89356 & 23.8 & \\
\hline 0.17401 & 0.00964 & 2.69382 \\
\hline 0.32788 & 0.01616 & 6.31234 \\
\hline 0.73708 & 0.02268 & 63.76185 \\
\hline 0.26601 & 0.03246 & 4.45511 \\
\hline 0.48499 & 0.02604 & 7.22615 \\
\hline 0.84341 & 0.06268 & \\
\hline 0.7815 & 0.48036 & 304.61307 \\
\hline 0.19476 & 0.01516 & 3.0612 \\
\hline 0.54366 & 0.08076 & 26.49921 \\
\hline 0.08993 & 0.00434 & 1.22983 \\
\hline 0.41425 & 0.02902 & 9.84986 \\
\hline 0.16564 & 0.01072 & 2.81007 \\
\hline 0.10949 & 0.00526 & 1.65789 \\
\hline 3.32061 & 0.26362 & 4.3043 \\
\hline 0.26158 & 0.02836 & 4.51147 \\
\hline 0.43406 & 0.01982 & 9.7395 \\
\hline 0.09149 & 0.00318 & 1.44988 \\
\hline 0.09466 & 0.00344 & 1.3585 \\
\hline 0.06339 & 0.00196 & 1.18944 \\
\hline 0.32915 & 0.0205 & 6.4152 \\
\hline 0.06124 & 0.06666 & 1.54329 \\
\hline 0.06026 & 0.00152 & 0.86043 \\
\hline 0.06083 & 0.0016 & 0.87607 \\
\hline 0.52258 & 0.0376 & 14.87858 \\
\hline 0.80939 & 0.1058 & 9.69346 \\
\hline 0.05816 & 0.00192 & 0.87486 \\
\hline 0.06117 & 0.002 & 0.95155 \\
\hline 0.32696 & 0.02176 & 6.43972 \\
\hline 0.35446 & 0.01968 & 7.14347 \\
\hline 0.27022 & 0.01444 & 4.69718 \\
\hline 0.23211 & 0.02076 & 4.17965 \\
\hline 0.63467 & 0.04426 & 35.61636 \\
\hline 0.31925 & 0.02884 & 6.8763 \\
\hline 0.06351 & 0.0022 & 1.26028 \\
\hline 0.15051 & 0.0061 & 2.06587 \\
\hline-0.69304 & 1.68616 & -87.11931 \\
\hline 0.07026 & 0.00202 & 1.19412 \\
\hline 0.17066 & 0.00964 & 2.6866 \\
\hline 0.16872 & 0.01888 & 2.42568 \\
\hline 0.11962 & 0.00604 & 1.72739 \\
\hline 0.06751 & 0.00254 & 1.25312 \\
\hline 0.0585 & 0.00316 & 0.76786 \\
\hline 0.1528 & 0.00854 & 2.25562 \\
\hline
\end{tabular}




$\begin{array}{lcccccc}\text { X2976S_G001 } & 3002 & 380.7 & 0.1441 & 0.06368 & 0.00152 & 1.1529 \\ \text { X2976S_G002 } & 98.7 & 32 & 1.3611 & 0.09875 & 0.00388 & 3.34317 \\ \text { X2976S_G003 } & 89.4 & 13.8 & 0.5963 & 0.0661 & 0.00252 & 1.27974 \\ \text { X2976S_G004 } & 3764.6 & 142.6 & 1.5314 & 0.07274 & 0.00194 & 0.31921 \\ \text { X2976S_G005 } & 122 & 14 & 0.603 & 0.06302 & 0.00256 & 0.90981 \\ \text { X2976S_G006 } & 46.1 & 5.1 & 0.1348 & 0.11907 & 0.00646 & 1.76848 \\ \text { X2976S_G007 } & 192.3 & 28 & 0.7767 & 0.06253 & 0.00206 & 1.08438 \\ \text { X2976S_G008 } & 159.2 & 25.4 & 0.7881 & 0.10531 & 0.00318 & 2.03732 \\ \text { X2976S_G009 } & 41 & 4.7 & 0.4442 & 0.06317 & 0.00398 & 0.94264 \\ \text { X2976S_G010 } & & & & -1.73864 & 13.66988 & 88.78706 \\ \text { X2976S_G011 } & & & & 2.75858 & 10.63546 & \\ \text { X2976S_G012 } & 711.9 & 166.3 & 0.9686 & 0.08762 & 0.00218 & 2.58652 \\ \text { X2976S_G013 } & & & & 0.84696 & 0.0243 & \\ \text { X2976S_G014 } & & & & 0.61836 & 0.04776 & 7.99551 \\ \text { X2976S_G015 } & 240.2 & 26.5 & 0.0774 & 0.2075 & 0.00928 & 2.88349 \\ \text { X2976S_G016 } & 1223.1 & 145.1 & 0.1351 & 0.07309 & 0.00186 & 1.24929 \\ \text { X2976S_G017 } & 210.9 & 34.4 & 0.7856 & 0.06602 & 0.00214 & 1.28153 \\ \text { X2976S_G018 } & & & & 0.92668 & 0.47222 & 50.50384\end{array}$




\section{$28.1 \%$ concordant}

\begin{tabular}{|c|c|c|c|c|c|c|}
\hline $2 \sigma 75$ & $\mathrm{Pb206/U238}$ & $2 \sigma 68$ & $\begin{array}{c}\text { ages } \\
\text { age } 206 / 238\end{array}$ & $2 \sigma$ age 68 & age $207 / 235$ & $2 \sigma$ age 75 \\
\hline 0.06824 & 0.09557 & 0.00382 & 588.4 & 22.4 & 572.3 & 44.4 \\
\hline 0.0357 & 0.13023 & 0.00324 & 789.2 & 18.4 & 784.6 & 21.2 \\
\hline 2.78122 & 0.74702 & 0.02812 & & & & \\
\hline 0.04988 & 0.10419 & 0.00316 & 638.9 & 18.4 & 695.7 & 30.2 \\
\hline 0.0298 & 0.10349 & 0.00266 & 634.8 & 15.6 & 624.2 & 20.2 \\
\hline 0.03566 & 0.10027 & 0.00278 & 616 & 16.2 & 607.6 & 23.8 \\
\hline 0.03436 & 0.10427 & 0.00282 & 639.4 & 16.4 & 615.3 & 22.8 \\
\hline 0.15 & 0.02244 & 0.00208 & & & & \\
\hline 0.053 & 0.10144 & 0.0033 & 622.8 & 19.4 & 652.3 & 33.2 \\
\hline 0.04966 & 0.09582 & 0.00268 & & & & \\
\hline 0.04808 & 0.09722 & 0.00268 & & & & \\
\hline 0.0233 & 0.10662 & 0.00254 & 653.1 & 14.8 & 643.8 & 16.2 \\
\hline 0.3021 & 0.15938 & 0.00662 & & & & \\
\hline 0.23026 & 0.13916 & 0.0063 & & & & \\
\hline 0.15642 & 0.11398 & 0.00386 & & & & \\
\hline 0.16384 & 0.11951 & 0.004 & & & & \\
\hline 0.33104 & 0.15007 & 0.00708 & & & & \\
\hline 0.0227 & 0.10692 & 0.0025 & 654.8 & 14.6 & 674.5 & 15.6 \\
\hline 0.0341 & 0.11947 & 0.00304 & 727.5 & 17.6 & 715.5 & 21.2 \\
\hline 0.04272 & 0.13771 & 0.00354 & 831.7 & 20 & 828.7 & 23.8 \\
\hline 0.03468 & 0.11981 & 0.003 & 729.5 & 17.2 & 754.7 & 21 \\
\hline 0.03364 & 0.11724 & 0.00298 & 714.7 & 17.2 & 713.7 & 21 \\
\hline 0.01798 & 0.04955 & 0.00132 & 311.8 & 8.2 & 381.5 & 15.2 \\
\hline 0.03318 & 0.13702 & 0.0033 & 827.8 & 18.8 & 809.8 & 19.6 \\
\hline 0.0318 & 0.13455 & 0.00322 & 813.8 & 18.2 & 792.7 & 19 \\
\hline 0.03326 & 0.11101 & 0.00288 & 678.6 & 16.8 & 669.5 & 21.4 \\
\hline 0.05888 & 0.10071 & 0.00302 & & & & \\
\hline 0.13272 & 0.11405 & 0.0039 & & & & \\
\hline 0.0252 & 0.11207 & 0.00264 & 684.8 & 15.4 & 711.6 & 16.6 \\
\hline 2.6494 & 0.6657 & 0.02726 & & & & \\
\hline 0.03172 & 0.12593 & 0.00306 & 764.6 & 17.6 & 762.7 & 19.4 \\
\hline 0.35718 & 0.14949 & 0.0075 & & & & \\
\hline 0.04988 & 0.09891 & 0.0029 & & & & \\
\hline 0.24952 & 0.13803 & 0.0051 & & & & \\
\hline 0.07216 & 0.08354 & 0.0025 & & & & \\
\hline 0.16702 & 0.02199 & 0.00228 & & & & \\
\hline 0.13522 & 0.17119 & 0.00708 & & & & \\
\hline 0.10634 & 0.10523 & 0.00374 & & & & \\
\hline 0.09524 & 0.10768 & 0.00388 & & & & \\
\hline 0.35624 & 0.16336 & 0.0073 & & & & \\
\hline 0.40432 & 0.15344 & 0.00804 & & & & \\
\hline 0.04292 & 0.12139 & 0.00316 & 738.6 & 18.2 & 827.9 & 24 \\
\hline 0.02604 & 0.10586 & 0.0026 & 648.7 & 15.2 & 631.4 & 17.8 \\
\hline 0.024 & 0.10502 & 0.00254 & 643.8 & 14.8 & 626.4 & 16.8 \\
\hline 0.0601 & 0.10193 & 0.00312 & & & & \\
\hline 0.00912 & 0.04216 & 0.00098 & 266.2 & 6 & 320.9 & 8.8 \\
\hline 0.03214 & 0.13002 & 0.00312 & 788 & 17.8 & 803.4 & 19.2 \\
\hline 0.0383 & 0.05238 & 0.00124 & 329.1 & 7.6 & 960.7 & 20.2 \\
\hline 0.3807 & 0.17269 & 0.00702 & & & & \\
\hline 0.02742 & 0.09886 & 0.00254 & 607.7 & 15 & 578.5 & 19.2 \\
\hline
\end{tabular}




\begin{tabular}{|c|c|c|c|c|c|c|}
\hline 0.0375 & 0.10564 & 0.00294 & 647.4 & 17.2 & 629 & 24.4 \\
\hline 18.2417 & 1.10158 & 0.19142 & & & & \\
\hline 0.81984 & 0.23202 & 0.01146 & & & & \\
\hline 0.04972 & 0.11981 & 0.00324 & & & & \\
\hline 0.05956 & 0.0962 & 0.0029 & & & & \\
\hline 0.098 & 0.10533 & 0.00384 & & & & \\
\hline 0.39596 & 0.15875 & 0.0069 & & & & \\
\hline 0.19692 & 0.15722 & 0.00424 & & & & \\
\hline 0.10264 & 0.11101 & 0.00366 & & & & \\
\hline 0.05136 & 0.1041 & 0.00294 & & & & \\
\hline 12.03134 & 1.62823 & 0.11346 & & & & \\
\hline 0.07154 & 0.07119 & 0.00202 & & & & \\
\hline 0.05962 & 0.12451 & 0.00338 & & & & \\
\hline 0.05674 & 0.10135 & 0.00284 & & & & \\
\hline 0.09262 & 0.0835 & 0.0028 & & & & \\
\hline 0.21818 & 0.15375 & 0.00416 & & & & \\
\hline 0.05982 & 0.10132 & 0.00314 & & & & \\
\hline 22.44522 & 0.8833 & 0.31518 & & & & \\
\hline 10.53562 & 1.58871 & 0.1272 & & & & \\
\hline 0.28972 & 0.14496 & 0.00484 & & & & \\
\hline 0.04702 & 0.12495 & 0.00352 & 759 & 20.2 & 738.6 & 27.4 \\
\hline 0.0475 & 0.12322 & 0.00348 & 749.1 & 20 & 732.9 & 27.8 \\
\hline 3.20334 & 0.58122 & 0.03418 & & & & \\
\hline 0.0119 & 0.0547 & 0.00128 & 343.3 & 7.8 & 395 & 10.8 \\
\hline 0.13438 & 0.11377 & 0.00444 & & & & \\
\hline 0.31436 & 0.14935 & 0.00622 & & & & \\
\hline 0.22266 & 0.13093 & 0.00506 & & & & \\
\hline 0.28032 & 0.14729 & 0.00538 & & & & \\
\hline 53.9596 & 1.27209 & 2.13884 & & & & \\
\hline 0.03836 & 0.1384 & 0.00344 & 835.6 & 19.4 & 822.5 & 21.6 \\
\hline 0.0958 & 0.1179 & 0.00446 & & & & \\
\hline 0.35134 & 0.1432 & 0.00644 & & & & \\
\hline 0.12466 & 0.12875 & 0.00436 & & & & \\
\hline 0.37124 & 0.1576 & 0.00728 & & & & \\
\hline 0.0383 & 0.09873 & 0.00264 & & & & \\
\hline 0.33046 & 0.15435 & 0.00646 & & & & \\
\hline 0.04336 & 0.1048 & 0.00304 & 642.5 & 17.8 & 653.5 & 27.2 \\
\hline 0.56386 & 0.17577 & 0.0095 & & & & \\
\hline 0.14696 & 0.12314 & 0.0046 & & & & \\
\hline 0.25148 & 0.14685 & 0.00646 & & & & \\
\hline 0.52454 & 0.19574 & 0.00784 & & & & \\
\hline 0.0348 & 0.09303 & 0.00232 & & & & \\
\hline 0.46406 & 0.21148 & 0.00658 & & & & \\
\hline 0.06404 & 0.09505 & 0.00238 & & & & \\
\hline 0.1599 & 0.10391 & 0.00684 & & & & \\
\hline 0.02198 & 0.09166 & 0.00218 & 565.3 & 12.8 & 622 & 15.6 \\
\hline 0.04468 & 0.10326 & 0.00312 & 633.5 & 18.2 & 626.3 & 28.6 \\
\hline 0.19824 & 0.11587 & 0.00514 & & & & \\
\hline 0.06474 & 0.1188 & 0.0035 & & & & \\
\hline 0.04648 & 0.1416 & 0.00374 & 853.7 & 21.2 & 831.8 & 25.4 \\
\hline 0.16812 & 0.11237 & 0.00504 & & & & \\
\hline 0.13274 & 0.11462 & 0.00388 & & & & \\
\hline 0.5114 & 0.15206 & 0.0089 & & & & \\
\hline 0.19604 & 0.13659 & 0.0058 & & & & \\
\hline
\end{tabular}




\begin{tabular}{|c|c|c|c|c|c|c|}
\hline 0.5952 & 0.17905 & 0.0091 & & & & \\
\hline 0.22396 & 0.13378 & 0.00526 & & & & \\
\hline 0.06384 & 0.10006 & 0.00284 & & & & \\
\hline 528.80274 & 2.35387 & 4.7347 & & & & \\
\hline 0.14596 & 0.12271 & 0.00386 & & & & \\
\hline 0.02796 & 0.1147 & 0.0028 & 700 & 16.2 & 684.9 & 18.2 \\
\hline 0.24822 & 0.12581 & 0.00584 & & & & \\
\hline 0.05226 & 0.10739 & 0.00308 & & & & \\
\hline 0.48712 & 0.17669 & 0.00874 & & & & \\
\hline \multirow[t]{2}{*}{0.11928} & 0.10929 & 0.00426 & & & & \\
\hline & -5.32653 & 174.91976 & & & & \\
\hline 0.1377 & 0.11239 & 0.00434 & & & & \\
\hline 0.27824 & 0.13977 & 0.0057 & & & & \\
\hline 2.18536 & 0.62803 & 0.02234 & & & & \\
\hline 0.46588 & 0.12159 & 0.01046 & & & & \\
\hline 0.32356 & 0.10817 & 0.005 & & & & \\
\hline 255.24976 & 2.82977 & 2.5612 & & & & \\
\hline 0.21368 & 0.11411 & 0.00592 & & & & \\
\hline 3.6167 & 0.35387 & 0.0517 & & & & \\
\hline 0.05752 & 0.09928 & 0.00306 & & & & \\
\hline 0.5934 & 0.17262 & 0.01026 & & & & \\
\hline 0.1671 & 0.12316 & 0.00532 & & & & \\
\hline 0.07676 & 0.10993 & 0.00352 & & & & \\
\hline 0.1549 & 0.00941 & 0.00072 & & & & \\
\hline 0.42272 & 0.12521 & 0.00964 & & & & \\
\hline 0.39764 & 0.1629 & 0.00662 & & & & \\
\hline 0.05076 & 0.11505 & 0.00308 & & & & \\
\hline 0.04944 & 0.10419 & 0.00284 & & & & \\
\hline 0.03812 & 0.13622 & 0.00342 & 823.3 & 19.4 & 795.7 & 22 \\
\hline 0.3486 & 0.1415 & 0.00708 & & & & \\
\hline 1.64034 & 0.18295 & 0.06022 & & & & \\
\hline 0.02354 & 0.10366 & 0.00248 & 635.8 & 14.4 & 630.4 & 16.4 \\
\hline 0.02462 & 0.10456 & 0.00252 & 641.1 & 14.8 & 638.9 & 17 \\
\hline 0.91476 & 0.2067 & 0.0136 & & & & \\
\hline 0.87326 & 0.08694 & 0.01002 & & & & \\
\hline 0.02966 & 0.10921 & 0.00276 & 668.2 & 16 & 638.2 & 19.8 \\
\hline 0.03204 & 0.11293 & 0.00286 & 689.7 & 16.6 & 678.9 & 20.4 \\
\hline 0.37292 & 0.14299 & 0.00754 & & & & \\
\hline 0.34966 & 0.1463 & 0.0067 & & & & \\
\hline 0.22514 & 0.12619 & 0.00524 & & & & \\
\hline 0.32988 & 0.13073 & 0.0081 & & & & \\
\hline 2.42654 & 0.4074 & 0.03024 & & & & \\
\hline 0.53634 & 0.15637 & 0.0109 & & & & \\
\hline 0.04468 & 0.14406 & 0.00374 & 867.6 & 21 & 828 & 24.6 \\
\hline 0.08072 & 0.09964 & 0.00304 & & & & \\
\hline 207.85294 & 0.91257 & 2.14782 & & & & \\
\hline 0.03598 & 0.12338 & 0.00306 & 750 & 17.6 & 797.9 & 21 \\
\hline 0.14046 & 0.11428 & 0.00444 & & & & \\
\hline 0.24664 & 0.10437 & 0.00676 & & & & \\
\hline 0.08332 & 0.10483 & 0.0035 & & & & \\
\hline 0.04762 & 0.13476 & 0.0036 & 815 & 20.4 & 824.8 & 26 \\
\hline 0.04064 & 0.09529 & 0.00286 & 586.7 & 16.8 & 578.5 & 27.2 \\
\hline 0.11752 & 0.10716 & 0.00402 & & & & \\
\hline
\end{tabular}




$\begin{array}{ccccccc}0.02916 & 0.13135 & 0.00298 & 795.6 & 17 & 778.6 & 18 \\ 0.13116 & 0.24562 & 0.007 & 1415.8 & 36.2 & 1491.2 & 38 \\ 0.04884 & 0.14046 & 0.00364 & 847.3 & 20.6 & 836.7 & 26.2 \\ 0.00882 & 0.03184 & 0.00074 & 202.1 & 4.6 & 281.3 & 8.6 \\ 0.03664 & 0.10474 & 0.00274 & 642.1 & 16 & 657 & 23.4 \\ 0.09082 & 0.10776 & 0.00366 & 659.7 & 21.2 & 1034 & 41.6 \\ 0.03616 & 0.12582 & 0.00308 & 764 & 17.6 & 745.8 & 21.6 \\ 0.06246 & 0.14036 & 0.00346 & 846.7 & 19.6 & 1128.1 & 26.6 \\ 0.0576 & 0.10826 & 0.00342 & 662.6 & 19.8 & 674.3 & 34.8 \\ 305.21586 & -0.3705 & 2.72262 & & & & \\ 0.068 & 0.21416 & 0.00494 & 1250.9 & 26.2 & 1296.8 & 24.8 \\ 89.1667 & 10.56119 & 0.76328 & & & & \\ 0.47154 & 0.09381 & 0.00626 & & & & \\ 0.11904 & 0.10082 & 0.00336 & 619.2 & 19.6 & 1377.6 & 42 \\ 0.03342 & 0.12401 & 0.00286 & 753.6 & 16.4 & 823.1 & 19.4 \\ 0.04206 & 0.14083 & 0.00346 & 849.4 & 19.6 & 837.5 & 23.2 \\ 20.82344 & 0.3954 & 0.2149 & & & & \end{array}$




\begin{tabular}{|c|c|c|c|c|c|}
\hline age $207 / 206$ & $2 \sigma$ age 76 & $\begin{array}{l}\text { discordance } \\
\Delta 68-75[\%]\end{array}$ & $\Delta 68-76[\%]$ & $\begin{array}{c}\text { preferred age } \\
\text { age }\end{array}$ & $2 \sigma$ age \\
\hline 511.1 & 204.8 & 2.8 & 15.1 & 588.4 & 22.4 \\
\hline 774 & 62.2 & 0.6 & 2 & 789.2 & 18.4 \\
\hline 886.1 & 107.2 & -8.2 & -27.9 & 638.9 & 18.4 \\
\hline 588.4 & 75 & 1.7 & 7.9 & 634.8 & 15.6 \\
\hline 578.9 & 95.2 & 1.4 & 6.4 & 616 & 16.2 \\
\hline 530.1 & 90.6 & 3.9 & 20.6 & 639.4 & 16.4 \\
\hline 758.1 & 128.2 & -4.5 & -17.8 & 622.8 & 19.4 \\
\hline 613.6 & 52.4 & 1.4 & 6.4 & 653.1 & 14.8 \\
\hline 743 & 45.6 & -2.9 & -11.9 & 654.8 & 14.6 \\
\hline 680.3 & 69.4 & 1.7 & 6.9 & 727.5 & 17.6 \\
\hline 823.1 & 69 & 0.4 & 1 & 831.7 & 20 \\
\hline 832.8 & 63.6 & -3.3 & -12.4 & 729.5 & 17.2 \\
\hline 712.9 & 68 & 0.1 & 0.2 & 714.7 & 17.2 \\
\hline 832.2 & 83 & -18.3 & -62.5 & & \\
\hline 763 & 53.4 & 2.2 & 8.5 & 827.8 & 18.8 \\
\hline 736.3 & 53 & 2.7 & 10.5 & 813.8 & 18.2 \\
\hline 641.3 & 75.4 & 1.4 & 5.8 & 678.6 & 16.8 \\
\hline 799.4 & 47.2 & -3.8 & -14.3 & 684.8 & 15.4 \\
\hline 759.7 & 56.2 & 0.2 & 0.6 & 764.6 & 17.6 \\
\hline
\end{tabular}

$\begin{array}{cccccc}1078.1 & 67.2 & -10.8 & -31.5 & 738.6 & 18.2 \\ 572.3 & 62.6 & 2.7 & 13.4 & 648.7 & 15.2 \\ 566.7 & 57.6 & 2.8 & 13.6 & 643.8 & 14.8 \\ & & & & & \\ 742 & 47 & -17 & -64.1 & & 17.8 \\ 848.6 & 51.2 & -1.9 & -7.1 & 788 & \\ 2969.2 & 35.6 & -65.7 & -88.9 & & \\ 467.8 & 77.8 & 5.1 & 29.9 & & \end{array}$


565.6

96

2.9

14.5

647.4

17.2

679.6

94.2

686.1

96.6

2.8

2.2

11.7

759

749.1

20.2

9.2

20

711.9

48.4

$-13.1$

$-51.8$

343.3

7.8

789.8

61.6

1.6

5.8

835.6

19.4

693.6

103.6

$-1.7$

$-7.4$

642.5

17.8

40

41

42

43

44

45

46

47

48

49

50

51

52

53

54

55

56

57

58

59

60

$\begin{array}{lrrrrr}836.2 & 49.8 & -9.1 & -32.4 & 565.3 & 12.8 \\ 602.5 & 115.4 & 1.1 & 5.1 & 633.5 & 18.2 \\ 776 & 75.6 & 2.6 & 10 & 853.7 & 21.2 \\ & & & \\ & & & \\ & & & \end{array}$


58.6

2.2

9.7

700

16.2

10

11

12

13

14

15

16

17

18

19

20

21

22

23

24

25

26

27

28

29

30

31

32

33

34

35

36

37

38

39

40

41

42

43

44

45

46

47

48

49

50

51

52

53

54

55

56

57

58

59

60
721.3

612.9

633.2

535.8

645.2

725.4

936

853.5

548.5
65.6

54.4

56.6

72.2

70.2

73.4

59

78.2

118
3.5

0.9

0.3

4.7

1.6

24.7

6.9

668.2

689.7

16

16.6

19.4

14.4

$641.1 \quad 14.8$
$867.6 \quad 21$

$750 \quad 17.6$

$\begin{array}{ll}-19.9 & 750\end{array}$

815

586.7
20.4

16.8 


$\begin{array}{cccccc}731 & 50.6 & 2.2 & 8.8 & 795.6 & 17 \\ 1600.6 & 73.4 & -5.1 & -11.5 & 1600.6 & 73.4 \\ 809.5 & 79.8 & 1.3 & 4.7 & 847.3 & 20.6 \\ 1006.7 & 54.2 & -28.2 & -79.9 & & \\ 708.9 & 86.4 & -2.3 & -9.4 & 642.1 & 16 \\ 1942.3 & 97 & -36.2 & -66 & & \\ 692.3 & 70.2 & 2.4 & 10.4 & 764 & 17.6 \\ 1719.7 & 55.4 & -24.9 & -50.8 & & 19.8 \\ 714 & 133.8 & -1.7 & -7.2 & 662.6 & \\ & & & & & \\ 1374.2 & 47.8 & -3.5 & -9 & 1374.2 & 47.8 \\ & & & & & \\ 2886.1 & 72.6 & -55.1 & -78.5 & & \\ 1016.4 & 51.6 & -8.4 & -25.9 & 753.6 & 16.4 \\ 807 & 67.8 & 1.4 & 5.2 & 849.4 & 19.6\end{array}$




\section{Sample 3295S Dabus @ Bambesi 3 grain analysed 2 concordant ages 66}

\begin{tabular}{cccc|ccc}
\cline { 2 - 7 } grain & $\begin{array}{c}\text { concentrations } \\
\mathrm{U}[\mathrm{ppm}]\end{array}$ & $\mathrm{Pb}[\mathrm{ppm}]$ & $\mathrm{Th} / \mathrm{U}$ & $\begin{array}{c}\text { isotopic ratios } \\
\mathrm{Pb207/Pb206}\end{array}$ & $\mathbf{2 \sigma} \mathbf{7 6}$ & $\mathrm{Pb207/U235}$ \\
\hline X3295S_G001 & 3016.8 & 172.5 & 1.0027 & 0.13499 & 0.004 & 0.93722 \\
X3295S_G002 & 595.1 & 80.9 & 0.7332 & 0.06536 & 0.00216 & 1.07774 \\
X3295S_G003 & 666.4 & 89.6 & 0.6329 & 0.06354 & 0.00206 & 1.06204
\end{tabular}




\section{$.7 \%$ concordant}

\begin{tabular}{ccc|cccc}
\hline $\mathbf{2 \sigma} \mathbf{7 5}$ & Pb206/U238 & $\mathbf{2 \sigma} 6 \mathbf{8}$ & $\begin{array}{c}\text { ages } \\
\text { age 206/238 }\end{array}$ & 20 age 68 & age 207/235 & 20 age 75 \\
\hline 0.02754 & 0.05037 & 0.00116 & 316.8 & 7.2 & 671.4 & 18.4 \\
0.03538 & 0.11963 & 0.00282 & 728.4 & 16.2 & 742.5 & 21.4 \\
0.03434 & 0.12127 & 0.00284 & 737.9 & 16.4 & 734.8 & 21
\end{tabular}




\begin{tabular}{cc|cc|cc|}
\hline & & discordance & preferred age & \\
age 207/206 & 20 age 76 & $\mathbf{\Delta 6 8 - 7 5 [ \% ]}$ & $\boldsymbol{\Delta}$ 68-76 [\%] & age & 20 age \\
\hline 2163.9 & 51.6 & -52.8 & -85.4 & & \\
785.9 & 69.4 & -1.9 & -7.3 & 728.4 & 16.2 \\
726.4 & 68.8 & 0.4 & 1.6 & 737.9 & 16.4
\end{tabular}

10 
Sample 2970S Beles @ Enat Beles 153 grain analysed 130 concordant ages

\begin{tabular}{|c|c|c|c|c|c|c|}
\hline \multirow[b]{2}{*}{ grain } & \multicolumn{3}{|c|}{ concentrations } & \multicolumn{3}{|l|}{ isotopic ratios } \\
\hline & U [ppm] & $\mathrm{Pb}$ [ppm] & $\mathrm{Th} / \mathrm{U}$ & $\mathrm{Pb} 207 / \mathrm{Pb} 206$ & $2 \sigma 76$ & $\mathrm{~Pb} 207 / \mathrm{U} 235$ \\
\hline X2970S_G001 & 1163.6 & 68.2 & 0.3015 & 0.0731 & 0.00192 & 0.61145 \\
\hline X2970S_G002 & 1126.4 & 99.9 & 0.3685 & 0.08253 & 0.00194 & 0.97458 \\
\hline X2970S_G003 & 2074.2 & 11.1 & 0.6642 & 0.04854 & 0.00248 & 0.03194 \\
\hline X2970n_G001 & 182.4 & 28 & 0.6375 & 0.06836 & 0.002 & 1.29603 \\
\hline X2970n_G002 & 18.1 & 3.7 & 0.4429 & 0.07017 & 0.00396 & 1.8709 \\
\hline X2970n G003 & 594.8 & 86.9 & 0.546 & 0.06706 & 0.00166 & 1.24368 \\
\hline X2970n_G004 & 76.5 & 10.9 & 0.3841 & 0.06859 & 0.00254 & 1.29702 \\
\hline X2970n_G005 & 5540.4 & & 1.5926 & 0.27861 & 0.0064 & 0.78425 \\
\hline X2970n_G006 & 136.5 & 16.5 & 0.7097 & 0.06478 & 0.0022 & 0.95479 \\
\hline X2970n_G007 & 441.8 & 64.7 & 0.5326 & 0.06912 & 0.00176 & 1.31361 \\
\hline X2970n_G008 & 389.1 & 54.7 & 0.5365 & 0.06773 & 0.00178 & 1.20897 \\
\hline X2970n_G009 & 68.5 & 8.5 & 0.7155 & 0.06335 & 0.00276 & 0.95675 \\
\hline X2970n_G010 & 108.2 & 13.5 & 0.6065 & 0.06288 & 0.0023 & 0.98478 \\
\hline X2970n_G011 & 1044.5 & 120.3 & 0.6239 & 0.06905 & 0.00166 & 0.98575 \\
\hline X2970n_G012 & 28.3 & & 2.1315 & -0.0005 & 0.00298 & -0.0281 \\
\hline X2970n_G013 & 136.5 & 15.8 & 0.3647 & 0.06148 & 0.0021 & 0.95095 \\
\hline X2970n_G014 & 307.6 & 36.1 & 0.4358 & 0.06262 & 0.00176 & 0.96461 \\
\hline X2970n_G015 & 1685.2 & 103.2 & 0.0529 & 0.07301 & 0.00186 & 0.65324 \\
\hline X2970n_G016 & 205.1 & 22.3 & 0.2517 & 0.06241 & 0.00194 & 0.94113 \\
\hline X2970n_G017 & 156.9 & 17.3 & 0.2912 & 0.06464 & 0.00214 & 0.97439 \\
\hline X2970n_G018 & 3334.7 & 18 & 0.9031 & 0.0472 & 0.00176 & 0.0294 \\
\hline X2970n_G019 & 850.8 & 80.3 & 0.4541 & 0.06694 & 0.00168 & 0.82269 \\
\hline X2970n_G020 & 1084.7 & 109.1 & 0.3947 & 0.06982 & 0.0017 & 0.94467 \\
\hline X2970n_G021 & 139.3 & 22.2 & 0.2703 & 0.0708 & 0.0022 & 1.54122 \\
\hline X2970n_G022 & 1518.6 & 104.7 & 0.4942 & 0.10012 & 0.00242 & 0.85569 \\
\hline X2970n_G023 & 46.4 & 8.8 & 0.597 & 0.07509 & 0.0031 & 1.77566 \\
\hline X2970n_G024 & 835.5 & 83.5 & 0.3602 & 0.07962 & 0.00198 & 1.08335 \\
\hline X2970n_G025 & 177.9 & 22 & 0.2572 & 0.05509 & 0.00178 & 0.94053 \\
\hline X2970n_G026 & 86.7 & 13.2 & 0.7551 & 0.06569 & 0.00244 & 1.19831 \\
\hline X2970n_G027 & 470.7 & 64.8 & 0.4543 & 0.06854 & 0.00176 & 1.22948 \\
\hline X2970n_G028 & 1204.8 & 171.7 & 0.7307 & 0.06806 & 0.00162 & 1.17665 \\
\hline X2970n_G029 & 360.3 & 49.9 & 0.5824 & 0.0682 & 0.00184 & 1.18329 \\
\hline X2970n_G030 & 3932.3 & 253.6 & 1.0307 & 0.10112 & 0.00236 & 0.72647 \\
\hline X2970n_G031 & 73.6 & 11.2 & 0.669 & 0.06697 & 0.00266 & 1.25575 \\
\hline X2970n_G032 & 80.4 & 8.9 & 0.4858 & 0.07075 & 0.0034 & 1.00116 \\
\hline X2970n_G033 & 226 & 27.5 & 0.4898 & 0.06254 & 0.00194 & 0.98636 \\
\hline X2970n_G034 & 551.2 & 80 & 0.7583 & 0.06809 & 0.00174 & 1.1801 \\
\hline X2970n_G035 & 47 & 0.3 & 0.5358 & 0.04346 & 0.0192 & 0.0325 \\
\hline X2970n_G036 & 145 & 22.3 & 0.6888 & 0.06617 & 0.00216 & 1.24401 \\
\hline X2970n_G037 & 181.8 & 23.1 & 0.4329 & 0.06498 & 0.00206 & 1.08562 \\
\hline X2970n_G038 & 36.8 & 4.7 & 0.848 & 0.06068 & 0.00366 & 0.89705 \\
\hline X2970n_G039 & 256 & 27.2 & 0.1127 & 0.06193 & 0.00188 & 0.95054 \\
\hline X2970n_G040 & 343.8 & 35.6 & 0.0833 & 0.0636 & 0.00182 & 0.96013 \\
\hline X2970n_G041 & 176.2 & 24.7 & 0.3495 & 0.0685 & 0.00212 & 1.28761 \\
\hline X2970n_G042 & 234.5 & 33.9 & 0.3577 & 0.06506 & 0.00188 & 1.25428 \\
\hline X2970n_G043 & 150.7 & 18.3 & 0.6306 & 0.0643 & 0.00224 & 0.96905 \\
\hline X2970n_G044 & 164.3 & 21.4 & 0.8773 & 0.06477 & 0.0022 & 0.98345 \\
\hline X2970n_G045 & 216.9 & 26.1 & 0.7392 & 0.06304 & 0.00204 & 0.91174 \\
\hline X2970n_G046 & 123.5 & 15.5 & 0.2788 & 0.06532 & 0.00232 & 1.12535 \\
\hline X2970n_G047 & 99.1 & 12.5 & 0.2781 & 0.0648 & 0.00244 & 1.12144 \\
\hline
\end{tabular}




\begin{tabular}{|c|c|c|c|c|c|c|}
\hline X2970n_G048 & 61.7 & 9.1 & 0.597 & 0.06507 & 0.0029 & 1.20669 \\
\hline X2970n_G049 & 309.8 & 1.6 & 0.6555 & 0.04459 & 0.00536 & 0.02903 \\
\hline X2970n_G050 & 108.2 & 14.1 & 0.7818 & 0.06065 & 0.00236 & 0.94362 \\
\hline X2970n_G051 & 132 & 15.8 & 0.8629 & 0.06324 & 0.00234 & 0.88725 \\
\hline X2970n_G052 & 51.5 & 6 & 0.557 & 0.08555 & 0.0039 & 1.24186 \\
\hline X2970n_G053 & 130.3 & 14.6 & 0.8468 & 0.06078 & 0.00232 & 0.87578 \\
\hline X2970n_G054 & 5248.7 & 391.3 & 0.3047 & 0.07039 & 0.00166 & 0.73372 \\
\hline X2970n_G055 & 1718.6 & 129.4 & 0.2609 & 0.06783 & 0.0017 & 0.70986 \\
\hline X2970n_G056 & 467.3 & 61.7 & 0.454 & 0.06543 & 0.00174 & 1.12575 \\
\hline X2970n_G057 & 452 & 59 & 0.4539 & 0.0647 & 0.00174 & 1.10102 \\
\hline X2970n_G058 & 271.3 & 29 & 0.5164 & 0.06577 & 0.002 & 0.91232 \\
\hline X2970n_G059 & 49.8 & 7.4 & 0.611 & 0.06689 & 0.00316 & 1.23933 \\
\hline X2970n G060 & 31.7 & 4.6 & 0.4908 & 0.0698 & 0.00396 & 1.29515 \\
\hline X2970n_G061 & 214.1 & 26.3 & 0.4193 & 0.06798 & 0.00208 & 1.09682 \\
\hline X2970n_G062 & 823 & 68.4 & 0.1613 & 0.06997 & 0.00184 & 0.82144 \\
\hline X2970n_G063 & 269.6 & 30.1 & 0.1804 & 0.06549 & 0.002 & 1.03011 \\
\hline X2970n_G064 & 260 & 37.3 & 0.5533 & 0.06725 & 0.002 & 1.22374 \\
\hline X2970n_G065 & 185.8 & 21.1 & 0.4811 & 0.06295 & 0.0021 & 0.92751 \\
\hline X2970n_G066 & 386.3 & 33.5 & 0.1358 & 0.06685 & 0.00196 & 0.83416 \\
\hline X2970n_G067 & 31.7 & 3.8 & 0.7155 & 0.06476 & 0.0057 & 0.93722 \\
\hline X2970n_G068 & 34 & 4.3 & 0.765 & 0.06226 & 0.00388 & 0.94723 \\
\hline X2970n_G069 & 175 & 20.9 & 0.5867 & 0.0619 & 0.0021 & 0.93054 \\
\hline X2970n_G070 & 221.5 & 32.9 & 0.5449 & 0.06901 & 0.00206 & 1.29796 \\
\hline X2970n_G071 & 191.5 & 23.2 & 0.5307 & 0.06288 & 0.00208 & 0.97156 \\
\hline X2970n_G072 & 229.4 & 31.6 & 0.3009 & 0.06573 & 0.00198 & 1.23283 \\
\hline X2970n_G073 & 305.9 & 43.3 & 0.3969 & 0.06729 & 0.00192 & 1.26225 \\
\hline X2970n_G074 & 629.9 & 84.3 & 0.3915 & 0.06972 & 0.00184 & 1.23213 \\
\hline X2970n_G075 & 294.6 & 55 & 0.577 & 0.07539 & 0.00208 & 1.76511 \\
\hline X2970n_G076 & 105.4 & 18.8 & 0.4641 & 0.07259 & 0.00244 & 1.67356 \\
\hline X2970n_G077 & 562.5 & 91.4 & 0.9809 & 0.06683 & 0.0018 & 1.22953 \\
\hline X2970n_G078 & 198.3 & 30.7 & 0.6908 & 0.06594 & 0.00208 & 1.2443 \\
\hline X2970n_G079 & 53.8 & 7.6 & 0.4485 & 0.06697 & 0.0031 & 1.24066 \\
\hline X2970n_G080 & 757.3 & 141.9 & 0.8587 & 0.07378 & 0.0019 & 1.63101 \\
\hline X2970n_G081 & 631.6 & 116.6 & 0.9029 & 0.07926 & 0.00212 & 1.71222 \\
\hline X2970n_G082 & 608.4 & 105.5 & 1.3218 & 0.07553 & 0.002 & 1.46313 \\
\hline X2970n_G083 & 88.9 & 10.6 & 0.7337 & 0.06352 & 0.00268 & 0.9149 \\
\hline X2970n_G084 & 129.1 & 18.3 & 0.322 & 0.06647 & 0.00228 & 1.27592 \\
\hline X2970n_G085 & 426 & 54.2 & 0.738 & 0.06666 & 0.0019 & 1.02282 \\
\hline X2970n_G086 & 149.5 & 18.6 & 0.78 & 0.06109 & 0.0022 & 0.9103 \\
\hline X2970n_G087 & 148.4 & 22.1 & 0.5026 & 0.06651 & 0.00222 & 1.26495 \\
\hline X2970n_G088 & 295.1 & 40.7 & 0.1648 & 0.07357 & 0.0021 & 1.43077 \\
\hline X2970n_G089 & 139.3 & 19.2 & 0.3392 & 0.06479 & 0.00222 & 1.19984 \\
\hline X2970n_G090 & 1292.6 & 6.3 & 0.5007 & 0.04574 & 0.00258 & 0.02883 \\
\hline X2970n_G091 & 168.2 & 23.4 & 1.0691 & 0.06596 & 0.00226 & 1.01795 \\
\hline X2970n_G092 & 638.4 & 71.6 & 0.604 & 0.07541 & 0.00206 & 1.06061 \\
\hline X2970n_G093 & 11669.3 & 137.6 & 0.237 & 0.24508 & 0.0062 & 0.35706 \\
\hline X2970n_G094 & 92.3 & 13.8 & 0.6158 & 0.06595 & 0.00258 & 1.22568 \\
\hline X2970n_G095 & 697.3 & 110.6 & 0.8087 & 0.06604 & 0.00178 & 1.24059 \\
\hline X2970n_G096 & 60 & 8.3 & 0.6846 & 0.06175 & 0.00312 & 1.03931 \\
\hline X2970n_G097 & 139.9 & 18.4 & 1.1988 & 0.06926 & 0.00254 & 0.98898 \\
\hline X2970n_G098 & 3572 & 184 & 0.3294 & 0.06992 & 0.00182 & 0.49131 \\
\hline X2970n_G099 & 45.9 & 9.8 & 0.8397 & 0.07492 & 0.00318 & 1.86837 \\
\hline X2970n_G100 & 43.6 & 5.3 & 0.5313 & 0.06193 & 0.0034 & 0.95451 \\
\hline X2970n_G101 & 495.6 & 70.9 & 0.3527 & 0.06641 & 0.00184 & 1.27193 \\
\hline X2970n_G102 & 132.5 & 18.4 & 1.0196 & 0.06309 & 0.00234 & 0.98563 \\
\hline
\end{tabular}




\begin{tabular}{|c|c|c|c|c|c|c|}
\hline X2970n_G103 & 982.8 & 132.4 & 0.4276 & 0.06598 & 0.00176 & 1.17212 \\
\hline X2970n_G104 & 89.5 & 10.6 & 0.6232 & 0.0612 & 0.00268 & 0.90384 \\
\hline X2970n_G105 & 1652.3 & 95.8 & 0.36 & 0.07669 & 0.00208 & 0.58101 \\
\hline X2970n_G106 & 211.9 & 23.7 & 0.4231 & 0.06488 & 0.00216 & 0.94834 \\
\hline X2970n_G107 & 215.2 & 25.1 & 0.5546 & 0.07137 & 0.00234 & 1.04365 \\
\hline X2970n_G108 & 264.5 & 30.4 & 0.529 & 0.06114 & 0.00198 & 0.90038 \\
\hline X2970n_G109 & 105.4 & 12 & 0.4186 & 0.06257 & 0.00252 & 0.94266 \\
\hline X2970n_G110 & 6853.4 & 91.1 & 0.4072 & 0.13355 & 0.00362 & 0.2306 \\
\hline X2970n G111 & 27.8 & 5.4 & 0.4914 & 0.07952 & 0.00398 & 1.96393 \\
\hline X2970n_G112 & 43.6 & 8.6 & 0.7605 & 0.07672 & 0.0034 & 1.81423 \\
\hline X2970n_G113 & 329.7 & 47.8 & 0.4913 & 0.06639 & 0.00198 & 1.24126 \\
\hline X2970n_G114 & 152.4 & 17.4 & 0.5066 & 0.07553 & 0.00264 & 1.09071 \\
\hline X2970n_G115 & 83.3 & 12.7 & 0.6899 & 0.06661 & 0.00266 & 1.24052 \\
\hline X2970n_G116 & 115 & 14.1 & 0.4758 & 0.06172 & 0.0024 & 0.978 \\
\hline X2970n_G117 & 193.7 & 26.9 & 0.3766 & 0.06799 & 0.00224 & 1.25753 \\
\hline X2970n_G118 & 288.9 & 45.4 & 0.6506 & 0.06662 & 0.00204 & 1.2917 \\
\hline X2970n_G119 & 57.8 & 6.9 & 0.5125 & 0.0627 & 0.00324 & 0.96734 \\
\hline X2970n_G120 & 215.2 & 35.2 & 0.3042 & 0.07096 & 0.00222 & 1.56617 \\
\hline X2970n_G121 & 392 & 49.6 & 0.6775 & 0.06253 & 0.00192 & 0.97195 \\
\hline X2970n_G122 & 33.4 & 3.7 & 0.3798 & 0.06569 & 0.00424 & 0.96125 \\
\hline X2970n_G123 & 96.3 & 13.6 & 0.6394 & 0.06445 & 0.0026 & 1.12556 \\
\hline X2970n_G124 & 270.2 & 44.9 & 1.0031 & 0.06613 & 0.00206 & 1.25261 \\
\hline X2970n_G125 & 210.2 & 32.8 & 0.767 & 0.06656 & 0.0022 & 1.24264 \\
\hline X2970n_G126 & 5024.4 & 270.2 & 0.2765 & 0.07522 & 0.00202 & 0.56198 \\
\hline X2970n_G127 & 209 & 24.4 & 0.5947 & 0.08872 & 0.0029 & 1.25896 \\
\hline X2970n_G128 & 1826.2 & 200.8 & 0.933 & 0.07001 & 0.00192 & 0.87695 \\
\hline X2970n_G129 & 55.5 & 8.2 & 0.1705 & 0.06966 & 0.00308 & 1.44759 \\
\hline X2970n_G130 & 872.9 & 103.9 & 0.3453 & 0.06677 & 0.00196 & 1.07704 \\
\hline X2970n_G131 & & & & 0.75887 & 0.29002 & 782.48004 \\
\hline X2970n_G132 & 57.2 & 10.1 & 0.5688 & 0.07268 & 0.00308 & 1.60912 \\
\hline X2970n_G133 & 128.6 & 15.3 & 0.3715 & 0.06211 & 0.00242 & 0.98554 \\
\hline X2970n_G134 & 282.7 & 36.6 & 0.1875 & 0.10705 & 0.00324 & 1.85312 \\
\hline X2970n_G135 & 283.8 & 41.3 & 0.5518 & 0.06846 & 0.00214 & 1.26054 \\
\hline X2970n_G136 & 162.6 & 19.7 & 0.6472 & 0.06198 & 0.00226 & 0.93018 \\
\hline X2970n_G137 & 1604.2 & 106.7 & 0.7298 & 0.12914 & 0.00366 & 1.04934 \\
\hline X2970n_G138 & 392.5 & 58.1 & 1.3994 & 0.06188 & 0.00192 & 0.94316 \\
\hline X2970n_G139 & 179 & 29.3 & 0.9218 & 0.06539 & 0.00222 & 1.23178 \\
\hline X2970n_G140 & 139.3 & 19.7 & 0.3543 & 0.06823 & 0.00242 & 1.29553 \\
\hline X2970n_G141 & 578.3 & 78.1 & 0.1583 & 0.06859 & 0.002 & 1.31152 \\
\hline X2970n_G142 & 55.5 & 5.9 & 0.2941 & 0.06261 & 0.00322 & 0.90761 \\
\hline X2970n_G143 & 97.4 & 11.7 & 0.5265 & 0.06531 & 0.00272 & 1.00804 \\
\hline X2970n_G144 & 514.9 & 72.7 & 0.4885 & 0.06653 & 0.00198 & 1.21189 \\
\hline X2970n_G145 & 231.7 & 30.2 & 0.8374 & 0.06222 & 0.00214 & 0.95634 \\
\hline X2970n_G146 & 112.2 & 16.4 & 0.5106 & 0.06618 & 0.00254 & 1.23705 \\
\hline X2970n_G147 & 139.9 & 16.6 & 0.5052 & 0.06055 & 0.00232 & 0.92704 \\
\hline X2970n_G148 & 159.7 & 19 & 0.5348 & 0.06302 & 0.00232 & 0.95817 \\
\hline X2970n_G149 & 2151.4 & 89.3 & 0.5611 & 0.08938 & 0.00264 & 0.47772 \\
\hline X2970n_G150 & 105.9 & 17.3 & 0.935 & 0.06568 & 0.00254 & 1.22981 \\
\hline
\end{tabular}


$85.0 \%$ concordant

\begin{tabular}{|c|c|c|c|c|c|c|}
\hline $2 \sigma 75$ & Pb206/U238 & $2 \sigma 68$ & $\begin{array}{c}\text { ages } \\
\text { age } 206 / 238\end{array}$ & $2 \sigma$ age 68 & age $207 / 235$ & $2 \sigma$ age 75 \\
\hline 0.01662 & 0.06063 & 0.0014 & 379.5 & 8.6 & 484.5 & 13.4 \\
\hline 0.02426 & 0.0856 & 0.00194 & 529.5 & 11.6 & 690.8 & 16.4 \\
\hline 0.0016 & 0.00477 & 0.00012 & 30.7 & 0.8 & 31.9 & 1.8 \\
\hline 0.03966 & 0.13756 & 0.00338 & 830.9 & 19.2 & 844 & 22 \\
\hline 0.10386 & 0.19346 & 0.00632 & 1140.1 & 34.2 & 1070.8 & 43.2 \\
\hline 0.0332 & 0.13456 & 0.00318 & 813.8 & 18 & 820.5 & 19.2 \\
\hline 0.04862 & 0.1372 & 0.00362 & 828.8 & 20.6 & 844.4 & 26 \\
\hline 0.01972 & 0.02042 & 0.00048 & 130.3 & 3 & 587.9 & 14.6 \\
\hline 0.03308 & 0.10694 & 0.00272 & 655 & 15.8 & 680.6 & 21 \\
\hline 0.03584 & 0.13789 & 0.00328 & 832.7 & 18.6 & 851.7 & 20 \\
\hline 0.03378 & 0.12951 & 0.0031 & 785.1 & 17.6 & 804.7 & 19.8 \\
\hline 0.04142 & 0.10957 & 0.00302 & 670.3 & 17.6 & 681.6 & 25.6 \\
\hline 0.03646 & 0.11363 & 0.00296 & 693.8 & 17.2 & 696.1 & 22.6 \\
\hline 0.02584 & 0.10358 & 0.00244 & 635.4 & 14.2 & 696.5 & 17 \\
\hline 0.16736 & 0.40798 & 0.01642 & 2205.7 & 75.2 & -28.9 & -175.2 \\
\hline 0.0331 & 0.11223 & 0.00284 & 685.7 & 16.4 & 678.6 & 21 \\
\hline 0.02866 & 0.11177 & 0.00272 & 683 & 15.8 & 685.7 & 18.6 \\
\hline 0.01778 & 0.06491 & 0.00154 & 405.4 & 9.4 & 510.5 & 14 \\
\hline 0.03018 & 0.1094 & 0.0027 & 669.3 & 15.6 & 673.5 & 19.6 \\
\hline 0.03316 & 0.10936 & 0.00276 & 669 & 16 & 690.7 & 20.8 \\
\hline 0.0011 & 0.00452 & 0.00012 & 29.1 & 0.8 & 29.4 & 1.4 \\
\hline 0.0222 & 0.08916 & 0.00212 & 550.6 & 12.6 & 609.5 & 15.8 \\
\hline 0.02494 & 0.09816 & 0.00232 & 603.6 & 13.6 & 675.3 & 16.8 \\
\hline 0.04944 & 0.15793 & 0.00396 & 945.3 & 22 & 947 & 24.6 \\
\hline 0.02234 & 0.06201 & 0.00146 & 387.8 & 8.8 & 627.8 & 15.8 \\
\hline 0.07322 & 0.17158 & 0.0048 & 1020.8 & 26.4 & 1036.6 & 32.4 \\
\hline 0.02894 & 0.09872 & 0.00234 & 606.9 & 13.8 & 745.3 & 18.2 \\
\hline 0.03144 & 0.12386 & 0.00308 & 752.7 & 17.6 & 673.2 & 20 \\
\hline 0.04498 & 0.13234 & 0.00348 & 801.2 & 19.8 & 799.8 & 25.2 \\
\hline 0.03394 & 0.13015 & 0.0031 & 788.7 & 17.6 & 814.1 & 19.6 \\
\hline 0.03056 & 0.12543 & 0.00294 & 761.7 & 16.8 & 789.8 & 18.4 \\
\hline 0.03396 & 0.12587 & 0.00304 & 764.3 & 17.4 & 792.8 & 20 \\
\hline 0.01852 & 0.05212 & 0.00122 & 327.5 & 7.4 & 554.5 & 14.2 \\
\hline 0.05016 & 0.13605 & 0.00368 & 822.3 & 20.8 & 826 & 27.2 \\
\hline 0.04716 & 0.10267 & 0.00298 & 630 & 17.4 & 704.4 & 28.6 \\
\hline 0.03176 & 0.11443 & 0.00284 & 698.4 & 16.4 & 696.9 & 20 \\
\hline 0.03248 & 0.12574 & 0.003 & 763.5 & 17.2 & 791.4 & 19.2 \\
\hline 0.01414 & 0.00543 & 0.00046 & 34.9 & 3 & 32.5 & 14.4 \\
\hline 0.04176 & 0.1364 & 0.00344 & 824.3 & 19.6 & 820.7 & 23.2 \\
\hline 0.03546 & 0.12122 & 0.00302 & 737.6 & 17.4 & 746.4 & 21.4 \\
\hline 0.05272 & 0.10725 & 0.00338 & 656.8 & 19.6 & 650.1 & 32.6 \\
\hline 0.02994 & 0.11135 & 0.00274 & 680.6 & 15.8 & 678.4 & 19.4 \\
\hline 0.02874 & 0.10953 & 0.00266 & 670 & 15.4 & 683.4 & 18.6 \\
\hline 0.0412 & 0.13638 & 0.0034 & 824.2 & 19.2 & 840.2 & 22.8 \\
\hline 0.038 & 0.13986 & 0.00342 & 843.9 & 19.4 & 825.3 & 21.4 \\
\hline 0.0344 & 0.10934 & 0.0028 & 668.9 & 16.2 & 688 & 21.6 \\
\hline 0.03398 & 0.11015 & 0.0028 & 673.6 & 16.2 & 695.4 & 21.4 \\
\hline 0.03024 & 0.10493 & 0.00262 & 643.2 & 15.2 & 658 & 19.8 \\
\hline 0.0405 & 0.125 & 0.00322 & 759.3 & 18.4 & 765.5 & 23.6 \\
\hline 0.04264 & 0.12555 & 0.0033 & 762.4 & 19 & 763.7 & 24.6 \\
\hline
\end{tabular}




\begin{tabular}{|c|c|c|c|c|c|c|}
\hline 0.05344 & 0.13455 & 0.0038 & 813.8 & 21.6 & 803.7 & 29.4 \\
\hline 0.00338 & 0.00472 & 0.00018 & 30.4 & 1.2 & 29.1 & 3.6 \\
\hline 0.0369 & 0.11289 & 0.00298 & 689.5 & 17.2 & 674.8 & 23.2 \\
\hline 0.03304 & 0.10179 & 0.00264 & 624.9 & 15.4 & 644.9 & 21.6 \\
\hline 0.05552 & 0.10532 & 0.0031 & 645.5 & 18 & 819.7 & 30.6 \\
\hline 0.0337 & 0.10454 & 0.0027 & 641 & 15.8 & 638.7 & 21.8 \\
\hline 0.01898 & 0.07563 & 0.00176 & 470 & 10.6 & 558.7 & 14.2 \\
\hline 0.01912 & 0.07593 & 0.0018 & 471.8 & 10.8 & 544.7 & 14.6 \\
\hline 0.0319 & 0.12483 & 0.00298 & 758.3 & 17 & 765.7 & 19.2 \\
\hline 0.03144 & 0.12346 & 0.00296 & 750.4 & 17 & 753.8 & 19.2 \\
\hline 0.0288 & 0.10063 & 0.00248 & 618.1 & 14.6 & 658.3 & 19 \\
\hline 0.058 & 0.13442 & 0.00388 & 813 & 22 & 818.6 & 31.2 \\
\hline 0.07184 & 0.13463 & 0.00426 & 814.2 & 24.2 & 843.6 & 37.2 \\
\hline 0.0348 & 0.11705 & 0.0029 & 713.6 & 16.8 & 751.8 & 21 \\
\hline 0.02306 & 0.08518 & 0.00204 & 527 & 12.2 & 608.9 & 16.2 \\
\hline 0.0325 & 0.11412 & 0.00282 & 696.6 & 16.4 & 719 & 20.2 \\
\hline 0.03784 & 0.13202 & 0.00324 & 799.4 & 18.4 & 811.5 & 21.6 \\
\hline 0.03166 & 0.10691 & 0.0027 & 654.8 & 15.8 & 666.3 & 20.4 \\
\hline 0.02552 & 0.09054 & 0.0022 & 558.7 & 13 & 615.9 & 17.6 \\
\hline 0.0794 & 0.10499 & 0.00422 & 643.6 & 24.6 & 671.4 & 47.6 \\
\hline 0.0575 & 0.11038 & 0.0036 & 675 & 21 & 676.7 & 34.8 \\
\hline 0.03228 & 0.10906 & 0.00276 & 667.3 & 16 & 667.9 & 20.8 \\
\hline 0.04048 & 0.13647 & 0.00336 & 824.7 & 19 & 844.8 & 22.2 \\
\hline 0.03284 & 0.1121 & 0.00282 & 684.9 & 16.4 & 689.3 & 20.8 \\
\hline 0.03846 & 0.13607 & 0.00334 & 822.4 & 19 & 815.6 & 21.8 \\
\hline 0.03776 & 0.1361 & 0.0033 & 822.6 & 18.8 & 828.9 & 21.2 \\
\hline 0.0345 & 0.12821 & 0.00306 & 777.6 & 17.4 & 815.3 & 20 \\
\hline 0.05126 & 0.16986 & 0.0041 & 1011.3 & 22.6 & 1032.7 & 23.8 \\
\hline 0.05762 & 0.16727 & 0.0043 & 997.1 & 23.8 & 998.5 & 26.8 \\
\hline 0.03506 & 0.13349 & 0.0032 & 807.7 & 18.2 & 814.1 & 20.2 \\
\hline 0.04068 & 0.13691 & 0.00342 & 827.2 & 19.4 & 820.8 & 22.6 \\
\hline 0.05698 & 0.1344 & 0.00386 & 812.9 & 22 & 819.2 & 30.6 \\
\hline 0.04482 & 0.16039 & 0.0038 & 958.9 & 21.2 & 982.2 & 22 \\
\hline 0.04852 & 0.15674 & 0.00376 & 938.6 & 21 & 1013.1 & 23 \\
\hline 0.04132 & 0.14054 & 0.00336 & 847.7 & 19 & 915.3 & 21.6 \\
\hline 0.03854 & 0.1045 & 0.00284 & 640.7 & 16.6 & 659.7 & 24.4 \\
\hline 0.04462 & 0.13926 & 0.00356 & 840.5 & 20.2 & 835 & 24.4 \\
\hline 0.03048 & 0.11132 & 0.0027 & 680.4 & 15.6 & 715.3 & 19.2 \\
\hline 0.03312 & 0.10811 & 0.00278 & 661.8 & 16.2 & 657.2 & 21.4 \\
\hline 0.04326 & 0.13799 & 0.0035 & 833.3 & 19.8 & 830.1 & 23.8 \\
\hline 0.04294 & 0.14109 & 0.00344 & 850.8 & 19.4 & 901.9 & 22.4 \\
\hline 0.04182 & 0.13435 & 0.00342 & 812.6 & 19.4 & 800.5 & 23.6 \\
\hline 0.0016 & 0.00457 & 0.00012 & 29.4 & 0.8 & 28.9 & 1.8 \\
\hline 0.03546 & 0.11197 & 0.00284 & 684.2 & 16.4 & 712.9 & 21.8 \\
\hline 0.03058 & 0.10204 & 0.00246 & 626.4 & 14.4 & 734.1 & 19 \\
\hline 0.00964 & 0.01057 & 0.00026 & 67.8 & 1.6 & 310 & 9.4 \\
\hline 0.04828 & 0.13484 & 0.0036 & 815.4 & 20.4 & 812.4 & 26.4 \\
\hline 0.0354 & 0.1363 & 0.00326 & 823.7 & 18.4 & 819.1 & 20.2 \\
\hline 0.05168 & 0.12212 & 0.00356 & 742.8 & 20.4 & 723.6 & 30.2 \\
\hline 0.03662 & 0.10359 & 0.0027 & 635.4 & 15.8 & 698.2 & 22.8 \\
\hline 0.01358 & 0.05098 & 0.0012 & 320.5 & 7.4 & 405.8 & 11.8 \\
\hline 0.07918 & 0.18092 & 0.0051 & 1072 & 27.8 & 1070 & 33.8 \\
\hline 0.05128 & 0.11181 & 0.00338 & 683.3 & 19.6 & 680.4 & 31 \\
\hline 0.03728 & 0.13896 & 0.00334 & 838.8 & 19 & 833.3 & 20.8 \\
\hline 0.03698 & 0.11335 & 0.00294 & 692.2 & 17 & 696.5 & 22.8 \\
\hline
\end{tabular}




\begin{tabular}{|c|c|c|c|c|c|c|}
\hline 0.0333 & 0.12889 & 0.00308 & 781.5 & 17.6 & 787.6 & 19.6 \\
\hline 0.03934 & 0.10714 & 0.00294 & 656.1 & 17.2 & 653.8 & 25 \\
\hline 0.0167 & 0.05497 & 0.00132 & 345 & 8 & 465.1 & 13.6 \\
\hline 0.03226 & 0.10604 & 0.00266 & 649.7 & 15.6 & 677.2 & 20.6 \\
\hline 0.03486 & 0.10609 & 0.00266 & 650 & 15.6 & 725.7 & 21.4 \\
\hline 0.03004 & 0.10684 & 0.00266 & 654.4 & 15.4 & 651.9 & 19.6 \\
\hline 0.03792 & 0.10931 & 0.0029 & 668.7 & 16.8 & 674.3 & 23.8 \\
\hline 0.00656 & 0.01253 & 0.0003 & 80.3 & 2 & 210.7 & 6.8 \\
\hline 0.09692 & 0.17919 & 0.00554 & 1062.5 & 30.2 & 1103.2 & 39.6 \\
\hline 0.07976 & 0.17157 & 0.00492 & 1020.8 & 27 & 1050.6 & 34.6 \\
\hline 0.03838 & 0.13566 & 0.00332 & 820.1 & 18.8 & 819.5 & 21.6 \\
\hline 0.03856 & 0.10477 & 0.0027 & 642.3 & 15.8 & 748.8 & 23 \\
\hline 0.04956 & 0.13511 & 0.00362 & 817 & 20.6 & 819.1 & 27 \\
\hline 0.03826 & 0.11496 & 0.00302 & 701.5 & 17.4 & 692.6 & 23.6 \\
\hline 0.0424 & 0.13419 & 0.00338 & 811.7 & 19.2 & 826.8 & 23.4 \\
\hline 0.04092 & 0.14068 & 0.00346 & 848.5 & 19.6 & 842.1 & 22.4 \\
\hline 0.04912 & 0.11194 & 0.0033 & 684 & 19.2 & 687.1 & 29.8 \\
\hline 0.05054 & 0.16014 & 0.00398 & 957.6 & 22.2 & 956.9 & 24.8 \\
\hline 0.0309 & 0.11278 & 0.00276 & 688.9 & 16 & 689.5 & 19.6 \\
\hline 0.0601 & 0.10617 & 0.00352 & 650.5 & 20.6 & 683.9 & 36.2 \\
\hline 0.0455 & 0.12671 & 0.0034 & 769.1 & 19.4 & 765.6 & 26 \\
\hline 0.04042 & 0.13743 & 0.0034 & 830.1 & 19.2 & 824.6 & 22.4 \\
\hline 0.04192 & 0.13545 & 0.0034 & 818.9 & 19.4 & 820.1 & 23.4 \\
\hline 0.01602 & 0.0542 & 0.00128 & 340.3 & 7.8 & 452.8 & 13 \\
\hline 0.04204 & 0.10296 & 0.0026 & 631.7 & 15.2 & 827.4 & 23.4 \\
\hline 0.0254 & 0.09087 & 0.00216 & 560.7 & 12.8 & 639.3 & 17.2 \\
\hline 0.0637 & 0.15077 & 0.00424 & 905.3 & 23.8 & 908.9 & 31.4 \\
\hline 0.03304 & 0.11704 & 0.00284 & 713.5 & 16.4 & 742.2 & 20 \\
\hline 957.08948 & 7.48082 & 9.2735 & & & & \\
\hline 0.06804 & 0.16062 & 0.00448 & 960.2 & 24.8 & 973.8 & 31.8 \\
\hline 0.03856 & 0.11512 & 0.00302 & 702.4 & 17.4 & 696.4 & 23.6 \\
\hline 0.05772 & 0.1256 & 0.0031 & 762.7 & 17.8 & 1064.5 & 25.8 \\
\hline 0.0408 & 0.13359 & 0.0033 & 808.3 & 18.8 & 828.2 & 22.6 \\
\hline 0.03422 & 0.10889 & 0.0028 & 666.3 & 16.2 & 667.7 & 21.8 \\
\hline 0.03106 & 0.05895 & 0.00142 & 369.2 & 8.6 & 728.6 & 19.4 \\
\hline 0.03034 & 0.11059 & 0.00272 & 676.2 & 15.8 & 674.5 & 19.6 \\
\hline 0.04266 & 0.13667 & 0.00344 & 825.8 & 19.6 & 815.1 & 23.6 \\
\hline 0.04664 & 0.13775 & 0.00354 & 831.9 & 20 & 843.7 & 25.2 \\
\hline 0.04002 & 0.13874 & 0.00336 & 837.5 & 19 & 850.8 & 21.8 \\
\hline 0.0459 & 0.10518 & 0.00308 & 644.7 & 18 & 655.8 & 28.6 \\
\hline 0.04174 & 0.11197 & 0.00302 & 684.2 & 17.6 & 707.9 & 25.2 \\
\hline 0.0376 & 0.13216 & 0.00322 & 800.2 & 18.4 & 806.1 & 21.4 \\
\hline 0.03342 & 0.11151 & 0.0028 & 681.5 & 16.2 & 681.4 & 21.2 \\
\hline 0.04764 & 0.13561 & 0.00356 & 819.8 & 20.2 & 817.5 & 26.2 \\
\hline 0.03572 & 0.11108 & 0.0029 & 679 & 16.8 & 666.1 & 22.6 \\
\hline 0.03564 & 0.11031 & 0.00284 & 674.5 & 16.4 & 682.3 & 22.4 \\
\hline 0.01462 & 0.03878 & 0.00094 & 245.3 & 5.8 & 396.5 & 12.6 \\
\hline 0.04774 & 0.13585 & 0.00358 & 821.2 & 20.4 & 814.3 & 26.2 \\
\hline
\end{tabular}




\begin{tabular}{|c|c|c|c|c|c|}
\hline age $207 / 206$ & $2 \sigma$ age 76 & $\begin{array}{l}\text { discordance } \\
\Delta 68-75[\%]\end{array}$ & $\Delta 68-76[\%]$ & $\begin{array}{c}\text { preferred age } \\
\text { age }\end{array}$ & $2 \sigma$ age \\
\hline 1016.7 & 53.2 & -21.7 & -62.7 & & \\
\hline 1258.1 & 46 & -23.4 & -57.9 & & \\
\hline 125.7 & 120.2 & -3.9 & -75.6 & 30.7 & 0.8 \\
\hline 879.5 & 60.6 & -1.6 & -5.5 & 830.9 & 19.2 \\
\hline 933.3 & 115.8 & 6.5 & 22.2 & & \\
\hline 839.6 & 51.6 & -0.8 & -3.1 & 813.8 & 18 \\
\hline 886.4 & 76.6 & -1.8 & -6.5 & 828.8 & 20.6 \\
\hline 3355.2 & 35.8 & -77.8 & -96.1 & & \\
\hline 767.2 & 71.6 & -3.8 & -14.6 & 655 & 15.8 \\
\hline 902.3 & 52.4 & -2.2 & -7.7 & 832.7 & 18.6 \\
\hline 860.3 & 54.6 & -2.4 & -8.7 & 785.1 & 17.6 \\
\hline 720 & 92.4 & -1.7 & -6.9 & 670.3 & 17.6 \\
\hline 704.2 & 77.8 & -0.3 & -1.5 & 693.8 & 17.2 \\
\hline 900.2 & 49.6 & $\begin{array}{c}-8.8 \\
-7721.3\end{array}$ & -29.4 & 635.4 & 14.2 \\
\hline 656.1 & 73.2 & 1 & 4.5 & 685.7 & 16.4 \\
\hline 695.4 & 59.8 & -0.4 & -1.8 & 683 & 15.8 \\
\hline 1014.2 & 51.6 & -20.6 & -60 & & \\
\hline 688.2 & 66.4 & -0.6 & -2.8 & 669.3 & 15.6 \\
\hline 762.6 & 69.8 & -3.1 & -12.3 & 669 & 16 \\
\hline 59.4 & 88.8 & -1.2 & -51 & 29.1 & 0.8 \\
\hline 835.9 & 52.2 & -9.7 & -34.1 & 550.6 & 12.6 \\
\hline 923.1 & 50 & -10.6 & -34.6 & 603.6 & 13.6 \\
\hline 951.6 & 63.6 & -0.2 & -0.7 & 945.3 & 22 \\
\hline 1626.3 & 45 & -38.2 & -76.2 & & \\
\hline 1070.9 & 83 & -1.5 & -4.7 & 1020.8 & 26.4 \\
\hline 1187.6 & 49.2 & -18.6 & -48.9 & & \\
\hline 415.9 & 72.2 & 11.8 & 81 & & \\
\hline 796.5 & 77.8 & 0.2 & 0.6 & 801.2 & 19.8 \\
\hline 884.9 & 53 & -3.1 & -10.9 & 788.7 & 17.6 \\
\hline 870.4 & 49.4 & -3.5 & -12.5 & 761.7 & 16.8 \\
\hline 874.6 & 55.8 & -3.6 & -12.6 & 764.3 & 17.4 \\
\hline 1644.8 & 43.2 & -40.9 & -80.1 & & \\
\hline 836.8 & 82.8 & -0.4 & -1.7 & 822.3 & 20.8 \\
\hline 950.2 & 98.4 & -10.6 & -33.7 & 630 & 17.4 \\
\hline 692.6 & 66.2 & 0.2 & 0.8 & 698.4 & 16.4 \\
\hline 871.3 & 53 & -3.5 & -12.4 & 763.5 & 17.2 \\
\hline-141.2 & 1094.2 & 7.5 & -124.7 & & \\
\hline 811.8 & 68.2 & 0.4 & 1.5 & 824.3 & 19.6 \\
\hline 773.7 & 66.8 & -1.2 & -4.7 & 737.6 & 17.4 \\
\hline 627.9 & 130 & 1 & 4.6 & 656.8 & 19.6 \\
\hline 671.7 & 65 & 0.3 & 1.3 & 680.6 & 15.8 \\
\hline 728.4 & 60.6 & -2 & -8 & 670 & 15.4 \\
\hline 883.7 & 64 & -1.9 & -6.7 & 824.2 & 19.2 \\
\hline 776.3 & 60.8 & 2.2 & 8.7 & 843.9 & 19.4 \\
\hline 751.5 & 73.6 & -2.8 & -11 & 668.9 & 16.2 \\
\hline 766.9 & 71.6 & -3.1 & -12.2 & 673.6 & 16.2 \\
\hline 709.6 & 68.8 & -2.2 & -9.3 & 643.2 & 15.2 \\
\hline 784.7 & 74.6 & -0.8 & -3.2 & 759.3 & 18.4 \\
\hline 767.8 & 79.4 & -0.2 & -0.7 & 762.4 & 19 \\
\hline
\end{tabular}




\begin{tabular}{|c|c|c|c|c|c|}
\hline 776.6 & 93.8 & 1.3 & 4.8 & 813.8 & 21.6 \\
\hline-78 & 294.2 & 4.5 & -138.9 & 30.4 & 1.2 \\
\hline 626.8 & 83.8 & 2.2 & 10 & 689.5 & 17.2 \\
\hline 716.3 & 78.6 & -3.1 & -12.8 & 624.9 & 15.4 \\
\hline 1328 & 88.2 & -21.3 & -51.4 & & \\
\hline 631.5 & 82.2 & 0.4 & 1.5 & 641 & 15.8 \\
\hline 939.8 & 48.4 & -15.9 & -50 & & \\
\hline 863.4 & 52 & -13.4 & -45.4 & 471.8 & 10.8 \\
\hline 788.2 & 55.8 & -1 & -3.8 & 758.3 & 17 \\
\hline 764.6 & 56.6 & -0.5 & -1.9 & 750.4 & 17 \\
\hline 799.1 & 63.8 & -6.1 & -22.6 & 618.1 & 14.6 \\
\hline 834.4 & 98.4 & -0.7 & -2.6 & 813 & 22 \\
\hline 922.5 & 116.6 & -3.5 & -11.7 & 814.2 & 24.2 \\
\hline 867.9 & 63.4 & -5.1 & -17.8 & 713.6 & 16.8 \\
\hline 927.5 & 54 & -13.4 & -43.2 & 527 & 12.2 \\
\hline 790.1 & 64 & -3.1 & -11.8 & 696.6 & 16.4 \\
\hline 845.5 & 61.8 & -1.5 & -5.5 & 799.4 & 18.4 \\
\hline 706.5 & 71 & -1.7 & -7.3 & 654.8 & 15.8 \\
\hline 833.1 & 61.2 & -9.3 & -32.9 & 558.7 & 13 \\
\hline 766.5 & 185.4 & -4.1 & -16 & 643.6 & 24.6 \\
\hline 683.1 & 133 & -0.3 & -1.2 & 675 & 21 \\
\hline 670.7 & 72.6 & -0.1 & -0.5 & 667.3 & 16 \\
\hline 899 & 61.6 & -2.4 & -8.3 & 824.7 & 19 \\
\hline 704.2 & 70.4 & -0.6 & -2.7 & 684.9 & 16.4 \\
\hline 797.8 & 63.2 & 0.8 & 3.1 & 822.4 & 19 \\
\hline 846.8 & 59.4 & -0.8 & -2.9 & 822.6 & 18.8 \\
\hline 920.1 & 54.2 & -4.6 & -15.5 & 777.6 & 17.4 \\
\hline 1078.9 & 55.4 & -2.1 & -6.3 & 1011.3 & 22.6 \\
\hline 1002.5 & 68.2 & -0.1 & -0.5 & 997.1 & 23.8 \\
\hline 832.5 & 56.2 & -0.8 & -3 & 807.7 & 18.2 \\
\hline 804.5 & 66 & 0.8 & 2.8 & 827.2 & 19.4 \\
\hline 836.8 & 96.4 & -0.8 & -2.9 & 812.9 & 22 \\
\hline 1035.5 & 52 & -2.4 & -7.4 & 958.9 & 21.2 \\
\hline 1178.6 & 52.8 & -7.4 & -20.4 & 938.6 & 21 \\
\hline 1082.6 & 53.2 & -7.4 & -21.7 & 847.7 & 19 \\
\hline 725.7 & 89.4 & -2.9 & -11.7 & 640.7 & 16. \\
\hline 821.2 & 71.6 & 0.7 & 2.3 & 840.5 & 20. \\
\hline 827.2 & 59.4 & -4.9 & -17.7 & 680.4 & 15. \\
\hline 642.4 & 77.4 & 0.7 & 3 & 661.8 & 16.2 \\
\hline 822.5 & 69.6 & 0.4 & 1.3 & 833.3 & 19.8 \\
\hline 1029.7 & 57.8 & -5.7 & -17.4 & 850.8 & 19.4 \\
\hline 767.5 & 72.2 & 1.5 & 5.9 & 812.6 & 19.4 \\
\hline-16.1 & 136.4 & 1.8 & -282.9 & 29.4 & 0.8 \\
\hline 805.1 & 71.8 & -4 & -15 & 684.2 & 16.4 \\
\hline 1079.5 & 54.8 & -14.7 & -42 & 626.4 & 14. \\
\hline 3153.3 & 40.2 & -78.1 & -97.9 & & \\
\hline 804.8 & 82 & 0.4 & 1.3 & 815.4 & 20. \\
\hline 807.6 & 56.4 & 0.6 & 2 & 823.7 & 18. \\
\hline 665.5 & 108.2 & 2.7 & 11.6 & 742.8 & 20. \\
\hline 906.5 & 75.6 & -9 & -29.9 & 635.4 & 15. \\
\hline 926 & 53.4 & -21 & -65.4 & & \\
\hline 1066.4 & 85.4 & 0.2 & 0.5 & 1072 & 27. \\
\hline 671.7 & 117.4 & 0.4 & 1.7 & 683.3 & 19.6 \\
\hline 819.3 & 57.8 & 0.7 & 2.4 & 838.8 & 19 \\
\hline 711.3 & 78.8 & -0.6 & -2.7 & 692.2 & 17 \\
\hline
\end{tabular}




\begin{tabular}{|c|c|c|c|c|c|}
\hline 805.7 & 55.8 & -0.8 & -3 & 781.5 & 17.6 \\
\hline 646.3 & 94 & 0.4 & 1.5 & 656.1 & 17.2 \\
\hline 1113.1 & 54.2 & -25.8 & -69 & & \\
\hline 770.4 & 70 & -4.1 & -15.7 & 649.7 & 15.6 \\
\hline 968 & 67 & -10.4 & -32.9 & 650 & 15.6 \\
\hline 644.2 & 69.6 & 0.4 & 1.6 & 654.4 & 15.4 \\
\hline 693.6 & 85.8 & -0.8 & -3.6 & 668.7 & 16.8 \\
\hline 2145.2 & 47.4 & -61.9 & -96.3 & & \\
\hline 1185.1 & 98.8 & -3.7 & -10.3 & 1062.5 & 30.2 \\
\hline 1113.9 & 88.4 & -2.8 & -8.4 & 1020.8 & 27 \\
\hline 818.7 & 62.4 & 0.1 & 0.2 & 820.1 & 18.8 \\
\hline 1082.6 & 70.2 & -14.2 & -40.7 & 642.3 & 15.8 \\
\hline 825.6 & 83.4 & -0.3 & -1 & 817 & 20.6 \\
\hline 664.4 & 83.2 & 1.3 & 5.6 & 701.5 & 17.4 \\
\hline 868.2 & 68.2 & -1.8 & -6.5 & 811.7 & 19.2 \\
\hline 825.9 & 63.8 & 0.8 & 2.7 & 848.5 & 19.6 \\
\hline 698.1 & 110 & -0.4 & -2 & 684 & 19.2 \\
\hline 956.3 & 64 & 0.1 & 0.1 & 957.6 & 22.2 \\
\hline 692.3 & 65.4 & -0.1 & -0.5 & 688.9 & 16 \\
\hline 796.5 & 135.4 & -4.9 & -18.3 & 650.5 & 20.6 \\
\hline 756.4 & 85.2 & 0.4 & 1.7 & 769.1 & 19.4 \\
\hline 810.5 & 65.2 & 0.7 & 2.4 & 830.1 & 19.2 \\
\hline 824 & 69 & -0.1 & -0.6 & 818.9 & 19.4 \\
\hline 1074.4 & 54 & -24.9 & -68.3 & & \\
\hline 1398.1 & 62.6 & -23.7 & -54.8 & & \\
\hline 928.6 & 56.4 & -12.3 & -39.6 & 560.7 & 12.8 \\
\hline 918.3 & 91 & -0.4 & -1.4 & 905.3 & 23.8 \\
\hline 830.6 & 61.2 & -3.9 & -14.1 & 713.5 & 16.4 \\
\hline 1005 & 86 & -1.4 & -4.5 & 960.2 & 24.8 \\
\hline 677.9 & 83.2 & 0.9 & 3.6 & 702.4 & 17.4 \\
\hline 1749.8 & 55.4 & -28.4 & -56.4 & & \\
\hline 882.5 & 64.6 & -2.4 & -8.4 & 808.3 & 18.8 \\
\hline 673.4 & 78 & -0.2 & -1.1 & 666.3 & 16.2 \\
\hline 2086.3 & 49.8 & -49.3 & -82.3 & & \\
\hline 670 & 66.4 & 0.2 & 0.9 & 676.2 & 15.8 \\
\hline 786.9 & 71.2 & 1.3 & 4.9 & 825.8 & 19.6 \\
\hline 875.5 & 73.4 & -1.4 & -5 & 831.9 & 20 \\
\hline 886.4 & 60.2 & -1.6 & -5.5 & 837.5 & 19 \\
\hline 695 & 109.6 & -1.7 & -7.2 & 644.7 & 18 \\
\hline 784.3 & 87.4 & -3.3 & -12.8 & 684.2 & 17.6 \\
\hline 823.1 & 62.2 & -0.7 & -2.8 & 800.2 & 18.4 \\
\hline 681.7 & 73.4 & 0 & 0 & 681.5 & 16.2 \\
\hline 812.1 & 80.2 & 0.3 & 1 & 819.8 & 20.2 \\
\hline 623.3 & 82.6 & 1.9 & 8.9 & 679 & 16.8 \\
\hline 708.9 & 78.2 & -1.1 & -4.8 & 674.5 & 16.4 \\
\hline 1412.3 & 56.6 & -38.1 & -82.6 & & \\
\hline 796.2 & 81 & 0.8 & 3.1 & 821.2 & 20.4 \\
\hline
\end{tabular}


Sample 2964S Blue Nile @ Bambudi 323 grain analysed 293 concordant ag

\begin{tabular}{|c|c|c|c|c|c|c|}
\hline \multirow[b]{2}{*}{ grain } & \multicolumn{3}{|c|}{ concentrations } & \multicolumn{3}{|l|}{ isotopic ratios } \\
\hline & U [ppm] & $\mathrm{Pb}$ [ppm] & $\mathrm{Th} / \mathrm{U}$ & $\mathrm{Pb} 207 / \mathrm{Pb} 206$ & $2 \sigma 76$ & $\mathrm{~Pb} 207 / \mathrm{U} 235$ \\
\hline X2964n_G001 & 139.8 & 18.1 & 0.3154 & 0.07491 & 0.0023 & 1.29007 \\
\hline X2964n_G002 & 89 & 13.6 & 0.4051 & 0.06722 & 0.00228 & 1.3535 \\
\hline X2964n_G003 & 46 & 5.9 & 0.6669 & 0.0664 & 0.00306 & 1.04201 \\
\hline X2964n_G004 & 260.4 & 27.4 & 0.223 & 0.06252 & 0.00172 & 0.91827 \\
\hline X2964n_G005 & 63.7 & 6.6 & 0.3221 & 0.06442 & 0.00314 & 0.9024 \\
\hline X2964n G006 & 185.3 & 97.7 & 0.157 & 0.18768 & 0.00418 & 12.98802 \\
\hline X2964n G007 & 131.6 & 16.1 & 0.3961 & 0.06521 & 0.0021 & 1.05079 \\
\hline X2964n_G008 & 317.4 & 44.5 & 0.3097 & 0.06515 & 0.00166 & 1.23519 \\
\hline X2964n_G009 & 132.1 & 20.2 & 0.549 & 0.06736 & 0.00204 & 1.3005 \\
\hline X2964n_G010 & 483.5 & 58.4 & 0.4485 & 0.06177 & 0.00154 & 0.97494 \\
\hline X2964n_G011 & 216.8 & 22.8 & 0.6161 & 0.05973 & 0.00182 & 0.78268 \\
\hline X2964n_G012 & 196.3 & 25.4 & 0.3952 & 0.06661 & 0.0019 & 1.14578 \\
\hline X2964n G013 & 111.5 & 19.1 & 0.5565 & 0.07762 & 0.00236 & 1.67053 \\
\hline X2964n_G014 & 42.1 & 0.3 & 1.3018 & 0.28573 & 0.03736 & 0.24477 \\
\hline X2964n_G015 & 1149.3 & 132.2 & 0.4484 & 0.06687 & 0.00152 & 1.00817 \\
\hline X2964n_G016 & 24.4 & 4.8 & 1.5348 & 0.06633 & 0.00386 & 1.2974 \\
\hline X2964n_G017 & 259 & 24.4 & 0.0258 & 0.06154 & 0.00178 & 0.86092 \\
\hline X2964n_G018 & 534.7 & 57.7 & 0.0373 & 0.0665 & 0.00162 & 1.06374 \\
\hline X2964n_G019 & 269 & 12.5 & 0.9112 & 0.05123 & 0.00208 & 0.27484 \\
\hline X2964n_G020 & 70.8 & 10.8 & 0.8592 & 0.06456 & 0.00254 & 1.14924 \\
\hline X2964n G021 & 101.5 & 10.3 & 0.1725 & 0.05922 & 0.0023 & 0.85161 \\
\hline X2964n_G022 & 233.1 & 24.4 & 0.4412 & 0.05854 & 0.00176 & 0.80315 \\
\hline X2964n_G023 & 161.3 & 34.3 & 0.7287 & 0.07613 & 0.00212 & 1.9443 \\
\hline X2964n_G024 & 485.4 & 62.4 & 1.727 & 0.06144 & 0.0019 & 0.76115 \\
\hline X2964n_G025 & 265.2 & 116.4 & 1.0474 & 0.12625 & 0.00286 & 6.03315 \\
\hline X2964n_G026 & 97.2 & 10 & 0.1017 & 0.06315 & 0.00236 & 0.93229 \\
\hline X2964n_G027 & 102 & 9.8 & 0.7074 & 0.05951 & 0.00248 & 0.68965 \\
\hline X2964n_G028 & 405.4 & 39.3 & 0.5099 & 0.05864 & 0.0016 & 0.72845 \\
\hline X2964n_G029 & 1667.7 & 251 & 0.799 & 0.09822 & 0.00226 & 1.86368 \\
\hline X2964n_G030 & 293 & 40.1 & 0.5852 & 0.06539 & 0.00174 & 1.12117 \\
\hline X2964n G031 & 127.8 & 18.2 & 0.4791 & 0.07396 & 0.00234 & 1.3481 \\
\hline X2964n_G032 & 380.6 & 51.6 & 0.3109 & 0.06682 & 0.00168 & 1.22556 \\
\hline X2964n_G033 & 62.7 & 8.9 & 0.3147 & 0.06862 & 0.00278 & 1.31965 \\
\hline X2964n_G034 & 606 & 61 & 0.0733 & 0.06204 & 0.0016 & 0.91509 \\
\hline X2964n_G035 & 306.8 & 42.8 & 1.1925 & 0.06275 & 0.00176 & 0.9392 \\
\hline X2964n_G036 & 194.3 & 31.8 & 0.843 & 0.06842 & 0.00198 & 1.31171 \\
\hline X2964n_G037 & 185.7 & 24.9 & 0.3819 & 0.07523 & 0.00222 & 1.32633 \\
\hline X2964n_G038 & 421.7 & 55.2 & 0.1593 & 0.06598 & 0.00164 & 1.22407 \\
\hline X2964n_G039 & 65.6 & 9.4 & 0.8938 & 0.06612 & 0.00278 & 1.09259 \\
\hline X2964n_G040 & 325.5 & 110.3 & 0.5501 & 0.11175 & 0.00258 & 4.69673 \\
\hline X2964n_G041 & 70.8 & 7.7 & 0.5809 & 0.05944 & 0.00268 & 0.81399 \\
\hline X2964n_G042 & 589.3 & 62.2 & 0.6224 & 0.07087 & 0.00238 & 0.94235 \\
\hline X2964n_G043 & 51.7 & 6.1 & 0.2335 & 0.06659 & 0.00304 & 1.09287 \\
\hline X2964n_G044 & 520.3 & 105.1 & 0.692 & 0.0856 & 0.00202 & 2.05591 \\
\hline X2964n_G045 & 139.8 & 16.6 & 0.5348 & 0.06213 & 0.0021 & 0.93812 \\
\hline X2964n_G046 & 213 & 26.9 & 0.3902 & 0.06675 & 0.00194 & 1.11394 \\
\hline X2964n_G047 & 162.8 & 24.1 & 0.7213 & 0.06779 & 0.00282 & 1.21496 \\
\hline X2964n_G048 & 98.6 & 13.2 & 0.3735 & 0.06687 & 0.00236 & 1.18759 \\
\hline X2964n_G049 & 80.4 & 12.1 & 0.6209 & 0.06348 & 0.00242 & 1.18598 \\
\hline X2964n G050 & 683.1 & 87 & 0.0853 & 0.06722 & 0.0016 & 1.24304 \\
\hline
\end{tabular}




\begin{tabular}{|c|c|c|c|c|c|c|}
\hline X2964n_G051 & 100 & 15.6 & 0.7725 & 0.06691 & 0.00232 & 1.23959 \\
\hline X2964n_G052 & 78 & 11.6 & 0.6791 & 0.06331 & 0.0024 & 1.14623 \\
\hline X2964n_G053 & 516 & 64.3 & 0.4576 & 0.06332 & 0.00158 & 1.02579 \\
\hline X2964n G054 & 223.1 & 21.4 & 0.3871 & 0.0583 & 0.00186 & 0.74662 \\
\hline X2964n_G055 & 270.9 & 1.3 & 1.1242 & 0.05772 & 0.00696 & 0.02996 \\
\hline X2964n_G056 & 78 & 10.8 & 0.3351 & 0.06617 & 0.00252 & 1.23343 \\
\hline X2964n_G057 & 244.1 & 27.5 & 0.8074 & 0.05987 & 0.00182 & 0.7992 \\
\hline X2964n_G058 & 328.9 & 41.4 & 0.6731 & 0.06222 & 0.0017 & 0.96538 \\
\hline X2964n_G059 & 57.9 & 8.3 & 0.3989 & 0.06581 & 0.00284 & 1.2472 \\
\hline X2964n G060 & 389.6 & 56.1 & 0.6736 & 0.06497 & 0.00168 & 1.14667 \\
\hline X2964n_G061 & 67 & 9.3 & 0.4881 & 0.06698 & 0.00346 & 1.19766 \\
\hline X2964n_G062 & 251.8 & 36.3 & 0.6417 & 0.0661 & 0.00184 & 1.18841 \\
\hline X2964n_G063 & 438 & 40.1 & 0.7986 & 0.05827 & 0.00162 & 0.63391 \\
\hline X2964n_G064 & 474.9 & 68.8 & 0.5119 & 0.06896 & 0.0017 & 1.28753 \\
\hline X2964n_G065 & 54.1 & & 1.6182 & 0.1545 & 0.0464 & 1.0337 \\
\hline X2964n G066 & 537.6 & 21.7 & 0.389 & 0.05481 & 0.0018 & 0.29457 \\
\hline X2964n_G067 & 208.2 & 38 & 0.3776 & 0.07347 & 0.00196 & 1.77567 \\
\hline X2964n_G068 & 121.6 & 17.5 & 0.5128 & 0.06967 & 0.00228 & 1.27781 \\
\hline X2964n_G069 & 99.1 & 11.1 & 0.5796 & 0.06017 & 0.00242 & 0.84848 \\
\hline X2964n_G070 & 64.1 & 10.1 & 0.706 & 0.06819 & 0.00274 & 1.30917 \\
\hline X2964n_G071 & 124.5 & 16.5 & 0.8387 & 0.05985 & 0.00224 & 0.92854 \\
\hline X2964n G072 & 37.8 & 5.7 & 0.8059 & 0.06762 & 0.00336 & 1.21088 \\
\hline X2964n_G073 & 459.5 & 54 & 0.1266 & 0.06497 & 0.0017 & 1.09567 \\
\hline X2964n_G074 & 73.2 & 0.3 & 0.555 & 0.04451 & 0.01622 & 0.02575 \\
\hline X2964n_G075 & 67 & 9.3 & 0.4 & 0.06827 & 0.0028 & 1.25392 \\
\hline X2964n_G076 & 74.7 & 10.5 & 0.5046 & 0.07856 & 0.00436 & 1.40835 \\
\hline X2964n_G077 & 1606.5 & 160.9 & 0.0305 & 0.06117 & 0.0014 & 0.90855 \\
\hline X2964n_G078 & 56 & 8 & 0.4076 & 0.06607 & 0.00288 & 1.23819 \\
\hline X2964n_G079 & 52.2 & 6.9 & 0.3212 & 0.06365 & 0.003 & 1.13002 \\
\hline X2964n_G080 & 459.1 & 75.7 & 0.9296 & 0.06632 & 0.00166 & 1.25392 \\
\hline X2964n_G081 & 125.9 & 19.3 & 0.6339 & 0.0656 & 0.0022 & 1.2453 \\
\hline X2964n_G082 & 1653.9 & & 1.882 & 0.10455 & 0.00252 & 0.76002 \\
\hline X2964n_G083 & & & & 0.53078 & 0.0663 & 20.09719 \\
\hline X2964n_G084 & 587.3 & 85.4 & 0.4027 & 0.06571 & 0.00158 & 1.25871 \\
\hline X2964n_G085 & 1433.2 & 124 & 0.4821 & 0.0708 & 0.00174 & 0.80592 \\
\hline X2964n_G086 & 19.6 & 3 & 1.1072 & 0.07099 & 0.00492 & 1.23214 \\
\hline X2964n_G087 & 59.8 & 5.8 & 0.7532 & 0.05902 & 0.00306 & 0.68296 \\
\hline X2964n_G088 & 83.3 & 10 & 0.4392 & 0.06548 & 0.0026 & 1.03277 \\
\hline X2964n_G089 & 501.2 & 86.9 & 0.62 & 0.07113 & 0.00172 & 1.52596 \\
\hline X2964n_G090 & 290.6 & 44.2 & 0.4006 & 0.06822 & 0.00218 & 1.365 \\
\hline X2964n_G091 & 52.7 & 7.4 & 0.3744 & 0.06315 & 0.00284 & 1.17799 \\
\hline X2964n_G092 & 793.7 & 252.5 & 0.6541 & 0.13412 & 0.00298 & 5.29084 \\
\hline X2964n_G093 & 210.6 & 30 & 0.3078 & 0.06454 & 0.00186 & 1.24663 \\
\hline X2964n_G094 & 45 & 6.3 & 0.5528 & 0.06272 & 0.00312 & 1.11492 \\
\hline X2964n_G095 & 109.1 & 12.1 & 0.5406 & 0.06063 & 0.00236 & 0.85795 \\
\hline X2964n_G096 & 164.7 & 24.2 & 0.4814 & 0.06583 & 0.002 & 1.25076 \\
\hline X2964n_G097 & 91.9 & 11.4 & 0.2394 & 0.06525 & 0.00244 & 1.118 \\
\hline X2964n_G098 & 52.2 & 5.9 & 0.7892 & 0.05565 & 0.0031 & 0.74173 \\
\hline X2964n_G099 & 83.8 & 12.1 & 0.5157 & 0.06699 & 0.0025 & 1.23405 \\
\hline X2964n_G100 & 61.3 & 12.1 & 1.064 & 0.07017 & 0.00274 & 1.54102 \\
\hline X2964n_G101 & 133.6 & 21.1 & 0.8338 & 0.06626 & 0.00208 & 1.22499 \\
\hline X2964n_G102 & 245.1 & 36.6 & 0.3765 & 0.06682 & 0.0018 & 1.32249 \\
\hline X2964n_G103 & 192.4 & 22.2 & 0.6936 & 0.06078 & 0.0019 & 0.85713 \\
\hline X2964n_G104 & 147 & 15.1 & 0.3182 & 0.06093 & 0.0021 & 0.8504 \\
\hline X2964n_G105 & 283.9 & 42 & 1.0038 & 0.06286 & 0.00172 & 1.04064 \\
\hline
\end{tabular}




\begin{tabular}{|c|c|c|c|c|c|c|}
\hline X2964n_G106 & 429.9 & 38.1 & 0.3037 & 0.05727 & 0.00158 & 0.69283 \\
\hline X2964n_G107 & 46.4 & 5.1 & 0.5427 & 0.06444 & 0.0032 & 0.91819 \\
\hline X2964n_G108 & 131.6 & 18.9 & 0.4134 & 0.06486 & 0.00204 & 1.21958 \\
\hline X2964n_G109 & 103.4 & 14.9 & 0.4611 & 0.06677 & 0.00226 & 1.2466 \\
\hline X2964n_G110 & 241.3 & 22 & 0.4 & 0.06242 & 0.00278 & 0.77374 \\
\hline X2964n_G111 & 88.1 & 14.9 & 1.3829 & 0.06814 & 0.00248 & 1.18298 \\
\hline X2964n_G112 & 131.2 & 10.5 & 0.4541 & 0.05722 & 0.0023 & 0.59944 \\
\hline X2964n_G113 & 62.7 & 9.6 & 0.8473 & 0.06661 & 0.00278 & 1.20445 \\
\hline X2964n_G114 & 133.1 & 23.1 & 2.7151 & 0.0678 & 0.0023 & 0.92664 \\
\hline X2964n_G115 & 282.9 & 28.5 & 1.2198 & 0.06862 & 0.00206 & 0.73678 \\
\hline X2964n_G116 & 183.8 & 4.6 & 0.6962 & 0.04924 & 0.00306 & 0.15104 \\
\hline X2964n_G117 & 305.4 & 57.7 & 0.9227 & 0.07297 & 0.0019 & 1.56665 \\
\hline X2964n_G118 & 312.1 & 44.3 & 0.7376 & 0.06439 & 0.00184 & 1.09819 \\
\hline X2964n_G119 & 38.3 & 5.6 & 0.4408 & 0.0643 & 0.0032 & 1.22706 \\
\hline X2964n_G120 & 38.8 & 5.7 & 0.4401 & 0.06487 & 0.00326 & 1.24139 \\
\hline X2964n_G121 & 356.1 & 39.5 & 0.4741 & 0.06108 & 0.00168 & 0.87711 \\
\hline X2964n_G122 & 79.9 & 10.8 & 0.5057 & 0.06476 & 0.0025 & 1.12206 \\
\hline X2964n_G123 & 63.2 & 9.2 & 0.3524 & 0.06639 & 0.0028 & 1.29166 \\
\hline X2964n_G124 & 151.3 & 23.8 & 0.5924 & 0.06778 & 0.00204 & 1.33073 \\
\hline X2964n_G125 & 243.7 & 32.2 & 0.178 & 0.06695 & 0.00188 & 1.24598 \\
\hline X2964n_G126 & 78.5 & 9.6 & 0.457 & 0.06104 & 0.00248 & 0.96968 \\
\hline X2964n_G127 & 958.8 & 111.2 & 0.504 & 0.06183 & 0.00148 & 0.9616 \\
\hline X2964n_G128 & 59.8 & 8.2 & 1.8042 & 0.06617 & 0.00328 & 0.89071 \\
\hline X2964n_G129 & 132.6 & 14.5 & 0.9014 & 0.0573 & 0.00222 & 0.72514 \\
\hline X2964n_G130 & 241.3 & 36.6 & 0.4946 & 0.06616 & 0.00188 & 1.28473 \\
\hline X2964n_G131 & 5532.2 & 620.9 & 4.3102 & 0.06665 & 0.00154 & 0.42337 \\
\hline X2964n_G132 & 21.5 & 2.4 & 0.6283 & 0.06079 & 0.00512 & 0.84901 \\
\hline X2964n_G133 & 100 & 14.4 & 0.5535 & 0.06541 & 0.0024 & 1.19259 \\
\hline X2964n_G134 & 205.4 & 37.8 & 0.7375 & 0.07115 & 0.00216 & 1.57316 \\
\hline X2964n_G135 & 190.5 & 34.2 & 0.5607 & 0.07013 & 0.00212 & 1.58828 \\
\hline X2964n_G136 & 135 & 18.8 & 0.3685 & 0.06473 & 0.00224 & 1.20337 \\
\hline X2964n_G137 & 119.7 & 12.4 & 0.3407 & 0.05832 & 0.00234 & 0.81579 \\
\hline X2964n_G138 & 68.9 & 10.7 & 0.4481 & 0.06841 & 0.00264 & 1.38525 \\
\hline X2964n_G139 & 37.8 & 4.9 & 0.3437 & 0.06531 & 0.00338 & 1.14501 \\
\hline X2964n_G140 & 93.3 & 11.4 & 0.8464 & 0.05841 & 0.00236 & 0.8304 \\
\hline X2964n_G141 & 38.8 & 4.7 & 0.1179 & 0.06602 & 0.00326 & 1.15556 \\
\hline X2964n_G142 & 111.1 & 16 & 0.4113 & 0.06605 & 0.00222 & 1.25235 \\
\hline X2964n_G143 & 3369 & 346.5 & 0.2849 & 0.06727 & 0.00158 & 0.95166 \\
\hline X2964n_G144 & 114.4 & 0.5 & 0.5335 & 0.05303 & 0.0139 & 0.02895 \\
\hline X2964n_G145 & 25.4 & 4.1 & 0.6174 & 0.06677 & 0.00382 & 1.32046 \\
\hline X2964n_G146 & 119.2 & 17.3 & 0.3986 & 0.06807 & 0.00218 & 1.30605 \\
\hline X2964n_G147 & 824.3 & 67.7 & 0.1621 & 0.07688 & 0.00188 & 0.90785 \\
\hline X2964n_G148 & 98.6 & 14.1 & 0.4094 & 0.06537 & 0.00222 & 1.22862 \\
\hline X2964n_G149 & 148.4 & 21.2 & 0.371 & 0.06649 & 0.002 & 1.26485 \\
\hline X2964n_G150 & 3589.7 & 182.6 & 0.3847 & 0.06168 & 0.00142 & 0.41767 \\
\hline X2964n_G151 & 69.9 & 10.5 & 0.5173 & 0.06504 & 0.0025 & 1.2504 \\
\hline X2964n_G152 & 170.4 & 31.4 & 0.4273 & 0.07116 & 0.00198 & 1.70803 \\
\hline X2964n_G153 & 583 & 29.9 & 0.1026 & 0.05297 & 0.0015 & 0.39593 \\
\hline X2964n_G154 & 584.5 & 29.7 & 0.1051 & 0.0532 & 0.0015 & 0.39463 \\
\hline X2964n_G155 & 52.2 & 8.7 & 0.3366 & 0.07244 & 0.00282 & 1.62034 \\
\hline X2964n_G156 & 789.8 & 5.1 & 2.4619 & 0.04631 & 0.00342 & 0.02446 \\
\hline X2964n_G157 & 171.4 & 30.1 & 0.7393 & 0.06901 & 0.002 & 1.45439 \\
\hline X2964n_G158 & 200.1 & 21.3 & 0.4189 & 0.05972 & 0.00188 & 0.83713 \\
\hline X2964n_G159 & 623.2 & 38.7 & 0.3646 & 0.05315 & 0.00194 & 0.44285 \\
\hline X2964n_G160 & 105.8 & 12 & 0.795 & 0.05831 & 0.0022 & 0.78332 \\
\hline
\end{tabular}




\begin{tabular}{|c|c|c|c|c|c|c|}
\hline X2964n_G161 & 508.4 & 46.5 & 0.0102 & 0.06119 & 0.00158 & 0.83743 \\
\hline X2964n_G162 & 253.2 & 33.7 & 0.422 & 0.06408 & 0.00178 & 1.11853 \\
\hline X2964n_G163 & 119.7 & 14.5 & 0.6417 & 0.06231 & 0.00248 & 0.93183 \\
\hline X2964n_G164 & 268.5 & 35.2 & 0.5089 & 0.06985 & 0.0019 & 1.15621 \\
\hline X2964n_G165 & 453.3 & 65.1 & 0.3436 & 0.06894 & 0.0017 & 1.3313 \\
\hline X2964n_G166 & 781.7 & 113 & 0.4436 & 0.06798 & 0.00162 & 1.28612 \\
\hline X2964n_G167 & 45.5 & 5.4 & 0.8692 & 0.06092 & 0.00318 & 0.84092 \\
\hline X2964n_G168 & 31.6 & 0.2 & 1.1915 & 0.05521 & 0.02388 & 0.03734 \\
\hline X2964n_G169 & 115.8 & 23.8 & 0.843 & 0.07391 & 0.00224 & 1.769 \\
\hline X2964n_G170 & 461.9 & 75.4 & 0.8896 & 0.06644 & 0.00168 & 1.25987 \\
\hline X2964n_G171 & 32.6 & 3.8 & 0.3957 & 0.05449 & 0.00342 & 0.85524 \\
\hline X2964n_G172 & 138.3 & 18.3 & 1.7645 & 0.05754 & 0.00212 & 0.72359 \\
\hline X2964n_G173 & 51.2 & 7.1 & 0.3561 & 0.06355 & 0.00284 & 1.17912 \\
\hline X2964n_G174 & 117.8 & 17.2 & 0.7785 & 0.06253 & 0.00208 & 1.08803 \\
\hline X2964n_G175 & 158.4 & 28.4 & 0.3935 & 0.07278 & 0.00204 & 1.71888 \\
\hline X2964n_G176 & 360.9 & 75.5 & 0.8801 & 0.07264 & 0.0018 & 1.75034 \\
\hline X2964n_G177 & 130.7 & 20.7 & 0.7257 & 0.06716 & 0.00208 & 1.27897 \\
\hline X2964n_G178 & 86.6 & 13.5 & 0.486 & 0.07968 & 0.003 & 1.58439 \\
\hline X2964n_G179 & 246 & 43.2 & 0.38 & 0.07286 & 0.00194 & 1.6844 \\
\hline X2964n_G180 & 55.5 & 7.8 & 0.4673 & 0.0695 & 0.00306 & 1.26651 \\
\hline X2964n_G181 & 47.9 & 8.9 & 1.7481 & 0.06378 & 0.003 & 1.12556 \\
\hline X2964n_G182 & 124.9 & 18.1 & 0.558 & 0.06515 & 0.00232 & 1.19563 \\
\hline X2964n_G183 & 39.7 & 5.7 & 0.469 & 0.065 & 0.00368 & 1.21369 \\
\hline X2964n_G184 & 142.6 & 11.9 & 0.4 & 0.05813 & 0.00236 & 0.64131 \\
\hline X2964n_G185 & 1070.3 & 178.9 & 0.7987 & 0.07204 & 0.00172 & 1.42278 \\
\hline X2964n_G186 & 1746.7 & 130.5 & 0.4785 & 0.06764 & 0.00164 & 0.66502 \\
\hline X2964n_G187 & 170.9 & 19.2 & 0.915 & 0.05939 & 0.00206 & 0.76592 \\
\hline X2964n_G188 & 11 & 0.1 & 2.1535 & 0.05344 & 0.06458 & 0.03352 \\
\hline X2964n_G189 & 310.2 & 44.2 & 0.573 & 0.06423 & 0.00174 & 1.15189 \\
\hline X2964n_G190 & 746.3 & 24.2 & 0.7559 & 0.05051 & 0.00166 & 0.19777 \\
\hline X2964n_G191 & 202.5 & 21.9 & 1.1955 & 0.05597 & 0.0019 & 0.6503 \\
\hline X2964n_G192 & 218.3 & 23.3 & 0.3605 & 0.05932 & 0.00184 & 0.85045 \\
\hline X2964n_G193 & 962.6 & 103.9 & 0.691 & 0.06141 & 0.00152 & 0.81308 \\
\hline X2964n_G194 & 82.3 & 13.4 & 0.4013 & 0.07112 & 0.0026 & 1.52111 \\
\hline X2964n_G195 & 51.2 & 8.5 & 0.7731 & 0.06699 & 0.00274 & 1.32325 \\
\hline X2964n_G196 & 14.4 & 1.9 & 0.4845 & 0.06823 & 0.00522 & 1.13545 \\
\hline X2964n_G197 & 131.2 & 17 & 0.1607 & 0.07005 & 0.00218 & 1.28056 \\
\hline X2964n_G198 & 270.5 & 54.9 & 0.4244 & 0.07498 & 0.00194 & 1.97991 \\
\hline X2964n_G199 & 107.2 & 11.8 & 0.7813 & 0.06004 & 0.00236 & 0.79002 \\
\hline X2964n_G200 & 139.3 & 20.2 & 0.4301 & 0.0658 & 0.00208 & 1.25031 \\
\hline X2964n_G201 & 90 & 14.2 & 1.089 & 0.06176 & 0.00234 & 1.0702 \\
\hline X2964n_G202 & 59.4 & 10.3 & 0.4237 & 0.06962 & 0.00262 & 1.57266 \\
\hline X2964n_G203 & 264.2 & 28.3 & 0.4018 & 0.06299 & 0.00208 & 0.88788 \\
\hline X2964n_G204 & 225.5 & 19.1 & 0.086 & 0.06103 & 0.00206 & 0.75673 \\
\hline X2964n_G205 & 57.9 & 7.2 & 0.829 & 0.06161 & 0.00298 & 0.90483 \\
\hline X2964n_G206 & 56 & 8.2 & 0.4333 & 0.08134 & 0.0042 & 1.54441 \\
\hline X2964n_G207 & 50.3 & 4.8 & 0.846 & 0.05688 & 0.00362 & 0.63225 \\
\hline X2964n_G208 & 113.9 & 15.5 & 0.6093 & 0.06358 & 0.00236 & 1.07585 \\
\hline X2964n_G209 & 63.7 & 2.1 & 0.7022 & 0.07797 & 0.01412 & 0.30909 \\
\hline X2964n_G210 & 228.3 & 34.3 & 0.6034 & 0.06562 & 0.00206 & 1.22924 \\
\hline X2964n_G211 & 1501.2 & 246.3 & 1.1601 & 0.06416 & 0.00156 & 1.13284 \\
\hline X2964n_G212 & 709.9 & 69.7 & 0.4095 & 0.06798 & 0.00192 & 0.86643 \\
\hline X2964n_G213 & 85.7 & 12.8 & 0.6696 & 0.06634 & 0.00272 & 1.21696 \\
\hline X2964n_G214 & 155.1 & 18 & 1.0099 & 0.05841 & 0.00234 & 0.76531 \\
\hline X2964n_G215 & 144.6 & 18.7 & 0.3849 & 0.0639 & 0.00254 & 1.09892 \\
\hline
\end{tabular}




\begin{tabular}{|c|c|c|c|c|c|c|}
\hline X2964n_G216 & 78 & 0.3 & 0.7402 & 0.04926 & 0.02424 & 0.02547 \\
\hline X2964n_G217 & 332.2 & 35.6 & 0.2737 & 0.0628 & 0.00194 & 0.93613 \\
\hline X2964n G218 & 54.6 & 7.6 & 0.7749 & 0.06011 & 0.00372 & 0.9891 \\
\hline X2964n G219 & 158.4 & 21.2 & 0.4709 & 0.06304 & 0.00218 & 1.09138 \\
\hline X2964n_G220 & 192.4 & 22.2 & 0.3277 & 0.06132 & 0.00208 & 0.95295 \\
\hline X2964n_G221 & 70.4 & 9.2 & 1.5537 & 0.06705 & 0.00444 & 0.87862 \\
\hline X2964n_G222 & 471 & 38.8 & 0.3687 & 0.06459 & 0.00178 & 0.72655 \\
\hline X2964n_G223 & 83.8 & 12.9 & 0.6082 & 0.06638 & 0.00246 & 1.27104 \\
\hline X2964n G224 & 78.5 & 11.8 & 0.5882 & 0.06529 & 0.00252 & 1.22177 \\
\hline X2964n G225 & 2262.7 & 118.3 & 0.0772 & 0.07043 & 0.00266 & 0.53811 \\
\hline X2964n_G226 & 1742.9 & 65.5 & 0.1985 & 0.09594 & 0.00338 & 0.49802 \\
\hline X2964n_G227 & 117.8 & 16.4 & 0.6311 & 0.06561 & 0.00224 & 1.12684 \\
\hline X2964n_G228 & 128.3 & 16.5 & 1.1629 & 0.06102 & 0.0024 & 0.85461 \\
\hline X2964n_G229 & 419.8 & 70.2 & 1.3134 & 0.06466 & 0.00172 & 1.12741 \\
\hline X2964n_G230 & 42.1 & 5.8 & 0.5654 & 0.06505 & 0.00316 & 1.128 \\
\hline X2964n_G231 & 185.7 & 81.4 & 1.1285 & 0.12213 & 0.00302 & 5.7383 \\
\hline X2964n_G232 & 114.4 & 12.4 & 1.034 & 0.0575 & 0.00228 & 0.70049 \\
\hline X2964n_G233 & 74.7 & 8.6 & 0.4264 & 0.05903 & 0.00252 & 0.89389 \\
\hline X2964n_G234 & 123.5 & 19.8 & 0.6352 & 0.06575 & 0.00214 & 1.29883 \\
\hline X2964n_G235 & 112 & 6 & 0.9802 & 0.05079 & 0.00274 & 0.30889 \\
\hline X2964n_G236 & 92.4 & 13.1 & 0.3821 & 0.06341 & 0.00224 & 1.19016 \\
\hline X2964n G237 & 155.1 & 16.8 & 0.7103 & 0.05944 & 0.00198 & 0.78056 \\
\hline X2964n_G238 & 203 & 27.4 & 0.3132 & 0.06665 & 0.00198 & 1.21734 \\
\hline X2964n_G239 & 339.9 & 48.6 & 0.4586 & 0.06582 & 0.00174 & 1.22092 \\
\hline X2964n_G240 & 123.5 & 17.9 & 0.5053 & 0.06444 & 0.00206 & 1.19258 \\
\hline X2964n_G241 & 164.7 & 24 & 0.4533 & 0.06526 & 0.00194 & 1.23623 \\
\hline X2964n_G242 & 546.7 & 58 & 0.3586 & 0.06098 & 0.00162 & 0.87035 \\
\hline X2964n_G243 & 55.5 & 7.3 & 0.3256 & 0.06478 & 0.00282 & 1.14343 \\
\hline X2964n_G244 & 94.3 & 12.3 & 0.127 & 0.06386 & 0.00234 & 1.19691 \\
\hline X2964n_G245 & 1696.9 & 66.5 & 0.2507 & 0.07786 & 0.00216 & 0.42762 \\
\hline X2964n_G246 & 75.6 & 11.9 & 0.791 & 0.06472 & 0.00248 & 1.21674 \\
\hline X2964n_G247 & 227.9 & 28.8 & 0.4118 & 0.06358 & 0.0019 & 1.06031 \\
\hline X2964n_G248 & 167.1 & 22.9 & 0.9762 & 0.06174 & 0.00202 & 0.95952 \\
\hline X2964n_G249 & 881.3 & 3.8 & 0.7447 & 0.12137 & 0.00638 & 0.06073 \\
\hline X2964n_G250 & 88.6 & 11 & 0.6157 & 0.06161 & 0.00242 & 0.94685 \\
\hline X2964n_G251 & 665.4 & 33.6 & 0.098 & 0.05258 & 0.00148 & 0.38827 \\
\hline X2964n_G252 & 81.9 & 11.6 & 0.5964 & 0.06511 & 0.00254 & 1.15154 \\
\hline X2964n_G253 & 20.6 & 2.6 & 0.3692 & 0.0642 & 0.00496 & 1.08963 \\
\hline X2964n_G254 & 4.3 & 0.8 & 0.2448 & 0.07678 & 0.01044 & 1.93111 \\
\hline X2964n_G255 & 98.1 & 11.9 & 0.253 & 0.06441 & 0.00248 & 1.08267 \\
\hline X2964n_G256 & 159.4 & 20 & 0.179 & 0.06503 & 0.00214 & 1.15296 \\
\hline X2964n_G257 & 290.6 & 39.6 & 0.3476 & 0.0673 & 0.0019 & 1.23576 \\
\hline X2964n_G258 & 106.3 & 15.4 & 0.5722 & 0.06906 & 0.00244 & 1.26389 \\
\hline X2964n_G259 & 1120.1 & 7.3 & 1.237 & 0.04647 & 0.0025 & 0.03209 \\
\hline X2964n_G260 & 342.3 & 40.2 & 0.917 & 0.05961 & 0.00174 & 0.80687 \\
\hline X2964n_G261 & 48.3 & 5.6 & 0.6416 & 0.06072 & 0.00326 & 0.87435 \\
\hline X2964n_G262 & 1960.2 & 64.1 & 0.4249 & 0.1677 & 0.00434 & 0.67079 \\
\hline X2964n_G263 & & & & -1.90734 & 2.44014 & \\
\hline X2964n_G264 & 131.2 & 20.2 & 0.5803 & 0.06812 & 0.00228 & 1.31514 \\
\hline X2964n_G265 & 104.8 & 15.1 & 0.3883 & 0.06678 & 0.0023 & 1.27174 \\
\hline X2964n_G266 & 79 & 13 & 0.2484 & 0.07005 & 0.00246 & 1.59119 \\
\hline X2964n_G267 & 13.9 & 1.8 & 1.2467 & 0.06103 & 0.00562 & 0.85184 \\
\hline X2964n_G268 & 59.8 & 8.6 & 0.4889 & 0.06583 & 0.00258 & 1.21161 \\
\hline X2964n_G269 & 79.9 & 11.9 & 0.5635 & 0.06582 & 0.00236 & 1.23246 \\
\hline X2964n_G270 & 51.2 & 12.1 & 1.7851 & 0.0725 & 0.00276 & 1.61346 \\
\hline
\end{tabular}




\begin{tabular}{|c|c|c|c|c|c|c|}
\hline \multicolumn{4}{|l|}{$\begin{array}{l}\text { X2964n_G271 } \\
\text { X2964n G272 }\end{array}$} & 0.98808 & 0.21196 & \\
\hline X2964n_G273 & 314 & 46.2 & 0.7403 & 0.06343 & 0.00174 & 1.12033 \\
\hline X2964n_G274 & 127.3 & 14.5 & 0.1518 & 0.06204 & 0.00208 & 1.00343 \\
\hline X2964n_G275 & 313.5 & 17.1 & 0.6362 & 0.12036 & 0.0037 & 0.80548 \\
\hline X2964n_G276 & 336.5 & 17.7 & 0.6201 & 0.11811 & 0.00346 & 0.76853 \\
\hline X2964n_G277 & 20.1 & 2.7 & 0.3529 & 0.06656 & 0.0043 & 1.2014 \\
\hline X2964n_G278 & 92.9 & 16.3 & 1.2417 & 0.06583 & 0.0023 & 1.19801 \\
\hline X2964n_G279 & 117.3 & 13.7 & 0.9431 & 0.06067 & 0.00226 & 0.81335 \\
\hline X2964n_G280 & 48.8 & 7.3 & 0.5476 & 0.06628 & 0.00288 & 1.24601 \\
\hline X2964n_G281 & & & & 0.17461 & 0.0108 & 3.48827 \\
\hline X2964n_G282 & 101 & 0.6 & 1.1734 & 0.21869 & 0.02732 & 0.14254 \\
\hline X2964n_G283 & 236.5 & 22.2 & 0.0888 & 0.06025 & 0.00188 & 0.8255 \\
\hline X2964n_G284 & 1812.3 & 116.5 & 0.139 & 0.07084 & 0.0018 & 0.65351 \\
\hline X2964n_G285 & 802.8 & 58.6 & 0.6261 & 0.08553 & 0.0026 & 0.74612 \\
\hline X2964n_G286 & 87.6 & 8.7 & 0.5711 & 0.05869 & 0.00248 & 0.73243 \\
\hline X2964n_G287 & 117.8 & 12.5 & 1.584 & 0.1177 & 0.00392 & 1.37087 \\
\hline X2964n_G288 & 91 & 11 & 0.4275 & 0.06248 & 0.00234 & 0.98836 \\
\hline X2964n_G289 & 54.6 & 7.8 & 0.6047 & 0.06658 & 0.00292 & 1.18971 \\
\hline X2964n_G290 & 69.4 & 9.7 & 0.4537 & 0.06949 & 0.00332 & 1.25933 \\
\hline X2964n_G291 & 138.8 & 17.5 & 1.3863 & 0.06136 & 0.00216 & 0.79284 \\
\hline X2964n_G292 & 207.7 & 29.4 & 0.3985 & 0.06674 & 0.00194 & 1.24416 \\
\hline X2964n_G293 & 64.6 & 9.4 & 0.5578 & 0.06628 & 0.00256 & 1.22308 \\
\hline X2964n_G294 & 57.4 & 7.5 & 1.0349 & 0.06023 & 0.00274 & 0.87854 \\
\hline X2964n_G295 & 199.6 & 32 & 0.7646 & 0.06706 & 0.00196 & 1.28884 \\
\hline X2964n_G296 & 52.7 & 6.2 & 0.2955 & 0.06339 & 0.0028 & 1.02373 \\
\hline X2964n_G297 & 178.5 & 19.5 & 0.2227 & 0.06001 & 0.00194 & 0.91497 \\
\hline X2964n_G298 & 51.2 & 6.9 & 0.2941 & 0.06508 & 0.00282 & 1.19766 \\
\hline X2964n_G299 & 66.5 & 0.4 & 1.1583 & 0.06167 & 0.01642 & 0.03501 \\
\hline X2964n_G300 & 538.5 & 53.8 & 0.2625 & 0.06148 & 0.00166 & 0.8479 \\
\hline X2964n_G301 & 95.7 & 13.5 & 0.4616 & 0.06674 & 0.00234 & 1.22175 \\
\hline X2964n_G302 & 120.2 & 16.4 & 0.5152 & 0.06316 & 0.00214 & 1.09897 \\
\hline X2964n_G303 & 61.3 & 6.6 & 0.7923 & 0.0622 & 0.00282 & 0.79881 \\
\hline X2964n_G304 & 51.7 & 5.6 & 0.7875 & 0.06003 & 0.0029 & 0.77108 \\
\hline X2964n_G305 & 232.2 & 24.3 & 0.5267 & 0.06612 & 0.002 & 0.8909 \\
\hline X2964n_G306 & 334.1 & 14.8 & 0.4352 & 0.05306 & 0.0019 & 0.30939 \\
\hline X2964n_G307 & 261.4 & 34.1 & 0.3647 & 0.06665 & 0.00194 & 1.16472 \\
\hline X2964n_G308 & 93.3 & 9.8 & 0.9361 & 0.07143 & 0.00278 & 0.90328 \\
\hline X2964n_G309 & 20.6 & 2.3 & 1.1561 & 0.06881 & 0.00498 & 0.84092 \\
\hline X2964n_G310 & 124.5 & 16.1 & 0.3269 & 0.06434 & 0.00208 & 1.12343 \\
\hline X2964n_G311 & 182.4 & 15.4 & 0.455 & 0.05885 & 0.00198 & 0.64974 \\
\hline X2964n_G312 & 189.1 & 20.4 & 0.3058 & 0.06354 & 0.002 & 0.94528 \\
\hline X2964n_G313 & 172.8 & 23.6 & 0.4471 & 0.06849 & 0.00222 & 1.22022 \\
\hline X2964n_G314 & 396.4 & 34.8 & 0.0944 & 0.06154 & 0.00184 & 0.78793 \\
\hline X2964n_G315 & 128.3 & 14.4 & 0.0673 & 0.06349 & 0.0021 & 1.04455 \\
\hline X2964n_G316 & 313.1 & 47.2 & 0.1779 & 0.07106 & 0.00196 & 1.50536 \\
\hline X2964n_G317 & 150.3 & 16.6 & 1.4128 & 0.05916 & 0.00212 & 0.67264 \\
\hline X2964n_G318 & 98.6 & 11.6 & 0.8484 & 0.06327 & 0.00264 & 0.87563 \\
\hline X2964n_G319 & 77.1 & 10.6 & 0.315 & 0.06553 & 0.00246 & 1.22091 \\
\hline X2964n_G320 & 109.1 & 25.5 & 1.3028 & 0.07476 & 0.00236 & 1.82636 \\
\hline X2964L_G001 & 294.6 & 1.7 & 0.9077 & 0.04876 & 0.0077 & 0.03259 \\
\hline X2964L_G002 & 138.6 & 60.1 & 1.6108 & 0.10922 & 0.0034 & 4.63314 \\
\hline X2964L_G003 & 16 & 1.6 & 0.0016 & 0.06652 & 0.00744 & 1.00847 \\
\hline
\end{tabular}


es $\quad 90.7 \%$ concordant

\begin{tabular}{|c|c|c|c|c|c|c|}
\hline $2 \sigma 75$ & Pb206/U238 & $2 \sigma 68$ & $\begin{array}{c}\text { ages } \\
\text { age } 206 / 238\end{array}$ & $2 \sigma$ age 68 & age $207 / 235$ & $2 \sigma$ age 75 \\
\hline 0.04136 & 0.12495 & 0.00318 & 759 & 18.2 & 841.3 & 22.8 \\
\hline 0.0475 & 0.14609 & 0.00382 & 879 & 21.4 & 869.1 & 25 \\
\hline 0.04766 & 0.11385 & 0.00328 & 695.1 & 19 & 724.9 & 28.2 \\
\hline 0.02718 & 0.10656 & 0.00262 & 652.7 & 15.2 & 661.4 & 18 \\
\hline 0.0434 & 0.10163 & 0.00298 & 624 & 17.4 & 653 & 27.4 \\
\hline 0.32812 & 0.50205 & 0.01214 & 2622.6 & 52.2 & 2678.8 & 31 \\
\hline 0.03526 & 0.1169 & 0.003 & 712.7 & 17.4 & 729.3 & 21.4 \\
\hline 0.03446 & 0.13755 & 0.00334 & 830.8 & 19 & 816.7 & 19.8 \\
\hline 0.0414 & 0.14006 & 0.00354 & 845 & 20 & 845.9 & 22.6 \\
\hline 0.02662 & 0.1145 & 0.00276 & 698.8 & 16 & 691 & 17.4 \\
\hline 0.025 & 0.09506 & 0.00238 & 585.4 & 14 & 587 & 17.6 \\
\hline 0.03462 & 0.1248 & 0.0031 & 758.1 & 17.8 & 775.2 & 20.6 \\
\hline 0.05312 & 0.15614 & 0.00398 & 935.3 & 22.2 & 997.4 & 25.2 \\
\hline 0.02558 & 0.00621 & 0.00052 & 39.9 & 3.4 & 222.3 & 31 \\
\hline 0.0258 & 0.10937 & 0.0026 & 669.1 & 15.2 & 707.9 & 16.8 \\
\hline 0.07416 & 0.14191 & 0.0046 & 855.5 & 26 & 844.6 & 38.2 \\
\hline 0.0264 & 0.1015 & 0.00252 & 623.2 & 14.8 & 630.6 & 18 \\
\hline 0.02864 & 0.11606 & 0.0028 & 707.8 & 16.2 & 735.7 & 18 \\
\hline 0.0112 & 0.03892 & 0.00102 & 246.1 & 6.4 & 246.6 & 10.6 \\
\hline 0.04566 & 0.12914 & 0.0035 & 783 & 20 & 776.9 & 26 \\
\hline 0.03364 & 0.10433 & 0.00278 & 639.7 & 16.2 & 625.5 & 22 \\
\hline 0.02544 & 0.09954 & 0.00248 & 611.7 & 14.6 & 598.6 & 17.6 \\
\hline 0.05798 & 0.18528 & 0.00464 & 1095.8 & 25.2 & 1096.5 & 25.2 \\
\hline 0.02454 & 0.08987 & 0.00226 & 554.8 & 13.4 & 574.7 & 17.4 \\
\hline 0.15412 & 0.3467 & 0.00836 & 1918.8 & 40 & 1980.6 & 28.8 \\
\hline 0.03546 & 0.1071 & 0.00284 & 655.9 & 16.6 & 668.8 & 22.4 \\
\hline 0.02884 & 0.08408 & 0.00228 & 520.4 & 13.6 & 532.6 & 20.6 \\
\hline 0.0213 & 0.09013 & 0.0022 & 556.3 & 13 & 555.6 & 15.6 \\
\hline 0.0478 & 0.13766 & 0.0033 & 831.4 & 18.6 & 1068.3 & 22 \\
\hline 0.03212 & 0.12438 & 0.00304 & 755.7 & 17.4 & 763.5 & 19.4 \\
\hline 0.04444 & 0.13224 & 0.0034 & 800.6 & 19.4 & 866.7 & 23.8 \\
\hline 0.03372 & 0.13307 & 0.00322 & 805.4 & 18.4 & 812.3 & 19.6 \\
\hline 0.05398 & 0.13951 & 0.00386 & 841.9 & 21.8 & 854.4 & 28.4 \\
\hline 0.02562 & 0.10701 & 0.0026 & 655.4 & 15.2 & 659.8 & 17.2 \\
\hline 0.02798 & 0.10859 & 0.00268 & 664.6 & 15.6 & 672.5 & 18.4 \\
\hline 0.04036 & 0.13909 & 0.00348 & 839.5 & 19.6 & 850.9 & 22 \\
\hline 0.04116 & 0.12791 & 0.00322 & 775.9 & 18.4 & 857.3 & 22.4 \\
\hline 0.03342 & 0.13459 & 0.00326 & 814 & 18.6 & 811.6 & 19.4 \\
\hline 0.04604 & 0.11989 & 0.00334 & 729.9 & 19.2 & 749.8 & 26.8 \\
\hline 0.12134 & 0.30491 & 0.00736 & 1715.6 & 36.4 & 1766.7 & 28 \\
\hline 0.03672 & 0.09935 & 0.00278 & 610.6 & 16.4 & 604.7 & 24.2 \\
\hline 0.03246 & 0.09647 & 0.0025 & 593.7 & 14.6 & 674.1 & 21 \\
\hline 0.0496 & 0.11907 & 0.00342 & 725.2 & 19.8 & 749.9 & 28.6 \\
\hline 0.05368 & 0.17424 & 0.00418 & 1035.4 & 23 & 1134.3 & 23 \\
\hline 0.03282 & 0.10954 & 0.00282 & 670.1 & 16.4 & 671.9 & 20.8 \\
\hline 0.03416 & 0.12107 & 0.00302 & 736.7 & 17.4 & 760.1 & 20.4 \\
\hline 0.05082 & 0.13003 & 0.00364 & 788 & 20.8 & 807.5 & 28 \\
\hline 0.04278 & 0.12884 & 0.00338 & 781.2 & 19.4 & 794.8 & 24.2 \\
\hline 0.0459 & 0.13554 & 0.00364 & 819.4 & 20.6 & 794.1 & 25.6 \\
\hline 0.03266 & 0.13416 & 0.00322 & 811.6 & 18.4 & 820.3 & 19 \\
\hline
\end{tabular}




\begin{tabular}{|c|c|c|c|c|c|c|}
\hline 0.04402 & 0.1344 & 0.00352 & 812.9 & 20 & 818.7 & 24.4 \\
\hline 0.0441 & 0.13136 & 0.00352 & 795.6 & 20 & 775.5 & 25.2 \\
\hline 0.02794 & 0.11753 & 0.00284 & 716.3 & 16.4 & 716.8 & 17.8 \\
\hline 0.0247 & 0.09291 & 0.00234 & 572.7 & 13.8 & 566.3 & 17.6 \\
\hline 0.00344 & 0.00377 & 0.00018 & 24.3 & 1.2 & 30 & 3.8 \\
\hline 0.0478 & 0.13524 & 0.00364 & 817.7 & 20.6 & 815.9 & 26.2 \\
\hline 0.02538 & 0.09685 & 0.00242 & 595.9 & 14.2 & 596.4 & 17.8 \\
\hline 0.02832 & 0.11256 & 0.00276 & 687.6 & 16 & 686.1 & 18.4 \\
\hline 0.05404 & 0.13749 & 0.00388 & 830.5 & 22 & 822.1 & 29 \\
\hline 0.03202 & 0.12804 & 0.0031 & 776.7 & 17.8 & 775.7 & 19.2 \\
\hline 0.06106 & 0.12972 & 0.00398 & 786.3 & 22.8 & 799.5 & 33.2 \\
\hline 0.03532 & 0.13044 & 0.00322 & 790.4 & 18.4 & 795.2 & 20.6 \\
\hline 0.01886 & 0.07893 & 0.00194 & 489.7 & 11.6 & 498.5 & 14.6 \\
\hline 0.03474 & 0.13546 & 0.00326 & 818.9 & 18.6 & 840.2 & 19.6 \\
\hline 0.2721 & 0.04854 & 0.0079 & 305.6 & 48.6 & 720.8 & 176.4 \\
\hline 0.01004 & 0.03899 & 0.00098 & 246.6 & 6 & 262.2 & 9.6 \\
\hline 0.0512 & 0.17535 & 0.00432 & 1041.5 & 23.6 & 1036.6 & 23.6 \\
\hline 0.04324 & 0.13305 & 0.00344 & 805.2 & 19.6 & 835.9 & 23.8 \\
\hline 0.03434 & 0.1023 & 0.00276 & 627.9 & 16.2 & 623.8 & 22.6 \\
\hline 0.05308 & 0.13928 & 0.00384 & 840.6 & 21.8 & 849.8 & 28 \\
\hline 0.03546 & 0.11255 & 0.00298 & 687.5 & 17.2 & 666.9 & 22.4 \\
\hline 0.05944 & 0.12991 & 0.0039 & 787.4 & 22.2 & 805.6 & 32.2 \\
\hline 0.03086 & 0.12234 & 0.00298 & 744 & 17.2 & 751.3 & 19 \\
\hline 0.00922 & 0.0042 & 0.0003 & 27 & 2 & 25.8 & 9.4 \\
\hline 0.05164 & 0.13324 & 0.00368 & 806.3 & 21 & 825.2 & 28 \\
\hline 0.07612 & 0.13006 & 0.00428 & 788.2 & 24.4 & 892.5 & 38.4 \\
\hline 0.02322 & 0.10776 & 0.00256 & 659.7 & 14.8 & 656.3 & 16 \\
\hline 0.05388 & 0.13596 & 0.00384 & 821.8 & 21.8 & 818.1 & 29.2 \\
\hline 0.05286 & 0.12881 & 0.00372 & 781.1 & 21.2 & 767.8 & 29.8 \\
\hline 0.03424 & 0.13716 & 0.0033 & 828.6 & 18.8 & 825.2 & 19.6 \\
\hline 0.0431 & 0.13771 & 0.00356 & 831.7 & 20.2 & 821.3 & 23.8 \\
\hline 0.02008 & 0.05274 & 0.00126 & 331.3 & 7.8 & 574 & 14.8 \\
\hline 2.21862 & 0.2747 & 0.03246 & & & & \\
\hline 0.03354 & 0.13897 & 0.00334 & 838.8 & 19 & 827.3 & 19.2 \\
\hline 0.02166 & 0.08259 & 0.00198 & 511.6 & 11.8 & 600.2 & 15.6 \\
\hline 0.08284 & 0.12592 & 0.00454 & 764.5 & 26 & 815.3 & 43.8 \\
\hline 0.03486 & 0.08394 & 0.00246 & 519.6 & 14.6 & 528.6 & 24.6 \\
\hline 0.04132 & 0.11443 & 0.0031 & 698.4 & 18 & 720.3 & 24.8 \\
\hline 0.04064 & 0.15563 & 0.00374 & 932.4 & 20.8 & 940.9 & 21 \\
\hline 0.04524 & 0.14517 & 0.00372 & 873.8 & 21 & 874 & 24 \\
\hline 0.05286 & 0.13534 & 0.00384 & 818.3 & 21.8 & 790.4 & 29.2 \\
\hline 0.1327 & 0.2862 & 0.0068 & 1622.5 & 34 & 1867.4 & 27.8 \\
\hline 0.03804 & 0.14014 & 0.00348 & 845.5 & 19.6 & 821.9 & 21.4 \\
\hline 0.05482 & 0.12897 & 0.00382 & 782 & 21.8 & 760.5 & 31 \\
\hline 0.03388 & 0.10265 & 0.00274 & 629.9 & 16 & 629 & 22.2 \\
\hline 0.03988 & 0.13784 & 0.00346 & 832.4 & 19.6 & 823.7 & 22.2 \\
\hline 0.0425 & 0.12431 & 0.00332 & 755.3 & 19 & 762 & 24.6 \\
\hline 0.04068 & 0.09669 & 0.00288 & 595 & 17 & 563.4 & 27.4 \\
\hline 0.04696 & 0.13365 & 0.00358 & 808.7 & 20.4 & 816.2 & 25.8 \\
\hline 0.06076 & 0.15933 & 0.00436 & 953.1 & 24.2 & 946.9 & 29.4 \\
\hline 0.04014 & 0.13413 & 0.0034 & 811.4 & 19.4 & 812.1 & 22.6 \\
\hline 0.03816 & 0.14359 & 0.00352 & 864.9 & 19.8 & 855.6 & 21 \\
\hline 0.02804 & 0.1023 & 0.00258 & 627.9 & 15 & 628.6 & 18.8 \\
\hline 0.03006 & 0.10126 & 0.0026 & 621.8 & 15.2 & 624.9 & 20 \\
\hline 0.03056 & 0.1201 & 0.00294 & 731.1 & 17 & 724.2 & 19 \\
\hline
\end{tabular}




\begin{tabular}{|c|c|c|c|c|c|c|}
\hline 0.02042 & 0.08777 & 0.00214 & 542.3 & 12.6 & 534.5 & 15.4 \\
\hline 0.045 & 0.10338 & 0.00304 & 634.2 & 17.8 & 661.4 & 28 \\
\hline 0.03986 & 0.13641 & 0.00346 & 824.3 & 19.6 & 809.6 & 22.6 \\
\hline 0.04358 & 0.13545 & 0.00352 & 818.9 & 20 & 821.9 & 24 \\
\hline 0.03416 & 0.08993 & 0.00252 & 555.1 & 15 & 581.9 & 23.4 \\
\hline 0.04372 & 0.12595 & 0.00334 & 764.7 & 19.2 & 792.7 & 24.8 \\
\hline 0.02434 & 0.07601 & 0.00202 & 472.3 & 12.2 & 476.9 & 18.4 \\
\hline 0.05056 & 0.13118 & 0.00366 & 794.6 & 20.8 & 802.6 & 27.8 \\
\hline 0.03228 & 0.09915 & 0.00256 & 609.4 & 15 & 665.9 & 20.8 \\
\hline 0.02306 & 0.0779 & 0.00194 & 483.6 & 11.6 & 560.5 & 16.8 \\
\hline 0.00916 & 0.02225 & 0.00066 & 141.9 & 4.2 & 142.8 & 9.2 \\
\hline 0.04416 & 0.15577 & 0.0038 & 933.2 & 21.2 & 957.1 & 22.2 \\
\hline 0.0333 & 0.12373 & 0.00306 & 752 & 17.6 & 752.5 & 20 \\
\hline 0.0606 & 0.13844 & 0.00412 & 835.8 & 23.4 & 813 & 32.4 \\
\hline 0.06172 & 0.13885 & 0.00414 & 838.2 & 23.4 & 819.5 & 32.8 \\
\hline 0.02574 & 0.10418 & 0.00254 & 638.9 & 14.8 & 639.4 & 17.4 \\
\hline 0.04378 & 0.12571 & 0.00338 & 763.3 & 19.4 & 764 & 25.2 \\
\hline 0.0546 & 0.14116 & 0.00394 & 851.2 & 22.2 & 842 & 29 \\
\hline 0.04198 & 0.14244 & 0.00358 & 858.4 & 20.2 & 859.2 & 22.8 \\
\hline 0.03706 & 0.13503 & 0.00332 & 816.5 & 18.8 & 821.6 & 21 \\
\hline 0.03974 & 0.11525 & 0.00312 & 703.2 & 18 & 688.3 & 24.4 \\
\hline 0.02532 & 0.11283 & 0.00268 & 689.2 & 15.6 & 684.1 & 16.8 \\
\hline 0.04358 & 0.09766 & 0.00288 & 600.7 & 17 & 646.8 & 27.6 \\
\hline 0.02846 & 0.09181 & 0.00242 & 566.2 & 14.2 & 553.7 & 20 \\
\hline 0.03882 & 0.14089 & 0.00348 & 849.7 & 19.6 & 839 & 21.6 \\
\hline 0.01084 & 0.04609 & 0.00108 & 290.5 & 6.6 & 358.5 & 10 \\
\hline 0.06928 & 0.10133 & 0.0038 & 622.2 & 22.2 & 624.1 & 43 \\
\hline 0.0444 & 0.13228 & 0.0035 & 800.9 & 20 & 797.2 & 25 \\
\hline 0.04996 & 0.16042 & 0.00404 & 959.1 & 22.4 & 959.7 & 24.4 \\
\hline 0.05034 & 0.16431 & 0.00414 & 980.7 & 23 & 965.6 & 24.6 \\
\hline 0.04268 & 0.13487 & 0.0035 & 815.6 & 19.8 & 802.1 & 24 \\
\hline 0.03306 & 0.10149 & 0.00272 & 623.1 & 16 & 605.7 & 22 \\
\hline 0.05414 & 0.14692 & 0.00398 & 883.7 & 22.4 & 882.7 & 27.8 \\
\hline 0.05842 & 0.12719 & 0.00384 & 771.8 & 22 & 774.9 & 32.4 \\
\hline 0.03368 & 0.10315 & 0.00276 & 632.8 & 16.2 & 613.8 & 22.4 \\
\hline 0.05626 & 0.127 & 0.00378 & 770.7 & 21.6 & 779.9 & 31.4 \\
\hline 0.0433 & 0.13755 & 0.00354 & 830.8 & 20 & 824.5 & 24 \\
\hline 0.02474 & 0.10264 & 0.00244 & 629.9 & 14.2 & 679 & 16.6 \\
\hline 0.0073 & 0.00396 & 0.0003 & 25.5 & 2 & 29 & 7.8 \\
\hline 0.07422 & 0.14349 & 0.0046 & 864.4 & 26 & 854.7 & 37.8 \\
\hline 0.04332 & 0.13921 & 0.00354 & 840.2 & 20 & 848.4 & 23.6 \\
\hline 0.02428 & 0.08567 & 0.00204 & 529.9 & 12.2 & 655.9 & 16.4 \\
\hline 0.043 & 0.13635 & 0.00352 & 824 & 20 & 813.7 & 23.8 \\
\hline 0.03974 & 0.13801 & 0.00344 & 833.4 & 19.4 & 830.1 & 22.2 \\
\hline 0.01074 & 0.04913 & 0.00116 & 309.2 & 7.2 & 354.4 & 9.8 \\
\hline 0.04876 & 0.13949 & 0.00374 & 841.8 & 21.2 & 823.6 & 26.4 \\
\hline 0.0504 & 0.17414 & 0.00428 & 1034.9 & 23.4 & 1011.5 & 23.8 \\
\hline 0.01182 & 0.05422 & 0.00132 & 340.4 & 8 & 338.7 & 10.8 \\
\hline 0.01182 & 0.05382 & 0.0013 & 337.9 & 8 & 337.7 & 10.6 \\
\hline 0.06364 & 0.16228 & 0.00444 & 969.4 & 24.6 & 978.1 & 29.8 \\
\hline 0.00174 & 0.00383 & 0.00012 & 24.6 & 0.8 & 24.5 & 2 \\
\hline 0.04434 & 0.1529 & 0.0038 & 917.2 & 21.2 & 911.7 & 23 \\
\hline 0.02748 & 0.10169 & 0.00254 & 624.3 & 14.8 & 617.6 & 18.6 \\
\hline 0.01646 & 0.06045 & 0.00156 & 378.4 & 9.4 & 372.3 & 14 \\
\hline 0.03006 & 0.09746 & 0.00256 & 599.5 & 15 & 587.4 & 20.6 \\
\hline
\end{tabular}




\begin{tabular}{|c|c|c|c|c|c|c|}
\hline 0.02346 & 0.09929 & 0.00238 & 610.2 & 14 & 617.7 & 16.4 \\
\hline 0.03298 & 0.12663 & 0.0031 & 768.6 & 17.8 & 762.3 & 19.8 \\
\hline 0.03742 & 0.1085 & 0.00292 & 664 & 17 & 668.6 & 23.6 \\
\hline 0.0335 & 0.12009 & 0.00292 & 731.1 & 16.8 & 780.2 & 19.8 \\
\hline 0.0359 & 0.1401 & 0.00336 & 845.2 & 19 & 859.4 & 20 \\
\hline 0.03378 & 0.13726 & 0.00326 & 829.1 & 18.4 & 839.6 & 19.2 \\
\hline 0.04332 & 0.10015 & 0.00296 & 615.3 & 17.4 & 619.7 & 27.8 \\
\hline 0.0157 & 0.00491 & 0.00052 & 31.6 & 3.4 & 37.2 & 16.2 \\
\hline 0.05608 & 0.17364 & 0.00438 & 1032.1 & 24 & 1034.2 & 25.6 \\
\hline 0.03448 & 0.13758 & 0.0033 & 831 & 18.8 & 827.8 & 19.8 \\
\hline 0.0527 & 0.11387 & 0.0036 & 695.2 & 20.8 & 627.5 & 33 \\
\hline 0.02708 & 0.09124 & 0.00236 & 562.9 & 14 & 552.8 & 19.2 \\
\hline 0.05242 & 0.13462 & 0.0038 & 814.2 & 21.6 & 790.9 & 29 \\
\hline 0.03744 & 0.12623 & 0.00322 & 766.3 & 18.4 & 747.5 & 22.2 \\
\hline 0.05122 & 0.17136 & 0.00424 & 1019.6 & 23.4 & 1015.6 & 24 \\
\hline 0.04742 & 0.17481 & 0.0042 & 1038.6 & 23 & 1027.3 & 22.4 \\
\hline 0.0414 & 0.13816 & 0.00348 & 834.2 & 19.8 & 836.4 & 22.8 \\
\hline 0.06028 & 0.14426 & 0.00394 & 868.7 & 22.2 & 964.1 & 29 \\
\hline 0.04806 & 0.16773 & 0.00408 & 999.6 & 22.6 & 1002.6 & 23 \\
\hline 0.05566 & 0.13222 & 0.00376 & 800.5 & 21.4 & 830.8 & 29.8 \\
\hline 0.05272 & 0.12803 & 0.00368 & 776.6 & 21 & 765.6 & 29.6 \\
\hline 0.04332 & 0.13316 & 0.00348 & 805.9 & 19.8 & 798.6 & 24.4 \\
\hline 0.06726 & 0.13546 & 0.00428 & 818.9 & 24.2 & 806.9 & 36 \\
\hline 0.02606 & 0.08004 & 0.00214 & 496.4 & 12.8 & 503.1 & 19.2 \\
\hline 0.03736 & 0.14329 & 0.0034 & 863.2 & 19.2 & 898.5 & 20 \\
\hline 0.0177 & 0.07133 & 0.0017 & 444.2 & 10.2 & 517.7 & 13.8 \\
\hline 0.02728 & 0.09357 & 0.0024 & 576.6 & 14.2 & 577.4 & 19 \\
\hline 0.04006 & 0.00455 & 0.00084 & 29.3 & 5.4 & 33.5 & 40.2 \\
\hline 0.03324 & 0.13011 & 0.00316 & 788.5 & 18 & 778.1 & 19.8 \\
\hline 0.0067 & 0.02841 & 0.0007 & 180.6 & 4.4 & 183.2 & 6.8 \\
\hline 0.02276 & 0.0843 & 0.00214 & 521.7 & 12.8 & 508.7 & 17 \\
\hline 0.02734 & 0.10401 & 0.00258 & 637.9 & 15 & 624.9 & 18.6 \\
\hline 0.02182 & 0.09606 & 0.00228 & 591.3 & 13.4 & 604.2 & 15.6 \\
\hline 0.05638 & 0.15518 & 0.00414 & 929.9 & 23.2 & 938.9 & 27.8 \\
\hline 0.05434 & 0.14331 & 0.00394 & 863.4 & 22.2 & 855.9 & 28.6 \\
\hline 0.08404 & 0.12074 & 0.00448 & 734.8 & 25.8 & 770.3 & 46 \\
\hline 0.04126 & 0.13264 & 0.00334 & 802.9 & 19 & 837.1 & 22.8 \\
\hline 0.0551 & 0.19157 & 0.00462 & 1129.9 & 25 & 1108.7 & 23.8 \\
\hline 0.03118 & 0.09547 & 0.00254 & 587.8 & 15 & 591.2 & 21.2 \\
\hline 0.04106 & 0.13786 & 0.00348 & 832.5 & 19.8 & 823.5 & 22.8 \\
\hline 0.04106 & 0.12572 & 0.00332 & 763.4 & 19 & 738.8 & 24.2 \\
\hline 0.06016 & 0.16389 & 0.0044 & 978.4 & 24.4 & 959.5 & 28.6 \\
\hline 0.03024 & 0.10226 & 0.0026 & 627.6 & 15.2 & 645.2 & 20 \\
\hline 0.02628 & 0.08996 & 0.00228 & 555.3 & 13.4 & 572.1 & 18.4 \\
\hline 0.04328 & 0.10655 & 0.00308 & 652.7 & 18 & 654.3 & 27.2 \\
\hline 0.07786 & 0.13776 & 0.00436 & 832 & 24.8 & 948.3 & 37.4 \\
\hline 0.03924 & 0.08064 & 0.00258 & 499.9 & 15.4 & 497.5 & 28 \\
\hline 0.0404 & 0.12277 & 0.00322 & 746.5 & 18.4 & 741.6 & 24 \\
\hline 0.0522 & 0.02876 & 0.00214 & 182.8 & 13.4 & 273.5 & 47 \\
\hline 0.0401 & 0.13591 & 0.00342 & 821.5 & 19.4 & 814 & 22.6 \\
\hline 0.03022 & 0.1281 & 0.00304 & 777 & 17.4 & 769.1 & 18.4 \\
\hline 0.0258 & 0.09247 & 0.00226 & 570.1 & 13.4 & 633.6 & 17.6 \\
\hline 0.04992 & 0.13309 & 0.00364 & 805.5 & 20.8 & 808.4 & 27.4 \\
\hline 0.0308 & 0.09507 & 0.00252 & 585.5 & 14.8 & 577.1 & 21.2 \\
\hline 0.04396 & 0.12478 & 0.00336 & 758 & 19.2 & 752.8 & 25.6 \\
\hline
\end{tabular}




\begin{tabular}{|c|c|c|c|c|c|c|}
\hline 0.0123 & 0.00375 & 0.00038 & 24.1 & 2.4 & 25.5 & 12.6 \\
\hline 0.02998 & 0.10815 & 0.00268 & 662 & 15.6 & 670.9 & 19.4 \\
\hline 0.05976 & 0.11939 & 0.00388 & 727.1 & 22.4 & 698.3 & 35.4 \\
\hline 0.03862 & 0.1256 & 0.00324 & 762.7 & 18.6 & 749.2 & 22.8 \\
\hline 0.03322 & 0.11276 & 0.00288 & 688.8 & 16.6 & 679.6 & 21 \\
\hline 0.0563 & 0.09507 & 0.00326 & 585.5 & 19.2 & 640.2 & 35.4 \\
\hline 0.02122 & 0.08161 & 0.00198 & 505.7 & 11.8 & 554.5 & 15.6 \\
\hline 0.04764 & 0.13892 & 0.00368 & 838.6 & 20.8 & 832.9 & 25.8 \\
\hline 0.04772 & 0.13577 & 0.00364 & 820.7 & 20.6 & 810.6 & 26.2 \\
\hline 0.02038 & 0.05544 & 0.00146 & 347.8 & 9 & 437.2 & 16.4 \\
\hline 0.0175 & 0.03766 & 0.001 & 238.3 & 6.2 & 410.4 & 14.8 \\
\hline 0.03954 & 0.1246 & 0.0032 & 757 & 18.4 & 766.2 & 23 \\
\hline 0.03388 & 0.10161 & 0.0027 & 623.8 & 15.8 & 627.2 & 22.2 \\
\hline 0.03196 & 0.12651 & 0.00304 & 767.9 & 17.4 & 766.5 & 19.2 \\
\hline 0.054 & 0.1258 & 0.00368 & 763.9 & 21 & 766.8 & 30.6 \\
\hline 0.15492 & 0.34091 & 0.00824 & 1891 & 39.6 & 1937.2 & 30 \\
\hline 0.02798 & 0.08838 & 0.00234 & 545.9 & 13.8 & 539.1 & 20 \\
\hline 0.03812 & 0.10987 & 0.003 & 672 & 17.4 & 648.5 & 24.2 \\
\hline 0.04356 & 0.14332 & 0.00364 & 863.4 & 20.6 & 845.2 & 23.6 \\
\hline 0.01638 & 0.04412 & 0.00126 & 278.3 & 7.8 & 273.3 & 14.6 \\
\hline 0.04308 & 0.13618 & 0.00354 & 823 & 20 & 796 & 24.2 \\
\hline 0.02664 & 0.09527 & 0.0024 & 586.6 & 14.2 & 585.8 & 18.6 \\
\hline 0.03796 & 0.13252 & 0.00328 & 802.2 & 18.6 & 808.6 & 21.6 \\
\hline 0.03452 & 0.13459 & 0.00324 & 814 & 18.4 & 810.2 & 20 \\
\hline 0.03938 & 0.13427 & 0.00338 & 812.2 & 19.2 & 797.2 & 22.4 \\
\hline 0.03858 & 0.13744 & 0.0034 & 830.2 & 19.2 & 817.2 & 21.8 \\
\hline 0.0247 & 0.10355 & 0.00248 & 635.2 & 14.4 & 635.8 & 17 \\
\hline 0.0495 & 0.12806 & 0.00356 & 776.8 & 20.4 & 774.1 & 28 \\
\hline 0.04454 & 0.136 & 0.00356 & 822 & 20.2 & 799.2 & 25 \\
\hline 0.01254 & 0.03985 & 0.00096 & 251.9 & 6 & 361.5 & 11.2 \\
\hline 0.04722 & 0.1364 & 0.00364 & 824.3 & 20.6 & 808.3 & 26 \\
\hline 0.03316 & 0.121 & 0.00298 & 736.3 & 17.2 & 734 & 20.2 \\
\hline 0.03234 & 0.11276 & 0.00284 & 688.8 & 16.4 & 683 & 20.6 \\
\hline 0.00296 & 0.00363 & 0.00012 & 23.4 & 0.8 & 59.9 & 3.6 \\
\hline 0.03734 & 0.11151 & 0.00296 & 681.5 & 17.2 & 676.5 & 23.4 \\
\hline 0.0116 & 0.05357 & 0.0013 & 336.4 & 8 & 333.1 & 10.6 \\
\hline 0.0452 & 0.12832 & 0.00344 & 778.3 & 19.6 & 778 & 25.8 \\
\hline 0.08164 & 0.12315 & 0.00456 & 748.7 & 26.2 & 748.3 & 45.4 \\
\hline 0.2525 & 0.18248 & 0.01142 & 1080.5 & 62.2 & 1091.9 & 100.2 \\
\hline 0.04196 & 0.12197 & 0.00324 & 741.9 & 18.6 & 744.9 & 24.6 \\
\hline 0.03902 & 0.12865 & 0.00326 & 780.2 & 18.6 & 778.6 & 22.6 \\
\hline 0.03678 & 0.13322 & 0.00324 & 806.2 & 18.4 & 817 & 21 \\
\hline 0.04532 & 0.13278 & 0.00346 & 803.7 & 19.6 & 829.7 & 24.8 \\
\hline 0.0017 & 0.00501 & 0.00014 & 32.2 & 0.8 & 32.1 & 2 \\
\hline 0.02474 & 0.09821 & 0.0024 & 603.9 & 14 & 600.7 & 17.2 \\
\hline 0.04602 & 0.10448 & 0.00314 & 640.6 & 18.4 & 637.9 & 29.2 \\
\hline 0.01842 & 0.02902 & 0.0007 & 184.4 & 4.4 & 521.2 & 14.4 \\
\hline 1207.4945 & 2.5389 & 3.9038 & & & & \\
\hline 0.04502 & 0.14007 & 0.00358 & 845.1 & 20.2 & 852.4 & 24.2 \\
\hline 0.04494 & 0.13817 & 0.00356 & 834.3 & 20.2 & 833.2 & 24.4 \\
\hline 0.05696 & 0.16481 & 0.0043 & 983.5 & 23.8 & 966.8 & 27.2 \\
\hline 0.07572 & 0.10127 & 0.00408 & 621.8 & 23.8 & 625.7 & 47 \\
\hline 0.04776 & 0.13354 & 0.00358 & 808 & 20.4 & 805.9 & 26.4 \\
\hline 0.04486 & 0.13586 & 0.00354 & 821.2 & 20 & 815.5 & 24.8 \\
\hline 0.06204 & 0.16147 & 0.00434 & 964.9 & 24 & 975.5 & 29.2 \\
\hline
\end{tabular}




\begin{tabular}{|c|c|c|c|c|c|c|}
\hline 1320.0393 & 11.74949 & 9.82606 & & & & \\
\hline 0.03256 & 0.12815 & 0.0031 & 777.3 & 17.8 & 763.1 & 19.6 \\
\hline 0.03442 & 0.11735 & 0.00298 & 715.3 & 17.2 & 705.5 & 21.4 \\
\hline 0.02518 & 0.04856 & 0.00124 & 305.7 & 7.6 & 599.9 & 18.2 \\
\hline 0.02316 & 0.04721 & 0.00118 & 297.4 & 7.2 & 578.9 & 17 \\
\hline 0.0756 & 0.13096 & 0.00444 & 793.3 & 25.4 & 801.2 & 40.4 \\
\hline 0.04284 & 0.13204 & 0.00342 & 799.5 & 19.4 & 799.7 & 24 \\
\hline 0.03062 & 0.09727 & 0.00252 & 598.4 & 14.8 & 604.3 & 20.6 \\
\hline 0.05388 & 0.13641 & 0.00382 & 824.3 & 21.6 & 821.6 & 29.2 \\
\hline 0.19986 & 0.14495 & 0.00614 & & & & \\
\hline 0.01484 & 0.00473 & 0.00036 & 30.4 & 2.4 & 135.3 & 18.6 \\
\hline 0.0266 & 0.09941 & 0.00246 & 611 & 14.4 & 611.1 & 18.2 \\
\hline 0.0179 & 0.06694 & 0.0016 & 417.7 & 9.6 & 510.6 & 14 \\
\hline 0.02336 & 0.06329 & 0.00158 & 395.6 & 9.6 & 566 & 17 \\
\hline 0.03086 & 0.09055 & 0.00244 & 558.8 & 14.4 & 558 & 21.6 \\
\hline 0.04586 & 0.08451 & 0.00222 & 523 & 13.2 & 876.5 & 25 \\
\hline 0.03728 & 0.11478 & 0.003 & 700.4 & 17.4 & 697.9 & 23 \\
\hline 0.05178 & 0.12965 & 0.00362 & 785.9 & 20.6 & 795.8 & 28.6 \\
\hline 0.0593 & 0.13149 & 0.00386 & 796.4 & 22 & 827.6 & 31.8 \\
\hline 0.02836 & 0.09375 & 0.0024 & 577.7 & 14.2 & 592.8 & 19.6 \\
\hline 0.038 & 0.13526 & 0.00332 & 817.8 & 18.8 & 820.8 & 21.4 \\
\hline 0.04764 & 0.13389 & 0.00358 & 810 & 20.4 & 811.2 & 26.2 \\
\hline 0.03962 & 0.10583 & 0.00294 & 648.5 & 17.2 & 640.2 & 25.4 \\
\hline 0.03956 & 0.13945 & 0.00342 & 841.6 & 19.4 & 840.8 & 21.8 \\
\hline 0.04508 & 0.11718 & 0.00326 & 714.3 & 18.8 & 715.8 & 26.8 \\
\hline 0.03054 & 0.11063 & 0.00276 & 676.4 & 16 & 659.7 & 19.8 \\
\hline 0.05168 & 0.13353 & 0.0037 & 808 & 21 & 799.5 & 28.4 \\
\hline 0.009 & 0.00412 & 0.0003 & 26.5 & 2 & 34.9 & 9.4 \\
\hline 0.02432 & 0.10007 & 0.0024 & 614.8 & 14 & 623.5 & 16.8 \\
\hline 0.0437 & 0.13282 & 0.00342 & 803.9 & 19.4 & 810.6 & 24.2 \\
\hline 0.03814 & 0.12626 & 0.0032 & 766.5 & 18.4 & 752.9 & 22.6 \\
\hline 0.03596 & 0.09319 & 0.0026 & 574.4 & 15.4 & 596.2 & 24 \\
\hline 0.03686 & 0.0932 & 0.00266 & 574.4 & 15.6 & 580.4 & 24.8 \\
\hline 0.02796 & 0.09777 & 0.0024 & 601.3 & 14 & 646.9 & 18.6 \\
\hline 0.0112 & 0.04231 & 0.00106 & 267.1 & 6.6 & 273.7 & 10.4 \\
\hline 0.0356 & 0.1268 & 0.0031 & 769.6 & 17.8 & 784.2 & 20.8 \\
\hline 0.03524 & 0.09175 & 0.00246 & 565.9 & 14.6 & 653.5 & 22.8 \\
\hline 0.05846 & 0.08867 & 0.00318 & 547.7 & 18.8 & 619.7 & 37.4 \\
\hline 0.03754 & 0.12669 & 0.00318 & 769 & 18.2 & 764.6 & 22 \\
\hline 0.02238 & 0.0801 & 0.00202 & 496.7 & 12 & 508.3 & 16.8 \\
\hline 0.03062 & 0.10795 & 0.00268 & 660.8 & 15.6 & 675.6 & 19.8 \\
\hline 0.0406 & 0.12928 & 0.00326 & 783.8 & 18.6 & 809.9 & 23 \\
\hline 0.02454 & 0.09291 & 0.00228 & 572.7 & 13.4 & 590 & 17.4 \\
\hline 0.03556 & 0.11938 & 0.00302 & 727 & 17.4 & 726.2 & 21.6 \\
\hline 0.04374 & 0.15371 & 0.00372 & 921.7 & 20.8 & 932.6 & 22.4 \\
\hline 0.02452 & 0.0825 & 0.0021 & 511 & 12.6 & 522.3 & 18 \\
\hline 0.03632 & 0.10042 & 0.00272 & 616.9 & 16 & 638.6 & 23.6 \\
\hline 0.0463 & 0.13518 & 0.00356 & 817.3 & 20.2 & 810.2 & 25.6 \\
\hline 0.0595 & 0.17726 & 0.00446 & 1052 & 24.4 & 1055 & 26.4 \\
\hline 0.005 & 0.00485 & 0.0002 & 31.2 & 1.2 & 32.6 & 5.2 \\
\hline 0.1421 & 0.30775 & 0.00706 & 1729.6 & 34.8 & 1755.3 & 32.2 \\
\hline 0.10908 & 0.10998 & 0.00474 & 672.6 & 27.6 & 708.1 & 61.2 \\
\hline
\end{tabular}




\begin{tabular}{|c|c|c|c|c|c|}
\hline age $207 / 206$ & $2 \sigma$ age 76 & $\begin{array}{l}\text { discordance } \\
\Delta 68-75[\%]\end{array}$ & $\Delta 68-76[\%]$ & $\begin{array}{c}\text { preferred age } \\
\text { age }\end{array}$ & $2 \sigma$ age \\
\hline 1066.1 & 61.8 & -9.8 & -28.8 & 759 & 18.2 \\
\hline 844.6 & 70.6 & 1.1 & 4.1 & 879 & 21.4 \\
\hline 819 & 96.2 & -4.1 & -15.1 & 695.1 & 19 \\
\hline 691.9 & 58.6 & -1.3 & -5.7 & 652.7 & 15.2 \\
\hline 755.4 & 102.8 & -4.4 & -17.4 & 624 & 17.4 \\
\hline 2721.9 & 36.6 & -2.1 & -3.6 & 2721.9 & 36.6 \\
\hline 781.1 & 67.6 & -2.3 & -8.8 & 712.7 & 17.4 \\
\hline 779.2 & 53.6 & 1.7 & 6.6 & 830.8 & 19 \\
\hline 848.9 & 63 & -0.1 & -0.5 & 845 & 20 \\
\hline 666.2 & 53.4 & 1.1 & 4.9 & 698.8 & 16 \\
\hline 593.8 & 66 & -0.3 & -1.4 & 585.4 & 14 \\
\hline 825.6 & 59.6 & -2.2 & -8.2 & 758.1 & 17.8 \\
\hline 1137.2 & 60.4 & -6.2 & -17.8 & 935.3 & 22.2 \\
\hline 3394.5 & 203.6 & -82 & -98.8 & & \\
\hline 833.7 & 47.4 & -5.5 & -19.7 & 669.1 & 15.2 \\
\hline 816.8 & 121.6 & 1.3 & 4.7 & 855.5 & 26 \\
\hline 658.2 & 62 & -1.2 & -5.3 & 623.2 & 14.8 \\
\hline 822.2 & 50.8 & -3.8 & -13.9 & 707.8 & 16.2 \\
\hline 251.2 & 93.4 & -0.2 & -2 & 246.1 & 6.4 \\
\hline 760 & 83 & 0.8 & 3 & 783 & 20 \\
\hline 575.2 & 84.4 & 2.3 & 11.2 & 639.7 & 16.2 \\
\hline 550 & 65.6 & 2.2 & 11.2 & 611.7 & 14.6 \\
\hline 1098.5 & 55.8 & -0.1 & -0.2 & 1095.8 & 25.2 \\
\hline 654.7 & 66.4 & -3.5 & -15.3 & 554.8 & 13.4 \\
\hline 2046.4 & 40 & -3.1 & -6.2 & 2046.4 & 40 \\
\hline 713.3 & 79.4 & -1.9 & -8 & 655.9 & 16.6 \\
\hline 585.8 & 90.4 & -2.3 & -11.2 & 520.4 & 13.6 \\
\hline 553.8 & 59.6 & 0.1 & 0.5 & 556.3 & 13 \\
\hline 1590.6 & 43 & -22.2 & -47.7 & & \\
\hline 786.9 & 55.8 & -1 & -4 & 755.7 & 17.4 \\
\hline 1040.4 & 63.8 & -7.6 & -23 & 800.6 & 19.4 \\
\hline 832.2 & 52.4 & -0.9 & -3.2 & 805.4 & 18.4 \\
\hline 887.3 & 83.8 & -1.5 & -5.1 & 841.9 & 21.8 \\
\hline 675.5 & 55.2 & -0.7 & -3 & 655.4 & 15.2 \\
\hline 699.8 & 59.8 & -1.2 & -5 & 664.6 & 15.6 \\
\hline 881.3 & 59.8 & -1.3 & -4.7 & 839.5 & 19.6 \\
\hline 1074.7 & 59.2 & -9.5 & -27.8 & 775.9 & 18.4 \\
\hline 805.7 & 52 & 0.3 & 1 & 814 & 18.6 \\
\hline 810.2 & 88 & -2.6 & -9.9 & 729.9 & 19.2 \\
\hline 1828.1 & 41.8 & -2.9 & -6.2 & 1828.1 & 41.8 \\
\hline 583.2 & 97.8 & 1 & 4.7 & 610.6 & 16.4 \\
\hline 953.7 & 68.6 & -11.9 & -37.7 & 593.7 & 14.6 \\
\hline 825 & 95.2 & -3.3 & -12.1 & 725.2 & 19.8 \\
\hline 1329.2 & 45.6 & -8.7 & -22.1 & 1035.4 & 23 \\
\hline 678.6 & 72.2 & -0.3 & -1.3 & 670.1 & 16.4 \\
\hline 830 & 60.6 & -3.1 & -11.2 & 736.7 & 17.4 \\
\hline 862.1 & 86.4 & -2.4 & -8.6 & 788 & 20.8 \\
\hline 833.7 & 73.6 & -1.7 & -6.3 & 781.2 & 19.4 \\
\hline 724.4 & 80.8 & 3.2 & 13.1 & 819.4 & 20.6 \\
\hline 844.6 & 49.6 & -1.1 & -3.9 & 811.6 & 18.4 \\
\hline
\end{tabular}




\begin{tabular}{|c|c|c|c|c|c|}
\hline 835 & 72.2 & -0.7 & -2.6 & 812.9 & 20 \\
\hline 718.7 & 80.4 & 2.6 & 10.7 & 795.6 & 20 \\
\hline 719 & 53 & -0.1 & -0.4 & 716.3 & 16.4 \\
\hline 541.1 & 69.8 & 1.1 & 5.9 & 572.7 & 13.8 \\
\hline 519.1 & 264.6 & -19.1 & -95.3 & & \\
\hline 811.8 & 79.6 & 0.2 & 0.7 & 817.7 & 20.6 \\
\hline 598.9 & 65.8 & -0.1 & -0.5 & 595.9 & 14.2 \\
\hline 681.7 & 58.4 & 0.2 & 0.9 & 687.6 & 16 \\
\hline 800.3 & 90.4 & 1 & 3.8 & 830.5 & 22 \\
\hline 773.4 & 54.4 & 0.1 & 0.4 & 776.7 & 17.8 \\
\hline 837.2 & 107.6 & -1.7 & -6.1 & 786.3 & 22.8 \\
\hline 809.5 & 58.2 & -0.6 & -2.4 & 790.4 & 18.4 \\
\hline 539.9 & 60.8 & -1.8 & -9.3 & 489.7 & 11.6 \\
\hline 897.5 & 50.8 & -2.5 & -8.8 & 818.9 & 18.6 \\
\hline 2396.3 & 510.8 & -57.6 & -87.2 & & \\
\hline 404.5 & 73.6 & -5.9 & -39 & 246.6 & 6 \\
\hline 1026.9 & 54 & 0.5 & 1.4 & 1041.5 & 23.6 \\
\hline 918.6 & 67.2 & -3.7 & -12.3 & 805.2 & 19.6 \\
\hline 609.7 & 87 & 0.7 & 3 & 627.9 & 16.2 \\
\hline 874.3 & 83.2 & -1.1 & -3.9 & 840.6 & 21.8 \\
\hline 598.2 & 81 & 3.1 & 14.9 & 687.5 & 17.2 \\
\hline 856.9 & 103.2 & -2.3 & -8.1 & 787.4 & 22.2 \\
\hline 773.4 & 55 & -1 & -3.8 & 744 & 17.2 \\
\hline-82.4 & 892.4 & 4.7 & -132.8 & 27 & 2 \\
\hline 876.8 & 84.8 & -2.3 & -8 & 806.3 & 21 \\
\hline 1161.1 & 110 & -11.7 & -32.1 & 788.2 & 24.4 \\
\hline 645.2 & 49.2 & 0.5 & 2.2 & 659.7 & 14.8 \\
\hline 808.6 & 91.2 & 0.5 & 1.6 & 821.8 & 21.8 \\
\hline 730 & 99.8 & 1.7 & 7 & 781.1 & 21.2 \\
\hline 816.5 & 52.4 & 0.4 & 1.5 & 828.6 & 18.8 \\
\hline 793.6 & 70.4 & 1.3 & 4.8 & 831.7 & 20.2 \\
\hline 1706.4 & 44.4 & -42.3 & -80.6 & & \\
\hline 797.2 & 50.4 & 1.4 & 5.2 & 838.8 & 19 \\
\hline 951.6 & 50.2 & -14.8 & -46.2 & 511.6 & 11.8 \\
\hline 957.1 & 141.6 & -6.2 & -20.1 & 764.5 & 26 \\
\hline 567.8 & 112.8 & -1.7 & -8.5 & 519.6 & 14.6 \\
\hline 789.8 & 83.4 & -3 & -11.6 & 698.4 & 18 \\
\hline 961.2 & 49.4 & -0.9 & -3 & 932.4 & 20.8 \\
\hline 875.2 & 66.2 & 0 & -0.2 & 873.8 & 21 \\
\hline 713.3 & 95.6 & 3.5 & 14.7 & 818.3 & 21.8 \\
\hline 2152.6 & 38.8 & -13.1 & -24.6 & & \\
\hline 759.4 & 60.8 & 2.9 & 11.3 & 845.5 & 19.6 \\
\hline 698.8 & 106 & 2.8 & 11.9 & 782 & 21.8 \\
\hline 626.1 & 84 & 0.1 & 0.6 & 629.9 & 16 \\
\hline 801 & 63.6 & 1.1 & 3.9 & 832.4 & 19.6 \\
\hline 782.4 & 78.6 & -0.9 & -3.5 & 755.3 & 19 \\
\hline 438.4 & 124 & 5.6 & 35.7 & & \\
\hline 837.5 & 77.8 & -0.9 & -3.4 & 808.7 & 20. \\
\hline 933.3 & 80.2 & 0.6 & 2.1 & 953.1 & 24. \\
\hline 814.6 & 65.6 & -0.1 & -0.4 & 811.4 & 19. \\
\hline 832.2 & 56.2 & 1.1 & 3.9 & 864.9 & 19.8 \\
\hline 631.5 & 67.4 & -0.1 & -0.6 & 627.9 & 15 \\
\hline 636.8 & 74.2 & -0.5 & -2.4 & 621.8 & 15.2 \\
\hline 703.5 & 58.2 & 1 & 3.9 & 731.1 & 17 \\
\hline
\end{tabular}




\begin{tabular}{|c|c|c|c|c|c|}
\hline 501.9 & 60.8 & 1.5 & 8 & 542.3 & 12.6 \\
\hline 756.1 & 104.8 & -4.1 & -16.1 & 634.2 & 17.8 \\
\hline 769.8 & 66.2 & 1.8 & 7.1 & 824.3 & 19.6 \\
\hline 830.6 & 70.6 & -0.4 & -1.4 & 818.9 & 20 \\
\hline 688.5 & 95 & -4.6 & -19.4 & 555.1 & 15 \\
\hline 872.8 & 75.4 & -3.5 & -12.4 & 764.7 & 19.2 \\
\hline 500 & 88.6 & -1 & -5.6 & 472.3 & 12.2 \\
\hline 825.6 & 87 & -1 & -3.8 & 794.6 & 20.8 \\
\hline 862.4 & 70.4 & -8.5 & -29.3 & 609.4 & 15 \\
\hline 887.3 & 62 & -13.7 & -45.5 & 483.6 & 11.6 \\
\hline 159.3 & 145.4 & -0.7 & -10.9 & 141.9 & 4.2 \\
\hline 1013.1 & 52.8 & -2.5 & -7.9 & 933.2 & 21.2 \\
\hline 754.5 & 60.4 & -0.1 & -0.3 & 752 & 17.6 \\
\hline 751.5 & 105 & 2.8 & 11.2 & 835.8 & 23.4 \\
\hline 770.1 & 105.8 & 2.3 & 8.8 & 838.2 & 23.4 \\
\hline 642.1 & 59.2 & -0.1 & -0.5 & 638.9 & 14.8 \\
\hline 766.5 & 81.4 & -0.1 & -0.4 & 763.3 & 19.4 \\
\hline 818.7 & 88.2 & 1.1 & 4 & 851.2 & 22.2 \\
\hline 861.8 & 62.4 & -0.1 & -0.4 & 858.4 & 20.2 \\
\hline 836.2 & 58.4 & -0.6 & -2.4 & 816.5 & 18.8 \\
\hline 640.6 & 87.4 & 2.2 & 9.8 & 703.2 & 18 \\
\hline 668.2 & 51.2 & 0.7 & 3.1 & 689.2 & 15.6 \\
\hline 811.8 & 103.6 & -7.1 & -26 & 600.7 & 17 \\
\hline 503.1 & 85.2 & 2.3 & 12.5 & 566.2 & 14.2 \\
\hline 811.4 & 59.4 & 1.3 & 4.7 & 849.7 & 19.6 \\
\hline 826.9 & 48.2 & -19 & -64.9 & & \\
\hline 631.8 & 181.4 & -0.3 & -1.5 & 622.2 & 22.2 \\
\hline 787.6 & 77 & 0.5 & 1.7 & 800.9 & 20 \\
\hline 961.7 & 62 & -0.1 & -0.3 & 959.1 & 22.4 \\
\hline 932.2 & 62 & 1.6 & 5.2 & 980.7 & 23 \\
\hline 765.6 & 73 & 1.7 & 6.5 & 815.6 & 19.8 \\
\hline 541.8 & 87.8 & 2.9 & 15 & 623.1 & 16 \\
\hline 881 & 79.8 & 0.1 & 0.3 & 883.7 & 22.4 \\
\hline 784.3 & 108.8 & -0.4 & -1.6 & 771.8 & 22 \\
\hline 545.2 & 88.2 & 3.1 & 16.1 & 632.8 & 16.2 \\
\hline 807 & 103.4 & -1.2 & -4.5 & 770.7 & 21.6 \\
\hline 808 & 70.4 & 0.8 & 2.8 & 830.8 & 20 \\
\hline 846.1 & 48.8 & -7.2 & -25.6 & 629.9 & 14.2 \\
\hline 330.1 & 594.6 & -12.1 & -92.3 & 25.5 & 2 \\
\hline 830.6 & 119.2 & 1.1 & 4.1 & 864.4 & 26 \\
\hline 870.7 & 66.4 & -1 & -3.5 & 840.2 & 20 \\
\hline 1118.1 & 48.8 & -19.2 & -52.6 & & \\
\hline 786.3 & 71.4 & 1.3 & 4.8 & 824 & 20 \\
\hline 821.8 & 62.8 & 0.4 & 1.4 & 833.4 & 19.4 \\
\hline 663 & 49.4 & -12.8 & -53.4 & 309.2 & 7.2 \\
\hline 775.6 & 80.8 & 2.2 & 8.5 & 841.8 & 21.2 \\
\hline 962 & 56.8 & 2.3 & 7.6 & 1034.9 & 23.4 \\
\hline 327.5 & 64.2 & 0.5 & 3.9 & 340.4 & 8 \\
\hline 337.3 & 63.8 & 0.1 & 0.2 & 337.9 & 8 \\
\hline 998.3 & 79 & -0.9 & -2.9 & 969.4 & 24.6 \\
\hline 13.8 & 177.6 & 0.4 & 78.7 & 24.6 & 0.8 \\
\hline 899 & 59.8 & 0.6 & 2 & 917.2 & 21.2 \\
\hline 593.4 & 68.2 & 1.1 & 5.2 & 624.3 & 14.8 \\
\hline 335.2 & 82.8 & 1.6 & 12.9 & 378.4 & 9.4 \\
\hline 541.4 & 82.4 & 2.1 & 10.7 & 599.5 & 15 \\
\hline
\end{tabular}




\begin{tabular}{|c|c|c|c|c|c|}
\hline 645.9 & 55.4 & -1.2 & -5.5 & 610.2 & 14 \\
\hline 744.3 & 58.8 & 0.8 & 3.3 & 768.6 & 17.8 \\
\hline 684.8 & 85 & -0.7 & -3 & 664 & 17 \\
\hline 923.9 & 55.8 & -6.3 & -20.9 & 731.1 & 16.8 \\
\hline 896.9 & 50.8 & -1.7 & -5.8 & 845.2 & 19 \\
\hline 867.9 & 49.4 & -1.2 & -4.5 & 829.1 & 18.4 \\
\hline 636.4 & 112.4 & -0.7 & -3.3 & 615.3 & 17.4 \\
\hline 420.7 & 965.6 & -15.2 & -92.5 & & \\
\hline 1039 & 61.2 & -0.2 & -0.7 & 1032.1 & 24 \\
\hline 820.3 & 52.8 & 0.4 & 1.3 & 831 & 18.8 \\
\hline 391.4 & 140.8 & 10.8 & 77.6 & & \\
\hline 512.3 & 81 & 1.8 & 9.9 & 562.9 & 14 \\
\hline 726.7 & 94.8 & 2.9 & 12 & 814.2 & 21.6 \\
\hline 692.3 & 71 & 2.5 & 10.7 & 766.3 & 18.4 \\
\hline 1007.8 & 56.8 & 0.4 & 1.2 & 1019.6 & 23.4 \\
\hline 1003.9 & 50.2 & 1.1 & 3.4 & 1038.6 & 23 \\
\hline 842.7 & 64.4 & -0.3 & -1 & 834.2 & 19.8 \\
\hline 1189.1 & 74.4 & -9.9 & -26.9 & 868.7 & 22.2 \\
\hline 1010.1 & 54 & -0.3 & -1 & 999.6 & 22.6 \\
\hline 913.6 & 90.6 & -3.6 & -12.4 & 800.5 & 21.4 \\
\hline 734.3 & 99.6 & 1.4 & 5.8 & 776.6 & 21 \\
\hline 779.2 & 74.8 & 0.9 & 3.4 & 805.9 & 19.8 \\
\hline 774.3 & 119.2 & 1.5 & 5.8 & 818.9 & 24.2 \\
\hline 534.7 & 88.8 & -1.3 & -7.2 & 496.4 & 12.8 \\
\hline 987.1 & 48.6 & -3.9 & -12.5 & 863.2 & 19.2 \\
\hline 857.5 & 50.4 & -14.2 & -48.2 & 444.2 & 10.2 \\
\hline 581.4 & 75.4 & -0.1 & -0.8 & 576.6 & 14.2 \\
\hline 347.5 & 2732.8 & -12.6 & -91.6 & 29.3 & 5.4 \\
\hline 749.2 & 57.2 & 1.3 & 5.2 & 788.5 & 18 \\
\hline 218.5 & 76 & -1.4 & -17.4 & 180.6 & 4.4 \\
\hline 451.2 & 75.4 & 2.6 & 15.6 & 521.7 & 12.8 \\
\hline 578.9 & 67.4 & 2.1 & 10.2 & 637.9 & 15 \\
\hline 653.6 & 53.2 & -2.1 & -9.5 & 591.3 & 13.4 \\
\hline 960.9 & 74.6 & -1 & -3.2 & 929.9 & 23.2 \\
\hline 837.5 & 85.2 & 0.9 & 3.1 & 863.4 & 22.2 \\
\hline 875.5 & 158.4 & -4.6 & -16.1 & 734.8 & 25.8 \\
\hline 929.8 & 63.8 & -4.1 & -13.6 & 802.9 & 19 \\
\hline 1068 & 52 & 1.9 & 5.8 & & \\
\hline 605 & 85 & -0.6 & -2.8 & 587.8 & 15 \\
\hline 800 & 66.2 & 1.1 & 4.1 & 832.5 & 19.8 \\
\hline 665.8 & 81.2 & 3.3 & 14.7 & 763.4 & 19 \\
\hline 917.2 & 77.4 & 2 & 6.7 & 978.4 & 24.4 \\
\hline 707.9 & 70.2 & -2.7 & -11.3 & 627.6 & 15.2 \\
\hline 640.3 & 72.6 & -2.9 & -13.3 & 555.3 & 13.4 \\
\hline 660.6 & 103.6 & -0.2 & -1.2 & 652.7 & 18 \\
\hline 1229.7 & 101.4 & -12.3 & -32.3 & 832 & 24.8 \\
\hline 486.9 & 140.4 & 0.5 & 2.7 & 499.9 & 15.4 \\
\hline 727.7 & 78.6 & 0.7 & 2.6 & 746.5 & 18.4 \\
\hline 1146.1 & 359.8 & -33.2 & -84.1 & & \\
\hline 794.3 & 65.8 & 0.9 & 3.4 & 821.5 & 19.4 \\
\hline 746.9 & 51.4 & 1 & 4 & 777 & 17.4 \\
\hline 867.9 & 58.6 & -10 & -34.3 & 570.1 & 13.4 \\
\hline 817.1 & 85.6 & -0.4 & -1.4 & 805.5 & 20.8 \\
\hline 545.2 & 87.6 & 1.5 & 7.4 & 585.5 & 14.8 \\
\hline 738.3 & 84.2 & 0.7 & 2.7 & 758 & 19.2 \\
\hline
\end{tabular}




\begin{tabular}{|c|c|c|c|c|c|}
\hline 160.2 & 1151.2 & -5.5 & -84.9 & 24.1 & 2.4 \\
\hline 701.5 & 65.8 & -1.3 & -5.6 & 662 & 15.6 \\
\hline 607.5 & 133.8 & 4.1 & 19.7 & 727.1 & 22.4 \\
\hline 709.6 & 73.6 & 1.8 & 7.5 & 762.7 & 18.6 \\
\hline 650.5 & 72.8 & 1.3 & 5.9 & 688.8 & 16.6 \\
\hline 839.3 & 137.8 & -8.6 & -30.2 & 585.5 & 19.2 \\
\hline 761 & 58.2 & -8.8 & -33.5 & 505.7 & 11.8 \\
\hline 818.4 & 77.4 & 0.7 & 2.5 & 838.6 & 20.8 \\
\hline 783.7 & 81 & 1.2 & 4.7 & 820.7 & 20.6 \\
\hline 940.9 & 77.4 & -20.4 & -63 & & \\
\hline 1546.6 & 66.2 & -41.9 & -84.6 & & \\
\hline 794 & 71.6 & -1.2 & -4.7 & 757 & 18.4 \\
\hline 639.9 & 84.6 & -0.5 & -2.5 & 623.8 & 15.8 \\
\hline 763.3 & 56 & 0.2 & 0.6 & 767.9 & 17.4 \\
\hline 776 & 102.2 & -0.4 & -1.6 & 763.9 & 21 \\
\hline 1987.6 & 44 & -2.4 & -4.9 & 1987.6 & 44 \\
\hline 510.8 & 87.2 & 1.3 & 6.9 & 545.9 & 13.8 \\
\hline 568.2 & 93 & 3.6 & 18.3 & 672 & 17.4 \\
\hline 798.4 & 68.2 & 2.2 & 8.1 & 863.4 & 20.6 \\
\hline 231.3 & 124.6 & 1.8 & 20.3 & 278.3 & 7.8 \\
\hline 722 & 75 & 3.4 & 14 & 823 & 20 \\
\hline 583.2 & 72.4 & 0.1 & 0.6 & 586.6 & 14.2 \\
\hline 826.9 & 62 & -0.8 & -3 & 802.2 & 18.6 \\
\hline 800.7 & 55.4 & 0.5 & 1.7 & 814 & 18.4 \\
\hline 756.1 & 67.4 & 1.9 & 7.4 & 812.2 & 19.2 \\
\hline 782.7 & 62.4 & 1.6 & 6.1 & 830.2 & 19.2 \\
\hline 638.5 & 57.2 & -0.1 & -0.5 & 635.2 & 14.4 \\
\hline 767.2 & 91.6 & 0.3 & 1.2 & 776.8 & 20.4 \\
\hline 737 & 77.6 & 2.9 & 11.5 & 822 & 20.2 \\
\hline 1143.3 & 55.2 & -30.3 & -78 & & \\
\hline 765.2 & 80.8 & 2 & 7.7 & 824.3 & 20.6 \\
\hline 727.7 & 63.4 & 0.3 & 1.2 & 736.3 & 17.2 \\
\hline 665.1 & 70 & 0.8 & 3.6 & 688.8 & 16.4 \\
\hline 1976.5 & 93.6 & -61 & -98.8 & & \\
\hline 660.6 & 84.2 & 0.7 & 3.2 & 681.5 & 17.2 \\
\hline 310.7 & 64 & 1 & 8.3 & 336.4 & 8 \\
\hline 777.9 & 82 & 0 & 0 & 778.3 & 19.6 \\
\hline 748.2 & 163.2 & 0 & 0.1 & 748.7 & 26.2 \\
\hline 1115.5 & 271.4 & -1 & -3.1 & 1080.5 & 62.2 \\
\hline 755.1 & 81.2 & -0.4 & -1.8 & 741.9 & 18.6 \\
\hline 775.3 & 69.2 & 0.2 & 0.6 & 780.2 & 18.6 \\
\hline 847.1 & 58.8 & -1.3 & -4.8 & 806.2 & 18.4 \\
\hline 900.5 & 72.8 & -3.1 & -10.8 & 803.7 & 19.6 \\
\hline 22.1 & 129.2 & 0.4 & 45.9 & 32.2 & 0.8 \\
\hline 589.4 & 63.4 & 0.5 & 2.5 & 603.9 & 14 \\
\hline 629.3 & 115.6 & 0.4 & 1.8 & 640.6 & 18.4 \\
\hline 2534.8 & 43.4 & -64.6 & -92.7 & & \\
\hline 872.2 & 69.4 & -0.9 & -3.1 & 845.1 & 20.2 \\
\hline 830.9 & 71.8 & 0.1 & 0.4 & 834.3 & 20.2 \\
\hline 929.8 & 72 & 1.7 & 5.8 & 983.5 & 23.8 \\
\hline 640.3 & 198 & -0.6 & -2.9 & 621.8 & 23.8 \\
\hline 801 & 82.2 & 0.3 & 0.9 & 808 & 20.4 \\
\hline 800.7 & 75.2 & 0.7 & 2.6 & 821.2 & 20 \\
\hline 1000 & 77.4 & -1.1 & -3.5 & 964.9 & 24 \\
\hline
\end{tabular}




\begin{tabular}{|c|c|c|c|c|c|}
\hline 722.7 & 58.2 & 1.9 & 7.6 & 777.3 & 17.8 \\
\hline 675.5 & 71.6 & 1.4 & 5.9 & 715.3 & 17.2 \\
\hline 1961.6 & 54.8 & -49 & -84.4 & & \\
\hline 1927.8 & 52.4 & -48.6 & -84.6 & & \\
\hline 824 & 134.8 & -1 & -3.7 & 793.3 & 25.4 \\
\hline 801 & 73.2 & 0 & -0.2 & 799.5 & 19.4 \\
\hline 627.6 & 80.2 & -1 & -4.6 & 598.4 & 14.8 \\
\hline 815.2 & 90.8 & 0.3 & 1.1 & 824.3 & 21.6 \\
\hline 2971.1 & 201.4 & -77.5 & -99 & & \\
\hline 612.6 & 67.4 & 0 & -0.3 & 611 & 14.4 \\
\hline 952.8 & 52 & -18.2 & -56.2 & & \\
\hline 1327.6 & 58.8 & -30.1 & -70.2 & & \\
\hline 555.6 & 92.2 & 0.1 & 0.6 & 558.8 & 14.4 \\
\hline 1921.6 & 59.8 & -40.3 & -72.8 & & \\
\hline 690.6 & 79.8 & 0.4 & 1.4 & 700.4 & 17.4 \\
\hline 824.7 & 91.6 & -1.3 & -4.7 & 785.9 & 20.6 \\
\hline 913.3 & 98.4 & -3.8 & -12.8 & 796.4 & 22 \\
\hline 651.9 & 75.6 & -2.5 & -11.4 & 577.7 & 14.2 \\
\hline 829.7 & 60.6 & -0.4 & -1.4 & 817.8 & 18.8 \\
\hline 815.2 & 80.8 & -0.1 & -0.6 & 810 & 20.4 \\
\hline 611.8 & 98.2 & 1.3 & 6 & 648.5 & 17.2 \\
\hline 839.6 & 60.8 & 0.1 & 0.2 & 841.6 & 19.4 \\
\hline 721.3 & 93.8 & -0.2 & -1 & 714.3 & 18.8 \\
\hline 603.9 & 70 & 2.5 & 12 & 676.4 & 16 \\
\hline 776.9 & 91.2 & 1.1 & 4 & 808 & 21 \\
\hline 662.7 & 570.4 & -24.1 & -96 & & \\
\hline 656.1 & 58 & -1.4 & -6.3 & 614.8 & 14 \\
\hline 829.7 & 73.2 & -0.8 & -3.1 & 803.9 & 19.4 \\
\hline 713.6 & 72 & 1.8 & 7.4 & 766.5 & 18.4 \\
\hline 681 & 96.8 & -3.7 & -15.7 & 574.4 & 15.4 \\
\hline 604.7 & 104.6 & -1 & -5 & 574.4 & 15.6 \\
\hline 810.2 & 63.2 & -7 & -25.8 & 601.3 & 14 \\
\hline 331.4 & 81.2 & -2.4 & -19.4 & 267.1 & 6.6 \\
\hline 826.9 & 60.8 & -1.9 & -6.9 & 769.6 & 17.8 \\
\hline 969.7 & 79.4 & -13.4 & -41.6 & 565.9 & 14.6 \\
\hline 893 & 149.4 & -11.6 & -38.7 & 547.7 & 18.8 \\
\hline 752.8 & 68.2 & 0.6 & 2.1 & 769 & 18.2 \\
\hline 561.6 & 73.4 & -2.3 & -11.5 & 496.7 & 12 \\
\hline 726.4 & 66.8 & -2.2 & -9 & 660.8 & 15.6 \\
\hline 883.4 & 67 & -3.2 & -11.3 & 783.8 & 18.6 \\
\hline 658.2 & 64.2 & -2.9 & -13 & 572.7 & 13.4 \\
\hline 724.7 & 70.2 & 0.1 & 0.3 & 727 & 17.4 \\
\hline 959.1 & 56.4 & -1.2 & -3.9 & 921.7 & 20.8 \\
\hline 573 & 78 & -2.2 & -10.8 & 511 & 12.6 \\
\hline 717.3 & 88.6 & -3.4 & -14 & 616.9 & 16 \\
\hline 791.4 & 78.8 & 0.9 & 3.3 & 817.3 & 20.2 \\
\hline 1062.1 & 63.6 & -0.3 & -0.9 & 1052 & 24.4 \\
\hline 136.3 & 371 & -4.2 & -77.1 & 31.2 & 1.2 \\
\hline 1786.4 & 56.8 & -1.5 & -3.2 & 1786.4 & 56. \\
\hline 822.8 & 233.4 & -5 & -18.2 & 672.6 & 27.6 \\
\hline
\end{tabular}




\section{Sample 2876S Rahad @ Hufeira 7 grain analysed 4 concordant ages 57.}

\begin{tabular}{cccc|ccc}
\cline { 2 - 7 } grain & $\begin{array}{c}\text { concentrations } \\
\mathrm{U}[\mathrm{ppm}]\end{array}$ & $\mathrm{Pb}[\mathrm{ppm}]$ & $\mathrm{Th} / \mathrm{U}$ & $\begin{array}{c}\text { isotopic ratios } \\
\mathrm{Pb207/Pb206}\end{array}$ & $\mathbf{2 \sigma} \mathbf{7 6}$ & $\mathbf{P b 2 0 7 / U 2 3 5}$ \\
\hline X2876S_G001 & 312.4 & 43.3 & 0.4962 & 0.06596 & 0.002 & 1.1764 \\
X2876S_G002 & 79.1 & 12.2 & 0.6183 & 0.06751 & 0.00294 & 1.2944 \\
X2876S_G003 & 2676.1 & 77.1 & 0.3435 & 0.17475 & 0.0046 & 0.6434 \\
X2876S_G004 & 2200.8 & 72.9 & 0.592 & 0.18985 & 0.00546 & 0.77387 \\
X2876S_G005 & 364.5 & 40.8 & 0.3764 & 0.0806 & 0.00244 & 1.19871 \\
X2876S_G006 & 561.9 & 89.1 & 0.0724 & 0.0733 & 0.00204 & 1.687 \\
X2876S_G007 & 380.8 & 48.8 & 0.7138 & 0.07286 & 0.00212 & 1.19124
\end{tabular}




\section{$1 \%$ concordant}

\begin{tabular}{ccc|cccc}
\hline $\mathbf{2 \sigma} \mathbf{7 5}$ & Pb206/U238 & $\mathbf{2 \sigma 6 8}$ & $\begin{array}{c}\text { ages } \\
\text { age 206/238 }\end{array}$ & $\mathbf{2 \sigma}$ age 68 & age 207/235 & 20 age 75 \\
\hline 0.03636 & 0.12941 & 0.00312 & 784.5 & 17.8 & 789.6 & 21.2 \\
0.05576 & 0.13911 & 0.00382 & 839.6 & 21.6 & 843.2 & 29.4 \\
0.01738 & 0.02671 & 0.00064 & 169.9 & 4 & 504.4 & 14.2 \\
0.02218 & 0.02957 & 0.00072 & 187.9 & 4.6 & 582 & 16.6 \\
0.03706 & 0.10791 & 0.00262 & 660.6 & 15.2 & 800 & 21.4 \\
0.0488 & 0.16697 & 0.00396 & 995.4 & 21.8 & 1003.6 & 23.4 \\
0.03568 & 0.11862 & 0.00284 & 722.6 & 16.4 & 796.5 & 20.8
\end{tabular}




\begin{tabular}{cc|cc|cc}
\hline & & discordance & & preferred age & \\
age $\mathbf{2 0 7 / 2 0 6}$ & 20 age 76 & $\mathbf{\Delta} \mathbf{6 8 - 7 5}[\%]$ & $\boldsymbol{\Delta} \mathbf{6 8 - 7 6}[\%]$ & age & 20 age \\
\hline 805.1 & 63.4 & -0.7 & -2.6 & 784.5 & 17.8 \\
853.5 & 90.4 & -0.4 & -1.6 & 839.6 & 21.6 \\
2603.7 & 43.8 & -66.3 & -93.5 & & \\
2740.9 & 47.4 & -67.7 & -93.1 & & \\
1211.7 & 59.6 & -17.4 & -45.5 & & \\
1022.3 & 56.4 & -0.8 & -2.6 & 995.4 & 21.8 \\
1010.1 & 59 & -9.3 & -28.5 & 722.6 & 16.4
\end{tabular}


Sample 3312S Tekeze @ Togo Ber 168 grain analysed 129 concordant age:

\begin{tabular}{|c|c|c|c|c|c|c|}
\hline \multirow[b]{2}{*}{ grain } & \multicolumn{3}{|c|}{ concentrations } & \multicolumn{3}{|l|}{ isotopic ratios } \\
\hline & U [ppm] & $\mathrm{Pb}$ [ppm] & $\mathrm{Th} / \mathrm{U}$ & Pb207/Pb206 & $2 \sigma 76$ & Pb207/U235 \\
\hline X3312n_G001 & 41.8 & 22.7 & 0.7586 & 0.15518 & 0.0051 & 9.5433 \\
\hline X3312n_G002 & 207.4 & 25.8 & 0.2875 & 0.06561 & 0.00186 & 1.11036 \\
\hline X3312n G003 & 266.7 & 43.5 & 0.456 & 0.07298 & 0.00198 & 1.55327 \\
\hline X3312n_G004 & 140.3 & 21.1 & 0.2312 & 0.0718 & 0.00202 & 1.4879 \\
\hline X3312n_G005 & 326.8 & 30.6 & 0.274 & 0.0586 & 0.00168 & 0.75432 \\
\hline X3312n_G006 & 1054.5 & 100.3 & 0.2452 & 0.06099 & 0.0014 & 0.80639 \\
\hline X3312n_G007 & 432.2 & 56.3 & 0.6157 & 0.06593 & 0.00168 & 1.07864 \\
\hline X3312n_G008 & 170.8 & 19.8 & 0.3505 & 0.05717 & 0.0019 & 0.88414 \\
\hline X3312n G009 & 159.5 & 28.2 & 0.4634 & 0.06548 & 0.00212 & 1.4912 \\
\hline X3312n G010 & 318.1 & 28.2 & 0.2497 & 0.05482 & 0.00166 & 0.67194 \\
\hline X3312n_G011 & 149.9 & 21.2 & 0.547 & 0.06332 & 0.00232 & 1.12945 \\
\hline X3312n_G012 & 413.1 & 48.4 & 0.6417 & 0.05758 & 0.00186 & 0.83151 \\
\hline X3312n_G013 & 313.7 & 175.5 & 0.304 & 0.19353 & 0.00428 & 13.69093 \\
\hline X3312n_G014 & 418.3 & 45.4 & 0.2287 & 0.05909 & 0.00158 & 0.89051 \\
\hline X3312n_G015 & 468 & 31.9 & 0.5102 & 0.0592 & 0.0019 & 0.51712 \\
\hline X3312n_G016 & 1051 & 119.8 & 0.7814 & 0.16884 & 0.00362 & 2.27187 \\
\hline X3312n_G017 & 6905.4 & 40.9 & 1.4818 & 0.04697 & 0.00166 & 0.02778 \\
\hline X3312n_G018 & 271.9 & 48.6 & 0.4765 & 0.06852 & 0.0018 & 1.57207 \\
\hline X3312n_G019 & 403.5 & 44.9 & 0.4255 & 0.074 & 0.0021 & 1.08246 \\
\hline X3312n_G020 & 38.3 & 4 & 0.7155 & 0.05722 & 0.00414 & 0.74494 \\
\hline X3312n_G021 & 383.4 & 60.4 & 0.3168 & 0.06618 & 0.00166 & 1.39857 \\
\hline X3312n_G022 & 136.8 & 72.8 & 0.6224 & 0.15569 & 0.00412 & 9.70954 \\
\hline X3312n_G023 & 420.9 & 38.9 & 0.1556 & 0.05851 & 0.00184 & 0.77086 \\
\hline X3312n_G024 & 641.4 & 97.8 & 0.0231 & 0.06793 & 0.0017 & 1.52358 \\
\hline X3312n_G025 & 413.1 & 57.3 & 0.3323 & 0.06363 & 0.00166 & 1.1867 \\
\hline X3312n_G026 & 136.8 & 20.2 & 0.3199 & 0.06564 & 0.00216 & 1.30888 \\
\hline X3312n_G027 & 1265.3 & 109.7 & 0.0596 & 0.06896 & 0.0016 & 0.88424 \\
\hline X3312n_G028 & 461 & 51.2 & 1.1241 & 0.06833 & 0.00222 & 0.88187 \\
\hline X3312n_G029 & 435.7 & 58.5 & 0.3582 & 0.06256 & 0.0017 & 1.12135 \\
\hline X3312n_G030 & 314.6 & 42.4 & 0.6076 & 0.06224 & 0.00176 & 1.04534 \\
\hline X3312n_G031 & 112.4 & 11.9 & 0.2698 & 0.05888 & 0.00236 & 0.85346 \\
\hline X3312n_G032 & 493.2 & 52.9 & 0.2848 & 0.05865 & 0.00154 & 0.86074 \\
\hline X3312n_G033 & 513.3 & 49.2 & 0.0066 & 0.0569 & 0.00156 & 0.81407 \\
\hline X3312n_G034 & 469.7 & 28.1 & 0.8275 & 0.08086 & 0.0037 & 0.60598 \\
\hline X3312n_G035 & 189.1 & 19 & 0.2348 & 0.06005 & 0.00222 & 0.84066 \\
\hline X3312n_G036 & 1139 & 87.7 & 0.1406 & 0.09133 & 0.0026 & 0.99351 \\
\hline X3312n_G037 & 160.3 & 96.5 & 0.5386 & 0.18665 & 0.0042 & 13.37021 \\
\hline X3312n_G038 & 20.9 & 1.9 & 0.4092 & 0.07397 & 0.00724 & 0.93091 \\
\hline X3312n_G039 & 495 & 88.7 & 0.4176 & 0.0703 & 0.00168 & 1.64103 \\
\hline X3312n_G040 & 112.4 & 16.5 & 0.5989 & 0.06641 & 0.00248 & 1.21777 \\
\hline X3312n_G041 & 236.2 & 32.4 & 0.5732 & 0.06253 & 0.00194 & 1.08062 \\
\hline X3312n_G042 & 258.8 & 38.2 & 0.2843 & 0.07368 & 0.002 & 1.48018 \\
\hline X3312n_G043 & 213.5 & 20.9 & 0.4044 & 0.05824 & 0.00198 & 0.75299 \\
\hline X3312n_G044 & 1736.8 & 107 & 1.1539 & 0.08682 & 0.00224 & 0.62914 \\
\hline X3312n_G045 & 348.6 & 35.4 & 0.1137 & 0.07282 & 0.002 & 1.05992 \\
\hline X3312n_G046 & 349.5 & 174.1 & 0.2997 & 0.15972 & 0.00376 & 10.07878 \\
\hline X3312n_G047 & 475.8 & 73.1 & 0.1673 & 0.0781 & 0.0019 & 1.71499 \\
\hline X3312n_G048 & 98.5 & 10.4 & 0.8313 & 0.05739 & 0.00288 & 0.71499 \\
\hline X3312n_G049 & 111.5 & 11.5 & 0.3362 & 0.05864 & 0.00252 & 0.81334 \\
\hline X3312n_G050 & 96.7 & 0.5 & 0.4001 & 0.03763 & 0.01808 & 0.02379 \\
\hline
\end{tabular}




\begin{tabular}{|c|c|c|c|c|c|c|}
\hline X3312n_G051 & 379.1 & 36.5 & 0.8744 & 0.05511 & 0.00174 & 0.61572 \\
\hline X3312n_G052 & 480.2 & 195.4 & 0.5002 & 0.13697 & 0.00304 & 6.92866 \\
\hline X3312n_G053 & 1341.2 & 50.2 & 0.4424 & 0.08065 & 0.00222 & 0.39794 \\
\hline X3312n_G054 & & & & 1.25485 & 6.76684 & \\
\hline X3312n_G055 & 145.5 & 14.6 & 0.2691 & 0.05896 & 0.0023 & 0.8102 \\
\hline X3312n_G056 & 706.7 & 90.8 & 0.0777 & 0.07071 & 0.00172 & 1.31613 \\
\hline X3312n_G057 & 604.8 & 95.9 & 0.1879 & 0.07105 & 0.00172 & 1.57379 \\
\hline X3312n_G058 & 129.8 & 16.8 & 0.2619 & 0.06413 & 0.00234 & 1.13543 \\
\hline X3312n_G059 & 1600.9 & 81.4 & 0.0593 & 0.1043 & 0.00248 & 0.76863 \\
\hline X3312n_G060 & 311.1 & 30.5 & 0.759 & 0.07483 & 0.0023 & 0.88334 \\
\hline X3312n_G061 & 982.1 & 72.7 & 0.5375 & 0.07782 & 0.00196 & 0.76181 \\
\hline X3312n_G062 & 76.7 & 10.6 & 0.2441 & 0.06303 & 0.0027 & 1.19976 \\
\hline X3312n G063 & 759.9 & 113.1 & 0.172 & 0.06896 & 0.00166 & 1.45202 \\
\hline X3312n_G064 & 99.3 & 12.9 & 0.2843 & 0.06264 & 0.00258 & 1.10441 \\
\hline X3312n_G065 & 95 & 14 & 0.3047 & 0.06557 & 0.00262 & 1.3045 \\
\hline X3312n_G066 & 393.9 & 25.6 & 0.3365 & 0.05843 & 0.002 & 0.51696 \\
\hline X3312n_G067 & 288.5 & 71.4 & 0.1978 & 0.10568 & 0.00262 & 3.62369 \\
\hline X3312n_G068 & 380 & 116.2 & 0.1454 & 0.10935 & 0.00258 & 4.61828 \\
\hline X3312n_G069 & 593.5 & 69.8 & 0.1705 & 0.06329 & 0.00166 & 1.0614 \\
\hline X3312n_G070 & 964.7 & 90.6 & 0.1903 & 0.05953 & 0.0015 & 0.79064 \\
\hline X3312n_G071 & 212.6 & 27.7 & 0.2515 & 0.06587 & 0.00206 & 1.18543 \\
\hline X3312n_G072 & 58.4 & 9.2 & 2.5328 & 0.06602 & 0.00384 & 0.8461 \\
\hline X3312n_G073 & 470.6 & 61.4 & 0.52 & 0.06859 & 0.00194 & 1.17437 \\
\hline X3312n_G074 & 598.7 & 90.2 & 0.069 & 0.07119 & 0.00178 & 1.55854 \\
\hline X3312n_G075 & 108.1 & 16.1 & 0.38 & 0.0892 & 0.0032 & 1.72285 \\
\hline X3312n_G076 & 447.9 & 39.9 & 0.1819 & 0.05756 & 0.00176 & 0.72637 \\
\hline X3312n_G077 & 566.4 & 50.4 & 0.4494 & 0.22245 & 0.00558 & 2.51576 \\
\hline X3312n_G078 & 52.3 & 0.3 & 0.319 & 0.06444 & 0.02134 & 0.04262 \\
\hline X3312n_G079 & 179.5 & 14.5 & 0.1609 & 0.05761 & 0.00224 & 0.66337 \\
\hline X3312n_G080 & 293.7 & 26.7 & 0.0777 & 0.05984 & 0.00192 & 0.79862 \\
\hline X3312n_G081 & 61.9 & 6.6 & 0.4799 & 0.06209 & 0.00354 & 0.86061 \\
\hline X3312n_G082 & 223.1 & 22 & 0.2499 & 0.05912 & 0.00198 & 0.80839 \\
\hline X3312n_G083 & 252.7 & 41.8 & 0.087 & 0.07446 & 0.00212 & 1.77789 \\
\hline X3312n_G084 & 196.1 & 37.5 & 0.5839 & 0.07331 & 0.00212 & 1.74262 \\
\hline X3312n_G085 & 534.2 & 64.3 & 0.3226 & 0.06792 & 0.00184 & 1.11366 \\
\hline X3312n_G086 & 853.2 & 45 & 0.5403 & 0.06639 & 0.0019 & 0.46994 \\
\hline X3312n_G087 & 995.2 & 99.2 & 0.3275 & 0.06038 & 0.00156 & 0.81668 \\
\hline X3312n_G088 & 288.5 & 46.1 & 0.2779 & 0.07136 & 0.00202 & 1.55038 \\
\hline X3312n_G089 & 217 & 24.4 & 0.1617 & 0.07169 & 0.00236 & 1.13752 \\
\hline X3312n_G090 & 461.9 & 40.1 & 0.3966 & 0.07569 & 0.00218 & 0.90802 \\
\hline X3312n_G091 & 352.9 & 32.1 & 0.4663 & 0.05982 & 0.00186 & 0.70692 \\
\hline X3312n_G092 & 488.9 & 55 & 0.8892 & 0.05777 & 0.00172 & 0.74971 \\
\hline X3312n G093 & 117.6 & 51.8 & 0.3898 & 0.18858 & 0.0048 & 10.67172 \\
\hline X3312n_G094 & 97.6 & 10.5 & 0.4343 & 0.05892 & 0.00278 & 0.83107 \\
\hline X3312n_G095 & 317.2 & 31 & 0.7485 & 0.06826 & 0.00224 & 0.80055 \\
\hline X3312n_G096 & 549 & 56.9 & 0.2464 & 0.05821 & 0.00168 & 0.83605 \\
\hline X3312n_G097 & 874.9 & 79.9 & 0.0546 & 0.08657 & 0.00236 & 1.15522 \\
\hline X3312n_G098 & 57.5 & 8.4 & 0.3277 & 0.06484 & 0.00324 & 1.27175 \\
\hline X3312n_G099 & 390.4 & 32.3 & 0.192 & 0.05685 & 0.00184 & 0.66537 \\
\hline X3312n_G100 & 310.2 & 35.9 & 0.1622 & 0.06901 & 0.0021 & 1.13732 \\
\hline X3312n_G101 & 990 & 84.2 & 0.1058 & 0.06096 & 0.00162 & 0.76265 \\
\hline X3312n_G102 & 26.1 & 0.2 & 0.4936 & 0.54851 & 0.0762 & 0.72547 \\
\hline X3312n_G103 & 50.5 & 5.5 & 0.9947 & 0.06174 & 0.00396 & 0.76878 \\
\hline X3312n_G104 & 47.1 & 5 & 0.1928 & 0.06066 & 0.0037 & 0.91203 \\
\hline X3312n_G105 & 74.1 & 11.2 & 0.6515 & 0.06838 & 0.00304 & 1.26206 \\
\hline
\end{tabular}




\begin{tabular}{|c|c|c|c|c|c|c|}
\hline X3312n_G106 & 189.1 & 17.3 & 0.4542 & 0.06375 & 0.00238 & 0.7659 \\
\hline X3312n G107 & 531.6 & 35 & 0.9322 & 0.07623 & 0.00238 & 0.63446 \\
\hline X3312n_G108 & 271 & 20.7 & 1.4029 & 0.07653 & 0.00278 & 0.67184 \\
\hline X3312n_G109 & 154.2 & 21.9 & 0.0758 & 0.06996 & 0.00244 & 1.44139 \\
\hline X3312n_G110 & 122 & 14.3 & 0.6828 & 0.05829 & 0.00258 & 0.82988 \\
\hline X3312n_G111 & 406.1 & 37.2 & 0.1865 & 0.06072 & 0.0019 & 0.78705 \\
\hline X3312n_G112 & 700.6 & 68.4 & 0.2269 & 0.07098 & 0.00198 & 0.97557 \\
\hline X3312n_G113 & 291.9 & 27.3 & 0.1493 & 0.05922 & 0.002 & 0.79037 \\
\hline X3312n_G114 & 377.3 & 76.8 & 0.4604 & 0.07655 & 0.0021 & 2.0087 \\
\hline X3312n_G115 & 1939.9 & 50.9 & 0.8283 & 0.13723 & 0.00404 & 0.4477 \\
\hline X3312n_G116 & 727.7 & 61.8 & 0.2039 & 0.08131 & 0.00226 & 0.96418 \\
\hline X3312n_G117 & 232.7 & 35.4 & 0.2351 & 0.07057 & 0.0022 & 1.49322 \\
\hline X3312n_G118 & 499.3 & 80.8 & 0.2448 & 0.07194 & 0.00196 & 1.60175 \\
\hline X3312n G119 & 146.4 & 20.3 & 0.5277 & 0.06801 & 0.00252 & 1.19898 \\
\hline X3312n_G120 & 56.6 & 5.2 & 0.2584 & 0.06708 & 0.00388 & 0.86403 \\
\hline X3312n_G121 & 18.3 & 2.5 & 0.2576 & 0.0706 & 0.00586 & 1.32403 \\
\hline X3312n_G122 & 233.5 & 33.9 & 0.5812 & 0.07103 & 0.00232 & 1.31613 \\
\hline X3312n_G123 & 1074.5 & 98.5 & 1.2295 & 0.06466 & 0.00182 & 0.67179 \\
\hline X3312n_G124 & 180.4 & 97.3 & 0.5115 & 0.18764 & 0.00486 & 12.3844 \\
\hline X3312n_G125 & 228.3 & 17.7 & 0.3787 & 0.07239 & 0.00276 & 0.74831 \\
\hline X3312n_G126 & 157.7 & 18.9 & 0.3964 & 0.06495 & 0.00242 & 1.03708 \\
\hline X3312n_G127 & 135.1 & 5.7 & 0.6447 & 0.05058 & 0.00332 & 0.26323 \\
\hline X3312n_G128 & 184.7 & 6.6 & 0.7318 & 0.02541 & 0.0139 & 0.11393 \\
\hline X3312n_G129 & 1107.6 & 90.3 & 0.3778 & 0.04287 & 0.0017 & 0.4514 \\
\hline X3312n_G130 & 244 & 19.3 & 0.3238 & 0.06865 & 0.00266 & 0.71705 \\
\hline X3312n_G131 & 1257.5 & 3.5 & 2.3124 & 0.04844 & 0.00542 & 0.0113 \\
\hline X3312n G132 & 1859.7 & 95 & 0.1223 & 0.10083 & 0.00272 & 0.73451 \\
\hline X3312n_G133 & 152.5 & 81.7 & 0.2339 & 0.17796 & 0.00476 & 12.18623 \\
\hline X3312n_G134 & 368.6 & 72 & 0.5944 & 0.07403 & 0.00214 & 1.79447 \\
\hline X3312n_G135 & 570.8 & 50.5 & 0.4299 & 0.05936 & 0.00184 & 0.6895 \\
\hline X3312n_G136 & 725 & 99 & 0.2145 & 0.07279 & 0.002 & 1.38479 \\
\hline X3312n_G137 & 110.7 & 13.3 & 0.9991 & 0.0624 & 0.0028 & 0.84023 \\
\hline X3312n_G138 & 36.6 & 0.6 & 0.5429 & 0.76495 & 0.04982 & 3.87665 \\
\hline X3312n_G139 & 289.3 & 13.5 & 0.6451 & 0.0551 & 0.00256 & 0.31838 \\
\hline X3312n_G140 & 325.1 & 30.7 & 0.2753 & 0.06325 & 0.00214 & 0.82745 \\
\hline X3312n_G141 & 252.7 & 101.9 & 0.3921 & 0.15935 & 0.00428 & 8.26436 \\
\hline X3312n_G142 & 146.4 & 15.1 & 0.3751 & 0.06224 & 0.00266 & 0.85635 \\
\hline X3312n_G143 & 397.4 & 46.4 & 0.5571 & 0.06392 & 0.00208 & 0.94438 \\
\hline X3312n_G144 & 295.4 & 134.5 & 0.3642 & 0.16373 & 0.00434 & 9.51822 \\
\hline X3312n_G145 & 189.1 & 19.7 & 0.4178 & 0.06649 & 0.00248 & 0.91127 \\
\hline X3312n_G146 & 1413.5 & 65.9 & 0.1489 & 0.11076 & 0.00312 & 0.72799 \\
\hline X3312n_G147 & 129 & 17.4 & 0.2443 & 0.06831 & 0.00262 & 1.27103 \\
\hline X3312n_G148 & 248.4 & 36.1 & 0.5146 & 0.06985 & 0.00228 & 1.30723 \\
\hline X3312n_G149 & 258 & 34.4 & 0.3511 & 0.06846 & 0.00224 & 1.22535 \\
\hline X3312n_G150 & 501.1 & 45.4 & 0.4124 & 0.05822 & 0.00188 & 0.69486 \\
\hline X3312n_G151 & 226.6 & 31.8 & 0.3562 & 0.06954 & 0.00234 & 1.30357 \\
\hline X3312n_G152 & 212.6 & 121.3 & 1.0415 & 0.16121 & 0.00442 & 9.87922 \\
\hline X3312n_G153 & 149 & 25 & 0.7392 & 0.0703 & 0.00256 & 1.41438 \\
\hline X3312n_G154 & 380 & 33.7 & 0.2976 & 0.06221 & 0.00212 & 0.75807 \\
\hline X3312n_G155 & 41.8 & 4.6 & 0.2649 & 0.07651 & 0.00456 & 1.15056 \\
\hline X3312n_G156 & 1094.5 & 85.1 & 0.4134 & 0.08997 & 0.0026 & 0.92178 \\
\hline X3312n_G157 & 912.4 & 294 & 0.1114 & 0.12442 & 0.00334 & 5.59961 \\
\hline X3312n_G158 & 301.5 & 61.2 & 0.9058 & 0.07524 & 0.00232 & 1.74988 \\
\hline X3312S_G001 & 99.2 & 11.3 & 0.6691 & 0.06749 & 0.00306 & 0.94226 \\
\hline X3312S_G002 & 58.8 & 6.2 & 0.4672 & 0.05904 & 0.00362 & 0.81273 \\
\hline
\end{tabular}




$\begin{array}{lcccccc}\text { X3312S_G003 } & 299 & 29.1 & 0.1997 & 0.0707 & 0.00248 & 0.96958 \\ \text { X3312S_G004 } & & & & 0.7748 & 0.21732 & \\ \text { X3312S_G005 } & 572.7 & 19.7 & 0.607 & 0.19195 & 0.01166 & 0.83047 \\ \text { X3312S_G006 } & 119 & 19 & 3.3174 & 0.05949 & 0.00274 & 0.68829 \\ \text { X3312S_G007 } & 24.3 & 5.3 & 1.0323 & 0.08112 & 0.00474 & 2.00083 \\ \text { X3312S_G008 } & & & & 0.05945 & 0.0018 & 0.70284 \\ \text { X3312S_G009 } & 68.9 & 13.3 & 0.8917 & 0.06879 & 0.00304 & 1.56117 \\ \text { X3312S_G010 } & 158 & 30.6 & 0.8983 & 0.07031 & 0.0027 & 1.58476\end{array}$




\section{5 $\quad 76.8 \%$ concordant}

\begin{tabular}{|c|c|c|c|c|c|c|}
\hline $2 \sigma 75$ & $\mathrm{~Pb} 206 / \mathrm{U} 238$ & $2 \sigma 68$ & $\begin{array}{c}\text { ages } \\
\text { age } 206 / 238\end{array}$ & $2 \sigma$ age 68 & age $207 / 235$ & $2 \sigma$ age 75 \\
\hline 0.33382 & 0.4469 & 0.01394 & 2381.5 & 62.2 & 2391.7 & 41.6 \\
\hline 0.03386 & 0.12298 & 0.0032 & 747.7 & 18.4 & 758.3 & 20.6 \\
\hline 0.04556 & 0.15467 & 0.00402 & 927.1 & 22.4 & 951.8 & 23.2 \\
\hline 0.04492 & 0.15057 & 0.00394 & 904.2 & 22 & 925.5 & 23.4 \\
\hline 0.02316 & 0.09353 & 0.00242 & 576.4 & 14.2 & 570.7 & 16.8 \\
\hline 0.02092 & 0.09607 & 0.0024 & 591.3 & 14.2 & 600.4 & 15.4 \\
\hline 0.03012 & 0.11886 & 0.00302 & 724 & 17.4 & 743 & 19 \\
\hline 0.0307 & 0.11235 & 0.003 & 686.4 & 17.4 & 643.2 & 20.4 \\
\hline 0.05056 & 0.16546 & 0.00446 & 987 & 24.6 & 926.8 & 25.6 \\
\hline 0.02154 & 0.08905 & 0.00232 & 549.9 & 13.8 & 521.9 & 16.4 \\
\hline 0.04254 & 0.12958 & 0.00358 & 785.5 & 20.4 & 767.5 & 24.8 \\
\hline 0.02818 & 0.1049 & 0.00278 & 643.1 & 16.2 & 614.4 & 19.2 \\
\hline 0.3505 & 0.51391 & 0.01318 & 2673.3 & 56.2 & 2728.6 & 32 \\
\hline 0.02598 & 0.10947 & 0.0028 & 669.7 & 16.2 & 646.6 & 17.8 \\
\hline 0.01732 & 0.06346 & 0.00166 & 396.6 & 10 & 423.2 & 14.4 \\
\hline 0.05606 & 0.09774 & 0.00244 & 601.2 & 14.4 & 1203.6 & 23.2 \\
\hline 0.00102 & 0.0043 & 0.00012 & 27.7 & 0.8 & 27.8 & 1.2 \\
\hline 0.045 & 0.16665 & 0.00426 & 993.6 & 23.6 & 959.2 & 22.8 \\
\hline 0.03274 & 0.10624 & 0.00276 & 650.9 & 16 & 744.8 & 20.4 \\
\hline 0.05222 & 0.09456 & 0.0034 & 582.4 & 20 & 565.3 & 35 \\
\hline 0.03882 & 0.15348 & 0.00388 & 920.4 & 21.6 & 888.3 & 21 \\
\hline 0.28398 & 0.45292 & 0.01244 & 2408.2 & 55.2 & 2407.6 & 35.2 \\
\hline 0.02546 & 0.09569 & 0.0025 & 589.1 & 14.8 & 580.3 & 18 \\
\hline 0.04238 & 0.16288 & 0.00412 & 972.8 & 22.8 & 939.9 & 21.8 \\
\hline 0.03388 & 0.13544 & 0.00344 & 818.8 & 19.6 & 794.4 & 20 \\
\hline 0.04496 & 0.14481 & 0.00388 & 871.8 & 21.8 & 849.6 & 24.4 \\
\hline 0.02314 & 0.09312 & 0.00232 & 574 & 13.6 & 643.3 & 16.2 \\
\hline 0.02976 & 0.09372 & 0.0025 & 577.5 & 14.8 & 642 & 20 \\
\hline 0.03304 & 0.13016 & 0.00332 & 788.8 & 19 & 763.6 & 20 \\
\hline 0.0318 & 0.12196 & 0.00314 & 741.8 & 18 & 726.6 & 19.8 \\
\hline 0.03474 & 0.10524 & 0.00294 & 645 & 17.2 & 626.5 & 22.8 \\
\hline 0.02482 & 0.10656 & 0.0027 & 652.7 & 15.8 & 630.5 & 17.2 \\
\hline 0.02408 & 0.10389 & 0.00264 & 637.2 & 15.4 & 604.7 & 17 \\
\hline 0.0271 & 0.05441 & 0.00164 & 341.5 & 10 & 481 & 21 \\
\hline 0.0317 & 0.10165 & 0.00278 & 624.1 & 16.2 & 619.5 & 21.4 \\
\hline 0.03 & 0.07898 & 0.00206 & 490 & 12.4 & 700.5 & 19.6 \\
\hline 0.34656 & 0.52005 & 0.01326 & 2699.4 & 56.2 & 2706.2 & 32.2 \\
\hline 0.08664 & 0.09136 & 0.00424 & 563.6 & 25 & 668.1 & 53 \\
\hline 0.044 & 0.16946 & 0.00424 & 1009.1 & 23.4 & 986.1 & 21.8 \\
\hline 0.04654 & 0.13313 & 0.0037 & 805.7 & 21 & 808.8 & 26 \\
\hline 0.03528 & 0.12545 & 0.00328 & 761.9 & 18.8 & 743.9 & 21.4 \\
\hline 0.04356 & 0.14582 & 0.00374 & 877.5 & 21 & 922.3 & 22.6 \\
\hline 0.0266 & 0.09384 & 0.0025 & 578.2 & 14.8 & 570 & 18.8 \\
\hline 0.01776 & 0.0526 & 0.00134 & 330.5 & 8.2 & 495.6 & 14.2 \\
\hline 0.03136 & 0.10565 & 0.0027 & 647.4 & 15.8 & 733.8 & 19.6 \\
\hline 0.26976 & 0.45802 & 0.01176 & 2430.8 & 52 & 2442 & 32.2 \\
\hline 0.04648 & 0.15938 & 0.004 & 953.3 & 22.2 & 1014.2 & 22.4 \\
\hline 0.03566 & 0.09043 & 0.00272 & 558.1 & 16 & 547.7 & 24.8 \\
\hline 0.03512 & 0.10066 & 0.00286 & 618.3 & 16.8 & 604.3 & 23.4 \\
\hline 0.01116 & 0.00459 & 0.0005 & 29.5 & 3.2 & 23.9 & 11.6 \\
\hline
\end{tabular}




\begin{tabular}{|c|c|c|c|c|c|c|}
\hline 0.02036 & 0.08108 & 0.0021 & 502.6 & 12.6 & 487.2 & 15.8 \\
\hline 0.17648 & 0.36712 & 0.0091 & 2015.8 & 43 & 2102.3 & 29.6 \\
\hline 0.01178 & 0.03581 & 0.00092 & 226.8 & 5.8 & 340.2 & 10.8 \\
\hline 0.03226 & 0.09972 & 0.00274 & 612.8 & 16 & 602.6 & 21.6 \\
\hline 0.0357 & 0.13507 & 0.00336 & 816.7 & 19 & 852.8 & 20 \\
\hline 0.0427 & 0.16075 & 0.004 & 960.9 & 22.2 & 959.9 & 21.6 \\
\hline 0.04256 & 0.12849 & 0.0035 & 779.2 & 20 & 770.3 & 24.6 \\
\hline 0.02044 & 0.05348 & 0.00134 & 335.9 & 8.2 & 579 & 15.2 \\
\hline 0.02848 & 0.08566 & 0.00224 & 529.8 & 13.4 & 642.8 & 19.2 \\
\hline 0.02118 & 0.07103 & 0.00178 & 442.4 & 10.8 & 575.1 & 15.6 \\
\hline 0.0518 & 0.13811 & 0.00398 & 834 & 22.6 & 800.5 & 28.6 \\
\hline 0.03918 & 0.15279 & 0.0038 & 916.6 & 21.2 & 910.7 & 20.8 \\
\hline 0.04606 & 0.12792 & 0.00362 & 776 & 20.6 & 755.5 & 26.6 \\
\hline 0.05316 & 0.14436 & 0.00408 & 869.3 & 23 & 847.7 & 28.2 \\
\hline 0.01828 & 0.06419 & 0.00168 & 401.1 & 10.2 & 423.1 & 15 \\
\hline 0.09978 & 0.24878 & 0.00628 & 1432.2 & 32.4 & 1554.7 & 28.2 \\
\hline 0.12286 & 0.30643 & 0.00764 & 1723.1 & 37.6 & 1752.6 & 28.6 \\
\hline 0.0304 & 0.12167 & 0.00304 & 740.2 & 17.4 & 734.5 & 19 \\
\hline 0.02208 & 0.09636 & 0.0024 & 593 & 14.2 & 591.5 & 15.8 \\
\hline 0.03922 & 0.13057 & 0.00342 & 791.1 & 19.6 & 793.8 & 22.4 \\
\hline 0.048 & 0.09298 & 0.00302 & 573.1 & 17.8 & 622.5 & 31 \\
\hline 0.03586 & 0.12422 & 0.00318 & 754.8 & 18.2 & 788.7 & 21 \\
\hline 0.04314 & 0.15883 & 0.00396 & 950.3 & 22 & 953.9 & 21.8 \\
\hline 0.06318 & 0.14012 & 0.0039 & 845.3 & 22 & 1017.1 & 29.2 \\
\hline 0.02356 & 0.09154 & 0.00234 & 564.6 & 13.8 & 554.4 & 17 \\
\hline 0.0687 & 0.08204 & 0.0021 & 508.3 & 12.6 & 1276.6 & 26 \\
\hline 0.01358 & 0.0048 & 0.00046 & 30.9 & 3 & 42.4 & 14.4 \\
\hline 0.02632 & 0.08353 & 0.00226 & 517.2 & 13.4 & 516.7 & 19.2 \\
\hline 0.0269 & 0.09681 & 0.00252 & 595.7 & 14.8 & 596 & 18.6 \\
\hline 0.04806 & 0.10054 & 0.0032 & 617.6 & 18.8 & 630.5 & 30.6 \\
\hline 0.02816 & 0.09918 & 0.0026 & 609.6 & 15.2 & 601.6 & 19.4 \\
\hline 0.05436 & 0.17321 & 0.00444 & 1029.8 & 24.4 & 1037.4 & 24.8 \\
\hline 0.0541 & 0.17242 & 0.00444 & 1025.4 & 24.4 & 1024.4 & 25 \\
\hline 0.0327 & 0.11894 & 0.00298 & 724.5 & 17.2 & 759.9 & 19.8 \\
\hline 0.01442 & 0.05135 & 0.0013 & 322.8 & 8 & 391.1 & 12.4 \\
\hline 0.0232 & 0.09811 & 0.00244 & 603.3 & 14.4 & 606.2 & 16.4 \\
\hline 0.04734 & 0.1576 & 0.00402 & 943.4 & 22.4 & 950.6 & 23.6 \\
\hline 0.03916 & 0.11509 & 0.00304 & 702.2 & 17.6 & 771.3 & 22.8 \\
\hline 0.028 & 0.08702 & 0.00222 & 537.9 & 13.2 & 656 & 18.6 \\
\hline 0.02316 & 0.08572 & 0.0022 & 530.2 & 13 & 542.9 & 17 \\
\hline 0.02376 & 0.09412 & 0.00238 & 579.9 & 14 & 568.1 & 17 \\
\hline 0.30136 & 0.41046 & 0.01058 & 2217 & 48.4 & 2495 & 33.6 \\
\hline 0.03918 & 0.10231 & 0.00298 & 627.9 & 17.4 & 614.2 & 25.6 \\
\hline 0.02728 & 0.08507 & 0.00222 & 526.3 & 13.2 & 597.1 & 19 \\
\hline 0.0259 & 0.10417 & 0.00262 & 638.8 & 15.2 & 617 & 17.8 \\
\hline 0.03408 & 0.09679 & 0.00244 & 595.6 & 14.4 & 779.7 & 20.2 \\
\hline 0.06336 & 0.14226 & 0.00434 & 857.4 & 24.4 & 833.2 & 33.2 \\
\hline 0.02268 & 0.08488 & 0.00218 & 525.2 & 13 & 517.9 & 16.8 \\
\hline 0.03666 & 0.11953 & 0.00308 & 727.9 & 17.8 & 771.2 & 21.6 \\
\hline 0.02218 & 0.09074 & 0.00226 & 559.9 & 13.4 & 575.5 & 16 \\
\hline 0.06926 & 0.00959 & 0.00102 & 61.5 & 6.6 & 553.9 & 74.6 \\
\hline 0.04804 & 0.09031 & 0.003 & 557.4 & 17.8 & 579.1 & 31.8 \\
\hline 0.0545 & 0.10904 & 0.00358 & 667.2 & 20.8 & 658.1 & 33.6 \\
\hline 0.05632 & 0.13385 & 0.00388 & 809.8 & 22 & 828.8 & 30 \\
\hline
\end{tabular}




\begin{tabular}{|c|c|c|c|c|c|c|}
\hline 0.02916 & 0.08713 & 0.00234 & 538.5 & 13.8 & 577.4 & 20.2 \\
\hline 0.02074 & 0.06036 & 0.00156 & 377.8 & 9.4 & 498.9 & 16 \\
\hline 0.02488 & 0.06367 & 0.00172 & 397.9 & 10.4 & 521.8 & 18.4 \\
\hline 0.05204 & 0.14941 & 0.004 & 897.7 & 22.4 & 906.3 & 26.4 \\
\hline 0.03682 & 0.10325 & 0.0029 & 633.4 & 17 & 613.5 & 24.2 \\
\hline 0.02602 & 0.094 & 0.0024 & 579.2 & 14.2 & 589.5 & 18 \\
\hline 0.02932 & 0.09967 & 0.0025 & 612.5 & 14.6 & 691.3 & 18.8 \\
\hline 0.02792 & 0.09678 & 0.00252 & 595.5 & 14.8 & 591.4 & 19.2 \\
\hline 0.05982 & 0.19029 & 0.00478 & 1122.9 & 25.8 & 1118.5 & 25.2 \\
\hline 0.01382 & 0.02366 & 0.00062 & 150.7 & 4 & 375.7 & 12.4 \\
\hline 0.02884 & 0.08599 & 0.00216 & 531.8 & 12.8 & 685.5 & 18.6 \\
\hline 0.04908 & 0.15344 & 0.00396 & 920.2 & 22.2 & 927.6 & 24.6 \\
\hline 0.04756 & 0.16146 & 0.00404 & 964.9 & 22.4 & 970.9 & 23.2 \\
\hline 0.04558 & 0.12785 & 0.00346 & 775.6 & 19.8 & 800.1 & 25.4 \\
\hline 0.04896 & 0.0934 & 0.003 & 575.6 & 17.6 & 632.3 & 31.2 \\
\hline 0.10634 & 0.136 & 0.00552 & 822 & 31.4 & 856.3 & 53.4 \\
\hline 0.04504 & 0.13438 & 0.0035 & 812.8 & 19.8 & 852.8 & 24.2 \\
\hline 0.02036 & 0.07535 & 0.00188 & 468.3 & 11.2 & 521.8 & 15.4 \\
\hline 0.35498 & 0.47861 & 0.01208 & 2521.2 & 52.6 & 2634 & 34 \\
\hline 0.02904 & 0.07496 & 0.00204 & 466 & 12.2 & 567.2 & 20.4 \\
\hline 0.0396 & 0.11579 & 0.0031 & 706.3 & 18 & 722.5 & 23.8 \\
\hline 0.01686 & 0.03774 & 0.00118 & 238.8 & 7.4 & 237.3 & 15.4 \\
\hline 0.062 & 0.03252 & 0.00212 & 206.3 & 13.2 & 109.6 & 57.2 \\
\hline 0.01866 & 0.07636 & 0.0019 & 474.4 & 11.4 & 378.3 & 14.8 \\
\hline 0.02822 & 0.07575 & 0.00204 & 470.7 & 12.2 & 548.9 & 20 \\
\hline 0.00122 & 0.00169 & 0.00006 & 10.9 & 0.4 & 11.4 & 1.4 \\
\hline 0.02156 & 0.05282 & 0.0013 & 331.8 & 8 & 559.2 & 15.6 \\
\hline 0.3577 & 0.49657 & 0.01262 & 2599 & 54.4 & 2618.8 & 34.6 \\
\hline 0.05562 & 0.17579 & 0.00442 & 1043.9 & 24.2 & 1043.5 & 25 \\
\hline 0.0226 & 0.08424 & 0.00212 & 521.4 & 12.6 & 532.5 & 16.6 \\
\hline 0.04138 & 0.13796 & 0.00342 & 833.1 & 19.4 & 882.5 & 21.8 \\
\hline 0.03774 & 0.09765 & 0.00276 & 600.6 & 16.2 & 619.3 & 24.6 \\
\hline 0.1841 & 0.03675 & 0.00204 & 232.7 & 12.6 & 1608.8 & 69 \\
\hline 0.01476 & 0.0419 & 0.00116 & 264.6 & 7.2 & 280.7 & 13.2 \\
\hline 0.02914 & 0.09488 & 0.00246 & 584.3 & 14.4 & 612.2 & 19.6 \\
\hline 0.24294 & 0.37611 & 0.00944 & 2058.1 & 44.2 & 2260.4 & 33.2 \\
\hline 0.03678 & 0.09977 & 0.00276 & 613.1 & 16.2 & 628.1 & 23.8 \\
\hline 0.03222 & 0.10715 & 0.00274 & 656.2 & 16 & 675.2 & 20.4 \\
\hline 0.27692 & 0.42158 & 0.01048 & 2267.6 & 47.6 & 2389.3 & 33.4 \\
\hline 0.03468 & 0.0994 & 0.00264 & 610.9 & 15.4 & 657.7 & 22.2 \\
\hline 0.02202 & 0.04767 & 0.00118 & 300.2 & 7.2 & 555.4 & 16 \\
\hline 0.04964 & 0.13495 & 0.00364 & 816 & 20.6 & 832.9 & 26.6 \\
\hline 0.04454 & 0.13572 & 0.00348 & 820.4 & 19.8 & 848.9 & 23.8 \\
\hline 0.04194 & 0.12981 & 0.00334 & 786.8 & 19 & 812.2 & 23.2 \\
\hline 0.02348 & 0.08655 & 0.0022 & 535.1 & 13 & 535.7 & 17.2 \\
\hline 0.04566 & 0.13595 & 0.00352 & 821.7 & 20 & 847.3 & 24.4 \\
\hline 0.29488 & 0.44442 & 0.01114 & 2370.4 & 49.8 & 2423.6 & 34.2 \\
\hline 0.05304 & 0.14591 & 0.00388 & 878 & 21.8 & 895 & 26.8 \\
\hline 0.02682 & 0.08837 & 0.00228 & 545.9 & 13.6 & 572.9 & 18.8 \\
\hline 0.06684 & 0.10906 & 0.00362 & 667.3 & 21 & 777.5 & 37 \\
\hline 0.0284 & 0.0743 & 0.00184 & 462 & 11 & 663.3 & 18.6 \\
\hline 0.16428 & 0.3264 & 0.008 & 1820.9 & 38.8 & 1916 & 31.4 \\
\hline 0.05704 & 0.16867 & 0.00428 & 1004.8 & 23.6 & 1027.1 & 25.8 \\
\hline 0.0418 & 0.1013 & 0.00276 & 622 & 16.2 & 674.1 & 26 \\
\hline 0.0482 & 0.09988 & 0.0031 & 613.7 & 18.2 & 604 & 31.2 \\
\hline
\end{tabular}




$\begin{array}{ccccccc}0.03386 & 0.0995 & 0.00244 & 611.5 & 14.4 & 688.2 & 21.4 \\ 978.01856 & -7.97338 & 9.22612 & & & & \\ 0.04456 & 0.03139 & 0.00122 & 199.2 & 7.6 & 613.9 & 33.2 \\ 0.03102 & 0.08394 & 0.00226 & 519.6 & 13.4 & 531.8 & 22 \\ 0.11346 & 0.17896 & 0.00594 & 1061.3 & 32.4 & 1115.8 & 45.6 \\ 0.0216 & 0.08577 & 0.002 & & & & \\ 0.06774 & 0.16466 & 0.0045 & 982.6 & 25 & 954.9 & 32.2 \\ 0.06018 & 0.16354 & 0.0042 & 976.4 & 23.2 & 964.2 & 28.8\end{array}$




\begin{tabular}{|c|c|c|c|c|c|}
\hline age $207 / 206$ & $2 \sigma$ age 76 & $\begin{array}{l}\text { discordance } \\
\Delta 68-75[\%]\end{array}$ & $\Delta 68-76[\%]$ & $\begin{array}{c}\text { preferred age } \\
\text { age }\end{array}$ & $2 \sigma$ age \\
\hline 2403.8 & 55.8 & -0.4 & -0.9 & 2403.8 & 55.8 \\
\hline 794 & 59.4 & -1.4 & -5.8 & 747.7 & 18.4 \\
\hline 1013.4 & 55 & -2.6 & -8.5 & 927.1 & 22.4 \\
\hline 980.3 & 57.4 & -2.3 & -7.8 & 904.2 & 22 \\
\hline 552.3 & 62.6 & 1 & 4.4 & 576.4 & 14.2 \\
\hline 638.9 & 49.4 & -1.5 & -7.4 & 591.3 & 14.2 \\
\hline 804.2 & 53.4 & -2.6 & -10 & 724 & 17.4 \\
\hline 498.1 & 73.2 & 6.7 & 37.8 & & \\
\hline 789.8 & 68 & 6.5 & 25 & & \\
\hline 404.9 & 67.8 & 5.4 & 35.8 & & \\
\hline 719 & 77.8 & 2.3 & 9.2 & 785.5 & 20.4 \\
\hline 513.8 & 71 & 4.7 & 25.2 & 643.1 & 16.2 \\
\hline 2772.4 & 36.2 & -2 & -3.6 & 2772.4 & 36.2 \\
\hline 570.4 & 58.2 & 3.6 & 17.4 & 669.7 & 16.2 \\
\hline 574.5 & 69.8 & -6.3 & -31 & 396.6 & 10 \\
\hline 2546.2 & 36 & -50.1 & -76.4 & & \\
\hline 47.7 & 84.4 & -0.6 & -42 & 27.7 & 0.8 \\
\hline 884.3 & 54.4 & 3.6 & 12.4 & 993.6 & 23.6 \\
\hline 1041.5 & 57.2 & -12.6 & -37.5 & 650.9 & 16 \\
\hline 500 & 159.4 & 3 & 16.5 & 582.4 & 20 \\
\hline 812.1 & 52.4 & 3.6 & 13.3 & 920.4 & 21.6 \\
\hline 2409.4 & 45 & 0 & 0 & 2409.4 & 45 \\
\hline 548.9 & 68.6 & 1.5 & 7.3 & 589.1 & 14.8 \\
\hline 866.4 & 51.8 & 3.5 & 12.3 & 972.8 & 22.8 \\
\hline 729.4 & 55.2 & 3.1 & 12.3 & 818.8 & 19.6 \\
\hline 794.9 & 69 & 2.6 & 9.7 & 871.8 & 21.8 \\
\hline 897.5 & 47.8 & -10.8 & -36.1 & 574 & 13.6 \\
\hline 878.6 & 67.2 & -10 & -34.3 & 577.5 & 14.8 \\
\hline 693.3 & 58 & 3.3 & 13.8 & 788.8 & 19 \\
\hline 682.4 & 60.4 & 2.1 & 8.7 & 741.8 & 18 \\
\hline 562.7 & 87.4 & 3 & 14.6 & 645 & 17.2 \\
\hline 554.1 & 57.2 & 3.5 & 17.8 & 652.7 & 15.8 \\
\hline 487.7 & 60.6 & 5.4 & 30.7 & & \\
\hline 1218 & 90 & -29 & -72 & & \\
\hline 605.4 & 80 & 0.7 & 3.1 & 624.1 & 16.2 \\
\hline 1453.5 & 54.2 & -30 & -66.3 & & \\
\hline 2712.9 & 37.2 & -0.2 & -0.5 & 2712.9 & 37.2 \\
\hline 1040.6 & 197.6 & -15.6 & -45.8 & & \\
\hline 937.1 & 49 & 2.3 & 7.7 & 1009.1 & 23.4 \\
\hline 819.3 & 78 & -0.4 & -1.7 & 805.7 & 21 \\
\hline 692.3 & 66.2 & 2.4 & 10 & 761.9 & 18.8 \\
\hline 1032.7 & 54.8 & -4.9 & -15 & 877.5 & 21 \\
\hline 538.8 & 74.4 & 1.4 & 7.3 & 578.2 & 14.8 \\
\hline 1356.5 & 49.8 & -33.3 & -75.6 & & \\
\hline 1008.9 & 55.8 & -11.8 & -35.8 & 647.4 & 15.8 \\
\hline 2452.7 & 39.8 & -0.5 & -0.9 & 2452.7 & 39.8 \\
\hline 1149.4 & 48.4 & -6 & -17.1 & 953.3 & 22.2 \\
\hline 506.6 & 110.4 & 1.9 & 10.2 & 558.1 & 16 \\
\hline 553.8 & 93.8 & 2.3 & 11.7 & 618.3 & 16.8 \\
\hline-511.3 & 1282.2 & 23.7 & -105.8 & & \\
\hline
\end{tabular}




\begin{tabular}{|c|c|c|c|c|c|}
\hline 416.7 & 70.6 & 3.2 & 20.6 & 502.6 & 12.6 \\
\hline 2189.2 & 38.6 & -4.1 & -7.9 & 2189.2 & 38.6 \\
\hline 1212.9 & 54.2 & -33.3 & -81.3 & & \\
\hline 565.6 & 85 & 1.7 & 8.3 & 612.8 & 16 \\
\hline 949 & 49.8 & -4.2 & -13.9 & 816.7 & 19 \\
\hline 958.9 & 49.4 & 0.1 & 0.2 & 960.9 & 22.2 \\
\hline 745.9 & 77.2 & 1.2 & 4.5 & 779.2 & 20 \\
\hline 1702 & 43.8 & -42 & -80.3 & & \\
\hline 1063.9 & 61.8 & -17.6 & -50.2 & & \\
\hline 1142.3 & 50 & -23.1 & -61.3 & & \\
\hline 709.2 & 91 & 4.2 & 17.6 & 834 & 22.6 \\
\hline 897.5 & 49.6 & 0.6 & 2.1 & 916.6 & 21.2 \\
\hline 696 & 87.8 & 2.7 & 11.5 & 776 & 20.6 \\
\hline 792.7 & 83.8 & 2.5 & 9.7 & 869.3 & 23 \\
\hline 545.9 & 74.8 & -5.2 & -26.5 & 401.1 & 10.2 \\
\hline 1726.2 & 45.6 & -7.9 & -17 & & \\
\hline 1788.6 & 43 & -1.7 & -3.7 & 1788.6 & 43 \\
\hline 718 & 55.6 & 0.8 & 3.1 & 740.2 & 17.4 \\
\hline 586.5 & 54.6 & 0.3 & 1.1 & 593 & 14.2 \\
\hline 802.2 & 65.6 & -0.3 & -1.4 & 791.1 & 19.6 \\
\hline 807 & 121.8 & -7.9 & -29 & 573.1 & 17.8 \\
\hline 886.4 & 58.4 & -4.3 & -14.8 & 754.8 & 18.2 \\
\hline 962.9 & 51 & -0.4 & -1.3 & 950.3 & 22 \\
\hline 1408.5 & 68.6 & -16.9 & -40 & & \\
\hline 513.1 & 67.2 & 1.8 & 10.1 & 564.6 & 13.8 \\
\hline 2998.5 & 40.4 & -60.2 & -83 & & \\
\hline 756.1 & 698.8 & -27.2 & -95.9 & & \\
\hline 515 & 85.4 & 0.1 & 0.4 & 517.2 & 13.4 \\
\hline 597.8 & 69.4 & -0.1 & -0.4 & 595.7 & 14.8 \\
\hline 677.2 & 121.8 & -2 & -8.8 & 617.6 & 18.8 \\
\hline 571.5 & 72.8 & 1.3 & 6.7 & 609.6 & 15.2 \\
\hline 1054 & 57.4 & -0.7 & -2.3 & 1029.8 & 24.4 \\
\hline 1022.5 & 58.6 & 0.1 & 0.3 & 1025.4 & 24.4 \\
\hline 866.1 & 56.2 & -4.7 & -16.4 & 724.5 & 17.2 \\
\hline 818.7 & 59.8 & -17.5 & -60.6 & & \\
\hline 617.2 & 55.8 & -0.5 & -2.3 & 603.3 & 14.4 \\
\hline 967.7 & 57.8 & -0.8 & -2.5 & 943.4 & 22.4 \\
\hline 977.2 & 67 & -9 & -28.1 & 702.2 & 17.6 \\
\hline 1086.9 & 57.8 & -18 & -50.5 & & \\
\hline 597.1 & 67.4 & -2.3 & -11.2 & 530.2 & 13 \\
\hline 521 & 65.4 & 2.1 & 11.3 & 579.9 & 14 \\
\hline 2729.8 & 42 & -11.1 & -18.8 & & \\
\hline 564.1 & 102.8 & 2.2 & 11.3 & 627.9 & 17.4 \\
\hline 876.5 & 68 & -11.9 & -39.9 & 526.3 & 13.2 \\
\hline 537.7 & 63.2 & 3.5 & 18.8 & 638.8 & 15.2 \\
\hline 1350.9 & 52.6 & -23.6 & -55.9 & & \\
\hline 769.1 & 105.2 & 2.9 & 11.5 & 857.4 & 24.4 \\
\hline 485.7 & 71.4 & 1.4 & 8.1 & 525.2 & 13 \\
\hline 899 & 62.8 & -5.6 & -19 & 727.9 & 17.8 \\
\hline 637.8 & 57.2 & -2.7 & -12.2 & 559.9 & 13.4 \\
\hline 4377.2 & 203.2 & -88.9 & -98.6 & & \\
\hline 665.1 & 137.4 & -3.7 & -16.2 & 557.4 & 17.8 \\
\hline 627.2 & 131.4 & 1.4 & 6.4 & 667.2 & 20.8 \\
\hline 880.1 & 92 & -2.3 & -8 & 809.8 & 22 \\
\hline
\end{tabular}




\begin{tabular}{|c|c|c|c|c|c|}
\hline 733.3 & 79 & -6.7 & -26.6 & 538.5 & 13.8 \\
\hline 1101.1 & 62.4 & -24.3 & -65.7 & & \\
\hline 1109 & 72.6 & -23.7 & -64.1 & & \\
\hline 927.2 & 71.6 & -1 & -3.2 & 897.7 & 22.4 \\
\hline 540.7 & 96.8 & 3.2 & 17.2 & 633.4 & 17 \\
\hline 629.3 & 67.4 & -1.8 & -8 & 579.2 & 14.2 \\
\hline 956.8 & 57 & -11.4 & -36 & 612.5 & 14.6 \\
\hline 575.2 & 73.4 & 0.7 & 3.5 & 595.5 & 14.8 \\
\hline 1109.5 & 54.8 & 0.4 & 1.2 & 1109.5 & 54.8 \\
\hline 2192.5 & 51.2 & -59.9 & -93.1 & & \\
\hline 1228.9 & 54.6 & -22.4 & -56.7 & & \\
\hline 945 & 63.8 & -0.8 & -2.6 & 920.2 & 22.2 \\
\hline 984.2 & 55.4 & -0.6 & -2 & 964.9 & 22.4 \\
\hline 868.9 & 76.8 & -3.1 & -10.7 & 775.6 & 19.8 \\
\hline 840.3 & 120.4 & -9 & -31.5 & 575.6 & 17.6 \\
\hline 945.9 & 170 & -4 & -13.1 & 822 & 31.4 \\
\hline 958.3 & 66.8 & -4.7 & -15.2 & 812.8 & 19.8 \\
\hline 763.3 & 59.4 & -10.3 & -38.6 & 468.3 & 11.2 \\
\hline 2721.6 & 42.6 & -4.3 & -7.4 & 2721.6 & 42.6 \\
\hline 996.9 & 77.4 & -17.9 & -53.3 & & \\
\hline 772.7 & 78.4 & -2.2 & -8.6 & 706.3 & 18 \\
\hline \multirow[t]{2}{*}{221.7} & 151.8 & 0.7 & 7.7 & 238.8 & 7.4 \\
\hline & & 88.3 & & & \\
\hline-175.2 & 98.8 & 25.4 & -370.8 & & \\
\hline 888.2 & 80 & -14.2 & -47 & 470.7 & 12.2 \\
\hline 120.8 & 263.6 & -4.6 & -91 & 10.9 & 0.4 \\
\hline 1639.4 & 50 & -40.7 & -79.8 & & \\
\hline 2634 & 44.4 & -0.8 & -1.3 & 2634 & 44.4 \\
\hline 1042.3 & 58.4 & 0 & 0.2 & 1043.9 & 24.2 \\
\hline 580.3 & 67.4 & -2.1 & -10.2 & 521.4 & 12.6 \\
\hline 1008.1 & 55.8 & -5.6 & -17.4 & 833.1 & 19.4 \\
\hline 687.8 & 95.8 & -3 & -12.7 & 600.6 & 16.2 \\
\hline 4857.8 & 93 & -85.5 & -95.2 & & \\
\hline 416.3 & 103.8 & -5.7 & -36.4 & 264.6 & 7.2 \\
\hline 716.6 & 71.8 & -4.6 & -18.5 & 584.3 & 14.4 \\
\hline 2448.8 & 45.4 & -9 & -16 & & \\
\hline 682.4 & 91.2 & -2.4 & -10.2 & 613.1 & 16.2 \\
\hline 739 & 68.8 & -2.8 & -11.2 & 656.2 & 16 \\
\hline 2494.5 & 44.6 & -5.1 & -9.1 & 2494.5 & 44.6 \\
\hline 821.8 & 77.8 & -7.1 & -25.7 & 610.9 & 15.4 \\
\hline 1811.9 & 51.2 & -45.9 & -83.4 & & \\
\hline 878 & 79.4 & -2 & -7.1 & 816 & 20.6 \\
\hline 923.9 & 67 & -3.4 & -11.2 & 820.4 & 19.8 \\
\hline 882.5 & 67.6 & -3.1 & -10.8 & 786.8 & 19 \\
\hline 538.1 & 70.6 & -0.1 & -0.5 & 535.1 & 13 \\
\hline 914.8 & 69.2 & -3 & -10.2 & 821.7 & 20 \\
\hline 2468.4 & 46.2 & -2.2 & -4 & 2468.4 & 46.2 \\
\hline 937.1 & 74.6 & -1.9 & -6.3 & 878 & 21.8 \\
\hline 681.3 & 72.8 & -4.7 & -19.9 & 545.9 & 13.6 \\
\hline 1108.4 & 119 & -14.2 & -39.8 & 667.3 & 21 \\
\hline 1424.9 & 55.2 & -30.3 & -67.6 & & \\
\hline 2020.6 & 47.6 & -5 & -9.9 & 2020.6 & 47.6 \\
\hline 1074.9 & 62 & -2.2 & -6.5 & 1004.8 & 23.6 \\
\hline 852.9 & 94.2 & -7.7 & -27.1 & 622 & 16.2 \\
\hline 568.6 & 133.4 & 1.6 & 7.9 & 613.7 & 18.2 \\
\hline
\end{tabular}




$\begin{array}{cccccc}948.8 & 71.8 & -11.2 & -35.5 & 611.5 & 14.4 \\ 2758.9 & 99.8 & -67.5 & -92.8 & & \\ 585.1 & 100 & -2.3 & -11.2 & 519.6 & 13.4 \\ 1224.4 & 114.8 & -4.9 & -13.3 & 1061.3 & 32.4 \\ & & & & & \\ 892.4 & 91.2 & 2.9 & 10.1 & 982.6 & 25 \\ 937.4 & 78.8 & 1.3 & 4.2 & 976.4 & 23.2\end{array}$


Sample 2873S Atbara @ Showak 64 grain analysed 53 concordant ages :

\begin{tabular}{|c|c|c|c|c|c|c|}
\hline \multirow[b]{2}{*}{ grain } & \multicolumn{3}{|c|}{ concentrations } & \multicolumn{3}{|l|}{ isotopic ratios } \\
\hline & U [ppm] & $\mathrm{Pb}$ [ppm] & $\mathrm{Th} / \mathrm{U}$ & $\mathrm{Pb} 207 / \mathrm{Pb} 206$ & $2 \sigma 76$ & $\mathrm{~Pb} 207 / \mathrm{U} 235$ \\
\hline$\overline{X 2873 S \_G 001}$ & 542.4 & 59 & 0.8116 & 0.05906 & 0.00188 & 0.7596 \\
\hline X2873S_G002 & 67 & 0.4 & 0.9184 & 0.05571 & 0.02744 & 0.03745 \\
\hline X2873S_G003 & 366.9 & 130.6 & 0.5788 & 0.11114 & 0.00314 & 4.85604 \\
\hline X2873S_G004 & 869.2 & 37.7 & 0.5491 & 0.05209 & 0.00206 & 0.28975 \\
\hline X2873S_G005 & 124.3 & 17.9 & 0.6196 & 0.0678 & 0.0028 & 1.21492 \\
\hline X2873S G006 & 58.2 & 0.4 & 0.6841 & 0.08096 & 0.03252 & 0.05948 \\
\hline X2873S G007 & 34.5 & 5 & 0.6535 & 0.06157 & 0.00506 & 1.11186 \\
\hline X2873S_G008 & 284.9 & 27.8 & 0.4091 & 0.05894 & 0.0023 & 0.76064 \\
\hline X2873S_G009 & 92.2 & 10.1 & 0.3801 & 0.0591 & 0.00304 & 0.86808 \\
\hline X2873S_G010 & 133.6 & 14.8 & 0.7055 & 0.05622 & 0.00256 & 0.76416 \\
\hline X2873S_G011 & 78.2 & 9.6 & 1.0036 & 0.0606 & 0.0032 & 0.84072 \\
\hline X2873S_G012 & 310.5 & 47.5 & 0.7562 & 0.06447 & 0.00212 & 1.18307 \\
\hline X2873S G013 & 1971.7 & 77.2 & 0.5716 & 0.14162 & 0.00408 & 0.68209 \\
\hline X2873S_G014 & 140.1 & 22.3 & 0.9238 & 0.06569 & 0.00278 & 1.19542 \\
\hline X2873S_G015 & 161.1 & 23.5 & 0.4764 & 0.06541 & 0.0028 & 1.22985 \\
\hline X2873S_G016 & 344.5 & 29.5 & 0.4133 & 0.06326 & 0.00244 & 0.71743 \\
\hline X2873S_G017 & 120.6 & 14.1 & 1.1977 & 0.05746 & 0.0033 & 0.72754 \\
\hline X2873S G018 & 129 & 14 & 1.0018 & 0.05605 & 0.0031 & 0.68998 \\
\hline X2873S G019 & 416.7 & 73.6 & 0.4003 & 0.07339 & 0.00234 & 1.70904 \\
\hline X2873S_G020 & 88 & 18.6 & 0.6017 & 0.07324 & 0.0031 & 1.92774 \\
\hline X2873S_G021 & 69.4 & 9.8 & 0.6599 & 0.06379 & 0.00352 & 1.11929 \\
\hline X2873S_G022 & 232.8 & 28.5 & 0.9663 & 0.06206 & 0.00246 & 0.86482 \\
\hline X2873S_G023 & 1164.9 & 97.2 & 2.877 & 0.0779 & 0.00248 & 0.52642 \\
\hline X2873S_G024 & 221.1 & 26.1 & 0.7048 & 0.05985 & 0.00238 & 0.86468 \\
\hline X2873S_G025 & 999.6 & 376.9 & 0.0249 & 0.12788 & 0.0036 & 6.74416 \\
\hline X2873S_G026 & 191.8 & 22.9 & 1.3661 & 0.05726 & 0.00234 & 0.70848 \\
\hline X2873S_G027 & 371.5 & 237.7 & 0.0257 & 0.302 & 0.00844 & 26.98064 \\
\hline X2873S_G028 & 492.1 & 52.4 & 0.2491 & 0.05967 & 0.00194 & 0.88207 \\
\hline X2873S_G029 & 778 & 97.6 & 0.2938 & 0.09418 & 0.0029 & 1.64584 \\
\hline X2873S_G030 & 468.8 & 53.6 & 0.1486 & 0.0629 & 0.00214 & 1.02646 \\
\hline X2873S G031 & 2204 & 298.3 & 0.2743 & 0.16869 & 0.00502 & 3.26594 \\
\hline X2873S_G032 & 340.8 & 50.1 & 0.2195 & 0.06923 & 0.00234 & 1.41627 \\
\hline X2873S_G033 & 312.9 & 55 & 0.3197 & 0.06948 & 0.00234 & 1.6159 \\
\hline X2873S_G034 & 296.1 & 45.9 & 0.1424 & 0.0674 & 0.00234 & 1.48344 \\
\hline X2873S_G035 & 162.5 & 24.2 & 0.6215 & 0.06723 & 0.0026 & 1.24651 \\
\hline X2873S_G036 & 61.9 & 10.2 & 0.4715 & 0.07253 & 0.00382 & 1.54779 \\
\hline X2873S_G037 & 255.1 & 25.3 & 0.1367 & 0.06032 & 0.0024 & 0.85892 \\
\hline X2873S_G038 & 666.2 & 61.7 & 0.0187 & 0.06524 & 0.00218 & 0.89586 \\
\hline X2873S_G039 & 190 & 20.9 & 0.5662 & 0.05929 & 0.00238 & 0.82705 \\
\hline X2873S_G040 & 422.3 & 55.8 & 0.5042 & 0.06762 & 0.0024 & 1.16354 \\
\hline X2873S_G041 & 96.4 & 10.9 & 0.6784 & 0.05941 & 0.0033 & 0.83226 \\
\hline X2873S_G042 & 48 & 7.3 & 0.7122 & 0.06523 & 0.00442 & 1.2069 \\
\hline X2873S_G043 & 13 & & 0.6788 & 0.27647 & 0.01082 & 26.98205 \\
\hline X2873S G044 & 120.1 & 6.6 & 0.5174 & 0.05081 & 0.00332 & 0.35824 \\
\hline X2873S_G045 & 141.5 & 7.9 & 0.5694 & 0.05086 & 0.00306 & 0.35984 \\
\hline X2873S_G046 & 177.4 & 9.9 & 0.5705 & 0.05086 & 0.0028 & 0.35986 \\
\hline X2873S_G047 & 27.5 & 0.2 & 1.3281 & 0.05927 & 0.04714 & 0.04786 \\
\hline X2873S_G048 & 43.3 & 5.9 & 1.3364 & 0.05771 & 0.00468 & 0.83595 \\
\hline X2873S_G049 & 69.4 & 7.9 & 0.4809 & 0.05809 & 0.00374 & 0.85493 \\
\hline X2873S G050 & 197.4 & 28.7 & 0.1903 & 0.07679 & 0.0031 & 1.52764 \\
\hline
\end{tabular}




$\begin{array}{llcccccc}1 & & & & & \\ 2 & \text { X2873S_G051 } & 563.8 & 64.6 & 0.2827 & 0.07723 & 0.00258 & 1.22119 \\ 3 & \text { X2873S_G052 } & 93.6 & 62.9 & 1.6725 & 0.15765 & 0.00524 & 10.0762 \\ 4 & \text { X2873S_G053 } & 207.2 & 25.2 & 0.5062 & 0.05954 & 0.00252 & 0.86424 \\ 5 & \text { X2873S_G054 } & 163.4 & 19.1 & 0.5965 & 0.0592 & 0.00274 & 0.87192 \\ 6 & \text { X2873S_G055 } & 63.3 & 7 & 0.4951 & 0.0625 & 0.00364 & 0.89132 \\ 7 & \text { X2873S_G056 } & 87.5 & 46 & 0.9935 & 0.16023 & 0.00526 & 9.29114 \\ 8 & \text { X2873S_G057 } & 136.9 & 36.7 & 0.2108 & 0.13775 & 0.00494 & 5.02522 \\ 9 & \text { X2873S_G058 } & 162.5 & 9.1 & 0.5474 & 0.05503 & 0.00366 & 0.39108 \\ 10 & \text { X2873S_G059 } & 470.2 & 30.1 & 1.0652 & 0.05272 & 0.00234 & 0.37833 \\ 11 & \text { X2873S_G060 } & 289.6 & 38.7 & 0.5261 & 0.06627 & 0.00252 & 1.12724 \\ 12 & \text { X2873S_G061 } & 517.2 & 47 & 0.3535 & 0.07128 & 0.00254 & 0.86538 \\ 13 & \text { X2873S_G062 } & 32.1 & 3.8 & 1.0804 & 0.05613 & 0.00518 & 0.73451 \\ 14 & \text { X2873S_G063 } & 63.3 & 9.1 & 0.4332 & 0.06445 & 0.00346 & 1.21385 \\ 15 & \text { X2873S_G064 } & 377.1 & 81 & 1.2825 & 0.06546 & 0.00224 & 1.49795 \\ 16 & & & & & & & \end{array}$




\section{$82.8 \%$ concordant}

\begin{tabular}{|c|c|c|c|c|c|c|}
\hline $2 \sigma 75$ & $\mathrm{~Pb} 206 / \mathrm{U} 238$ & $2 \sigma 68$ & $\begin{array}{c}\text { ages } \\
\text { age } 206 / 238\end{array}$ & $2 \sigma$ age 68 & age $207 / 235$ & $2 \sigma$ age 75 \\
\hline 0.02452 & 0.09331 & 0.00226 & 575.1 & 13.4 & 573.8 & 17.6 \\
\hline 0.01792 & 0.00488 & 0.00058 & 31.4 & 3.8 & 37.3 & 18.6 \\
\hline 0.1423 & 0.31698 & 0.00764 & 1775 & 37.4 & 1794.7 & 31.2 \\
\hline 0.01138 & 0.04036 & 0.00102 & 255.1 & 6.4 & 258.4 & 10.8 \\
\hline 0.0498 & 0.13001 & 0.00348 & 787.9 & 19.8 & 807.4 & 27.4 \\
\hline 0.02294 & 0.00533 & 0.00062 & 34.3 & 4 & 58.7 & 23.8 \\
\hline 0.08846 & 0.13101 & 0.00494 & 793.6 & 28.2 & 759.1 & 48.4 \\
\hline 0.02954 & 0.09363 & 0.0024 & 577 & 14.2 & 574.4 & 20.4 \\
\hline 0.04372 & 0.10657 & 0.00306 & 652.8 & 17.8 & 634.5 & 27.8 \\
\hline 0.03424 & 0.09861 & 0.00266 & 606.3 & 15.6 & 576.4 & 23.2 \\
\hline 0.04328 & 0.10065 & 0.00292 & 618.2 & 17.2 & 619.5 & 28 \\
\hline 0.03958 & 0.13313 & 0.00328 & 805.7 & 18.6 & 792.7 & 22.6 \\
\hline 0.02012 & 0.03494 & 0.00084 & 221.4 & 5.2 & 528 & 15.4 \\
\hline 0.05006 & 0.13202 & 0.00356 & 799.4 & 20.2 & 798.5 & 27.8 \\
\hline 0.05228 & 0.1364 & 0.0037 & 824.3 & 21 & 814.3 & 28.4 \\
\hline 0.02744 & 0.08228 & 0.00212 & 509.7 & 12.6 & 549.1 & 19.6 \\
\hline 0.04064 & 0.09185 & 0.00276 & 566.5 & 16.2 & 555.1 & 27.8 \\
\hline 0.03726 & 0.08931 & 0.00262 & 551.5 & 15.6 & 532.8 & 26 \\
\hline 0.05538 & 0.16895 & 0.00414 & 1006.3 & 22.8 & 1011.9 & 25.8 \\
\hline 0.08104 & 0.19096 & 0.00528 & 1126.6 & 28.6 & 1090.8 & 33.8 \\
\hline 0.0604 & 0.1273 & 0.00384 & 772.4 & 22 & 762.6 & 33.8 \\
\hline 0.03408 & 0.1011 & 0.00262 & 620.9 & 15.4 & 632.8 & 22.4 \\
\hline 0.01694 & 0.04903 & 0.0012 & 308.6 & 7.4 & 429.4 & 14 \\
\hline 0.03436 & 0.10482 & 0.00272 & 642.6 & 15.8 & 632.7 & 22.4 \\
\hline 0.19726 & 0.38261 & 0.00908 & 2088.5 & 42.4 & 2078.4 & 32.6 \\
\hline 0.02882 & 0.08976 & 0.00234 & 554.1 & 13.8 & 543.8 & 20.4 \\
\hline 0.78626 & 0.64817 & 0.01544 & 3221.1 & 60.4 & 3382.8 & 36 \\
\hline 0.02916 & 0.10725 & 0.00262 & 656.8 & 15.2 & 642.1 & 19.4 \\
\hline 0.05182 & 0.12679 & 0.00308 & 769.5 & 17.6 & 988 & 24.8 \\
\hline 0.03528 & 0.11839 & 0.00294 & 721.3 & 17 & 717.2 & 21.6 \\
\hline 0.09946 & 0.14046 & 0.00342 & 847.3 & 19.4 & 1473 & 29.8 \\
\hline 0.04858 & 0.14842 & 0.0037 & 892.1 & 20.8 & 895.8 & 25 \\
\hline 0.05498 & 0.16874 & 0.0042 & 1005.2 & 23.2 & 976.4 & 26.2 \\
\hline 0.05206 & 0.15967 & 0.00402 & 954.9 & 22.4 & 923.6 & 26 \\
\hline 0.04804 & 0.13452 & 0.0035 & 813.6 & 19.8 & 821.8 & 26.2 \\
\hline 0.07952 & 0.15483 & 0.00472 & 928 & 26.4 & 949.6 & 37.6 \\
\hline 0.03392 & 0.10331 & 0.00268 & 633.8 & 15.6 & 629.5 & 22.2 \\
\hline 0.03028 & 0.09963 & 0.00246 & 612.2 & 14.4 & 649.5 & 20 \\
\hline 0.03298 & 0.1012 & 0.00264 & 621.4 & 15.4 & 612 & 22 \\
\hline 0.04162 & 0.12483 & 0.00314 & 758.3 & 18 & 783.6 & 23.8 \\
\hline 0.04516 & 0.10163 & 0.00302 & 624 & 17.6 & 614.9 & 29 \\
\hline 0.0794 & 0.13424 & 0.00462 & 812 & 26.2 & 803.8 & 42.2 \\
\hline 1.1295 & 0.70805 & 0.02532 & 3451.1 & 95.6 & 3382.8 & 52 \\
\hline 0.0227 & 0.05115 & 0.00156 & 321.6 & 9.6 & 310.9 & 19.4 \\
\hline 0.0211 & 0.05133 & 0.00152 & 322.7 & 9.4 & 312.1 & 18 \\
\hline 0.01934 & 0.05133 & 0.00146 & 322.7 & 9 & 312.1 & 16.6 \\
\hline 0.03738 & 0.00586 & 0.0009 & 37.7 & 5.8 & 47.5 & 37.6 \\
\hline 0.06566 & 0.10509 & 0.0038 & 644.2 & 22.2 & 616.9 & 41 \\
\hline 0.05366 & 0.10677 & 0.00342 & 654 & 20 & 627.4 & 33.6 \\
\hline 0.06128 & 0.14433 & 0.00388 & 869.1 & 21.8 & 941.6 & 29.8 \\
\hline
\end{tabular}




$\begin{array}{lcccccc}0.04132 & 0.11472 & 0.00284 & 700.1 & 16.4 & 810.3 & 23.2 \\ 0.34428 & 0.46372 & 0.01224 & 2456 & 54 & 2441.8 & 39.2 \\ 0.03614 & 0.10531 & 0.0028 & 645.5 & 16.4 & 632.4 & 23.6 \\ 0.03966 & 0.10685 & 0.00294 & 654.4 & 17.2 & 636.6 & 25.4 \\ 0.05042 & 0.10347 & 0.00318 & 634.7 & 18.6 & 647.1 & 31.6 \\ 0.31188 & 0.42069 & 0.01086 & 2263.6 & 49.2 & 2367.1 & 38.2 \\ 0.18146 & 0.26467 & 0.00712 & 1513.7 & 36.2 & 1823.6 & 38 \\ 0.02514 & 0.05156 & 0.00162 & 324.1 & 10 & 335.2 & 21 \\ 0.0166 & 0.05207 & 0.00138 & 327.2 & 8.4 & 325.8 & 14.4 \\ 0.0429 & 0.1234 & 0.00318 & 750.1 & 18.2 & 766.4 & 24.8 \\ 0.03106 & 0.08808 & 0.00222 & 544.2 & 13.2 & 633.1 & 20.6 \\ 0.06546 & 0.09494 & 0.00368 & 584.7 & 21.6 & 559.2 & 43 \\ 0.06384 & 0.13665 & 0.00412 & 825.7 & 23.4 & 807 & 34.2 \\ 0.05212 & 0.16602 & 0.00414 & 990.1 & 22.8 & 929.6 & 25.8\end{array}$




\begin{tabular}{|c|c|c|c|c|c|}
\hline age $207 / 206$ & $2 \sigma$ age 76 & $\begin{array}{l}\text { discordance } \\
\Delta 68-75[\%]\end{array}$ & $\Delta 68-76[\%]$ & $\begin{array}{c}\text { preferred age } \\
\text { age }\end{array}$ & $2 \sigma$ age \\
\hline 569.3 & 69.2 & 0.2 & 1 & 575.1 & 13.4 \\
\hline 440.8 & 1095.8 & -15.9 & -92.9 & & \\
\hline 1818.1 & 51.2 & -1.1 & -2.4 & 1818.1 & 51.2 \\
\hline 289.4 & 90.4 & -1.3 & -11.9 & 255.1 & 6.4 \\
\hline 862.4 & 85.6 & -2.4 & -8.6 & 787.9 & 19.8 \\
\hline 1220.5 & 789.6 & -41.6 & -97.2 & & \\
\hline 659.2 & 176.2 & 4.6 & 20.4 & 793.6 & 28.2 \\
\hline 564.9 & 85 & 0.5 & 2.1 & 577 & 14.2 \\
\hline 570.8 & 112 & 2.9 & 14.4 & 652.8 & 17.8 \\
\hline 461.1 & 101 & 5.2 & 31.5 & & \\
\hline 625.1 & 113.8 & -0.2 & -1.1 & 618.2 & 17.2 \\
\hline 757.1 & 69.4 & 1.6 & 6.4 & 805.7 & 18.6 \\
\hline 2247.1 & 49.8 & -58.1 & -90.1 & & \\
\hline 796.5 & 88.8 & 0.1 & 0.4 & 799.4 & 20.2 \\
\hline 787.6 & 89.8 & 1.2 & 4.7 & 824.3 & 21 \\
\hline 717 & 82 & -7.2 & -28.9 & 509.7 & 12.6 \\
\hline 509.2 & 126.2 & 2 & 11.2 & 566.5 & 16.2 \\
\hline 454.3 & 122.8 & 3.5 & 21.4 & 551.5 & 15.6 \\
\hline 1024.7 & 64.6 & -0.6 & -1.8 & 1006.3 & 22.8 \\
\hline 1020.6 & 85.6 & 3.3 & 10.4 & & \\
\hline 734.7 & 116.8 & 1.3 & 5.1 & 772.4 & 22 \\
\hline 676.2 & 84.8 & -1.9 & -8.2 & 620.9 & 15.4 \\
\hline 1144.3 & 63.2 & -28.1 & -73 & & \\
\hline 598.2 & 86.2 & 1.6 & 7.4 & 642.6 & 15.8 \\
\hline 2069 & 49.6 & 0.5 & 0.9 & 2069 & 49.6 \\
\hline 501.6 & 90 & 1.9 & 10.5 & 554.1 & 13.8 \\
\hline 3480.5 & 43.2 & -4.8 & -7.5 & 3480.5 & 43.2 \\
\hline 591.6 & 70.4 & 2.3 & 11 & 656.8 & 15.2 \\
\hline 1511.7 & 58.2 & -22.1 & -49.1 & & \\
\hline 704.9 & 72.4 & 0.6 & 2.3 & 721.3 & 17 \\
\hline 2544.7 & 49.8 & -42.5 & -66.7 & & \\
\hline 905.6 & 69.6 & -0.4 & -1.5 & 892.1 & 20.8 \\
\hline 913 & 69.4 & 2.9 & 10.1 & 1005.2 & 23.2 \\
\hline 850.2 & 72.2 & 3.4 & 12.3 & 954.9 & 22.4 \\
\hline 844.9 & 80.4 & -1 & -3.7 & 813.6 & 19.8 \\
\hline 1000.8 & 107 & -2.3 & -7.3 & 928 & 26.4 \\
\hline 615.1 & 86 & 0.7 & 3 & 633.8 & 15.6 \\
\hline 782.1 & 70.2 & -5.7 & -21.7 & 612.2 & 14.4 \\
\hline 577.8 & 87.2 & 1.5 & 7.6 & 621.4 & 15.4 \\
\hline 856.9 & 73.6 & -3.2 & -11.5 & 758.3 & 18 \\
\hline 582.2 & 120.6 & 1.5 & 7.2 & 624 & 17.6 \\
\hline 781.8 & 142.4 & 1 & 3.9 & 812 & 26.2 \\
\hline 3343.1 & 61.2 & 2 & 3.2 & 3343.1 & 61.2 \\
\hline 232.2 & 150.8 & 3.4 & 38.5 & 321.6 & 9.6 \\
\hline 234.5 & 138.8 & 3.4 & 37.6 & 322.7 & 9.4 \\
\hline 234.5 & 127 & 3.4 & 37.6 & 322.7 & 9 \\
\hline 577 & 1728.6 & -20.7 & -93.5 & & \\
\hline 518.8 & 178 & 4.4 & 24.2 & 644.2 & 22.2 \\
\hline 533.2 & 141 & 4.2 & 22.7 & 654 & 20 \\
\hline 1115.7 & 80.6 & -7.7 & -22.1 & 869.1 & 21.8 \\
\hline
\end{tabular}




$\begin{array}{cccccc}1127.1 & 66.6 & -13.6 & -37.9 & 700.1 & 16.4 \\ 2430.6 & 56.4 & 0.6 & 1 & 2430.6 & 56.4 \\ 586.9 & 91.8 & 2.1 & 10 & 645.5 & 16.4 \\ 574.5 & 100.6 & 2.8 & 13.9 & 654.4 & 17.2 \\ 691.3 & 124.2 & -1.9 & -8.2 & 634.7 & 18.6 \\ 2458.1 & 55.4 & -4.4 & -7.9 & 2458.1 & 55.4 \\ 2199.1 & 62.2 & -17 & -31.2 & & \\ 413.4 & 148.6 & -3.3 & -21.6 & 324.1 & 10 \\ 316.8 & 101 & 0.4 & 3.3 & 327.2 & 8.4 \\ 814.9 & 79.4 & -2.1 & -8 & 750.1 & 18.2 \\ 965.5 & 72.8 & -14 & -43.6 & 544.2 & 13.2 \\ 457.5 & 204.8 & 4.6 & 27.8 & 584.7 & 21.6 \\ 756.4 & 113.2 & 2.3 & 9.2 & 825.7 & 23.4 \\ 789.2 & 71.8 & 6.5 & 25.5 & & \end{array}$


Sample 2858S Atbara @ Abu Ammar 75 grain analysed 31 concordant age:

\begin{tabular}{|c|c|c|c|c|c|c|}
\hline \multirow[b]{2}{*}{ grain } & \multicolumn{3}{|c|}{ concentrations } & \multicolumn{3}{|l|}{ isotopic ratios } \\
\hline & U [ppm] & $\mathrm{Pb}[\mathrm{ppm}]$ & $\mathrm{Th} / \mathrm{U}$ & Pb207/Pb206 & $2 \sigma 76$ & Pb207/U235 \\
\hline X2858n_G001 & 369.6 & 36.1 & 0.3019 & 0.06152 & 0.00168 & 0.8195 \\
\hline X2858n_G002 & 21.2 & 2 & 0.1501 & 0.06323 & 0.00494 & 0.83509 \\
\hline X2858n_G003 & 276.1 & 37.4 & 0.6591 & 0.07052 & 0.0019 & 1.18805 \\
\hline X2858n_G004 & 157.2 & 15.6 & 0.422 & 0.06529 & 0.00224 & 0.84751 \\
\hline X2858n_G005 & 579.9 & 149.6 & 0.1794 & 0.11519 & 0.00256 & 4.14383 \\
\hline X2858n_G006 & 59.5 & 9.7 & 0.3002 & 0.08174 & 0.00434 & 1.7908 \\
\hline X2858n_G007 & 72.2 & 11.3 & 0.3042 & 0.07276 & 0.00304 & 1.55264 \\
\hline X2858n_G008 & 1467.8 & 82 & 0.3843 & 0.24044 & 0.0057 & 1.74753 \\
\hline X2858n G009 & 78.6 & 12.1 & 0.3351 & 0.06882 & 0.00248 & 1.42505 \\
\hline X2858n G010 & & & & 0.83792 & 0.73142 & 218.59854 \\
\hline X2858n_G011 & 70.1 & 9.7 & 0.3927 & 0.06798 & 0.00262 & 1.23964 \\
\hline X2858n_G012 & & & & 1.69823 & 1.72138 & 619.20087 \\
\hline X2858n_G013 & 210.3 & 19.6 & 0.5271 & 0.06062 & 0.00194 & 0.72022 \\
\hline X2858n_G014 & 97.7 & 12.6 & 1.5956 & 0.06256 & 0.00254 & 0.79684 \\
\hline X2858n_G015 & 102 & 14.4 & 0.4415 & 0.06703 & 0.00232 & 1.23667 \\
\hline X2858n_G016 & & & & 0.82199 & 0.04166 & 483.47055 \\
\hline X2858n_G017 & & & & 0.79723 & 0.03304 & 244.76753 \\
\hline X2858n_G018 & & & & 0.81605 & 0.03124 & 267.39194 \\
\hline X2858n_G019 & & & & -0.35377 & 0.43898 & \\
\hline X2858n_G020 & & & & 4.13849 & 3.0015 & \\
\hline X2858n_G021 & & & & 1.0522 & 0.7681 & \\
\hline X2858n_G022 & 1826.8 & 126.4 & 0.1239 & 0.06225 & 0.00138 & 0.62979 \\
\hline X2858n_G023 & 223 & 24.2 & 0.5988 & 0.06179 & 0.00188 & 0.84602 \\
\hline X2858n_G024 & 475.8 & 24.4 & 0.3336 & 0.07183 & 0.00206 & 0.50847 \\
\hline X2858n_G025 & 616 & 67.6 & 0.5001 & 0.06886 & 0.00164 & 0.98726 \\
\hline X2858n_G026 & 201.8 & 25.6 & 0.5168 & 0.06788 & 0.00192 & 1.11092 \\
\hline X2858n_G027 & 131.7 & 18.5 & 0.4181 & 0.07181 & 0.00216 & 1.32978 \\
\hline X2858n_G028 & 265.5 & 35.1 & 0.5526 & 0.07424 & 0.00222 & 1.26627 \\
\hline X2858n_G029 & & & & 0.95429 & 1.17772 & 427.40057 \\
\hline X2858n_G030 & 930.4 & 98.6 & 0.3905 & 0.06951 & 0.0016 & 0.99422 \\
\hline X2858n_G031 & & & & 1.52282 & 1.97128 & 171.67656 \\
\hline X2858n_G032 & & & & 0.65368 & 0.45812 & 94.57461 \\
\hline X2858n_G033 & 1457.2 & 63 & 0.2892 & 0.0858 & 0.00222 & 0.50396 \\
\hline X2858n_G034 & 856 & 4.5 & 0.5961 & 0.04859 & 0.0056 & 0.03209 \\
\hline X2858n_G035 & 972.9 & 124.8 & 0.4393 & 0.06406 & 0.00148 & 1.08963 \\
\hline X2858n_G036 & 3812.9 & 89.9 & 0.0438 & 0.07962 & 0.00184 & 0.27662 \\
\hline X2858n_G037 & 184.8 & 26.6 & 0.4559 & 0.06188 & 0.00228 & 1.17088 \\
\hline X2858n_G038 & 206 & 28.4 & 0.3398 & 0.06827 & 0.00198 & 1.26864 \\
\hline X2858n_G039 & 193.3 & 24.7 & 0.3827 & 0.0632 & 0.00194 & 1.08591 \\
\hline X2858n_G040 & 159.3 & 17.7 & 0.143 & 0.06349 & 0.00214 & 1.01415 \\
\hline X2858n_G041 & 3617.5 & 89.8 & 0.1746 & 0.14478 & 0.00332 & 0.49827 \\
\hline X2858n_G042 & 424.8 & 41.6 & 0.3044 & 0.0568 & 0.00156 & 0.76463 \\
\hline X2858n_G043 & 756.2 & 97.8 & 0.3306 & 0.07267 & 0.00166 & 1.2925 \\
\hline X2858n_G044 & 159.3 & 7.4 & 0.1498 & 0.06777 & 0.00424 & 0.44839 \\
\hline X2858n_G045 & & & & 1.16994 & 2.02076 & \\
\hline X2858n_G046 & 197.5 & 32.1 & 0.3027 & 0.07228 & 0.00186 & 1.5968 \\
\hline X2858n_G047 & & & & 0.7312 & 0.30506 & 164.04414 \\
\hline X2858n_G048 & & & & 0.49909 & 0.17006 & 203.42905 \\
\hline X2858n_G049 & & & & -14.6524 & 2.4326 & \\
\hline X2858n_G050 & 492.8 & 3.4 & 1.445 & 0.25523 & 0.03552 & 0.19934 \\
\hline
\end{tabular}




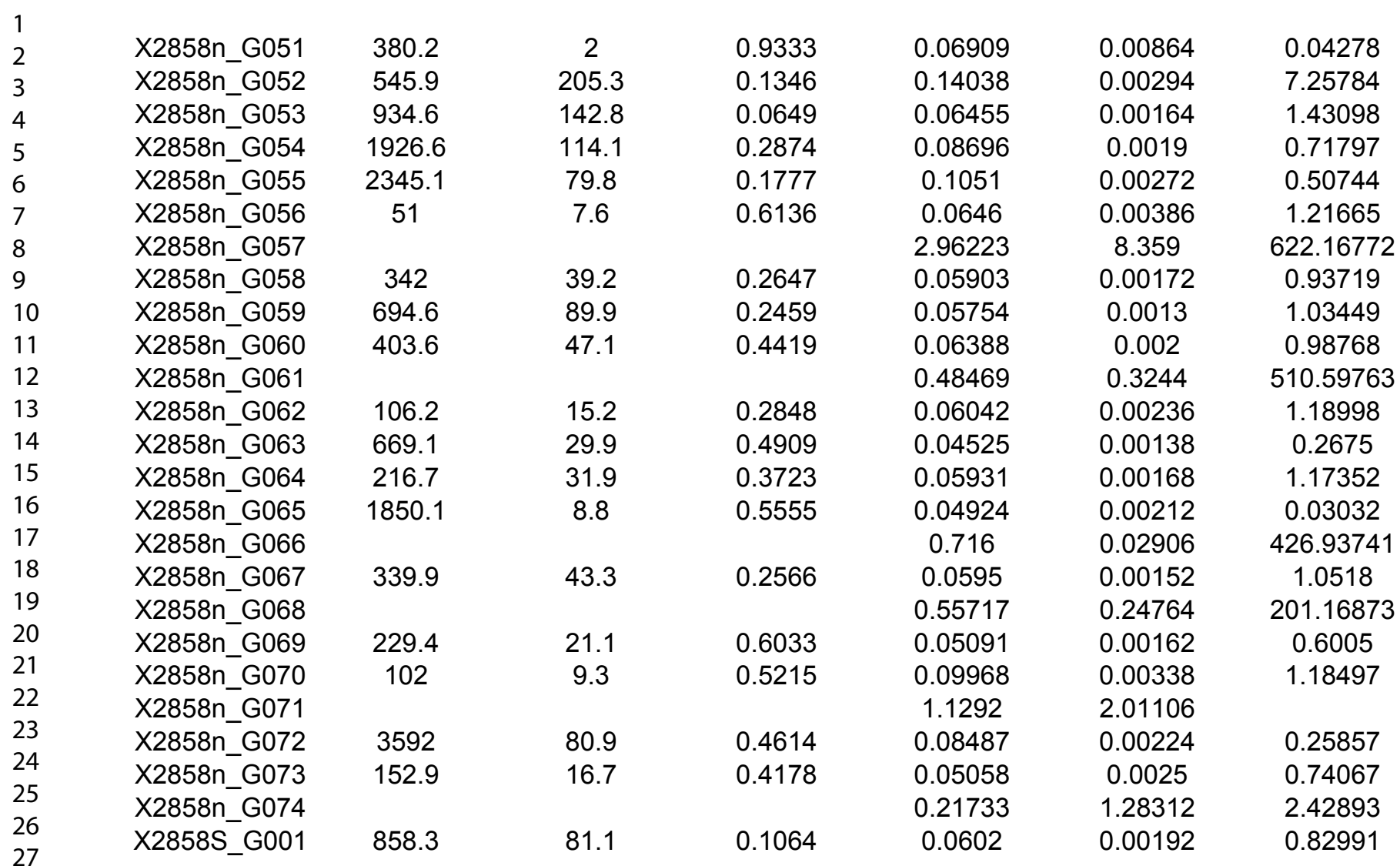




\section{$5 \quad 41.3 \%$ concordant}

\begin{tabular}{|c|c|c|c|c|c|c|}
\hline $2 \sigma 75$ & $\mathrm{~Pb} 206 / \mathrm{U} 238$ & $2 \sigma 68$ & $\begin{array}{c}\text { ages } \\
\text { age } 206 / 238\end{array}$ & $2 \sigma$ age 68 & age $207 / 235$ & $2 \sigma$ age 75 \\
\hline 0.0237 & 0.09655 & 0.00232 & 594.2 & 13.6 & 607.8 & 16.6 \\
\hline 0.0632 & 0.09573 & 0.00348 & 589.3 & 20.4 & 616.4 & 39.8 \\
\hline 0.034 & 0.12213 & 0.00296 & 742.8 & 17 & 795.1 & 20 \\
\hline 0.02966 & 0.0941 & 0.0024 & 579.7 & 14.2 & 623.3 & 20 \\
\hline 0.10298 & 0.26084 & 0.00614 & 1494.1 & 31.4 & 1663 & 26.4 \\
\hline 0.09302 & 0.15886 & 0.00516 & 950.4 & 28.8 & 1042.1 & 40.6 \\
\hline 0.06478 & 0.15474 & 0.00436 & 927.5 & 24.4 & 951.5 & 31.2 \\
\hline 0.0444 & 0.05271 & 0.00128 & 331.1 & 7.8 & 1026.3 & 22 \\
\hline 0.0521 & 0.15018 & 0.00396 & 902 & 22.2 & 899.5 & 26.6 \\
\hline 259.53922 & 1.89217 & 2.46782 & & & & \\
\hline 0.04812 & 0.13226 & 0.00356 & 800.7 & 20.2 & 818.7 & 26.4 \\
\hline 799.04102 & 2.6449 & 4.19668 & & & & \\
\hline 0.0238 & 0.08618 & 0.00216 & 532.9 & 12.8 & 550.8 & 17.2 \\
\hline 0.03236 & 0.0924 & 0.00248 & 569.7 & 14.6 & 595 & 22 \\
\hline 0.04364 & 0.13385 & 0.00348 & 809.8 & 19.8 & 817.4 & 24.4 \\
\hline 64.68664 & 4.26784 & 0.58268 & & & & \\
\hline 17.33048 & 2.22797 & 0.16432 & & & & \\
\hline \multirow[t]{2}{*}{17.76292} & 2.37794 & 0.16422 & & & & \\
\hline & -68.67284 & & & & & \\
\hline \multirow[t]{2}{*}{1872.8225} & 3.24685 & 3.98084 & & & & \\
\hline & -8.04471 & 20.22486 & & & & \\
\hline 0.01538 & 0.07345 & 0.00172 & 456.9 & 10.4 & 496 & 12.6 \\
\hline 0.02656 & 0.0994 & 0.00248 & 610.9 & 14.6 & 622.5 & 18.4 \\
\hline 0.01512 & 0.0514 & 0.00128 & 323.1 & 7.8 & 417.4 & 13 \\
\hline 0.0254 & 0.10411 & 0.00248 & 638.5 & 14.4 & 697.3 & 17 \\
\hline 0.0328 & 0.11884 & 0.00294 & 723.9 & 17 & 758.6 & 20 \\
\hline 0.04148 & 0.13449 & 0.0034 & 813.4 & 19.4 & 858.8 & 22.8 \\
\hline 0.03926 & 0.12388 & 0.00314 & 752.9 & 18 & 830.7 & 22.2 \\
\hline 563.26984 & 3.2533 & 5.0959 & & & & \\
\hline 0.02498 & 0.10391 & 0.00248 & 637.3 & 14.4 & 700.9 & 16.8 \\
\hline 154.5346 & 0.81906 & 1.11888 & & & & \\
\hline 64.10874 & 1.05124 & 0.72586 & & & & \\
\hline 0.01376 & 0.04268 & 0.00104 & 269.4 & 6.4 & 414.4 & 12.2 \\
\hline 0.00358 & 0.0048 & 0.00018 & 30.9 & 1.2 & 32.1 & 3.8 \\
\hline 0.02744 & 0.12363 & 0.00296 & 751.4 & 17 & 748.3 & 17.6 \\
\hline 0.00694 & 0.02525 & 0.0006 & 160.8 & 3.8 & 248 & 7.4 \\
\hline 0.04354 & 0.13755 & 0.0037 & 830.8 & 21 & 787.1 & 25 \\
\hline 0.0383 & 0.13509 & 0.0034 & 816.8 & 19.4 & 831.8 & 21.8 \\
\hline 0.03438 & 0.12493 & 0.00318 & 758.9 & 18.2 & 746.5 & 21.2 \\
\hline 0.03482 & 0.11614 & 0.00304 & 708.3 & 17.6 & 711 & 21.8 \\
\hline 0.01222 & 0.02503 & 0.0006 & 159.4 & 3.8 & 410.5 & 11.2 \\
\hline 0.02192 & 0.09793 & 0.00242 & 602.3 & 14.2 & 576.7 & 16.2 \\
\hline 0.03204 & 0.1294 & 0.00312 & 784.4 & 17.8 & 842.4 & 19 \\
\hline \multirow[t]{2}{*}{0.02674} & 0.04814 & 0.00162 & 303.1 & 10 & 376.2 & 22.4 \\
\hline & -4.18009 & 14.10796 & & & & \\
\hline 0.04348 & 0.16077 & 0.00398 & 961.1 & 22.2 & 969 & 22.4 \\
\hline 84.42198 & 1.63287 & 0.90896 & & & & \\
\hline \multirow[t]{2}{*}{107.72168} & 2.96693 & 1.57224 & & & & \\
\hline & 6.24922 & 3.8681 & & & & \\
\hline 0.02172 & 0.00569 & 0.00052 & 36.6 & 3.4 & 184.6 & 28.2 \\
\hline
\end{tabular}




\begin{tabular}{|c|c|c|c|c|c|c|}
\hline 0.00498 & 0.00451 & 0.00024 & 29 & 1.6 & 42.5 & 5.6 \\
\hline 0.16852 & 0.37651 & 0.00906 & 2060 & 42.4 & 2143.6 & 28.4 \\
\hline 0.03848 & 0.16146 & 0.00398 & 964.9 & 22 & 902 & 21.2 \\
\hline 0.0171 & 0.06014 & 0.00144 & 376.5 & 8.8 & 549.5 & 13.8 \\
\hline 0.01358 & 0.03517 & 0.00088 & 222.8 & 5.4 & 416.7 & 12.4 \\
\hline 0.0704 & 0.13722 & 0.00464 & 828.9 & 26.4 & 808.2 & 38.4 \\
\hline 1756.08544 & 1.53039 & 6.03016 & & & & \\
\hline 0.02814 & 0.1157 & 0.00292 & 705.8 & 16.8 & 671.4 & 19 \\
\hline 0.02542 & 0.13104 & 0.00316 & 793.8 & 18 & 721.2 & 17.2 \\
\hline 0.03146 & 0.11269 & 0.00292 & 688.4 & 17 & 697.5 & 20.6 \\
\hline 1120.29248 & 7.67945 & 16.833 & & & & \\
\hline 0.0464 & 0.14359 & 0.004 & 864.9 & 22.6 & 796 & 26.6 \\
\hline 0.0083 & 0.0431 & 0.00108 & 272 & 6.6 & 240.7 & 8.4 \\
\hline 0.03454 & 0.14429 & 0.00366 & 868.9 & 20.6 & 788.3 & 20.8 \\
\hline 0.00128 & 0.00449 & 0.00012 & 28.9 & 0.8 & 30.3 & 1.6 \\
\hline 41.02508 & 4.34943 & 0.42868 & & & & \\
\hline 0.0283 & 0.12896 & 0.0032 & 781.9 & 18.2 & 729.8 & 18.6 \\
\hline 156.61838 & 2.6343 & 2.05014 & & & & \\
\hline 0.01948 & 0.08608 & 0.0022 & 532.3 & 13 & 477.6 & 15.6 \\
\hline \multirow[t]{2}{*}{0.03956} & 0.08675 & 0.0024 & 536.3 & 14.2 & 793.6 & 24.2 \\
\hline & 233.21083 & & & & & \\
\hline 0.007 & 0.02224 & 0.00056 & 141.8 & 3.6 & 233.5 & 7.6 \\
\hline 0.03574 & 0.10691 & 0.00316 & 654.8 & 18.4 & 562.8 & 25 \\
\hline 13.09166 & 0.08161 & 0.19732 & & & & \\
\hline 0.02606 & 0.10002 & 0.00222 & 614.5 & 13 & 613.6 & 17.8 \\
\hline
\end{tabular}




\begin{tabular}{ccccccc}
\hline age $\mathbf{2 0 7 / 2 0 6}$ & 20 age $\mathbf{7 6}$ & $\begin{array}{c}\text { discordance } \\
\mathbf{\Delta} \mathbf{6 8 - 7 5}[\%]\end{array}$ & $\boldsymbol{\Delta} \mathbf{6 8 - 7 6}[\%]$ & $\begin{array}{c}\text { preferred age } \\
\text { age }\end{array}$ & $\mathbf{2 \sigma}$ age \\
\hline 657.5 & 58.6 & -2.2 & -9.6 & 594.2 & 13.6 \\
716 & 166 & -4.4 & -17.7 & 589.3 & 20.4 \\
943.5 & 55.2 & -6.6 & -21.3 & 742.8 & 17 \\
783.7 & 72 & -7 & -26 & 579.7 & 14.2 \\
1882.9 & 40 & -10.2 & -20.6 & & \\
1239.3 & 104 & -8.8 & -23.3 & 950.4 & 28.8 \\
1007.3 & 84.8 & -2.5 & -7.9 & 927.5 & 24.4 \\
3122.9 & 37.8 & -67.7 & -89.4 & & \\
893.3 & 74.4 & 0.3 & 1 & 902 & 22.2 \\
867.9 & 79.8 & -2.2 & -7.7 & 800.7 & 20.2 \\
625.8 & 69 & -3.2 & -14.8 & 532.9 & 12.8 \\
693.3 & 86.6 & -4.3 & -17.8 & 569.7 & 14.6 \\
838.7 & 72 & -0.9 & -3.4 & 809.8 & 19.8
\end{tabular}

\begin{tabular}{|c|c|c|c|c|c|}
\hline 682.7 & 47.4 & -7.9 & -33.1 & 456.9 & 10.4 \\
\hline 666.8 & 65.2 & -1.9 & -8.4 & 610.9 & 14.6 \\
\hline 981.1 & 58.4 & -22.6 & -67.1 & & \\
\hline 894.5 & 49.2 & -8.4 & -28.6 & 638.5 & 14.4 \\
\hline 864.9 & 58.6 & -4.6 & -16.3 & 723.9 & 17 \\
\hline 980.6 & 61.2 & -5.3 & -17 & 813.4 & 19.4 \\
\hline 1048 & 60.2 & -9.4 & -28.2 & 752.9 & 18 \\
\hline 913.9 & 47.4 & -9.1 & -30.3 & 637.3 & 14.4 \\
\hline 1333.7 & 50 & -35 & -79.8 & & \\
\hline 128.1 & 271.2 & -3.8 & -75.9 & 30.9 & 1.2 \\
\hline 743.6 & 48.8 & 0.4 & 1.1 & 751.4 & 17 \\
\hline 1187.6 & 45.6 & -35.2 & -86.5 & & \\
\hline 670 & 78.8 & 5.6 & 24 & & \\
\hline 876.8 & 60 & -1.8 & -6.8 & 816.8 & 19.4 \\
\hline 715 & 65.2 & 1.7 & 6.1 & 758.9 & 18.2 \\
\hline 724.7 & 71.4 & -0.4 & -2.3 & 708.3 & 17.6 \\
\hline 2285.2 & 39.4 & -61.2 & -93 & & \\
\hline 483.8 & 60.6 & 4.4 & 24.5 & 602.3 & 14.2 \\
\hline 1004.8 & 46.4 & -6.9 & -21.9 & 784.4 & 17.8 \\
\hline 861.5 & 129.8 & -19.4 & -64.8 & & \\
\hline 993.8 & 52.4 & -0.8 & -3.3 & 961.1 & 22.2 \\
\hline 3217.5 & 219.8 & -80.2 & -98.9 & & \\
\hline
\end{tabular}




$\begin{array}{cccccc}901.4 & 257.8 & -31.8 & -96.8 & & 36.2 \\ 2231.9 & 36.2 & -3.9 & -7.7 & 2231.9 & \\ 759.7 & 53.6 & 7 & 27 & & \\ 1359.6 & 42.2 & -31.5 & -72.3 & & \\ 1716.1 & 47.6 & -46.5 & -87 & & \\ 761.3 & 126 & 2.6 & 8.9 & 828.9 & \\ & & & & & \\ 568.2 & 63.4 & 5.1 & 24.2 & & \\ 512.3 & 49.6 & 10.1 & 55 & & \\ 737.7 & 66.2 & -1.3 & -6.7 & 688.4 & \\ & & & & & \\ 618.6 & 84.4 & 8.7 & 39.8 & & \\ -42.2 & 74.2 & 13 & -745 & & \\ 578.5 & 61.6 & 10.2 & 50.2 & & \\ 159.3 & 100.8 & -4.8 & -81.9 & 28.9 & \\ & & & & & \\ 585.4 & 55.4 & 7.1 & 33.6 & & \\ & & & & & \\ 236.8 & 73.4 & 11.5 & 124.8 & & \\ 1618.1 & 63.2 & -32.4 & -66.9 & & \\ 1312.6 & 51.2 & -39.3 & -89.2 & & \\ 221.7 & 114.4 & 16.3 & 195.3 & & \\ 610.8 & 69 & 0.2 & 0.6 & 614.5 & \end{array}$


Sample 2852S Main Nile @ Karima 1 grain analysed 1 concordant ages 1

\begin{tabular}{cccc|ccc}
\cline { 2 - 7 } grain & $\begin{array}{c}\text { concentrations } \\
\mathrm{U}[\mathrm{ppm}]\end{array}$ & $\mathrm{Pb}[\mathrm{ppm}]$ & $\mathrm{Th} / \mathrm{U}$ & $\begin{array}{c}\text { isotopic ratios } \\
\mathrm{Pb207} / \mathrm{Pb206}\end{array}$ & $\mathbf{2 \sigma}$ 76 & $\mathrm{Pb207/ \textrm {U } 2 3 5}$ \\
\hline X2852L_G001 & 94.9 & 10.4 & 0.3176 & 0.07632 & 0.00366 & 1.10837
\end{tabular}




\section{L00.0\% concordant}

\begin{tabular}{|c|c|c|c|c|c|c|}
\hline $2 \sigma 75$ & Pb206/U238 & $2 \sigma 68$ & $\begin{array}{c}\text { ages } \\
\text { age } 206 / 238\end{array}$ & $2 \sigma$ age 68 & age $207 / 235$ & $2 \sigma$ age 75 \\
\hline 0.05078 & 0.10537 & 0.00286 & 645.8 & 16.6 & 757.4 & 29.4 \\
\hline
\end{tabular}




\begin{tabular}{cc|cc|cc|}
\hline & & discordance & preferred age & \\
age 207/206 & 20 age 76 & $\Delta$ 68-75 [\%] & $\Delta$ 68-76 [\%] & age & 20 age \\
\hline 1103.5 & 95.8 & -14.7 & -41.5 & 645.8 & 16.6
\end{tabular}


Sample 2847S Wadi Milk @ Ed Debba 170 grain analysed 136 concordant :

\begin{tabular}{|c|c|c|c|c|c|c|}
\hline \multirow[b]{2}{*}{ grain } & \multicolumn{3}{|c|}{ concentrations } & \multicolumn{3}{|l|}{ isotopic ratios } \\
\hline & U [ppm] & $\mathrm{Pb}$ [ppm] & Th/U & Pb207/Pb206 & $2 \sigma 76$ & Pb207/U235 \\
\hline S2847_G001 & 63.4 & 11.8 & 0.6935 & 0.07835 & 0.00298 & 1.7616 \\
\hline S2847_G002 & 210.8 & 32.6 & 0.5305 & 0.06781 & 0.00198 & 1.33947 \\
\hline S2847_G003 & 107.5 & 19.9 & 5.6777 & 0.06262 & 0.00304 & 0.56785 \\
\hline S2847_G004 & 125.8 & 14.8 & 0.2502 & 0.06725 & 0.00232 & 1.10022 \\
\hline S2847_G005 & 230.6 & 32.4 & 1.7704 & 0.05866 & 0.00186 & 0.78811 \\
\hline S2847_G006 & 7207.5 & 163.9 & 0.0463 & 0.14084 & 0.00332 & 0.43613 \\
\hline S2847 G007 & 163 & 31.1 & 1.1043 & 0.0791 & 0.00232 & 1.67552 \\
\hline S2847_G008 & 310 & 33.7 & 0.7083 & 0.05923 & 0.00182 & 0.78059 \\
\hline S2847_G009 & 94.2 & 12.3 & 0.3735 & 0.06715 & 0.00258 & 1.16983 \\
\hline S2847_G010 & 61.5 & 8 & 0.8686 & 0.06377 & 0.00296 & 0.9738 \\
\hline S2847_G011 & 158.5 & 17.6 & 0.4021 & 0.06171 & 0.00214 & 0.9059 \\
\hline S2847 G012 & 148.3 & 11.8 & 0.7739 & 0.05648 & 0.0023 & 0.53634 \\
\hline S2847_G013 & 382.6 & 6.4 & 0.2323 & 0.0507 & 0.00286 & 0.11866 \\
\hline S2847_G014 & 734.4 & 83 & 0.344 & 0.06087 & 0.00152 & 0.92831 \\
\hline S2847_G015 & 938.8 & 96.2 & 0.5955 & 0.0682 & 0.00168 & 0.94008 \\
\hline S2847_G016 & 149.3 & 15.2 & 0.4944 & 0.05933 & 0.0021 & 0.77983 \\
\hline S2847_G017 & 136.9 & 26.3 & 0.6527 & 0.0757 & 0.00226 & 1.78777 \\
\hline S2847_G018 & 27.6 & 4.4 & 0.911 & 0.11228 & 0.0056 & 2.02378 \\
\hline S2847_G019 & 141.5 & 23.4 & 0.7109 & 0.07066 & 0.00216 & 1.40739 \\
\hline S2847_G020 & 311.9 & 24.8 & 0.4268 & 0.06436 & 0.002 & 0.67846 \\
\hline S2847_G021 & 187.4 & 17.6 & 0.1388 & 0.05905 & 0.00202 & 0.79718 \\
\hline S2847_G022 & 288.4 & 30.8 & 0.3148 & 0.06118 & 0.00178 & 0.88851 \\
\hline S2847_G023 & 223.7 & 5.4 & 1.9284 & 0.05128 & 0.00376 & 0.11362 \\
\hline S2847_G024 & 113 & 20.8 & 1.1453 & 0.07289 & 0.00242 & 1.46156 \\
\hline S2847_G025 & 187.4 & 8.3 & 0.5172 & 0.05056 & 0.00248 & 0.28991 \\
\hline S2847_G026 & 293.9 & 26.3 & 0.0798 & 0.06083 & 0.00188 & 0.80553 \\
\hline S2847_G027 & 163 & 23.6 & 0.5581 & 0.06603 & 0.00218 & 1.2095 \\
\hline S2847_G028 & 93.2 & 23.4 & 0.6906 & 0.11045 & 0.00314 & 3.35789 \\
\hline S2847_G029 & 37.7 & 3.4 & 0.3045 & 0.05945 & 0.0042 & 0.73557 \\
\hline S2847_G030 & 70.7 & 5.6 & 0.5877 & 0.05385 & 0.00346 & 0.53773 \\
\hline S2847_G031 & 72.1 & 9.5 & 0.5586 & 0.06537 & 0.00288 & 1.09127 \\
\hline S2847_G032 & 65.7 & 7.8 & 0.992 & 0.05777 & 0.00322 & 0.77741 \\
\hline S2847_G033 & 103.8 & & 1.0831 & 0.32035 & 0.0093 & 5.94173 \\
\hline S2847_G034 & 85 & 12.5 & 0.7737 & 0.06367 & 0.00276 & 1.11652 \\
\hline S2847_G035 & 88.2 & 11.1 & 0.942 & 0.06141 & 0.0028 & 0.8876 \\
\hline S2847_G036 & 198.4 & 20.2 & 0.3942 & 0.05912 & 0.00208 & 0.80263 \\
\hline S2847_G037 & 164 & 19.7 & 0.4597 & 0.06455 & 0.00226 & 1.0183 \\
\hline S2847_G038 & 167.2 & 28.4 & 1.2008 & 0.06593 & 0.0021 & 1.20345 \\
\hline S2847_G039 & 146.5 & 19 & 0.3446 & 0.06555 & 0.00216 & 1.14148 \\
\hline S2847_G040 & 264.1 & 27.7 & 0.4346 & 0.05826 & 0.00178 & 0.80403 \\
\hline S2847_G041 & 395 & 53.5 & 0.8528 & 0.06862 & 0.00186 & 1.10769 \\
\hline S2847_G042 & 89.1 & 10.1 & 0.7393 & 0.06043 & 0.00262 & 0.82802 \\
\hline S2847_G043 & 81.3 & 10.8 & 1.6201 & 0.06166 & 0.00274 & 0.81163 \\
\hline S2847_G044 & 96 & 2.1 & 1.6565 & 0.04438 & 0.00616 & 0.09166 \\
\hline S2847_G045 & 54.2 & 6.3 & 0.3828 & 0.06199 & 0.0031 & 0.95993 \\
\hline S2847_G046 & 153.4 & 18.3 & 0.4339 & 0.06195 & 0.0021 & 0.9683 \\
\hline S2847_G047 & 53.3 & 12.5 & 1.8418 & 0.07122 & 0.00298 & 1.55114 \\
\hline S2847_G048 & 31.2 & 8.4 & 1.8767 & 0.07514 & 0.00352 & 1.8707 \\
\hline S2847_G049 & & & & 0.08111 & 0.04394 & 1.48923 \\
\hline S2847_G050 & 44.1 & 10.3 & 1.7076 & 0.07176 & 0.00304 & 1.61569 \\
\hline
\end{tabular}




\begin{tabular}{|c|c|c|c|c|c|c|}
\hline S2847_G051 & 109.8 & 18.8 & 0.8286 & 0.06685 & 0.00228 & 1.3464 \\
\hline S2847_G052 & 48.7 & 6.4 & 1.41 & 0.06002 & 0.00346 & 0.8154 \\
\hline S2847_G053 & 63.8 & 8.8 & 1.7105 & 0.0577 & 0.00304 & 0.77131 \\
\hline S2847_G054 & 187.8 & 33.2 & 0.4912 & 0.07422 & 0.00214 & 1.68241 \\
\hline S2847_G055 & 119.9 & 12.9 & 0.732 & 0.05789 & 0.00258 & 0.75738 \\
\hline S2847_G056 & 287 & 37.8 & 0.3048 & 0.06433 & 0.00198 & 1.15058 \\
\hline S2847_G057 & 40.9 & 5 & 0.3787 & 0.06127 & 0.00352 & 0.99828 \\
\hline S2847_G058 & 1455.9 & 126.6 & 0.5779 & 0.28656 & 0.0067 & 2.9888 \\
\hline S2847_G059 & 84.5 & 7.9 & 0.3555 & 0.05925 & 0.00278 & 0.74783 \\
\hline S2847_G060 & 588.3 & 58 & 0.5733 & 0.06065 & 0.00168 & 0.75196 \\
\hline S2847_G061 & 138.2 & 59.4 & 0.8933 & 0.1219 & 0.00314 & 5.94078 \\
\hline S2847_G062 & 70.7 & 7.4 & 1.2555 & 0.05709 & 0.00332 & 0.63772 \\
\hline S2847_G063 & 62 & 8.4 & 0.3332 & 0.06583 & 0.0028 & 1.19602 \\
\hline S2847_G064 & 158 & 20 & 0.7864 & 0.06146 & 0.00216 & 0.92622 \\
\hline S2847_G065 & 352.3 & 49.4 & 0.8356 & 0.06789 & 0.0019 & 1.12234 \\
\hline S2847_G066 & 132.7 & 21.7 & 0.7784 & 0.09289 & 0.00306 & 1.7769 \\
\hline S2847_G067 & 156.2 & 16.1 & 0.257 & 0.05918 & 0.00212 & 0.84423 \\
\hline S2847_G068 & 336.7 & 35.3 & 0.151 & 0.06197 & 0.00176 & 0.92789 \\
\hline S2847_G069 & 198.9 & 24.9 & 1.1903 & 0.06314 & 0.00202 & 0.85442 \\
\hline S2847_G070 & 186.9 & 26 & 0.5832 & 0.06485 & 0.00204 & 1.13446 \\
\hline S2847_G071 & 81.3 & 8.3 & 0.5786 & 0.05891 & 0.00284 & 0.757 \\
\hline S2847_G072 & 148.8 & 23 & 0.4788 & 0.07258 & 0.0023 & 1.4431 \\
\hline S2847_G073 & 103.3 & 11 & 0.4201 & 0.06186 & 0.00256 & 0.86867 \\
\hline S2847_G074 & & & & -1.14686 & 5.2828 & 61.31481 \\
\hline S2847_G075 & 110.7 & 14.5 & 0.3052 & 0.06584 & 0.00246 & 1.17371 \\
\hline S2847_G076 & & & & 18.39355 & 950.43842 & \\
\hline S2847_G077 & 882.7 & 71.8 & 0.2671 & 0.08595 & 0.00222 & 0.95028 \\
\hline S2847_G078 & 488.2 & 67.2 & 1.6185 & 0.05869 & 0.00164 & 0.79305 \\
\hline S2847_G079 & 47.8 & 5 & 0.4807 & 0.06089 & 0.00378 & 0.83264 \\
\hline S2847_G080 & 204.8 & 27.2 & 0.9395 & 0.06102 & 0.00206 & 0.93072 \\
\hline S2847_G081 & 22.5 & 2.1 & 0.3587 & 0.06166 & 0.00656 & 0.78957 \\
\hline S2847_G082 & 54.7 & 0.3 & 0.363 & 0.14452 & 0.03556 & 0.0906 \\
\hline S2847_G083 & 57.4 & 6.4 & 0.9338 & 0.05637 & 0.00368 & 0.71803 \\
\hline S2847_G084 & 47.3 & 7.6 & 0.7412 & 0.06565 & 0.00382 & 1.26474 \\
\hline S2847_G085 & 452.4 & 42.4 & 0.5424 & 0.08044 & 0.0023 & 0.95744 \\
\hline S2847_G086 & 26.2 & 3.7 & 0.5108 & 0.07936 & 0.005 & 1.4183 \\
\hline S2847_G087 & 1172.5 & 84.2 & 0.132 & 0.07228 & 0.00186 & 0.74608 \\
\hline S2847_G088 & 89.6 & 9 & 0.6912 & 0.05862 & 0.00278 & 0.71869 \\
\hline S2847_G089 & 105.2 & 13.5 & 0.3035 & 0.06526 & 0.00244 & 1.14329 \\
\hline S2847_G090 & 195.7 & 21.8 & 0.7518 & 0.0582 & 0.00204 & 0.78306 \\
\hline S2847_G091 & 296.7 & 38 & 0.429 & 0.06839 & 0.00196 & 1.15852 \\
\hline S2847_G092 & 122.2 & 16.6 & 0.4576 & 0.06669 & 0.00238 & 1.17881 \\
\hline S2847_G093 & 276.9 & 34.8 & 1.3902 & 0.07638 & 0.00234 & 1.0978 \\
\hline S2847_G094 & 219.1 & 17.8 & 0.7686 & 0.0547 & 0.00204 & 0.53227 \\
\hline S2847_G095 & 404.2 & 74 & 5.3193 & 0.05763 & 0.00188 & 0.53616 \\
\hline S2847_G096 & 345.8 & 174.5 & 1.1455 & 0.16924 & 0.00402 & 9.33081 \\
\hline S2847_G097 & 13.3 & 1.9 & 0.4686 & 0.05673 & 0.00594 & 1.02612 \\
\hline S2847_G098 & 150.6 & 14 & 0.2157 & 0.06125 & 0.0023 & 0.7995 \\
\hline S2847_G099 & 225.5 & 35.2 & 0.7281 & 0.06585 & 0.00206 & 1.24222 \\
\hline S2847_G100 & 185.5 & 61.3 & 0.7673 & 0.09641 & 0.0026 & 3.75297 \\
\hline S2847_G101 & 235.6 & 24.9 & 0.6278 & 0.05875 & 0.00212 & 0.77552 \\
\hline S2847_G102 & 270.1 & 29.2 & 0.5197 & 0.05904 & 0.00196 & 0.81909 \\
\hline S2847_G103 & 82.7 & 9 & 0.7829 & 0.05916 & 0.00304 & 0.76623 \\
\hline S2847_G104 & 85.4 & 9.1 & 0.7881 & 0.06344 & 0.0036 & 0.80686 \\
\hline S2847_G105 & 46.8 & 7 & 0.8043 & 0.06735 & 0.00408 & 1.17267 \\
\hline
\end{tabular}




\begin{tabular}{|c|c|c|c|c|c|c|}
\hline S2847_G106 & 78.5 & 8.8 & 0.2685 & 0.06578 & 0.00306 & 1.01426 \\
\hline S2847_G107 & 11.5 & 1.3 & 0.3184 & 0.07062 & 0.0085 & 1.05117 \\
\hline S2847_G108 & 32.1 & 4.8 & 0.3944 & 0.06402 & 0.00416 & 1.26759 \\
\hline S2847_G109 & 112.5 & 17.9 & 0.4769 & 0.06867 & 0.00248 & 1.40793 \\
\hline S2847_G110 & 69.4 & 10.7 & 0.4767 & 0.07059 & 0.00298 & 1.39807 \\
\hline S2847_G111 & 185.1 & 32.3 & 0.6266 & 0.0697 & 0.0022 & 1.50684 \\
\hline S2847 G112 & 1454.5 & 100 & 0.2471 & 0.0869 & 0.00222 & 0.82305 \\
\hline S2847_G113 & 39.5 & 3.8 & 0.4419 & 0.06081 & 0.00442 & 0.77589 \\
\hline S2847_G114 & 1272.2 & 107.2 & 0.8133 & 0.13517 & 0.00336 & 1.32188 \\
\hline S2847_G115 & 929.6 & 50.9 & 0.4982 & 0.07229 & 0.0021 & 0.51125 \\
\hline S2847_G116 & 79 & 7.5 & 0.4148 & 0.05955 & 0.00316 & 0.74694 \\
\hline S2847_G117 & 334.8 & 39 & 0.629 & 0.06102 & 0.00194 & 0.88427 \\
\hline S2847_G118 & 239.7 & 32.6 & 0.3732 & 0.06859 & 0.00216 & 1.24245 \\
\hline S2847_G119 & 52.8 & 7 & 0.4444 & 0.06955 & 0.00332 & 1.20487 \\
\hline S2847_G120 & 204.4 & 18.5 & 0.018 & 0.05839 & 0.00216 & 0.78975 \\
\hline S2847_G121 & 392.7 & 52.5 & 0.3591 & 0.06447 & 0.00186 & 1.15271 \\
\hline S2847_G122 & 96.4 & 13.7 & 0.7503 & 0.0596 & 0.00278 & 1.01639 \\
\hline S2847_G123 & 113 & 15 & 0.5102 & 0.06114 & 0.0026 & 1.04388 \\
\hline S2847 G124 & 24.8 & 2.9 & 0.3754 & 0.0634 & 0.00494 & 0.99707 \\
\hline S2847_G125 & 65.2 & 12.7 & 0.6848 & 0.07249 & 0.00288 & 1.71817 \\
\hline S2847_G126 & 86.8 & 11.8 & 0.513 & 0.06573 & 0.00276 & 1.14436 \\
\hline S2847_G127 & 60.2 & 7.5 & 0.6288 & 0.06121 & 0.00294 & 0.94974 \\
\hline S2847_G128 & 276 & 98.6 & 0.5366 & 0.12181 & 0.00304 & 5.42053 \\
\hline S2847_G129 & 254 & 29.2 & 0.4244 & 0.06212 & 0.00192 & 0.94163 \\
\hline S2847_G130 & 101 & 9.9 & 0.3263 & 0.05666 & 0.0023 & 0.75584 \\
\hline S2847_G131 & 156.6 & 18.7 & 0.5898 & 0.05941 & 0.00216 & 0.8896 \\
\hline S2847_G132 & 30.3 & 4.8 & 0.7594 & 0.06781 & 0.00428 & 1.26855 \\
\hline S2847_G133 & 632 & 67.8 & 1.3784 & 0.06938 & 0.00192 & 0.88633 \\
\hline S2847_G134 & 924.5 & 80.1 & 0.5555 & 0.11552 & 0.00298 & 1.30579 \\
\hline S2847_G135 & 328.8 & 58.6 & 1.1715 & 0.06691 & 0.00192 & 1.29242 \\
\hline S2847_G136 & 181.4 & 14.7 & 0.7145 & 0.05555 & 0.00234 & 0.54664 \\
\hline S2847_G137 & 37.7 & 4.5 & 0.5949 & 0.06543 & 0.00438 & 0.99072 \\
\hline S2847_G138 & 132.7 & 13.4 & 0.8165 & 0.06178 & 0.00266 & 0.73413 \\
\hline S2847_G139 & 971.8 & 74 & 0.401 & 0.07319 & 0.00204 & 0.76689 \\
\hline S2847_G140 & 948.4 & 60.8 & 1.3568 & 0.052 & 0.00156 & 0.3472 \\
\hline S2847_G141 & & & & 0.94567 & 0.7807 & 121.75606 \\
\hline S2847_G142 & 257.7 & 29.1 & 0.3202 & 0.10629 & 0.00322 & 1.58331 \\
\hline S2847_G143 & 29.9 & 20 & 1.6938 & 0.17264 & 0.00528 & 11.08545 \\
\hline S2847_G144 & 63.4 & 5 & 0.3404 & 0.0557 & 0.00356 & 0.5879 \\
\hline S2847_G145 & 113.4 & 12.3 & 0.4563 & 0.05958 & 0.00264 & 0.84446 \\
\hline S2847_G146 & 546.5 & 83.6 & 0.6173 & 0.06532 & 0.0018 & 1.24298 \\
\hline S2847_G147 & 794.6 & 350.4 & 0.3424 & 0.16815 & 0.00406 & 9.63739 \\
\hline S2847_G148 & 61.1 & 6.7 & 0.6591 & 0.05923 & 0.00312 & 0.80507 \\
\hline S2847_G149 & 26.6 & 2.4 & 0.2797 & 0.05296 & 0.00512 & 0.66656 \\
\hline S2847_G150 & 344 & 55.2 & 0.3745 & 0.07078 & 0.00198 & 1.5017 \\
\hline X2847S_G001 & 44.3 & 3.9 & 0.2761 & 0.06239 & 0.00528 & 0.7642 \\
\hline X2847S_G002 & 284.2 & 6.9 & 0.7542 & 0.047 & 0.00346 & 0.13883 \\
\hline X2847S_G003 & & & & 0.77435 & 0.05008 & 453.14423 \\
\hline X2847S_G004 & 145.8 & 16.6 & 0.7597 & 0.06076 & 0.00268 & 0.83234 \\
\hline X2847S_G005 & & & & 0.80614 & 0.06214 & 452.76517 \\
\hline X2847S_G006 & 7197.3 & 354.3 & 1.7807 & 0.09014 & 0.00236 & 0.49234 \\
\hline X2847S_G007 & 53.2 & 5.5 & 0.5096 & 0.06084 & 0.00448 & 0.81784 \\
\hline X2847S_G008 & 60.6 & 6.9 & 0.4883 & 0.05923 & 0.0037 & 0.87819 \\
\hline X2847S_G009 & 220.2 & 30.8 & 1.329 & 0.05882 & 0.00228 & 0.86617 \\
\hline X2847S_G010 & 214.3 & 12.4 & 0.9823 & 0.04833 & 0.00284 & 0.31782 \\
\hline
\end{tabular}




$\begin{array}{lcccccc}\text { X2847S_G011 } & 107.9 & 16 & 0.344 & 0.0654 & 0.003 & 1.30077 \\ \text { X2847S_G012 } & 257.6 & 138.6 & 0.542 & 0.16966 & 0.00442 & 10.98983 \\ \text { X2847S_G013 } & 281.7 & 41.2 & 0.6096 & 0.06591 & 0.0022 & 1.20738 \\ \text { X2847S_G014 } & 3440.5 & 138 & 0.5643 & 0.09809 & 0.00272 & 0.52955 \\ \text { X2847S_G015 } & 3694.7 & 19 & 0.8255 & 0.67268 & 0.0203 & 0.82013 \\ \text { X2847S_G016 } & 1106.3 & 63.6 & 0.3343 & 0.075 & 0.00228 & 0.59022 \\ \text { X2847S_G017 } & 155.2 & 20.2 & 0.2865 & 0.06037 & 0.0025 & 1.07105 \\ \text { X2847S_G018 } & 188.7 & 23.3 & 0.474 & 0.06154 & 0.0025 & 0.98825 \\ \text { X2847S_G019 } & 3903.5 & & 1.4576 & 0.55954 & 0.01494 & 1.68927 \\ \text { X2847S_G020 } & 4123.2 & & 1.7643 & 0.4978 & 0.01384 & 0.95387\end{array}$




\section{tges $\quad \mathbf{8 0 . 0} \%$ concordant}

\begin{tabular}{|c|c|c|c|c|c|c|}
\hline $2 \sigma 75$ & $\mathrm{~Pb} 206 / \mathrm{U} 238$ & $2 \sigma 68$ & $\begin{array}{c}\text { ages } \\
\text { age } 206 / 238\end{array}$ & $2 \sigma$ age 68 & age $207 / 235$ & $2 \sigma$ age 75 \\
\hline 0.06786 & 0.16312 & 0.00448 & 974.1 & 24.8 & 1031.4 & 30.4 \\
\hline 0.04104 & 0.1433 & 0.00356 & 863.3 & 20 & 863 & 22.2 \\
\hline 0.02712 & 0.06579 & 0.00188 & 410.7 & 11.4 & 456.6 & 20.8 \\
\hline 0.03878 & 0.11869 & 0.00308 & 723 & 17.8 & 753.5 & 23 \\
\hline 0.0259 & 0.09747 & 0.00244 & 599.6 & 14.4 & 590.1 & 18 \\
\hline 0.01126 & 0.02247 & 0.00054 & 143.2 & 3.4 & 367.5 & 10.4 \\
\hline 0.05166 & 0.15368 & 0.00386 & 921.6 & 21.6 & 999.3 & 24.6 \\
\hline 0.02508 & 0.09561 & 0.00238 & 588.6 & 14 & 585.8 & 17.6 \\
\hline 0.04544 & 0.12639 & 0.0034 & 767.2 & 19.4 & 786.6 & 25.6 \\
\hline 0.04494 & 0.11079 & 0.00314 & 677.3 & 18.2 & 690.4 & 27.2 \\
\hline 0.03208 & 0.10651 & 0.00274 & 652.4 & 16 & 654.9 & 20.8 \\
\hline 0.02198 & 0.0689 & 0.00184 & 429.5 & 11 & 436 & 17.2 \\
\hline 0.00654 & 0.01698 & 0.00048 & 108.5 & 3 & 113.9 & 6.8 \\
\hline 0.0252 & 0.11065 & 0.00266 & 676.5 & 15.4 & 666.7 & 17 \\
\hline 0.02524 & 0.10001 & 0.0024 & 614.5 & 14 & 672.9 & 17 \\
\hline 0.02822 & 0.09537 & 0.00246 & 587.2 & 14.4 & 585.4 & 19.4 \\
\hline 0.05582 & 0.17135 & 0.00434 & 1019.5 & 23.8 & 1041 & 25.4 \\
\hline 0.09778 & 0.13077 & 0.00426 & 792.3 & 24.2 & 1123.5 & 40.4 \\
\hline 0.04488 & 0.14451 & 0.00366 & 870.1 & 20.6 & 892.1 & 23.6 \\
\hline 0.0219 & 0.07648 & 0.00192 & 475.1 & 11.4 & 525.8 & 16.4 \\
\hline 0.02792 & 0.09795 & 0.0025 & 602.4 & 14.6 & 595.2 & 19.2 \\
\hline 0.02734 & 0.10537 & 0.0026 & 645.8 & 15.2 & 645.6 & 18.2 \\
\hline 0.00808 & 0.01608 & 0.00052 & 102.8 & 3.2 & 109.3 & 8.4 \\
\hline 0.05 & 0.14548 & 0.00378 & 875.6 & 21.2 & 914.7 & 25.4 \\
\hline 0.0141 & 0.0416 & 0.00114 & 262.7 & 7 & 258.5 & 12.8 \\
\hline 0.02588 & 0.09608 & 0.0024 & 591.4 & 14.2 & 599.9 & 18 \\
\hline 0.04134 & 0.13291 & 0.00342 & 804.4 & 19.4 & 805 & 23.2 \\
\hline 0.10084 & 0.22057 & 0.00564 & 1284.9 & 29.8 & 1494.6 & 30 \\
\hline 0.05076 & 0.08977 & 0.00298 & 554.2 & 17.6 & 559.8 & 33.6 \\
\hline 0.03378 & 0.07245 & 0.00226 & 450.9 & 13.6 & 436.9 & 25.4 \\
\hline 0.04806 & 0.12111 & 0.00342 & 736.9 & 19.6 & 749.1 & 27.8 \\
\hline 0.0428 & 0.09764 & 0.00292 & 600.6 & 17.2 & 584 & 28 \\
\hline 0.17448 & 0.13457 & 0.0037 & 813.9 & 21 & 1967.4 & 34.8 \\
\hline 0.04856 & 0.12723 & 0.00356 & 772 & 20.4 & 761.3 & 27.6 \\
\hline 0.04032 & 0.10487 & 0.00296 & 642.9 & 17.2 & 645.1 & 25.6 \\
\hline 0.02878 & 0.09851 & 0.00254 & 605.7 & 15 & 598.3 & 19.8 \\
\hline 0.03636 & 0.11446 & 0.00298 & 698.6 & 17.2 & 713.1 & 22.4 \\
\hline 0.03992 & 0.13243 & 0.00338 & 801.7 & 19.2 & 802.2 & 22.6 \\
\hline 0.03898 & 0.12635 & 0.00324 & 767 & 18.6 & 773.2 & 22.6 \\
\hline 0.02576 & 0.10013 & 0.0025 & 615.2 & 14.6 & 599.1 & 17.8 \\
\hline 0.03196 & 0.11713 & 0.00286 & 714 & 16.6 & 757.1 & 19.4 \\
\hline 0.03592 & 0.09942 & 0.00274 & 611 & 16 & 612.5 & 23.6 \\
\hline 0.03604 & 0.09551 & 0.00266 & 588 & 15.6 & 603.4 & 23.8 \\
\hline 0.01242 & 0.01498 & 0.00062 & 95.9 & 4 & 89 & 12.4 \\
\hline 0.0476 & 0.11236 & 0.00328 & 686.4 & 19 & 683.3 & 28.8 \\
\hline 0.03382 & 0.1134 & 0.00292 & 692.5 & 17 & 687.6 & 21.2 \\
\hline 0.0652 & 0.15801 & 0.00446 & 945.7 & 24.8 & 950.9 & 31.2 \\
\hline 0.0872 & 0.18063 & 0.0054 & 1070.4 & 29.4 & 1070.8 & 36.8 \\
\hline 0.77134 & 0.13322 & 0.02646 & & & & \\
\hline 0.06868 & 0.16335 & 0.00462 & 975.4 & 25.6 & 976.3 & 32 \\
\hline
\end{tabular}




\begin{tabular}{|c|c|c|c|c|c|c|}
\hline 0.04738 & 0.14612 & 0.0038 & 879.2 & 21.4 & 866 & 25 \\
\hline 0.04608 & 0.09857 & 0.00302 & 606 & 17.8 & 605.5 & 29.8 \\
\hline 0.04018 & 0.09699 & 0.00286 & 596.7 & 16.8 & 580.5 & 26.8 \\
\hline 0.0514 & 0.16447 & 0.00412 & 981.6 & 22.8 & 1001.9 & 24.4 \\
\hline 0.03362 & 0.09492 & 0.00264 & 584.6 & 15.6 & 572.5 & 23 \\
\hline 0.03716 & 0.12976 & 0.00328 & 786.5 & 18.8 & 777.5 & 21.6 \\
\hline 0.05636 & 0.11821 & 0.00366 & 720.3 & 21 & 702.9 & 33.2 \\
\hline 0.07698 & 0.07567 & 0.00182 & 470.2 & 11 & 1404.8 & 25.6 \\
\hline 0.03484 & 0.09158 & 0.0026 & 564.9 & 15.4 & 567 & 23.8 \\
\hline 0.02226 & 0.08996 & 0.0022 & 555.3 & 13 & 569.4 & 16.2 \\
\hline 0.1666 & 0.35359 & 0.00884 & 1951.7 & 42.2 & 1967.2 & 31.2 \\
\hline 0.03634 & 0.08104 & 0.00244 & 502.3 & 14.6 & 500.9 & 25.8 \\
\hline 0.05104 & 0.13181 & 0.0037 & 798.2 & 21 & 798.7 & 28.2 \\
\hline 0.03334 & 0.10933 & 0.00284 & 668.9 & 16.6 & 665.6 & 21.4 \\
\hline 0.03334 & 0.11994 & 0.00296 & 730.2 & 17 & 764.1 & 20 \\
\hline 0.06012 & 0.1388 & 0.00366 & 837.9 & 20.8 & 1037 & 27.4 \\
\hline 0.03086 & 0.1035 & 0.0027 & 634.9 & 15.8 & 621.5 & 20.6 \\
\hline 0.0279 & 0.10863 & 0.00268 & 664.8 & 15.6 & 666.5 & 18.4 \\
\hline 0.02836 & 0.09819 & 0.0025 & 603.8 & 14.6 & 627.1 & 19.2 \\
\hline 0.0373 & 0.12692 & 0.00322 & 770.3 & 18.4 & 769.9 & 21.8 \\
\hline 0.03632 & 0.09323 & 0.00266 & 574.6 & 15.6 & 572.3 & 24.6 \\
\hline 0.04762 & 0.14425 & 0.0037 & 868.7 & 20.8 & 907 & 24.4 \\
\hline 0.03602 & 0.10188 & 0.00278 & 625.4 & 16.2 & 634.8 & 23.4 \\
\hline 142.46438 & -0.3879 & 1.63824 & & & & \\
\hline 0.04456 & 0.12935 & 0.00346 & 784.2 & 19.8 & 788.4 & 25.2 \\
\hline 0.0265 & 0.08022 & 0.00196 & 497.4 & 11.6 & 678.2 & 17.6 \\
\hline 0.0235 & 0.09804 & 0.0024 & 602.9 & 14 & 592.9 & 16.6 \\
\hline 0.05064 & 0.09922 & 0.00316 & 609.8 & 18.6 & 615.1 & 32.2 \\
\hline 0.0324 & 0.11068 & 0.00284 & 676.7 & 16.4 & 668 & 20.8 \\
\hline 0.08118 & 0.09291 & 0.004 & 572.7 & 23.6 & 590.9 & 51.4 \\
\hline 0.02008 & 0.00455 & 0.0005 & 29.3 & 3.2 & 88.1 & 22.8 \\
\hline 0.04586 & 0.09242 & 0.003 & 569.8 & 17.8 & 549.5 & 31 \\
\hline 0.0723 & 0.13977 & 0.0045 & 843.4 & 25.4 & 830 & 37.8 \\
\hline 0.02882 & 0.08636 & 0.00216 & 534 & 12.8 & 682 & 18.8 \\
\hline 0.0867 & 0.12967 & 0.00452 & 786 & 25.8 & 896.6 & 42.8 \\
\hline 0.02076 & 0.07489 & 0.00182 & 465.5 & 11 & 565.9 & 15.4 \\
\hline 0.03392 & 0.08895 & 0.00252 & 549.3 & 15 & 549.9 & 23.4 \\
\hline 0.04334 & 0.1271 & 0.00338 & 771.3 & 19.4 & 774.1 & 24.8 \\
\hline 0.02814 & 0.09761 & 0.00252 & 600.4 & 14.8 & 587.2 & 19.4 \\
\hline 0.0352 & 0.12291 & 0.00306 & 747.3 & 17.6 & 781.3 & 20.6 \\
\hline 0.0431 & 0.12825 & 0.00338 & 777.9 & 19.4 & 790.8 & 24.4 \\
\hline 0.03518 & 0.10429 & 0.00266 & 639.5 & 15.6 & 752.3 & 21.2 \\
\hline 0.02016 & 0.0706 & 0.00184 & 439.8 & 11 & 433.3 & 16 \\
\hline 0.01818 & 0.0675 & 0.00172 & 421.1 & 10.4 & 435.9 & 14.6 \\
\hline 0.24658 & 0.40002 & 0.00972 & 2169.1 & 44.8 & 2371.1 & 31.2 \\
\hline 0.10482 & 0.13124 & 0.00546 & 794.9 & 31.2 & 717 & 58 \\
\hline 0.03042 & 0.0947 & 0.0025 & 583.3 & 14.8 & 596.5 & 20.8 \\
\hline 0.0406 & 0.13688 & 0.00348 & 827 & 19.8 & 819.9 & 22.6 \\
\hline 0.10896 & 0.28244 & 0.00708 & 1603.6 & 35.6 & 1582.7 & 29.6 \\
\hline 0.02854 & 0.09578 & 0.0025 & 589.6 & 14.8 & 582.9 & 19.8 \\
\hline 0.02816 & 0.10066 & 0.00258 & 618.3 & 15.2 & 607.5 & 19.2 \\
\hline 0.039 & 0.09398 & 0.00276 & 579 & 16.2 & 577.6 & 26 \\
\hline 0.04482 & 0.09228 & 0.00288 & 569 & 17 & 600.7 & 29.4 \\
\hline 0.06934 & 0.12633 & 0.00416 & 766.9 & 23.8 & 787.9 & 37.8 \\
\hline
\end{tabular}




\begin{tabular}{|c|c|c|c|c|c|c|}
\hline 0.047 & 0.11188 & 0.00322 & 683.7 & 18.6 & 711 & 28 \\
\hline 0.12168 & 0.10801 & 0.00542 & 661.2 & 31.6 & 729.5 & 67.8 \\
\hline 0.08066 & 0.14365 & 0.00488 & 865.3 & 27.6 & 831.3 & 41.6 \\
\hline 0.05218 & 0.14876 & 0.00398 & 894 & 22.4 & 892.3 & 26.6 \\
\hline 0.05932 & 0.1437 & 0.00406 & 865.6 & 22.8 & 888.1 & 30 \\
\hline 0.04978 & 0.15685 & 0.00402 & 939.2 & 22.4 & 933.2 & 24.8 \\
\hline 0.02288 & 0.06872 & 0.00168 & 428.4 & 10.2 & 609.7 & 16.2 \\
\hline 0.05476 & 0.09258 & 0.0032 & 570.8 & 18.8 & 583.1 & 35.8 \\
\hline 0.0359 & 0.07096 & 0.00174 & 441.9 & 10.4 & 855.3 & 20.2 \\
\hline 0.01564 & 0.05131 & 0.00128 & 322.6 & 7.8 & 419.3 & 13.2 \\
\hline 0.03916 & 0.091 & 0.00272 & 561.4 & 16 & 566.4 & 26.4 \\
\hline 0.02942 & 0.10515 & 0.00268 & 644.5 & 15.6 & 643.3 & 19.4 \\
\hline 0.04074 & 0.13143 & 0.00336 & 796 & 19.2 & 820 & 22.8 \\
\hline 0.0571 & 0.12569 & 0.0037 & 763.2 & 21.2 & 802.8 & 31.2 \\
\hline 0.02978 & 0.09814 & 0.00258 & 603.5 & 15.2 & 591 & 20.4 \\
\hline 0.03534 & 0.12974 & 0.00324 & 786.4 & 18.4 & 778.5 & 20.8 \\
\hline 0.04722 & 0.12373 & 0.00354 & 752 & 20.4 & 712.1 & 28 \\
\hline 0.04468 & 0.12388 & 0.00344 & 752.9 & 19.8 & 725.8 & 26.4 \\
\hline 0.07552 & 0.11411 & 0.00412 & 696.6 & 23.8 & 702.3 & 43.6 \\
\hline 0.06934 & 0.17198 & 0.0048 & 1023 & 26.4 & 1015.3 & 31.2 \\
\hline 0.04816 & 0.12633 & 0.00352 & 766.9 & 20.2 & 774.6 & 27.4 \\
\hline 0.04536 & 0.11259 & 0.00326 & 687.8 & 18.8 & 678 & 27.8 \\
\hline 0.14874 & 0.32288 & 0.00792 & 1803.8 & 38.6 & 1888.1 & 30 \\
\hline 0.03052 & 0.10998 & 0.00278 & 672.6 & 16.2 & 673.7 & 19.6 \\
\hline 0.0311 & 0.09679 & 0.00262 & 595.6 & 15.4 & 571.6 & 21.4 \\
\hline 0.03312 & 0.10865 & 0.00286 & 664.9 & 16.6 & 646.2 & 21.4 \\
\hline 0.07852 & 0.13574 & 0.00452 & 820.5 & 25.6 & 831.7 & 40.6 \\
\hline 0.02616 & 0.09269 & 0.0023 & 571.4 & 13.6 & 644.4 & 17.8 \\
\hline 0.03654 & 0.08201 & 0.00202 & 508.1 & 12 & 848.3 & 20.6 \\
\hline 0.03944 & 0.14016 & 0.0035 & 845.6 & 19.8 & 842.4 & 21.8 \\
\hline 0.02308 & 0.0714 & 0.00194 & 444.6 & 11.6 & 442.8 & 18 \\
\hline 0.0648 & 0.10987 & 0.00372 & 672 & 21.6 & 699.1 & 38 \\
\hline 0.03156 & 0.08623 & 0.00238 & 533.2 & 14.2 & 559 & 22 \\
\hline 0.02276 & 0.07602 & 0.00188 & 472.3 & 11.2 & 578 & 16.4 \\
\hline 0.011 & 0.04844 & 0.0012 & 304.9 & 7.4 & 302.6 & 10.2 \\
\hline 89.7697 & 0.93419 & 0.84974 & & & & \\
\hline 0.05008 & 0.10809 & 0.0028 & 661.6 & 16.2 & 963.7 & 24.8 \\
\hline 0.36312 & 0.46592 & 0.01316 & 2465.6 & 57.8 & 2530.3 & 38.8 \\
\hline 0.03684 & 0.07659 & 0.00242 & 475.7 & 14.4 & 469.5 & 26.8 \\
\hline 0.03744 & 0.10284 & 0.00286 & 631 & 16.8 & 621.6 & 24.4 \\
\hline 0.0368 & 0.13806 & 0.00342 & 833.7 & 19.4 & 820.2 & 20.8 \\
\hline 0.25808 & 0.41586 & 0.01008 & 2241.7 & 45.8 & 2400.7 & 31.4 \\
\hline 0.04194 & 0.09862 & 0.00292 & 606.3 & 17.2 & 599.7 & 27.4 \\
\hline 0.06276 & 0.09133 & 0.00352 & 563.4 & 20.8 & 518.6 & 42.2 \\
\hline 0.04502 & 0.15394 & 0.00384 & 923 & 21.4 & 931.1 & 22.8 \\
\hline 0.0621 & 0.08887 & 0.00322 & 548.9 & 19 & 576.4 & 40.4 \\
\hline 0.00988 & 0.02143 & 0.00064 & 136.7 & 4 & 132 & 9.8 \\
\hline 74.24446 & 4.24608 & 0.70756 & & & & \\
\hline 0.0356 & 0.09939 & 0.00254 & 610.8 & 14.8 & 614.9 & 23.6 \\
\hline 86.5441 & 4.07519 & 0.79522 & & & & \\
\hline 0.01288 & 0.03963 & 0.00086 & 250.5 & 5.4 & 406.5 & 11.4 \\
\hline 0.05798 & 0.09754 & 0.00326 & 600 & 19.2 & 606.8 & 37 \\
\hline 0.05314 & 0.10759 & 0.00326 & 658.7 & 19 & 640 & 33 \\
\hline 0.03272 & 0.10685 & 0.0026 & 654.4 & 15.2 & 633.5 & 21.6 \\
\hline 0.01804 & 0.04772 & 0.00132 & 300.5 & 8.2 & 280.2 & 16 \\
\hline
\end{tabular}




$\begin{array}{ccccccc}0.05826 & 0.14432 & 0.00388 & 869 & 21.8 & 846.1 & 30.6 \\ 0.29032 & 0.46999 & 0.01056 & 2483.5 & 46.4 & 2522.3 & 32 \\ 0.03988 & 0.13291 & 0.00312 & 804.4 & 17.8 & 804 & 22.6 \\ 0.0146 & 0.03917 & 0.00088 & 247.7 & 5.4 & 431.5 & 12.6 \\ 0.02242 & 0.00885 & 0.00022 & 56.8 & 1.4 & 608.1 & 17.8 \\ 0.01776 & 0.0571 & 0.0013 & 358 & 8 & 471 & 14.4 \\ 0.04322 & 0.12872 & 0.00324 & 780.6 & 18.6 & 739.3 & 25.4 \\ 0.03924 & 0.11652 & 0.00292 & 710.5 & 16.8 & 697.8 & 24 \\ 0.04408 & 0.02191 & 0.0005 & 139.7 & 3.2 & 1004.5 & 22.4 \\ 0.02544 & 0.0139 & 0.00032 & 89 & 2 & 680.1 & 17.8\end{array}$




\begin{tabular}{|c|c|c|c|c|c|}
\hline age $207 / 206$ & $2 \sigma$ age 76 & $\begin{array}{l}\text { discordance } \\
\Delta 68-75[\%]\end{array}$ & $\Delta$ 68-76 [\%] & $\begin{array}{c}\text { preferred age } \\
\text { age }\end{array}$ & $2 \sigma$ age \\
\hline 1155.8 & 75.4 & -5.6 & -15.7 & 974.1 & 24.8 \\
\hline 862.8 & 60.6 & 0 & 0.1 & 863.3 & 20 \\
\hline 695.4 & 103.4 & -10 & -40.9 & 410.7 & 11.4 \\
\hline 845.5 & 71.8 & -4 & -14.5 & 723 & 17.8 \\
\hline 554.5 & 69.2 & 1.6 & 8.1 & 599.6 & 14.4 \\
\hline 2237.6 & 40.8 & -61 & -93.6 & & \\
\hline 1174.6 & 58 & -7.8 & -21.5 & 921.6 & 21.6 \\
\hline 575.6 & 66.8 & 0.5 & 2.3 & 588.6 & 14 \\
\hline 842.4 & 80 & -2.5 & -8.9 & 767.2 & 19.4 \\
\hline 734 & 98.2 & -1.9 & -7.7 & 677.3 & 18.2 \\
\hline 664.1 & 74.2 & -0.4 & -1.8 & 652.4 & 16 \\
\hline 471.3 & 90.2 & -1.5 & -8.9 & 429.5 & 11 \\
\hline 227.2 & 130.4 & -4.7 & -52.2 & 108.5 & 3 \\
\hline 634.6 & 53.8 & 1.5 & 6.6 & 676.5 & 15.4 \\
\hline 874.6 & 51 & -8.7 & -29.7 & 614.5 & 14 \\
\hline 579.2 & 77 & 0.3 & 1.4 & 587.2 & 14.4 \\
\hline 1087.1 & 59.8 & -2.1 & -6.2 & 1019.5 & 23.8 \\
\hline 1836.6 & 90.4 & -29.5 & -56.9 & & \\
\hline 947.6 & 62.6 & -2.5 & -8.2 & 870.1 & 20.6 \\
\hline 753.5 & 65.6 & -9.7 & -36.9 & 475.1 & 11.4 \\
\hline 568.9 & 74.4 & 1.2 & 5.9 & 602.4 & 14.6 \\
\hline 645.6 & 62.6 & 0 & 0 & 645.8 & 15.2 \\
\hline 253.4 & 168.6 & -5.9 & -59.4 & 102.8 & 3.2 \\
\hline 1010.9 & 67.4 & -4.3 & -13.4 & 875.6 & 21.2 \\
\hline 220.8 & 113.4 & 1.6 & 19 & 262.7 & 7 \\
\hline 633.2 & 66.6 & -1.4 & -6.6 & 591.4 & 14.2 \\
\hline 807.3 & 69 & -0.1 & -0.4 & 804.4 & 19.4 \\
\hline 1806.8 & 51.6 & -14 & -28.9 & & \\
\hline 583.6 & 153.4 & -1 & -5 & 554.2 & 17.6 \\
\hline 364.8 & 144.8 & 3.2 & 23.6 & 450.9 & 13.6 \\
\hline 786.3 & 92.6 & -1.6 & -6.3 & 736.9 & 19.6 \\
\hline 521 & 122.4 & 2.8 & 15.3 & 600.6 & 17.2 \\
\hline 3571.5 & 44.6 & -58.6 & -77.2 & & \\
\hline 730.7 & 91.8 & 1.4 & 5.7 & 772 & 20.4 \\
\hline 653.6 & 97.8 & -0.3 & -1.6 & 642.9 & 17.2 \\
\hline 571.5 & 76.6 & 1.2 & 6 & 605.7 & 15 \\
\hline 759.7 & 73.8 & -2 & -8 & 698.6 & 17.2 \\
\hline 804.2 & 66.6 & -0.1 & -0.3 & 801.7 & 19.2 \\
\hline 792 & 69.2 & -0.8 & -3.2 & 767 & 18.6 \\
\hline 539.6 & 66.8 & 2.7 & 14 & 615.2 & 14.6 \\
\hline 887.3 & 56 & -5.7 & -19.5 & 714 & 16.6 \\
\hline 619 & 93.6 & -0.2 & -1.3 & 611 & 16 \\
\hline 662.3 & 95.2 & -2.5 & -11.2 & 588 & 15.6 \\
\hline-89.6 & 340.4 & 7.6 & -207 & & \\
\hline 673.8 & 107 & 0.5 & 1.9 & 686.4 & 19 \\
\hline 672.4 & 72.6 & 0.7 & 3 & 692.5 & 17 \\
\hline 963.7 & 85.4 & -0.6 & -1.9 & 945.7 & 24.8 \\
\hline 1072.2 & 94.2 & 0 & -0.2 & 1070.4 & 29.4 \\
\hline 979.1 & 86.4 & -0.1 & -0.4 & 975.4 & 25.6 \\
\hline
\end{tabular}




\begin{tabular}{|c|c|c|c|c|c|}
\hline 833.1 & 71 & 1.5 & 5.5 & 879.2 & 21.4 \\
\hline 604.3 & 124.8 & 0.1 & 0.3 & 606 & 17.8 \\
\hline 518.4 & 115.6 & 2.8 & 15.1 & 596.7 & 16.8 \\
\hline 1047.5 & 58.2 & -2 & -6.3 & 981.6 & 22.8 \\
\hline 525.6 & 97.8 & 2.1 & 11.2 & 584.6 & 15.6 \\
\hline 752.5 & 65 & 1.2 & 4.5 & 786.5 & 18.8 \\
\hline 648.7 & 123.4 & 2.5 & 11 & 720.3 & 21 \\
\hline 3399 & 36.4 & -66.5 & -86.2 & & \\
\hline 576.3 & 102 & -0.4 & -2 & 564.9 & 15.4 \\
\hline 626.8 & 59.8 & -2.5 & -11.4 & 555.3 & 13 \\
\hline 1984.2 & 45.8 & -0.8 & -1.6 & 1984.2 & 45.8 \\
\hline 495 & 128.2 & 0.3 & 1.5 & 502.3 & 14.6 \\
\hline 801 & 89.2 & -0.1 & -0.3 & 798.2 & 21 \\
\hline 655.4 & 75.4 & 0.5 & 2.1 & 668.9 & 16.6 \\
\hline 865.2 & 58 & -4.4 & -15.6 & 730.2 & 17 \\
\hline 1485.6 & 62.4 & -19.2 & -43.6 & & \\
\hline 573.7 & 78 & 2.2 & 10.7 & 634.9 & 15.8 \\
\hline 673.1 & 60.8 & -0.3 & -1.2 & 664.8 & 15.6 \\
\hline 712.9 & 68 & -3.7 & -15.3 & 603.8 & 14.6 \\
\hline 769.5 & 66.2 & 0.1 & 0.1 & 770.3 & 18.4 \\
\hline 563.8 & 105 & 0.4 & 1.9 & 574.6 & 15.6 \\
\hline 1002.2 & 64.4 & -4.2 & -13.3 & 868.7 & 20.8 \\
\hline 669.3 & 88.6 & -1.5 & -6.6 & 625.4 & 16.2 \\
\hline 801.3 & 78.2 & -0.5 & -2.1 & 784.2 & 19.8 \\
\hline 1337.1 & 50 & -26.7 & -62.8 & & \\
\hline 555.6 & 61 & 1.7 & 8.5 & 602.9 & 14 \\
\hline 635.4 & 133.6 & -0.9 & -4 & 609.8 & 18.6 \\
\hline 639.9 & 72.6 & 1.3 & 5.7 & 676.7 & 16.4 \\
\hline 662.3 & 228 & -3.1 & -13.5 & 572.7 & 23.6 \\
\hline 2282.1 & 423.6 & -66.8 & -98.7 & & \\
\hline 467 & 144.6 & 3.7 & 22 & 569.8 & 17.8 \\
\hline 795.2 & 122 & 1.6 & 6.1 & 843.4 & 25 \\
\hline 1207.8 & 56.4 & -21.7 & -55.8 & & \\
\hline 1181.1 & 124.6 & -12.3 & -33.5 & 786 & 25.8 \\
\hline 993.8 & 52.4 & -17.7 & -53.2 & & \\
\hline 553 & 103.4 & -0.1 & -0.7 & 549.3 & 15 \\
\hline 782.7 & 78.6 & -0.4 & -1.5 & 771.3 & 19.4 \\
\hline 537.3 & 76.6 & 2.2 & 11.7 & 600.4 & 14.8 \\
\hline 880.4 & 59.2 & -4.3 & -15.1 & 747.3 & 17.6 \\
\hline 828.1 & 74.4 & -1.6 & -6.1 & 777.9 & 19.4 \\
\hline 1105 & 61.2 & -15 & -42.1 & 639.5 & 15.6 \\
\hline 400 & 83.6 & 1.5 & 9.9 & 439.8 & 11 \\
\hline 515.7 & 71.6 & -3.4 & -18.4 & 421.1 & 10.4 \\
\hline 2550.1 & 39.8 & -8.5 & -14.9 & 2550.1 & 39.8 \\
\hline 481.1 & 231.4 & 10.9 & 65.2 & & \\
\hline 648 & 80.6 & -2.2 & -10 & 583.3 & 14.8 \\
\hline 801.6 & 65.6 & 0.9 & 3.2 & 827 & 19.8 \\
\hline 1555.8 & 50.6 & 1.3 & 3.1 & 1555.8 & 50.6 \\
\hline 557.8 & 78.6 & 1.2 & 5.7 & 589.6 & 14.8 \\
\hline 568.6 & 72.2 & 1.8 & 8.7 & 618.3 & 15. \\
\hline 573 & 111.8 & 0.2 & 1.1 & 579 & 16.2 \\
\hline 723 & 120.4 & -5.3 & -21.3 & 569 & 17 \\
\hline 848.6 & 126 & -2.7 & -9.6 & 766.9 & 23.8 \\
\hline
\end{tabular}




\begin{tabular}{|c|c|c|c|c|c|}
\hline 799.4 & 97.4 & -3.8 & -14.5 & 683.7 & 18.6 \\
\hline 946.4 & 246.4 & -9.4 & -30.1 & 661.2 & 31.6 \\
\hline 742.3 & 137.4 & 4.1 & 16.6 & 865.3 & 27.6 \\
\hline 888.8 & 74.6 & 0.2 & 0.6 & 894 & 22.4 \\
\hline 945.6 & 86.4 & -2.5 & -8.5 & 865.6 & 22.8 \\
\hline 919.5 & 64.8 & 0.7 & 2.1 & 939.2 & 22.4 \\
\hline 1358.3 & 49.2 & -29.7 & -68.5 & & \\
\hline 632.5 & 156.6 & -2.1 & -9.8 & 570.8 & 18.8 \\
\hline 2166.2 & 43.4 & -48.3 & -79.6 & & \\
\hline 994.1 & 59 & -23.1 & -67.6 & & \\
\hline 587.3 & 115.2 & -0.9 & -4.4 & 561.4 & 16 \\
\hline 639.9 & 68.4 & 0.2 & 0.7 & 644.5 & 15.6 \\
\hline 886.4 & 65 & -2.9 & -10.2 & 796 & 19.2 \\
\hline 915.1 & 98.2 & -4.9 & -16.6 & 763.2 & 21.2 \\
\hline 544.4 & 80.8 & 2.1 & 10.9 & 603.5 & 15.2 \\
\hline 757.1 & 60.8 & 1 & 3.9 & 786.4 & 18.4 \\
\hline 589.1 & 101.2 & 5.6 & 27.7 & & \\
\hline 644.2 & 91.4 & 3.7 & 16.9 & 752.9 & 19.8 \\
\hline 721.7 & 165.4 & -0.8 & -3.5 & 696.6 & 23.8 \\
\hline 999.7 & 80.6 & 0.8 & 2.3 & 1023 & 26.4 \\
\hline 797.8 & 88 & -1 & -3.9 & 766.9 & 20.2 \\
\hline 646.6 & 103.2 & 1.4 & 6.4 & 687.8 & 18.8 \\
\hline 1982.9 & 44.4 & -4.5 & -9 & 1982.9 & 44.4 \\
\hline 678.2 & 66 & -0.2 & -0.8 & 672.6 & 16.2 \\
\hline 478.3 & 89.8 & 4.2 & 24.5 & 595.6 & 15.4 \\
\hline 582.2 & 79 & 2.9 & 14.2 & 664.9 & 16.6 \\
\hline 862.8 & 131 & -1.3 & -4.9 & 820.5 & 25.6 \\
\hline 910.1 & 57 & -11.3 & -37.2 & 571.4 & 13.6 \\
\hline 1888 & 46.4 & -40.1 & -73.1 & & \\
\hline 835 & 59.8 & 0.4 & 1.3 & 845.6 & 19.8 \\
\hline 434.4 & 93.8 & 0.4 & 2.3 & 444.6 & 11.6 \\
\hline 788.2 & 140.6 & -3.9 & -14.7 & 672 & 21.6 \\
\hline 666.5 & 92.2 & -4.6 & -20 & 533.2 & 14. \\
\hline 1019.2 & 56.4 & -18.3 & -53.7 & & \\
\hline 285.4 & 68.6 & 0.8 & 6.8 & 304.9 & 7.4 \\
\hline 1736.7 & 55.6 & -31.3 & -61.9 & & \\
\hline 2583.4 & 51 & -2.6 & -4.6 & 2583.4 & 51 \\
\hline 440.4 & 142.2 & 1.3 & 8 & 475.7 & 14.4 \\
\hline 588.4 & 96.2 & 1.5 & 7.3 & 631 & 16.8 \\
\hline 784.7 & 57.8 & 1.6 & 6.2 & 833.7 & 19.4 \\
\hline 2539.3 & 40.4 & -6.6 & -11.7 & 2539.3 & 40.4 \\
\hline 575.6 & 114.6 & 1.1 & 5.3 & 606.3 & 17.2 \\
\hline 327.1 & 219.4 & 8.6 & 72.2 & & \\
\hline 951.1 & 57.2 & -0.9 & -2.9 & 923 & 21.4 \\
\hline 687.5 & 180.6 & -4.8 & -20.2 & 548.9 & 19 \\
\hline 49.2 & 175.8 & 3.5 & 177.7 & 136.7 & 4 \\
\hline 630.7 & 95 & -0.7 & -3.2 & 610.8 & 14.8 \\
\hline 1428.5 & 50 & -38.4 & -82.5 & & \\
\hline 633.6 & 158.6 & -1.1 & -5.3 & 600 & 19.2 \\
\hline 575.6 & 135.8 & 2.9 & 14.5 & 658.7 & 19 \\
\hline 560.4 & 84.4 & 3.3 & 16.8 & 654.4 & 15.2 \\
\hline 115.5 & 138.6 & 7.2 & 160.3 & & \\
\hline
\end{tabular}




$\begin{array}{cccccc}787.2 & 96.4 & 2.7 & 10.4 & 869 & 21.8 \\ 2554.3 & 43.6 & -1.5 & -2.8 & 2554.3 & 43.6 \\ 803.5 & 69.8 & 0.1 & 0.1 & 804.4 & 17.8 \\ 1588.1 & 51.8 & -42.6 & -84.4 & & \\ 4673.4 & 43.4 & -90.7 & -98.8 & & \\ 1068.5 & 61.2 & -24 & -66.5 & & \\ 616.9 & 89.4 & 5.6 & 26.5 & & \\ 658.2 & 87.2 & 1.8 & 8 & 710.5 & 16.8 \\ 4406.3 & 39 & -86.1 & -96.8 & & \\ 4234.7 & 41 & -86.9 & -97.9 & & \end{array}$


Sample 2899S Main Nile @ 3th cataract 231 grain analysed 44 concordant

\begin{tabular}{|c|c|c|c|c|c|c|}
\hline \multirow[b]{2}{*}{ grain } & \multicolumn{3}{|c|}{ concentrations } & \multicolumn{3}{|l|}{ isotopic ratios } \\
\hline & U [ppm] & $\mathrm{Pb}$ [ppm] & $\mathrm{Th} / \mathrm{U}$ & Pb207/Pb206 & $2 \sigma 76$ & Pb207/U235 \\
\hline X2899S_G001 & 47.1 & 0.3 & 0.9618 & 0.16299 & 0.05142 & 0.09746 \\
\hline X2899S_G002 & 130.3 & 18.2 & 0.6215 & 0.06579 & 0.00232 & 1.13273 \\
\hline X2899S_G003 & 582 & 68 & 0.6418 & 0.07083 & 0.00196 & 1.01428 \\
\hline X2899S_G004 & 93.3 & 13.8 & 0.7407 & 0.06363 & 0.00248 & 1.13623 \\
\hline X2899S_G005 & & & & 1.03481 & 0.44828 & 638.98126 \\
\hline X2899S_G006 & 327.9 & 54.1 & 0.6718 & 0.07991 & 0.00212 & 1.60376 \\
\hline X2899S_G007 & 979 & 212.8 & 0.3578 & 0.13985 & 0.00318 & 4.04282 \\
\hline X2899S_G008 & 145.1 & 48.3 & 0.4083 & 0.15718 & 0.00414 & 6.92922 \\
\hline X2899S G009 & & & & 0.81466 & 0.12272 & 188.68019 \\
\hline X2899S G010 & 153.8 & 21.1 & 0.6174 & 0.06587 & 0.00224 & 1.12315 \\
\hline X2899S_G011 & 510.8 & 69.9 & 0.6181 & 0.069 & 0.00172 & 1.17549 \\
\hline X2899S_G012 & 894.2 & 83.8 & 0.9229 & 0.076 & 0.0019 & 0.83932 \\
\hline X2899S_G013 & & & & 0.88491 & 0.7431 & 197.73334 \\
\hline X2899S_G014 & 134.1 & 14.1 & 1.3194 & 0.06065 & 0.00304 & 0.67763 \\
\hline X2899S_G015 & 1440.9 & 72.2 & 0.769 & 0.16947 & 0.0042 & 1.034 \\
\hline X2899S_G016 & 835.8 & 28.4 & 0.7604 & 0.04258 & 0.00472 & 0.17097 \\
\hline X2899n_G001 & 209.1 & 28.7 & 0.676 & 0.06225 & 0.002 & 1.04539 \\
\hline X2899n_G002 & 89.1 & 12 & 0.5622 & 0.07054 & 0.00292 & 1.19126 \\
\hline X2899n_G003 & 718.8 & 79.9 & 0.406 & 0.06515 & 0.00168 & 0.97549 \\
\hline X2899n_G004 & & & & 0.36201 & 0.71888 & -65.06522 \\
\hline X2899n_G005 & & & & 0.15519 & 0.22524 & 67.97797 \\
\hline X2899n_G006 & 226.4 & 30.1 & 0.3437 & 0.06457 & 0.00196 & 1.15045 \\
\hline X2899n_G007 & & & & 0.68408 & 0.01986 & 39.51293 \\
\hline X2899n_G008 & & & & 0.43658 & 0.05556 & 10.70723 \\
\hline X2899n_G009 & & & & 0.7515 & 0.09262 & 9.15391 \\
\hline X2899n_G010 & & & & 0.20579 & 0.01614 & 3.70401 \\
\hline X2899n_G011 & & & & 0.12698 & 0.342 & 498.18579 \\
\hline X2899n_G012 & & & & -11.05178 & 112.38534 & \\
\hline X2899n_G013 & & & & 0.8502 & 1.91008 & 264.39786 \\
\hline X2899n_G014 & & & & 0.86088 & 0.02472 & \\
\hline X2899n_G015 & & & & 1.19391 & 1.31928 & \\
\hline X2899n_G016 & 400.1 & 42.7 & 0.5399 & 0.0589 & 0.0017 & 0.80025 \\
\hline X2899n_G017 & & & & 0.81467 & 0.01962 & 645.89978 \\
\hline X2899n_G018 & & & & 0.69852 & 0.53748 & 169.67809 \\
\hline X2899n_G019 & & & & 0.16557 & 0.20788 & 68.40109 \\
\hline X2899n_G020 & 252 & 25.3 & 0.2362 & 0.05988 & 0.00196 & 0.83442 \\
\hline X2899n_G021 & 153.3 & 16.4 & 0.5211 & 0.06013 & 0.00226 & 0.81936 \\
\hline X2899n_G022 & & & & 0.47416 & 0.30434 & 608.03705 \\
\hline X2899n_G023 & & & & 0.24679 & 0.59518 & \\
\hline X2899n_G024 & & & & & & \\
\hline X2899n_G025 & & & & 0.74635 & 0.05168 & \\
\hline X2899n_G026 & 102.6 & 12.1 & 0.4503 & 0.06166 & 0.00262 & 0.94653 \\
\hline X2899n_G027 & & & & 4.33022 & 18.3277 & 102.5185 \\
\hline X2899n_G028 & & & & 1.05041 & 0.3053 & \\
\hline X2899n_G029 & & & & 0.82282 & 0.03508 & \\
\hline X2899n_G030 & & & & 0.59319 & 1.63282 & \\
\hline X2899n_G031 & & & & 1.07117 & 0.77606 & 672.27496 \\
\hline X2899n_G032 & & & & 0.84813 & 0.03644 & \\
\hline X2899n_G033 & & & & 0.82167 & 0.65162 & 231.13699 \\
\hline X2899n_G034 & & & & 0.05811 & 0.80922 & 2.57551 \\
\hline
\end{tabular}




\begin{tabular}{|c|c|c|c|c|c|c|}
\hline X2899n_G035 & & & & 1.12601 & 4.85896 & 324.15161 \\
\hline X2899n_G036 & 438 & 45.3 & 0.6441 & 0.06014 & 0.00172 & 0.78314 \\
\hline X2899n_G037 & 1212.6 & 118.1 & 0.2621 & 0.0588 & 0.00148 & 0.79042 \\
\hline X2899n_G038 & & & & 0.80258 & 0.01874 & \\
\hline X2899n_G039 & & & & 0.79348 & 0.08346 & 174.88936 \\
\hline X2899n_G040 & & & & 0.58088 & 0.01584 & 4.40591 \\
\hline X2899n_G041 & & & & 0.74453 & 0.99542 & 468.07867 \\
\hline X2899n_G042 & 96.8 & 14 & 0.6401 & 0.06336 & 0.00248 & 1.12962 \\
\hline X2899n_G043 & 421.3 & 67.2 & 0.6945 & 0.069 & 0.00414 & 1.3392 \\
\hline X2899n_G044 & & & & 0.62147 & 0.46584 & 145.94135 \\
\hline X2899n_G045 & & & & 0.90379 & 0.05686 & \\
\hline X2899n_G046 & & & & 0.67143 & 1.88348 & 223.73691 \\
\hline X2899n_G047 & & & & 0.75429 & 0.66996 & 75.91071 \\
\hline X2899n_G048 & 222.5 & 31.5 & 0.3877 & 0.06858 & 0.00216 & 1.27898 \\
\hline X2899n_G049 & 388 & 66.4 & 2.1547 & 0.06911 & 0.00194 & 1.15596 \\
\hline X2899n_G050 & & & & 0.86584 & 0.09644 & 799.14575 \\
\hline X2899n_G051 & & & & 0.65025 & 0.13756 & 3.38508 \\
\hline X2899n_G052 & & & & 0.31023 & 0.0221 & 6.79099 \\
\hline X2899n_G053 & & & & 7.97857 & 9.41644 & \\
\hline X2899n_G054 & & & & 0.80856 & 0.0244 & 455.0097 \\
\hline X2899n_G055 & & & & 0.69387 & 0.8906 & \\
\hline X2899n_G056 & 40.4 & 4.7 & 1.026 & 0.06159 & 0.00426 & 0.8217 \\
\hline X2899n_G057 & & & & 0.67794 & 0.14744 & 293.92026 \\
\hline X2899n_G058 & & & & 0.83191 & 0.32244 & 306.95905 \\
\hline X2899n_G059 & & & & -12.71757 & 708.6482 & 226.19861 \\
\hline X2899n_G060 & & & & 0.78417 & 0.06556 & 891.87738 \\
\hline X2899n_G061 & 110.9 & 46 & 1.0287 & 0.11877 & 0.00326 & 5.42665 \\
\hline X2899n_G062 & 344.4 & 42 & 0.9004 & 0.06042 & 0.00184 & 0.85366 \\
\hline X2899n_G063 & & & & 0.52004 & 0.40386 & 128.00943 \\
\hline X2899n_G064 & & & & 1.00044 & 0.88348 & \\
\hline X2899n_G065 & & & & 0.4685 & 0.02308 & 13.40717 \\
\hline X2899n_G066 & & & & 0.79776 & 0.0607 & 246.96307 \\
\hline X2899n_G067 & & & & -0.16527 & 1.10946 & \\
\hline X2899n_G068 & & & & 0.3643 & 0.04158 & 7.83264 \\
\hline X2899n_G069 & & & & 0.87158 & 0.72942 & 204.55707 \\
\hline X2899n_G070 & & & & 0.92839 & 0.61476 & 867.91357 \\
\hline X2899n_G071 & & & & 0.73629 & 0.1557 & 357.1062 \\
\hline X2899n_G072 & & & & 0.84107 & 0.05716 & 272.8002 \\
\hline X2899n_G073 & & & & 0.75175 & 2.07974 & \\
\hline X2899n_G074 & & & & 5.6728 & 31.1876 & \\
\hline X2899n_G075 & & & & 0.16259 & 0.56176 & \\
\hline X2899n_G076 & 25 & 3.5 & 0.3479 & 0.06997 & 0.0045 & 1.30906 \\
\hline X2899n_G077 & & & & 0.20592 & 0.01626 & 4.07449 \\
\hline X2899n_G078 & & & & 0.90774 & 0.0893 & \\
\hline X2899n_G079 & & & & 0.88933 & 0.04732 & \\
\hline X2899n_G080 & & & & 0.71273 & 0.01782 & 74.76343 \\
\hline X2899n_G081 & & & & 2.96345 & 8.62018 & 714.61377 \\
\hline X2899n_G082 & & & & 0.57074 & 0.58156 & 334.10168 \\
\hline X2899n_G083 & 189.8 & 22.2 & 0.1976 & 0.06797 & 0.00222 & 1.1226 \\
\hline X2899n_G084 & & & & -0.57814 & 1.17878 & 225.71655 \\
\hline X2899n_G085 & & & & 0.96066 & 0.95152 & 983.50586 \\
\hline X2899n_G086 & & & & 0.1434 & 0.00988 & 2.29022 \\
\hline X2899n_G087 & & & & 3.26487 & 8.6962 & 270.33612 \\
\hline X2899n_G088 & & & & 0.53381 & 0.3849 & 59.41283 \\
\hline X2899n G089 & & & & 3.03087 & 4.45508 & 369.55569 \\
\hline
\end{tabular}




\begin{tabular}{|c|c|c|c|c|c|c|}
\hline & 0.20433 & 0.00826 & 3.36812 \\
\hline \multicolumn{4}{|l|}{ X2899n_G091 } & 0.62772 & 0.23326 & 5.4442 \\
\hline \multicolumn{4}{|l|}{ X2899n_G092 } & 0.66362 & 0.51552 & 1.89333 \\
\hline \multicolumn{4}{|l|}{ X2899n_G093 } & 6.9358 & 34.09284 & 200.37692 \\
\hline \multicolumn{4}{|l|}{ X2899n_G094 } & -0.07565 & 0.95708 & -12.94398 \\
\hline \multicolumn{4}{|l|}{ X2899n_G095 } & -1.00625 & 2.0364 & 266.11096 \\
\hline X2899n_G096 & 209.1 & 29.7 & 0.2614 & 0.07993 & 0.00246 & 1.53923 \\
\hline \multicolumn{4}{|l|}{ X2899n G097 } & 1.68075 & 1.9004 & 866.30176 \\
\hline \multicolumn{4}{|l|}{ X2899n_G098 } & 0.81686 & 0.11416 & \\
\hline \multicolumn{4}{|l|}{ X2899n_G099 } & 0.15042 & 0.00796 & 2.39018 \\
\hline \multicolumn{4}{|l|}{ X2899n_G100 } & 0.41396 & 0.46112 & 256.88214 \\
\hline \multicolumn{4}{|l|}{ X2899n_G101 } & 1.18063 & 1.59328 & \\
\hline \multicolumn{4}{|l|}{ X2899n_G102 } & 0.16719 & 0.00978 & 2.74595 \\
\hline \multicolumn{4}{|l|}{ X2899n_G103 } & 0.81035 & 0.1256 & \\
\hline \multicolumn{4}{|l|}{ X2899n_G104 } & 0.15485 & 0.01184 & 2.56358 \\
\hline X2899n_G105 & 130.2 & 19.3 & 0.601 & 0.06668 & 0.00246 & 1.2215 \\
\hline \multicolumn{4}{|l|}{ X2899n_G106 } & 0.50481 & 0.2936 & 78.91154 \\
\hline \multicolumn{4}{|l|}{ X2899n_G107 } & 3.38121 & 35.8958 & 98.45258 \\
\hline \multicolumn{4}{|l|}{ X2899n G108 } & 0.24269 & 0.84214 & \\
\hline \multicolumn{4}{|l|}{ X2899n G109 } & 0.19471 & 0.02202 & 2.58933 \\
\hline X2899n_G110 & & & & 0.577 & 0.56688 & 117.05179 \\
\hline X2899n_G111 & & & & 0.296 & 0.34958 & \\
\hline X2899n_G112 & & & & 0.69383 & 0.11796 & 61.02545 \\
\hline X2899n_G113 & & & & 0.69966 & 0.08572 & 66.51342 \\
\hline X2899n_G114 & & & & 0.73697 & 0.66558 & 120.44802 \\
\hline X2899n_G115 & & & & 0.86415 & 0.0247 & \\
\hline X2899n_G116 & & & & -0.37514 & 0.56714 & \\
\hline X2899n_G117 & & & & 0.92371 & 0.56756 & 153.74809 \\
\hline X2899n_G118 & & & & 1.08789 & 0.92232 & 372.44092 \\
\hline X2899n_G119 & & & & 0.56814 & 0.04934 & 19.05741 \\
\hline X2899n_G120 & & & & 1.3723 & 1.56772 & \\
\hline X2899n_G121 & & & & 0.16372 & 0.00896 & 3.17064 \\
\hline X2899n_G122 & & & & 0.78331 & 0.18004 & \\
\hline X2899n_G123 & & & & 0.40245 & 0.05856 & 8.10071 \\
\hline X2899n_G124 & & & & -0.6272 & 3.70258 & \\
\hline X2899n_G125 & & & & 1.12 & 3.14884 & \\
\hline X2899n_G126 & & & & 3.75576 & 7.6763 & 764.43945 \\
\hline X2899n_G127 & & & & 0.82318 & 0.40052 & 750.40167 \\
\hline X2899n_G128 & & & & 0.12645 & 0.15404 & 2.97506 \\
\hline X2899n_G129 & & & & 2.92393 & 9.98636 & \\
\hline X2899n_G130 & & & & 0.13589 & 0.00586 & 2.06491 \\
\hline X2899n_G131 & & & & 0.81056 & 0.18038 & 796.11493 \\
\hline X2899n_G132 & 2146.3 & 95.9 & 0.1123 & 0.15943 & 0.00446 & 1.01979 \\
\hline X2899n_G133 & & & & 1.25254 & 0.56444 & \\
\hline X2899n_G134 & & & & 0.73578 & 0.28354 & 700.46521 \\
\hline X2899n_G135 & & & & 0.16646 & 0.01196 & 2.16211 \\
\hline X2899n_G136 & & & & 1.09488 & 0.68256 & 456.96021 \\
\hline X2899n_G137 & & & & 3.63021 & 15.71174 & \\
\hline X2899n_G138 & & & & 3.52041 & 9.85572 & 127.8897 \\
\hline X2899n_G139 & & & & 0.83362 & 0.03008 & 170.47064 \\
\hline X2899n_G140 & & & & 0.86696 & 0.04012 & 377.55725 \\
\hline X2899n_G141 & & & & 0.88467 & 0.06536 & \\
\hline X2899n_G142 & 105.8 & 10.1 & 0.4169 & 0.05812 & 0.00264 & 0.73173 \\
\hline X2899n_G143 & & & & -0.41809 & 0.83356 & \\
\hline X2899n_G144 & & & & 0.1475 & 0.13676 & 309.37341 \\
\hline
\end{tabular}




\begin{tabular}{|c|c|c|c|c|c|c|}
\hline \multicolumn{4}{|l|}{ X2899n_G145 } & 0.73077 & 0.24668 & 430.3959 \\
\hline \multicolumn{4}{|l|}{ X2899n_G146 } & 0.46366 & 0.5735 & 175.64935 \\
\hline \multicolumn{4}{|l|}{ X2899n_G147 } & 1.12475 & 2.49124 & 121.30037 \\
\hline \multicolumn{4}{|l|}{ X2899n_G148 } & 0.77341 & 0.48518 & \\
\hline \multicolumn{4}{|l|}{ X2899n_G149 } & 0.88875 & 1.43912 & 262.1579 \\
\hline \multicolumn{4}{|l|}{ X2899n_G150 } & 0.32004 & 0.23868 & 141.47784 \\
\hline \multicolumn{4}{|l|}{ X2899n_G151 } & 0.8041 & 0.07866 & 66.92515 \\
\hline \multicolumn{4}{|l|}{ X2899n_G152 } & 0.80261 & 0.9018 & 353.29575 \\
\hline \multicolumn{4}{|l|}{ X2899n_G153 } & -0.46607 & 1.30224 & 344.37973 \\
\hline \multicolumn{4}{|l|}{ X2899n_G154 } & 0.68137 & 0.83784 & 145.52046 \\
\hline \multicolumn{4}{|l|}{ X2899n_G155 } & 1.08724 & 1.40114 & \\
\hline X2899n_G156 & 106.4 & 16.6 & 0.587 & 0.065 & 0.0026 & 1.2661 \\
\hline \multicolumn{4}{|l|}{ X2899n_G157 } & 0.2262 & 0.01078 & 4.09485 \\
\hline \multicolumn{4}{|l|}{ X2899n_G158 } & 0.50931 & 0.0414 & 16.12952 \\
\hline \multicolumn{4}{|l|}{ X2899n_G159 } & 1.0103 & 1.16218 & \\
\hline \multicolumn{4}{|l|}{ X2899n_G160 } & 0.80689 & 0.2061 & 94.88361 \\
\hline \multicolumn{4}{|l|}{ X2899n_G161 } & 0.6766 & 0.30602 & 10.50132 \\
\hline \multicolumn{4}{|l|}{ X2899n_G162 } & 2.30481 & 9.82806 & \\
\hline \multicolumn{4}{|l|}{ X2899n_G163 } & 0.79693 & 0.02662 & 143.69821 \\
\hline X2899n_G164 & & & & 1.10328 & 0.5475 & \\
\hline X2899n_G165 & & & & 0.10204 & 0.00654 & 1.87101 \\
\hline X2899n_G166 & & & & 0.82 & 0.05612 & 5.22906 \\
\hline X2899n_G167 & & & & 0.76627 & 0.03444 & 37.69232 \\
\hline X2899n_G168 & & & & 0.42739 & 0.35146 & 92.40526 \\
\hline X2899n_G169 & & & & -2.46953 & 7.36894 & \\
\hline X2899n_G170 & & & & 0.52568 & 0.81372 & 249.36731 \\
\hline X2899n_G171 & & & & 0.2709 & 0.02006 & 4.27858 \\
\hline X2899n_G172 & & & & 0.90796 & 0.54454 & 435.18747 \\
\hline X2899n_G173 & & & & 0.92896 & 0.2574 & 395.99155 \\
\hline X2899n_G174 & & & & 0.82183 & 0.41056 & \\
\hline X2899n_G175 & 271.3 & 35.8 & 0.4391 & 0.06408 & 0.00204 & 1.10623 \\
\hline X2899n_G176 & & & & 0.83579 & 0.10584 & \\
\hline X2899n_G177 & & & & 0.23557 & 0.5562 & 24.26844 \\
\hline X2899n_G178 & & & & 0.65177 & 1.1432 & \\
\hline X2899n_G179 & & & & 0.10009 & 0.00454 & 1.65501 \\
\hline X2899n_G180 & & & & 0.57512 & 0.7503 & 451.65479 \\
\hline X2899n_G181 & & & & -29.97249 & 1018.77588 & \\
\hline X2899n_G182 & & & & 0.54418 & 0.28484 & 227.03661 \\
\hline X2899n_G183 & 170.6 & 17.6 & 0.4574 & 0.06152 & 0.00244 & 0.82497 \\
\hline X2899n_G184 & & & & 0.24671 & 0.0145 & 3.99326 \\
\hline X2899n_G185 & & & & 1.24703 & 1.32886 & 107.48511 \\
\hline X2899n_G186 & & & & 1.65901 & 2.8003 & 572.55524 \\
\hline X2899n_G187 & & & & 1.32409 & 2.12652 & 97.6114 \\
\hline X2899n_G188 & & & & 4.91036 & 17.08904 & 613.5813 \\
\hline X2899n_G189 & & & & 1.93647 & 4.4182 & \\
\hline X2899n_G190 & & & & 0.51726 & 0.0326 & 15.19784 \\
\hline X2899n_G191 & & & & -0.51439 & 0.50122 & -13.69502 \\
\hline X2899n_G192 & & & & 0.88538 & 0.03068 & \\
\hline X2899n_G193 & & & & 0.58418 & 0.0182 & 4.96716 \\
\hline X2899n_G194 & & & & 0.8206 & 0.14082 & \\
\hline X2899n_G195 & & & & -0.28076 & 0.9724 & \\
\hline X2899n_G196 & & & & 0.81977 & 0.71728 & \\
\hline X2899n_G197 & & & & 0.7765 & 0.50166 & 929.07703 \\
\hline X2899n_G198 & & & & 0.96652 & 1.35612 & \\
\hline X2899n_G199 & & & & 0.15813 & 0.24064 & \\
\hline
\end{tabular}




$\begin{array}{llcccccc}1 & & & & & \\ 2 & & & & & \\ 2 & & & & \\ 3 & \text { X2899L_G001 } & 1270.8 & 114.3 & 1.202 & 0.16809 & 0.0048 & 1.7096 \\ 3 & \text { X2899L_G002 } & 385.8 & 38.9 & 0.3784 & 0.06009 & 0.00206 & 0.8082 \\ 4 & \text { X2899L_G003 } & 392.5 & 43.1 & 0.5376 & 0.06601 & 0.00226 & 0.92733 \\ 5 & \text { X2899L_G004 } & 21.3 & 2.4 & 1.0073 & 0.06536 & 0.00778 & 0.83155 \\ 6 & \text { X2899L_G005 } & & & & 0.93436 & 0.67472 & \\ 7 & \text { X2899L_G006 } & & & & 1.712 & 1.65684 & 125.21554 \\ 8 & \text { X2899L_G007 } & 225.9 & 28.3 & 1.1769 & 0.05742 & 0.00234 & 0.77909 \\ 9 & \text { X2899L_G008 } & 248.6 & 32.7 & 0.4621 & 0.06604 & 0.00308 & 1.12907 \\ 10 & \text { X2899L_G009 } & 98.6 & 14.3 & 1.0337 & 0.06634 & 0.00328 & 1.08112 \\ 11 & \text { X2899L_G010 } & 92 & 13.4 & 0.9384 & 0.06558 & 0.00332 & 1.09755 \\ 12 & \text { X2899L_G011 } & 255.9 & 147.9 & 0.9127 & 0.16216 & 0.00454 & 10.34409 \\ 13 & \text { X2899L_G012 } & 78 & 12.2 & 0.8906 & 0.06089 & 0.0035 & 1.10017 \\ 14 & \text { X2899L_G013 } & 65.3 & 11 & 0.7056 & 0.07734 & 0.00384 & 1.56321 \\ 15 & \text { X2899L_G014 } & 88.6 & 14.4 & 0.9184 & 0.06592 & 0.00316 & 1.22871 \\ 16 & \text { X2899L_G015 } & 173.9 & 28.7 & 0.8311 & 0.06718 & 0.0026 & 1.30006 \\ 17 & \text { X2899L_G016 } & 465.8 & 36 & 0.1057 & 0.06052 & 0.00268 & 0.67788 \\ 18 & & & & & & & \end{array}$




\section{ages $\quad 19.0 \%$ concordant}

\begin{tabular}{|c|c|c|c|c|c|c|}
\hline $2 \sigma 75$ & $\mathrm{~Pb} 206 / \mathrm{U} 238$ & $2 \sigma 68$ & $\begin{array}{c}\text { ages } \\
\text { age } 206 / 238\end{array}$ & $2 \sigma$ age 68 & age $207 / 235$ & $2 \sigma$ age 75 \\
\hline 0.0276 & 0.00433 & 0.00062 & 27.9 & 4 & 94.4 & 31.2 \\
\hline 0.04 & 0.1248 & 0.00314 & 758.1 & 18 & 769.1 & 23.4 \\
\hline 0.02876 & 0.10379 & 0.00244 & 636.6 & 14.2 & 711 & 18.6 \\
\hline 0.04388 & 0.12943 & 0.00336 & 784.6 & 19.2 & 770.7 & 25.2 \\
\hline 541.58966 & 4.47573 & 4.01112 & & & & \\
\hline 0.04406 & 0.14546 & 0.0034 & 875.5 & 19.2 & 971.7 & 22.2 \\
\hline 0.09768 & 0.20953 & 0.0048 & 1226.3 & 25.6 & 1642.9 & 26.2 \\
\hline 0.1906 & 0.31953 & 0.00796 & 1787.4 & 38.8 & 2102.4 & 32.2 \\
\hline 31.03444 & 1.67874 & 0.3083 & & & & \\
\hline 0.03824 & 0.1236 & 0.00306 & 751.3 & 17.6 & 764.5 & 22.6 \\
\hline 0.03058 & 0.12348 & 0.00282 & 750.6 & 16.2 & 789.2 & 18.6 \\
\hline 0.02188 & 0.08005 & 0.00184 & 496.4 & 11 & 618.8 & 15.8 \\
\hline 217.68134 & 1.61964 & 2.0507 & & & & \\
\hline 0.03302 & 0.08098 & 0.00228 & 502 & 13.6 & 525.3 & 23.6 \\
\hline 0.02626 & 0.04422 & 0.00104 & 278.9 & 6.4 & 720.9 & 17.6 \\
\hline 0.0182 & 0.02911 & 0.00118 & 185 & 7.4 & 160.3 & 17.4 \\
\hline 0.03482 & 0.12192 & 0.00308 & 741.6 & 17.6 & 726.6 & 21.2 \\
\hline 0.04928 & 0.12259 & 0.00342 & 745.5 & 19.6 & 796.5 & 27.6 \\
\hline 0.02698 & 0.1087 & 0.00262 & 665.2 & 15.2 & 691.3 & 17.8 \\
\hline 220.73962 & -1.30475 & 3.62054 & & & & \\
\hline 110.92202 & 3.17978 & 3.24986 & & & & \\
\hline 0.0365 & 0.12934 & 0.00324 & 784.1 & 18.4 & 777.5 & 21.4 \\
\hline 1.21332 & 0.41931 & 0.013 & & & & \\
\hline 1.13986 & 0.17804 & 0.0193 & & & & \\
\hline 0.80802 & 0.08843 & 0.00946 & & & & \\
\hline \multirow[t]{3}{*}{0.25968} & 0.13066 & 0.00712 & & & & \\
\hline & 28.48181 & 202.37266 & & & & \\
\hline & 2.42725 & 33.8739 & & & & \\
\hline 527.5617 & 2.25753 & 5.0815 & & & & \\
\hline \multirow[t]{2}{*}{1917.5774} & 78.5456 & 16.17778 & & & & \\
\hline & 11.80613 & 121.11244 & & & & \\
\hline 0.02436 & 0.09863 & 0.00242 & 606.4 & 14.2 & 597 & 17.2 \\
\hline 22.97928 & 5.7554 & 0.2007 & & & & \\
\hline 141.9478 & 1.76336 & 1.66624 & & & & \\
\hline 95.99546 & 2.99904 & 2.3377 & & & & \\
\hline 0.0281 & 0.10116 & 0.00256 & 621.2 & 15 & 616.1 & 19.2 \\
\hline 0.03122 & 0.09892 & 0.0026 & 608.1 & 15.2 & 607.7 & 21 \\
\hline \multirow[t]{3}{*}{909.78564} & 9.30875 & 13.72466 & & & & \\
\hline & -2.17823 & 47.196 & & & & \\
\hline & 76.47248 & 67.7305 & & & & \\
\hline 0.04008 & 0.11143 & 0.00306 & 681 & 17.8 & 676.3 & 25 \\
\hline 65.05688 & 0.17186 & 0.72938 & & & & \\
\hline \multirow[t]{2}{*}{1515.65992} & 9.26528 & 10.6283 & & & & \\
\hline & 431.91614 & 386.66058 & & & & \\
\hline 586.23876 & -2.07317 & 6.97316 & & & & \\
\hline 1861.1792 & 4.55584 & 12.8092 & & & & \\
\hline 314.33908 & 15.27643 & 2.7068 & & & & \\
\hline 356.44468 & 2.04198 & 3.23206 & & & & \\
\hline 35.7168 & 0.32172 & 0.46238 & & & & \\
\hline
\end{tabular}




\begin{tabular}{|c|c|c|c|c|c|c|}
\hline 941.60566 & 2.0897 & 8.911 & & & & \\
\hline 0.02354 & 0.09452 & 0.00232 & 582.2 & 13.6 & 587.3 & 16.8 \\
\hline 0.0215 & 0.09757 & 0.00234 & 600.2 & 13.8 & 591.4 & 15.6 \\
\hline 34.63668 & 9.88722 & 0.30076 & & & & \\
\hline 28.52166 & 1.59993 & 0.27636 & & & & \\
\hline \multirow[t]{2}{*}{0.12076} & 0.05506 & 0.00146 & & & & \\
\hline & 4.56359 & 19.85274 & & & & \\
\hline 0.04468 & 0.12942 & 0.0035 & 784.6 & 20 & 767.6 & 25.6 \\
\hline 0.07828 & 0.14088 & 0.00474 & 849.6 & 26.8 & 862.9 & 40 \\
\hline \multirow[t]{2}{*}{120.77504} & 1.70461 & 1.49248 & & & & \\
\hline & 53.97356 & 28.0241 & & & & \\
\hline 785.35108 & 2.41884 & 8.82412 & & & & \\
\hline 63.95938 & 0.73052 & 0.63922 & & & & \\
\hline 0.04176 & 0.13538 & 0.00342 & 818.5 & 19.4 & 836.4 & 23 \\
\hline 0.03426 & 0.12141 & 0.00298 & 738.7 & 17.2 & 780.1 & 20.4 \\
\hline 300.41378 & 6.69961 & 2.55564 & & & & \\
\hline 0.47128 & 0.03779 & 0.00646 & & & & \\
\hline \multirow[t]{2}{*}{0.42492} & 0.15889 & 0.00884 & & & & \\
\hline & -3.76391 & 11.8142 & & & & \\
\hline \multirow[t]{2}{*}{24.86766} & 4.08476 & 0.22414 & & & & \\
\hline & -8.65181 & 47.87836 & & & & \\
\hline 0.05514 & 0.09684 & 0.00332 & 595.9 & 19.6 & 609 & 35.4 \\
\hline 130.66796 & 3.14696 & 1.43382 & & & & \\
\hline 216.53228 & 2.67828 & 1.97212 & & & & \\
\hline 453.49412 & -0.1291 & 7.19078 & & & & \\
\hline 257.46694 & 8.25556 & 2.41038 & & & & \\
\hline 0.1596 & 0.33165 & 0.0084 & 1846.4 & 40.6 & 1889.1 & 32 \\
\hline 0.02718 & 0.10256 & 0.00256 & 629.4 & 15 & 626.7 & 18.4 \\
\hline \multirow[t]{2}{*}{145.43966} & 1.78669 & 2.04724 & & & & \\
\hline & 8.28272 & 49.68608 & & & & \\
\hline 0.59894 & 0.20772 & 0.00928 & & & & \\
\hline 34.08996 & 2.24701 & 0.3226 & & & & \\
\hline 0.76104 & 0.15606 & 0.01372 & & & & \\
\hline 177.8369 & 1.70352 & 1.77698 & & & & \\
\hline 1268.77966 & 6.78556 & 10.29552 & & & & \\
\hline 169.32236 & 3.52035 & 1.71674 & & & & \\
\hline \multirow[t]{2}{*}{34.17068} & 2.35423 & 0.30658 & & & & \\
\hline & -7.46419 & 55.18332 & & & & \\
\hline 675.63946 & -0.38691 & 2.27918 & & & & \\
\hline 0.08184 & 0.13579 & 0.00468 & 820.8 & 26.6 & 849.7 & 42 \\
\hline \multirow[t]{3}{*}{0.29058} & 0.14362 & 0.00778 & & & & \\
\hline & 76.50069 & 125.96022 & & & & \\
\hline & 217.23091 & 234.0906 & & & & \\
\hline 2.08402 & 0.76137 & 0.01984 & & & & \\
\hline 1049.20948 & 1.75026 & 5.61892 & & & & \\
\hline 741.71784 & 4.24878 & 9.40134 & & & & \\
\hline 0.03788 & 0.11987 & 0.00306 & 729.8 & 17.6 & 764.2 & 22.2 \\
\hline \multirow[t]{2}{*}{457.02148} & -2.83373 & 5.70864 & & & & \\
\hline & 7.43069 & 25.59956 & & & & \\
\hline 0.14614 & 0.11592 & 0.00504 & & & & \\
\hline 268.96586 & 0.60098 & 1.66824 & & & & \\
\hline 40.8827 & 0.80783 & 0.53762 & & & & \\
\hline 297.17252 & 0.88498 & 1.44268 & & & & \\
\hline
\end{tabular}




$\begin{array}{ccc}0.13026 & 0.11964 & 0.00378 \\ 1.4071 & 0.06295 & 0.01902 \\ 0.91648 & 0.02071 & 0.01276 \\ 162.87342 & 0.20968 & 1.03792 \\ 163.2131 & 1.24185 & 2.17292 \\ 225.89538 & -1.91942 & 4.01508 \\ 0.04922 & 0.13976 & 0.00354 \\ & 3.74089 & 11.6603 \\ & & \\ 0.11914 & 0.11533 & 0.00414 \\ 653.01008 & 4.50378 & 11.32744\end{array}$

$\begin{array}{ccc}0.14938 & 0.1192 & 0.0047 \\ & -22.95223 & 23.13534 \\ 0.18024 & 0.12015 & 0.00576 \\ 0.04572 & 0.13294 & 0.00352 \\ 51.01302 & 1.13451 & 0.6859 \\ 198.53904 & 0.21132 & 2.22282 \\ & & \\ 0.25784 & 0.09652 & 0.0069 \\ 142.17526 & 1.4723 & 1.70448 \\ & -12.69963 & 83.63446 \\ 10.87604 & 0.63833 & 0.12382 \\ 8.9994 & 0.68994 & 0.10182 \\ 97.427 & 1.18614 & 1.16342\end{array}$

$\begin{array}{ccc}307.69174 & 3.38218 & 4.60686 \\ 100.9246 & 1.20798 & 0.90616 \\ 493.56818 & 2.4846 & 3.58998 \\ 1.4493 & 0.24344 & 0.01968 \\ & 6.32404 & 14.28356 \\ 0.1634 & 0.14054 & 0.00532 \\ & 26.36552 & 73.76222 \\ 0.97156 & 0.14608 & 0.01714 \\ & 3.75955 & 65.75554\end{array}$

$\begin{array}{lll}1826.98034 & 1.47712 & 4.5864\end{array}$

$\begin{array}{lll}896.66784 & 6.6156 & 8.03808\end{array}$

$\begin{array}{lll}3.52366 & 0.17075 & 0.06218\end{array}$

$\begin{array}{lll}1073.30334 & -1.02923 & 4.31204\end{array}$

$\begin{array}{lll}0.08628 & 0.11028 & 0.00338\end{array}$

$\begin{array}{lll}649.7221 & 7.12778 & 5.87924\end{array}$

$\begin{array}{lll}0.02992 & 0.04642 & 0.00116\end{array}$

$8.48321 \quad 13.19496$

$\begin{array}{lll}1055.07128 & 6.90871 & 10.44266\end{array}$

$\begin{array}{lll}0.1416 & 0.09426 & 0.00428\end{array}$

$\begin{array}{lll}641.65436 & 3.02878 & 4.4505\end{array}$

$\begin{array}{ccc}116.33194 & 0.26363 & 0.75796 \\ 8.40156 & 1.48401 & 0.07406 \\ 34.2106 & 3.16037 & 0.29266 \\ & 229.23752 & 451.07342 \\ 0.03318 & 0.09137 & 0.00254\end{array}$

$1164.87292 \quad 15.22031 \quad 55.92194$ $\begin{array}{llll}843.3 & 20 & 946.2 & 24.6\end{array}$

$\begin{array}{llll}804.6 & 20 & 810.5 & 25.4\end{array}$ 


\begin{tabular}{|c|c|c|c|c|c|c|}
\hline 301.04648 & 4.27397 & 3.06504 & & & & \\
\hline 310.48722 & 2.74908 & 4.50096 & & & & \\
\hline \multirow[t]{2}{*}{163.26088} & 0.78261 & 1.69578 & & & & \\
\hline & 14.67141 & 30.10684 & & & & \\
\hline 539.57904 & 2.14055 & 5.07756 & & & & \\
\hline 168.19286 & 3.20794 & 3.37136 & & & & \\
\hline 7.09372 & 0.60397 & 0.0708 & & & & \\
\hline \multirow{2}{*}{737.51954} & 3.19424 & 6.7785 & & & & \\
\hline & -5.36193 & 32.47276 & & & & \\
\hline 200.09056 & 1.54979 & 2.2052 & & & & \\
\hline 0.05104 & 0.14135 & 0.00384 & 852.3 & 21.6 & 830.6 & 27.4 \\
\hline 0.18252 & 0.13136 & 0.00474 & & & & \\
\hline \multirow[t]{2}{*}{1.14662} & 0.22981 & 0.01684 & & & & \\
\hline & 7.54744 & 16.79362 & & & & \\
\hline 26.69068 & 0.85329 & 0.26702 & & & & \\
\hline \multirow[t]{2}{*}{3.32678} & 0.11262 & 0.04188 & & & & \\
\hline & 6.52949 & 165.77358 & & & & \\
\hline 6.099 & 1.30842 & 0.05486 & & & & \\
\hline 1582.6029 & 8.86202 & 10.97636 & & & & \\
\hline 0.11492 & 0.13305 & 0.00498 & & & & \\
\hline 0.2552 & 0.04627 & 0.00274 & & & & \\
\hline 1.65626 & 0.35693 & 0.01648 & & & & \\
\hline 83.01722 & 1.56886 & 1.284 & & & & \\
\hline 987.4237 & 3.44211 & 13.30822 & & & & \\
\hline 0.27696 & 0.1146 & 0.00618 & & & & \\
\hline 748.9516 & 3.47787 & 6.13234 & & & & \\
\hline 201.62052 & 3.09309 & 1.66228 & & & & \\
\hline 525.01464 & -4.94568 & 4.87548 & & & & \\
\hline 0.0367 & 0.12527 & 0.00316 & 760.8 & 18.2 & 756.4 & 21.6 \\
\hline 1122.92724 & 16.73432 & 9.81522 & & & & \\
\hline 56.50022 & 0.74752 & 0.71282 & & & & \\
\hline 1617.406 & -3.81543 & 17.91312 & & & & \\
\hline 0.07366 & 0.11998 & 0.0036 & & & & \\
\hline 999.5431 & 5.69823 & 12.49076 & & & & \\
\hline 915.6853 & 0.08779 & 2.99136 & & & & \\
\hline 192.2272 & 3.02722 & 2.53394 & & & & \\
\hline 0.03306 & 0.0973 & 0.0026 & 598.6 & 15.2 & 610.8 & 22 \\
\hline 0.2133 & 0.11744 & 0.00496 & & & & \\
\hline 84.45468 & 0.62539 & 0.68094 & & & & \\
\hline 867.05798 & 2.50409 & 5.34522 & & & & \\
\hline 84.7107 & 0.53489 & 0.85484 & & & & \\
\hline 1638.56726 & 0.90664 & 3.94444 & & & & \\
\hline 1320.521 & -2.35068 & 6.96374 & & & & \\
\hline 0.8477 & 0.21318 & 0.01202 & & & & \\
\hline 10.82862 & 0.19317 & 0.12474 & & & & \\
\hline 462.29742 & 25.90498 & 3.78544 & & & & \\
\hline \multirow[t]{3}{*}{0.15282} & 0.06169 & 0.00172 & & & & \\
\hline & 28.51645 & 28.7878 & & & & \\
\hline & 23.78641 & 203.33814 & & & & \\
\hline 1370.73754 & 8.6811 & 12.99666 & & & & \\
\hline \multirow[t]{2}{*}{1109.65198} & -3.89682 & 9.05538 & & & & \\
\hline & -90.82253 & & & & & \\
\hline
\end{tabular}




$\begin{array}{ccccccc}0.04836 & 0.07379 & 0.0017 & 458.9 & 10.2 & 1012.1 & 23.6 \\ 0.0274 & 0.09759 & 0.00232 & 600.3 & 13.6 & 601.4 & 19 \\ 0.03138 & 0.10192 & 0.00244 & 625.7 & 14.2 & 666.2 & 20.4 \\ 0.095 & 0.0923 & 0.0043 & 569.1 & 25.4 & 614.5 & 59 \\ & & & & & & \\ 70.37664 & 0.53065 & 0.5454 & & & 585 & 21.2 \\ 0.03106 & 0.09844 & 0.00246 & 605.3 & 14.4 & 767.3 & 29 \\ 0.05108 & 0.12404 & 0.00338 & 753.8 & 19.4 & 744.2 & 30 \\ 0.05176 & 0.11823 & 0.00328 & 720.4 & 19 & 752.2 & 30.8 \\ 0.05398 & 0.12143 & 0.00344 & 738.8 & 19.8 & 2466.1 & 33.6 \\ 0.29312 & 0.4628 & 0.01068 & 2451.9 & 47 & 753.4 & 34.6 \\ 0.06134 & 0.13108 & 0.00394 & 794 & 22.4 & 955.7 & 35.6 \\ 0.07528 & 0.14665 & 0.00426 & 882.2 & 24 & 813.8 & 31 \\ 0.0573 & 0.13523 & 0.00374 & 817.6 & 21.2 & 845.7 & 26.4 \\ 0.04942 & 0.1404 & 0.00352 & 846.9 & 19.8 & 525.5 & 21 \\ 0.0292 & 0.08126 & 0.00212 & 503.6 & 12.6 & & \end{array}$




\begin{tabular}{|c|c|c|c|c|c|}
\hline age $207 / 206$ & $2 \sigma$ age 76 & $\begin{array}{l}\text { discordance } \\
\Delta 68-75[\%]\end{array}$ & $\Delta 68-76[\%]$ & $\begin{array}{c}\text { preferred age } \\
\text { age }\end{array}$ & $2 \sigma$ age \\
\hline 2486.9 & 531.8 & -70.5 & -98.9 & & \\
\hline 799.7 & 73.8 & -1.4 & -5.2 & 758.1 & 18 \\
\hline 952.5 & 56.6 & -10.5 & -33.2 & 636.6 & 14.2 \\
\hline 729.4 & 82.6 & 1.8 & 7.6 & 784.6 & 19.2 \\
\hline 1194.8 & 52.4 & -9.9 & -26.7 & 875.5 & 19.2 \\
\hline 2225.4 & 39.4 & -25.4 & -44.9 & & \\
\hline 2425.5 & 44.6 & -15 & -26.3 & & \\
\hline 802.2 & 71.2 & -1.7 & -6.4 & 751.3 & 17.6 \\
\hline 898.7 & 51.4 & -4.9 & -16.5 & 750.6 & 16.2 \\
\hline 1095.1 & 50 & -19.8 & -54.7 & & \\
\hline 626.8 & 108 & -4.4 & -19.9 & 502 & 13.6 \\
\hline 2552.4 & 41.6 & -61.3 & -89.1 & & \\
\hline-192.1 & 277.4 & 15.4 & -196.3 & & \\
\hline 682.7 & 68.6 & 2.1 & 8.6 & 741.6 & 17.6 \\
\hline 944.1 & 84.8 & -6.4 & -21 & 745.5 & 19.6 \\
\hline 779.2 & 54.2 & -3.8 & -14.6 & 665.2 & 15.2 \\
\hline 760.4 & 64 & 0.9 & 3.1 & 784.1 & 18.4 \\
\hline
\end{tabular}

563.4

62.8

1.6

7.6

606.4

14.2

599.2

70.8

0.8

3.7

621.2

15

608.3

81.2

0.1

608.1

15.2

662.3

91

0.7

2.8

681

17.8 


\begin{tabular}{|c|c|c|c|c|c|}
\hline 608.6 & 61.8 & -0.9 & -4.3 & 582.2 & 13.6 \\
\hline 559.7 & 54.8 & 1.5 & 7.2 & 600.2 & 13.8 \\
\hline 720.3 & 83 & 2.2 & 8.9 & 784.6 & 20 \\
\hline 898.7 & 123.8 & -1.5 & -5.5 & 849.6 & 26.8 \\
\hline 886.1 & 65 & -2.1 & -7.6 & 818.5 & 19.4 \\
\hline 902 & 57.8 & -5.3 & -18.1 & 738.7 & 17.2 \\
\hline 659.9 & 148.2 & -2.2 & -9.7 & 595.9 & 19.6 \\
\hline 1937.8 & 49.2 & -2.3 & -4.7 & 1937.8 & 49.2 \\
\hline 618.6 & 65.8 & 0.4 & 1.7 & 629.4 & 15 \\
\hline
\end{tabular}

67.8

$-4.5$

$-15.9$

729.8

17.6 


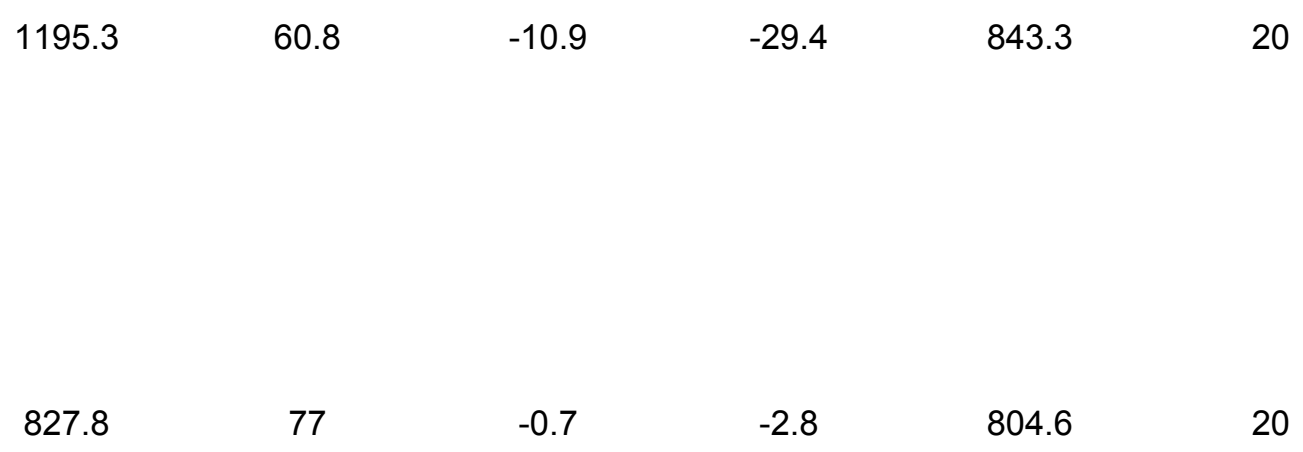

55

56

57

$-88.1$ 
$\begin{array}{llllll}774.3 & 84.2 & 2.6 & 10.1 & 852.3 & 21.6\end{array}$

$\begin{array}{llllll}744.3 & 67.4 & 0.6 & 2.2 & 760.8 & 18.2\end{array}$

$\begin{array}{llllll}657.5 & 85 & -2 & -9 & 598.6 & 15.2\end{array}$ 


$\begin{array}{cccccc}2538.7 & 47.8 & -54.7 & -81.9 & & \\ 606.8 & 74.2 & -0.2 & -1.1 & 600.3 & 13.6 \\ 806.7 & 71.6 & -6.1 & -22.4 & 625.7 & 14.2 \\ 785.9 & 250 & -7.4 & -27.6 & 569.1 & 25.4 \\ & & & & & \\ 507.7 & 89.6 & 3.5 & 19.2 & 605.3 & 14.4 \\ 807.6 & 97.6 & -1.8 & -6.7 & 753.8 & 19.4 \\ 817.1 & 103.4 & -3.2 & -11.8 & 720.4 & 19 \\ 793 & 106.2 & -1.8 & -6.8 & 738.8 & 19.8 \\ 2478.3 & 47.2 & -0.6 & -1.1 & 2478.3 & 47.2 \\ 635.4 & 123.8 & 5.4 & 25 & & \\ 1130 & 98.8 & -7.7 & -21.9 & 882.2 & 24 \\ 803.8 & 100.4 & 0.5 & 1.7 & 817.6 & 21.2 \\ 843.4 & 80.6 & 0.1 & 0.4 & 846.9 & 19.8 \\ 622.2 & 95.6 & -4.2 & -19.1 & 503.6 & 12.6\end{array}$


Sample 45 Main Nile @ Wadi Halfa 59 grain analysed 47 concordant ages

\begin{tabular}{|c|c|c|c|c|c|c|}
\hline \multirow[b]{2}{*}{ grain } & \multicolumn{3}{|c|}{ concentrations } & \multicolumn{3}{|l|}{ isotopic ratios } \\
\hline & U [ppm] & $\mathrm{Pb}$ [ppm] & Th/U & Pb207/Pb206 & $2 \sigma 76$ & Pb207/U235 \\
\hline X45 G001 & 295.2 & 50.3 & 0.7037 & 0.08421 & 0.0027 & 1.73869 \\
\hline X45_G002 & 116.6 & 14.8 & 0.2394 & 0.06427 & 0.00342 & 1.13158 \\
\hline X45_G003 & 33.3 & 3.6 & 0.2733 & 0.06349 & 0.0064 & 0.93864 \\
\hline X45_G004 & 551.1 & 55.6 & 0.6827 & 0.0584 & 0.00196 & 0.72365 \\
\hline X45_G005 & 64 & 28.8 & 1.0256 & 0.11888 & 0.00388 & 5.89592 \\
\hline X45_G006 & 363.2 & 52.3 & 0.6011 & 0.06829 & 0.00222 & 1.23788 \\
\hline X45 G007 & 74 & 6.4 & 0.3081 & 0.05951 & 0.00406 & 0.69971 \\
\hline X45 G008 & 235.9 & 34.1 & 0.5884 & 0.06467 & 0.00208 & 1.1749 \\
\hline X45_G009 & 88.6 & 12.2 & 0.3147 & 0.06618 & 0.00258 & 1.23777 \\
\hline X45_G010 & & & & -20.68902 & 927.17578 & 203.1976 \\
\hline X45_G011 & 107.3 & 10.3 & 0.071 & 0.06071 & 0.00256 & 0.8535 \\
\hline X45_G012 & 195.3 & 23.2 & 0.3526 & 0.0669 & 0.00252 & 1.07023 \\
\hline X45 G013 & 142.6 & 19 & 0.4849 & 0.06415 & 0.00284 & 1.10196 \\
\hline X45_G014 & 247.9 & 31.3 & 0.4422 & 0.06899 & 0.00228 & 1.1387 \\
\hline X45_G015 & 68.6 & 7.9 & 0.2948 & 0.0637 & 0.0041 & 0.99704 \\
\hline X45_G016 & 138.6 & 18.2 & 0.6034 & 0.06923 & 0.00278 & 1.13765 \\
\hline X45_G017 & & & & & & \\
\hline X45_G018 & 79.3 & 9.4 & 0.705 & 0.12907 & 0.00608 & 1.92096 \\
\hline X45_G019 & 441.2 & 55.6 & 0.4407 & 0.06378 & 0.00194 & 1.06488 \\
\hline X45_G020 & 697.7 & 79 & 0.3474 & 0.07241 & 0.00206 & 1.10781 \\
\hline X45_G021 & 197.9 & 27.6 & 0.677 & 0.06737 & 0.00258 & 1.15345 \\
\hline X45_G022 & 335.9 & 32.1 & 0.013 & 0.0624 & 0.00194 & 0.8926 \\
\hline X45_G023 & 273.2 & 39 & 0.2721 & 0.06878 & 0.00218 & 1.34823 \\
\hline X45_G024 & 118 & 20.4 & 0.3015 & 0.07624 & 0.00274 & 1.77872 \\
\hline X45_G025 & 347.2 & 43.1 & 0.2734 & 0.07169 & 0.00224 & 1.22312 \\
\hline X45_G026 & 102 & 12.1 & 0.9987 & 0.06006 & 0.00322 & 0.81284 \\
\hline X45_G027 & 388.5 & 35.1 & 0.2421 & 0.13097 & 0.0039 & 1.65235 \\
\hline X45_G028 & 11188.9 & 211.8 & 1.004 & 0.09646 & 0.00264 & 0.22339 \\
\hline X45_G029 & 1043.6 & 180.4 & 1.4629 & 0.06907 & 0.00184 & 1.32792 \\
\hline X45_G030 & 1534.1 & 100.1 & 0.8707 & 0.08069 & 0.00216 & 0.67149 \\
\hline X45_G031 & 1311.5 & 116.8 & 0.4268 & 0.06219 & 0.0017 & 0.72206 \\
\hline X45_G032 & 58.6 & 8 & 0.6401 & 0.06404 & 0.00388 & 1.076 \\
\hline X45_G033 & 86 & 0.4 & 0.7652 & 0.05854 & 0.03152 & 0.03092 \\
\hline X45_G034 & 193.3 & 20.4 & 0.7872 & 0.06069 & 0.00274 & 0.76814 \\
\hline X45_G035 & 149.9 & 14.2 & 0.1995 & 0.06239 & 0.00296 & 0.82703 \\
\hline X45_G036 & 283.2 & 38.7 & 0.5234 & 0.06651 & 0.00216 & 1.16135 \\
\hline X45_G037 & 116.6 & 16.3 & 0.5512 & 0.06579 & 0.0029 & 1.16851 \\
\hline X45_G038 & 425.2 & 53.3 & 0.439 & 0.06318 & 0.00196 & 1.03645 \\
\hline X45_G039 & 363.2 & 54.6 & 0.8724 & 0.07776 & 0.00238 & 1.35411 \\
\hline X45_G040 & 195.9 & 26.3 & 0.3897 & 0.06722 & 0.00232 & 1.19811 \\
\hline X45_G041 & 392.5 & 44.8 & 0.3948 & 0.06476 & 0.00202 & 0.97166 \\
\hline X45_G042 & 1258.8 & 116.7 & 0.1569 & 0.13132 & 0.00354 & 1.64305 \\
\hline X45_G043 & 1788.6 & 248.8 & 0.2375 & 0.06797 & 0.00182 & 1.30799 \\
\hline X45_G044 & 179.9 & 28.3 & 0.7195 & 0.06573 & 0.00246 & 1.25892 \\
\hline X45_G045 & 6109.6 & 259.2 & 0.8158 & 0.05882 & 0.00162 & 0.31544 \\
\hline X45_G046 & 181.9 & 24.4 & 0.4112 & 0.06688 & 0.00266 & 1.18549 \\
\hline X45_G047 & 337.9 & 76 & 1.4418 & 0.07595 & 0.0025 & 1.75122 \\
\hline X45_G048 & 74.6 & 33.4 & 0.3794 & 0.17641 & 0.00566 & 10.16329 \\
\hline X45_G049 & 371.2 & 46.3 & 0.5509 & 0.06453 & 0.0023 & 1.02229 \\
\hline X45_G050 & 87.3 & 0.5 & 1.3502 & 0.10573 & 0.03558 & 0.0616 \\
\hline
\end{tabular}




$\begin{array}{lcccccc}\text { X45_G051 } & 169.9 & 26.2 & 0.8132 & 0.06694 & 0.00252 & 1.218 \\ \text { X45_G052 } & 196.6 & 28.4 & 0.3944 & 0.06628 & 0.00238 & 1.26726 \\ \text { X45_G053 } & 246.6 & & 1.8967 & 0.13709 & 0.00422 & 2.88071 \\ \text { X45_G054 } & 102 & 14.1 & 0.5389 & 0.06425 & 0.00292 & 1.12243 \\ \text { X45_G055 } & 134.6 & 31.3 & 0.9232 & 0.07428 & 0.00264 & 1.97145 \\ \text { X45_G056 } & 369.2 & 43.6 & 0.8269 & 0.06257 & 0.00222 & 0.87238 \\ \text { X45_G057 } & 122 & 14.7 & 0.7931 & 0.06205 & 0.00308 & 0.898 \\ \text { X45_G058 } & 122.6 & 18.6 & 0.6928 & 0.06681 & 0.00322 & 1.24149 \\ \text { X45_G059 } & 4194.4 & 54 & 0.5728 & 0.25071 & 0.0075 & 0.39384\end{array}$




\section{7\% concordant}

\begin{tabular}{|c|c|c|c|c|c|c|}
\hline $2 \sigma 75$ & $\mathrm{~Pb} 206 / \mathrm{U} 238$ & $2 \sigma 68$ & $\begin{array}{c}\text { ages } \\
\text { age } 206 / 238\end{array}$ & $2 \sigma$ age 68 & age $207 / 235$ & $2 \sigma$ age 75 \\
\hline 0.05324 & 0.1498 & 0.00348 & 899.8 & 19.6 & 1023 & 25.6 \\
\hline 0.05828 & 0.12773 & 0.00352 & 774.9 & 20.2 & 768.5 & 32.4 \\
\hline 0.0913 & 0.10725 & 0.0042 & 656.8 & 24.4 & 672.2 & 53.2 \\
\hline 0.02356 & 0.08989 & 0.002 & 554.9 & 11.8 & 552.8 & 17.2 \\
\hline 0.1912 & 0.35982 & 0.00916 & 1981.3 & 43.4 & 1960.6 & 36 \\
\hline 0.03902 & 0.13151 & 0.00296 & 796.5 & 16.8 & 817.9 & 22.2 \\
\hline 0.04606 & 0.0853 & 0.00258 & 527.7 & 15.4 & 538.6 & 31.2 \\
\hline 0.03658 & 0.13181 & 0.00294 & 798.2 & 16.8 & 788.9 & 21.4 \\
\hline 0.04674 & 0.13569 & 0.00328 & 820.2 & 18.6 & 817.9 & 25.8 \\
\hline 511.51822 & -0.07125 & 3.1949 & & & & \\
\hline 0.03466 & 0.102 & 0.0025 & 626.1 & 14.6 & 626.6 & 22.8 \\
\hline 0.03894 & 0.11606 & 0.00276 & 707.8 & 16 & 738.9 & 23.4 \\
\hline 0.04712 & 0.12462 & 0.00314 & 757.1 & 18 & 754.3 & 27.2 \\
\hline 0.03636 & 0.11974 & 0.00272 & 729.1 & 15.6 & 771.9 & 21.6 \\
\hline 0.06194 & 0.11356 & 0.00344 & 693.4 & 20 & 702.3 & 36 \\
\hline 0.04416 & 0.11922 & 0.00292 & 726.1 & 16.8 & 771.4 & 25.4 \\
\hline 0.0846 & 0.10798 & 0.0032 & 661 & 18.6 & 1088.4 & 37.2 \\
\hline 0.03168 & 0.12113 & 0.00266 & 737.1 & 15.2 & 736.2 & 19.6 \\
\hline 0.0307 & 0.111 & 0.0024 & 678.6 & 14 & 757.1 & 19 \\
\hline 0.04276 & 0.12421 & 0.00298 & 754.7 & 17 & 778.9 & 24.6 \\
\hline 0.02706 & 0.10378 & 0.00228 & 636.5 & 13.4 & 647.8 & 18.2 \\
\hline 0.04164 & 0.14222 & 0.00318 & 857.2 & 18 & 866.8 & 22.6 \\
\hline 0.06226 & 0.16926 & 0.00406 & 1008 & 22.4 & 1037.7 & 28 \\
\hline 0.03732 & 0.12378 & 0.00276 & 752.3 & 15.8 & 811.2 & 21.4 \\
\hline 0.04198 & 0.09819 & 0.00268 & 603.8 & 15.8 & 604 & 27.4 \\
\hline 0.0473 & 0.09153 & 0.00208 & 564.6 & 12.2 & 990.5 & 23.6 \\
\hline 0.00594 & 0.0168 & 0.00036 & 107.4 & 2.2 & 204.7 & 6.4 \\
\hline 0.03474 & 0.13949 & 0.00296 & 841.8 & 16.8 & 858 & 19.8 \\
\hline 0.0176 & 0.06037 & 0.00128 & 377.9 & 7.8 & 521.6 & 14 \\
\hline 0.01934 & 0.08423 & 0.00178 & 521.3 & 10.6 & 551.9 & 14.8 \\
\hline 0.06298 & 0.12189 & 0.00362 & 741.4 & 20.8 & 741.7 & 35.6 \\
\hline 0.0164 & 0.00383 & 0.00038 & 24.6 & 2.4 & 30.9 & 16.6 \\
\hline 0.03352 & 0.09183 & 0.00232 & 566.4 & 13.6 & 578.7 & 22.8 \\
\hline 0.03766 & 0.09616 & 0.0025 & 591.9 & 14.8 & 612 & 24.8 \\
\hline 0.03664 & 0.12667 & 0.00284 & 768.8 & 16.2 & 782.6 & 21.6 \\
\hline 0.0496 & 0.12885 & 0.0033 & 781.3 & 18.8 & 785.9 & 27.8 \\
\hline 0.03134 & 0.11901 & 0.00262 & 724.9 & 15 & 722.1 & 19.6 \\
\hline 0.0404 & 0.12634 & 0.0028 & 767 & 16 & 869.3 & 22 \\
\hline 0.0402 & 0.1293 & 0.00298 & 783.9 & 17 & 799.7 & 23 \\
\hline 0.02964 & 0.10886 & 0.00242 & 666.1 & 14 & 689.3 & 19.2 \\
\hline 0.0434 & 0.09077 & 0.00194 & 560.1 & 11.4 & 986.9 & 21.8 \\
\hline 0.0344 & 0.1396 & 0.00296 & 842.4 & 16.8 & 849.2 & 19.6 \\
\hline 0.04586 & 0.13896 & 0.00332 & 838.8 & 18.8 & 827.4 & 25.2 \\
\hline 0.00856 & 0.03891 & 0.00082 & 246.1 & 5 & 278.4 & 8.4 \\
\hline 0.04574 & 0.12859 & 0.00314 & 779.8 & 18 & 793.9 & 25.8 \\
\hline 0.05642 & 0.16729 & 0.00384 & 997.2 & 21.2 & 1027.6 & 26 \\
\hline 0.32418 & 0.41797 & 0.01064 & 2251.3 & 48.4 & 2449.7 & 37.8 \\
\hline 0.03552 & 0.11493 & 0.00268 & 701.3 & 15.4 & 715.1 & 21.8 \\
\hline 0.01984 & 0.00423 & 0.00042 & 27.2 & 2.6 & 60.7 & 20.6 \\
\hline
\end{tabular}




$\begin{array}{ccccccc}0.04468 & 0.132 & 0.00316 & 799.3 & 18 & 808.9 & 24.8 \\ 0.0444 & 0.13871 & 0.00326 & 837.4 & 18.4 & 831.2 & 24.4 \\ 0.08616 & 0.15245 & 0.0035 & 914.7 & 19.6 & 1376.9 & 29 \\ 0.04936 & 0.12674 & 0.00326 & 769.2 & 18.6 & 764.1 & 28 \\ 0.06838 & 0.19256 & 0.00458 & 1135.2 & 24.8 & 1105.8 & 28.8 \\ 0.03012 & 0.10115 & 0.00234 & 621.1 & 13.6 & 636.9 & 20 \\ 0.04298 & 0.105 & 0.0028 & 643.6 & 16.4 & 650.7 & 27 \\ 0.05792 & 0.13481 & 0.00364 & 815.2 & 20.6 & 819.6 & 31 \\ 0.01134 & 0.0114 & 0.00026 & 73.1 & 1.6 & 337.2 & 10.8\end{array}$




\begin{tabular}{|c|c|c|c|c|c|}
\hline age $207 / 206$ & $2 \sigma$ age 76 & $\begin{array}{l}\text { discordance } \\
\Delta 68-75[\%]\end{array}$ & $\Delta$ 68-76 [\%] & $\begin{array}{c}\text { preferred age } \\
\text { age }\end{array}$ & $2 \sigma$ age \\
\hline 1297.4 & 62.4 & -12 & -30.6 & 899.8 & 19.6 \\
\hline 750.5 & 112.4 & 0.8 & 3.2 & 774.9 & 20.2 \\
\hline 724.7 & 213.8 & -2.3 & -9.4 & 656.8 & 24.4 \\
\hline 544.8 & 73.4 & 0.4 & 1.9 & 554.9 & 11.8 \\
\hline 1939.5 & 58.4 & 1.1 & 2.2 & 1939.5 & 58.4 \\
\hline 877.4 & 67.2 & -2.6 & -9.2 & 796.5 & 16.8 \\
\hline 585.8 & 148 & -2 & -9.9 & 527.7 & 15.4 \\
\hline 763.6 & 67.8 & 1.2 & 4.5 & 798.2 & 16.8 \\
\hline 812.1 & 81.6 & 0.3 & 1 & 820.2 & 18.6 \\
\hline 629 & 90.8 & -0.1 & -0.5 & 626.1 & 14.6 \\
\hline 834.7 & 78.4 & -4.2 & -15.2 & 707.8 & 16 \\
\hline 746.6 & 93.6 & 0.4 & 1.4 & 757.1 & 18 \\
\hline 898.4 & 68.2 & -5.5 & -18.9 & 729.1 & 15.6 \\
\hline 731.7 & 136.4 & -1.3 & -5.2 & 693.4 & 20 \\
\hline 905.6 & 82.8 & -5.9 & -19.8 & 726.1 & 16.8 \\
\hline 2085.3 & 82.8 & -39.3 & -68.3 & & \\
\hline 734.3 & 64.4 & 0.1 & 0.4 & 737.1 & 15.2 \\
\hline 997.5 & 57.8 & -10.4 & -32 & 678.6 & 14 \\
\hline 849.2 & 79.6 & -3.1 & -11.1 & 754.7 & 17 \\
\hline 687.8 & 66.4 & -1.7 & -7.5 & 636.5 & 13.4 \\
\hline 892.1 & 65.4 & -1.1 & -3.9 & 857.2 & 18 \\
\hline 1101.4 & 71.8 & -2.9 & -8.5 & 1008 & 22.4 \\
\hline 977.2 & 63.6 & -7.3 & -23 & 752.3 & 15.8 \\
\hline 605.7 & 116 & 0 & -0.3 & 603.8 & 15.8 \\
\hline 2111 & 52.2 & -43 & -73.3 & & \\
\hline 1556.7 & 51.4 & -47.5 & -93.1 & & \\
\hline 900.8 & 55 & -1.9 & -6.6 & 841.8 & 16.8 \\
\hline 1213.9 & 52.6 & -27.6 & -68.9 & & \\
\hline 680.6 & 58.4 & -5.5 & -23.4 & 521.3 & 10.6 \\
\hline 743 & 128.2 & 0 & -0.2 & 741.4 & 20.8 \\
\hline 550 & 1175.6 & -20.3 & -95.5 & & \\
\hline 628.3 & 97.2 & -2.1 & -9.9 & 566.4 & 13.6 \\
\hline 687.5 & 101.2 & -3.3 & -13.9 & 591.9 & 14.8 \\
\hline 822.5 & 67.8 & -1.8 & -6.5 & 768.8 & 16.2 \\
\hline 799.7 & 92.4 & -0.6 & -2.3 & 781.3 & 18.8 \\
\hline 714.3 & 66 & 0.4 & 1.5 & 724.9 & 15 \\
\hline 1140.7 & 60.8 & -11.8 & -32.8 & 767 & 16 \\
\hline 844.6 & 71.8 & -2 & -7.2 & 783.9 & 17 \\
\hline 766.5 & 65.8 & -3.4 & -13.1 & 666.1 & 14 \\
\hline 2115.7 & 47.2 & -43.2 & -73.5 & & \\
\hline 867.6 & 55.6 & -0.8 & -2.9 & 842.4 & 16.8 \\
\hline 797.8 & 78.4 & 1.4 & 5.1 & 838.8 & 18.8 \\
\hline 560.4 & 60 & -11.6 & -56.1 & 246.1 & 5 \\
\hline 834 & 82.8 & -1.8 & -6.5 & 779.8 & 18 \\
\hline 1093.8 & 66 & -3 & -8.8 & 997.2 & 21.2 \\
\hline 2619.4 & 53.4 & -8.1 & -14.1 & 2619.4 & 53.4 \\
\hline 759 & 75.2 & -1.9 & -7.6 & 701.3 & 15.4 \\
\hline 1727 & 617.8 & -55.2 & -98.4 & & \\
\hline
\end{tabular}




$\begin{array}{cccccc}835.9 & 78.4 & -1.2 & -4.4 & 799.3 & 18 \\ 815.2 & 75 & 0.7 & 2.7 & 837.4 & 18.4 \\ 2190.8 & 53.6 & -33.6 & -58.2 & & \\ 749.9 & 96 & 0.7 & 2.6 & 769.2 & 18.6 \\ 1049.1 & 71.6 & 2.7 & 8.2 & & \\ 693.6 & 75.6 & -2.5 & -10.5 & 621.1 & 13.6 \\ 675.8 & 106.2 & -1.1 & -4.8 & 643.6 & 16.4 \\ 831.9 & 100.4 & -0.5 & -2 & 815.2 & 20.6 \\ 3189.3 & 47.4 & -78.3 & -97.7 & & \end{array}$


Sample 46 Main Nile @ Aswan 4 grain analysed 1 concordant ages 25.0 !

\begin{tabular}{ccccccc}
\cline { 2 - 7 } grain & $\begin{array}{c}\text { concentrations } \\
\mathbf{U}[\mathrm{ppm}]\end{array}$ & $\mathrm{Pb}[\mathbf{p p m}]$ & $\mathrm{Th} / \mathrm{U}$ & $\begin{array}{c}\text { isotopic ratios } \\
\mathrm{Pb207/Pb206}\end{array}$ & $\mathbf{2 \sigma} \mathbf{7 6}$ & $\mathrm{Pb207/U235}$ \\
\hline X46_G001 & & & & 0.43384 & 0.1174 & 14.3692 \\
X46_G002 & 702.4 & 68.9 & 0.449 & 0.06998 & 0.00224 & 0.88448 \\
X46_G003 & & & & 0.04472 & 0.06686 & 27.49713 \\
X46_G004 & \multirow{2}{*}{1506.1} & 115.8 & 0.7112 & 0.08052 & 0.00248 & 0.77839
\end{tabular}




\section{$\%$ concordant}

\begin{tabular}{|c|c|c|c|c|c|c|}
\hline $2 \sigma 75$ & $\mathrm{~Pb} 206 / \mathrm{U} 238$ & $2 \sigma 68$ & $\begin{array}{c}\text { ages } \\
\text { age } 206 / 238\end{array}$ & $2 \sigma$ age 68 & age $207 / 235$ & $2 \sigma$ age 75 \\
\hline 3.2134 & 0.2403 & 0.0451 & & & & \\
\hline 0.02816 & 0.0917 & 0.00214 & 565.6 & 12.6 & 643.4 & 18.8 \\
\hline 48.4406 & 4.46092 & 4.29558 & & & & \\
\hline 0.02374 & 0.07013 & 0.00162 & 436.9 & 9.8 & 584.6 & 17.2 \\
\hline
\end{tabular}




\begin{tabular}{|c|c|c|c|c|c|}
\hline age $207 / 206$ & $2 \sigma$ age 76 & $\begin{array}{l}\text { discordance } \\
\Delta 68-75[\%]\end{array}$ & $\Delta 68-76[\%]$ & $\begin{array}{c}\text { preferred age } \\
\text { age }\end{array}$ & $2 \sigma$ age \\
\hline 927.8 & 65.8 & -12.1 & -39 & 565.6 & 12.6 \\
\hline 1209.8 & 60.6 & -25.3 & -63.9 & & \\
\hline
\end{tabular}




\section{Sample 2408L Main Nile @ Aswan 32 grain analysed 27 concordant ages}

\begin{tabular}{ccccccc} 
& \multicolumn{1}{c}{ concentrations } & & & isotopic ratios & & \\
grain & $\mathrm{U}[\mathrm{ppm}]$ & $\mathrm{Pb}[\mathrm{ppm}]$ & $\mathrm{Th} / \mathrm{U}$ & $\mathrm{Pb207/Pb206}$ & $\mathbf{2 \sigma} \mathbf{7 6}$ & $\mathrm{Pb207/U235}$ \\
\hline X2408L_G001 & 53.6 & 5.5 & 0.8557 & 0.05832 & 0.00404 & 0.69406 \\
X2408L_G002 & 353.8 & 28.2 & 0.3237 & 0.05523 & 0.00184 & 0.59993 \\
X2408L_G003 & 53.6 & 0.2 & 0.6788 & 0.0472 & 0.04218 & 0.024 \\
X2408L_G004 & 265.8 & 28.6 & 0.3004 & 0.06179 & 0.00202 & 0.90678 \\
X2408L_G005 & 206.1 & 22.1 & 0.7544 & 0.06018 & 0.00224 & 0.77825 \\
X2408L_G006 & 407.4 & 79.5 & 0.9755 & 0.07274 & 0.00192 & 1.60523 \\
X2408L_G007 & 949 & 17.8 & 20.3946 & 0.30268 & 0.01222 & 0.20395 \\
X2408L_G008 & & & & -0.21735 & 1.03982 & \\
X2408L_G009 & 211.8 & 30.6 & 0.4476 & 0.06543 & 0.00204 & 1.23979 \\
X2408L_G010 & 75.8 & 11.6 & 0.8343 & 0.1245 & 0.00482 & 2.15532 \\
X2408L_G011 & 55.3 & 7.9 & 0.4394 & 0.07342 & 0.00348 & 1.36067 \\
X2408L_G012 & & & & -0.69132 & 2.96764 & -76.76031 \\
X2408L_G013 & 35.3 & 3.6 & 0.9113 & 0.05648 & 0.00444 & 0.6607 \\
X2408L_G014 & 5475.3 & 378.7 & 0.099 & 0.06332 & 0.00156 & 0.63306 \\
X2408L_G015 & 166.4 & 20.7 & 0.5933 & 0.06568 & 0.00262 & 1.01866 \\
X2408L_G016 & 758.2 & 81.6 & 0.6923 & 0.06448 & 0.00188 & 0.84987 \\
X2408L_G017 & 99.3 & 13.9 & 0.501 & 0.06878 & 0.00302 & 1.23248 \\
X2408L_G018 & 103.7 & 13.5 & 0.3822 & 0.0652 & 0.00264 & 1.12981 \\
X2408L_G019 & 60.1 & 7.1 & 0.2649 & 0.06691 & 0.00386 & 1.09236 \\
X2408L_G020 & 418.3 & 41 & 0.3334 & 0.05806 & 0.00192 & 0.77038 \\
X2408L_G021 & 85 & 17.1 & 0.7719 & 0.07299 & 0.00282 & 1.74254 \\
X2408L_G022 & 128.1 & 18.3 & 0.746 & 0.06539 & 0.0025 & 1.11813 \\
X2408L_G023 & 65.8 & 8.3 & 0.461 & 0.07175 & 0.00348 & 1.16471 \\
X2408L_G024 & 163.4 & 17.6 & 0.3435 & 0.06558 & 0.00224 & 0.95291 \\
X2408L_G025 & 198.3 & 28.6 & 0.6938 & 0.06257 & 0.00198 & 1.09857 \\
X2408L_G026 & 69.7 & 9.2 & 0.4361 & 0.06551 & 0.003 & 1.1336 \\
X2408L_G027 & 205.7 & 45.8 & 0.6122 & 0.07864 & 0.00226 & 2.17273 \\
X2408L_G028 & 31.4 & 0.1 & 1.1 & 0.09884 & 0.08704 & 0.03466 \\
X2408L_G029 & 54.9 & 6.9 & 0.7568 & 0.06509 & 0.00384 & 0.99105 \\
X2408L_G030 & 139.9 & 19 & 0.8775 & 0.06226 & 0.00258 & 0.96047 \\
X2408L_G031 & 599.1 & 79 & 1.0747 & 0.0603 & 0.0018 & 0.88458 \\
X2408L_G032 & 634.4 & 92.4 & 0.4599 & 0.06499 & 0.00182 & 1.22893
\end{tabular}




\section{$84.4 \%$ concordant}

\begin{tabular}{ccc|cccc}
\hline $\mathbf{2 \sigma} \mathbf{7 5}$ & Pb206/U238 & $\mathbf{2 \sigma} \mathbf{6 8}$ & $\begin{array}{c}\text { ages } \\
\text { age } \mathbf{2 0 6 / 2 3 8}\end{array}$ & $\mathbf{2 \sigma}$ age $\mathbf{6 8}$ & age 207/235 & 20 age 75 \\
\hline 0.04612 & 0.08634 & 0.00268 & 533.9 & 16 & 535.2 & 31.6 \\
0.01944 & 0.0788 & 0.00176 & 489 & 10.6 & 477.2 & 15.2 \\
0.02116 & 0.00369 & 0.00054 & 23.7 & 3.4 & 24.1 & 21.6 \\
0.0288 & 0.10646 & 0.0024 & 652.2 & 14 & 655.3 & 19.2 \\
0.02806 & 0.09382 & 0.0022 & 578.1 & 13 & 584.5 & 19.6 \\
0.04156 & 0.1601 & 0.00342 & 957.3 & 19 & 972.3 & 21.2 \\
0.00698 & 0.00489 & 0.00014 & 31.4 & 0.8 & 188.5 & 8.6 \\
0.03778 & 0.13745 & 0.00308 & 830.2 & 17.4 & 818.8 & 21.6 \\
0.07884 & 0.12559 & 0.00334 & 762.7 & 19.2 & 1166.8 & 32.6 \\
0.06212 & 0.13445 & 0.00368 & 813.2 & 21 & 872.2 & 32 \\
346.93338 & 0.80551 & 1.97316 & & & & \\
0.0499 & 0.08486 & 0.00284 & 525.1 & 16.8 & 515 & 34.6 \\
0.01538 & 0.07253 & 0.00152 & 451.4 & 9.2 & 498 & 12.8 \\
0.03922 & 0.11252 & 0.00276 & 687.4 & 16 & 713.2 & 24 \\
0.02412 & 0.09563 & 0.00208 & 588.7 & 12.2 & 624.6 & 17 \\
0.05202 & 0.13 & 0.00336 & 787.9 & 19.2 & 815.5 & 28.6 \\
0.04422 & 0.1257 & 0.0031 & 763.3 & 17.8 & 767.7 & 25.6 \\
0.0605 & 0.11844 & 0.00352 & 721.6 & 20.2 & 749.6 & 34.4 \\
0.02472 & 0.09626 & 0.00216 & 592.5 & 12.8 & 580 & 17.6 \\
0.0652 & 0.1732 & 0.00432 & 1029.7 & 23.8 & 1024.4 & 29.6 \\
0.04148 & 0.12405 & 0.003 & 753.8 & 17.2 & 762.1 & 24.2 \\
0.05434 & 0.11776 & 0.00322 & 717.7 & 18.6 & 784.2 & 30.4 \\
0.0316 & 0.10541 & 0.00242 & 646 & 14.2 & 679.6 & 20.4 \\
0.03386 & 0.12738 & 0.00286 & 772.9 & 16.4 & 752.7 & 20.6 \\
0.04994 & 0.12554 & 0.0033 & 762.4 & 19 & 769.5 & 28.4 \\
0.06122 & 0.20044 & 0.00444 & 1177.7 & 23.8 & 1172.4 & 25.2 \\
0.0297 & 0.00254 & 0.00052 & 16.4 & 3.4 & 34.6 & 30.8 \\
0.05598 & 0.11046 & 0.0033 & 675.4 & 19.2 & 699.3 & 33.4 \\
0.03854 & 0.11191 & 0.00278 & 683.8 & 16.2 & 683.5 & 24 \\
0.02568 & 0.10642 & 0.00232 & 651.9 & 13.6 & 643.5 & 17.6 \\
0.03374 & 0.13719 & 0.00296 & 828.8 & 16.8 & 813.9 & 19.8
\end{tabular}




\begin{tabular}{|c|c|c|c|c|c|}
\hline age $207 / 206$ & $2 \sigma$ age 76 & $\begin{array}{l}\text { discordance } \\
\Delta 68-75[\%]\end{array}$ & $\Delta 68-76[\%]$ & $\begin{array}{c}\text { preferred age } \\
\text { age }\end{array}$ & $2 \sigma$ age \\
\hline 541.8 & $\overline{151.4}$ & -0.3 & -1.5 & 533.9 & 16 \\
\hline 421.5 & 74.4 & 2.5 & 16 & 489 & 10.6 \\
\hline 59.4 & 2130 & -1.4 & -60 & 23.7 & 3.4 \\
\hline 666.8 & 70 & -0.5 & -2.2 & 652.2 & 14 \\
\hline 610.1 & 80.4 & -1.1 & -5.2 & 578.1 & 13 \\
\hline 1006.7 & 53.6 & -1.5 & -4.9 & 957.3 & 19 \\
\hline 3484 & 62.4 & -83.3 & -99.1 & & \\
\hline 788.2 & 65.4 & 1.4 & 5.3 & 830.2 & 17.4 \\
\hline 2021.7 & 68.6 & -34.6 & -62.3 & & \\
\hline 1025.6 & 95.8 & -6.8 & -20.7 & 813.2 & 21 \\
\hline 471.3 & 174 & 1.9 & 11.4 & 525.1 & 16.8 \\
\hline 719 & 52.4 & -9.4 & -37.2 & 451.4 & 9.2 \\
\hline 796.2 & 83.6 & -3.6 & -13.7 & 687.4 & 16 \\
\hline 757.4 & 61.6 & -5.7 & -22.3 & 588.7 & 12.2 \\
\hline 892.1 & 90.6 & -3.4 & -11.7 & 787.9 & 19.2 \\
\hline 780.8 & 85.2 & -0.6 & -2.2 & 763.3 & 17.8 \\
\hline 835 & 120.2 & -3.7 & -13.6 & 721.6 & 20.2 \\
\hline 532 & 72.4 & 2.2 & 11.4 & 592.5 & 12.8 \\
\hline 1013.7 & 78.2 & 0.5 & 1.6 & 1029.7 & 23.8 \\
\hline 786.9 & 80.2 & -1.1 & -4.2 & 753.8 & 17.2 \\
\hline 978.9 & 98.8 & -8.5 & -26.7 & 717.7 & 18.6 \\
\hline 793 & 71.6 & -4.9 & -18.5 & 646 & 14.2 \\
\hline 693.6 & 67.4 & 2.7 & 11.4 & 772.9 & 16.4 \\
\hline 790.8 & 96 & -0.9 & -3.6 & 762.4 & 19 \\
\hline 1163.1 & 57 & 0.5 & 1.3 & 1163.1 & 57 \\
\hline 1602.3 & 1642.6 & -52.7 & -99 & & \\
\hline 777.2 & 124 & -3.4 & -13.1 & 675.4 & 19.2 \\
\hline 683.1 & 88.4 & 0 & 0.1 & 683.8 & 16.2 \\
\hline 614.4 & 64.4 & 1.3 & 6.1 & 651.9 & 13.6 \\
\hline 774 & 59 & 1.8 & 7.1 & 828.8 & 16.8 \\
\hline
\end{tabular}


Sample 2401B Main Nile @ Luxor 20 grain analysed 15 concordant ages

\begin{tabular}{|c|c|c|c|c|c|c|}
\hline \multirow[b]{2}{*}{ grain } & \multicolumn{3}{|c|}{ concentrations } & \multicolumn{3}{|l|}{ isotopic ratios } \\
\hline & U [ppm] & $\mathrm{Pb}$ [ppm] & Th/U & $\mathrm{Pb} 207 / \mathrm{Pb} 206$ & $2 \sigma 76$ & $\mathrm{~Pb} 207 / \mathrm{U} 235$ \\
\hline X2401S G001 & 55.7 & 4.9 & 0.6105 & 0.12364 & 0.0075 & 1.29691 \\
\hline X2401S_G002 & 325.1 & 22.6 & 0.1849 & 0.05712 & 0.00216 & 0.56545 \\
\hline X2401S_G003 & 201.5 & 26.7 & 0.6005 & 0.06163 & 0.00226 & 1.02235 \\
\hline X2401S_G004 & 198.5 & 24.8 & 0.2818 & 0.06354 & 0.00236 & 1.08526 \\
\hline X2401S_G005 & 73.9 & 8.3 & 0.287 & 0.06783 & 0.00366 & 1.03266 \\
\hline X2401S_G006 & 155.6 & 20.7 & 1.5383 & 0.0568 & 0.0025 & 0.74639 \\
\hline X2401S G007 & 84.7 & 8 & 0.2614 & 0.06049 & 0.00364 & 0.7911 \\
\hline X2401S_G008 & 2635.7 & 262.5 & 0.1199 & 0.06155 & 0.00158 & 0.88563 \\
\hline X2401S_G009 & 1775.7 & 146.8 & 2.4026 & 0.08572 & 0.00238 & 0.6752 \\
\hline X2401S_G010 & 96.5 & 11.9 & 0.4582 & 0.06055 & 0.00296 & 0.97706 \\
\hline X2401S_G011 & 162.1 & 24 & 0.3465 & 0.06514 & 0.0024 & 1.29736 \\
\hline X2401S_G012 & 529 & 61.1 & 0.2349 & 0.06616 & 0.00198 & 1.05773 \\
\hline X2401S_G013 & 42.9 & 12.7 & 0.7979 & 0.02125 & 0.00168 & 0.7641 \\
\hline X2401S_G014 & 81.8 & 0.4 & 0.5692 & 0.0645 & 0.02772 & 0.04067 \\
\hline X2401S_G015 & 463 & 62.9 & 0.4053 & 0.19457 & 0.00524 & 3.27577 \\
\hline X2401S_G016 & 789.1 & 88.8 & 0.1252 & 0.06332 & 0.0018 & 1.02584 \\
\hline X2401S_G017 & 146.3 & 6.2 & 0.506 & 0.05011 & 0.0039 & 0.27434 \\
\hline X2401S_G018 & 145.3 & 17.5 & 0.5493 & 0.06145 & 0.00264 & 0.94747 \\
\hline X2401S G019 & 118.2 & 11.3 & 0.3342 & 0.0601 & 0.0033 & 0.78002 \\
\hline X2401S_G020 & 83.2 & 9.6 & 1.1215 & 0.05909 & 0.00364 & 0.74564 \\
\hline
\end{tabular}




\section{$75.0 \%$ concordant}

\begin{tabular}{ccc|cccc}
\hline $\mathbf{2 \sigma} \mathbf{7 5}$ & Pb206/U238 & $\mathbf{2 \sigma} \mathbf{6 8}$ & $\begin{array}{c}\text { ages } \\
\text { age } \mathbf{2 0 6 / 2 3 8}\end{array}$ & $\mathbf{2 \sigma}$ age $\mathbf{6 8}$ & age $\mathbf{2 0 7 / 2 3 5}$ & 20 age $\mathbf{7 5}$ \\
\hline 0.0729 & 0.07613 & 0.00264 & 473 & 15.8 & 844.4 & 40 \\
0.02082 & 0.07184 & 0.0017 & 447.2 & 10.2 & 455.1 & 16.4 \\
0.03672 & 0.12039 & 0.00286 & 732.8 & 16.4 & 715.1 & 22.4 \\
0.03924 & 0.12395 & 0.00298 & 753.3 & 17 & 746.2 & 23.4 \\
0.0538 & 0.11048 & 0.00318 & 675.5 & 18.4 & 720.3 & 31.6 \\
0.03196 & 0.09536 & 0.0024 & 587.2 & 14.2 & 566.1 & 22 \\
0.04586 & 0.0949 & 0.0028 & 584.5 & 16.4 & 591.8 & 30 \\
0.0228 & 0.10442 & 0.00224 & 640.3 & 13 & 644 & 16 \\
0.01852 & 0.05716 & 0.00126 & 358.3 & 7.6 & 523.9 & 14.6 \\
0.04618 & 0.1171 & 0.00314 & 713.9 & 18.2 & 692.1 & 28 \\
0.04664 & 0.14453 & 0.00348 & 870.2 & 19.6 & 844.6 & 25.2 \\
0.03128 & 0.11601 & 0.0026 & 707.6 & 15 & 732.7 & 19.6 \\
0.0599 & 0.26088 & 0.00736 & 1494.3 & 37.6 & 576.4 & 37 \\
0.01704 & 0.00458 & 0.00046 & 29.5 & 3 & 40.5 & 17.6 \\
0.08692 & 0.12217 & 0.00274 & 743 & 15.8 & 1475.3 & 27.2 \\
0.02912 & 0.11755 & 0.0026 & 716.4 & 15 & 716.8 & 18.6 \\
0.02062 & 0.03973 & 0.00124 & 251.2 & 7.6 & 246.2 & 18.4 \\
0.03946 & 0.11187 & 0.00284 & 683.6 & 16.4 & 676.8 & 24.6 \\
0.04136 & 0.09417 & 0.00266 & 580.2 & 15.6 & 585.5 & 27.4 \\
0.04426 & 0.09156 & 0.00272 & 564.8 & 16 & 565.7 & 29.6
\end{tabular}




\begin{tabular}{cccc|cc}
\hline age $\mathbf{2 0 7 / 2 0 6}$ & $\mathbf{2 \sigma}$ age $\mathbf{7 6}$ & $\begin{array}{c}\text { discordance } \\
\mathbf{\Delta} \mathbf{6 8 - 7 5}[\%]\end{array}$ & $\mathbf{\Delta} \mathbf{6 8 - 7 6}[\%]$ & $\begin{array}{c}\text { preferred age } \\
\text { age }\end{array}$ & $\mathbf{2 \sigma}$ age \\
\hline 2009.4 & 107.6 & -44 & -76.5 & & \\
496.2 & 83.4 & -1.7 & -9.9 & 447.2 & 10.2 \\
661.3 & 78.6 & 2.5 & 10.8 & 732.8 & 16.4 \\
726.4 & 78.8 & 0.9 & 3.7 & 753.3 & 17 \\
863.4 & 112 & -6.2 & -21.8 & 675.5 & 18.4 \\
483.8 & 97.2 & 3.7 & 21.4 & 587.2 & 14.2 \\
621.1 & 129.8 & -1.2 & -5.9 & 584.5 & 16.4 \\
658.5 & 55 & -0.6 & -2.8 & 640.3 & 13 \\
1331.9 & 53.8 & -31.6 & -73.1 & & \\
623.3 & 105.4 & 3.1 & 14.5 & 713.9 & 18.2 \\
778.9 & 77.4 & 3 & 11.7 & 870.2 & 19.6 \\
811.4 & 62.6 & -3.4 & -12.8 & 707.6 & 15 \\
758.1 & 906.6 & -27.2 & -96.1 & & \\
2781.2 & 44.2 & -49.6 & -73.3 & & \\
719 & 60.4 & -0.1 & -0.4 & 716.4 & 15 \\
200.1 & 180.8 & 2 & 25.5 & 251.2 & 7.6 \\
655 & 92.2 & 1 & 4.4 & 683.6 & 16.4 \\
607.2 & 118.8 & -0.9 & -4.5 & 580.2 & 15.6 \\
570.4 & 134 & -0.2 & -1 & 564.8 & 16
\end{tabular}


Sample 1989SWadi Qena @ Qena 120 grain analysed 88 concordant ages

\begin{tabular}{|c|c|c|c|c|c|c|}
\hline \multirow[b]{2}{*}{ grain } & \multicolumn{3}{|c|}{ concentrations } & \multicolumn{3}{|l|}{ isotopic ratios } \\
\hline & U [ppm] & $\mathrm{Pb}$ [ppm] & Th/U & Pb207/Pb206 & $2 \sigma 76$ & Pb207/U235 \\
\hline X1989S_G001 & 320.1 & 117 & 0.263 & 0.1176 & 0.00262 & 5.69632 \\
\hline X1989S_G002 & 134.3 & 15.2 & 1.1166 & 0.07447 & 0.00294 & 0.9209 \\
\hline X1989S G003 & 51.4 & 6.1 & 0.4076 & 0.06274 & 0.0037 & 0.98796 \\
\hline X1989S_G004 & 131.2 & 17.5 & 0.5309 & 0.06364 & 0.00246 & 1.08303 \\
\hline X1989S_G005 & 152.3 & 25.5 & 1.2125 & 0.06616 & 0.00228 & 1.18792 \\
\hline X1989S_G006 & 123.3 & 15.1 & 0.9741 & 0.05847 & 0.00246 & 0.81246 \\
\hline X1989S_G007 & 150.3 & 23.6 & 2.0407 & 0.07915 & 0.003 & 1.11532 \\
\hline X1989S_G008 & 273.9 & 35.5 & 0.3294 & 0.06396 & 0.002 & 1.12242 \\
\hline X1989S G009 & 68.9 & 13.6 & 0.4429 & 0.07353 & 0.00318 & 1.86941 \\
\hline X1989S G010 & 29.5 & 8.4 & 2.1436 & 0.07683 & 0.00452 & 1.92468 \\
\hline X1989S_G011 & 187.1 & 31.5 & 0.7918 & 0.06842 & 0.00228 & 1.36642 \\
\hline X1989S_G012 & 301.5 & 35.8 & 0.8737 & 0.06175 & 0.00194 & 0.85398 \\
\hline X1989S_G013 & 47.5 & 6 & 1.1297 & 0.05973 & 0.0044 & 0.82129 \\
\hline X1989S_G014 & 341.5 & 64.1 & 0.3683 & 0.07332 & 0.00182 & 1.82281 \\
\hline X1989S_G015 & 570 & 95.4 & 0.3626 & 0.07175 & 0.0017 & 1.59808 \\
\hline X1989S_G016 & 2184.3 & 91.1 & 0.221 & 0.13101 & 0.00354 & 0.71876 \\
\hline X1989S_G017 & 68.6 & 9.4 & 0.3605 & 0.06413 & 0.00304 & 1.16777 \\
\hline X1989S_G018 & 39.4 & 6.7 & 0.3452 & 0.07484 & 0.00372 & 1.69843 \\
\hline X1989S_G019 & 195.3 & 26.8 & 0.8153 & 0.06563 & 0.00216 & 1.06414 \\
\hline X1989S_G020 & 304.9 & & 2.023 & 0.34191 & 0.00806 & 5.17154 \\
\hline X1989S G021 & 232 & 78.1 & 0.8876 & 0.10205 & 0.00242 & 3.94513 \\
\hline X1989S G022 & 216.4 & 26.8 & 0.4871 & 0.06 & 0.00192 & 0.95733 \\
\hline X1989S_G023 & 74.9 & 11 & 0.4904 & 0.06484 & 0.00278 & 1.22071 \\
\hline X1989S_G024 & 71.8 & 11 & 0.572 & 0.07055 & 0.0031 & 1.36044 \\
\hline X1989S_G025 & 438.3 & 44.9 & 0.3362 & 0.05987 & 0.00166 & 0.82878 \\
\hline X1989S_G026 & 102.7 & 12 & 0.4677 & 0.06332 & 0.00266 & 0.96331 \\
\hline X1989S_G027 & 47.1 & 5.4 & 0.7648 & 0.05699 & 0.00364 & 0.77705 \\
\hline X1989S_G028 & 557.4 & 62.8 & 0.0161 & 0.06394 & 0.0016 & 1.07302 \\
\hline X1989S_G029 & 1072.4 & 68.4 & 0.3062 & 0.08349 & 0.00218 & 0.74963 \\
\hline X1989S_G030 & 378.3 & 40.9 & 0.7347 & 0.05899 & 0.00178 & 0.77218 \\
\hline X1989S_G031 & 188.2 & 19.4 & 0.4571 & 0.05817 & 0.00204 & 0.78503 \\
\hline X1989S_G032 & 10831.4 & 256.1 & 0.2899 & 0.08755 & 0.00192 & 0.27302 \\
\hline X1989S_G033 & 669.6 & 79.8 & 0.7086 & 0.07115 & 0.002 & 1.04037 \\
\hline X1989S_G034 & 52.2 & 7.5 & 0.6599 & 0.06656 & 0.00348 & 1.17672 \\
\hline X1989S_G035 & 254.9 & 32.5 & 0.8366 & 0.05978 & 0.00188 & 0.89612 \\
\hline X1989S_G036 & 92.9 & 44.2 & 0.5854 & 0.16048 & 0.00394 & 9.26199 \\
\hline X1989S_G037 & 227.5 & 36.1 & 0.8722 & 0.06508 & 0.00198 & 1.2053 \\
\hline X1989S_G038 & 558 & 94.1 & 1.1499 & 0.06676 & 0.00166 & 1.22375 \\
\hline X1989S_G039 & 509.7 & 46.7 & 1.1955 & 0.07577 & 0.00216 & 0.78659 \\
\hline X1989S_G040 & 141.1 & 62.8 & 1.042 & 0.12345 & 0.00308 & 5.98119 \\
\hline X1989S_G041 & 332 & 7 & 0.9237 & 0.30043 & 0.01116 & 0.72649 \\
\hline X1989S_G042 & 58.8 & 6.5 & 0.8291 & 0.06031 & 0.0041 & 0.78378 \\
\hline X1989S_G043 & 2696.1 & 108.7 & 2.4267 & 0.09925 & 0.0028 & 0.38887 \\
\hline X1989S_G044 & 164.8 & 17 & 0.454 & 0.05934 & 0.00268 & 0.80011 \\
\hline X1989S_G045 & 126.3 & 10.3 & 0.0261 & 0.06005 & 0.00256 & 0.72842 \\
\hline X1989S_G046 & 761.1 & 110.8 & 0.7914 & 0.08796 & 0.0021 & 1.52203 \\
\hline X1989S_G047 & 58.9 & 5.7 & 0.7361 & 0.07272 & 0.00418 & 0.86301 \\
\hline X1989S_G048 & 698.8 & 80.3 & 0.1309 & 0.06392 & 0.00156 & 1.06723 \\
\hline X1989S_G049 & 478.4 & 60.8 & 0.6845 & 0.06035 & 0.00158 & 0.93787 \\
\hline X1989S_G050 & 868.4 & 68.8 & 0.0799 & 0.06368 & 0.00208 & 0.74344 \\
\hline
\end{tabular}




\begin{tabular}{|c|c|c|c|c|c|c|}
\hline X1989S_G051 & 102.7 & 14.1 & 0.3039 & 0.06461 & 0.00286 & 1.20867 \\
\hline X1989S_G052 & & & & 1.02221 & 0.5418 & 776.35217 \\
\hline X1989S_G053 & 1170 & 114.7 & 1.5767 & 0.07762 & 0.00188 & 0.87647 \\
\hline X1989S_G054 & & & & 1.53112 & 2.0154 & \\
\hline X1989S_G055 & 240.2 & 111.2 & 1.2963 & 0.1475 & 0.00336 & 7.10728 \\
\hline X1989S_G056 & 169.8 & 34.3 & 0.7704 & 0.07376 & 0.00222 & 1.7693 \\
\hline X1989S_G057 & 112 & 11.6 & 0.3936 & 0.05677 & 0.00252 & 0.78214 \\
\hline X1989S_G058 & 73.7 & 8.9 & 0.9672 & 0.05839 & 0.00322 & 0.80621 \\
\hline X1989S_G059 & 156.6 & 22.7 & 0.4174 & 0.07074 & 0.00232 & 1.34194 \\
\hline X1989S_G060 & 342 & 47 & 0.4659 & 0.06386 & 0.00178 & 1.13747 \\
\hline X1989S_G061 & 171.3 & 49.5 & 0.1666 & 0.11226 & 0.00278 & 4.48885 \\
\hline X1989S_G062 & 171.5 & 20.7 & 0.352 & 0.06218 & 0.00218 & 1.01127 \\
\hline X1989S_G063 & 17.1 & 2.1 & 1.1561 & 0.06578 & 0.00742 & 0.89095 \\
\hline X1989S_G064 & & & & 0.79091 & 0.06724 & 59.31997 \\
\hline X1989S_G065 & 221.8 & 33.8 & 0.2515 & 0.07169 & 0.00208 & 1.50717 \\
\hline X1989S_G066 & 220.1 & 50.9 & 1.2709 & 0.07562 & 0.00208 & 1.80638 \\
\hline X1989S_G067 & & & & 0.11812 & 0.00574 & 1.68413 \\
\hline X1989S_G068 & 4170.5 & 332.5 & 0.3476 & 0.16114 & 0.00342 & 1.58478 \\
\hline X1989S_G069 & & & & 0.94464 & 0.15378 & 226.61081 \\
\hline X1989S_G070 & 472.8 & 168 & 0.595 & 0.10925 & 0.00246 & 4.78218 \\
\hline X1989S_G071 & & & & 0.78952 & 0.03122 & \\
\hline X1989S_G072 & 119 & 12.5 & 0.4733 & 0.05952 & 0.0026 & 0.81157 \\
\hline X1989S_G073 & & & & 0.9289 & 0.16392 & 682.80847 \\
\hline X1989S_G074 & 468.8 & 50.3 & 0.5808 & 0.05795 & 0.0016 & 0.78309 \\
\hline X1989S_G075 & & & & 0.75023 & 0.08592 & 495.71719 \\
\hline X1989S_G076 & 68.1 & 10.8 & 0.726 & 0.07123 & 0.00316 & 1.37177 \\
\hline X1989S_G077 & 132.1 & 22.4 & 0.3609 & 0.07128 & 0.00236 & 1.61108 \\
\hline X1989S_G078 & 301.1 & 30.7 & 0.3904 & 0.05932 & 0.00186 & 0.80256 \\
\hline X1989S_G079 & 326.7 & 43.7 & 0.6585 & 0.06193 & 0.00178 & 1.02706 \\
\hline X1989S_G080 & 91.3 & 12.6 & 1.6675 & 0.0613 & 0.00298 & 0.81669 \\
\hline X1989S_G081 & 462.7 & 53.4 & 0.1056 & 0.06353 & 0.00168 & 1.06093 \\
\hline X1989S_G082 & 148.8 & 31.8 & 1.3024 & 0.07168 & 0.00228 & 1.60761 \\
\hline X1989S_G083 & 389.4 & 75.6 & 0.5136 & 0.07242 & 0.00182 & 1.79343 \\
\hline X1989S_G084 & 60 & 6.9 & 0.8021 & 0.06008 & 0.00356 & 0.82107 \\
\hline X1989S_G085 & & & & 0.23 & 0.01684 & 3.84316 \\
\hline X1989S_G086 & 141.9 & 14.4 & 0.0997 & 0.06337 & 0.00234 & 0.93567 \\
\hline X1989S_G087 & 265.9 & 148.3 & 0.9402 & 0.17563 & 0.0038 & 10.92314 \\
\hline X1989S_G088 & 3107.4 & 86.5 & 0.8335 & 0.12066 & 0.00288 & 0.36722 \\
\hline X1989S_G089 & 327.4 & 46.6 & 0.4607 & 0.06484 & 0.00178 & 1.207 \\
\hline X1989S_G090 & & & & 0.76863 & 0.0605 & \\
\hline X1989S_G091 & 89.2 & 10.3 & 0.3636 & 0.06421 & 0.00292 & 0.98722 \\
\hline X1989S_G092 & 389.5 & 45.8 & 0.1807 & 0.06412 & 0.00178 & 1.06227 \\
\hline X1989S_G093 & & & & 0.80798 & 0.0179 & 476.44827 \\
\hline X1989S_G094 & 41 & 4.5 & 0.6717 & 0.06207 & 0.00436 & 0.84097 \\
\hline X1989S_G095 & 567.9 & 56.6 & 0.0924 & 0.06672 & 0.0017 & 0.97088 \\
\hline X1989S_G096 & 184.7 & 65.9 & 0.4506 & 0.16222 & 0.00388 & 7.53586 \\
\hline X1989S_G097 & 1265.1 & 115.5 & 0.7848 & 0.06076 & 0.0018 & 0.70569 \\
\hline X1989S_G098 & 3044.6 & 121 & 1.3246 & 0.06504 & 0.00192 & 0.29888 \\
\hline X1989S_G099 & 267.9 & 30.4 & 0.5719 & 0.0604 & 0.00222 & 0.86278 \\
\hline X1989S_G100 & 348.1 & & 1.8112 & 0.05908 & 0.00184 & 0.75314 \\
\hline X1989S_G101 & 38.6 & 4 & 0.4481 & 0.05238 & 0.00408 & 0.70196 \\
\hline X1989S_G102 & 245.6 & 29.9 & 0.6772 & 0.0609 & 0.00196 & 0.91079 \\
\hline X1989S_G103 & 151.3 & 14.2 & 0.1066 & 0.05993 & 0.00232 & 0.81525 \\
\hline X1989S_G104 & 455.2 & 35.6 & 0.6741 & 0.05576 & 0.00172 & 0.53688 \\
\hline X1989S_G105 & 252.3 & 26.9 & 0.5882 & 0.06018 & 0.00204 & 0.80584 \\
\hline
\end{tabular}




$\begin{array}{lccccccc}1 & & & & & & \\ 2 & \text { X1989S_G106 } & 270.8 & 25.5 & 0.0572 & 0.05901 & 0.00192 & 0.81865 \\ 3 & \text { X1989S_G107 } & 288.6 & 167.6 & 0.5085 & 0.17342 & 0.00374 & 11.97164 \\ 4 & \text { X1989S_G108 } & 310.8 & 43.3 & 0.6648 & 0.06459 & 0.00178 & 1.11048 \\ 5 & \text { X1989S_G109 } & 123.4 & 71.1 & 0.8562 & 0.15746 & 0.0037 & 10.07179 \\ 6 & \text { X1989S_G110 } & 306.8 & 38.1 & 0.5737 & 0.06371 & 0.00186 & 0.99634 \\ 7 & \text { X1989S_G111 } & 431.4 & 52.5 & 0.5611 & 0.0605 & 0.00164 & 0.93306 \\ 8 & \text { X1989S_G112 } & 168.9 & 22.5 & 0.3105 & 0.06466 & 0.00216 & 1.16989 \\ 9 & \text { X1989S_G113 } & & & & 0.81727 & 0.01736 & \\ 10 & \text { X1989S_G114 } & 418.4 & 169.3 & 0.5867 & 0.12599 & 0.00274 & 6.21136 \\ 11 & \text { X1989S_G115 } & 154.8 & 17.7 & 0.9008 & 0.07072 & 0.0027 & 0.9257 \\ 12 & \text { X1989S_G116 } & 159.8 & 16.8 & 0.4532 & 0.06294 & 0.00292 & 0.86786 \\ 13 & \text { X1989S_G117 } & 1197.8 & 309.7 & 0.3877 & 0.14997 & 0.0032 & 5.44482 \\ 14 & \text { X1989S_G118 } & 593 & 60.4 & 0.0522 & 0.06451 & 0.00174 & 0.97931 \\ 15 & \text { X1989S_G119 } & 14.3 & 3.9 & 0.076 & 0.13088 & 0.00688 & 5.10743 \\ 16 & \text { X1989S_G120 } & 663.5 & 76.1 & 0.3553 & 0.06988 & 0.00184 & 1.0722 \\ 17 & & & & & & & \end{array}$




\section{3\% concordant}

\begin{tabular}{|c|c|c|c|c|c|c|}
\hline $2 \sigma 75$ & Pb206/U238 & $2 \sigma 68$ & $\begin{array}{c}\text { ages } \\
\text { age } 206 / 238\end{array}$ & $2 \sigma$ age 68 & age $207 / 235$ & $2 \sigma$ age 75 \\
\hline 0.13632 & 0.35109 & 0.008 & 1939.8 & 38.2 & 1930.8 & 27.6 \\
\hline 0.0357 & 0.08963 & 0.00236 & 553.3 & 14 & 662.8 & 23 \\
\hline 0.05668 & 0.11414 & 0.00352 & 696.7 & 20.4 & 697.7 & 33.6 \\
\hline 0.04172 & 0.12334 & 0.00318 & 749.8 & 18.2 & 745.1 & 24.6 \\
\hline 0.04118 & 0.13015 & 0.00324 & 788.7 & 18.4 & 795 & 23.4 \\
\hline 0.03368 & 0.10073 & 0.00264 & 618.7 & 15.4 & 603.8 & 22.6 \\
\hline 0.0417 & 0.10214 & 0.00268 & 626.9 & 15.6 & 760.7 & 24.6 \\
\hline 0.03552 & 0.12719 & 0.00306 & 771.8 & 17.6 & 764.1 & 21.2 \\
\hline 0.07996 & 0.18427 & 0.00518 & 1090.3 & 28.2 & 1070.3 & 34.2 \\
\hline 0.1103 & 0.18158 & 0.00608 & 1075.6 & 33.2 & 1089.7 & 45.2 \\
\hline 0.04588 & 0.14475 & 0.00358 & 871.5 & 20.2 & 874.6 & 24.4 \\
\hline 0.02712 & 0.10024 & 0.0024 & 615.8 & 14 & 626.8 & 18.4 \\
\hline 0.05852 & 0.09967 & 0.00342 & 612.5 & 20 & 608.8 & 37.2 \\
\hline 0.04778 & 0.18019 & 0.00416 & 1068 & 22.8 & 1053.7 & 22.2 \\
\hline 0.03998 & 0.16143 & 0.00366 & 964.7 & 20.4 & 969.5 & 20.4 \\
\hline 0.01958 & 0.03977 & 0.00094 & 251.4 & 5.8 & 549.9 & 15.2 \\
\hline 0.05434 & 0.13199 & 0.00372 & 799.2 & 21.2 & 785.6 & 30.2 \\
\hline 0.08276 & 0.16449 & 0.00494 & 981.7 & 27.4 & 1007.9 & 37.2 \\
\hline 0.0353 & 0.11753 & 0.00288 & 716.3 & 16.6 & 735.9 & 21.4 \\
\hline 0.12572 & 0.10964 & 0.00262 & 670.7 & 15.2 & 1847.9 & 28.6 \\
\hline 0.09964 & 0.28022 & 0.00648 & 1592.5 & 32.6 & 1623 & 26.8 \\
\hline 0.03098 & 0.11564 & 0.0028 & 705.4 & 16.2 & 681.9 & 20 \\
\hline 0.05158 & 0.13645 & 0.00368 & 824.6 & 20.8 & 810.1 & 28.2 \\
\hline 0.0588 & 0.13977 & 0.00388 & 843.4 & 22 & 872.1 & 30.4 \\
\hline 0.0236 & 0.10034 & 0.00234 & 616.4 & 13.8 & 612.9 & 16.6 \\
\hline 0.03994 & 0.11027 & 0.00292 & 674.3 & 17 & 685 & 24.8 \\
\hline 0.04828 & 0.09883 & 0.00312 & 607.5 & 18.4 & 583.8 & 31.6 \\
\hline 0.02804 & 0.12164 & 0.00278 & 740 & 16 & 740.2 & 17.8 \\
\hline 0.02016 & 0.06508 & 0.0015 & 406.4 & 9 & 568 & 15.2 \\
\hline 0.02366 & 0.09488 & 0.00224 & 584.3 & 13.2 & 581 & 17 \\
\hline 0.02772 & 0.09782 & 0.00242 & 601.6 & 14.2 & 588.3 & 19.2 \\
\hline 0.0064 & 0.0226 & 0.0005 & 144.1 & 3.2 & 245.1 & 6.8 \\
\hline 0.03008 & 0.10598 & 0.0025 & 649.4 & 14.6 & 724.1 & 19 \\
\hline 0.06014 & 0.12814 & 0.0038 & 777.2 & 21.8 & 789.8 & 33 \\
\hline 0.02862 & 0.10866 & 0.0026 & 665 & 15.2 & 649.7 & 19 \\
\hline 0.24252 & 0.41834 & 0.01016 & 2252.9 & 46.2 & 2364.3 & 31.6 \\
\hline 0.0373 & 0.13423 & 0.00322 & 812 & 18.4 & 803 & 21.4 \\
\hline 0.03198 & 0.13287 & 0.00304 & 804.2 & 17.2 & 811.5 & 18.8 \\
\hline 0.02278 & 0.07524 & 0.00178 & 467.6 & 10.6 & 589.2 & 16.6 \\
\hline 0.15782 & 0.3512 & 0.0084 & 1940.3 & 40 & 1973.1 & 30 \\
\hline 0.0238 & 0.01753 & 0.00052 & 112 & 3.2 & 554.5 & 20.4 \\
\hline 0.0516 & 0.0942 & 0.0031 & 580.3 & 18.2 & 587.6 & 33.6 \\
\hline 0.01106 & 0.0284 & 0.00068 & 180.5 & 4.2 & 333.5 & 10.6 \\
\hline 0.03556 & 0.09774 & 0.00264 & 601.2 & 15.6 & 596.9 & 23.8 \\
\hline 0.03056 & 0.08792 & 0.00232 & 543.2 & 13.8 & 555.6 & 21.4 \\
\hline 0.03838 & 0.12542 & 0.00286 & 761.7 & 16.4 & 939.3 & 20.2 \\
\hline 0.04766 & 0.08602 & 0.00268 & 532 & 16 & 631.8 & 30.8 \\
\hline 0.0273 & 0.12102 & 0.00276 & 736.4 & 15.8 & 737.4 & 17.6 \\
\hline 0.02568 & 0.11263 & 0.0026 & 688 & 15 & 671.8 & 17.2 \\
\hline 0.02442 & 0.08462 & 0.00206 & 523.6 & 12.2 & 564.4 & 17.6 \\
\hline
\end{tabular}




\begin{tabular}{|c|c|c|c|c|c|c|}
\hline 0.05256 & 0.1356 & 0.00372 & 819.7 & 21.2 & 804.6 & 29 \\
\hline 1130.65394 & 5.50495 & 8.0915 & & & & \\
\hline \multirow[t]{2}{*}{0.02226} & 0.08184 & 0.00186 & 507.1 & 11 & 639.1 & 15.8 \\
\hline & -5.03547 & 28.53604 & & & & \\
\hline 0.17312 & 0.34926 & 0.00808 & 1931.1 & 38.6 & 2125 & 28.8 \\
\hline 0.05424 & 0.17387 & 0.00422 & 1033.4 & 23.2 & 1034.3 & 25 \\
\hline 0.03418 & 0.09985 & 0.00266 & 613.5 & 15.6 & 586.7 & 23 \\
\hline 0.04336 & 0.10007 & 0.00294 & 614.8 & 17.2 & 600.3 & 28.4 \\
\hline 0.0445 & 0.13751 & 0.0034 & 830.6 & 19.2 & 864.1 & 23.8 \\
\hline 0.03262 & 0.12912 & 0.00302 & 782.8 & 17.2 & 771.3 & 19.6 \\
\hline 0.11716 & 0.28984 & 0.00684 & 1640.7 & 34.2 & 1728.9 & 28.4 \\
\hline 0.03546 & 0.11789 & 0.00294 & 718.4 & 17 & 709.5 & 22 \\
\hline 0.09648 & 0.09817 & 0.00452 & 603.7 & 26.6 & 646.9 & 58.2 \\
\hline 5.29938 & 0.54364 & 0.05454 & & & & \\
\hline 0.04474 & 0.15237 & 0.00364 & 914.2 & 20.4 & 933.3 & 23 \\
\hline 0.05146 & 0.17315 & 0.0041 & 1029.4 & 22.6 & 1047.8 & 23.8 \\
\hline 0.07774 & 0.10335 & 0.00326 & & & & \\
\hline 0.03626 & 0.07128 & 0.0016 & 443.9 & 9.6 & 964.2 & 19.2 \\
\hline 63.32246 & 1.73881 & 0.51264 & & & & \\
\hline 0.11586 & 0.31728 & 0.00724 & 1776.4 & 35.4 & 1781.8 & 27 \\
\hline 230.12312 & 13.59534 & 2.12784 & & & & \\
\hline 0.03488 & 0.09884 & 0.00264 & 607.6 & 15.4 & 603.3 & 23.2 \\
\hline 337.95246 & 5.32802 & 2.70278 & & & & \\
\hline 0.02242 & 0.09794 & 0.00228 & 602.3 & 13.4 & 587.2 & 16 \\
\hline 147.77878 & 4.78936 & 1.4486 & & & & \\
\hline 0.0599 & 0.13959 & 0.0039 & 842.3 & 22 & 876.9 & 30.8 \\
\hline 0.054 & 0.16383 & 0.0041 & 978 & 22.8 & 974.5 & 26 \\
\hline 0.0256 & 0.09806 & 0.00234 & 603 & 13.8 & 598.3 & 17.8 \\
\hline 0.03022 & 0.12021 & 0.00282 & 731.8 & 16.2 & 717.5 & 19 \\
\hline 0.03884 & 0.09657 & 0.0027 & 594.3 & 15.8 & 606.2 & 25.6 \\
\hline 0.02914 & 0.12104 & 0.0028 & 736.5 & 16.2 & 734.3 & 18.4 \\
\hline 0.05178 & 0.16256 & 0.004 & 971 & 22.2 & 973.2 & 25.2 \\
\hline 0.04714 & 0.17951 & 0.00414 & 1064.3 & 22.6 & 1043.1 & 22.2 \\
\hline 0.04724 & 0.09906 & 0.00304 & 608.9 & 17.8 & 608.6 & 30.6 \\
\hline 0.24906 & 0.12111 & 0.00622 & & & & \\
\hline 0.03454 & 0.10703 & 0.00272 & 655.5 & 15.8 & 670.6 & 22 \\
\hline 0.257 & 0.4508 & 0.01028 & 2398.8 & 45.6 & 2516.6 & 29.2 \\
\hline 0.00914 & 0.02206 & 0.0005 & 140.7 & 3.2 & 317.6 & 9 \\
\hline \multirow[t]{2}{*}{0.03416} & 0.13492 & 0.00316 & 815.9 & 18 & 803.8 & 20 \\
\hline & 100.08569 & 89.75538 & & & & \\
\hline 0.04394 & 0.11145 & 0.00306 & 681.2 & 17.8 & 697.3 & 26.8 \\
\hline 0.0303 & 0.12008 & 0.0028 & 731 & 16.2 & 734.9 & 19 \\
\hline 15.11756 & 4.27417 & 0.13656 & & & & \\
\hline 0.05708 & 0.09821 & 0.0033 & 603.9 & 19.4 & 619.7 & 36.2 \\
\hline 0.02588 & 0.10547 & 0.00242 & 646.4 & 14.2 & 688.9 & 17.2 \\
\hline 0.19142 & 0.33673 & 0.008 & 1870.9 & 38.6 & 2177.3 & 30.2 \\
\hline 0.02134 & 0.08419 & 0.00198 & 521.1 & 11.8 & 542.2 & 15.8 \\
\hline 0.00892 & 0.03331 & 0.00078 & 211.2 & 4.8 & 265.5 & 8.8 \\
\hline 0.03158 & 0.10354 & 0.0026 & 635.1 & 15.2 & 631.6 & 21 \\
\hline 0.02372 & 0.09241 & 0.0022 & 569.8 & 13 & 570 & 17 \\
\hline 0.05308 & 0.09714 & 0.00326 & 597.6 & 19.2 & 540 & 35.6 \\
\hline 0.02976 & 0.10841 & 0.00262 & 663.5 & 15.2 & 657.5 & 19.4 \\
\hline 0.03144 & 0.0986 & 0.00252 & 606.2 & 14.8 & 605.4 & 21.2 \\
\hline 0.01688 & 0.06979 & 0.00166 & 434.9 & 10 & 436.4 & 13.8 \\
\hline 0.02734 & 0.09706 & 0.00238 & 597.2 & 14 & 600.1 & 19 \\
\hline
\end{tabular}




$\begin{array}{ccccccc}0.02702 & 0.10056 & 0.00244 & 617.7 & 14.2 & 607.3 & 18.6 \\ 0.2806 & 0.50037 & 0.0114 & 2615.4 & 49 & 2602.2 & 29.4 \\ 0.03172 & 0.12462 & 0.00292 & 757.1 & 16.8 & 758.4 & 19.4 \\ 0.25374 & 0.46362 & 0.011 & 2455.5 & 48.4 & 2441.4 & 30.8 \\ 0.02966 & 0.11335 & 0.00268 & 692.2 & 15.6 & 702 & 19 \\ 0.02622 & 0.11179 & 0.0026 & 683.1 & 15 & 669.2 & 17.6 \\ 0.03928 & 0.13114 & 0.00324 & 794.4 & 18.4 & 786.6 & 22.8 \\ 245.63978 & 41.38657 & 2.17914 & & & & \\ 0.1463 & 0.35735 & 0.00808 & 1969.6 & 38.4 & 2006 & 27.4 \\ 0.03484 & 0.09487 & 0.00244 & 584.3 & 14.4 & 665.4 & 22.4 \\ 0.03934 & 0.09995 & 0.00274 & 614.1 & 16 & 634.4 & 25.4 \\ 0.12558 & 0.26316 & 0.0059 & 1506 & 30.2 & 1891.9 & 26.6 \\ 0.02722 & 0.11004 & 0.00256 & 673 & 14.8 & 693.3 & 17.8 \\ 0.26308 & 0.28286 & 0.01052 & 1605.7 & 52.8 & 1837.3 & 54.6 \\ 0.02924 & 0.11121 & 0.00258 & 679.8 & 15 & 739.8 & 18.4\end{array}$




\begin{tabular}{|c|c|c|c|c|c|}
\hline age $207 / 206$ & $2 \sigma$ age 76 & $\begin{array}{l}\text { discordance } \\
\Delta 68-75[\%]\end{array}$ & $\Delta 68-76[\%]$ & $\begin{array}{c}\text { preferred age } \\
\text { age }\end{array}$ & $2 \sigma$ age \\
\hline 1920.1 & 40 & 0.5 & 1 & 1920.1 & 40 \\
\hline 1054.2 & 79.6 & -16.5 & -47.5 & & \\
\hline 699.4 & 125.6 & -0.1 & -0.4 & 696.7 & 20.4 \\
\hline 729.7 & 82 & 0.6 & 2.8 & 749.8 & 18.2 \\
\hline 811.4 & 72 & -0.8 & -2.8 & 788.7 & 18.4 \\
\hline 547.4 & 92 & 2.5 & 13 & 618.7 & 15.4 \\
\hline 1175.9 & 75 & -17.6 & -46.7 & & \\
\hline 740.3 & 66.2 & 1 & 4.3 & 771.8 & 17.6 \\
\hline 1028.6 & 87.4 & 1.9 & 6 & 1090.3 & 28.2 \\
\hline 1116.8 & 117.4 & -1.3 & -3.7 & 1075.6 & 33.2 \\
\hline 881.3 & 69 & -0.4 & -1.1 & 871.5 & 20.2 \\
\hline 665.5 & 67.2 & -1.8 & -7.5 & 615.8 & 14 \\
\hline 593.8 & 159.6 & 0.6 & 3.1 & 612.5 & 20 \\
\hline 1022.8 & 50.2 & 1.4 & 4.4 & 1068 & 22.8 \\
\hline 978.9 & 48.2 & -0.5 & -1.4 & 964.7 & 20.4 \\
\hline 2111.5 & 47.4 & -54.3 & -88.1 & & \\
\hline 745.9 & 100.2 & 1.7 & 7.1 & 799.2 & 21.2 \\
\hline 1064.2 & 100 & -2.6 & -7.8 & 981.7 & 27.4 \\
\hline 794.6 & 69 & -2.7 & -9.8 & 716.3 & 16.6 \\
\hline 3671.3 & 36 & -63.7 & -81.7 & & \\
\hline 1661.7 & 43.8 & -1.9 & -4.2 & 1661.7 & 43.8 \\
\hline 603.6 & 69.2 & 3.4 & 16.9 & 705.4 & 16.2 \\
\hline 769.1 & 90.2 & 1.8 & 7.2 & 824.6 & 20.8 \\
\hline 944.4 & 90 & -3.3 & -10.7 & 843.4 & 22 \\
\hline 598.9 & 60 & 0.6 & 2.9 & 616.4 & 13.8 \\
\hline 719 & 89.2 & -1.6 & -6.2 & 674.3 & 17 \\
\hline 491.1 & 140.8 & 4.1 & 23.7 & 607.5 & 18.4 \\
\hline 739.6 & 53 & 0 & 0 & 740 & 16 \\
\hline 1280.7 & 50.8 & -28.4 & -68.3 & & \\
\hline 566.7 & 65.6 & 0.6 & 3.1 & 584.3 & 13.2 \\
\hline 536.2 & 76.8 & 2.3 & 12.2 & 601.6 & 14.2 \\
\hline 1372.6 & 42.2 & -41.2 & -89.5 & & \\
\hline 961.7 & 57.4 & -10.3 & -32.5 & 649.4 & 14.6 \\
\hline 824 & 109.2 & -1.6 & -5.7 & 777.2 & 21.8 \\
\hline 595.6 & 68.2 & 2.4 & 11.6 & 665 & 15.2 \\
\hline 2460.7 & 41.4 & -4.7 & -8.4 & 2460.7 & 41.4 \\
\hline 776.9 & 64 & 1.1 & 4.5 & 812 & 18.4 \\
\hline 830.3 & 51.8 & -0.9 & -3.1 & 804.2 & 17.2 \\
\hline 1089 & 57.2 & -20.6 & -57.1 & & \\
\hline 2006.7 & 44.2 & -1.7 & -3.3 & 2006.7 & 44.2 \\
\hline 3472.4 & 57.6 & -79.8 & -96.8 & & \\
\hline 614.7 & 146.8 & -1.2 & -5.6 & 580.3 & 18.2 \\
\hline 1610.1 & 52.6 & -45.9 & -88.8 & & \\
\hline 579.6 & 98.2 & 0.7 & 3.7 & 601.2 & 15.6 \\
\hline 605.4 & 92.2 & -2.2 & -10.3 & 543.2 & 13.8 \\
\hline 1381.6 & 45.8 & -18.9 & -44.9 & & \\
\hline 1006.2 & 116.6 & -15.8 & -47.1 & & \\
\hline 739 & 51.6 & -0.1 & -0.3 & 736.4 & 15.8 \\
\hline 616.1 & 56.6 & 2.4 & 11.7 & 688 & 15 \\
\hline 731 & 69.2 & -7.2 & -28.4 & 523.6 & 12.2 \\
\hline
\end{tabular}




\begin{tabular}{|c|c|c|c|c|c|}
\hline 761.7 & 93.4 & 1.9 & 7.6 & 819.7 & 21.2 \\
\hline 1137.2 & 48.2 & -20.7 & -55.4 & & \\
\hline 2317.1 & 39 & -9.1 & -16.7 & & \\
\hline 1034.9 & 60.8 & -0.1 & -0.1 & 1033.4 & 23.2 \\
\hline 482.6 & 98 & 4.6 & 27.1 & 613.5 & 15.6 \\
\hline 544.4 & 120.6 & 2.4 & 12.9 & 614.8 & 17.2 \\
\hline 949.9 & 67.2 & -3.9 & -12.6 & 830.6 & 19.2 \\
\hline 737 & 59 & 1.5 & 6.2 & 782.8 & 17.2 \\
\hline 1836.3 & 44.8 & -5.1 & -10.7 & 1836.3 & 44.8 \\
\hline 680.3 & 75 & 1.3 & 5.6 & 718.4 & 17 \\
\hline 799.4 & 236.4 & -6.7 & -24.5 & 603.7 & 26.6 \\
\hline 977.2 & 59.2 & -2 & -6.4 & 914.2 & 20.4 \\
\hline 1085 & 55.2 & -1.7 & -5.1 & 1029.4 & 22.6 \\
\hline 2467.7 & 35.8 & -54 & -82 & & \\
\hline 1786.9 & 41 & -0.3 & -0.6 & 1786.9 & 41 \\
\hline 586.2 & 94.8 & 0.7 & 3.7 & 607.6 & 15.4 \\
\hline 527.9 & 60.6 & 2.6 & 14.1 & 602.3 & 13.4 \\
\hline 964 & 90.6 & -3.9 & -12.6 & 842.3 & 22 \\
\hline 965.5 & 67.6 & 0.4 & 1.3 & 978 & 22.8 \\
\hline 578.9 & 68.2 & 0.8 & 4.2 & 603 & 13.8 \\
\hline 671.7 & 61.4 & 2 & 8.9 & 731.8 & 16.2 \\
\hline 649.8 & 104.4 & -2 & -8.5 & 594.3 & 15.8 \\
\hline 726 & 56 & 0.3 & 1.4 & 736.5 & 16.2 \\
\hline 976.9 & 64.8 & -0.2 & -0.6 & 971 & 22.2 \\
\hline 997.8 & 51 & 2 & 6.7 & 1064.3 & 22.6 \\
\hline 606.5 & 128.2 & 0 & 0.4 & 608.9 & 17.8 \\
\hline 720.7 & 78.4 & -2.3 & -9 & 655.5 & 15.8 \\
\hline 2612 & 36 & -4.7 & -8.2 & 2612 & 36 \\
\hline 1966 & 42.6 & -55.7 & -92.8 & & \\
\hline 769.1 & 57.8 & 1.5 & 6.1 & 815.9 & 18 \\
\hline 748.6 & 96 & -2.3 & -9 & 681.2 & 17.8 \\
\hline 745.6 & 58.6 & -0.5 & -2 & 731 & 16.2 \\
\hline 676.5 & 150.2 & -2.5 & -10.7 & 603.9 & 19.4 \\
\hline 829 & 53.2 & -6.2 & -22 & 646.4 & 14.2 \\
\hline 2478.9 & 40.4 & -14.1 & -24.5 & & \\
\hline 630.7 & 63.8 & -3.9 & -17.4 & 521.1 & 11 \\
\hline 775.6 & 62 & -20.4 & -72.8 & & \\
\hline 617.9 & 79.4 & 0.6 & 2.8 & 635.1 & 15.2 \\
\hline 570 & 67.8 & 0 & 0 & 569.8 & 13 \\
\hline 302 & 177.6 & 10.7 & 97.9 & & \\
\hline 635.7 & 69.2 & 0.9 & 4.4 & 663.5 & 15.2 \\
\hline 601 & 83.8 & 0.1 & 0.9 & 606.2 & 14.8 \\
\hline 442.8 & 68.6 & -0.3 & -1.8 & 434.9 & 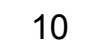 \\
\hline 610.1 & 73.2 & -0.5 & -2.1 & 597.2 & \\
\hline
\end{tabular}




$\begin{array}{cccccc}567.5 & 70.8 & 1.7 & 8.9 & 617.7 & 14.2 \\ 2590.9 & 36 & 0.5 & 0.9 & 2590.9 & 36 \\ 761 & 58.2 & -0.2 & -0.5 & 757.1 & 16.8 \\ 2428.6 & 39.8 & 0.6 & 1.1 & 2428.6 & 39.8 \\ 732 & 61.8 & -1.4 & -5.4 & 692.2 & 15.6 \\ 621.5 & 58.4 & 2.1 & 9.9 & 683.1 & 15 \\ 763.3 & 70.4 & 1 & 4.1 & 794.4 & 18.4 \\ & & & & & \\ 2042.8 & 38.4 & -1.8 & -3.6 & 2042.8 & 38.4 \\ 949.3 & 78.2 & -12.2 & -38.5 & 584.3 & 14.4 \\ 706.2 & 98.6 & -3.2 & -13 & 614.1 & 16 \\ 2345.6 & 36.4 & -20.4 & -35.8 & & \\ 758.4 & 56.8 & -2.9 & -11.3 & 673 & 14.8 \\ 2109.8 & 92.2 & -12.6 & -23.9 & & \\ 924.8 & 54 & -8.1 & -26.5 & 679.8 & 15\end{array}$


Sample IV Main Nile @ Tora 118 grain analysed 97 concordant ages 82.:

\begin{tabular}{|c|c|c|c|c|c|c|}
\hline \multirow[b]{2}{*}{ grain } & \multicolumn{3}{|l|}{ concentrations } & \multicolumn{3}{|l|}{ isotopic ratios } \\
\hline & U [ppm] & $\mathrm{Pb}[p p m]$ & $T h / U$ & Pb207/Pb206 & $2 \sigma 76$ & Pb207/U235 \\
\hline X14_G001 & 59.4 & 6.2 & 0.5289 & 0.05932 & 0.00316 & 0.78644 \\
\hline X14_G002 & 117.9 & 18.2 & 0.5391 & 0.06874 & 0.00266 & 1.37295 \\
\hline X14 G003 & 46.5 & 7.6 & 0.6589 & 0.06927 & 0.00328 & 1.38875 \\
\hline X14_G004 & & & & 0.83448 & 0.04516 & 461.34439 \\
\hline X14_G005 & 282.2 & 28.4 & 0.6618 & 0.0601 & 0.00188 & 0.77897 \\
\hline X14_G006 & 422 & 46.3 & 0.0914 & 0.06105 & 0.0017 & 0.97358 \\
\hline X14_G007 & 765.8 & 87.1 & 0.3449 & 0.06495 & 0.0017 & 1.02163 \\
\hline X14 G008 & 93.4 & 12.5 & 1.4513 & 0.06109 & 0.0029 & 0.84459 \\
\hline X14 G009 & 461.6 & 42.3 & 0.9451 & 0.07059 & 0.00204 & 0.82469 \\
\hline X14_G010 & 110.1 & 22.2 & 0.489 & 0.0751 & 0.00236 & 1.95084 \\
\hline X14_G011 & 293.8 & 50.9 & 1.4313 & 0.06476 & 0.00184 & 1.18895 \\
\hline X14_G012 & 104.1 & 18.8 & 0.6331 & 0.07089 & 0.00238 & 1.58402 \\
\hline X14_G013 & 279.2 & 169.6 & 0.5739 & 0.19156 & 0.00454 & 13.74306 \\
\hline X14_G014 & 926.7 & 40.2 & 0.508 & 0.05558 & 0.00164 & 0.31434 \\
\hline X14_G015 & 250 & 40.8 & 0.9138 & 0.06444 & 0.00188 & 1.21801 \\
\hline X14_G016 & 468.9 & 124.5 & 0.6811 & 0.1429 & 0.00344 & 4.8008 \\
\hline X14_G017 & 407.4 & 137.5 & 0.5883 & 0.12735 & 0.00308 & 5.48827 \\
\hline X14_G018 & 691.3 & 78.8 & 0.6695 & 0.05966 & 0.00158 & 0.84148 \\
\hline X14_G019 & 168.6 & 11.9 & 0.5758 & 0.05996 & 0.00296 & 0.53526 \\
\hline X14_G020 & 59.8 & 6.3 & 0.5477 & 0.05937 & 0.00354 & 0.80583 \\
\hline X14_G021 & 749 & 75.3 & 0.0392 & 0.06141 & 0.00168 & 0.91285 \\
\hline X14_G022 & 2056 & 81.1 & 1.1849 & 0.11707 & 0.00334 & 0.5259 \\
\hline X14_G023 & 1474.8 & 106.2 & 0.2839 & 0.08479 & 0.00218 & 0.86945 \\
\hline X14_G024 & 57.6 & 19.5 & 0.6292 & 0.11435 & 0.00394 & 4.76682 \\
\hline X14_G025 & 171.7 & 16.1 & 0.0003 & 0.05851 & 0.00206 & 0.82247 \\
\hline X14_G026 & 29.3 & 4.1 & 0.5139 & 0.06741 & 0.00416 & 1.20473 \\
\hline X14_G027 & 987.8 & 55.1 & 0.57 & 0.07494 & 0.00226 & 0.53781 \\
\hline X14_G028 & 123.9 & 15 & 1.0707 & 0.05836 & 0.00234 & 0.78954 \\
\hline X14_G029 & 118.3 & 18.6 & 0.2389 & 0.07212 & 0.0024 & 1.56623 \\
\hline X14_G030 & 90.8 & 7.9 & 0.5396 & 0.05649 & 0.00274 & 0.62756 \\
\hline X14_G031 & 93.4 & 18.3 & 1.9323 & 0.06622 & 0.00256 & 1.19259 \\
\hline X14_G032 & 169.1 & 68.4 & 0.5443 & 0.11876 & 0.00306 & 5.9111 \\
\hline X14_G033 & 311.9 & 1.3 & 0.3972 & 0.05465 & 0.00764 & 0.02955 \\
\hline X14_G034 & 684.5 & 100.5 & 0.0403 & 0.09717 & 0.00244 & 2.10986 \\
\hline X14_G035 & 165.6 & 19.7 & 0.8314 & 0.08242 & 0.00286 & 1.13602 \\
\hline X14_G036 & 166.9 & 27 & 0.8454 & 0.06707 & 0.00216 & 1.27641 \\
\hline X14_G037 & 89.9 & & 1.8571 & 0.22941 & 0.00914 & 3.1943 \\
\hline X14_G038 & 51.6 & 8 & 0.5902 & 0.09521 & 0.00496 & 1.81739 \\
\hline X14_G039 & 216 & 31.5 & 0.344 & 0.06723 & 0.00206 & 1.31449 \\
\hline X14_G040 & 46.9 & 1.5 & 0.8928 & 0.05415 & 0.00682 & 0.20586 \\
\hline X14_G041 & 256 & 34.9 & 0.6136 & 0.06343 & 0.002 & 1.0795 \\
\hline X14_G042 & 158.7 & 18.3 & 0.5175 & 0.06002 & 0.00222 & 0.88854 \\
\hline X14_G043 & 265.4 & 33.6 & 0.964 & 0.06042 & 0.00196 & 0.86824 \\
\hline X14_G044 & 53.3 & 9 & 0.8056 & 0.06672 & 0.00348 & 1.33315 \\
\hline X14_G045 & 364 & 54.3 & 0.7994 & 0.06594 & 0.00198 & 1.19211 \\
\hline X14_G046 & 5810.9 & 197.4 & 0.0553 & 0.06137 & 0.00166 & 0.30741 \\
\hline X14_G047 & 236.2 & 28.5 & 0.2719 & 0.06234 & 0.00202 & 1.03462 \\
\hline X14_G048 & 216.8 & 32.7 & 1.0639 & 0.06418 & 0.00224 & 1.07799 \\
\hline X14_G049 & 266.7 & 118.2 & 1.0809 & 0.12347 & 0.00326 & 6.12513 \\
\hline X14_G050 & 74.9 & 14.8 & 0.9239 & 0.07042 & 0.00292 & 1.59929 \\
\hline
\end{tabular}




\begin{tabular}{|c|c|c|c|c|c|c|}
\hline X14_G051 & 183.7 & 20.6 & 0.81 & 0.05778 & 0.0022 & 0.77268 \\
\hline X14_G052 & 437.5 & 42.7 & 0.0759 & 0.0624 & 0.00202 & 0.88632 \\
\hline X14_G053 & 119.6 & 12.1 & 0.4511 & 0.05911 & 0.00256 & 0.78597 \\
\hline X14_G054 & 70.6 & 9.2 & 1.2775 & 0.05838 & 0.003 & 0.80095 \\
\hline X14_G055 & 762.8 & 111.8 & 0.8211 & 0.06893 & 0.00186 & 1.26758 \\
\hline X14_G056 & 281.4 & 15.1 & 1.4065 & 0.05284 & 0.0023 & 0.28959 \\
\hline X14_G057 & 206.1 & 32.2 & 0.6129 & 0.06566 & 0.00208 & 1.27745 \\
\hline X14_G058 & 96.4 & 14.1 & 0.8702 & 0.07212 & 0.00284 & 1.21722 \\
\hline X14_G059 & 431.9 & 119.8 & 0.3789 & 0.14314 & 0.0039 & 5.30343 \\
\hline X14_G060 & 493.9 & 34.5 & 0.3671 & 0.06512 & 0.0021 & 0.60221 \\
\hline X14 G061 & 179.4 & 30.4 & 0.3945 & 0.07148 & 0.0024 & 1.60328 \\
\hline X14_G062 & 2833.4 & 128.4 & 0.4342 & 0.08941 & 0.00242 & 0.53618 \\
\hline X14_G063 & 119.6 & 13.7 & 0.7389 & 0.07728 & 0.00298 & 1.08116 \\
\hline X14_G064 & 260.3 & 25.7 & 0.0123 & 0.0641 & 0.00258 & 0.9442 \\
\hline X14_G065 & 270.6 & 40.3 & 0.4397 & 0.06607 & 0.002 & 1.28739 \\
\hline X14_G066 & 55.5 & 6.5 & 0.5442 & 0.05854 & 0.00342 & 0.87349 \\
\hline X14_G067 & 189.3 & 22.7 & 1.0546 & 0.08458 & 0.00352 & 1.10368 \\
\hline X14_G068 & 537.3 & 70.7 & 0.3661 & 0.06528 & 0.00186 & 1.14967 \\
\hline X14_G069 & 305 & 43.9 & 0.7753 & 0.06586 & 0.00202 & 1.13235 \\
\hline X14_G070 & 330.4 & 45.4 & 0.3593 & 0.06461 & 0.00194 & 1.18751 \\
\hline X14_G071 & 56.4 & 9.1 & 0.7869 & 0.06571 & 0.00316 & 1.26693 \\
\hline X14_G072 & 1008.8 & 65.8 & 0.3786 & 0.06464 & 0.0019 & 0.57431 \\
\hline X14 G073 & 146.3 & 47.9 & 0.7136 & 0.11812 & 0.00348 & 4.71598 \\
\hline X14_G074 & 49.5 & 4.5 & 0.0045 & 0.06132 & 0.00392 & 0.83532 \\
\hline X14_G075 & 1192.5 & 63.2 & 1.1088 & 0.07616 & 0.00278 & 0.47963 \\
\hline X14_G076 & 307.2 & 42.4 & 0.3791 & 0.06573 & 0.00206 & 1.2093 \\
\hline X14_G077 & 55.1 & 11.7 & 1.34 & 0.07536 & 0.0034 & 1.67396 \\
\hline X14_G078 & 486.6 & 49.8 & 0.3056 & 0.06587 & 0.0021 & 0.91845 \\
\hline X14_G079 & 237 & 168.3 & 1.4196 & 0.17464 & 0.00472 & 12.15558 \\
\hline X14_G080 & 460.8 & 55.8 & 0.5173 & 0.06934 & 0.00208 & 1.06213 \\
\hline X14_G081 & 142 & 22.9 & 2.8079 & 0.058 & 0.00238 & 0.72431 \\
\hline X14_G082 & 759.8 & 50 & 0.6547 & 0.07422 & 0.00228 & 0.61642 \\
\hline X14_G083 & 347.2 & 64.1 & 0.5828 & 0.07163 & 0.00218 & 1.65518 \\
\hline X14_G084 & 110.1 & 44.8 & 0.8775 & 0.12163 & 0.0037 & 5.64714 \\
\hline X14 G085 & 63.2 & 8.8 & 0.7697 & 0.06628 & 0.00316 & 1.10458 \\
\hline X14_G086 & 112.7 & 94.9 & 0.9049 & 0.30915 & 0.00858 & 29.34567 \\
\hline X14_G087 & 248.2 & 36.3 & 0.6844 & 0.07036 & 0.0023 & 1.27004 \\
\hline X14_G088 & 37.9 & 4.3 & 0.5172 & 0.05711 & 0.0038 & 0.8285 \\
\hline X14_G089 & 77 & 11.4 & 0.7826 & 0.06952 & 0.00298 & 1.23961 \\
\hline X14_G090 & 144.6 & 103.1 & 1.2023 & 0.17887 & 0.00508 & 13.02824 \\
\hline X14_G091 & 260.7 & 0.4 & 0.7092 & 0.15675 & 0.02784 & 0.02536 \\
\hline X14_G092 & 129.9 & 45.6 & 0.564 & 0.12201 & 0.00372 & 5.30093 \\
\hline X14_G093 & 54.2 & 9.9 & 2.1024 & 0.06365 & 0.00332 & 1.03012 \\
\hline X14_G094 & 467.2 & 47.9 & 0.7863 & 0.07124 & 0.00278 & 0.89095 \\
\hline X14_G095 & 263.3 & 114.2 & 0.3581 & 0.14248 & 0.0041 & 7.95041 \\
\hline X14_G096 & 191.4 & 19.8 & 0.247 & 0.0595 & 0.0023 & 0.85437 \\
\hline X14_G097 & 206.9 & 31.1 & 0.6078 & 0.06436 & 0.00226 & 1.21384 \\
\hline X14_G098 & 294.7 & 31 & 0.2958 & 0.05875 & 0.00212 & 0.8454 \\
\hline X14_G099 & & & & 0.57443 & 0.03292 & 32.87436 \\
\hline X14_G100 & 344.2 & 39.7 & 0.6883 & 0.06064 & 0.0021 & 0.85702 \\
\hline X14_G101 & 282.6 & 35.4 & 1.4893 & 0.06115 & 0.00226 & 0.78922 \\
\hline X14_G102 & 98.9 & 9.8 & 0.0628 & 0.06014 & 0.00284 & 0.87472 \\
\hline X14_G103 & 216.4 & 42.2 & 0.9501 & 0.07103 & 0.0024 & 1.58782 \\
\hline X14_G104 & 571.3 & 57.9 & 0.3515 & 0.05802 & 0.00188 & 0.79226 \\
\hline X14_G105 & 77.9 & 11.3 & 0.5535 & 0.06415 & 0.00294 & 1.17507 \\
\hline
\end{tabular}




$\begin{array}{lcccccc}\text { X14_G106 } & 93.8 & 13.7 & 0.5385 & 0.06406 & 0.00282 & 1.1814 \\ \text { X14_G107 } & 59.8 & 6.4 & 0.1838 & 0.06593 & 0.0047 & 0.99316 \\ \text { X14_G108 } & 837.2 & 54.2 & 2.3142 & 0.05315 & 0.00188 & 0.29141 \\ \text { X14_G109 } & 1818.1 & 176.2 & 0.8624 & 0.06474 & 0.00198 & 0.79558 \\ \text { X14_G110 } & 317.5 & 42.5 & 0.3012 & 0.06456 & 0.00216 & 1.17696 \\ \text { X14_G111 } & 805.8 & 165.4 & 0.3574 & 0.08694 & 0.00262 & 2.36559 \\ \text { X14_G112 } & 214.7 & 21.3 & 0.4321 & 0.06079 & 0.00232 & 0.7916 \\ \text { X14_G113 } & 290 & 31 & 0.4358 & 0.06327 & 0.0023 & 0.88661 \\ \text { X14_G114 } & 861.7 & 107.8 & 0.3545 & 0.07966 & 0.0025 & 1.32675 \\ \text { X14_G115 } & 144.1 & 21.4 & 0.5217 & 0.06756 & 0.00258 & 1.27906 \\ \text { X14_G116 } & & & & 0.77638 & 0.09482 & 818.84454 \\ \text { X14_G117 } & 312.8 & 34.4 & 0.4104 & 0.05954 & 0.00212 & 0.86514 \\ \text { X14_G118 } & 300.7 & 1.9 & 0.7366 & 0.08688 & 0.00914 & 0.06446\end{array}$




\section{$2 \%$ concordant}

\begin{tabular}{|c|c|c|c|c|c|c|}
\hline $2 \sigma 75$ & $\mathrm{~Pb} 206 / \mathrm{U} 238$ & $2 \sigma 68$ & $\begin{array}{c}\text { ages } \\
\text { age } 206 / 238\end{array}$ & $2 \sigma$ age 68 & age $207 / 235$ & $2 \sigma$ age 75 \\
\hline 0.04058 & 0.09616 & 0.00272 & 591.9 & 16 & 589.2 & 27 \\
\hline 0.0519 & 0.14486 & 0.00368 & 872.1 & 20.8 & 877.4 & 27.2 \\
\hline 0.06408 & 0.14543 & 0.00406 & 875.3 & 22.8 & 884.2 & 32.4 \\
\hline 60.87112 & 4.01011 & 0.53988 & & & & \\
\hline 0.0242 & 0.09402 & 0.00218 & 579.3 & 12.8 & 584.9 & 17.4 \\
\hline 0.02728 & 0.11567 & 0.0026 & 705.6 & 15 & 690.3 & 18 \\
\hline 0.0269 & 0.11409 & 0.00252 & 696.5 & 14.6 & 714.7 & 17.6 \\
\hline 0.0389 & 0.10029 & 0.00272 & 616.1 & 16 & 621.7 & 25.4 \\
\hline 0.02378 & 0.08475 & 0.00192 & 524.4 & 11.4 & 610.7 & 16.8 \\
\hline 0.06118 & 0.18842 & 0.00448 & 1112.8 & 24.4 & 1098.7 & 26.4 \\
\hline 0.03396 & 0.13317 & 0.00302 & 805.9 & 17.2 & 795.5 & 20 \\
\hline 0.05272 & 0.16209 & 0.00392 & 968.4 & 21.8 & 964 & 25.8 \\
\hline 0.33512 & 0.52041 & 0.01148 & 2700.9 & 48.6 & 2732.2 & 30.6 \\
\hline 0.00924 & 0.04103 & 0.00092 & 259.2 & 5.6 & 277.5 & 9 \\
\hline 0.03566 & 0.1371 & 0.00314 & 828.2 & 17.8 & 808.9 & 20.6 \\
\hline 0.11836 & 0.24369 & 0.00536 & 1405.9 & 27.8 & 1785 & 27.4 \\
\hline 0.13594 & 0.3126 & 0.0069 & 1753.5 & 33.8 & 1898.8 & 28.2 \\
\hline 0.02266 & 0.10232 & 0.00228 & 628 & 13.4 & 620 & 16 \\
\hline 0.02542 & 0.06475 & 0.00176 & 404.4 & 10.6 & 435.3 & 20 \\
\hline 0.04646 & 0.09845 & 0.00296 & 605.3 & 17.4 & 600.1 & 30.2 \\
\hline 0.02526 & 0.10783 & 0.00242 & 660.1 & 14 & 658.6 & 17.2 \\
\hline 0.0148 & 0.03259 & 0.00076 & 206.7 & 4.8 & 429.1 & 12.8 \\
\hline 0.02266 & 0.07438 & 0.00164 & 462.5 & 9.8 & 635.3 & 16 \\
\hline 0.1639 & 0.30238 & 0.00804 & 1703.1 & 39.8 & 1779.1 & 36.6 \\
\hline 0.02866 & 0.10197 & 0.00244 & 625.9 & 14.2 & 609.4 & 19.6 \\
\hline 0.0718 & 0.12963 & 0.00412 & 785.8 & 23.6 & 802.8 & 38.6 \\
\hline 0.01612 & 0.05206 & 0.0012 & 327.2 & 7.4 & 437 & 13.4 \\
\hline 0.03088 & 0.09813 & 0.00244 & 603.4 & 14.4 & 590.9 & 21.2 \\
\hline 0.05172 & 0.15753 & 0.0038 & 943 & 21.2 & 956.9 & 25.4 \\
\hline 0.02948 & 0.08058 & 0.00214 & 499.6 & 12.8 & 494.6 & 21.6 \\
\hline 0.04516 & 0.13065 & 0.00328 & 791.6 & 18.8 & 797.2 & 25.4 \\
\hline 0.15508 & 0.36105 & 0.00818 & 1987.1 & 38.8 & 1962.9 & 29.8 \\
\hline 0.00396 & 0.00392 & 0.00018 & 25.2 & 1.2 & 29.6 & 4.2 \\
\hline 0.0538 & 0.15751 & 0.00348 & 942.9 & 19.4 & 1152 & 23 \\
\hline 0.03856 & 0.09999 & 0.00244 & 614.4 & 14.2 & 770.6 & 23 \\
\hline 0.04082 & 0.13805 & 0.00326 & 833.6 & 18.4 & 835.3 & 22.8 \\
\hline 0.11688 & 0.10101 & 0.00306 & 620.3 & 18 & 1455.8 & 38.8 \\
\hline 0.09038 & 0.13847 & 0.00436 & 836 & 24.6 & 1051.7 & 39.8 \\
\hline 0.04026 & 0.14185 & 0.0033 & 855.1 & 18.6 & 852.1 & 22.2 \\
\hline 0.02494 & 0.02758 & 0.0012 & 175.4 & 7.6 & 190.1 & 23.2 \\
\hline 0.03394 & 0.12346 & 0.00288 & 750.4 & 16.6 & 743.4 & 20.6 \\
\hline 0.0324 & 0.10738 & 0.00262 & 657.5 & 15.2 & 645.6 & 21.2 \\
\hline 0.02782 & 0.10424 & 0.00242 & 639.2 & 14.2 & 634.6 & 18.8 \\
\hline 0.06764 & 0.14495 & 0.00422 & 872.6 & 23.8 & 860.3 & 34.6 \\
\hline 0.03576 & 0.13115 & 0.00302 & 794.4 & 17.2 & 796.9 & 20.8 \\
\hline 0.00836 & 0.03634 & 0.0008 & 230.1 & 5 & 272.2 & 8.4 \\
\hline 0.03328 & 0.1204 & 0.00282 & 732.9 & 16.2 & 721.2 & 20.6 \\
\hline 0.03722 & 0.12185 & 0.00294 & 741.2 & 16.8 & 742.7 & 22.4 \\
\hline 0.16426 & 0.35988 & 0.00818 & 1981.6 & 38.8 & 1993.8 & 30.4 \\
\hline 0.06486 & 0.16476 & 0.00434 & 983.2 & 24 & 969.9 & 30.6 \\
\hline
\end{tabular}




\begin{tabular}{|c|c|c|c|c|c|c|}
\hline 0.0289 & 0.09701 & 0.00238 & 596.9 & 14 & 581.3 & 20 \\
\hline 0.02842 & 0.10304 & 0.0024 & 632.2 & 14 & 644.4 & 19 \\
\hline 0.03314 & 0.09647 & 0.00248 & 593.7 & 14.6 & 588.9 & 22.6 \\
\hline 0.03992 & 0.09952 & 0.00276 & 611.6 & 16.2 & 597.4 & 26.4 \\
\hline 0.03442 & 0.13342 & 0.00298 & 807.3 & 17 & 831.3 & 19.8 \\
\hline 0.01226 & 0.03976 & 0.001 & 251.3 & 6.2 & 258.2 & 11.4 \\
\hline 0.04042 & 0.14115 & 0.0033 & 851.2 & 18.6 & 835.7 & 22.4 \\
\hline 0.04676 & 0.12245 & 0.00312 & 744.6 & 18 & 808.5 & 26.2 \\
\hline 0.1457 & 0.26879 & 0.00616 & 1534.7 & 31.2 & 1869.4 & 30.4 \\
\hline 0.0193 & 0.06709 & 0.00156 & 418.6 & 9.4 & 478.6 & 15.2 \\
\hline 0.05338 & 0.16271 & 0.00392 & 971.8 & 21.8 & 971.5 & 25.8 \\
\hline 0.01464 & 0.04351 & 0.00098 & 274.6 & 6 & 435.9 & 12.4 \\
\hline 0.04056 & 0.1015 & 0.00256 & 623.2 & 15 & 744.2 & 24.4 \\
\hline 0.03704 & 0.10687 & 0.0027 & 654.5 & 15.8 & 675.1 & 23.4 \\
\hline 0.03898 & 0.14137 & 0.00326 & 852.4 & 18.4 & 840.1 & 21.8 \\
\hline 0.04952 & 0.10826 & 0.00322 & 662.6 & 18.8 & 637.5 & 31 \\
\hline 0.0444 & 0.09467 & 0.0025 & 583.1 & 14.8 & 755.1 & 26.2 \\
\hline 0.03284 & 0.12776 & 0.00288 & 775.1 & 16.4 & 777.1 & 19.8 \\
\hline 0.03478 & 0.12473 & 0.00288 & 757.7 & 16.6 & 768.9 & 20.8 \\
\hline 0.03582 & 0.13333 & 0.00306 & 806.8 & 17.4 & 794.8 & 20.8 \\
\hline 0.05912 & 0.13988 & 0.00388 & 844 & 22 & 831 & 31.6 \\
\hline 0.01684 & 0.06445 & 0.00146 & 402.6 & 8.8 & 460.8 & 13.8 \\
\hline 0.13944 & 0.28965 & 0.00682 & 1639.8 & 34 & 1770.1 & 31.6 \\
\hline 0.0515 & 0.09884 & 0.0031 & 607.6 & 18.2 & 616.6 & 33 \\
\hline 0.01706 & 0.04569 & 0.00112 & 288 & 7 & 397.8 & 14.4 \\
\hline 0.03788 & 0.13347 & 0.0031 & 807.6 & 17.6 & 804.9 & 21.6 \\
\hline 0.0735 & 0.16116 & 0.00442 & 963.2 & 24.6 & 998.7 & 33.6 \\
\hline 0.02902 & 0.10116 & 0.00234 & 621.2 & 13.6 & 661.5 & 19.2 \\
\hline 0.33444 & 0.50497 & 0.01144 & 2635.1 & 49 & 2616.5 & 33 \\
\hline 0.0319 & 0.11113 & 0.00254 & 679.3 & 14.8 & 734.9 & 19.8 \\
\hline 0.02912 & 0.0906 & 0.00228 & 559.1 & 13.4 & 553.2 & 20.6 \\
\hline 0.01884 & 0.06025 & 0.00138 & 377.1 & 8.4 & 487.6 & 14.8 \\
\hline 0.05028 & 0.16766 & 0.00386 & 999.2 & 21.4 & 991.5 & 24.2 \\
\hline 0.1726 & 0.33684 & 0.00806 & 1871.4 & 38.8 & 1923.3 & 33.4 \\
\hline 0.05124 & 0.1209 & 0.00332 & 735.7 & 19 & 755.6 & 29.4 \\
\hline 0.83108 & 0.68869 & 0.01602 & 3377.6 & 61.2 & 3465.2 & 35.6 \\
\hline 0.0411 & 0.13095 & 0.00308 & 793.3 & 17.6 & 832.4 & 22.8 \\
\hline 0.05336 & 0.10526 & 0.0033 & 645.2 & 19.2 & 612.8 & 33.8 \\
\hline 0.0519 & 0.12937 & 0.0034 & 784.3 & 19.4 & 818.7 & 28.2 \\
\hline 0.37554 & 0.52844 & 0.01224 & 2734.9 & 51.6 & 2681.7 & 34.6 \\
\hline 0.00408 & 0.00117 & 0.0001 & 7.5 & 0.6 & 25.4 & 5 \\
\hline 0.16184 & 0.31521 & 0.00746 & 1766.3 & 36.6 & 1869 & 33 \\
\hline 0.05224 & 0.11742 & 0.00334 & 715.7 & 19.2 & 719 & 30.6 \\
\hline 0.03404 & 0.09074 & 0.00228 & 559.9 & 13.4 & 646.9 & 22.2 \\
\hline 0.23062 & 0.40486 & 0.00928 & 2191.4 & 42.6 & 2225.4 & 33.2 \\
\hline 0.03252 & 0.10418 & 0.00256 & 638.9 & 15 & 627 & 21.4 \\
\hline 0.04206 & 0.13685 & 0.00328 & 826.8 & 18.6 & 807 & 23.6 \\
\hline 0.03016 & 0.1044 & 0.0025 & 640.1 & 14.6 & 622.1 & 20.2 \\
\hline 1.8756 & 0.41522 & 0.02396 & & & & \\
\hline 0.02928 & 0.10254 & 0.00242 & 629.3 & 14.2 & 628.5 & 19.6 \\
\hline 0.02864 & 0.09365 & 0.00226 & 577.1 & 13.4 & 590.7 & 19.8 \\
\hline 0.0402 & 0.10553 & 0.00282 & 646.7 & 16.4 & 638.1 & 25.8 \\
\hline 0.0532 & 0.16218 & 0.00386 & 968.9 & 21.4 & 965.4 & 25.8 \\
\hline 0.02554 & 0.09907 & 0.0023 & 609 & 13.4 & 592.5 & 17.8 \\
\hline 0.05248 & 0.13291 & 0.00356 & 804.4 & 20.2 & 789 & 29.2 \\
\hline
\end{tabular}




$\begin{array}{ccccccc}0.0509 & 0.1338 & 0.00352 & 809.5 & 20 & 792 & 28.2 \\ 0.06796 & 0.10929 & 0.00376 & 668.6 & 21.8 & 700.3 & 40 \\ 0.01016 & 0.03978 & 0.00094 & 251.5 & 5.8 & 259.7 & 9.8 \\ 0.02434 & 0.08916 & 0.00204 & 550.6 & 12 & 594.3 & 17.2 \\ 0.0391 & 0.13228 & 0.0031 & 800.9 & 17.6 & 789.9 & 22.4 \\ 0.07126 & 0.19741 & 0.0045 & 1161.4 & 24.2 & 1232.3 & 27 \\ 0.0297 & 0.09448 & 0.0023 & 582 & 13.6 & 592.1 & 20.4 \\ 0.03184 & 0.10168 & 0.00246 & 624.2 & 14.4 & 644.5 & 20.8 \\ 0.0416 & 0.12084 & 0.00278 & 735.4 & 16 & 857.5 & 22.6 \\ 0.04792 & 0.13736 & 0.0034 & 829.7 & 19.2 & 836.4 & 26 \\ 316.72082 & 7.65251 & 2.99676 & & & & \\ 0.03036 & 0.10543 & 0.0025 & 646.2 & 14.6 & 632.9 & 20.2 \\ 0.00632 & 0.00538 & 0.00024 & 34.6 & 1.6 & 63.4 & 7\end{array}$




\begin{tabular}{|c|c|c|c|c|c|}
\hline age $207 / 206$ & $2 \sigma$ age 76 & $\begin{array}{l}\text { discordance } \\
\Delta 68-75[\%]\end{array}$ & $\Delta 68-76[\%]$ & $\begin{array}{c}\text { preferred age } \\
\text { age }\end{array}$ & $2 \sigma$ age \\
\hline 578.9 & 115.8 & 0.5 & 2.2 & 591.9 & 16 \\
\hline 890.9 & 80 & -0.6 & -2.1 & 872.1 & 20.8 \\
\hline 906.8 & 97.6 & -1 & -3.5 & 875.3 & 22.8 \\
\hline 607.2 & 67.6 & -1 & -4.6 & 579.3 & 12.8 \\
\hline 641 & 59.8 & 2.2 & 10.1 & 705.6 & 15 \\
\hline 772.7 & 55 & -2.6 & -9.9 & 696.5 & 14.6 \\
\hline 642.4 & 102 & -0.9 & -4.1 & 616.1 & 16 \\
\hline 945.6 & 59.2 & -14.1 & -44.5 & 524.4 & 11.4 \\
\hline 1071.2 & 63.2 & 1.3 & 3.9 & 1071.2 & 63.2 \\
\hline 766.5 & 59.8 & 1.3 & 5.1 & 805.9 & 17.2 \\
\hline 954.2 & 68.6 & 0.5 & 1.5 & 968.4 & 21.8 \\
\hline 2755.6 & 39 & -1.1 & -2 & 2755.6 & 39 \\
\hline 435.6 & 65.8 & -6.6 & -40.5 & 259.2 & 5.6 \\
\hline 756.1 & 61.6 & 2.4 & 9.5 & 828.2 & 17.8 \\
\hline 2262.6 & 41.6 & -21.2 & -37.9 & & \\
\hline 2061.7 & 42.6 & -7.7 & -14.9 & 2061.7 & 42.6 \\
\hline 591.3 & 57.4 & 1.3 & 6.2 & 628 & 13.4 \\
\hline 602.1 & 106.8 & -7.1 & -32.8 & 404.4 & 10.6 \\
\hline 580.7 & 129.6 & 0.9 & 4.2 & 605.3 & 17.4 \\
\hline 653.6 & 58.8 & 0.2 & 1 & 660.1 & 14 \\
\hline 1912 & 51.2 & -51.8 & -89.2 & & \\
\hline 1310.7 & 49.8 & -27.2 & -64.7 & & \\
\hline 1869.7 & 62.2 & -4.3 & -8.9 & 1869.7 & 62.2 \\
\hline 548.9 & 76.8 & 2.7 & 14 & 625.9 & 14.2 \\
\hline 850.5 & 128.2 & -2.1 & -7.6 & 785.8 & 23.6 \\
\hline 1066.9 & 60.6 & -25.1 & -69.3 & & \\
\hline 543.3 & 87.6 & 2.1 & 11.1 & 603.4 & 14.4 \\
\hline 989.3 & 67.6 & -1.5 & -4.7 & 943 & 21.2 \\
\hline 471.7 & 107.4 & 1 & 5.9 & 499.6 & 12.8 \\
\hline 813.3 & 80.8 & -0.7 & -2.7 & 791.6 & 18.8 \\
\hline 1937.7 & 46.2 & 1.2 & 2.6 & 1937.7 & 46.2 \\
\hline 397.9 & 313.4 & -14.7 & -93.7 & 25.2 & 1.2 \\
\hline 1570.5 & 47 & -18.2 & -40 & & \\
\hline 1255.5 & 67.8 & -20.3 & -51.1 & & \\
\hline 839.9 & 67 & -0.2 & -0.8 & 833.6 & 18.4 \\
\hline 3047.9 & 63.8 & -57.4 & -79.6 & & \\
\hline 1532.2 & 98 & -20.5 & -45.4 & & \\
\hline 844.9 & 63.8 & 0.4 & 1.2 & 855.1 & 18.6 \\
\hline 377.3 & 283.4 & -7.7 & -53.5 & 175.4 & 7.6 \\
\hline 722.7 & 66.8 & 0.9 & 3.8 & 750.4 & 16.6 \\
\hline 604.3 & 80 & 1.8 & 8.8 & 657.5 & 15.2 \\
\hline 618.6 & 70 & 0.7 & 3.3 & 639.2 & 14.2 \\
\hline 829 & 108.8 & 1.4 & 5.3 & 872.6 & 23.8 \\
\hline 804.5 & 62.8 & -0.3 & -1.2 & 794.4 & 17.2 \\
\hline 652.2 & 58 & -15.5 & -64.7 & & \\
\hline 685.8 & 69.2 & 1.6 & 6.9 & 732.9 & 16.2 \\
\hline 747.6 & 73.8 & -0.2 & -0.9 & 741.2 & 16.8 \\
\hline 2007 & 46.8 & -0.6 & -1.3 & 2007 & 46.8 \\
\hline 940.6 & 85 & 1.4 & 4.5 & 983.2 & 24 \\
\hline
\end{tabular}




\begin{tabular}{|c|c|c|c|c|c|}
\hline 521.4 & 83.6 & 2.7 & 14.5 & 596.9 & 14 \\
\hline 687.8 & 69 & -1.9 & -8.1 & 632.2 & 14 \\
\hline 571.1 & 94.2 & 0.8 & 3.9 & 593.7 & 14.6 \\
\hline 544.1 & 112.4 & 2.4 & 12.4 & 611.6 & 16.2 \\
\hline 896.6 & 55.6 & -2.9 & -10 & 807.3 & 17 \\
\hline 321.9 & 98.8 & -2.7 & -21.9 & 251.3 & 6.2 \\
\hline 795.6 & 66.4 & 1.8 & 7 & 851.2 & 18.6 \\
\hline 989.3 & 80.2 & -7.9 & -24.7 & 744.6 & 18 \\
\hline 2265.5 & 47 & -17.9 & -32.3 & & \\
\hline 778.2 & 67.8 & -12.5 & -46.2 & 418.6 & 9.4 \\
\hline 971.2 & 68.4 & 0 & 0.1 & 971.8 & 21.8 \\
\hline 1413 & 51.8 & -37 & -80.6 & & \\
\hline 1128.4 & 76.8 & -16.3 & -44.8 & & \\
\hline 744.9 & 85 & -3 & -12.1 & 654.5 & 15.8 \\
\hline 808.6 & 63.4 & 1.5 & 5.4 & 852.4 & 18.4 \\
\hline 550 & 127.6 & 3.9 & 20.5 & 662.6 & 18.8 \\
\hline 1305.9 & 80.8 & -22.8 & -55.3 & & \\
\hline 783.4 & 59.8 & -0.3 & -1.1 & 775.1 & 16.4 \\
\hline 801.9 & 64.2 & -1.4 & -5.5 & 757.7 & 16.6 \\
\hline 761.7 & 63.4 & 1.5 & 5.9 & 806.8 & 17.4 \\
\hline 797.2 & 100.8 & 1.6 & 5.9 & 844 & 22 \\
\hline 762.6 & 62 & -12.6 & -47.2 & 402.6 & 8.8 \\
\hline 1928 & 52.8 & -7.4 & -14.9 & 1928 & 52.8 \\
\hline 650.5 & 137.2 & -1.5 & -6.6 & 607.6 & 18.2 \\
\hline 1099.3 & 73 & -27.6 & -73.8 & & \\
\hline 797.8 & 65.6 & 0.3 & 1.2 & 807.6 & 17.6 \\
\hline 1078.1 & 90.6 & -3.6 & -10.7 & 963.2 & 24.6 \\
\hline 802.2 & 66.8 & -6.1 & -22.6 & 621.2 & 13.6 \\
\hline 2602.6 & 45 & 0.7 & 1.2 & 2602.6 & 45 \\
\hline 908.9 & 61.8 & -7.6 & -25.3 & 679.3 & 14.8 \\
\hline 529.8 & 90 & 1.1 & 5.5 & 559.1 & 13.4 \\
\hline 1047.5 & 62 & -22.7 & -64 & & \\
\hline 975.4 & 62 & 0.8 & 2.4 & 999.2 & 21.4 \\
\hline 1980.3 & 54.2 & -2.7 & -5.5 & 1980.3 & 54.2 \\
\hline 815.2 & 99.6 & -2.6 & -9.8 & 735.7 & 19 \\
\hline 3516.7 & 42.8 & -2.5 & -4 & 3516.7 & 42.8 \\
\hline 938.9 & 67 & -4.7 & -15.5 & 793.3 & 17.6 \\
\hline 495.8 & 146.6 & 5.3 & 30.1 & & \\
\hline 914.2 & 88.2 & -4.2 & -14.2 & 784.3 & 19.4 \\
\hline 2642.4 & 47.2 & 2 & 3.5 & 2642.4 & 47.2 \\
\hline 2420.9 & 301.4 & -70.4 & -99.7 & & \\
\hline 1985.8 & 54.2 & -5.5 & -11.1 & 1985.8 & 54.2 \\
\hline 730 & 110.6 & -0.5 & -2 & 715.7 & 19.2 \\
\hline 964.3 & 79.6 & -13.4 & -41.9 & 559.9 & 13.4 \\
\hline 2257.6 & 49.6 & -1.5 & -2.9 & 2257.6 & 49.6 \\
\hline 585.4 & 83.8 & 1.9 & 9.1 & 638.9 & 15 \\
\hline 753.5 & 74.2 & 2.5 & 9.7 & 826.8 & 18.6 \\
\hline 557.8 & 78.6 & 2.9 & 14.8 & 640.1 & 14.6 \\
\hline 626.5 & 74.6 & 0.1 & 0.4 & 629.3 & 14.2 \\
\hline 644.5 & 79.4 & -2.3 & -10.5 & 577.1 & 13.4 \\
\hline 608.6 & 102 & 1.3 & 6.3 & 646.7 & 16.4 \\
\hline 958.3 & 69 & 0.4 & 1.1 & 968.9 & 21.4 \\
\hline 530.5 & 71 & 2.8 & 14.8 & 609 & 13.4 \\
\hline 746.6 & 96.8 & 2 & 7.8 & 804.4 & 20.2 \\
\hline
\end{tabular}




$\begin{array}{cccccc}743.6 & 93 & 2.2 & 8.9 & 809.5 & 20 \\ 804.2 & 149.2 & -4.5 & -16.9 & 668.6 & 21.8 \\ 335.2 & 80.2 & -3.2 & -25 & 251.5 & 5.8 \\ 765.9 & 64.4 & -7.4 & -28.1 & 550.6 & 12 \\ 760 & 70.6 & 1.4 & 5.4 & 800.9 & 17.6 \\ 1359.2 & 58 & -5.8 & -14.6 & 1359.2 & 58 \\ 631.8 & 82.2 & -1.7 & -7.9 & 582 & 13.6 \\ 717.3 & 77.2 & -3.1 & -13 & 624.2 & 14.4 \\ 1188.6 & 62 & -14.2 & -38.1 & 735.4 & 16 \\ 855.1 & 79.4 & -0.8 & -3 & 829.7 & 19.2 \\ & & & & & \\ 586.9 & 77.2 & 2.1 & 10.1 & 646.2 & 14.6 \\ 1357.8 & 202.8 & -45.5 & -97.5 & & \end{array}$

15

16

17

18

19

20

21

22

23

24

25

26

27

28

29

30

31

32

33

34

35

36

37

44

45

46

47

48

49

50

51

52

53

54

55

56

57

58

59

60 


\section{Sample 51 Main Nile @ Cairo Ma'adi 10 grain analysed 5 concordant ages}

\begin{tabular}{cccc|ccc} 
& concentrations & & & isotopic ratios & & \\
grain & $\mathbf{U}[\mathbf{p p m}]$ & $\mathrm{Pb}[\mathbf{p p m}]$ & Th/U & Pb207/Pb206 & $\mathbf{2 \sigma} \mathbf{7 6}$ & Pb207/U235 \\
\hline X51_G001 & 89.6 & 14 & 0.6199 & 0.06281 & 0.00296 & 1.22487 \\
X51_G002 & 44.3 & 17.9 & 0.774 & -0.00403 & 0.00476 & -0.17637 \\
X51_G003 & 163.5 & 27.7 & 0.9901 & 0.06147 & 0.0028 & 1.17208 \\
X51_G004 & 287.2 & 44.3 & 1.1935 & 0.064 & 0.00246 & 1.05602 \\
X51_G005 & 79.8 & 13.1 & 0.6212 & 0.06566 & 0.00336 & 1.34248 \\
X51_G006 & 53.7 & 8 & 0.3887 & 0.06644 & 0.00368 & 1.31058 \\
X51_G007 & 400.5 & 45 & 0.5965 & 0.05699 & 0.00208 & 0.80184 \\
X51_G008 & 121.7 & 15.9 & 0.5518 & 0.05787 & 0.0027 & 0.96006 \\
X51_G009 & 325.1 & 70.2 & 0.2968 & 0.08595 & 0.00278 & 2.50824 \\
X51_G010 & 40.9 & 4.8 & 0.3963 & 0.05475 & 0.00456 & 0.85732
\end{tabular}


$50.0 \%$ concordant

\begin{tabular}{ccc|cccc}
\hline $\mathbf{2 \sigma} \mathbf{7 5}$ & Pb206/U238 & $\mathbf{2 \sigma} \mathbf{6 8}$ & $\begin{array}{c}\text { ages } \\
\text { age } \mathbf{2 0 6 / 2 3 8}\end{array}$ & $\mathbf{2 \sigma}$ age 68 & age 207/235 & 20 age $\mathbf{7 5}$ \\
\hline 0.05652 & 0.14161 & 0.0039 & 853.8 & 22 & 812 & 30.6 \\
0.20852 & 0.31776 & 0.00958 & 1778.8 & 46.8 & -197 & -257.2 \\
0.0522 & 0.13847 & 0.00376 & 836 & 21.2 & 787.6 & 29 \\
0.04008 & 0.11983 & 0.00302 & 729.6 & 17.4 & 731.9 & 24 \\
0.06684 & 0.1485 & 0.0043 & 892.5 & 24.2 & 864.3 & 34.2 \\
0.07068 & 0.14326 & 0.00434 & 863.1 & 24.4 & 850.4 & 36.4 \\
0.0291 & 0.10219 & 0.0025 & 627.2 & 14.6 & 597.9 & 19.8 \\
0.04394 & 0.1205 & 0.00326 & 733.4 & 18.8 & 683.3 & 26.8 \\
0.08162 & 0.21197 & 0.0051 & 1239.3 & 27.2 & 1274.4 & 29.2 \\
0.0691 & 0.11373 & 0.00412 & 694.4 & 23.8 & 628.7 & 42.6
\end{tabular}




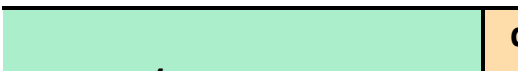

\begin{tabular}{cc} 
age $207 / 206$ & 20 age 76 \\
\hline 701.8 & 100.4
\end{tabular}

discordance

$\Delta$ 68-75 [\%]

$\Delta 68-76[\%]$

preferred age age

$2 \sigma$ age

655.7

97.8

741.6

81.2

107.2

820.3

115.6

491.1

524.8

80.4

102.4

1337.1

62.6

402

186.6 $-1002.8$

6.1

$-0.3$

3.3

1.5

21.7

27.5

$-1.6$

12.2

5.2

27.7

39.7

7.3

$-2.8$

$-7.3$

10.5

72.7

729.6

892.5

17.4

24.2

24.4

863.1

14.6

18

19

20

21

22

23

24

25

26

27

28

29

30

31

32

33

34

35

36

37

38

39

40

41

42

43

44

45

46

47

48

49

50

51

52

53

54

55

56

57

58

59

60

FOR REVIEW PURPOSES ONLY 
Sample 3290S Nile Delta beach @ Idku east 150 grain analysed 124 concor

\begin{tabular}{|c|c|c|c|c|c|c|}
\hline \multirow[b]{2}{*}{ grain } & \multicolumn{3}{|c|}{ concentrations } & \multicolumn{3}{|l|}{ isotopic ratios } \\
\hline & U [ppm] & $\mathrm{Pb}$ [ppm] & $T h / U$ & Pb207/Pb206 & $2 \sigma 76$ & Pb207/U235 \\
\hline S3290_G001 & 253.1 & 36.6 & 0.7726 & 0.06252 & 0.00178 & 1.07699 \\
\hline S3290_G002 & 139.1 & 25 & 0.354 & 0.07322 & 0.00216 & 1.74865 \\
\hline S3290_G003 & 62.7 & 7.8 & 0.2538 & 0.06441 & 0.00286 & 1.10602 \\
\hline S3290_G004 & 171.5 & 19.7 & 0.8731 & 0.06128 & 0.00254 & 0.82194 \\
\hline S3290_G005 & 78.4 & 11.2 & 0.4743 & 0.06243 & 0.0026 & 1.14647 \\
\hline S3290_G006 & 789.5 & 151.8 & 1.5302 & 0.0668 & 0.00158 & 1.27534 \\
\hline S3290_G007 & 228.5 & 34.9 & 0.5491 & 0.06769 & 0.0019 & 1.30557 \\
\hline S3290 G008 & 6706.4 & 122.9 & 1.1952 & 0.10169 & 0.00242 & 0.21638 \\
\hline S3290_G009 & 59.6 & 9 & 0.5275 & 0.06714 & 0.00288 & 1.29163 \\
\hline S3290_G010 & 198.2 & 30 & 1.9952 & 0.06061 & 0.002 & 0.82757 \\
\hline S3290_G011 & 91.5 & 12.1 & 0.676 & 0.06697 & 0.0026 & 1.07802 \\
\hline S3290_G012 & 325.2 & 51 & 0.6945 & 0.06723 & 0.00178 & 1.28395 \\
\hline S3290_G013 & 603.9 & 35.9 & 0.2406 & 0.10927 & 0.00284 & 0.86965 \\
\hline S3290_G014 & 61.7 & 7.6 & 0.5533 & 0.0623 & 0.003 & 0.96268 \\
\hline S3290_G015 & 586.1 & 58.9 & 0.4022 & 0.06081 & 0.00156 & 0.80905 \\
\hline S3290_G016 & 251.5 & 42 & 0.322 & 0.0714 & 0.00192 & 1.59891 \\
\hline S3290_G017 & 201.3 & 15.9 & 0.5015 & 0.06123 & 0.00218 & 0.61854 \\
\hline S3290_G018 & 43.4 & 6.5 & 0.4389 & 0.0743 & 0.00346 & 1.44161 \\
\hline S3290_G019 & 423.5 & 18.6 & 0.2599 & 0.05348 & 0.00182 & 0.32775 \\
\hline S3290_G020 & 94.1 & 14.8 & 0.5094 & 0.08553 & 0.00288 & 1.6878 \\
\hline S3290_G021 & 133.9 & 19 & 0.4011 & 0.06527 & 0.00216 & 1.21946 \\
\hline S3290_G022 & 3558.1 & 290.8 & 0.0407 & 0.05953 & 0.00132 & 0.72201 \\
\hline S3290_G023 & 135.9 & 18.1 & 0.6196 & 0.06275 & 0.00224 & 1.03628 \\
\hline S3290_G024 & 471.1 & 58.2 & 0.3812 & 0.06469 & 0.00166 & 1.06185 \\
\hline S3290_G025 & 221.7 & 25.2 & 0.7777 & 0.06095 & 0.00194 & 0.82127 \\
\hline S3290_G026 & 91 & 9.8 & 0.7451 & 0.05968 & 0.00266 & 0.77132 \\
\hline S3290_G027 & 108.2 & 13.2 & 1.1542 & 0.05977 & 0.00248 & 0.79081 \\
\hline S3290_G028 & 180.4 & 19.8 & 0.5256 & 0.05937 & 0.00202 & 0.83134 \\
\hline S3290_G029 & 158.4 & 16.1 & 0.3036 & 0.05862 & 0.00216 & 0.80854 \\
\hline S3290_G030 & 324.2 & 48 & 0.4384 & 0.06895 & 0.00182 & 1.33689 \\
\hline S3290_G031 & 151.6 & 64.1 & 0.4614 & 0.13295 & 0.00318 & 7.01126 \\
\hline S3290_G032 & 511.4 & 59.7 & 1.0065 & 0.05929 & 0.00158 & 0.77844 \\
\hline S3290_G033 & 232.7 & 30.5 & 0.1978 & 0.06586 & 0.0019 & 1.21024 \\
\hline S3290_G034 & 68 & 28.2 & 1.1163 & 0.11646 & 0.00454 & 5.19894 \\
\hline S3290_G035 & 286.5 & 93.9 & 0.1873 & 0.11715 & 0.0028 & 5.2226 \\
\hline S3290_G036 & 122.4 & 15.4 & 1.0233 & 0.06013 & 0.0024 & 0.84504 \\
\hline S3290_G037 & 229.5 & 24.7 & 0.7771 & 0.05904 & 0.00192 & 0.75699 \\
\hline S3290_G038 & 98.3 & 0.5 & 0.523 & 0.05199 & 0.01474 & 0.03573 \\
\hline S3290_G039 & 668.2 & 239.7 & 0.2998 & 0.15224 & 0.00336 & 7.31259 \\
\hline S3290_G040 & 314.8 & 25.7 & 0.0176 & 0.05904 & 0.0018 & 0.72033 \\
\hline S3290_G041 & 82.6 & 16.7 & 0.646 & 0.07378 & 0.00266 & 1.83474 \\
\hline S3290_G042 & 40.8 & 0.2 & 0.601 & 0.03279 & 0.03546 & 0.02163 \\
\hline S3290_G043 & 265.6 & 51.7 & 3.0041 & 0.06055 & 0.00182 & 0.87704 \\
\hline S3290_G044 & 121.8 & 49.3 & 0.852 & 0.11348 & 0.0029 & 5.23142 \\
\hline S3290_G045 & 950.6 & 77.9 & 0.7142 & 0.07569 & 0.00188 & 0.78715 \\
\hline S3290_G046 & 123.4 & 12 & 0.4225 & 0.05983 & 0.0029 & 0.76972 \\
\hline S3290_G047 & 203.4 & 78.1 & 0.4056 & 0.1202 & 0.00302 & 5.85795 \\
\hline S3290_G048 & 95.2 & 10.3 & 0.1711 & 0.06271 & 0.00266 & 0.96104 \\
\hline S3290_G049 & 79 & 11.6 & 0.5257 & 0.06709 & 0.00278 & 1.25193 \\
\hline S3290_G050 & 30502.8 & 75 & 0.6571 & 0.56754 & 0.01264 & 0.23805 \\
\hline
\end{tabular}




\begin{tabular}{|c|c|c|c|c|c|c|}
\hline S3290_G051 & 120.3 & 15.1 & 0.4545 & 0.08536 & 0.00292 & 1.37792 \\
\hline S3290_G052 & 159 & 20.8 & 0.6394 & 0.06646 & 0.0022 & 1.07018 \\
\hline S3290 G053 & 235.3 & 26.3 & 0.3327 & 0.06343 & 0.00198 & 0.95678 \\
\hline S3290 G054 & 213.9 & 21.8 & 0.4249 & 0.05977 & 0.002 & 0.80655 \\
\hline S3290_G055 & 116.1 & 16.7 & 0.4571 & 0.06528 & 0.00234 & 1.21603 \\
\hline S3290_G056 & 116.6 & 14.9 & 0.5496 & 0.06309 & 0.00236 & 1.01962 \\
\hline S3290_G057 & 586.7 & 70.3 & 0.8402 & 0.06014 & 0.00156 & 0.8462 \\
\hline S3290_G058 & 533.3 & 75.6 & 0.6364 & 0.0642 & 0.00162 & 1.12486 \\
\hline S3290_G059 & 243.1 & 30.5 & 0.5626 & 0.06215 & 0.00188 & 0.9815 \\
\hline $\mathrm{S} 3290^{\circ} \mathrm{G} 060$ & & & & 0.3076 & 0.01918 & 5.62695 \\
\hline S3290_G061 & 297 & 28.5 & 0.1 & 0.06055 & 0.00182 & 0.84376 \\
\hline S3290_G062 & 2021.9 & 109 & 0.5837 & 0.07835 & 0.00188 & 0.51797 \\
\hline S3290_G063 & 129.7 & 19.6 & 0.1952 & 0.09906 & 0.00306 & 2.0183 \\
\hline S3290_G064 & 615.9 & 88.8 & 0.272 & 0.06776 & 0.00166 & 1.33266 \\
\hline S3290_G065 & 64.3 & 7.7 & 0.6115 & 0.06245 & 0.00306 & 0.93552 \\
\hline S3290_G066 & 289.7 & 122 & 0.8852 & 0.12398 & 0.00288 & 5.96796 \\
\hline S3290_G067 & 96.2 & 9.9 & 0.6436 & 0.06048 & 0.00272 & 0.76563 \\
\hline S3290_G068 & 253.6 & 95.6 & 0.4855 & 0.11264 & 0.00268 & 5.2879 \\
\hline S3290_G069 & 305.9 & 38.5 & 0.3696 & 0.06757 & 0.00192 & 1.13191 \\
\hline S3290_G070 & 115.6 & 12 & 0.5801 & 0.05897 & 0.00248 & 0.76922 \\
\hline S3290_G071 & 89.4 & 9.9 & 1.1181 & 0.05955 & 0.00284 & 0.72577 \\
\hline S3290 G072 & 232.2 & 35 & 0.4224 & 0.06845 & 0.002 & 1.34954 \\
\hline S3290_G073 & 159.5 & 19.4 & 0.2021 & 0.0654 & 0.0022 & 1.11407 \\
\hline S3290_G074 & 102.5 & 14.9 & 0.6616 & 0.06945 & 0.00254 & 1.23551 \\
\hline S3290_G075 & 731 & 75.4 & 0.3753 & 0.06026 & 0.00154 & 0.82832 \\
\hline S3290_G076 & 283.4 & 44 & 0.412 & 0.06887 & 0.00192 & 1.40405 \\
\hline S3290_G077 & 473.2 & 48.4 & 0.2588 & 0.06028 & 0.00164 & 0.85273 \\
\hline S3290_G078 & 85.2 & 9.2 & 0.6265 & 0.06069 & 0.00284 & 0.81142 \\
\hline S3290_G079 & 622.2 & 56.4 & 0.6577 & 0.06667 & 0.00178 & 0.75912 \\
\hline S3290_G080 & 6273.9 & 123.9 & 0.0547 & 0.25616 & 0.00582 & 0.64125 \\
\hline S3290_G081 & 82.6 & 9.6 & 0.7376 & 0.05886 & 0.00284 & 0.82603 \\
\hline S3290_G082 & 121.8 & 11.5 & 0.8259 & 0.05674 & 0.00252 & 0.62784 \\
\hline S3290_G083 & 83.7 & 8.5 & 0.8871 & 0.05848 & 0.00302 & 0.68143 \\
\hline S3290_G084 & 32.4 & 0.2 & 0.4918 & -0.00216 & 0.0383 & -0.00146 \\
\hline S3290_G085 & 72.2 & 9.7 & 0.3758 & 0.06808 & 0.00312 & 1.21649 \\
\hline S3290_G086 & 286.5 & 38.9 & 1.2079 & 0.06116 & 0.00184 & 0.88719 \\
\hline S3290_G087 & 197.1 & 22.8 & 0.8412 & 0.06279 & 0.00216 & 0.85289 \\
\hline S3290_G088 & 1369.4 & 95.8 & 0.497 & 0.14986 & 0.00354 & 1.33457 \\
\hline S3290_G089 & 221.2 & 28.5 & 0.3766 & 0.06525 & 0.00198 & 1.11833 \\
\hline S3290_G090 & 2039.2 & 161.3 & 0.3725 & 0.07465 & 0.00176 & 0.7934 \\
\hline S3290_G091 & 711.6 & 82 & 0.3567 & 0.06268 & 0.00158 & 0.98283 \\
\hline S3290_G092 & 492.5 & 55.7 & 0.3046 & 0.19456 & 0.0047 & 2.75788 \\
\hline S3290_G093 & 2209.7 & 85.6 & 1.3224 & 0.08605 & 0.00224 & 0.37562 \\
\hline S3290_G094 & 53.9 & 23.1 & 0.8497 & 0.12918 & 0.00382 & 6.30041 \\
\hline S3290_G095 & 549 & 51.6 & 0.0918 & 0.06045 & 0.00164 & 0.82689 \\
\hline S3290 G096 & 19.3 & 2.4 & 0.3246 & 0.06628 & 0.00544 & 1.13237 \\
\hline S3290_G097 & 198.2 & 23.9 & 0.4149 & 0.06654 & 0.00212 & 1.05331 \\
\hline S3290_G098 & 280.8 & 38.1 & 0.5587 & 0.06544 & 0.0019 & 1.12287 \\
\hline S3290_G099 & 6523.3 & & 1.571 & 0.31971 & 0.0081 & 0.21074 \\
\hline S3290_G100 & & & & 0.01141 & 0.93964 & 10.77196 \\
\hline S3290_G101 & 1392.9 & 126.5 & 0.2806 & 0.06475 & 0.00156 & 0.80203 \\
\hline S3290_G102 & 788.5 & 70.4 & 0.1052 & 0.06164 & 0.00158 & 0.80972 \\
\hline S3290_G103 & 55.9 & 7.9 & 0.2457 & 0.06317 & 0.003 & 1.21874 \\
\hline S3290_G104 & 1168.1 & 108.9 & 0.0318 & 0.05997 & 0.00146 & 0.83037 \\
\hline S3290_G105 & 145.9 & 19.2 & 1.3836 & 0.06168 & 0.0023 & 0.83689 \\
\hline
\end{tabular}




\begin{tabular}{|c|c|c|c|c|c|c|}
\hline S3290_G106 & 108.8 & 12.8 & 1.0185 & 0.05767 & 0.00252 & 0.76387 \\
\hline S3290_G107 & 285 & 38.6 & 0.418 & 0.06497 & 0.00192 & 1.15858 \\
\hline S3290_G108 & 340.4 & 50.8 & 0.6478 & 0.06408 & 0.0018 & 1.17842 \\
\hline S3290_G109 & 29.8 & 3.3 & 0.8063 & 0.06369 & 0.005 & 0.83981 \\
\hline S3290_G110 & 53.3 & 7.4 & 0.4514 & 0.06302 & 0.00328 & 1.13338 \\
\hline S3290_G111 & 76.9 & 9.9 & 0.5249 & 0.06523 & 0.00296 & 1.06786 \\
\hline S3290_G112 & 2294.9 & 71.7 & 0.4752 & 0.10181 & 0.00256 & 0.42834 \\
\hline S3290_G113 & 517.6 & 55.5 & 0.1727 & 0.06149 & 0.00166 & 0.93538 \\
\hline $\mathrm{S} 3290 \mathrm{G} 114$ & 82.1 & 11.8 & 0.4026 & 0.07044 & 0.00296 & 1.33714 \\
\hline S3290_G115 & 74.2 & 8.4 & 0.8783 & 0.05832 & 0.00306 & 0.77224 \\
\hline S3290_G116 & 114 & 14.7 & 1.4995 & 0.05565 & 0.00254 & 0.72302 \\
\hline S3290_G117 & 130.2 & 16.1 & 0.6885 & 0.05911 & 0.00238 & 0.89622 \\
\hline S3290_G118 & 454.9 & 208.8 & 0.9149 & 0.12594 & 0.0029 & 6.45836 \\
\hline S3290_G119 & 99.9 & 23.8 & 1.5619 & 0.07573 & 0.00266 & 1.79452 \\
\hline S3290_G120 & 789 & 71.1 & 0.2584 & 0.05859 & 0.00154 & 0.73054 \\
\hline S3290_G121 & 603.4 & 59.3 & 0.2334 & 0.06028 & 0.0016 & 0.82629 \\
\hline S3290_G122 & 139.6 & 21.1 & 0.3689 & 0.06842 & 0.00234 & 1.37143 \\
\hline S3290_G123 & 77.9 & 8.1 & 0.6577 & 0.05646 & 0.003 & 0.72183 \\
\hline S3290_G124 & 194.5 & 27.4 & 0.3766 & 0.06752 & 0.0021 & 1.25921 \\
\hline S3290_G125 & 99.9 & 14.5 & 0.4848 & 0.06603 & 0.00262 & 1.23538 \\
\hline S3290_G126 & 25.6 & 2.5 & 0.4118 & 0.05745 & 0.00562 & 0.75946 \\
\hline S3290_G127 & 61.2 & 8.7 & 0.4599 & 0.06324 & 0.00302 & 1.17198 \\
\hline S3290_G128 & 208.6 & 50.7 & 0.9803 & 0.10461 & 0.0028 & 2.9102 \\
\hline S3290_G129 & 434 & 60.1 & 0.5178 & 0.06565 & 0.0018 & 1.18193 \\
\hline S3290_G130 & 1228.7 & 38.8 & 0.7284 & 0.04942 & 0.00156 & 0.18918 \\
\hline S3290_G131 & 789 & 89.5 & 0.3038 & 0.0667 & 0.0017 & 1.02378 \\
\hline S3290_G132 & 463.8 & 95.4 & 0.6366 & 0.10544 & 0.0026 & 2.70794 \\
\hline S3290_G133 & 79.5 & 15.2 & 0.8162 & 0.07459 & 0.00286 & 1.679 \\
\hline S3290_G134 & 80 & 8.5 & 0.6007 & 0.06077 & 0.00296 & 0.81179 \\
\hline S3290_G135 & 1038.9 & 105.8 & 0.1657 & 0.08535 & 0.0021 & 1.22135 \\
\hline S3290_G136 & 283.4 & 24.3 & 0.261 & 0.05779 & 0.0019 & 0.68469 \\
\hline S3290_G137 & 95.2 & 11 & 0.5899 & 0.05985 & 0.00262 & 0.86938 \\
\hline S3290_G138 & 50.2 & 6.1 & 0.4431 & 0.06289 & 0.00388 & 0.98814 \\
\hline S3290_G139 & 118.7 & 11.8 & 0.3493 & 0.05887 & 0.00252 & 0.78384 \\
\hline S3290_G140 & 78.4 & 11.7 & 0.6296 & 0.06454 & 0.00282 & 1.19161 \\
\hline S3290_G141 & 32.4 & 0.1 & 0.415 & 0.04529 & 0.04716 & 0.02648 \\
\hline S3290_G142 & & & & -0.26686 & 0.70824 & \\
\hline S3290_G143 & & & & 0.52829 & 0.02218 & 23.32735 \\
\hline S3290_G144 & 1121 & 93.5 & 0.4338 & 0.06309 & 0.0016 & 0.74 \\
\hline S3290_G145 & 2961 & 146.4 & 1.0728 & 0.29085 & 0.00676 & 1.63908 \\
\hline S3290_G146 & 615.4 & 96.6 & 0.5931 & 0.07649 & 0.00196 & 1.57346 \\
\hline S3290_G147 & 76.3 & 14.7 & 1.132 & 0.0681 & 0.00284 & 1.4324 \\
\hline S3290_G148 & 541.7 & 55.4 & 0.2692 & 0.05789 & 0.00166 & 0.81625 \\
\hline S3290_G149 & 878.4 & 115.3 & 1.0551 & 0.07041 & 0.0018 & 1.07149 \\
\hline S3290_G150 & 767.1 & 65.8 & 1.4041 & 0.06547 & 0.00188 & 0.6381 \\
\hline
\end{tabular}


dant ages $\quad 82.7 \%$ concordant

\begin{tabular}{|c|c|c|c|c|c|c|}
\hline $2 \sigma 75$ & Pb206/U238 & $2 \sigma 68$ & $\begin{array}{c}\text { ages } \\
\text { age } 206 / 238\end{array}$ & $2 \sigma$ age 68 & age $207 / 235$ & $2 \sigma$ age 75 \\
\hline 0.03256 & 0.12497 & 0.00312 & 759.1 & 17.8 & 742.2 & 20 \\
\hline 0.05416 & 0.17324 & 0.0044 & 1029.9 & 24.2 & 1026.7 & 25.2 \\
\hline 0.0489 & 0.12456 & 0.00352 & 756.8 & 20.2 & 756.3 & 28 \\
\hline 0.03404 & 0.0973 & 0.00268 & 598.6 & 15.8 & 609.1 & 22.8 \\
\hline 0.04786 & 0.13322 & 0.00368 & 806.2 & 21 & 775.6 & 27.2 \\
\hline 0.03316 & 0.13849 & 0.00332 & 836.1 & 18.8 & 834.8 & 19.2 \\
\hline 0.0389 & 0.13992 & 0.00348 & 844.2 & 19.6 & 848.2 & 21.6 \\
\hline 0.00562 & 0.01544 & 0.00038 & 98.8 & 2.4 & 198.9 & 6.2 \\
\hline 0.05532 & 0.13955 & 0.00396 & 842.1 & 22.4 & 842 & 29.4 \\
\hline 0.02826 & 0.09905 & 0.00254 & 608.8 & 14.8 & 612.3 & 19.2 \\
\hline 0.0422 & 0.11678 & 0.00318 & 712 & 18.4 & 742.7 & 25 \\
\hline 0.03654 & 0.13855 & 0.0034 & 836.5 & 19.2 & 838.6 & 20.6 \\
\hline 0.02416 & 0.05774 & 0.00142 & 361.9 & 8.6 & 635.4 & 17 \\
\hline 0.04592 & 0.1121 & 0.00326 & 684.9 & 18.8 & 684.7 & 28 \\
\hline 0.02244 & 0.09652 & 0.00234 & 594 & 13.8 & 601.9 & 16 \\
\hline 0.04592 & 0.16244 & 0.00402 & 970.3 & 22.2 & 969.8 & 22.8 \\
\hline 0.02246 & 0.07329 & 0.00192 & 455.9 & 11.6 & 488.9 & 17.2 \\
\hline 0.0666 & 0.14076 & 0.0042 & 849 & 23.8 & 906.4 & 33.2 \\
\hline 0.01146 & 0.04446 & 0.00114 & 280.4 & 7 & 287.8 & 10.6 \\
\hline 0.05818 & 0.14315 & 0.00382 & 862.5 & 21.6 & 1003.9 & 27.4 \\
\hline 0.04174 & 0.13553 & 0.00352 & 819.3 & 20 & 809.5 & 23.4 \\
\hline 0.01806 & 0.08799 & 0.0021 & 543.6 & 12.4 & 551.9 & 13.8 \\
\hline 0.03774 & 0.11981 & 0.00316 & 729.5 & 18.2 & 722.1 & 23 \\
\hline 0.02958 & 0.11908 & 0.0029 & 725.3 & 16.8 & 734.7 & 18.6 \\
\hline 0.02726 & 0.09775 & 0.0025 & 601.2 & 14.6 & 608.8 & 18.6 \\
\hline 0.03424 & 0.09375 & 0.00262 & 577.7 & 15.4 & 580.5 & 23.2 \\
\hline 0.0329 & 0.09599 & 0.00262 & 590.9 & 15.4 & 591.6 & 22.2 \\
\hline 0.0292 & 0.10158 & 0.00262 & 623.7 & 15.4 & 614.4 & 19.6 \\
\hline 0.03036 & 0.10006 & 0.00264 & 614.8 & 15.4 & 601.6 & 20.6 \\
\hline 0.03812 & 0.14066 & 0.00346 & 848.4 & 19.6 & 861.9 & 21 \\
\hline 0.18592 & 0.38259 & 0.00948 & 2088.4 & 44.2 & 2112.9 & 30.6 \\
\hline 0.02236 & 0.09525 & 0.00234 & 586.5 & 13.8 & 584.6 & 16.2 \\
\hline 0.03686 & 0.13331 & 0.00334 & 806.7 & 19 & 805.3 & 21.2 \\
\hline 0.2071 & 0.32385 & 0.01004 & 1808.5 & 48.8 & 1852.4 & 42.4 \\
\hline 0.13822 & 0.32341 & 0.00796 & 1806.4 & 38.8 & 1856.3 & 29.2 \\
\hline 0.03402 & 0.10195 & 0.00276 & 625.8 & 16.2 & 621.9 & 22.4 \\
\hline 0.02546 & 0.09303 & 0.00238 & 573.4 & 14 & 572.3 & 18.2 \\
\hline 0.00988 & 0.00499 & 0.00034 & 32.1 & 2.2 & 35.6 & 10.2 \\
\hline 0.18218 & 0.34847 & 0.00836 & 1927.3 & 40 & 2150.3 & 29.2 \\
\hline 0.02298 & 0.08851 & 0.00222 & 546.7 & 13.2 & 550.9 & 16.8 \\
\hline 0.06752 & 0.18042 & 0.00488 & 1069.3 & 26.6 & 1058 & 29.6 \\
\hline 0.0232 & 0.00479 & 0.00066 & 30.8 & 4.2 & 21.7 & 23.4 \\
\hline 0.02778 & 0.10509 & 0.00264 & 644.2 & 15.4 & 639.4 & 18.6 \\
\hline 0.14606 & 0.33444 & 0.00844 & 1859.9 & 40.8 & 1857.7 & 30.6 \\
\hline 0.02138 & 0.07544 & 0.00184 & 468.8 & 11 & 589.6 & 15.6 \\
\hline 0.03682 & 0.09334 & 0.0027 & 575.3 & 16 & 579.6 & 25 \\
\hline 0.16156 & 0.35357 & 0.0089 & 1951.6 & 42.4 & 1955 & 30.8 \\
\hline 0.04096 & 0.11118 & 0.0031 & 679.6 & 18 & 683.8 & 25.2 \\
\hline 0.05208 & 0.13537 & 0.0038 & 818.4 & 21.6 & 824.3 & 28.2 \\
\hline 0.0059 & 0.00304 & 0.00008 & 19.6 & 0.6 & 216.8 & 6.8 \\
\hline
\end{tabular}




\begin{tabular}{|c|c|c|c|c|c|c|}
\hline 0.048 & 0.11711 & 0.00312 & 713.9 & 18 & 879.6 & 25.6 \\
\hline 0.0367 & 0.11683 & 0.00304 & 712.3 & 17.6 & 738.8 & 22.2 \\
\hline 0.03124 & 0.10944 & 0.0028 & 669.5 & 16.2 & 681.6 & 20 \\
\hline 0.02774 & 0.0979 & 0.00252 & 602.1 & 14.8 & 600.5 & 19.2 \\
\hline 0.04444 & 0.13515 & 0.00358 & 817.2 & 20.4 & 808 & 24.8 \\
\hline 0.03868 & 0.11725 & 0.00314 & 714.7 & 18.2 & 713.7 & 23.6 \\
\hline 0.02384 & 0.10208 & 0.0025 & 626.6 & 14.6 & 622.6 & 16.6 \\
\hline 0.03106 & 0.12711 & 0.0031 & 771.4 & 17.8 & 765.3 & 18.8 \\
\hline 0.0313 & 0.11458 & 0.0029 & 699.3 & 16.8 & 694.4 & 19.8 \\
\hline 0.30776 & 0.13271 & 0.00646 & & & & \\
\hline 0.02656 & 0.10109 & 0.00254 & 620.8 & 14.8 & 621.2 & 18.2 \\
\hline 0.0137 & 0.04796 & 0.00116 & 302 & 7.2 & 423.8 & 11.8 \\
\hline 0.06486 & 0.14781 & 0.00388 & 888.7 & 21.8 & 1121.7 & 27.6 \\
\hline 0.03604 & 0.14268 & 0.00348 & 859.8 & 19.6 & 860 & 20 \\
\hline 0.04536 & 0.10868 & 0.0032 & 665.1 & 18.6 & 670.5 & 28 \\
\hline 0.1548 & 0.34923 & 0.00852 & 1930.9 & 40.8 & 1971.2 & 29.2 \\
\hline 0.03432 & 0.09185 & 0.0026 & 566.5 & 15.4 & 577.3 & 23.4 \\
\hline 0.13986 & 0.3406 & 0.00838 & 1889.6 & 40.2 & 1866.9 & 29.2 \\
\hline 0.03428 & 0.12153 & 0.00304 & 739.4 & 17.4 & 768.7 & 20.4 \\
\hline 0.03244 & 0.09464 & 0.0026 & 582.9 & 15.4 & 579.3 & 22.2 \\
\hline 0.0343 & 0.08841 & 0.00252 & 546.1 & 15 & 554.1 & 23.8 \\
\hline 0.04174 & 0.14304 & 0.00362 & 861.8 & 20.4 & 867.4 & 22.6 \\
\hline 0.03872 & 0.12359 & 0.00322 & 751.2 & 18.4 & 760.1 & 22.8 \\
\hline 0.04594 & 0.12906 & 0.00348 & 782.5 & 19.8 & 816.8 & 25.6 \\
\hline 0.02308 & 0.09973 & 0.00244 & 612.8 & 14.4 & 612.7 & 16.2 \\
\hline 0.0419 & 0.1479 & 0.0037 & 889.2 & 20.8 & 890.6 & 22.2 \\
\hline 0.02482 & 0.10264 & 0.00254 & 629.9 & 14.8 & 626.1 & 17.2 \\
\hline 0.03768 & 0.097 & 0.00278 & 596.8 & 16.4 & 603.3 & 25 \\
\hline 0.0218 & 0.08261 & 0.00204 & 511.7 & 12.2 & 573.5 & 16 \\
\hline 0.01622 & 0.01816 & 0.00044 & 116 & 2.8 & 503.1 & 13.2 \\
\hline 0.03956 & 0.10182 & 0.00292 & 625.1 & 17 & 611.4 & 25.8 \\
\hline 0.02776 & 0.08027 & 0.00224 & 497.7 & 13.4 & 494.7 & 20.6 \\
\hline 0.03474 & 0.08454 & 0.00248 & 523.2 & 14.8 & 527.6 & 24.4 \\
\hline 0.02594 & 0.00491 & 0.00062 & 31.6 & 4 & -1.5 & -26.4 \\
\hline 0.05534 & 0.12965 & 0.00378 & 785.9 & 21.6 & 808.2 & 30.2 \\
\hline 0.02806 & 0.10524 & 0.00266 & 645 & 15.6 & 644.9 & 18.8 \\
\hline 0.03016 & 0.09854 & 0.00258 & 605.8 & 15.2 & 626.2 & 20.2 \\
\hline 0.03486 & 0.06461 & 0.00158 & 403.6 & 9.6 & 860.9 & 19.8 \\
\hline 0.03564 & 0.12435 & 0.00316 & 755.6 & 18.2 & 762.2 & 21.2 \\
\hline 0.02082 & 0.07711 & 0.00186 & 478.8 & 11.2 & 593.1 & 15.2 \\
\hline 0.02708 & 0.11376 & 0.00278 & 694.5 & 16 & 695.1 & 17.6 \\
\hline 0.07282 & 0.10284 & 0.00254 & 631 & 14.8 & 1344.2 & 25.8 \\
\hline 0.01052 & 0.03167 & 0.00078 & 201 & 4.8 & 323.8 & 10 \\
\hline 0.1982 & 0.35386 & 0.0096 & 1953 & 45.8 & 2018.5 & 35.2 \\
\hline 0.02408 & 0.09924 & 0.00246 & 610 & 14.4 & 611.9 & 16.8 \\
\hline 0.08996 & 0.12396 & 0.00482 & 753.3 & 27.6 & 768.9 & 49 \\
\hline 0.03508 & 0.11484 & 0.00296 & 700.8 & 17.2 & 730.5 & 21.4 \\
\hline 0.03448 & 0.12448 & 0.00314 & 756.3 & 18 & 764.3 & 20.6 \\
\hline 0.00564 & 0.00478 & 0.00012 & 30.7 & 0.8 & 194.2 & 6.4 \\
\hline 886.84876 & 6.84662 & 17.0622 & & & & \\
\hline 0.02144 & 0.08986 & 0.00218 & 554.7 & 12.8 & 598 & 15.4 \\
\hline 0.02274 & 0.09531 & 0.00234 & 586.9 & 13.8 & 602.3 & 16.2 \\
\hline 0.05762 & 0.13997 & 0.00412 & 844.5 & 23.2 & 809.2 & 31.2 \\
\hline 0.0224 & 0.10046 & 0.00244 & 617.1 & 14.2 & 613.8 & 15.8 \\
\hline 0.03188 & 0.09844 & 0.00264 & 605.3 & 15.4 & 617.4 & 21.2 \\
\hline
\end{tabular}




\begin{tabular}{|c|c|c|c|c|c|c|}
\hline 0.03356 & 0.0961 & 0.00268 & 591.5 & 15.8 & 576.2 & 22.8 \\
\hline 0.03612 & 0.12937 & 0.00328 & 784.3 & 18.8 & 781.3 & 21.2 \\
\hline 0.03528 & 0.13343 & 0.00334 & 807.4 & 19 & 790.6 & 20.6 \\
\hline 0.06392 & 0.09567 & 0.00352 & 589 & 20.8 & 619 & 40.2 \\
\hline 0.05842 & 0.13047 & 0.00398 & 790.5 & 22.6 & 769.4 & 32.6 \\
\hline 0.04842 & 0.11877 & 0.00342 & 723.5 & 19.8 & 737.7 & 28.2 \\
\hline 0.01172 & 0.03052 & 0.00076 & 193.8 & 4.8 & 362 & 10.8 \\
\hline 0.02714 & 0.11036 & 0.00274 & 674.8 & 16 & 670.5 & 18 \\
\hline 0.05662 & 0.13772 & 0.00392 & 831.8 & 22.2 & 862 & 29.4 \\
\hline 0.04008 & 0.09607 & 0.00288 & 591.3 & 17 & 581 & 26.8 \\
\hline 0.03298 & 0.09427 & 0.00266 & 580.7 & 15.6 & 552.4 & 22.8 \\
\hline 0.03644 & 0.11001 & 0.003 & 672.8 & 17.4 & 649.7 & 23.4 \\
\hline 0.16786 & 0.37206 & 0.00908 & 2039.1 & 42.6 & 2040.2 & 29.6 \\
\hline 0.06502 & 0.17193 & 0.00466 & 1022.7 & 25.6 & 1043.5 & 29 \\
\hline 0.02084 & 0.09046 & 0.00222 & 558.3 & 13.2 & 556.9 & 15.4 \\
\hline 0.02388 & 0.09945 & 0.00246 & 611.2 & 14.4 & 611.6 & 16.6 \\
\hline 0.04856 & 0.14542 & 0.00386 & 875.2 & 21.8 & 876.8 & 25.4 \\
\hline 0.03786 & 0.09275 & 0.00276 & 571.8 & 16.2 & 551.7 & 26 \\
\hline 0.0411 & 0.13531 & 0.00348 & 818.1 & 19.8 & 827.6 & 22.8 \\
\hline 0.04976 & 0.13575 & 0.00376 & 820.6 & 21.4 & 816.8 & 27.2 \\
\hline 0.07214 & 0.0959 & 0.00388 & 590.3 & 22.8 & 573.7 & 46.4 \\
\hline 0.05562 & 0.13446 & 0.00398 & 813.3 & 22.6 & 787.6 & 30.8 \\
\hline 0.0841 & 0.20184 & 0.00512 & 1185.2 & 27.4 & 1384.6 & 27.8 \\
\hline 0.03496 & 0.13063 & 0.00326 & 791.5 & 18.6 & 792.2 & 20.4 \\
\hline 0.00624 & 0.02778 & 0.0007 & 176.6 & 4.4 & 175.9 & 6.6 \\
\hline 0.02848 & 0.11137 & 0.00274 & 680.7 & 15.8 & 715.8 & 18.2 \\
\hline 0.07378 & 0.18634 & 0.0046 & 1101.5 & 25 & 1330.6 & 25.8 \\
\hline 0.06548 & 0.16331 & 0.00454 & 975.1 & 25.2 & 1000.6 & 30.2 \\
\hline 0.03914 & 0.09692 & 0.00284 & 596.3 & 16.6 & 603.5 & 25.8 \\
\hline 0.03312 & 0.10382 & 0.00254 & 636.8 & 14.8 & 810.4 & 19.4 \\
\hline 0.02348 & 0.08596 & 0.00222 & 531.6 & 13.2 & 529.6 & 17.2 \\
\hline 0.03828 & 0.10539 & 0.00296 & 645.9 & 17.2 & 635.2 & 24.6 \\
\hline 0.05964 & 0.114 & 0.0037 & 695.9 & 21.4 & 697.8 & 35.2 \\
\hline 0.03378 & 0.0966 & 0.00268 & 594.5 & 15.8 & 587.7 & 22.8 \\
\hline 0.05216 & 0.13397 & 0.00382 & 810.5 & 21.8 & 796.7 & 28.8 \\
\hline 0.02732 & 0.00424 & 0.0006 & 27.3 & 3.8 & 26.5 & 27.6 \\
\hline 541.8678 & 5.13813 & 8.63322 & & & & \\
\hline 0.95312 & 0.32037 & 0.01342 & & & & \\
\hline 0.02064 & 0.0851 & 0.0021 & 526.5 & 12.4 & 562.4 & 15.2 \\
\hline 0.04258 & 0.04089 & 0.001 & 258.3 & 6.2 & 985.4 & 21.2 \\
\hline 0.04406 & 0.14924 & 0.0037 & 896.7 & 20.8 & 959.8 & 22.2 \\
\hline 0.06024 & 0.15262 & 0.00434 & 915.6 & 24.2 & 902.6 & 30.2 \\
\hline 0.02506 & 0.1023 & 0.00256 & 627.9 & 15 & 606 & 17.4 \\
\hline 0.03004 & 0.11041 & 0.00272 & 675.1 & 15.8 & 739.5 & 18.6 \\
\hline 0.0196 & 0.07072 & 0.00178 & 440.5 & 10.8 & 501.1 & 15.2 \\
\hline
\end{tabular}




\begin{tabular}{|c|c|c|c|c|c|}
\hline age $207 / 206$ & $2 \sigma$ age 76 & $\begin{array}{l}\text { discordance } \\
\Delta 68-75[\%]\end{array}$ & $\Delta 68-76[\%]$ & $\begin{array}{c}\text { preferred age } \\
\text { age }\end{array}$ & $2 \sigma$ age \\
\hline 691.9 & 60.8 & 2.3 & 9.7 & 759.1 & 17.8 \\
\hline 1020 & 59.8 & 0.3 & 1 & 1029.9 & 24.2 \\
\hline 755.1 & 93.8 & 0.1 & 0.2 & 756.8 & 20.2 \\
\hline 649.1 & 89 & -1.7 & -7.8 & 598.6 & 15.8 \\
\hline 688.9 & 88.8 & 3.9 & 17 & 806.2 & 21 \\
\hline 831.5 & 49.4 & 0.2 & 0.6 & 836.1 & 18.8 \\
\hline 859.1 & 58.2 & -0.5 & -1.7 & 844.2 & 19.6 \\
\hline 1655.2 & 44 & -50.3 & -94 & & \\
\hline 842.1 & 89.2 & 0 & 0 & 842.1 & 22.4 \\
\hline 625.4 & 71.2 & -0.6 & -2.7 & 608.8 & 14.8 \\
\hline 836.8 & 80.8 & -4.1 & -14.9 & 712 & 18.4 \\
\hline 844.9 & 55 & -0.3 & -1 & 836.5 & 19.2 \\
\hline 1787.3 & 47.4 & -43 & -79.8 & & \\
\hline 684.4 & 102.8 & 0 & 0.1 & 684.9 & 18.8 \\
\hline 632.5 & 55.2 & -1.3 & -6.1 & 594 & 13.8 \\
\hline 968.9 & 54.8 & 0.1 & 0.1 & 970.3 & 22.2 \\
\hline 647.3 & 76.4 & -6.7 & -29.6 & 455.9 & 11.6 \\
\hline 1049.6 & 93.8 & -6.3 & -19.1 & 849 & 23.8 \\
\hline 349.2 & 77 & -2.6 & -19.7 & 280.4 & 7 \\
\hline 1327.6 & 65.2 & -14.1 & -35 & 862.5 & 21.6 \\
\hline 783.1 & 69.6 & 1.2 & 4.6 & 819.3 & 20 \\
\hline 586.5 & 48.2 & -1.5 & -7.3 & 543.6 & 12.4 \\
\hline 699.8 & 76 & 1 & 4.2 & 729.5 & 18.2 \\
\hline 764.3 & 54 & -1.3 & -5.1 & 725.3 & 16.8 \\
\hline 637.5 & 68.4 & -1.2 & -5.7 & 601.2 & 14.6 \\
\hline 592 & 96.6 & -0.5 & -2.4 & 577.7 & 15.4 \\
\hline 595.3 & 90 & -0.1 & -0.7 & 590.9 & 15.4 \\
\hline 580.7 & 74 & 1.5 & 7.4 & 623.7 & 15.4 \\
\hline 553 & 80.4 & 2.2 & 11.2 & 614.8 & 15.4 \\
\hline 897.2 & 54.4 & -1.6 & -5.4 & 848.4 & 19.6 \\
\hline 2137.3 & 41.8 & -1.2 & -2.3 & 2137.3 & 41.8 \\
\hline 577.8 & 58 & 0.3 & 1.5 & 586.5 & 13.8 \\
\hline 801.9 & 60.4 & 0.2 & 0.6 & 806.7 & 19 \\
\hline 1902.6 & 70 & -2.4 & -4.9 & 1902.6 & 70 \\
\hline 1913.2 & 42.8 & -2.7 & -5.6 & 1913.2 & 42.8 \\
\hline 608.3 & 86.2 & 0.6 & 2.9 & 625.8 & 16.2 \\
\hline 568.6 & 70.8 & 0.2 & 0.9 & 573.4 & 14 \\
\hline 285 & 648.4 & -10 & -88.7 & 32.1 & 2.2 \\
\hline 2371.2 & 37.6 & -10.4 & -18.7 & & \\
\hline 568.6 & 66.4 & -0.8 & -3.8 & 546.7 & 13.2 \\
\hline 1035.5 & 72.8 & 1.1 & 3.3 & 1069.3 & 26.6 \\
\hline-893.7 & 3131.6 & 41.8 & -103.4 & & \\
\hline 623.3 & 64.8 & 0.7 & 3.4 & 644.2 & 15.4 \\
\hline 1855.9 & 46.2 & 0.1 & 0.2 & 1855.9 & 46.2 \\
\hline 1086.9 & 49.8 & -20.5 & -56.9 & & \\
\hline 597.4 & 105 & -0.7 & -3.7 & 575.3 & 16 \\
\hline 1959.2 & 44.8 & -0.2 & -0.4 & 1959.2 & 44.8 \\
\hline 698.4 & 90.4 & -0.6 & -2.7 & 679.6 & 18 \\
\hline 840.6 & 86.2 & -0.7 & -2.6 & 818.4 & 21.6 \\
\hline 4427 & 32.4 & -91 & -99.6 & & \\
\hline
\end{tabular}




\begin{tabular}{|c|c|c|c|c|c|}
\hline 1323.7 & 66.2 & -18.8 & -46.1 & & \\
\hline 820.9 & 69.2 & -3.6 & -13.2 & 712.3 & 17.6 \\
\hline 722.7 & 66.2 & -1.8 & -7.4 & 669.5 & 16.2 \\
\hline 595.3 & 72.6 & 0.3 & 1.1 & 602.1 & 14.8 \\
\hline 783.4 & 75.4 & 1.1 & 4.3 & 817.2 & 20.4 \\
\hline 711.3 & 79.6 & 0.1 & 0.5 & 714.7 & 18.2 \\
\hline 608.6 & 56 & 0.6 & 3 & 626.6 & 14.6 \\
\hline 748.2 & 53.4 & 0.8 & 3.1 & 771.4 & 17.8 \\
\hline 679.3 & 64.6 & 0.7 & 2.9 & 699.3 & 16.8 \\
\hline 623.3 & 64.8 & -0.1 & -0.4 & 620.8 & 14.8 \\
\hline 1155.8 & 47.6 & -28.7 & -73.9 & & \\
\hline 1606.5 & 57.6 & -20.8 & -44.7 & & \\
\hline 861.2 & 50.8 & 0 & -0.2 & 859.8 & 19.6 \\
\hline 689.6 & 104.6 & -0.8 & -3.5 & 665.1 & 18.6 \\
\hline 2014.3 & 41.2 & -2 & -4.1 & 2014.3 & 41.2 \\
\hline 620.8 & 97 & -1.9 & -8.8 & 566.5 & 15.4 \\
\hline 1842.4 & 43 & 1.2 & 2.6 & 1842.4 & 43 \\
\hline 855.4 & 59 & -3.8 & -13.6 & 739.4 & 17.4 \\
\hline 566 & 91.6 & 0.6 & 3 & 582.9 & 15.4 \\
\hline 587.3 & 103.4 & -1.4 & -7 & 546.1 & 15 \\
\hline 882.2 & 60.4 & -0.6 & -2.3 & 861.8 & 20.4 \\
\hline 787.2 & 70.6 & -1.2 & -4.6 & 751.2 & 18.4 \\
\hline 912.1 & 75.2 & -4.2 & -14.2 & 782.5 & 19.8 \\
\hline 612.9 & 55.2 & 0 & 0 & 612.8 & 14.4 \\
\hline 894.8 & 57.6 & -0.2 & -0.6 & 889.2 & 20.8 \\
\hline 613.6 & 58.8 & 0.6 & 2.6 & 629.9 & 14.8 \\
\hline 628.3 & 100.8 & -1.1 & -5 & 596.8 & 16.4 \\
\hline 827.5 & 55.6 & -10.8 & -38.2 & 511.7 & 12.2 \\
\hline 3223.2 & 35.8 & -76.9 & -96.4 & & \\
\hline 561.9 & 105.2 & 2.2 & 11.2 & 625.1 & 17 \\
\hline 481.4 & 98.2 & 0.6 & 3.4 & 497.7 & 13.4 \\
\hline 547.8 & 112.8 & $\begin{array}{c}-0.8 \\
-2228.3\end{array}$ & -4.5 & 523.2 & 14.8 \\
\hline 871 & 95 & -2.8 & -9.8 & 785.9 & 21.6 \\
\hline 644.9 & 64.6 & 0 & 0 & 645 & 15.6 \\
\hline 701.1 & 73.2 & -3.3 & -13.6 & 605.8 & 15.2 \\
\hline 2344.3 & 40.4 & -53.1 & -82.8 & & \\
\hline 782.4 & 63.8 & -0.9 & -3.4 & 755.6 & 18.2 \\
\hline 1059.1 & 47.4 & -19.3 & -54.8 & & \\
\hline 697.4 & 53.8 & -0.1 & -0.4 & 694.5 & 16 \\
\hline 2781.1 & 39.6 & -53.1 & -77.3 & & \\
\hline 1339.3 & 50.4 & -37.9 & -85 & & \\
\hline 2086.8 & 52 & -3.2 & -6.4 & 2086.8 & 52 \\
\hline 619.7 & 58.6 & -0.3 & -1.6 & 610 & 14.4 \\
\hline 815.2 & 171.6 & -2 & -7.6 & 753.3 & 27.6 \\
\hline 823.4 & 66.6 & -4.1 & -14.9 & 700.8 & 17.2 \\
\hline 788.5 & 61 & -1.1 & -4.1 & 756.3 & 18 \\
\hline 3568.4 & 39 & -84.2 & -99.1 & & \\
\hline 766.2 & 50.8 & -7.2 & -27.6 & 554.7 & 12.8 \\
\hline 661.6 & 55 & -2.6 & -11.3 & 586.9 & 13.8 \\
\hline 714 & 101 & 4.4 & 18.3 & 844.5 & 23.2 \\
\hline 602.5 & 52.6 & 0.5 & 2.4 & 617.1 & 14.2 \\
\hline 663 & 79.8 & -2 & -8.7 & 605.3 & 15.4 \\
\hline
\end{tabular}




\begin{tabular}{|c|c|c|c|c|c|}
\hline 517.2 & 96 & 2.7 & 14.4 & 591.5 & 15.8 \\
\hline 773.4 & 62.2 & 0.4 & 1.4 & 784.3 & 18.8 \\
\hline 744.3 & 59.4 & 2.1 & 8.5 & 807.4 & 19 \\
\hline 731.4 & 166.4 & -4.9 & -19.5 & 589 & 20.8 \\
\hline 708.9 & 110.6 & 2.8 & 11.5 & 790.5 & 22.6 \\
\hline 781.8 & 95.4 & -1.9 & -7.5 & 723.5 & 19.8 \\
\hline 1657.4 & 46.6 & -46.5 & -88.3 & & \\
\hline 656.4 & 58 & 0.7 & 2.8 & 674.8 & 16 \\
\hline 941.2 & 86.2 & -3.5 & -11.6 & 831.8 & 22.2 \\
\hline 541.8 & 114.8 & 1.8 & 9.1 & 591.3 & 17 \\
\hline 438.4 & 101.6 & 5.1 & 32.5 & & \\
\hline 571.1 & 87.6 & 3.6 & 17.8 & 672.8 & 17.4 \\
\hline 2042.1 & 40.8 & -0.1 & -0.1 & 2042.1 & 40.8 \\
\hline 1087.9 & 70.4 & -2 & -6 & 1022.7 & 25.6 \\
\hline 551.9 & 57.4 & 0.2 & 1.2 & 558.3 & 13.2 \\
\hline 613.6 & 57.4 & -0.1 & -0.4 & 611.2 & 14.4 \\
\hline 881.3 & 70.8 & -0.2 & -0.7 & 875.2 & 21.8 \\
\hline 470.5 & 117.6 & 3.6 & 21.5 & 571.8 & 16.2 \\
\hline 853.9 & 64.6 & -1.1 & -4.2 & 818.1 & 19.8 \\
\hline 807.3 & 83 & 0.5 & 1.6 & 820.6 & 21.4 \\
\hline 508.8 & 215 & 2.9 & 16 & 590.3 & 22.8 \\
\hline 716.3 & 101.4 & 3.3 & 13.5 & 813.3 & 22.6 \\
\hline 1707.5 & 49.2 & -14.4 & -30.6 & & \\
\hline 795.2 & 57.4 & -0.1 & -0.5 & 791.5 & 18.6 \\
\hline 167.8 & 73.8 & 0.4 & 5.3 & 176.6 & 4.4 \\
\hline 828.4 & 53.2 & -4.9 & -17.8 & 680.7 & 15.8 \\
\hline 1722 & 45.2 & -17.2 & -36 & & \\
\hline 1057.5 & 77.2 & -2.5 & -7.8 & 975.1 & 25.2 \\
\hline 631.1 & 105 & -1.2 & -5.5 & 596.3 & 16.6 \\
\hline 1323.5 & 47.6 & -21.4 & -51.9 & & \\
\hline 521.8 & 72.2 & 0.4 & 1.9 & 531.6 & 13.2 \\
\hline 598.2 & 94.8 & 1.7 & 8 & 645.9 & 17.2 \\
\hline 704.5 & 131.2 & -0.3 & -1.2 & 695.9 & 21.4 \\
\hline 562.3 & 93.2 & 1.2 & 5.7 & 594.5 & 15.8 \\
\hline 759.4 & 92.2 & 1.7 & 6.7 & 810.5 & 21.8 \\
\hline-40 & 2529.2 & 2.8 & -168.1 & 27.3 & 3.8 \\
\hline 711.3 & 54 & -6.4 & -26 & 526.5 & 12.4 \\
\hline 3422.2 & 36.2 & -73.8 & -92.5 & & \\
\hline 1107.9 & 51.2 & -6.6 & -19.1 & 896.7 & 20.8 \\
\hline 871.6 & 86.4 & 1.4 & 5.1 & 915.6 & 24.2 \\
\hline 525.6 & 62.8 & 3.6 & 19.5 & 627.9 & 15 \\
\hline 940.3 & 52.4 & -8.7 & -28.2 & 675.1 & 15.8 \\
\hline 789.5 & 60.2 & -12.1 & -44.2 & 440.5 & 10.8 \\
\hline
\end{tabular}


Sample 3709P Nile Delta beach placer @ Rosetta 150 grain analysed 122 c

\begin{tabular}{|c|c|c|c|c|c|c|}
\hline \multirow[b]{2}{*}{ grain } & \multicolumn{3}{|c|}{ concentrations } & \multicolumn{3}{|l|}{ isotopic ratios } \\
\hline & U [ppm] & $\mathrm{Pb}$ [ppm] & $\mathrm{Th} / \mathrm{U}$ & $\mathrm{Pb} 207 / \mathrm{Pb} 206$ & $2 \sigma 76$ & $\mathrm{~Pb} 207 / \mathrm{U} 235$ \\
\hline X3709P G001 & 2823 & 128.6 & 0.4072 & 0.06959 & 0.00166 & 0.42916 \\
\hline X3709P_G002 & 133.1 & 21.9 & 0.5161 & 0.07381 & 0.00228 & 1.54755 \\
\hline X3709P_G003 & 113.3 & 14.9 & 0.5024 & 0.06596 & 0.00242 & 1.11956 \\
\hline X3709P_G004 & 259.9 & 99.1 & 0.694 & 0.15942 & 0.00368 & 7.45365 \\
\hline X3709P_G005 & 2398.9 & 187.2 & 0.6334 & 0.06127 & 0.00142 & 0.60388 \\
\hline X3709P G006 & 500.6 & 155.8 & 0.4309 & 0.11867 & 0.00274 & 4.88366 \\
\hline X3709P G007 & 94.9 & 17 & 0.4445 & 0.07219 & 0.00252 & 1.68465 \\
\hline X3709P_G008 & 121.8 & 14.6 & 1.3753 & 0.05875 & 0.00248 & 0.72709 \\
\hline X3709P_G009 & 546.6 & 96 & 0.2293 & 0.07335 & 0.00178 & 1.78228 \\
\hline X3709P_G010 & 140.9 & 17.1 & 1.224 & 0.05764 & 0.00236 & 0.75312 \\
\hline X3709P_G011 & 47.4 & 6.2 & 0.4538 & 0.06321 & 0.00344 & 1.08352 \\
\hline X3709P_G012 & 99.1 & 11 & 0.7217 & 0.06137 & 0.00276 & 0.82751 \\
\hline X3709P_G013 & 266.9 & 81.4 & 0.2083 & 0.13928 & 0.0033 & 5.85835 \\
\hline X3709P_G014 & 77.2 & 6.3 & 0.2399 & 0.05931 & 0.00312 & 0.67709 \\
\hline X3709P_G015 & 102 & 58.5 & 1.2227 & 0.16529 & 0.00408 & 10.0217 \\
\hline X3709P_G016 & 58.8 & 6.4 & 1.2335 & 0.05829 & 0.00358 & 0.68751 \\
\hline X3709P_G017 & 275.4 & 44.6 & 0.5055 & 0.0696 & 0.00196 & 1.44656 \\
\hline X3709P_G018 & 87.1 & 11.4 & 0.78 & 0.06358 & 0.00286 & 0.99679 \\
\hline X3709P G019 & 150.8 & 20.3 & 0.5352 & 0.06376 & 0.00224 & 1.09858 \\
\hline X3709P_G020 & 237.9 & 25.8 & 0.7472 & 0.05603 & 0.00196 & 0.73481 \\
\hline X3709P_G021 & 44.6 & 5.1 & 0.5571 & 0.06109 & 0.00392 & 0.88214 \\
\hline X3709P_G022 & 328.5 & 56.2 & 0.4838 & 0.06903 & 0.00186 & 1.52395 \\
\hline X3709P_G023 & 160 & 17.3 & 0.4639 & 0.05804 & 0.00222 & 0.81978 \\
\hline X3709P_G024 & 372.4 & 47.9 & 0.6425 & 0.08705 & 0.0038 & 1.39678 \\
\hline X3709P_G025 & 46 & 6.5 & 1.0704 & -0.23053 & 0.01146 & -3.2368 \\
\hline X3709P_G026 & 1084 & 72.9 & 1.099 & 0.07395 & 0.00202 & 0.58584 \\
\hline X3709P_G027 & 163.6 & 21.2 & 0.2887 & 0.06846 & 0.00234 & 1.20348 \\
\hline X3709P_G028 & 67.3 & 10.2 & 0.7976 & 0.06335 & 0.00308 & 1.14427 \\
\hline X3709P_G029 & 148 & 13.8 & 0.6753 & 0.09002 & 0.00334 & 1.04725 \\
\hline X3709P_G030 & 86.4 & 9.2 & 0.6704 & 0.05849 & 0.00292 & 0.76494 \\
\hline X3709P G031 & 179.8 & 16.3 & 0.5024 & 0.05789 & 0.00222 & 0.6768 \\
\hline X3709P_G032 & 81.4 & 18.7 & 1.3703 & 0.07098 & 0.0027 & 1.69301 \\
\hline X3709P_G033 & 273.3 & 25.5 & 0.1028 & 0.06043 & 0.0019 & 0.81918 \\
\hline X3709P_G034 & 785.9 & 15.9 & 0.5235 & 0.04664 & 0.00194 & 0.12191 \\
\hline X3709P_G035 & 265.5 & 26 & 0.2059 & 0.05843 & 0.00188 & 0.80337 \\
\hline X3709P_G036 & 445.4 & -42.5 & 0.6273 & 0.04776 & 0.00538 & 0.03041 \\
\hline X3709P_G037 & 34.7 & 3.4 & 0.3333 & 0.06009 & 0.0045 & 0.80169 \\
\hline X3709P_G038 & 119.7 & 13.8 & 1.0886 & 0.0585 & 0.00244 & 0.75203 \\
\hline X3709P_G039 & 332.8 & 33.6 & 0.2507 & 0.05809 & 0.00172 & 0.81518 \\
\hline X3709P_G040 & 14.2 & 6.2 & 1.5633 & 0.1102 & 0.00544 & 4.72752 \\
\hline X3709P_G041 & 131 & 16.9 & 0.4241 & 0.06458 & 0.0023 & 1.10132 \\
\hline X3709P_G042 & 88.5 & 10 & 0.9948 & 0.06331 & 0.00292 & 0.80654 \\
\hline X3709P_G043 & 148 & 107 & 2.1035 & 0.16904 & 0.004 & 10.64897 \\
\hline X3709P G044 & 177 & 16.2 & 0.3658 & 0.06012 & 0.00216 & 0.73969 \\
\hline X3709P_G045 & 613.2 & 75.8 & 0.3906 & 0.06578 & 0.00168 & 1.07839 \\
\hline X3709P_G046 & 436.9 & 38.9 & 0.0967 & 0.05845 & 0.00164 & 0.75877 \\
\hline X3709P_G047 & 52.4 & 5.6 & 0.546 & 0.06016 & 0.0035 & 0.8212 \\
\hline X3709P_G048 & 486.4 & 60.5 & 0.1064 & 0.06355 & 0.00174 & 1.14204 \\
\hline X3709P_G049 & 46.7 & 6.4 & 0.5115 & 0.06381 & 0.00354 & 1.11978 \\
\hline X3709P G050 & 571.4 & 58.5 & 0.2302 & 0.06339 & 0.00166 & 0.90361 \\
\hline
\end{tabular}




\begin{tabular}{|c|c|c|c|c|c|c|}
\hline X3709P_G051 & 73.6 & 7.9 & 0.3107 & 0.07065 & 0.00318 & 1.02681 \\
\hline X3709P_G052 & 80.7 & 8.7 & 1.0907 & 0.05832 & 0.00292 & 0.69688 \\
\hline X3709P_G053 & 252.1 & 26.3 & 0.2547 & 0.0628 & 0.002 & 0.9097 \\
\hline X3709P_G054 & & & & -0.20382 & 1.76162 & 24.7821 \\
\hline X3709P_G055 & 120.4 & 15.3 & 0.6413 & 0.06541 & 0.00254 & 1.02896 \\
\hline X3709P_G056 & 119.7 & 12.4 & 0.6295 & 0.05736 & 0.00252 & 0.74297 \\
\hline X3709P_G057 & 73.6 & 34.1 & 0.2835 & 0.21167 & 0.00542 & 13.13029 \\
\hline X3709P_G058 & 204.6 & 74.4 & 0.5006 & 0.17912 & 0.00428 & 8.45249 \\
\hline X3709P_G059 & 45.3 & 4.8 & 0.4063 & 0.05947 & 0.0038 & 0.83962 \\
\hline X3709P_G060 & 1147.8 & & 1.0947 & 0.53908 & 0.01218 & 9.16412 \\
\hline X3709P_G061 & 115.4 & 11.6 & 0.4339 & 0.05766 & 0.00252 & 0.76096 \\
\hline X3709P_G062 & 103.4 & 7.5 & 0.5308 & 0.05532 & 0.00308 & 0.51419 \\
\hline X3709P_G063 & 51 & 4.3 & 0.6675 & 0.05256 & 0.00404 & 0.54417 \\
\hline X3709P_G064 & 114 & 16.4 & 0.4777 & 0.06553 & 0.00246 & 1.22402 \\
\hline X3709P_G065 & 259.1 & 40.2 & 0.4218 & 0.06836 & 0.00202 & 1.39506 \\
\hline X3709P_G066 & 237.9 & 38.9 & 0.1332 & 0.07191 & 0.0021 & 1.67406 \\
\hline X3709P_G067 & 73.6 & 38.9 & 0.9108 & 0.16386 & 0.00436 & 9.75353 \\
\hline X3709P_G068 & 207.5 & 39.4 & 0.6553 & 0.07194 & 0.00216 & 1.68561 \\
\hline X3709P_G069 & 461.7 & 225 & 0.7063 & 0.16991 & 0.00388 & 9.89974 \\
\hline X3709P_G070 & 43.9 & 5.5 & 0.634 & 0.06521 & 0.00408 & 1.02682 \\
\hline X3709P_G071 & 44.6 & 5.5 & 0.5662 & 0.06352 & 0.00408 & 0.99726 \\
\hline X3709P_G072 & 36.1 & 2.9 & 0.2241 & 0.05927 & 0.00496 & 0.67391 \\
\hline X3709P_G073 & 492.1 & 158 & 0.0155 & 0.14597 & 0.00338 & 6.77911 \\
\hline X3709P_G074 & 157.2 & & 0.4419 & 0.71417 & 0.01658 & 59.45008 \\
\hline X3709P_G075 & 271.9 & 24.9 & 0.1375 & 0.05998 & 0.00204 & 0.79018 \\
\hline X3709P_G076 & 262.7 & 117.8 & 0.2522 & 0.18114 & 0.00426 & 10.7745 \\
\hline X3709P_G077 & 121.1 & 15.6 & 0.7072 & 0.07243 & 0.00284 & 1.13637 \\
\hline X3709P_G078 & & & & 0.52975 & 1.1656 & -53.41385 \\
\hline X3709P_G079 & 355.4 & 37.5 & 0.4679 & 0.05935 & 0.0018 & 0.81612 \\
\hline X3709P_G080 & 231.5 & 25.4 & 0.7939 & 0.06823 & 0.0023 & 0.89878 \\
\hline X3709P_G081 & 53.8 & 6.9 & 0.3362 & 0.06382 & 0.0034 & 1.10861 \\
\hline X3709P_G082 & 310.1 & 28.8 & 0.1675 & 0.06088 & 0.00188 & 0.80673 \\
\hline X3709P_G083 & 131.7 & 24.1 & 0.3786 & 0.07386 & 0.00232 & 1.7812 \\
\hline X3709P_G084 & 64.4 & 9.4 & 0.447 & 0.06346 & 0.00292 & 1.20217 \\
\hline X3709P_G085 & 104.8 & 10.5 & 0.4455 & 0.05817 & 0.0026 & 0.76765 \\
\hline X3709P_G086 & 77.2 & 15.3 & 0.6977 & 0.07029 & 0.00266 & 1.6984 \\
\hline X3709P_G087 & 153.6 & 20 & 0.4006 & 0.06508 & 0.00224 & 1.11822 \\
\hline X3709P_G088 & 70.8 & 9.2 & 1.4627 & 0.06137 & 0.0034 & 0.81338 \\
\hline X3709P_G089 & 110.5 & 10.4 & 0.8448 & 0.05737 & 0.00274 & 0.63476 \\
\hline X3709P_G090 & 289.6 & 26.3 & 0.0282 & 0.05918 & 0.00196 & 0.79848 \\
\hline X3709P_G091 & 190.5 & 19 & 0.6002 & 0.05875 & 0.00226 & 0.73319 \\
\hline X3709P_G092 & 57.4 & 5.8 & 0.2337 & 0.058 & 0.00346 & 0.81604 \\
\hline X3709P_G093 & 36.1 & 20.3 & 0.9004 & 0.16638 & 0.00506 & 10.43345 \\
\hline X3709P_G094 & 51.7 & 6.2 & 0.1802 & 0.05893 & 0.00342 & 0.99009 \\
\hline X3709P_G095 & 39.7 & 6.5 & 0.3994 & 0.06829 & 0.00374 & 1.46248 \\
\hline X3709P_G096 & 486.4 & 55.5 & 0.6306 & 0.06754 & 0.00192 & 0.95542 \\
\hline X3709P_G097 & 191.2 & 77.5 & 0.7182 & 0.16232 & 0.00402 & 7.99504 \\
\hline X3709P_G098 & 5607.8 & 77.1 & 0.6446 & 0.18476 & 0.00456 & 0.30319 \\
\hline X3709P_G099 & 116.1 & 11.1 & 0.3245 & 0.05708 & 0.00266 & 0.74321 \\
\hline X3709P_G100 & 43.9 & 6.3 & 1.0521 & 0.06668 & 0.004 & 1.07469 \\
\hline X3709P_G101 & 502 & 51.2 & 0.1344 & 0.06095 & 0.00174 & 0.89309 \\
\hline X3709P_G102 & 506.3 & 112.2 & 0.2526 & 0.09913 & 0.00242 & 2.97079 \\
\hline X3709P G103 & 38.9 & 18.9 & 1.826 & 0.109 & 0.00394 & 4.83484 \\
\hline X3709P_G104 & 191.2 & 26 & 0.3515 & 0.06639 & 0.0023 & 1.20607 \\
\hline X3709P_G105 & 286.1 & 38.5 & 0.4601 & 0.06231 & 0.00206 & 1.08826 \\
\hline
\end{tabular}




\begin{tabular}{|c|c|c|c|c|c|c|}
\hline X3709P_G106 & 195.4 & 24.2 & 0.3331 & 0.07009 & 0.00244 & 1.16796 \\
\hline X3709P_G107 & 134.5 & 23.7 & 0.7579 & 0.07123 & 0.00234 & 1.4926 \\
\hline X3709P_G108 & & & & -0.41889 & 3.748 & \\
\hline X3709P_G109 & 659.9 & 183.5 & 0.1153 & 0.10369 & 0.00246 & 4.06994 \\
\hline X3709P G110 & 99.8 & 10.2 & 0.4251 & 0.06555 & 0.00292 & 0.8748 \\
\hline X3709P_G111 & 169.2 & & 1.5489 & 0.31886 & 0.0088 & 5.78937 \\
\hline X3709P_G112 & 45.3 & 5 & 0.7885 & 0.06085 & 0.00404 & 0.80741 \\
\hline X3709P_G113 & 416.3 & 59 & 0.4725 & 0.06759 & 0.00188 & 1.24327 \\
\hline X3709P_G114 & 68.7 & 6.9 & 0.4289 & 0.06071 & 0.0035 & 0.80917 \\
\hline X3709P_G115 & 79.3 & 8.3 & 0.7344 & 0.06027 & 0.00332 & 0.76118 \\
\hline X3709P_G116 & 78.6 & 7.5 & 0.481 & 0.05627 & 0.00318 & 0.69359 \\
\hline X3709P_G117 & 383.8 & 158 & 0.8762 & 0.14781 & 0.00354 & 7.16346 \\
\hline X3709P_G118 & 87.8 & 10 & 0.7986 & 0.05333 & 0.0029 & 0.72451 \\
\hline X3709P_G119 & 41.1 & 4.9 & 1.168 & 0.05943 & 0.00448 & 0.76523 \\
\hline X3709P_G120 & 746.3 & 63.1 & 0.0693 & 0.05737 & 0.0016 & 0.71303 \\
\hline X3709P_G121 & 315.8 & 33.1 & 0.0549 & 0.06185 & 0.00192 & 0.95174 \\
\hline X3709P_G122 & 386.6 & 73.7 & 1.4474 & 0.11731 & 0.003 & 2.73061 \\
\hline X3709P_G123 & 183.4 & 67.3 & 0.6421 & 0.11988 & 0.00308 & 5.3338 \\
\hline X3709P_G124 & 230.8 & 21.9 & 0.4508 & 0.05978 & 0.0021 & 0.74328 \\
\hline X3709P_G125 & 160.7 & 15.1 & 0.2444 & 0.05953 & 0.00248 & 0.7734 \\
\hline X3709P_G126 & 278.3 & 40.6 & 0.3914 & 0.06782 & 0.00206 & 1.31155 \\
\hline X3709P_G127 & 266.2 & 25.6 & 0.4685 & 0.05725 & 0.00196 & 0.71515 \\
\hline X3709P_G128 & 199.7 & 30.9 & 0.6784 & 0.08434 & 0.00262 & 1.58678 \\
\hline X3709P_G129 & 66.6 & 5.9 & 0.3808 & 0.05937 & 0.0034 & 0.706 \\
\hline X3709P_G130 & 288.9 & 37.4 & 0.4647 & 0.06319 & 0.00194 & 1.06314 \\
\hline X3709P_G131 & 102 & 14 & 0.4869 & 0.29558 & 0.00884 & 4.9678 \\
\hline X3709P_G132 & 72.2 & 30.2 & 0.8726 & 0.11985 & 0.0036 & 5.70353 \\
\hline X3709P_G133 & 473.7 & 40.7 & 0.0262 & 0.05867 & 0.0018 & 0.75023 \\
\hline X3709P_G134 & 333.5 & 101.4 & 0.3421 & 0.12207 & 0.00306 & 4.92073 \\
\hline X3709P_G135 & 348.4 & 56.7 & 0.2794 & 0.0706 & 0.00202 & 1.57138 \\
\hline X3709P_G136 & 240 & 26.9 & 0.4697 & 0.06076 & 0.00208 & 0.87468 \\
\hline X3709P_G137 & 152.9 & 15.6 & 0.5848 & 0.05521 & 0.00246 & 0.70666 \\
\hline X3709P_G138 & 194 & 72.1 & 0.6827 & 0.11239 & 0.00298 & 4.9944 \\
\hline X3709P_G139 & 62.3 & 8.5 & 0.2539 & 0.06576 & 0.0033 & 1.23561 \\
\hline X3709P_G140 & 162.1 & 22.7 & 0.891 & 0.06167 & 0.0024 & 1.00801 \\
\hline X3709P_G141 & 400.1 & 52.4 & 0.5922 & 0.06375 & 0.00194 & 1.04913 \\
\hline X3709P_G142 & 321.5 & 1.6 & 0.6458 & 0.0482 & 0.0079 & 0.02928 \\
\hline X3709P_G143 & 186.9 & 18.1 & 0.2494 & 0.06168 & 0.0024 & 0.82457 \\
\hline X3709P_G144 & 728.6 & 69.4 & 0.0632 & 0.06155 & 0.00172 & 0.86861 \\
\hline X3709P_G145 & 271.9 & 31.9 & 0.5146 & 0.05938 & 0.00204 & 0.89381 \\
\hline X3709P_G146 & 429.8 & 45.3 & 0.2197 & 0.05953 & 0.00178 & 0.87798 \\
\hline X3709P_G147 & 2931.3 & 332.3 & 0.2332 & 0.06529 & 0.00158 & 0.93186 \\
\hline X3709P_G148 & 407.8 & 154.9 & 0.2565 & 0.15158 & 0.00368 & 7.71838 \\
\hline X3709P_G149 & 36.8 & 4.7 & 0.3477 & 0.06612 & 0.00426 & 1.12159 \\
\hline X3709P_G150 & 144.4 & 14.2 & 0.2109 & 0.09425 & 0.0054 & 1.2587 \\
\hline
\end{tabular}




\section{oncordant ages $\quad 81.3 \%$ concordant}

\begin{tabular}{|c|c|c|c|c|c|c|}
\hline $2 \sigma 75$ & Pb206/U238 & $2 \sigma 68$ & $\begin{array}{c}\text { ages } \\
\text { age } 206 / 238\end{array}$ & $2 \sigma$ age 68 & age $207 / 235$ & $2 \sigma$ age 75 \\
\hline 0.00992 & 0.04474 & 0.00092 & 282.1 & 5.6 & 362.6 & 9.6 \\
\hline 0.04634 & 0.1521 & 0.0034 & 912.7 & 19 & 949.5 & 23.6 \\
\hline 0.03954 & 0.12313 & 0.0029 & 748.6 & 16.6 & 762.8 & 23.4 \\
\hline 0.16862 & 0.3392 & 0.00704 & 1882.8 & 33.8 & 2167.4 & 27.8 \\
\hline 0.01366 & 0.07151 & 0.00144 & 445.2 & 8.6 & 479.7 & 11.8 \\
\hline 0.11064 & 0.29855 & 0.00616 & 1684.1 & 30.6 & 1799.4 & 26 \\
\hline 0.05682 & 0.1693 & 0.00398 & 1008.3 & 22 & 1002.7 & 26.8 \\
\hline 0.02944 & 0.08979 & 0.00218 & 554.3 & 12.8 & 554.8 & 20.8 \\
\hline 0.0421 & 0.17629 & 0.00364 & 1046.7 & 20 & 1039 & 20.8 \\
\hline 0.02964 & 0.0948 & 0.00228 & 583.9 & 13.4 & 570 & 20.8 \\
\hline 0.05664 & 0.12435 & 0.0035 & 755.6 & 20 & 745.4 & 32.4 \\
\hline 0.03582 & 0.09782 & 0.00248 & 601.6 & 14.6 & 612.2 & 23.8 \\
\hline 0.1359 & 0.30514 & 0.0064 & 1716.7 & 31.6 & 1955.1 & 27.4 \\
\hline 0.03406 & 0.08282 & 0.00224 & 512.9 & 13.4 & 525 & 24.2 \\
\hline 0.24498 & 0.43986 & 0.00968 & 2350 & 43.4 & 2436.8 & 30.6 \\
\hline 0.04052 & 0.08558 & 0.00248 & 529.3 & 14.8 & 531.3 & 28.2 \\
\hline 0.03946 & 0.15079 & 0.00324 & 905.4 & 18.2 & 908.4 & 21.2 \\
\hline 0.04306 & 0.11374 & 0.0029 & 694.4 & 16.8 & 702.2 & 26.2 \\
\hline 0.03732 & 0.12499 & 0.00288 & 759.2 & 16.6 & 752.7 & 22.4 \\
\hline 0.0248 & 0.09515 & 0.00214 & 585.9 & 12.6 & 559.4 & 17.8 \\
\hline 0.05446 & 0.10476 & 0.00318 & 642.2 & 18.6 & 642.1 & 33.8 \\
\hline 0.03996 & 0.16016 & 0.0034 & 957.7 & 18.8 & 940.1 & 21 \\
\hline 0.0302 & 0.10246 & 0.0024 & 628.8 & 14 & 607.9 & 20.6 \\
\hline 0.05752 & 0.11641 & 0.00314 & 709.9 & 18.2 & 887.6 & 30.4 \\
\hline 0.15 & 0.10186 & 0.0031 & 625.3 & 18.2 & & 85.6 \\
\hline 0.0154 & 0.05747 & 0.00122 & 360.2 & 7.4 & 468.2 & 13 \\
\hline 0.03978 & 0.12754 & 0.00294 & 773.8 & 16.8 & 802.2 & 22.8 \\
\hline 0.05338 & 0.13104 & 0.0035 & 793.8 & 20 & 774.5 & 30 \\
\hline 0.03694 & 0.0844 & 0.00206 & 522.3 & 12.2 & 727.5 & 23 \\
\hline 0.0367 & 0.09489 & 0.0025 & 584.4 & 14.8 & 576.9 & 24.8 \\
\hline 0.02492 & 0.08482 & 0.00198 & 524.8 & 11.8 & 524.8 & 18.4 \\
\hline 0.0625 & 0.17306 & 0.00424 & 1028.9 & 23.4 & 1005.9 & 28.8 \\
\hline 0.02498 & 0.09835 & 0.00216 & 604.7 & 12.6 & 607.6 & 17.6 \\
\hline 0.00486 & 0.01896 & 0.00044 & 121.1 & 2.8 & 116.8 & 5.2 \\
\hline 0.02506 & 0.09975 & 0.0022 & 612.9 & 12.8 & 598.7 & 17.6 \\
\hline 0.0033 & 0.00462 & 0.00016 & 29.7 & 1 & 30.4 & 3.6 \\
\hline 0.0576 & 0.09679 & 0.0032 & 595.6 & 18.8 & 597.8 & 37 \\
\hline 0.03024 & 0.09327 & 0.00226 & 574.8 & 13.4 & 569.4 & 21 \\
\hline 0.02332 & 0.10181 & 0.00218 & 625 & 12.8 & 605.4 & 16.6 \\
\hline 0.2286 & 0.31124 & 0.01034 & 1746.8 & 50.8 & 1772.1 & 49.8 \\
\hline 0.03774 & 0.12373 & 0.00286 & 752 & 16.4 & 754 & 22.6 \\
\hline 0.03558 & 0.09243 & 0.00236 & 569.9 & 14 & 600.5 & 24 \\
\hline 0.24896 & 0.45703 & 0.00972 & 2426.4 & 43 & 2493 & 29.6 \\
\hline 0.0255 & 0.08927 & 0.00204 & 551.2 & 12 & 562.2 & 18.4 \\
\hline 0.02674 & 0.11894 & 0.00248 & 724.5 & 14.2 & 742.8 & 17.4 \\
\hline 0.02074 & 0.09418 & 0.002 & 580.2 & 11.8 & 573.3 & 15.4 \\
\hline 0.0458 & 0.09904 & 0.00284 & 608.8 & 16.6 & 608.7 & 29.8 \\
\hline 0.0304 & 0.13037 & 0.00276 & 790 & 15.8 & 773.5 & 18.8 \\
\hline 0.05964 & 0.12733 & 0.00366 & 772.6 & 21 & 762.9 & 33.6 \\
\hline 0.02306 & 0.10342 & 0.00216 & 634.4 & 12.6 & 653.7 & 16.2 \\
\hline
\end{tabular}




\begin{tabular}{|c|c|c|c|c|c|c|}
\hline 0.0441 & 0.10545 & 0.00274 & 646.3 & 16 & 717.3 & 26.8 \\
\hline 0.0335 & 0.0867 & 0.00228 & 536 & 13.6 & 536.9 & 23.6 \\
\hline 0.0279 & 0.1051 & 0.00232 & 644.2 & 13.6 & 656.9 & 18.8 \\
\hline 208.18956 & -0.88214 & 2.74294 & & & & \\
\hline 0.03856 & 0.11413 & 0.00274 & 696.7 & 15.8 & 718.4 & 23.6 \\
\hline 0.03142 & 0.09398 & 0.00232 & 579 & 13.6 & 564.1 & 21.8 \\
\hline 0.3341 & 0.45004 & 0.01034 & 2395.4 & 46 & 2689.1 & 32.4 \\
\hline 0.19866 & 0.34236 & 0.00728 & 1898 & 35 & 2280.8 & 29 \\
\hline 0.05164 & 0.10244 & 0.0031 & 628.7 & 18.2 & 618.9 & 32.8 \\
\hline 0.20182 & 0.12334 & 0.00254 & 749.8 & 14.6 & 2354.5 & 28 \\
\hline 0.03198 & 0.09575 & 0.00236 & 589.5 & 13.8 & 574.6 & 22 \\
\hline 0.0275 & 0.06744 & 0.00182 & 420.7 & 11 & 421.3 & 21.4 \\
\hline 0.0402 & 0.07512 & 0.0024 & 466.9 & 14.4 & 441.2 & 29.8 \\
\hline 0.0443 & 0.13553 & 0.00322 & 819.3 & 18.2 & 811.6 & 24.8 \\
\hline 0.04024 & 0.14807 & 0.00324 & 890.1 & 18.2 & 886.8 & 21.8 \\
\hline 0.04746 & 0.1689 & 0.0037 & 1006 & 20.4 & 998.7 & 23.2 \\
\hline 0.25766 & 0.43187 & 0.00998 & 2314.1 & 45 & 2411.8 & 32.4 \\
\hline 0.04904 & 0.16999 & 0.00376 & 1012.1 & 20.8 & 1003.1 & 23.8 \\
\hline 0.223 & 0.42272 & 0.00872 & 2272.8 & 39.6 & 2425.5 & 28.4 \\
\hline 0.06172 & 0.11425 & 0.0035 & 697.4 & 20.2 & 717.3 & 35.8 \\
\hline 0.0616 & 0.1139 & 0.00348 & 695.4 & 20.2 & 702.4 & 36 \\
\hline 0.05422 & 0.08249 & 0.0029 & 511 & 17.2 & 523.1 & 37.2 \\
\hline 0.15492 & 0.33694 & 0.00698 & 1871.9 & 33.6 & 2083 & 27.4 \\
\hline 1.3818 & 0.60396 & 0.01326 & 3045.8 & 53.2 & 4164.9 & 32 \\
\hline 0.02594 & 0.09559 & 0.00216 & 588.5 & 12.8 & 591.3 & 18.4 \\
\hline 0.25036 & 0.43157 & 0.0091 & 2312.8 & 41 & 2503.9 & 29.4 \\
\hline 0.04274 & 0.11382 & 0.00278 & 694.9 & 16 & 770.8 & 25 \\
\hline 105.01892 & -0.73154 & 1.3823 & & & & \\
\hline 0.02412 & 0.09977 & 0.00216 & 613.1 & 12.6 & 605.9 & 17 \\
\hline 0.02916 & 0.09557 & 0.00218 & 588.4 & 12.8 & 651.1 & 19.6 \\
\hline 0.05698 & 0.12603 & 0.00354 & 765.2 & 20.2 & 757.5 & 32.2 \\
\hline 0.0242 & 0.09614 & 0.0021 & 591.7 & 12.4 & 600.6 & 17.2 \\
\hline 0.05432 & 0.17498 & 0.00396 & 1039.5 & 21.8 & 1038.6 & 25.2 \\
\hline 0.05342 & 0.13744 & 0.00358 & 830.2 & 20.2 & 801.6 & 29.4 \\
\hline 0.03294 & 0.09574 & 0.00238 & 589.4 & 14 & 578.4 & 22.6 \\
\hline 0.06208 & 0.17531 & 0.00428 & 1041.3 & 23.4 & 1007.9 & 28.8 \\
\hline 0.03738 & 0.12467 & 0.00286 & 757.4 & 16.4 & 762.1 & 22.2 \\
\hline 0.04316 & 0.09616 & 0.00268 & 591.9 & 15.8 & 604.3 & 28.2 \\
\hline 0.0291 & 0.08027 & 0.00204 & 497.7 & 12.2 & 499.1 & 21.4 \\
\hline 0.0255 & 0.09788 & 0.00218 & 602 & 12.8 & 596 & 18 \\
\hline 0.02724 & 0.09054 & 0.00212 & 558.7 & 12.6 & 558.4 & 19.4 \\
\hline 0.04678 & 0.10207 & 0.00292 & 626.5 & 17 & 605.8 & 30.2 \\
\hline 0.31728 & 0.45498 & 0.0117 & 2417.4 & 51.8 & 2474 & 37 \\
\hline 0.05536 & 0.1219 & 0.00354 & 741.5 & 20.4 & 698.8 & 32.8 \\
\hline 0.07712 & 0.15538 & 0.00456 & 931.1 & 25.4 & 915 & 37.4 \\
\hline 0.02626 & 0.10263 & 0.0022 & 629.8 & 12.8 & 680.9 & 17.6 \\
\hline 0.19476 & 0.35737 & 0.0077 & 1969.7 & 36.6 & 2230.5 & 29.6 \\
\hline 0.0072 & 0.01191 & 0.00026 & 76.3 & 1.6 & 268.9 & 7.8 \\
\hline 0.03342 & 0.09447 & 0.0024 & 581.9 & 14.2 & 564.3 & 23 \\
\hline 0.06168 & 0.11694 & 0.00356 & 712.9 & 20.6 & 741 & 35.4 \\
\hline 0.02496 & 0.10631 & 0.00228 & 651.3 & 13.2 & 648 & 17.2 \\
\hline 0.07132 & 0.21744 & 0.00454 & 1268.3 & 24 & 1400.2 & 24.4 \\
\hline 0.17156 & 0.32183 & 0.00852 & 1798.7 & 41.6 & 1791 & 37.6 \\
\hline 0.0406 & 0.1318 & 0.00304 & 798.1 & 17.4 & 803.4 & 23.2 \\
\hline 0.03484 & 0.12671 & 0.00286 & 769.1 & 16.4 & 747.7 & 21.2 \\
\hline
\end{tabular}




\begin{tabular}{|c|c|c|c|c|c|c|}
\hline 0.0391 & 0.1209 & 0.0028 & 735.7 & 16.2 & 785.7 & 22.8 \\
\hline 0.04742 & 0.15203 & 0.00346 & 912.3 & 19.4 & 927.4 & 24.4 \\
\hline 0.09526 & 0.28477 & 0.00588 & 1615.3 & 29.6 & 1648.3 & 25.6 \\
\hline 0.03732 & 0.09682 & 0.00246 & 595.7 & 14.4 & 638.2 & 24.4 \\
\hline 0.15062 & 0.13173 & 0.0031 & 797.7 & 17.6 & 1944.8 & 31.4 \\
\hline 0.0513 & 0.09627 & 0.00298 & 592.5 & 17.6 & 601 & 33.2 \\
\hline 0.03386 & 0.13347 & 0.00286 & 807.6 & 16.2 & 820.4 & 19.8 \\
\hline 0.0448 & 0.09671 & 0.00276 & 595.1 & 16.2 & 602 & 29.2 \\
\hline 0.0401 & 0.09164 & 0.00256 & 565.2 & 15.2 & 574.7 & 27.2 \\
\hline 0.03784 & 0.08944 & 0.00248 & 552.2 & 14.6 & 535 & 26.2 \\
\hline 0.16958 & 0.35164 & 0.00736 & 1942.4 & 35.2 & 2132 & 28.4 \\
\hline 0.0381 & 0.09857 & 0.00266 & 606 & 15.6 & 553.3 & 26 \\
\hline 0.05534 & 0.09343 & 0.00308 & 575.8 & 18.2 & 577 & 36.2 \\
\hline 0.01946 & 0.09017 & 0.00192 & 556.5 & 11.4 & 546.5 & 14.8 \\
\hline 0.0286 & 0.11164 & 0.00246 & 682.3 & 14.2 & 679 & 18.8 \\
\hline 0.0683 & 0.16889 & 0.0036 & 1006 & 19.8 & 1336.8 & 24.8 \\
\hline 0.13488 & 0.32281 & 0.00698 & 1803.4 & 34 & 1874.3 & 28.8 \\
\hline 0.02514 & 0.09021 & 0.00206 & 556.8 & 12.2 & 564.3 & 18.2 \\
\hline 0.031 & 0.09426 & 0.0023 & 580.7 & 13.6 & 581.7 & 21.4 \\
\hline 0.0387 & 0.14032 & 0.0031 & 846.5 & 17.6 & 850.8 & 21.6 \\
\hline 0.02366 & 0.09063 & 0.00204 & 559.3 & 12 & 547.8 & 17.4 \\
\hline 0.04712 & 0.13651 & 0.00314 & 824.9 & 17.8 & 965 & 24 \\
\hline 0.0388 & 0.08627 & 0.00244 & 533.4 & 14.4 & 542.4 & 26.8 \\
\hline 0.03172 & 0.12207 & 0.00268 & 742.5 & 15.4 & 735.4 & 19.8 \\
\hline 0.13832 & 0.12194 & 0.003 & 741.7 & 17.2 & 1813.9 & 32.8 \\
\hline 0.16898 & 0.34529 & 0.00822 & 1912.1 & 39.4 & 1931.9 & 33.2 \\
\hline 0.0224 & 0.09278 & 0.00202 & 572 & 12 & 568.4 & 16.4 \\
\hline 0.12154 & 0.29248 & 0.00622 & 1653.9 & 31 & 1805.8 & 27.8 \\
\hline 0.0439 & 0.16149 & 0.0035 & 965.1 & 19.4 & 959 & 22.2 \\
\hline 0.02906 & 0.10445 & 0.00236 & 640.4 & 13.8 & 638.1 & 19.4 \\
\hline 0.03042 & 0.09287 & 0.0023 & 572.5 & 13.6 & 542.8 & 21.4 \\
\hline 0.13074 & 0.32242 & 0.00706 & 1801.5 & 34.4 & 1818.4 & 29 \\
\hline 0.05978 & 0.13632 & 0.00372 & 823.8 & 21.2 & 816.9 & 32 \\
\hline 0.03786 & 0.1186 & 0.00284 & 722.5 & 16.4 & 707.9 & 23.2 \\
\hline 0.03104 & 0.11941 & 0.00262 & 727.2 & 15 & 728.5 & 19.6 \\
\hline 0.00466 & 0.00441 & 0.0002 & 28.4 & 1.2 & 29.3 & 5 \\
\hline 0.03102 & 0.09699 & 0.0023 & 596.7 & 13.6 & 610.6 & 21 \\
\hline 0.02358 & 0.10239 & 0.00218 & 628.4 & 12.8 & 634.8 & 16.6 \\
\hline 0.0299 & 0.1092 & 0.00248 & 668.1 & 14.4 & 648.4 & 19.8 \\
\hline 0.02546 & 0.107 & 0.00232 & 655.3 & 13.6 & 639.9 & 17.6 \\
\hline 0.0223 & 0.10355 & 0.00214 & 635.2 & 12.6 & 668.6 & 15.6 \\
\hline 0.18512 & 0.36945 & 0.00774 & 2026.8 & 36.4 & 2198.7 & 28.8 \\
\hline 0.06944 & 0.12307 & 0.0039 & 748.2 & 22.4 & 763.7 & 38.6 \\
\hline 0.06766 & 0.09689 & 0.0031 & 596.2 & 18.2 & 827.3 & 37.2 \\
\hline
\end{tabular}




\begin{tabular}{|c|c|c|c|c|c|}
\hline age $207 / 206$ & $2 \sigma$ age 76 & $\begin{array}{l}\text { discordance } \\
\Delta 68-75[\%]\end{array}$ & $\Delta 68-76[\%]$ & $\begin{array}{c}\text { preferred age } \\
\text { age }\end{array}$ & $2 \sigma$ age \\
\hline 916.3 & 49 & -22.2 & -69.2 & & \\
\hline 1036.3 & 62.4 & -3.9 & -11.9 & 912.7 & 19 \\
\hline 805.1 & 76.8 & -1.9 & -7 & 748.6 & 16.6 \\
\hline 2449.5 & 39 & -13.1 & -23.1 & & \\
\hline 648.7 & 49.8 & -7.2 & -31.4 & 445.2 & 8.6 \\
\hline 1936.3 & 41.4 & -6.4 & -13 & 1936.3 & 41.4 \\
\hline 991.3 & 71 & 0.5 & 1.7 & 1008.3 & 22 \\
\hline 557.8 & 92 & -0.1 & -0.6 & 554.3 & 12.8 \\
\hline 1023.6 & 49.2 & 0.7 & 2.3 & 1046.7 & 20 \\
\hline 516.1 & 90 & 2.4 & 13.1 & 583.9 & 13.4 \\
\hline 715.3 & 115.6 & 1.4 & 5.6 & 755.6 & 20 \\
\hline 652.2 & 96.6 & -1.7 & -7.8 & 601.6 & 14.6 \\
\hline 2218.3 & 41 & -12.2 & -22.6 & & \\
\hline 578.5 & 114.4 & -2.3 & -11.3 & 512.9 & 13.4 \\
\hline 2510.5 & 41.6 & -3.6 & -6.4 & 2510.5 & 41.6 \\
\hline 540.7 & 134.4 & -0.4 & -2.1 & 529.3 & 14.8 \\
\hline 916.6 & 58 & -0.3 & -1.2 & 905.4 & 18.2 \\
\hline 727.7 & 95.4 & -1.1 & -4.6 & 694.4 & 16.8 \\
\hline 733.7 & 74.4 & 0.9 & 3.5 & 759.2 & 16.6 \\
\hline 453.6 & 77.6 & 4.7 & 29.2 & 585.9 & 12.6 \\
\hline 642.4 & 138 & 0 & 0 & 642.2 & 18.6 \\
\hline 899.6 & 55.6 & 1.9 & 6.5 & 957.7 & 18.8 \\
\hline 531.3 & 83.8 & 3.4 & 18.4 & 628.8 & 14 \\
\hline 1361.6 & 84.2 & -20 & -47.9 & & \\
\hline 1040.1 & 55.2 & -23.1 & -65.4 & & \\
\hline 882.5 & 70.6 & -3.5 & -12.3 & 773.8 & 16.8 \\
\hline 720 & 103.2 & 2.5 & 10.2 & 793.8 & 20 \\
\hline 1426 & 70.8 & -28.2 & -63.4 & & \\
\hline 548.2 & 109 & 1.3 & 6.6 & 584.4 & 14.8 \\
\hline 525.6 & 84 & 0 & -0.1 & 524.8 & 11.8 \\
\hline 956.8 & 77.8 & 2.3 & 7.5 & 1028.9 & 23.4 \\
\hline 619 & 67.8 & -0.5 & -2.3 & 604.7 & 12.6 \\
\hline 30.8 & 99.6 & 3.7 & 292.7 & 121.1 & 2.8 \\
\hline 545.9 & 70.4 & 2.4 & 12.3 & 612.9 & 12.8 \\
\hline 87.4 & 267 & -2.3 & -66 & 29.7 & 1 \\
\hline 606.8 & 162 & -0.4 & -1.9 & 595.6 & 18.8 \\
\hline 548.5 & 91 & 1 & 4.8 & 574.8 & 13.4 \\
\hline 533.2 & 64.8 & 3.2 & 17.2 & 625 & 12.8 \\
\hline 1802.7 & 89.8 & -1.4 & -3.1 & 1802.7 & 89.8 \\
\hline 760.7 & 75 & -0.3 & -1.1 & 752 & 16.4 \\
\hline 718.7 & 98 & -5.1 & -20.7 & 569.9 & 14 \\
\hline 2548.2 & 39.6 & -2.7 & -4.8 & 2548.2 & 39.6 \\
\hline 607.9 & 77.6 & -2 & -9.3 & 551.2 & 12 \\
\hline 799.4 & 53.6 & -2.5 & -9.4 & 724.5 & 14.2 \\
\hline 546.7 & 61.2 & 1.2 & 6.1 & 580.2 & 11.8 \\
\hline 609.3 & 125.8 & 0 & -0.1 & 608.8 & 16.6 \\
\hline 726.7 & 58 & 2.1 & 8.7 & 790 & 15.8 \\
\hline 735.3 & 117.4 & 1.3 & 5.1 & 772.6 & 21 \\
\hline 721.3 & 55.6 & -2.9 & -12 & 634.4 & 12.6 \\
\hline
\end{tabular}




\begin{tabular}{|c|c|c|c|c|c|}
\hline 947.3 & 92.2 & -9.9 & -31.8 & 646.3 & 16 \\
\hline 541.8 & 109.4 & -0.2 & -1.1 & 536 & 13.6 \\
\hline 701.5 & 67.8 & -1.9 & -8.2 & 644.2 & 13.6 \\
\hline 787.6 & 81.6 & -3 & -11.5 & 696.7 & 15.8 \\
\hline 505.4 & 96.6 & 2.6 & 14.6 & 579 & 13.6 \\
\hline 2918.4 & 41.4 & -10.9 & -17.9 & & \\
\hline 2644.7 & 39.6 & -16.8 & -28.2 & & \\
\hline 584.3 & 138.8 & 1.6 & 7.6 & 628.7 & 18.2 \\
\hline 4351.8 & 33.2 & -68.2 & -82.8 & & \\
\hline 516.9 & 96 & 2.6 & 14 & 589.5 & 13.8 \\
\hline 425.2 & 124.2 & -0.1 & -1.1 & 420.7 & 11 \\
\hline 309.8 & 175 & 5.8 & 50.7 & & \\
\hline 791.4 & 78.8 & 1 & 3.5 & 819.3 & 18.2 \\
\hline 879.5 & 61.2 & 0.4 & 1.2 & 890.1 & 18.2 \\
\hline 983.4 & 59.4 & 0.7 & 2.3 & 1006 & 20.4 \\
\hline 2495.9 & 44.8 & -4 & -7.3 & 2495.9 & 44.8 \\
\hline 984.2 & 61.2 & 0.9 & 2.8 & 1012.1 & 20.8 \\
\hline 2556.8 & 38.2 & -6.3 & -11.1 & 2556.8 & 38.2 \\
\hline 781.1 & 131.4 & -2.8 & -10.7 & 697.4 & 20.2 \\
\hline 725.7 & 136.2 & -1 & -4.2 & 695.4 & 20.2 \\
\hline 577 & 181.8 & -2.3 & -11.4 & 511 & 17.2 \\
\hline 2299.2 & 39.8 & -10.1 & -18.6 & & \\
\hline 4759.5 & 33.4 & -26.9 & -36 & & \\
\hline 602.9 & 73.6 & -0.5 & -2.4 & 588.5 & 12.8 \\
\hline 2663.3 & 39 & -7.6 & -13.2 & 2663.3 & 39 \\
\hline 998 & 79.6 & -9.8 & -30.4 & 694.9 & 16 \\
\hline 580 & 65.8 & 1.2 & 5.7 & 613.1 & 12.6 \\
\hline 875.5 & 69.8 & -9.6 & -32.8 & 588.4 & 12.8 \\
\hline 735.7 & 112.8 & 1 & 4 & 765.2 & 20.2 \\
\hline 635 & 66.4 & -1.5 & -6.8 & 591.7 & 12.4 \\
\hline 1037.6 & 63.4 & 0.1 & 0.2 & 1039.5 & 21.8 \\
\hline 723.7 & 97.6 & 3.6 & 14.7 & 830.2 & 20.2 \\
\hline 536.2 & 97.8 & 1.9 & 9.9 & 589.4 & 14 \\
\hline 936.8 & 77.6 & 3.3 & 11.2 & 1041.3 & 23.4 \\
\hline 776.9 & 72.4 & -0.6 & -2.5 & 757.4 & 16.4 \\
\hline 652.2 & 119 & -2.1 & -9.3 & 591.9 & 15.8 \\
\hline 505.8 & 105 & -0.3 & -1.6 & 497.7 & 12.2 \\
\hline 573.7 & 72 & 1 & 4.9 & 602 & 12.8 \\
\hline 557.8 & 83.8 & 0.1 & 0.2 & 558.7 & 12.6 \\
\hline 529.8 & 130.8 & 3.4 & 18.3 & 626.5 & 17 \\
\hline 2521.6 & 51 & -2.3 & -4.1 & 2521.6 & 51 \\
\hline 564.5 & 126.4 & 6.1 & 31.4 & & \\
\hline 877.4 & 113.4 & 1.8 & 6.1 & 931.1 & 25.4 \\
\hline 854.5 & 59 & -7.5 & -26.3 & 629.8 & 12.8 \\
\hline 2480 & 41.8 & -11.7 & -20.6 & & \\
\hline 2696.1 & 40.8 & -71.6 & -97.2 & & \\
\hline 494.6 & 102.8 & 3.1 & 17.6 & 581.9 & 14.2 \\
\hline 827.8 & 125.2 & -3.8 & -13.9 & 712.9 & 20.6 \\
\hline 637.5 & 61.4 & 0.5 & 2.2 & 651.3 & 13.2 \\
\hline 1607.8 & 45.6 & -9.4 & -21.1 & & \\
\hline 1782.8 & 66 & 0.4 & 0.9 & 1782.8 & 66 \\
\hline 818.7 & 72.4 & -0.7 & -2.5 & 798.1 & 17.4 \\
\hline 684.8 & 70.6 & 2.9 & 12.3 & 769.1 & 16.4 \\
\hline
\end{tabular}




\begin{tabular}{|c|c|c|c|c|c|}
\hline 931 & 71.4 & -6.4 & -21 & 735.7 & 16.2 \\
\hline 964 & 67 & -1.6 & -5.4 & 912.3 & 19.4 \\
\hline 1691.2 & 43.8 & -2 & -4.5 & 1691.2 & 43.8 \\
\hline 792 & 93.4 & -6.6 & -24.8 & 595.7 & 14.4 \\
\hline 3564.3 & 42.4 & -59 & -77.6 & & \\
\hline 633.9 & 143 & -1.4 & -6.5 & 592.5 & 17.6 \\
\hline 856 & 57.8 & -1.6 & -5.7 & 807.6 & 16.2 \\
\hline 629 & 124.2 & -1.1 & -5.4 & 595.1 & 16.2 \\
\hline 613.3 & 119 & -1.6 & -7.8 & 565.2 & 15.2 \\
\hline 463 & 125.2 & 3.2 & 19.3 & 552.2 & 14.6 \\
\hline 2320.7 & 41 & -8.9 & -16.3 & & \\
\hline 342.9 & 123 & 9.5 & 76.8 & & \\
\hline 582.9 & 163.6 & -0.2 & -1.2 & 575.8 & 18.2 \\
\hline 505.8 & 61.4 & 1.8 & 10 & 556.5 & 11.4 \\
\hline 668.9 & 66.4 & 0.5 & 2 & 682.3 & 14.2 \\
\hline 1915.6 & 45.8 & -24.7 & -47.5 & & \\
\hline 1954.4 & 45.8 & -3.8 & -7.7 & 1954.4 & 45.8 \\
\hline 595.6 & 76.2 & -1.3 & -6.5 & 556.8 & 12.2 \\
\hline 586.5 & 90.4 & -0.2 & -1 & 580.7 & 13.6 \\
\hline 863.1 & 63 & -0.5 & -1.9 & 846.5 & 17.6 \\
\hline 501.2 & 75.4 & 2.1 & 11.6 & 559.3 & 12 \\
\hline 1300.4 & 60.4 & -14.5 & -36.6 & 824.9 & 17.8 \\
\hline 580.7 & 124.4 & -1.6 & -8.1 & 533.4 & 14.4 \\
\hline 714.6 & 65.2 & 1 & 3.9 & 742.5 & 15.4 \\
\hline 3447.2 & 46.4 & -59.1 & -78.5 & & \\
\hline 1954 & 53.6 & -1 & -2.1 & 1954 & 53.6 \\
\hline 554.9 & 67 & 0.6 & 3.1 & 572 & 12 \\
\hline 1986.7 & 44.6 & -8.4 & -16.8 & & \\
\hline 945.9 & 58.6 & 0.6 & 2 & 965.1 & 19.4 \\
\hline 630.7 & 73.8 & 0.4 & 1.5 & 640.4 & 13.8 \\
\hline 420.7 & 99.4 & 5.5 & 36.1 & & \\
\hline 1838.4 & 48 & -0.9 & -2 & 1838.4 & 48 \\
\hline 798.7 & 105.2 & 0.8 & 3.1 & 823.8 & 21.2 \\
\hline 662.7 & 83.4 & 2.1 & 9 & 722.5 & 16.4 \\
\hline 733.3 & 64.4 & -0.2 & -0.8 & 727.2 & 15 \\
\hline 109.1 & 387 & -3.2 & -74 & 28.4 & 1.2 \\
\hline 663 & 83.4 & -2.3 & -10 & 596.7 & 13.6 \\
\hline 658.5 & 60 & -1 & -4.6 & 628.4 & 12.8 \\
\hline 581.1 & 74.6 & 3 & 15 & 668.1 & 14.4 \\
\hline 586.5 & 64.8 & 2.4 & 11.7 & 655.3 & 13.6 \\
\hline 783.7 & 50.8 & -5 & -19 & 635.2 & 12.6 \\
\hline 2363.8 & 41.4 & -7.8 & -14.3 & 2363.8 & 41.4 \\
\hline 810.2 & 134.8 & -2 & -7.6 & 748.2 & 22.4 \\
\hline 1513.1 & 108.2 & -27.9 & -60.6 & & \\
\hline
\end{tabular}


Sample 3289S Nile Delta beach @ Ras El Barr 150 grain analysed 123 conce

\begin{tabular}{|c|c|c|c|c|c|c|}
\hline \multirow[b]{2}{*}{ grain } & \multicolumn{3}{|c|}{ concentrations } & \multicolumn{3}{|l|}{ isotopic ratios } \\
\hline & U [ppm] & $\mathrm{Pb}$ [ppm] & $T h / U$ & Pb207/Pb206 & $2 \sigma 76$ & Pb207/U235 \\
\hline S3289_G001 & 640.1 & 51.3 & 0.156 & 0.05747 & 0.00158 & 0.65942 \\
\hline S3289_G002 & 43.8 & 7.9 & 0.6311 & 0.07048 & 0.0039 & 1.56446 \\
\hline S3289_G003 & 289.9 & 59.4 & 0.4898 & 0.12789 & 0.00312 & 3.44327 \\
\hline S3289_G004 & 185.9 & 30.1 & 1.071 & 0.06471 & 0.0019 & 1.15636 \\
\hline S3289_G005 & 256.4 & 31.6 & 0.702 & 0.12081 & 0.00324 & 1.74097 \\
\hline S3289_G006 & 157.1 & 20.6 & 0.5013 & 0.06321 & 0.0024 & 1.06354 \\
\hline S3289_G007 & 123.1 & 15.4 & 0.7093 & 0.06205 & 0.00244 & 0.94773 \\
\hline S3289 G008 & 172.5 & 21.9 & 0.3764 & 0.06739 & 0.00216 & 1.13816 \\
\hline S3289 G009 & 857.4 & 90.3 & 0.2911 & 0.0655 & 0.0016 & 0.94257 \\
\hline S3289_G010 & 78.3 & 8.5 & 0.4312 & 0.06032 & 0.00294 & 0.85966 \\
\hline S3289_G011 & 234.3 & 55.2 & 1.3323 & 0.07395 & 0.00208 & 1.81587 \\
\hline S3289_G012 & 322.4 & 34.3 & 0.3465 & 0.06007 & 0.00174 & 0.85944 \\
\hline S3289_G013 & 95.8 & 12.8 & 0.3406 & 0.06478 & 0.00256 & 1.1648 \\
\hline S3289_G014 & 42.2 & 4.9 & 0.2332 & 0.06167 & 0.0039 & 0.98924 \\
\hline S3289_G015 & 176.1 & 21.1 & 0.2592 & 0.06195 & 0.00208 & 1.02361 \\
\hline S3289_G016 & 231.7 & 18.6 & 1.1433 & 0.06447 & 0.00242 & 0.58997 \\
\hline S3289_G017 & 82.4 & 9.3 & 0.8099 & 0.06656 & 0.00356 & 0.88868 \\
\hline S3289_G018 & 587 & 76.2 & 0.6236 & 0.06271 & 0.00158 & 1.01061 \\
\hline S3289_G019 & 1040.2 & 116.7 & 0.408 & 0.06328 & 0.00146 & 0.9421 \\
\hline S3289_G020 & 120 & 12.9 & 0.3724 & 0.062 & 0.00246 & 0.89224 \\
\hline S3289_G021 & 132.3 & 15.4 & 0.6582 & 0.06113 & 0.0023 & 0.88149 \\
\hline S3289_G022 & & & & -1.43077 & 13.44876 & \\
\hline S3289_G023 & 246.7 & 32.2 & 0.4615 & 0.06418 & 0.00196 & 1.09185 \\
\hline S3289_G024 & 241 & 31.3 & 1.3561 & 0.05993 & 0.00212 & 0.80998 \\
\hline S3289_G025 & 60.8 & 27.3 & 0.8536 & 0.12574 & 0.00388 & 6.4038 \\
\hline S3289_G026 & & & & 0.85966 & 0.1049 & \\
\hline S3289_G027 & 57.7 & 6.5 & 0.7701 & 0.05767 & 0.00334 & 0.7732 \\
\hline S3289_G028 & 156.5 & 22.1 & 0.4625 & 0.0685 & 0.00234 & 1.24409 \\
\hline S3289_G029 & 224 & 16 & 0.8036 & 0.0537 & 0.00226 & 0.45688 \\
\hline S3289_G030 & 1801.3 & 261.3 & 2.073 & 0.06978 & 0.0016 & 0.99202 \\
\hline S3289_G031 & 246.7 & 33.4 & 0.5495 & 0.06274 & 0.00188 & 1.07302 \\
\hline S3289_G032 & 75.2 & 10.5 & 0.3201 & 0.0649 & 0.00278 & 1.22158 \\
\hline S3289_G033 & 38.6 & 5.5 & 0.4784 & 0.06946 & 0.00412 & 1.27571 \\
\hline S3289_G034 & 93.2 & 18.5 & 0.7723 & 0.07273 & 0.00254 & 1.71793 \\
\hline S3289_G035 & 247.7 & 101.6 & 0.8217 & 0.11457 & 0.00262 & 5.39725 \\
\hline S3289_G036 & 120.5 & & 1.8316 & 0.1037 & 0.00364 & 1.41813 \\
\hline S3289_G037 & 167.9 & 22.6 & 0.4128 & 0.06382 & 0.0022 & 1.13343 \\
\hline S3289_G038 & 119.5 & 14.1 & 1.3019 & 0.05848 & 0.0025 & 0.72911 \\
\hline S3289_G039 & 387.8 & 0 & 0.9408 & 0.27882 & 0.52694 & 0.0013 \\
\hline S3289_G040 & 108.7 & 16.9 & 0.7281 & 0.06882 & 0.00248 & 1.29925 \\
\hline S3289_G041 & 43.3 & 6.3 & 0.393 & 0.06833 & 0.00376 & 1.31191 \\
\hline S3289_G042 & 174.6 & 24.9 & 0.4126 & 0.06652 & 0.0021 & 1.24774 \\
\hline S3289_G043 & 113.8 & 17 & 0.9007 & 0.06472 & 0.00256 & 1.11932 \\
\hline S3289_G044 & 37.1 & 4.1 & 0.8025 & 0.06041 & 0.00498 & 0.7968 \\
\hline S3289_G045 & 418.1 & 70.6 & 1.0919 & 0.06634 & 0.0017 & 1.24088 \\
\hline S3289_G046 & 100.4 & 36.9 & 0.6491 & 0.118 & 0.00306 & 5.24905 \\
\hline S3289_G047 & 23.2 & 3.3 & 0.3871 & 0.06651 & 0.00534 & 1.27296 \\
\hline S3289_G048 & 33 & 4.2 & 0.5659 & 0.06176 & 0.00426 & 0.99202 \\
\hline S3289_G049 & 23.7 & 5.4 & 0.899 & 0.07233 & 0.00456 & 1.89472 \\
\hline S3289_G050 & 216.3 & 43.4 & 0.2993 & 0.16471 & 0.00394 & 4.64469 \\
\hline
\end{tabular}




\begin{tabular}{|c|c|c|c|c|c|c|}
\hline S3289_G051 & 178.2 & 18.3 & 0.4753 & 0.05915 & 0.00216 & 0.78685 \\
\hline S3289_G052 & 287.3 & 32.1 & 0.5424 & 0.05969 & 0.0018 & 0.85158 \\
\hline S3289 G053 & 174.1 & 22.5 & 1.5219 & 0.05942 & 0.0022 & 0.77289 \\
\hline S3289 G054 & & & & 0.37459 & 0.03636 & 9.28942 \\
\hline S3289_G055 & & & & -0.15999 & 5.1057 & -19.60884 \\
\hline S3289_G056 & 128.7 & 26.5 & 1.2122 & 0.07283 & 0.00246 & 1.60919 \\
\hline S3289_G057 & 625.1 & 82.6 & 1.0016 & 0.09942 & 0.0024 & 1.51906 \\
\hline S3289_G058 & 460.9 & 58.4 & 1.0662 & 0.05946 & 0.00172 & 0.83908 \\
\hline S3289_G059 & 47.9 & 9.4 & 1.0363 & 0.07159 & 0.00342 & 1.5781 \\
\hline S3289 G060 & 133.4 & 15.7 & 0.9154 & 0.05945 & 0.00236 & 0.80854 \\
\hline S3289_G061 & 214.7 & 21.1 & 0.1939 & 0.05684 & 0.00196 & 0.78941 \\
\hline S3289_G062 & 26.3 & 3 & 0.1231 & 0.06242 & 0.00498 & 1.01104 \\
\hline S3289_G063 & 305.9 & 38.2 & 2.8292 & 0.16143 & 0.00444 & 1.67399 \\
\hline S3289_G064 & 71.6 & 13.4 & 0.4625 & 0.07406 & 0.00282 & 1.79866 \\
\hline S3289_G065 & 163.2 & 21 & 1.2961 & 0.0613 & 0.00232 & 0.83193 \\
\hline S3289_G066 & 115.3 & 17 & 0.9473 & 0.06437 & 0.00242 & 1.08504 \\
\hline S3289_G067 & 85.5 & 10.6 & 0.5423 & 0.06456 & 0.00306 & 1.01875 \\
\hline S3289_G068 & 102 & 11.8 & 0.5386 & 0.06072 & 0.00262 & 0.89311 \\
\hline S3289_G069 & 287.3 & 49.6 & 1.274 & 0.0649 & 0.00186 & 1.18888 \\
\hline S3289_G070 & 426.9 & 229.1 & 0.4801 & 0.1778 & 0.0039 & 11.69771 \\
\hline S3289_G071 & 138 & 15.8 & 1.0083 & 0.06026 & 0.00242 & 0.77945 \\
\hline S3289_G072 & 536.6 & 47.4 & 0.2185 & 0.05935 & 0.00162 & 0.74912 \\
\hline S3289_G073 & 31.9 & 6 & 1.7298 & 0.06616 & 0.00428 & 1.19987 \\
\hline S3289_G074 & 47.4 & 7.7 & 0.1036 & 0.07169 & 0.00334 & 1.67313 \\
\hline S3289_G075 & 26.8 & 2.8 & 0.3539 & 0.06254 & 0.0057 & 0.88108 \\
\hline S3289_G076 & 216.8 & 29.7 & 0.3652 & 0.06517 & 0.00192 & 1.19201 \\
\hline S3289_G077 & & & & 0.82107 & 0.01824 & 420.51776 \\
\hline S3289_G078 & 89.6 & 12.5 & 0.4478 & 0.07372 & 0.0029 & 1.34488 \\
\hline S3289_G079 & 56.1 & 6.8 & 1.1091 & 0.05958 & 0.0035 & 0.79373 \\
\hline S3289_G080 & 134.4 & 15.6 & 0.5991 & 0.06228 & 0.0024 & 0.90627 \\
\hline S3289_G081 & 4134.5 & 159.5 & 0.1575 & 0.06653 & 0.00152 & 0.37484 \\
\hline S3289_G082 & 129.8 & 20.6 & 0.8139 & 0.06718 & 0.00232 & 1.25775 \\
\hline S3289_G083 & 69.5 & 10.3 & 1.8741 & 0.05976 & 0.00312 & 0.82009 \\
\hline S3289_G084 & 247.2 & 31.4 & 0.409 & 0.06631 & 0.00208 & 1.1127 \\
\hline S3289_G085 & & & & 0.21178 & 0.01258 & 3.52038 \\
\hline S3289_G086 & 821.3 & 230.1 & 0.2422 & 0.16726 & 0.00376 & 6.48633 \\
\hline S3289_G087 & 22.7 & 2.3 & 0.4137 & 0.06296 & 0.00584 & 0.83748 \\
\hline S3289_G088 & 257.5 & 44.7 & 0.9299 & 0.06817 & 0.00188 & 1.35731 \\
\hline S3289_G089 & 174.1 & 23.7 & 0.7922 & 0.06388 & 0.00222 & 1.04074 \\
\hline S3289_G090 & 403.2 & 49.5 & 0.3078 & 0.07096 & 0.00184 & 1.20882 \\
\hline S3289_G091 & 58.2 & 6.1 & 0.5565 & 0.05767 & 0.00314 & 0.77322 \\
\hline S3289_G092 & 79.3 & 10.1 & 0.4379 & 0.06238 & 0.00284 & 1.04492 \\
\hline S3289_G093 & 41.7 & 4.4 & 0.6924 & 0.05645 & 0.0044 & 0.73625 \\
\hline S3289_G094 & 147.8 & 18.3 & 1.2412 & 0.05818 & 0.0022 & 0.78807 \\
\hline S3289_G095 & 22.1 & 2.5 & 0.3997 & 0.07095 & 0.0065 & 1.04722 \\
\hline S3289_G096 & 686.4 & 82.6 & 0.1107 & 0.06978 & 0.00172 & 1.20321 \\
\hline S3289 G097 & 74.7 & 9.7 & 1.1438 & 0.06212 & 0.00306 & 0.88361 \\
\hline S3289_G098 & 89.1 & 11.9 & 0.1818 & 0.06259 & 0.00258 & 1.17704 \\
\hline S3289_G099 & 152.4 & 19.2 & 0.2097 & 0.06406 & 0.00222 & 1.13043 \\
\hline S3289_G100 & 299.7 & 44.4 & 1.4761 & 0.06145 & 0.00186 & 0.92941 \\
\hline S3289_G101 & 127.7 & 19.8 & 0.6456 & 0.06506 & 0.0022 & 1.25197 \\
\hline S3289_G102 & 52.5 & 5.5 & 0.5295 & 0.05676 & 0.00344 & 0.75993 \\
\hline S3289_G103 & 351.2 & 158.4 & 1.7747 & 0.15178 & 0.00356 & 6.57509 \\
\hline S3289_G104 & 20.1 & 0.4 & 0.8268 & 0.07475 & 0.02398 & 0.15699 \\
\hline S3289_G105 & 781.7 & 40.7 & 1.5356 & 0.05047 & 0.0016 & 0.26349 \\
\hline
\end{tabular}




\begin{tabular}{|c|c|c|c|c|c|c|}
\hline S3289_G106 & 148.8 & 18.4 & 0.9633 & 0.06025 & 0.00222 & 0.85571 \\
\hline S3289_G107 & 439.8 & 47.5 & 0.1734 & 0.06087 & 0.00168 & 0.93222 \\
\hline S3289_G108 & 245.1 & 25.3 & 0.4652 & 0.05652 & 0.00198 & 0.76138 \\
\hline S3289_G109 & 78.8 & 7.8 & 0.4576 & 0.05867 & 0.00308 & 0.7628 \\
\hline S3289_G110 & 829.1 & 79.4 & 0.1529 & 0.05845 & 0.0015 & 0.80075 \\
\hline S3289_G111 & 192.1 & 32.1 & 0.1686 & 0.0719 & 0.0021 & 1.69076 \\
\hline S3289_G112 & 2748.3 & 76.6 & 0.2083 & 0.1288 & 0.0031 & 0.48202 \\
\hline S3289_G113 & 66.4 & 9.4 & 0.4328 & 0.06569 & 0.00282 & 1.21615 \\
\hline S3289_G114 & 117.9 & 16.9 & 0.5996 & 0.06454 & 0.0023 & 1.15929 \\
\hline S3289_G115 & 277.6 & 27.8 & 0.5354 & 0.05796 & 0.00184 & 0.74226 \\
\hline S3289_G116 & 102 & 14.5 & 0.4103 & 0.06568 & 0.00258 & 1.23532 \\
\hline S3289_G117 & 167.4 & 3 & 0.4473 & 0.04584 & 0.00462 & 0.10757 \\
\hline S3289_G118 & 68.5 & 8.2 & 0.7867 & 0.06018 & 0.00346 & 0.85814 \\
\hline S3289_G119 & 265.7 & 93.2 & 0.5548 & 0.11848 & 0.00292 & 5.16602 \\
\hline S3289_G120 & 59.2 & 9.4 & 0.6408 & 0.06606 & 0.00316 & 1.29607 \\
\hline S3289_G121 & 211.1 & 36.1 & 0.2044 & 0.07121 & 0.0021 & 1.71572 \\
\hline S3289_G122 & 35.5 & 4.9 & 0.3402 & 0.06665 & 0.00376 & 1.22902 \\
\hline S3289_G123 & 95.8 & 66 & 1.4292 & 0.17051 & 0.00428 & 11.54803 \\
\hline S3289_G124 & 258 & 35.4 & 0.8265 & 0.06254 & 0.00186 & 1.01468 \\
\hline S3289_G125 & 44.8 & 5.2 & 0.8077 & 0.05971 & 0.00394 & 0.81999 \\
\hline S3289_G126 & 210.1 & 22.1 & 0.4331 & 0.05963 & 0.00208 & 0.82735 \\
\hline S3289_G127 & 106.6 & 5.5 & 0.5225 & 0.04894 & 0.00338 & 0.32429 \\
\hline S3289_G128 & 83.4 & 11.3 & 0.6963 & 0.06412 & 0.00296 & 1.06151 \\
\hline S3289_G129 & 97.3 & 44.4 & 1.1216 & 0.14256 & 0.00382 & 7.14247 \\
\hline S3289_G130 & 488.2 & 50.1 & 0.4425 & 0.05412 & 0.00296 & 0.72911 \\
\hline S3289_G131 & 200.8 & 31.9 & 0.469 & 0.06866 & 0.00214 & 1.4158 \\
\hline S3289_G132 & 588.6 & 3.3 & 0.9268 & 0.04641 & 0.00466 & 0.03067 \\
\hline S3289_G133 & 72.1 & 10.4 & 0.5139 & 0.0643 & 0.0029 & 1.19531 \\
\hline S3289_G134 & 36 & 3.9 & 0.6277 & 0.05557 & 0.0044 & 0.74434 \\
\hline S3289_G135 & 48.9 & 5.3 & 0.6259 & 0.06024 & 0.00388 & 0.81944 \\
\hline S3289_G136 & 25.2 & 3.5 & 0.3685 & 0.06123 & 0.00484 & 1.14704 \\
\hline S3289_G137 & 83.9 & 10.3 & 1.1653 & 0.06149 & 0.0029 & 0.82609 \\
\hline S3289_G138 & 231.2 & 23.5 & 1.0167 & 0.05705 & 0.00214 & 0.65515 \\
\hline S3289_G139 & 533.5 & 54.7 & 0.1895 & 0.06058 & 0.0017 & 0.87771 \\
\hline S3289_G140 & 124.6 & 16 & 0.189 & 0.06647 & 0.00242 & 1.20732 \\
\hline S3289_G141 & 488.2 & 78.3 & 9.0774 & 0.09942 & 0.00288 & 0.73069 \\
\hline S3289_G142 & 51 & 7.4 & 0.5466 & 0.07207 & 0.00352 & 1.36192 \\
\hline S3289_G143 & 315.7 & 56.8 & 0.3306 & 0.07128 & 0.00196 & 1.72301 \\
\hline S3289_G144 & 80.3 & 8 & 0.4034 & 0.05782 & 0.0029 & 0.76265 \\
\hline S3289_G145 & 54.6 & 26.3 & 0.5641 & 0.14265 & 0.00422 & 8.3022 \\
\hline S3289_G146 & 89.1 & 16 & 0.4724 & 0.07112 & 0.00272 & 1.64969 \\
\hline S3289_G147 & 2630.4 & 195.9 & 1.0209 & 0.09121 & 0.00228 & 0.83435 \\
\hline S3289_G148 & & & & 0.85063 & 0.02026 & \\
\hline S3289_G149 & 152.9 & 28.4 & 3.3188 & 0.05763 & 0.00216 & 0.76726 \\
\hline S3289_G150 & 38.6 & 3.9 & 0.0779 & 0.05698 & 0.00388 & 0.8357 \\
\hline
\end{tabular}


Jrdant ages $\quad \mathbf{8 2 . 0} \%$ concordant

\begin{tabular}{|c|c|c|c|c|c|c|}
\hline $2 \sigma 75$ & Pb206/U238 & $2 \sigma 68$ & $\begin{array}{c}\text { ages } \\
\text { age } 206 / 238\end{array}$ & $2 \sigma$ age 68 & age $207 / 235$ & $2 \sigma$ age 75 \\
\hline 0.01936 & 0.08324 & 0.00202 & 515.4 & 12 & 514.3 & 14.8 \\
\hline 0.08514 & 0.16104 & 0.00516 & 962.6 & 28.6 & 956.2 & 39.6 \\
\hline 0.09118 & 0.19533 & 0.0048 & 1150.2 & 25.8 & 1514.3 & 27.2 \\
\hline 0.03554 & 0.12964 & 0.00322 & 785.8 & 18.4 & 780.2 & 21 \\
\hline 0.04922 & 0.10455 & 0.00262 & 641 & 15.2 & 1023.8 & 23.6 \\
\hline 0.04104 & 0.12206 & 0.00326 & 742.4 & 18.8 & 735.6 & 24.2 \\
\hline 0.0376 & 0.11081 & 0.00296 & 677.5 & 17.2 & 676.9 & 23.4 \\
\hline 0.0378 & 0.12252 & 0.00312 & 745.1 & 18 & 771.6 & 22.2 \\
\hline 0.02512 & 0.1044 & 0.0025 & 640.1 & 14.6 & 674.2 & 16.8 \\
\hline 0.04154 & 0.1034 & 0.00298 & 634.3 & 17.4 & 629.9 & 26.6 \\
\hline 0.05402 & 0.17813 & 0.00444 & 1056.8 & 24.2 & 1051.2 & 24.6 \\
\hline 0.02616 & 0.10379 & 0.00256 & 636.6 & 15 & 629.8 & 17.8 \\
\hline 0.0464 & 0.13044 & 0.00354 & 790.4 & 20.2 & 784.2 & 26.2 \\
\hline 0.0611 & 0.11637 & 0.00376 & 709.6 & 21.8 & 698.3 & 35.8 \\
\hline 0.0354 & 0.11987 & 0.00308 & 729.8 & 17.8 & 715.7 & 21.8 \\
\hline 0.02242 & 0.06639 & 0.00174 & 414.4 & 10.6 & 470.8 & 17.2 \\
\hline 0.0465 & 0.09686 & 0.00296 & 596 & 17.4 & 645.7 & 29.4 \\
\hline 0.02754 & 0.11691 & 0.00282 & 712.8 & 16.2 & 709.2 & 17.8 \\
\hline 0.02418 & 0.10801 & 0.00258 & 661.2 & 15 & 674 & 16.4 \\
\hline 0.03568 & 0.1044 & 0.00282 & 640.1 & 16.4 & 647.6 & 23 \\
\hline 0.03372 & 0.10461 & 0.00276 & 641.4 & 16.2 & 641.8 & 21.8 \\
\hline 0.03492 & 0.12342 & 0.00312 & 750.2 & 18 & 749.4 & 21 \\
\hline 0.02932 & 0.09804 & 0.00254 & 602.9 & 15 & 602.4 & 20 \\
\hline 0.20832 & 0.36946 & $\begin{array}{c}0.01024 \\
359.49036\end{array}$ & 2026.9 & 48.2 & 2032.8 & 36.4 \\
\hline 0.04404 & 0.09726 & 0.00298 & 598.3 & 17.6 & 581.6 & 29 \\
\hline 0.04372 & 0.13176 & 0.00344 & 797.9 & 19.6 & 820.7 & 24.2 \\
\hline 0.01924 & 0.06172 & 0.00166 & 386.1 & 10 & 382.1 & 16 \\
\hline 0.02524 & 0.10313 & 0.00246 & 632.7 & 14.4 & 699.8 & 16.8 \\
\hline 0.03384 & 0.12407 & 0.00312 & 753.9 & 17.8 & 740.2 & 20.6 \\
\hline 0.05226 & 0.13655 & 0.00382 & 825.1 & 21.6 & 810.5 & 28.6 \\
\hline 0.0741 & 0.13324 & 0.00438 & 806.3 & 25 & 834.9 & 38.6 \\
\hline 0.0616 & 0.17135 & 0.00456 & 1019.5 & 25 & 1015.3 & 28.2 \\
\hline 0.13748 & 0.34175 & 0.00828 & 1895.1 & 39.8 & 1884.4 & 28.6 \\
\hline 0.05006 & 0.0992 & 0.0027 & 609.7 & 15.8 & 896.6 & 26.4 \\
\hline 0.04012 & 0.12883 & 0.00336 & 781.2 & 19.2 & 769.4 & 23.4 \\
\hline 0.03114 & 0.09045 & 0.00246 & 558.2 & 14.6 & 556 & 21.6 \\
\hline 0.00214 & 0.00003 & 0.00004 & 0.2 & 0.2 & 1.3 & 2.8 \\
\hline 0.04772 & 0.13695 & 0.00364 & 827.4 & 20.6 & 845.4 & 25.6 \\
\hline 0.07092 & 0.13929 & 0.00436 & 840.6 & 24.6 & 851 & 36.4 \\
\hline 0.04092 & 0.13607 & 0.00348 & 822.4 & 19.8 & 822.4 & 23 \\
\hline 0.04466 & 0.12546 & 0.0034 & 761.9 & 19.4 & 762.6 & 25.8 \\
\hline 0.06382 & 0.09569 & 0.00354 & 589.1 & 20.8 & 595 & 40.6 \\
\hline 0.03442 & 0.13569 & 0.0033 & 820.2 & 18.8 & 819.3 & 19.8 \\
\hline 0.14796 & 0.32272 & 0.0082 & 1803 & 40 & 1860.6 & 31 \\
\hline 0.0996 & 0.13885 & 0.00522 & 838.2 & 29.6 & 833.7 & 50.4 \\
\hline 0.0668 & 0.11652 & 0.00396 & 710.5 & 22.8 & 699.8 & 38.8 \\
\hline 0.11714 & 0.19003 & 0.00662 & 1121.5 & 35.8 & 1079.2 & 47.8 \\
\hline 0.12166 & 0.20457 & 0.00506 & 1199.8 & 27 & 1757.3 & 28.8 \\
\hline
\end{tabular}




\begin{tabular}{|c|c|c|c|c|c|c|}
\hline 0.02938 & 0.0965 & 0.00254 & 593.9 & 15 & 589.4 & 20.2 \\
\hline 0.0269 & 0.10349 & 0.0026 & 634.8 & 15.2 & 625.5 & 18.4 \\
\hline 0.02918 & 0.09436 & 0.00248 & 581.3 & 14.6 & 581.4 & 20.2 \\
\hline 0.77102 & 0.17991 & 0.01384 & & & & \\
\hline 600.20892 & 0.88916 & 8.41218 & & & & \\
\hline 0.05602 & 0.16029 & 0.00422 & 958.4 & 23.4 & 973.8 & 26.8 \\
\hline 0.04014 & 0.11084 & 0.0027 & 677.6 & 15.6 & 938.1 & 21 \\
\hline 0.02576 & 0.10238 & 0.00254 & 628.3 & 14.8 & 618.6 & 17.6 \\
\hline 0.07468 & 0.15991 & 0.0048 & 956.3 & 26.6 & 961.6 & 35 \\
\hline 0.0323 & 0.09867 & 0.00266 & 606.6 & 15.6 & 601.6 & 21.8 \\
\hline 0.02794 & 0.10076 & 0.0026 & 618.9 & 15.2 & 590.8 & 19.2 \\
\hline 0.0783 & 0.11751 & 0.00436 & 716.2 & 25.2 & 709.4 & 45 \\
\hline 0.04792 & 0.07523 & 0.00194 & 467.6 & 11.6 & 998.7 & 24 \\
\hline 0.06956 & 0.17619 & 0.00486 & 1046.1 & 26.6 & 1045 & 30.6 \\
\hline 0.03192 & 0.09846 & 0.00262 & 605.4 & 15.4 & 614.7 & 21.4 \\
\hline 0.04134 & 0.12229 & 0.00328 & 743.7 & 18.8 & 746.1 & 24.4 \\
\hline 0.04782 & 0.11448 & 0.00334 & 698.7 & 19.4 & 713.3 & 28.6 \\
\hline 0.03866 & 0.10671 & 0.00298 & 653.6 & 17.4 & 648 & 24.6 \\
\hline 0.03598 & 0.13291 & 0.00332 & 804.4 & 18.8 & 795.4 & 21 \\
\hline 0.29062 & 0.4773 & 0.01148 & 2515.5 & 50 & 2580.5 & 30.4 \\
\hline 0.0315 & 0.09384 & 0.00254 & 578.2 & 15 & 585.2 & 21.6 \\
\hline 0.02192 & 0.09157 & 0.00226 & 564.8 & 13.4 & 567.7 & 16 \\
\hline 0.07568 & 0.13157 & 0.00446 & 796.8 & 25.4 & 800.5 & 40.4 \\
\hline 0.07744 & 0.16931 & 0.00504 & 1008.3 & 27.8 & 998.4 & 35.2 \\
\hline 0.07796 & 0.10221 & 0.00396 & 627.3 & 23.2 & 641.6 & 47.2 \\
\hline 0.037 & 0.1327 & 0.00334 & 803.3 & 19 & 796.9 & 21.4 \\
\hline 11.81596 & 3.71568 & 0.103 & & & & \\
\hline 0.0534 & 0.13236 & 0.00366 & 801.3 & 20.8 & 865.3 & 28 \\
\hline 0.04588 & 0.09664 & 0.003 & 594.7 & 17.6 & 593.3 & 29.8 \\
\hline 0.0354 & 0.10556 & 0.00284 & 646.9 & 16.6 & 655.1 & 22.6 \\
\hline 0.0096 & 0.04088 & 0.00098 & 258.3 & 6 & 323.2 & 9.2 \\
\hline 0.04444 & 0.13582 & 0.00358 & 821 & 20.4 & 826.9 & 24.6 \\
\hline 0.04224 & 0.09956 & 0.00296 & 611.8 & 17.4 & 608.1 & 27.4 \\
\hline 0.0363 & 0.12173 & 0.00312 & 740.5 & 18 & 759.5 & 21.6 \\
\hline 0.19058 & 0.1206 & 0.00508 & & & & \\
\hline 0.16346 & 0.28134 & 0.00678 & 1598.1 & 34.2 & 2044 & 29 \\
\hline 0.07522 & 0.0965 & 0.00384 & 593.9 & 22.6 & 617.8 & 46.8 \\
\hline 0.04008 & 0.14446 & 0.0036 & 869.8 & 20.2 & 870.7 & 21.8 \\
\hline 0.037 & 0.1182 & 0.0031 & 720.2 & 17.8 & 724.3 & 22.6 \\
\hline 0.03394 & 0.1236 & 0.00304 & 751.3 & 17.4 & 804.6 & 19.8 \\
\hline 0.04164 & 0.09728 & 0.0029 & 598.4 & 17 & 581.6 & 27.4 \\
\hline 0.04728 & 0.12153 & 0.00346 & 739.4 & 19.8 & 726.4 & 27.8 \\
\hline 0.056 & 0.09462 & 0.0033 & 582.8 & 19.4 & 560.2 & 36.8 \\
\hline 0.0303 & 0.09828 & 0.00262 & 604.3 & 15.4 & 590.1 & 20.8 \\
\hline 0.0927 & 0.10708 & 0.00436 & 655.8 & 25.4 & 727.5 & 52 \\
\hline 0.03238 & 0.1251 & 0.00304 & 759.9 & 17.4 & 802.1 & 19.2 \\
\hline 0.0431 & 0.1032 & 0.00302 & 633.1 & 17.6 & 642.9 & 27.2 \\
\hline 0.04896 & 0.13644 & 0.00378 & 824.5 & 21.4 & 789.9 & 27.2 \\
\hline 0.04016 & 0.12803 & 0.00336 & 776.6 & 19.2 & 768 & 23.4 \\
\hline 0.02966 & 0.10973 & 0.00278 & 671.2 & 16.2 & 667.3 & 19.4 \\
\hline 0.0438 & 0.13961 & 0.00366 & 842.5 & 20.8 & 824.3 & 24.2 \\
\hline 0.04518 & 0.09713 & 0.00304 & 597.6 & 17.8 & 574 & 30 \\
\hline 0.1717 & 0.31431 & 0.00768 & 1761.9 & 37.6 & 2056 & 29.8 \\
\hline 0.04872 & 0.01524 & 0.00134 & 97.5 & 8.6 & 148.1 & 45.8 \\
\hline 0.00872 & 0.03788 & 0.00096 & 239.7 & 6 & 237.5 & 8.6 \\
\hline
\end{tabular}




\begin{tabular}{|c|c|c|c|c|c|c|}
\hline 0.03202 & 0.10305 & 0.00272 & 632.3 & 15.8 & 627.8 & 21.2 \\
\hline 0.02748 & 0.11111 & 0.00276 & 679.2 & 16 & 668.8 & 18.2 \\
\hline 0.02746 & 0.09774 & 0.00254 & 601.2 & 15 & 574.8 & 19.2 \\
\hline 0.0395 & 0.09434 & 0.0028 & 581.2 & 16.4 & 575.6 & 26.6 \\
\hline 0.02242 & 0.0994 & 0.00244 & 610.9 & 14.4 & 597.3 & 16 \\
\hline 0.05234 & 0.17062 & 0.00434 & 1015.5 & 23.8 & 1005.1 & 24.8 \\
\hline 0.01276 & 0.02715 & 0.00066 & 172.7 & 4.2 & 399.5 & 11.2 \\
\hline 0.05246 & 0.13432 & 0.0038 & 812.5 & 21.6 & 808 & 28.6 \\
\hline 0.04238 & 0.13032 & 0.00346 & 789.7 & 19.8 & 781.6 & 24.2 \\
\hline 0.02458 & 0.09293 & 0.00236 & 572.8 & 14 & 563.7 & 17.6 \\
\hline 0.049 & 0.13646 & 0.00374 & 824.6 & 21.2 & 816.8 & 26.8 \\
\hline 0.01052 & 0.01703 & 0.00062 & 108.9 & 4 & 103.7 & 10.6 \\
\hline 0.04848 & 0.10347 & 0.00322 & 634.7 & 18.8 & 629.1 & 30.6 \\
\hline 0.14042 & 0.31636 & 0.00784 & 1771.9 & 38.4 & 1847 & 29.8 \\
\hline 0.06166 & 0.14235 & 0.00424 & 857.9 & 24 & 844 & 32.4 \\
\hline 0.05368 & 0.17483 & 0.00446 & 1038.7 & 24.4 & 1014.4 & 25 \\
\hline 0.06812 & 0.1338 & 0.00424 & 809.5 & 24.2 & 813.9 & 36.2 \\
\hline 0.32042 & 0.4914 & 0.0125 & 2576.7 & 54 & 2568.5 & 33.4 \\
\hline 0.03178 & 0.11773 & 0.00298 & 717.5 & 17.2 & 711.2 & 20 \\
\hline 0.05292 & 0.09965 & 0.0033 & 612.4 & 19.4 & 608 & 33.8 \\
\hline 0.02972 & 0.10068 & 0.00264 & 618.4 & 15.4 & 612.1 & 20 \\
\hline 0.02182 & 0.04808 & 0.00154 & 302.7 & 9.4 & 285.2 & 19 \\
\hline 0.04864 & 0.12012 & 0.00348 & 731.3 & 20 & 734.6 & 28.6 \\
\hline 0.20738 & 0.36355 & 0.0094 & 1999 & 44.4 & 2129.4 & 33.2 \\
\hline 0.03922 & 0.09776 & 0.00298 & 601.3 & 17.4 & 556 & 26.8 \\
\hline 0.04602 & 0.14963 & 0.00386 & 898.9 & 21.6 & 895.6 & 24 \\
\hline 0.00298 & 0.00479 & 0.00018 & 30.8 & 1.2 & 30.7 & 3.2 \\
\hline 0.05404 & 0.1349 & 0.00388 & 815.8 & 22 & 798.4 & 29.6 \\
\hline 0.05744 & 0.0972 & 0.00346 & 598 & 20.4 & 564.9 & 37.6 \\
\hline 0.05146 & 0.09871 & 0.00324 & 606.8 & 19 & 607.7 & 33 \\
\hline 0.08842 & 0.13594 & 0.00502 & 821.7 & 28.4 & 775.8 & 47.4 \\
\hline 0.03876 & 0.09748 & 0.00282 & 599.6 & 16.6 & 611.4 & 25.4 \\
\hline 0.02492 & 0.08332 & 0.0022 & 515.9 & 13 & 511.6 & 18.4 \\
\hline 0.02626 & 0.10513 & 0.00262 & 644.4 & 15.2 & 639.7 & 17.8 \\
\hline 0.045 & 0.13179 & 0.00354 & 798.1 & 20.2 & 804 & 25.2 \\
\hline 0.02216 & 0.05333 & 0.00136 & 334.9 & 8.4 & 557 & 16.6 \\
\hline 0.06572 & 0.13712 & 0.00418 & 828.4 & 23.6 & 872.7 & 33.8 \\
\hline 0.05104 & 0.17539 & 0.0044 & 1041.7 & 24.2 & 1017.1 & 24 \\
\hline 0.03788 & 0.09571 & 0.0028 & 589.2 & 16.4 & 575.5 & 25.6 \\
\hline 0.26316 & 0.42233 & 0.01152 & 2271 & 52.2 & 2264.6 & 36.4 \\
\hline 0.06396 & 0.16831 & 0.00464 & 1002.8 & 25.6 & 989.4 & 29.8 \\
\hline 0.02282 & 0.06638 & 0.00162 & 414.3 & 9.8 & 616 & 16.2 \\
\hline 43.18768 & 12.81978 & 0.34402 & & & & \\
\hline 0.02942 & 0.09661 & 0.00258 & 594.5 & 15.2 & 578.2 & 20.2 \\
\hline 0.0558 & 0.10643 & 0.00354 & 652 & 20.6 & 616.8 & 35 \\
\hline
\end{tabular}




\begin{tabular}{|c|c|c|c|c|c|}
\hline age $207 / 206$ & $2 \sigma$ age 76 & $\begin{array}{l}\text { discordance } \\
\Delta 68-75[\%]\end{array}$ & $\Delta 68-76[\%]$ & $\begin{array}{c}\text { preferred age } \\
\text { age }\end{array}$ & $2 \sigma$ age \\
\hline 509.6 & 60.4 & 0.2 & 1.1 & 515.4 & 12 \\
\hline 942.4 & 113.4 & 0.7 & 2.1 & 962.6 & 28.6 \\
\hline 2069.2 & 43 & -24 & -44.4 & & \\
\hline 764.9 & 61.8 & 0.7 & 2.7 & 785.8 & 18.4 \\
\hline 1968.2 & 47.8 & -37.4 & -67.4 & & \\
\hline 715.3 & 80.6 & 0.9 & 3.8 & 742.4 & 18.8 \\
\hline 675.8 & 84 & 0.1 & 0.2 & 677.5 & 17.2 \\
\hline 849.9 & 66.6 & -3.4 & -12.3 & 745.1 & 18 \\
\hline 790.4 & 51.2 & -5.1 & -19 & 640.1 & 14.6 \\
\hline 615.1 & 105.2 & 0.7 & 3.1 & 634.3 & 17.4 \\
\hline 1040.1 & 56.8 & 0.5 & 1.6 & 1056.8 & 24.2 \\
\hline 606.1 & 62.6 & 1.1 & 5 & 636.6 & 15 \\
\hline 767.2 & 83.2 & 0.8 & 3 & 790.4 & 20.2 \\
\hline 662.7 & 135.4 & 1.6 & 7.1 & 709.6 & 21.8 \\
\hline 672.4 & 71.8 & 2 & 8.5 & 729.8 & 17.8 \\
\hline 757.1 & 79.2 & -12 & -45.3 & 414.4 & 10.6 \\
\hline 824 & 111.6 & -7.7 & -27.7 & 596 & 17.4 \\
\hline 698.4 & 53.6 & 0.5 & 2.1 & 712.8 & 16.2 \\
\hline 717.7 & 49 & -1.9 & -7.9 & 661.2 & 15 \\
\hline 674.1 & 84.8 & -1.1 & -5 & 640.1 & 16.4 \\
\hline 643.8 & 80.8 & -0.1 & -0.4 & 641.4 & 16.2 \\
\hline 747.6 & 64.6 & 0.1 & 0.4 & 750.2 & 18 \\
\hline 601 & 76.6 & 0.1 & 0.3 & 602.9 & 15 \\
\hline 2039.2 & 54.6 & -0.3 & -0.6 & 2039.2 & 54.6 \\
\hline 517.2 & 127.2 & 2.9 & 15.7 & 598.3 & 17.6 \\
\hline 883.7 & 70.6 & -2.8 & -9.7 & 797.9 & 19.6 \\
\hline 358.5 & 95 & 1 & 7.7 & 386.1 & 10 \\
\hline 921.9 & 47.2 & -9.6 & -31.4 & 632.7 & 14.4 \\
\hline 699.4 & 63.8 & 1.9 & 7.8 & 753.9 & 17.8 \\
\hline 771.1 & 90.2 & 1.8 & 7 & 825.1 & 21.6 \\
\hline 912.4 & 122 & -3.4 & -11.6 & 806.3 & 25 \\
\hline 1006.4 & 70.8 & 0.4 & 1.3 & 1019.5 & 25 \\
\hline 1873.1 & 41.2 & 0.6 & 1.2 & 1873.1 & 41.2 \\
\hline 1691.4 & 64.8 & -32 & -64 & & \\
\hline 735.7 & 73 & 1.5 & 6.2 & 781.2 & 19.2 \\
\hline 547.8 & 93.4 & 0.4 & 1.9 & 558.2 & 14.6 \\
\hline 3356.3 & 2952.2 & -85.3 & -100 & & \\
\hline 893.3 & 74.4 & -2.1 & -7.4 & 827.4 & 20.6 \\
\hline 878.6 & 113.8 & -1.2 & -4.3 & 840.6 & 24.6 \\
\hline 822.8 & 66 & 0 & 0 & 822.4 & 19.8 \\
\hline 765.2 & 83.4 & -0.1 & -0.4 & 761.9 & 19.4 \\
\hline 618.3 & 178 & -1 & -4.7 & 589.1 & 20.8 \\
\hline 817.1 & 53.6 & 0.1 & 0.4 & 820.2 & 18.8 \\
\hline 1926.2 & 46.4 & -3.1 & -6.4 & 1926.2 & 46.4 \\
\hline 822.5 & 167.6 & 0.5 & 1.9 & 838.2 & 29.6 \\
\hline 665.8 & 147.8 & 1.5 & 6.7 & 710.5 & 22.8 \\
\hline 995.2 & 128.2 & 3.9 & 12.7 & & \\
\hline 2504.6 & 40.2 & -31.7 & -52.1 & & \\
\hline
\end{tabular}




\begin{tabular}{|c|c|c|c|c|c|}
\hline 572.6 & 79.4 & 0.8 & 3.7 & 593.9 & 15 \\
\hline 592.4 & 65.4 & 1.5 & 7.2 & 634.8 & 15.2 \\
\hline 582.5 & 80.4 & 0 & -0.2 & 581.3 & 14.6 \\
\hline 1009.2 & 68.4 & -1.6 & -5 & 958.4 & 23.4 \\
\hline 1613.3 & 45 & -27.8 & -58 & & \\
\hline 584 & 62.8 & 1.6 & 7.6 & 628.3 & 14.8 \\
\hline 974.3 & 97.4 & -0.6 & -1.9 & 956.3 & 26.6 \\
\hline 583.6 & 86.2 & 0.8 & 3.9 & 606.6 & 15.6 \\
\hline 485.3 & 76.2 & 4.7 & 27.5 & 618.9 & 15.2 \\
\hline 688.5 & 170.2 & 1 & 4 & 716.2 & 25.2 \\
\hline 2470.7 & 46.4 & -53.2 & -81.1 & & \\
\hline 1043.1 & 76.8 & 0.1 & 0.3 & 1046.1 & 26.6 \\
\hline 649.8 & 81.2 & -1.5 & -6.8 & 605.4 & 15.4 \\
\hline 753.8 & 79.4 & -0.3 & -1.3 & 743.7 & 18.8 \\
\hline 760 & 100 & -2 & -8.1 & 698.7 & 19.4 \\
\hline 629.3 & 93 & 0.9 & 3.9 & 653.6 & 17.4 \\
\hline 771.1 & 60.4 & 1.1 & 4.3 & 804.4 & 18.8 \\
\hline 2632.5 & 36.4 & -2.5 & -4.4 & 2632.5 & 36.4 \\
\hline 612.9 & 86.8 & -1.2 & -5.7 & 578.2 & 15 \\
\hline 580 & 59.2 & -0.5 & -2.6 & 564.8 & 13.4 \\
\hline 811.4 & 135.4 & -0.5 & -1.8 & 796.8 & 25.4 \\
\hline 977.2 & 95 & 1 & 3.2 & 1008.3 & 27.8 \\
\hline 692.6 & 194.4 & -2.2 & -9.4 & 627.3 & 23.2 \\
\hline 779.8 & 62 & 0.8 & 3 & 803.3 & 19 \\
\hline 1033.8 & 79.4 & -7.4 & -22.5 & 801.3 & 20.8 \\
\hline 588.4 & 127.4 & 0.2 & 1.1 & 594.7 & 17.6 \\
\hline 683.7 & 82.2 & -1.2 & -5.4 & 646.9 & 16.6 \\
\hline 823.1 & 47.6 & -20.1 & -68.6 & & \\
\hline 843.4 & 71.8 & -0.7 & -2.7 & 821 & 20.4 \\
\hline 594.9 & 113.2 & 0.6 & 2.8 & 611.8 & 17.4 \\
\hline 816.2 & 65.6 & -2.5 & -9.3 & 740.5 & 18 \\
\hline 2530.4 & 37.8 & -21.8 & -36.8 & & \\
\hline 706.9 & 197.4 & -3.9 & -16 & 593.9 & 22.6 \\
\hline 873.7 & 57.2 & -0.1 & -0.4 & 869.8 & 20.2 \\
\hline 737.7 & 73.6 & -0.6 & -2.4 & 720.2 & 17.8 \\
\hline 956.3 & 53 & -6.6 & -21.4 & 751.3 & 17.4 \\
\hline 517.2 & 119.6 & 2.9 & 15.7 & 598.4 & 17 \\
\hline 687.2 & 97.2 & 1.8 & 7.6 & 739.4 & 19.8 \\
\hline 470.1 & 172.6 & 4 & 24 & 582.8 & 19.4 \\
\hline 536.5 & 82.8 & 2.4 & 12.6 & 604.3 & 15.4 \\
\hline 956 & 187.4 & -9.9 & -31.4 & 655.8 & 25.4 \\
\hline 921.9 & 50.6 & -5.3 & -17.6 & 759.9 & 17.4 \\
\hline 678.2 & 105.2 & -1.5 & -6.7 & 633.1 & 17.6 \\
\hline 694.3 & 87.8 & 4.4 & 18.7 & 824.5 & 21.4 \\
\hline 743.6 & 73.2 & 1.1 & 4.4 & 776.6 & 19.2 \\
\hline 655 & 65 & 0.6 & 2.5 & 671.2 & 16.2 \\
\hline 776.3 & 71.2 & 2.2 & 8.5 & 842.5 & 20.8 \\
\hline 482.2 & 133.8 & 4.1 & 23.9 & 597.6 & 17.8 \\
\hline 2366.1 & 40 & -14.3 & -25.5 & & \\
\hline 1061.8 & 645.4 & -34.1 & -90.8 & & \\
\hline 216.7 & 73.4 & 0.9 & 10.6 & 239.7 & 6 \\
\hline
\end{tabular}




\begin{tabular}{|c|c|c|c|c|c|}
\hline 612.6 & 79.6 & 0.7 & 3.2 & 632.3 & 15.8 \\
\hline 634.6 & 59.4 & 1.6 & 7 & 679.2 & 16 \\
\hline 472.9 & 77.6 & 4.6 & 27.1 & 601.2 & 15 \\
\hline 554.9 & 114.6 & 1 & 4.7 & 581.2 & 16.4 \\
\hline 546.7 & 56 & 2.3 & 11.7 & 610.9 & 14.4 \\
\hline 983.1 & 59.4 & 1 & 3.3 & 1015.5 & 23.8 \\
\hline 2081.7 & 42.4 & -56.8 & -91.7 & & \\
\hline 796.5 & 90 & 0.6 & 2 & 812.5 & 21.6 \\
\hline 759.4 & 75.2 & 1 & 4 & 789.7 & 19.8 \\
\hline 528.3 & 69.6 & 1.6 & 8.4 & 572.8 & 14 \\
\hline 796.2 & 82.4 & 1 & 3.6 & 824.6 & 21.2 \\
\hline-10.8 & 243.4 & 4.9 & -1108.7 & 108.9 & 4 \\
\hline 610.1 & 124.2 & 0.9 & 4 & 634.7 & 18.8 \\
\hline 1933.4 & 44.2 & -4.1 & -8.4 & 1933.4 & 44.2 \\
\hline 808.3 & 100 & 1.7 & 6.1 & 857.9 & 24 \\
\hline 963.4 & 60.2 & 2.4 & 7.8 & 1038.7 & 24.4 \\
\hline 826.9 & 117.6 & -0.5 & -2.1 & 809.5 & 24.2 \\
\hline 2562.7 & 42 & 0.3 & 0.5 & 2562.7 & 42 \\
\hline 692.6 & 63.4 & 0.9 & 3.6 & 717.5 & 17.2 \\
\hline 593.1 & 143 & 0.7 & 3.3 & 612.4 & 19.4 \\
\hline 590.2 & 75.6 & 1 & 4.8 & 618.4 & 15.4 \\
\hline 145 & 162 & 6.1 & 108.8 & & \\
\hline 745.6 & 97.6 & -0.5 & -1.9 & 731.3 & 20 \\
\hline 2258.5 & 46.2 & -6.1 & -11.5 & 2258.5 & 46.2 \\
\hline 376 & 123 & 8.1 & 59.9 & & \\
\hline 888.5 & 64.4 & 0.4 & 1.2 & 898.9 & 21.6 \\
\hline 19 & 241.2 & 0.4 & 62.4 & 30.8 & 1.2 \\
\hline 751.5 & 95.2 & 2.2 & 8.5 & 815.8 & 22 \\
\hline 435.2 & 176.4 & 5.8 & 37.4 & & \\
\hline 612.2 & 139.2 & -0.1 & -0.9 & 606.8 & 19 \\
\hline 647.3 & 169.8 & 5.9 & 26.9 & & \\
\hline 656.4 & 101.2 & -1.9 & -8.7 & 599.6 & 16.6 \\
\hline 493.5 & 82.6 & 0.8 & 4.5 & 515.9 & 13 \\
\hline 624.4 & 60.6 & 0.7 & 3.2 & 644.4 & 15.2 \\
\hline 821.2 & 76 & -0.7 & -2.8 & 798.1 & 20.2 \\
\hline 1613.3 & 54 & -39.9 & -79.2 & & \\
\hline 987.9 & 99.4 & -5.1 & -16.2 & 828.4 & 23.6 \\
\hline 965.5 & 56.2 & 2.4 & 7.9 & 1041.7 & 24.2 \\
\hline 522.9 & 110 & 2.4 & 12.7 & 589.2 & 16.4 \\
\hline 2259.6 & 51 & 0.3 & 0.5 & 2259.6 & 51 \\
\hline 960.9 & 78.2 & 1.4 & 4.4 & 1002.8 & 25.6 \\
\hline 1451 & 47.6 & -32.7 & -71.4 & & \\
\hline 515.7 & 82.4 & 2.8 & 15.3 & 594.5 & 15.2 \\
\hline 490.8 & 150.2 & 5.7 & 32.9 & & \\
\hline
\end{tabular}

